\title{
ANALYTICAL RESULTS, DATABASE MANAGEMENT AND QUALITY ASSURANCE FOR ANALYSIS OF SOIL AND GROUNDWATER SAMPLES COLLECTED BY CONE PENETROMETER FROM THE F AND H AREA SEEPAGE BASINS (U)
}

October 1994

UNCLASSIFIED

Does Not Contain Unclassified Controlled Nuclear Information (UCNI)

Westinghouse Savannah River Company

Savannah River Site

Aiken, SC 29808

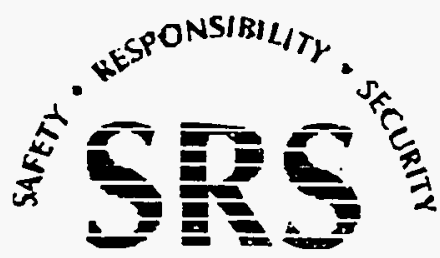

Prepared for the U. S. Department of Energy under Contract No. DE-AC09-89SR18035

SAVANNAH RIVER SITE

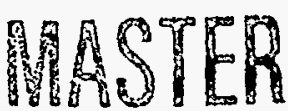

DISTRIBUTION OF THIS DOCUMENT IS UNLIMITED 
WSRC-TR-94-0484

Rev. 0

\section{ANALYTICAL RESULTS, DATABASE MANAGEMENT AND QUALITY ASSURANCE FOR ANALYSIS OF SOIL AND GROUNDWATER SAMPLES COLLECTED BY CONE PENETROMETER FROM THE F AND H AREA SEEPAGE BASINS (U)}

Revision 0, Prepared by:

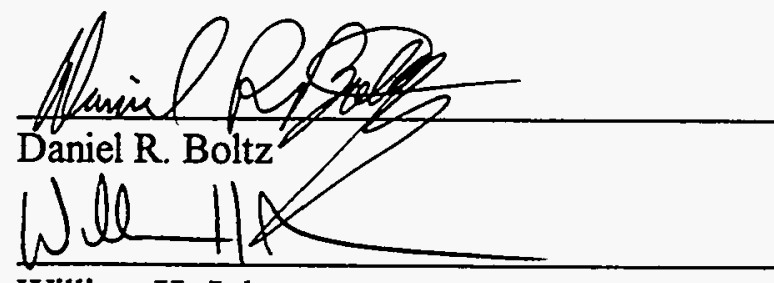

William $\mathrm{H}$. Johnson

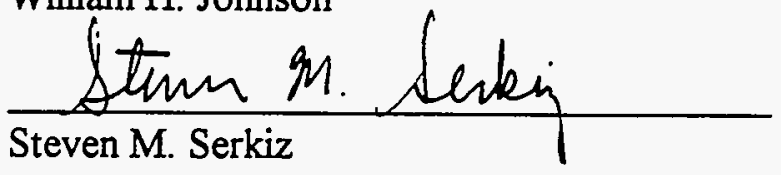

Reviewed and Approved by:

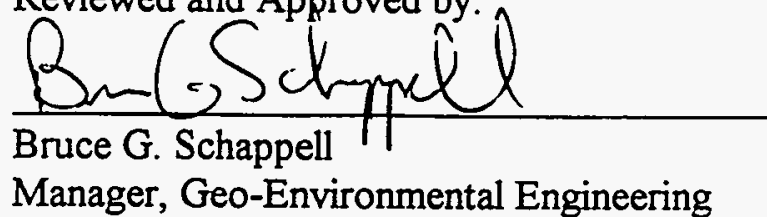

Manager, Geo-Environmental Engineering

$$
\begin{aligned}
& \frac{10 / 26 / 94}{\text { Date }} \\
& \frac{10 / 26 / 94}{\text { Date }} \\
& \frac{10 / 26 / 94}{\text { Date }}
\end{aligned}
$$

$$
\frac{10 / 26 / 94}{\text { Date }}
$$

Westinghouse Savannah River Company

Savannah River Site

Aiken, SC 29808

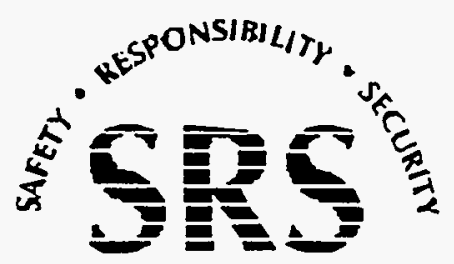

Prepared for the U. S. Department of Energy under Contract No. DE-AC09-89SR18035 


\section{DISCLAIMER}

This report was prepared by Westinghouse Savannah River Company (WSRC) for the United States Department of Energy under Contract No. DE-AC09-89SR18035 and is an account of work performed under that contract. Reference herein to any specific commercial product, process, or service by the trade name, trademark, manufacturer or otherwise does not necessarily constitute or imply endorsement, recommendation, or favoring of same by WSRC or by the United States Government or any agency thereof.

\section{DISCLAIMER}

This report was prepared as an account of work sponsored by an agency of the United States This reportes Government nor any agency thereof, nor any of their Government. makes any warranty, express or implied, or assumes any legal liability or responsiemployees, maks any wertust, or bility for the accuracy, completess, or use would not infringe privately owned rights. Referprocess disclosed, or represents that its use would not infinge pr service by trade name, trademark, ence herein to any specific commercial product, process, or server or imply its endorsement, recommanufacturer, or otherwise does not necssatly constitue or any agency thereof. The views mendation, or favoring by the United States Government or any agence or reflect those of the and opinions of authors expressed herein do not

Westinghouse Savannah River Company

Savannah River Site

Aiken, SC 29808

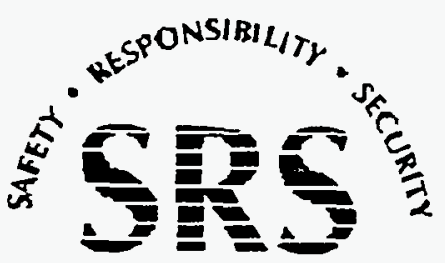

Prepared for the U.S. Department of Energy under Contract No. DE-AC09-89SR18035 


\section{DISCLAIMER}

Portions of this document may be illegible in electronic image products. Images are produced from the best available original document. 


\section{EXECUTIVE SUMMARY}

The Quantification of Soil Source Terms and Determination of the Geochemistry Controlling Distribution Coefficients $\left(\mathrm{K}_{d}\right.$ values) of Contaminants at the $\mathrm{F}$ - and $\mathrm{H}$-Area Seepage Basins (FHSB) study was designed to generate site-specific contaminant transport factors for contaminated groundwater downgradient of the Basins. The experimental approach employed in this study was to collect soil and its associated porewater from contaminated areas downgradient of the FHSB. Samples were collected over a wide range of geochemical conditions (e.g., $\mathrm{pH}$, conductivity, and contaminant concentration) and were used to describe the partitioning of contaminants between the aqueous phase and soil surfaces at the site. The partitioning behavior may be used to develop site-specific transport factors.

This report summarizes the analytical procedures and results for both soil and porewater samples collected as part of this study and the database management of these data.

Keywords: Analytical results, F and H Seepage Basins, soil, groundwater 
WSRC-TR-94-0484 
CONTENTS

EXECUTIVE SUMMARY

CONTENTS

$\mathrm{v}$

LIST OF FIGURES vii

LIST OF TABLES $\quad$ ix

1.0 INTRODUCTION 1

2.0 EXPERIMENTAL APPROACH

2.1 Sampling 2

2.2 Field Analysis 3

2.3 Laboratory Analysis 3

2.3.1 Groundwater Samples 3

2.3.2 Soil Samples 4

2.3.3 ICP-MS Analysis 5

2.3.3.1 Elemental Response/Interference $\quad 6$

3.0 MATHEMATICAL MANIPULATIONS AND DATABASE MANAGEMENT

$\begin{array}{ll}\text { 3.1 Weighted Average Method } & 19\end{array}$

4.0 ANALYTICAL RESULTS 21

4.1 Soil 21

4.2 Groundwater 21

5.0 QUALITY CONTROL/QUALITY ASSURANCE

6.0 REFERENCES 27

Appendix A ANALYTICAL PROCEDURES A-1

Appendix B RESULTS OF F AND H AREA BASIN SOIL ANALYSES B-1

Appendix C RESULTS OF F AND H AREA BASIN WATER ANALYSES C-1

Appendix D DATABASE DICTIONARY D-1

Appendix E MICROSOFT ACCESS BASIC MODULES F-1 
WSRC-TR-94-0484 


\section{LIST OF FIGURES}

2-1 Areal view of F-Area Sampling Transects

2-2 Areal view of H-Area Sampling Transects

12

2-3 Cross section of 'Transect $A-A^{\prime}$

2-4 Cross section of Transect B-B'

2-5 Cross section of 'Transect C-C'

2-6 Cross section of Transect D-D'

2-7 Cross section of Transect $\mathbf{E}-\mathbf{E}^{\prime}$

2-8 Cross section of Transect F-F'

4-1 Soil Classification for the F-Area soil samples.

4-2 Soil Classification for the H-Area soil samples. 


\section{LIST OF TABLES}

2-1 Cone penetrometer test sampling locations and depths.

2-2 Analysis performed on groundwater samples 9

2-3 Analysis performed on soil samples 9

2-4 Elements analyzed for in soil and water samples using ICP-MS 9

2-5 Approximate ICP-MS detection limits 10

5-1 QAVC controls on analyses $\quad 26$ 


\subsection{INTRODUCTION}

To properly assess the risk and potential remedial alternatives at a waste site, knowledge of the geochemical transport characteristics of each contaminant is required.

Contaminants that are soluble in water may not travel through the subsurface environment at the same velocity as the water due to the processes of solid-phase sorption and precipitation. Therefore, to accurately predict contaminant transport a knowledge of both aqueous-phase and surface speciation is necessary.

This report summarizes the analytical results for both soil and porewater samples collected as part of this study and the database management of these data. In particular, this report addresses the following subjects:

- the analytical methods used to characterize soil and porewater samples;

- analytical results for porewater and soil contaminant concentrations and physical soil properties;

- mathematical manipulations and database management of the porewater and soil data ; and

- the quality assurance/quality control (QA/QC) activities associated with this project.

This report is organized as follows:

- Section 1 is the introduction including a brief description of the project;

- Section 2 describes the experimental procedures employed to collect and characterize soil and porewater samples ;

- a description of the mathematical manipulations and database management of the porewater and soil data is contained in Section 3;

- the analytical results are summarized in Section 4; and

- Section 5 is a description of the QAQQC activities associated with this project. 


\subsection{EXPERIMENTAL PROCEDURES}

\subsection{Sample Collection}

Electric cone penetrometer testing (ECPT) and direct push technology (DPT) were performed in the F and H Areas between May 24, 1993 and September 10, 1993. Ebasco Environmental supplied technical, and health and safety oversight for the task and maintained all pertinent field logs and documentation. All appropriate data collected throughout the project is included in the appendices to this report. Subsurface Technology, Inc. (STI) supplied a 20 ton cone penetrometer rig with on earth anchoring system. This minimally invasive site characterization technique was carried out at 28 locations, including 19 in F Area and 9 in H Area. Results of the field activities are presented in the Cone Penetrometer Sampling Report (Ebasco 1993).

Geotechnical data collected included sleeve resistance, tip pressure and pore pressure. Four sites were tested with the ECPT probe and the data was used to generate soil classification plots allowing for correlation across the various lithologic units in the immediate area. The bulk of the task dealt with the collection of groundwater and vadose and saturated soil samples. These samples were collected under an inert gas stream (argon) to minimize chemical reactions with the atmosphere. Altogether 54 groundwater samples, 32 vadose and 52 saturated soil samples were collected for a total of 138 sampling events. Replicate samples (i.e. saturated zone sample pairs and soil samples from the vadose zone) were collected at ten percent of the locations selected on a random basis. Two deep groundwater samples were collected from the top of the Green Clay interval.

Sampling locations and depths were selected such that they spanned a representative range of groundwater $\mathrm{pH}(\mathrm{pH}=3-7)$ and major ion chemistry. In addition, locations were selected which previously exhibited measurable groundwater contaminant concentrations. The areal view of the sampling transects, along with $\mathrm{pH}$ and RCRA metals concentration contours are shown in Figures 2-1 and 2-2 for the $\mathrm{F}$ and the $\mathrm{H}$ Areas respectively. The cross sections for each of these sampling transects are shown in Figures 2-3 through 2-8 for transects A through F respectively. Sampling depths and site coordinates of CPT sampling locations are summarized in Table 2-1.

All samples collected in the field were identified by a 4 digit sample identification number. The first letter in a sample name (A-F) represents the transect from which it came. The second number (1-5) is the hole in the transect, whereas the third number $(1-4)$ is the sampling depth from which the sample came. The forth digit represents the type of sample; ' $S$ ' for soil, ' $W$ ' for porewater. For example, sample $C 42 S$ is a soil sample from the second depth ( 50 feet) in the second hole of transect $C$. Replicate samples had a fifth digit represented by the symbol $R$. Because of difficulties, the replicate soil sample D13SR, was collected at two separate intervals (102 feet and 106 feet). These subsamples are identified individually as D13SRA and D13SRB, respectively. The two deep groundwater samples collected from the top of the Green Clay interval at sites A5 and B4 are labeled as A55V and B45V respectively. 
Two uncontaminated soil samples representative of the lithographic unit sampled downgradient of the FHSB were collected from the Little Grand Canyon area which is located upgradient of F-Area. The soils were collected from an outcropping at a height of approximately 20 feet above the tan clay layer. This depth is equivalent to that of the deeper samples in this work. These samples were given the designation GC4S and GC5S after a sample labeling scheme already in use in another study at the collection site.

Multiple refusals were encountered at various locations and were related to localized lithologic conditions as well as construction material associated with road buildings, cut and fill trenching operations. Refusals tended to occur in the 110' to 120' range, although one penetration successfully reached $145^{\prime}$. Two methods to drill past refusal were attempted. One method involved spinning the drill pipe and cutting a new hole with a rotary drill bit. The other employed a vibratory motor that sent sonic waves down the push rods to vibrate past refusal zones. Both methods had merit and met with partial success but need further development and experimentation. The test holes were subsequently grouted to surface and the topography returned to its natural state.

\subsection{Field Analyses}

Immediately upon collecting porewater samples, the temperature, $\mathrm{pH}$, redox potential, and conductivity of the sample were measured in the field. Temperature and $\mathrm{pH}$ were measured using a pH meter with a built in temperature probe. Redox potential was measured directly using a combination platinum electrode. Porewater samples were stored in acid-washed 1-liter LDPE bottles. Soil samples were double bagged into 4-1 zip-lock bags. Both porewater and soil samples were then stored in a cooler on ice until the end of the day. After Health Protection personnel verified that the gross beta and gamma count rates, as measured by a portable count rate meter, were not above background levels, the samples were transported to a darkened sample storage cooler which was maintained at a temperature $4^{\circ} \mathrm{C}$.

\subsection{Laboratory Analysis}

Tables 2-2 and 2-3 list all analysis performed on groundwater and soil samples collected in this study.

\subsubsection{Groundwater Samples}

Within a week of sampling, two $10-\mathrm{ml}$ aliquots of each porewater sample were filtered using $0.45 \mu \mathrm{m}$ syringe filters. Both of these aliquots were screened for ${ }^{3} \mathrm{H}$ using liquid scintillation counting (ADS-2424), and $\mathrm{Cl}^{-}, \mathrm{F}^{-}, \mathrm{NO}_{2}{ }^{-}, \mathrm{NO}_{3}{ }^{-}, \mathrm{PO}_{4}{ }^{3-}$, and $\mathrm{SO}_{4}{ }^{2-}$ concentrations using ion chromatography (ADS-2306; ADS-2204). A filtered and 
unacidified 60-ml aliquot of the sample was analyzed for organic and inorganic carbon using an automated carbon analyzer (ADS-2200). The remaining sample was filtered through a $0.45 \mu \mathrm{m}$ cellulose nitrate membrane and acidified to $1 \% \mathrm{v} / \mathrm{v}$ with $70 \%$ ultrapure $\mathrm{HNO}_{3}$. The acidified samples were analyzed quantitatively using inductively coupled plasma (argon) mass spectrometry (see section 2.3.3).

Selected water samples were analyzed with gamma ray spectroscopy analysis to determine radionuclide concentrations. For this analysis, a $500 \mathrm{ml}$ aliquot of the acidifed sample was placed in a 1-liter Marinelli beaker. The samples were then counted with a low background detection system for 20,000 to 50,000 seconds. The radionuclide analyses obtained by gamma spectroscopy are listed in Table D-7.

\subsubsection{Soil Samples}

Physical and chemical properties of the soil samples examined included total elemental analysis, soil $\mathrm{pH}$, particle size distribution, carbon content, cation exchange capacity and hygroscopic moisture content. Additionally, gamma ray spectroscopy was performed on a $200 \mathrm{~cm}^{3}$ aliquot of most soil samples.

Each soil sample was prepared for total elemental analysis using the hydrofluoric acid total digestion procedure outlined by $\operatorname{Lim}(1982)$. Samples were prepared in triplicate by digesting approximately $200 \mathrm{mg}$ of soil in $1 \mathrm{ml}$ of ultra-pure aqua regia and $10 \mathrm{ml}$ of ultrapure HF. Samples were then heated at $100^{\circ} \mathrm{C}$ for 3 hours, filtered through $0.45 \mu \mathrm{m}$ Teflon filters, and diluted to $50 \mathrm{ml}$ with deionized water. After ICP-MS analysis, as described below, contaminant concentrations in the total digestion extract were converted to soil concentrations by multiplying by a conversion factor equal to the extract volume divided by the digested sample weight (see Appendix A, procedure 3.3.1.7, section III.J).

Particle size analysis of each soil sample was performed using either the method presented by Gee (1986) (presented as procedure 3.3.1.5 in Appendix A) or Colquhon (1993) as modified by Johnson (1994). 30 or 40-g aliquots of each sample were prepared for analysis by removing all surface coatings and cementing agents. Organic matter was removed by washing the soils with hydrogen peroxide or sodium hypochlorite; carbonates with sodium acetate or ammonium citrate; and iron oxides using a heated bicarbonate buffered, sodium dithionate-citrate solution. Additionally, EDTA was used in the Colquhon method to remove sulfate. Dispersion of the soil particles was facilitated by shaking the soil overnight in a sodium hexametaphosphate solution. The distribution of the sand fraction $(>53 \mu \mathrm{m})$ of each sample was determined by washing and shaking the dispersed sample through a series of brass sieves. All soil particles passing through the smallest $(53 \mu \mathrm{m})$ sieve were analyzed to determine amount and distribution of the clay $(<2$ $\mu \mathrm{m})$ and silt $(2-53 \mu \mathrm{m})$ fractions using a pipet-sedimentation method. Data from replicate analyses were averaged to obtain the fraction of sand, clay and silt in the sample. 
The cation exchange capacity (CEC) of each soil sample was measured in duplicate using the barium-magnesium exchange technique outlined by Rhoades (1982). A 2-g portion of soil was saturated with barium by equilibration with a $\mathrm{BaCl}_{2}$ solution at an ionic strength of 0.012 and a $\mathrm{pH}$ of 4.5. The soil was then reacted with $\mathrm{MgSO}_{4}$ which replaced the sorbed barium with magnesium. After allowing one hour for initial exchange to occur, the solution conductivity was adjusted to $5 \mathrm{~S} \mathrm{~m}^{-2}$ by the addition of deionized water or additional $\mathrm{MgSO}_{4}$ solution. Samples were then shaken overnight to allow equilibrium to occur. After centrifuging, the concentration of magnesium in the supernate was analyzed using ICP-ES (ADS-1509). The concentration of magnesium exchanged per unit weight of soil is equivalent to the cation exchange capacity and was reported in units of meq $\mathrm{kg}^{-1}$. Procedure 3.3.1.6 in Appendix A details the method used to measure CEC.

Procedure 3.3.1.1 in Appendix A presents the methodology used to determine soil pH. The procedure encompasses the requirements of ASTM procedure D4972-89 (ASTM 1989) and EPA method 9045 (EPA 1986). Approximately $10 \mathrm{~g}$ of soil was allowed to equilibrate with $20 \mathrm{ml}$ of deionized water. The $\mathrm{pH}$ of the water was measured directly. Additionally, soil $\mathrm{pH}$ was measured using a $0.01 \mathrm{M} \mathrm{CaCl}_{2}$ solution instead of deionized water. This allowed the replacement and hydrolyzation of aluminum ions from the soil.

\subsubsection{ICP-MS Analysis}

The acidified samples were analyzed quantitatively using inductively coupled plasma (argon) mass spectrometry (ICP-MS). This analysis method uses a plasma torch to ionize the sample by heating it to $8000^{\circ} \mathrm{K}$. The cations are drawn through a low vacuum interface into a mass spectrometer which determines the isotopic concentration by the charge to mass ratio of the cations. Elemental concentrations can then be calculated by correcting for the natural abundance of an isotope and the ionization efficiency of the element. Before analysis of a sample, standard or blank, each is spiked with a series of internal standards (i.e., elements at known concentrations). Based on counts obtained from these internal standards, calculated concentrations are normalized to correct for instrument response changes.

ICP-MS allows the determination of elemental concentrations of up to 70 elements at $\mathrm{ng} \mathrm{I}^{-1}$ concentrations with minimal sample volume. Because the ICP-MS is designed to detect individual atoms instead of solution activity, the detection limit of ICP-MS is outstanding for metals and long-lived radionuclides. Many elements may be analyzed for by monitoring more than one isotope. ICP-MS analyses were conducted for 51 isotopes representing the 30 elements listed in Table 2-4. Additionally, a semi-quantitative calculation for the isotopes of $\mathrm{Hg}$ and the major actinide isotopes with an atomic mass less than 246 was performed on most water samples and a limited number of soil samples.

The detection limit of ICP-MS is influenced by the alignment and sensitivity of the instrument, the background counts and natural isotopic abundance of the isotope being examined, and ionization potential of the element. 
The lower detection limit (LDL) for ICP-MS analysis may be calculated using the definition set forth by the American Chemical Society (1980), where the LDL is given by the equation:

$$
\mathrm{LDL}=\frac{3 \sigma_{\text {blenk }}}{\left(\mathrm{C}_{\text {tanderd }}-\mathrm{C}_{\text {bleak }}\right)} *[\text { Standard }]
$$

where: $\sigma_{\text {blank }}$ is the standard deviation of a blank sample;

$\mathrm{C}_{\text {atandard }}$ and $\mathrm{C}_{\text {blenk }}$ are the integrated counts obtained from a standard

and the blank respectively; and

[Standard] is the concentration of the standard of the analyte being examined with a concentration of approximately $10 \sigma_{b \text { bank }}$

Typical LDL values for ICP-MS based on these calculations are presented in Table 2-5 for two days when analysis were performed.

In this work, the LDL was assumed to be equal to one-half the lower limit of quantification. The lower limit of quantification was estimated daily based on the linearity of instrument response to standards of known concentration, or if more limiting, was assumed equal to the minimum standard concentration examined that day. The LDL values determined using this method were nearly always greater than the values reported in Table 2-5. Additionally, the concentrations of samples which had large standard deviations, as based on three or more analyses of that sample, were assumed to be less than the LDL.

\subsubsection{Elemental Response/Interference}

The ability of an ICP-MS instrument to produce a detectable and quantifiable signal in response to an element at a given concentration is influenced by many things. Major influencing factors include detector sensitivity, gas flow, integrity of interface components, alignment of the electrostatic tuning fields and component age.

Interferences occur at atomic mass numbers where two or more elements have isotopes of similar mass or doubly ionized atoms are present. Ionized atoms and molecules of atmospheric or ICP gas (argon), or combinations of these atoms and molecules with elements in the sample matrix, may also produce interferences. Common among these species are oxides. The formation of oxides will reduce the response of the instrument to certain elements in the sample as a percentage of the atoms for those elements will be detected at the mass associated with the ionized oxide molecule. Other potential interferences can be caused by a poor mass alignment of the instrument or very large concentrations which will tend cause ions detected to be associated with, and reported as, an element of similar mass. 
Other problems may reduce the analytical sensitivity of a given element or series of elements. These are related to the chemistry of the element and include the formation of insoluble complexes (particularly $\mathrm{PbCl}$ and $\mathrm{AgCl}$ ), a poor ability to ionize and a chemical incompatibility with the structural components instrument (i.e., glassware, tubing, interface cones).

Below is a listing, by element, of common response and interference problems that occurred during analysis of the FHSB groundwater and soil samples.

- Aluminum - common element in the DDI water matrix.

- Arsenic - interference from $\mathrm{ArCl}$; corrected based on detected ${ }^{77} \mathrm{Se}$ to ${ }^{78} \mathrm{Se}$ concentration ratio

- Barium - Interferences from tin oxides

- Cadmium - Accumulates on glassware

- ${ }^{114} \mathrm{Cd}$ has an isobaric interference from tin and $\mathrm{MoO}$; corrected bäsed on ${ }^{98} \mathrm{Mo}$ and ${ }^{118} \mathrm{Sn}$ concentrations.

- Calcium - common element in DDI water matrix

- interference at ${ }^{40} \mathrm{Ca}$ from $\mathrm{Ar}$ in plasma gas; must use ${ }^{44} \mathrm{Ca}$ which has only a $2.1 \%$ abundance and an interference from SiO

- Copper - interferences for titanium oxides and hydroxides in samples with high Ti concentrations

- Iron - common element in DDI water matrix; interference from calcium oxides

- Lead - may form insoluble lead chlorides in a chloride matrix; problems may be present in results for soil extractions which were in $1 \%$ aqua regia

- Magnesium $-{ }^{26} \mathrm{Mg}$ response is affected by high Al concentration; only ${ }^{2 \mathrm{Mg}} \mathrm{M}$ and ${ }^{25} \mathrm{Mg}$ are reported in this work.

- Manganese - response effected by high Fe concentrations; interference from $\mathrm{Cd}^{2+}$ ions

- Mercury - accumulates on glassware; forms insoluble chloride salt

- Nickel - interference from oxides of $\mathrm{Ca}, \mathrm{Sc}$, and $\mathrm{Ti}$ and $\mathrm{Sn}^{2+}$ ions

- Selenium - interferences from oxides of $\mathrm{Zn}$ and $\mathrm{Ni}$

- ${ }^{80} \mathrm{Se}$ has interference from $\mathrm{Ar}_{2}$ molecule in plasma gas, must use ${ }^{77} \mathrm{Se}(7.5 \%$ abundance) and ${ }^{82} \mathrm{Se}(9.0 \%)$

- ${ }^{77} \mathrm{Se}$ has $\mathrm{ArCl}$ interference; corrected based on ${ }^{88} \mathrm{Se}$ and ${ }^{76} \mathrm{Se}$

- Silver - forms insoluble salts, especially $\mathrm{AgCl} ;{ }^{91} \mathrm{ZrO}$ interference

- Sodium - common element in DDI water matrix; accumulates on glassware and interface components

- Thorium - forms oxides easily

- Uranium - forms oxides easily

- Vanadium - ClO and SO interferences; corrected based on detected ${ }^{53} \mathrm{Cr}$ to ${ }^{52} \mathrm{Cr}$ ratio

- Zinc - interferences from oxides of $\mathrm{Ti}, \mathrm{Cr}, \mathrm{V}$ and $\mathrm{Fe}$ and $\mathrm{Ba}^{2+}$ ions 
Table 2-1. Cone penetrometer test sampling locations and depths.

\begin{tabular}{|c|c|c|c|c|c|c|c|c|c|c|}
\hline $\begin{array}{c}\text { CPT } \\
\text { label }\end{array}$ & $\begin{array}{l}\text { East } \\
\text { Site } \\
\text { ceordinute }\end{array}$ & $\begin{array}{l}\text { North } \\
\text { Site } \\
\text { Coordinte }\end{array}$ & $\begin{array}{c}\text { Ground } \\
\text { Elevation } \\
\text { (feet) }\end{array}$ & $\begin{array}{l}\text { depth to } \\
\text { water } \\
\text { table } \\
\text { fleefll } \\
\end{array}$ & $\begin{array}{c}\text { Pirst } \\
\text { vadose } \\
\text { zone } \\
\text { Soll } \\
\text { sample } \\
\text { dieetl } \\
\end{array}$ & $\begin{array}{c}\text { second } \\
\text { vadose } \\
\text { zone } \\
\text { Soll } \\
\text { Sample } \\
\text { Uleetl } \\
\end{array}$ & $\begin{array}{c}\text { depth to } \\
\text { first } \\
\text { Water } \\
\text { Sample } \\
\text { (feetll } \\
\end{array}$ & $\begin{array}{l}\text { depth to } \\
\text { first } \\
\text { saturated } \\
\text { Soll } \\
\text { Sample } \\
\text { (feell } \\
\end{array}$ & $\begin{array}{l}\text { depth to } \\
\text { second } \\
\text { Water } \\
\text { Sample } \\
\text { (foetl } \\
\end{array}$ & $\begin{array}{c}\text { depth to } \\
\text { second } \\
\text { saturated } \\
\text { Soll } \\
\text { Sample } \\
\text { (10ell) }\end{array}$ \\
\hline "A1 & 50095 & 75396 & 281 & 68 & 63 & NA & 73 & 75 & 88 & 90 \\
\hline$A 2$ & 50041 & 74990 & 282 & 71 & 68 & NA & 76 & 78 & 91 & 83 \\
\hline A3 & 49897 & 74633 & 261 & 51 & NA & NA & 56 & 58 & 71 & 73 \\
\hline A4 & 50005 & 74227 & 241 & 34 & NA & NA & 39 & 41 & 54 & 56 \\
\hline AG & 49899 & 73896 & 215 & 24 & 18 & NA & 28 & 30 & 38 & 40 \\
\hline B1 & 51100 & 75372 & 277 & 63 & 48 & 58 & 68 & 70 & 78 & 80 \\
\hline B2 & 51088 & 75269 & 276 & 62 & 47 & 57 & 67 & 69 & 77 & 78 \\
\hline B3 & 51072 & 75125 & 268 & 56 & 51 & NA & 61 & 63 & 71 & 73 \\
\hline B4 & 51049 & 74919 & 259 & 48 & 43 & NA & 53 & 55 & 73 & 75 \\
\hline B6 & 51016 & 74623 & 245 & 35 & NA & NA & 40 & 42 & 55 & 57 \\
\hline C1 & 51374 & 75563 & 278 & 62 & 24 & 57 & 67 & & 82 & 84 \\
\hline C2 & 51403 & 75443 & 277 & 60 & 55 & NA & 65 & 67 & 80 & 82 \\
\hline C3 & 51433 & 75318 & 287 & 51 & 46 & NA & 56 & 58 & 71 & 73 \\
\hline C4 & 51482 & 75075 & 258 & 43 & 38 & NA & 48 & 50 & 63 & 65 \\
\hline D1 & 50215 & 75568 & 282 & 68 & 32 & 63 & 102 & 104 & NA & NA \\
\hline D2 & 50158 & 75486 & 282 & 68 & 63 & NA & NA & NA & NA & NA \\
\hline D3 & 50095 & 75396 & 281 & 68 & 63 & NA & NA & NA & NA & NA \\
\hline D4 & 49952 & 75181 & 284 & 72 & 67 & NA & NA & NA & NA & NA \\
\hline D6 & 49786 & 74953 & 280 & 69 & 64 & NA & NA & NA & NA & NA \\
\hline E1 & 57029 & 71706 & 262 & 39 & 29 & 34 & 44 & 46 & 49 & 51 \\
\hline E2 & 56965 & 71612 & 259 & 37 & 27 & 32 & 42 & 44 & 47 & 49 \\
\hline EJ & 56880 & 71487 & 243 & 23 & 13 & 18 & $2 B$ & 30 & 33 & 35 \\
\hline$E_{4}$ & 56795 & 71242 & 222 & 4 & NA & NA & 9 & 11 & 14 & 16 \\
\hline F1 & 58688 & 72057 & 259 & 28 & 23 & NA & 30 & 32 & 35 & 37 \\
\hline F2 & 58621 & 71982 & 257 & 27 & 22 & NA & 29 & 31 & 34 & 36 \\
\hline F3 & 58480 & 71874 & 254 & 25 & 20 & NA & 27 & 29 & 32 & 34 \\
\hline F4 & 58223 & 71535 & 247 & 22 & 17 & NA & 27 & 29 & 32 & 34 \\
\hline F6 & 57882 & 71227 & 238 & 18 & 13 & NA & 21 & 23 & 26 & 28 \\
\hline
\end{tabular}

Intersection of transects ' $A$ ' and ' $D$ '. 
Table 2-2. Analysis performed on water samples.

\section{$\mathrm{pH}$}

Redox potential (Eh)

Conductivity

Temperature

Total organic carbon

Total inorganic carbon

Tritium concentration

Anion concentration $\left(\mathrm{Cl}, \mathrm{F}, \mathrm{NO}_{2}{ }^{-}, \mathrm{NO}_{3}{ }^{-}, \mathrm{PO}_{4}{ }^{3-}, \mathrm{SO}_{4}{ }^{2-}\right)$

Table 2-3. Analysis performed on soil samples.

Particle Size Analysis

Cation Exchange Capacity

Total Carbon

$\mathrm{pH}$

$\mathrm{X}$-ray Diffraction Analysis

Table 2-4. Elements analyzed for in soil and water samples using ICP-MS.

\begin{tabular}{|l|l|l|}
\hline All Samples & All Samples & Selected Samples \\
\hline Aluminum & Manganese & Bismuth \\
Arsenic & Nickel & Gold \\
Barium & Selenium & Hafnium \\
Cadmium & Silver & Mercury \\
Calcium & Sodium & Neptunium $^{1}$ \\
Cesium & Strontium & Plutonium \\
Chromium & Thorium & Scandium \\
Cobalt & Uranium & Tin \\
Copper & Yttrium & Titanium \\
Iron & Zinc & Tungsten \\
Lead & Zirconium & Vanadium \\
Magnesium & & \\
\hline
\end{tabular}

${ }^{1}$ Elements analyzed for semi-quantitatively using a thallium internal standard. 
Table 2-5. Approximate ICP-MS detection limits. Units are $\mu \mathrm{g} \mathrm{I}^{-1}$. Numbers in parentheses are the isotope monitored for polyisotopic elements. The instrument had reduced sensitivity at high masses on 8-24-94.

\begin{tabular}{|c|c|c|c|c|c|c|c|c|}
\hline Element & $8-24-94$ & $6-29-94$ & Element & $\begin{array}{c}\text { 8-24- } \\
94 \\
\end{array}$ & $6-29-94$ & Element & $8-24-94$ & $6-29-94$ \\
\hline $\mathrm{Na}$ & 20 & 0.7 & Ni (60) & 4 & 0.1 & $\mathrm{Cs}$ & 0.2 & 0.003 \\
\hline $\operatorname{Mg}(24)$ & 0.2 & 0.09 & $\mathrm{Cu}(63)$ & NA & 0.02 & $\mathrm{Ba}(138)$ & 0.03 & 0.03 \\
\hline Al & $>100$ & $>100$ & $\operatorname{Zn}(66)$ & NA & 0.1 & $\operatorname{Sm}(147)$ & 0.03 & NA \\
\hline K (39) & $>100$ & $>100$ & As & 0.2 & 0.2 & Yb (173) & NA & 0.04 \\
\hline Ca (44) & $>100$ & $>100$ & Se (77) & 4 & 2 & W (184) & 0.02 & 0.006 \\
\hline Sc (45) & 0.3 & $>5$ & Sr (88) & NA & 0.02 & $\mathrm{~Pb}(208)$ & 0.4 & 0.3 \\
\hline$V(51)$ & 0.2 & 0.01 & $\mathbf{Y}$ & 0.01 & 0.002 & Th & 0.009 & 0.006 \\
\hline $\operatorname{Cr}(52)$ & 0.3 & 0.06 & $\operatorname{Zr}(90)$ & 0.08 & 0.02 & U-235 & 0.004 & $<0.004$ \\
\hline Mn & 4 & 0.2 & Ag (107) & 0.007 & 0.2 & U-238 & 0.05 & 0.02 \\
\hline Fe (56) & 30 & 4 & Cd (111) & 0.07 & 0.05 & & & \\
\hline Co & 0.04 & 0.03 & $\operatorname{Sn}(118)$ & NA & 0.005 & & & \\
\hline
\end{tabular}

NA-Not analyzed for. 


\section{LEGEND}

- Sampling Transect

- - SREL Colloidal Characterization

$-\mathrm{pH}$

- Ba - at $1000 \mathrm{ug} / 1$

Cd - at $10 \mathrm{ug} / \mathrm{l}$ *

- $\mathrm{Hg}-$ ot $2 \mathrm{ug} / \mathrm{l}$

NOTES: $\mathrm{Pb}$ - at $50 \mathrm{ug} / 1$

1. * Barnwell/McBean 'Áquifer

2. Isocontours weré' drawn from 2091 datá
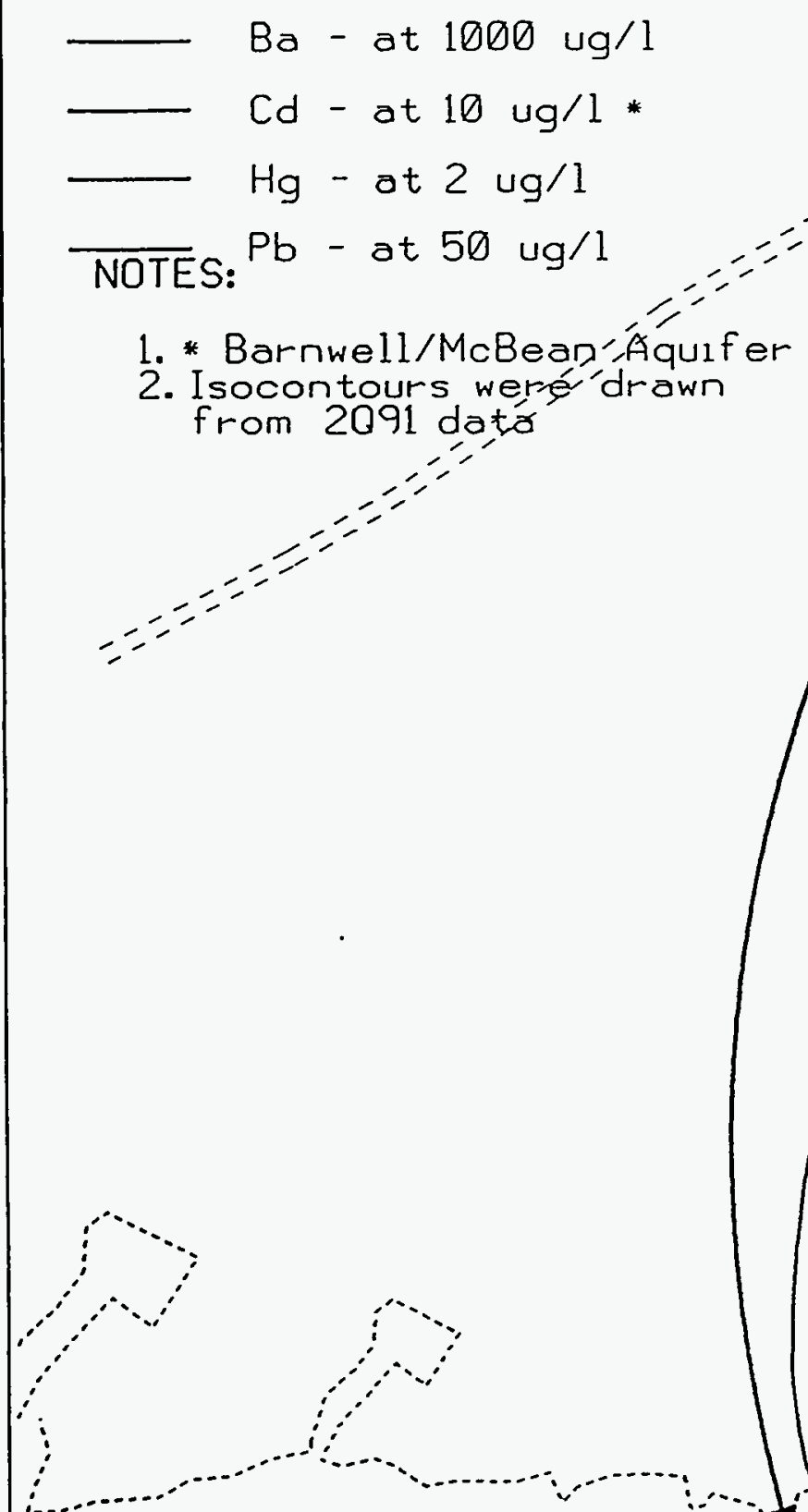


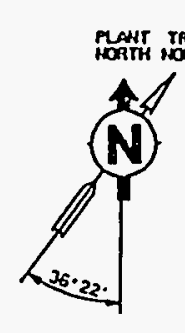

$\mathrm{pH}$ and RCRA Metals Isocontours and H-Area Seepag!

Figure 2-2. Areal view of

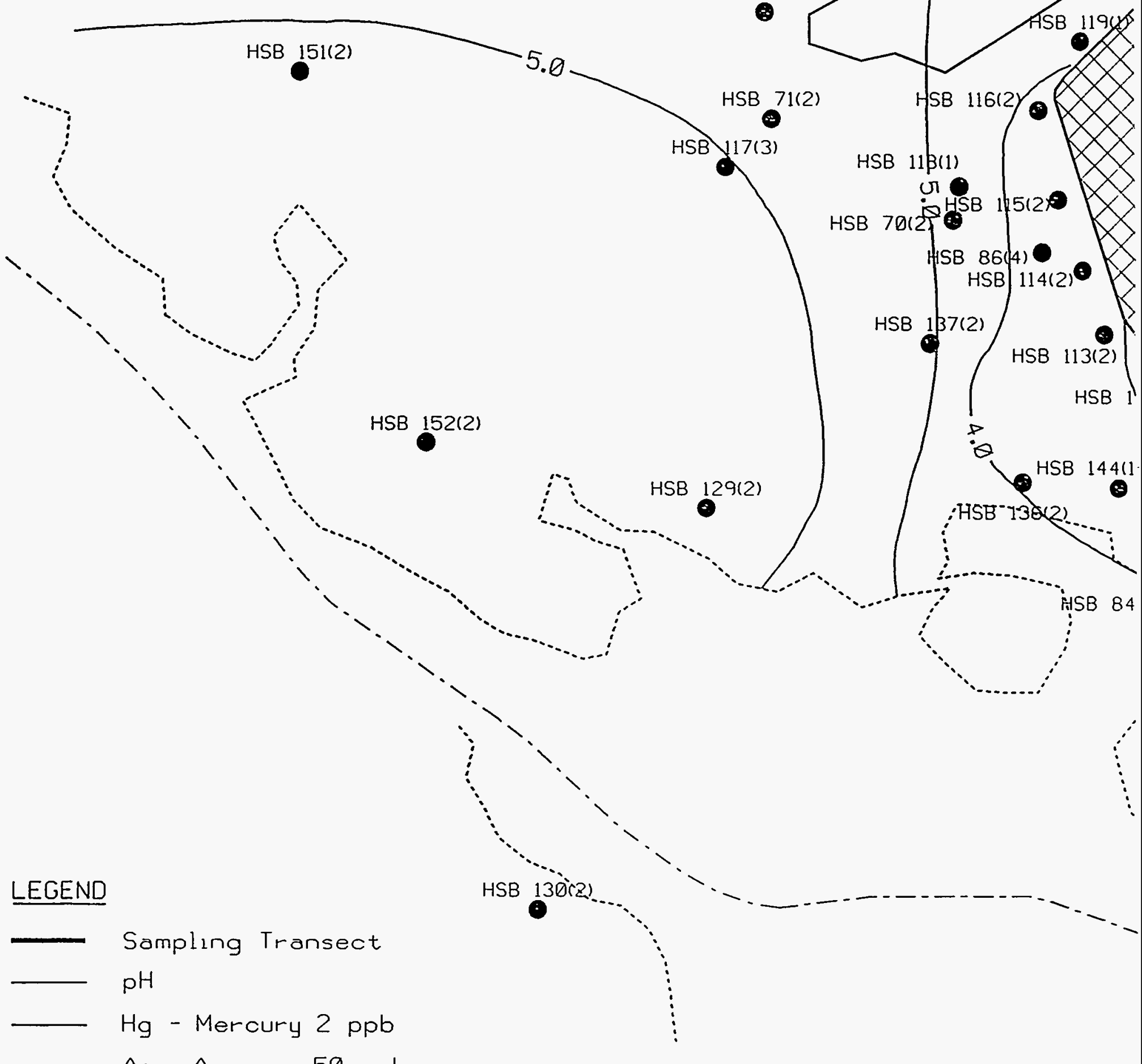


Sampling Transect Locations Basins

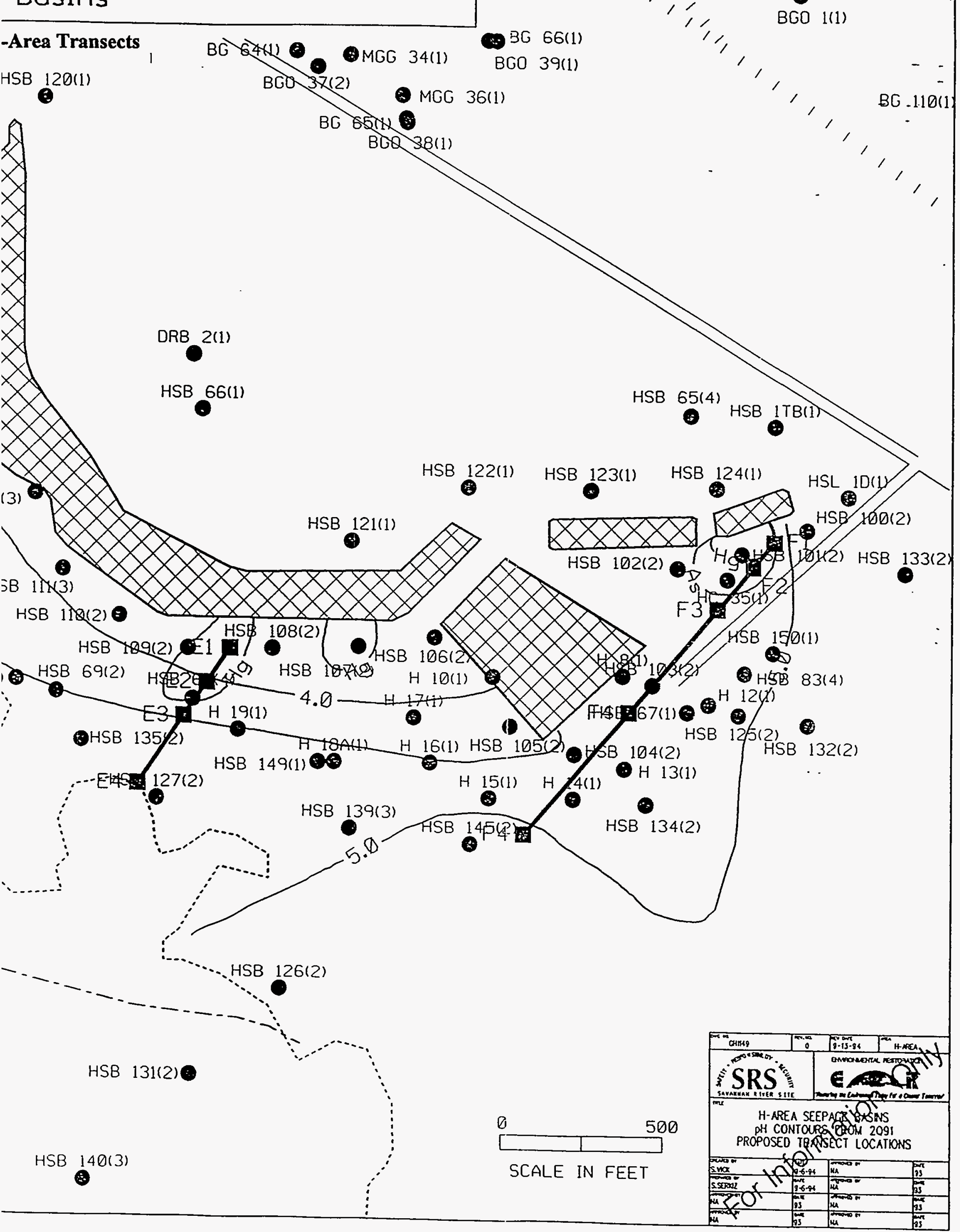


Figure 2-3. Cross section of Transect A-A'

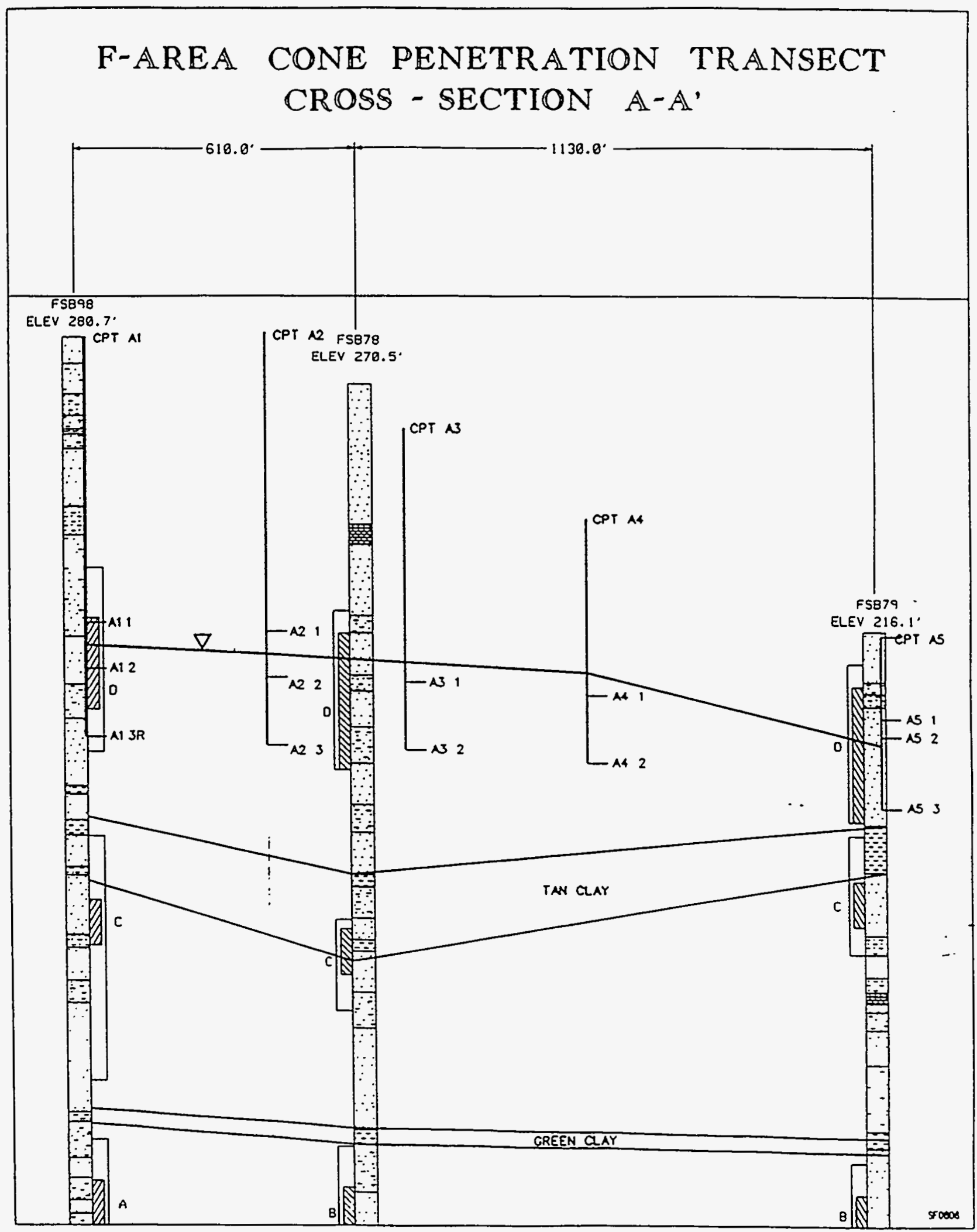


Figure 2-4. Cross section of Transect B-B'

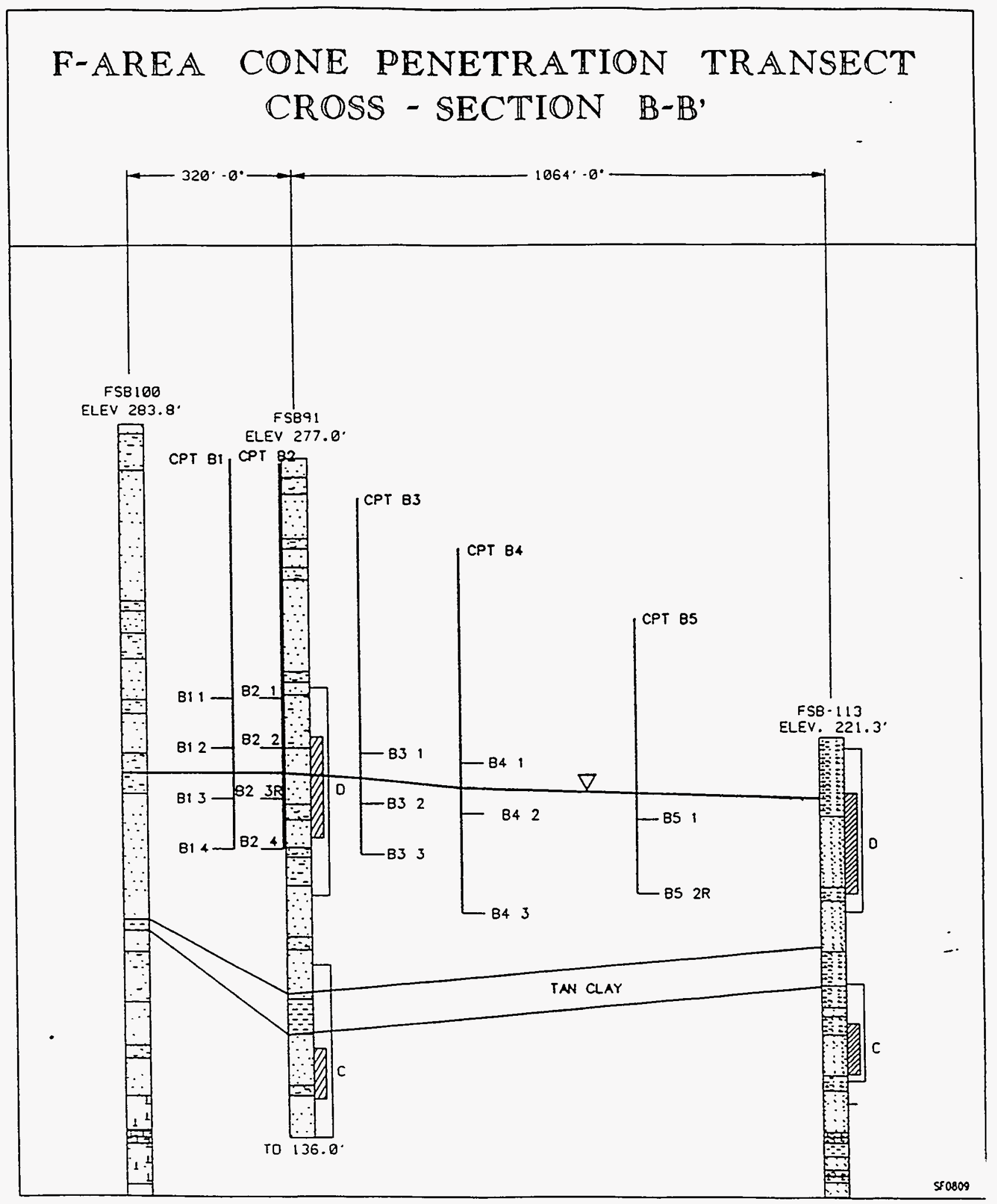


Figure 2-5. Cross section of Transect C-C'

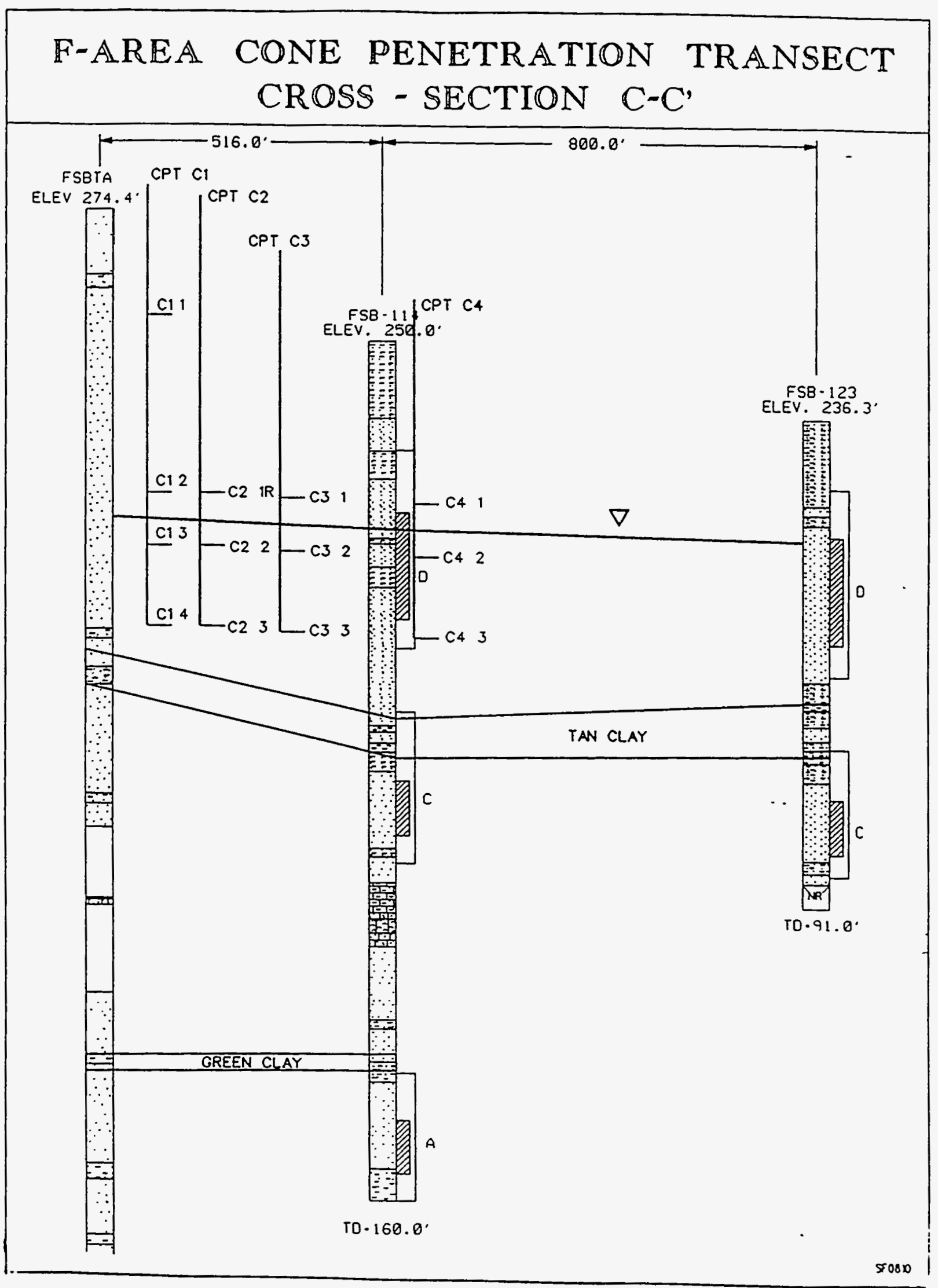


Figure 2-6. Cross section of Transect D-D'

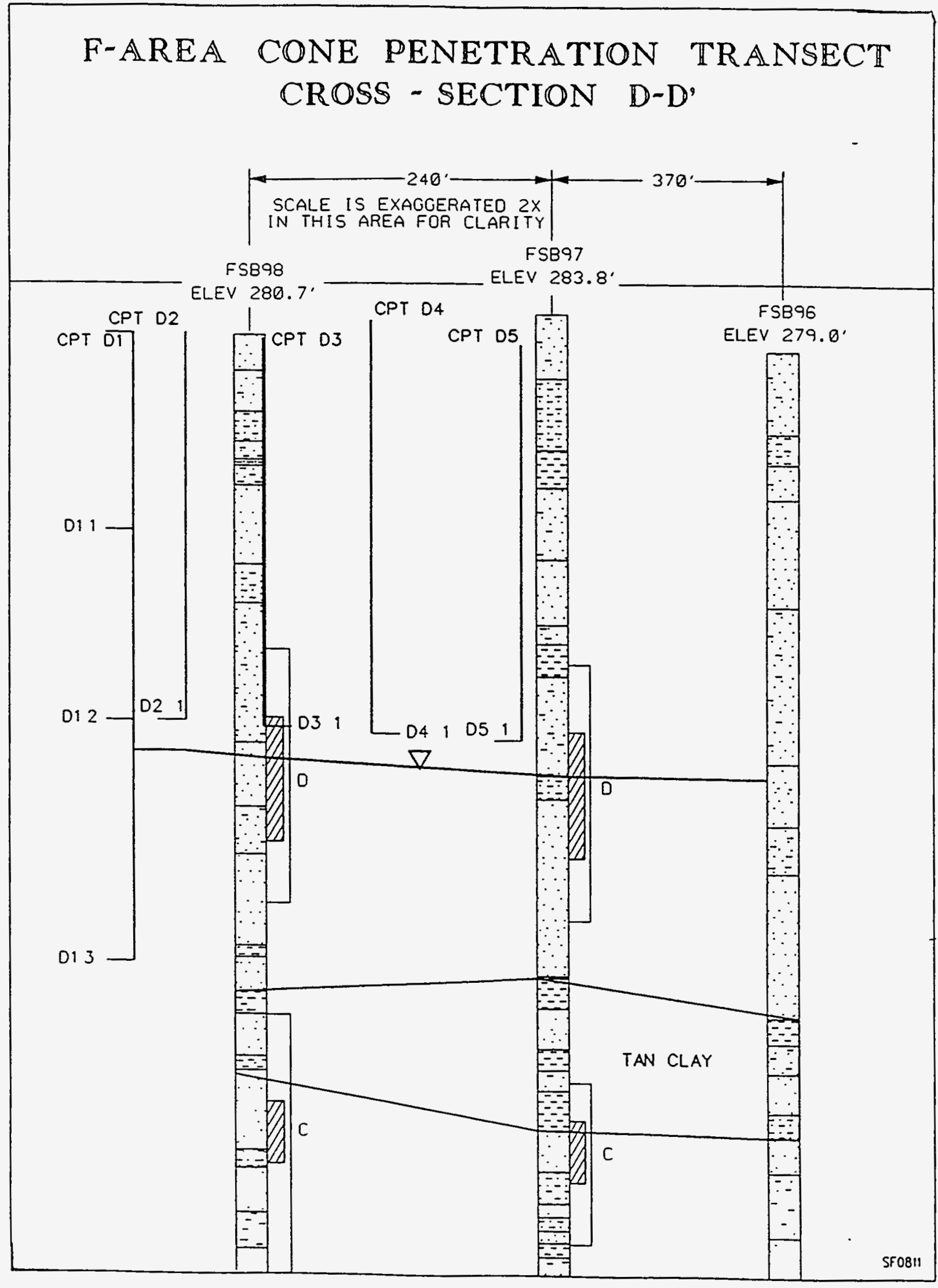


Figure 2-7. Cross section of Transect E-E'

\section{H-AREA CONE PENETRATION TRANSECT CROSS - SECTION E-E'}

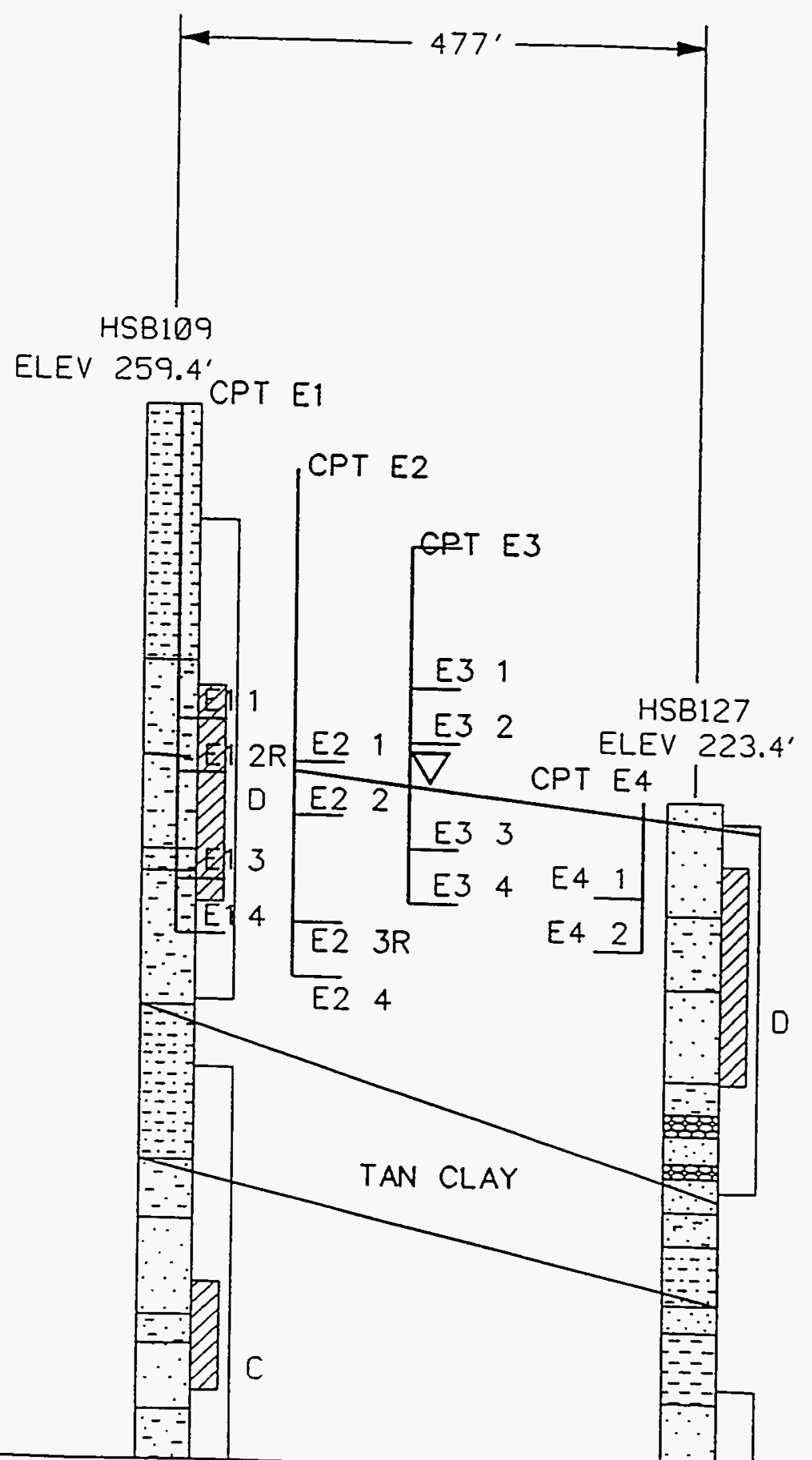


Figure 2-8. Cross section of Transect F-F'

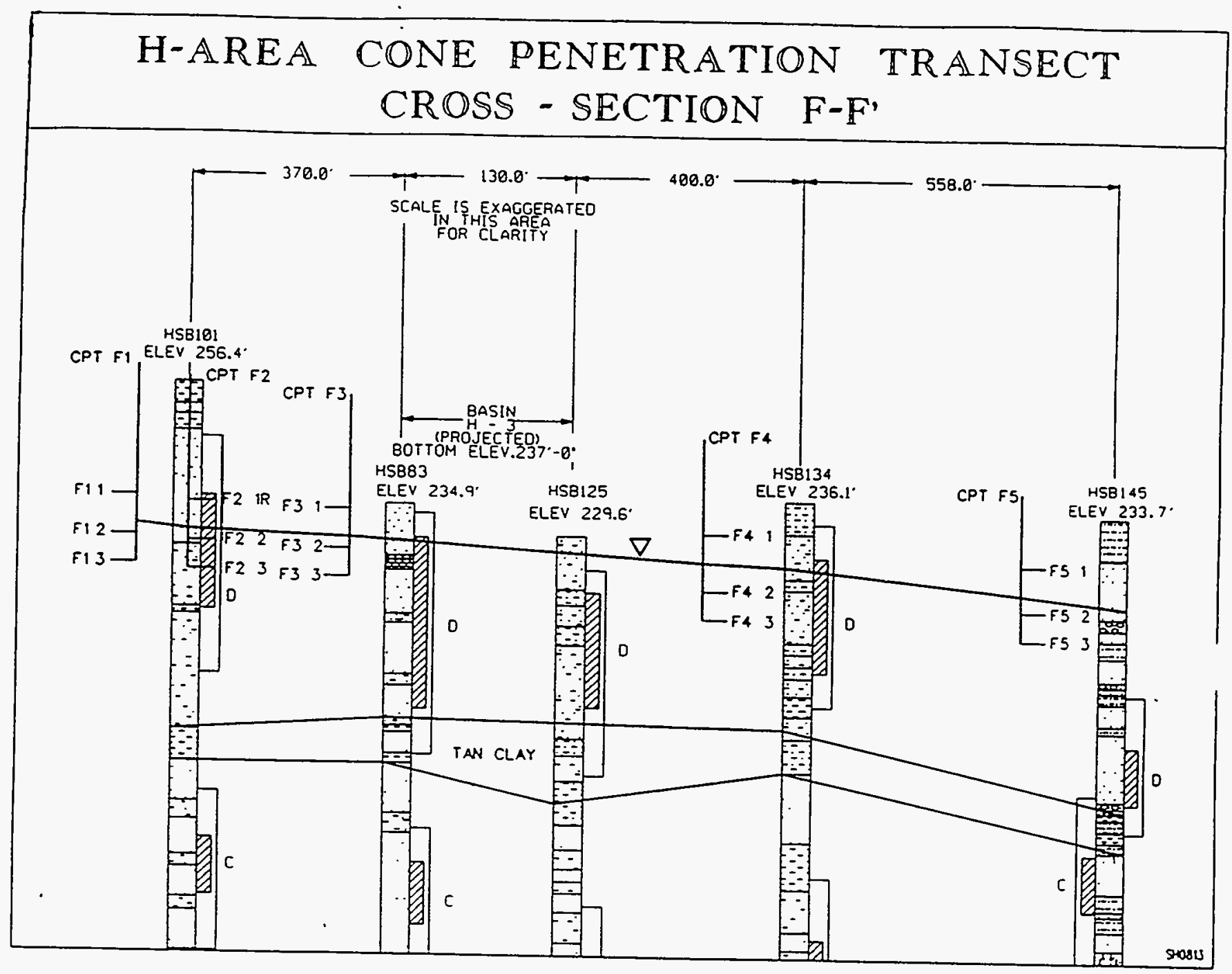




\subsection{MATHEMATICAL MANIPULATIONS AND DATABASE MANAGEMENT}

The establishment of a computerized database and data reporting in table forms was incorporated into the database management program. A database of analytical results for soil physical properties, radionuclides, cations, and anions was constructed using the Relational Database Management System (RDBMS) Microsoft Access on an IBM 486 compatible computer.

The Relational Database Management System (RDBMS) Microsoft Access Version 2.0 for Windows 3.1, was used to create the database F\&HDB.MDB used to store and perform analysis of the data. MS Access uses database objects, such as tables, queries, forms, and modules (MS Access Basic subprograms) to organize, analyze and present the data.

The tables that contain the data have been named in accordance with the six transects along which samples were collected, transects A, B, C, D, E, and F. Each transect is associated with three tables, a transect table, an isotope table, and a cross-section table. Transect tables contain information that is generic to all sample locations along the entire transect. The isotope tables contain the concentration and standard deviation for all runs of an experiment at each location where a sample was taken along a transect. Crosssection tables contain the X-and Y-coordinates of each sample location taken from Intergraph CADD design (.DGN) files.

Queries of the transect and isotope tables were constructed to compile weighted averages of concentrations of isotopes of each sample. The weighted averages were calculated from statistical methods for analyzing error propagation (explained below) using the Microsoft Access Basic programming language. MS Basic allows functions and procedures to be written by the user. The functions are combined into modules which can be called from a query. Therefore, queries were constructed which incorporated both the raw data and calls to statistical functions which determined the weighted averages of the concentrations of isotopes. The result of a query on a table for information on a particular isotope allowed the raw analytical data, as well as weighted averages of all isotopes, to be presented in a report.

The implementation of MS Access to create the database is explained in the F\&H Database Dictionary located in Appendix $D$, while the computer code used to perform the data manipulations is presented in Appendix E.

\subsection{Weighted Averaging Method}

Knoll (1989) presents a method of combining the measurements from a counting experiment with unequal errors. This weighted average method places greatest emphasis on values with small errors and less weight on measurements for which the estimated error is greatest. The best estimate of the concentration ( $\langle$ Conc $\rangle$ ) of the sample is given by the equation: 


$$
\langle\text { Conc. }\rangle=\sum_{i=1}^{n} a_{i} \text { Conc }_{i}
$$

where: Conc $_{i}$ is the concentration reported for measure $I$ of the sample; $n$ is the total number of measurements; and

$a_{i}$ is the normalized weighting factor of measurement $i$ which is inversely proportional to the square of the standard deviation of the measurement $\left(\sigma_{i}\right)$ and may be calculated from the equation:

$$
\mathrm{a}_{i}=\frac{1}{\sigma_{1}^{2}}\left(\sum_{j=1}^{n} \frac{1}{\sigma_{j}^{2}}\right)^{-1}
$$

The expected standard deviation of the sample based on the combination of measurements is equal to

$$
\sigma_{\langle\text {Conc }\rangle}=\frac{1}{\sqrt{\sum_{i=1}^{n} \frac{1}{\sigma_{i}^{2}}}}
$$




\subsection{ANALYTICAL RESULTS}

\subsection{Soil}

The results of chemical and physical analysis performed in this project are presented in Appendix B. Tables B-1 through B-7 summarize the chemical and physical properties of the soil samples collected in this work. Tables B-8 to B-14 provide details of the particle size analysis performed on the samples. Because particle size analysis work was performed by two different laboratories, data for each transect is presented in two tables according to the sieve series used for the individual samples. Figures 4-1 and 4-2 are soil classification charts based on the USDA soil classification scheme (Soil Survey Staff 1975) for the F and H area soil samples collected in this work.

Table B-15 summarizes the gamma ray spectroscopy data for selected soil samples.

The remaining tables in Appendix B present the elemental concentrations of soil samples by element for each transect. For elements for which more than one isotope was monitored using ICP-MS, the results of all isotopes are presented and then averaged as explained in section 3. Isotopic and elemental concentrations for which no standard deviation is reported are less than the lower limit of detection. The concentration reported is one-half the lower limit of quantification associated used with that element or isotope.

In cases which the ICP-MS analysis showed a detectable concentration of an element based on one isotope and a less than detectable concentration of the element based on a different isotope (usually an isotope with smaller natural abundance), the reported mean concentration is the average of the detected concentration and one-half of the lower limit of quantification. 27 such calculations are present in the soil sample concentration data, primarily in the reported values for cadmium (14) and silver (10). Additionally, this method was used to determine the silver concentration of a single water sample, D13SR

\subsection{Groundwater}

The results of chemical and physical analysis performed in this project are presented in Appendix C. Results of field analysis of groundwater were reported by Ebasco (1993) and are summarized by sample, along with laboratory data on the tritium, carbon and anion concentrations in tables $\mathrm{C}-1$ to $\mathrm{C}-6$. Groundwater concentrations of $\mathrm{NO}_{2}{ }^{-}$and $\mathrm{PO}_{4}{ }^{4}$ were not detected or less than the detection limit (typically $1-5 \mathrm{mg} \mathrm{I}^{-1}$ ) on all samples, expect sample E23W(R) which had a $\mathrm{NO}_{2}^{-}$concentration of $1.6 \mathrm{mg} \mathrm{I}^{-1}$.

Table C-7 summarizes the gamma ray spectroscopy data for selected samples.

The remaining table in Appendix $\mathrm{C}$ present elemental concentrations of the water samples by element and transect. For elements for which more than one isotope was monitored using 
ICP-MS, the results of all isotopes are presented and then averaged as explained in section 3. Isotopic and elemental concentrations for which no standard deviation is reported are less than the lower limit of detection. The concentration reported is the one-half the lower limit of quantification limit associated used with that element or isotope. Groundwater concentration data for samples F43W, F52W and F53W, have no reported standard deviation values. Data for these samples which are above the detection limit are noted. 
WSRC-TR-94-0484

Figure 4-1. Soil Classification for the F-Area soil samples.

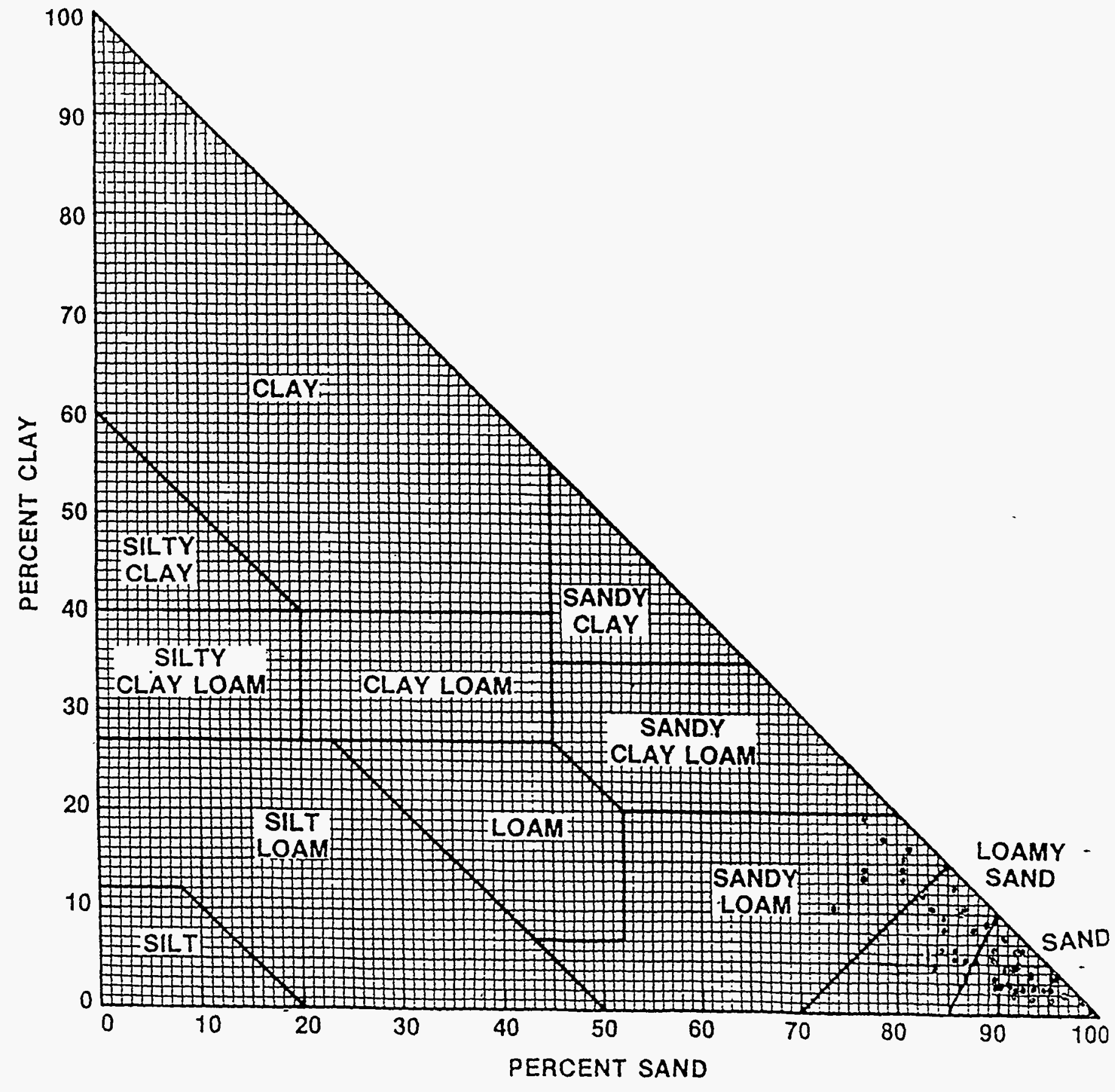


Figure 4-2. Soil Classification for the H-Area soil samples.

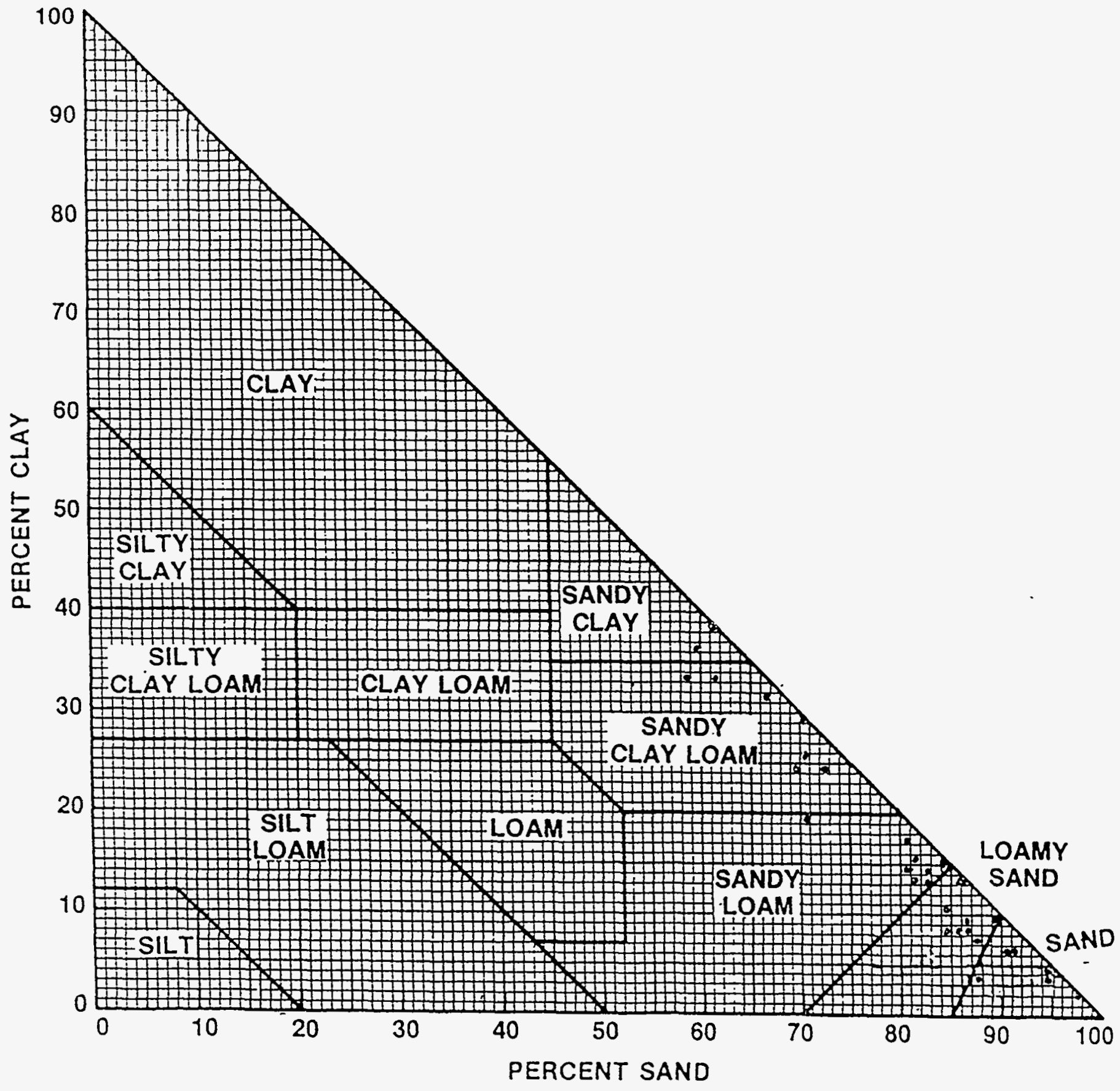




\subsection{QUALITY CONTROL/QUALITY ASSURANCE}

Soil and groundwater samples were using the procedures and with the QAVC controls contained in the WSRC 3Q5 Manual - "Hydrologic Data Collection (U)". Included in this procedure were: the use of chain-of-custody protocols to document sample handling; sample packaging and storage requirements; and sample collection procedures.

After sampling per the 3Q5 Manual requirements and Health Protection personnel verified that the beta and gamma count rates were not above background levels, samples were transported to SRTC along with appropriate chain-of-custody documentation. The samples were stored in a secured cooler which was maintained at a temperature $4^{\circ} \mathrm{C}$ prior to sample preparation and analysis.

To evaluate the amount of variability in this sampling method, replicate samples (i.e. saturated zone sample pairs and soil samples from the vadose zone) were collected at ten percent of the locations selected on a random basis. These samples are denoted with an " $R$ " as the last letter in the sample identification number.

In the laboratory, sample QA/QC was dependent on the type of analysis and the performing organization. Table 5-1 summarizes the laboratory QA/QC controls and the performing organization for each analysis.

Data entry into the transect and isotope tables of the F\&HDB.MDB MS Access database was performed by personnel at SRTC and SREL. Ten percent of the data input to the tables were audited for accuracy, misplaced or omitted data. The MS Access database, F\&HDB.MDB, was designed to use the validation rule properties which are standard attributes of the MS Access software. These validation rules assured that all data fell within a range of expected numerical values. 
Table 5-1. QA/QC controls on analyses

\begin{tabular}{|l|c|c|}
\hline Analysis & No. of Analyses & $\begin{array}{c}\text { Performing } \\
\text { Organization }\end{array}$ \\
\hline Field Measurements & 1 & Ebasco \\
$\mathrm{pH}$ & 1 & Ebasco \\
Redox Potential & 1 & Ebasco \\
Conductivity & 1 & Ebasco \\
Temperature & & \\
Soil Analyses & 3 & IWT/SGS \\
Digestions/ICP-MS & 2 & IWT/SGS/UF \\
Particle Size & 2 & IWT/SGS \\
CEC & 3 & IWT/SGS \\
Total Carbon & 1 & ADS/GERC \\
Gamma Spec & & \\
\hline Porewater Analysis & 2 & Ebasco/IWT/SGS \\
pH & 1 & ADS \\
Organic Carbon & 1 & ADS \\
Inorganic Carbon & 2 & ADS \\
Tritium & 2 & ADS \\
Major Anions & 3 & IWT/SGS \\
ICP/MS & 1 & ADS/GERC \\
Gamma Spec & & \\
\hline
\end{tabular}

Note:Ebasco - Ebasco Services Company

IWT - Interim Waste Technology in SRTC

SGS - Site Geotechnical Services in E\&PD

ADS - Analytical Development Section in SRTC

UF - University of Florida, Department of Geology

GERC - Georgia Environmental Resources Center at Georgia Institute of Technology 


\subsection{REFERENCES}

American Chemical Society. 1980. Guidelines for data acquisition and data quality evaluation in environmental chemistry. Anal. Chem. 52:2242-2249.

ADS-1502 Atomic absorption spectrometer - Varian AA-475 (U)

ADS-1509 Inductively coupled plasma - emission spectrometer for aqueous sample analysis (U)

ADS-2200 LECO CS-044 Analyzer (U)

ADS-2204 Preparation of standards for analysis of solutions by ion chromatography $(U)$

ADS-2306 Analysis of solutions by ion chromatography (U)

ADS-2424 Gross alpha/beta determination by liquid scintillation counting (U)

ASTM D4972-89. 1989. Standard test method for $\mathrm{pH}$ of soils.

Colquhon, D. J., J. Rine, M. J. Segall, M. G. Waddell, M. T. Katuna, and A. D. Cohen. 1993. Sedimentology and stratigraphy of the Upland Unit, SCUREF Cooperative Agreement, Task Order 53, Progress Report, December 2, 1992, Volume I Text. Geological Sciences Dept. Earth Sciences and Resources Institute, University of South Carolina.

Ebasco Services Incorporated. 1993. Savannah River Site F and H Seepage Basins soil/groundwater cone penetrometer sampling report (U). 2 Vols. USDOE Report WSRC-TR-93-537. Savannah River Site, Aiken, SC.

EPA. 1986. Method 9045 - Soil pH.

Gee, G. W. and J.W. Bauder. 1986. Particle-size analysis. In: Klute, A. ed. Methods of soil analysis, Part 1. Madison, WI: American Society of Agronomy, 383-412.

Johnson, N. A. 1994. Clay mineralogy of the surficial aquifer and confining system, Fand H-Area Seepage Basins, Savannah River Site. Dept. of Geology report, University of Florida, Gainesville, Fl.

Knoll, G. F. 1989. Radiation Detection and Measurement, 2nd ed., Wiley \& Sons, 87-96.

Lim, C. H. and M. L. Jackson. 1982. Dissolution for total elemental analysis. In: Page, A. L., Miller, R. H., and Keeney, D. R. eds. Methods of soil analysis, part 2. Madison, WI: Soil Science Society of America, 1-12. 
Rhoades, J. D. 1982. Cation Exchange Capacity. In: Page, A. L., Miller, R. H., and Keeney, D. R. eds. Methods of soil analysis, part 2. Madison, WI: Soil Science Society of America, 149-157.

Soil Survey Staff. 1975. Soil Taxonomy: A basic system of soil classification for marking and interpreting soil surveys. USDA-SCS Agric. Handbook 436.

Washington, DC: U.S. Government Printing Office. 
Appendix A

ANALYTICAL METHODOLOGY 


\subsubsection{Soil pH and Conductivity}

\section{PURPOSE}

This procedure provides an analytical method to measure the $\mathrm{pH}$ of a noncalcareous soil.

\section{REQUIRED EQUIPMENT/REAGENTS}
A. Equipment
1. $\mathrm{pH}$ meter with a micro tip, combination electrode.
2. 30 -ml beakers
3. \#10 (2-mm) sieve
B. Reagents
1. Secondary $\mathrm{pH}$ standard solutions with a $\mathrm{pH}$ of $2.0,4.0,7.0$ and 10.0.
2. Distilled, deionized (DDI) water
3. Calcium Chloride $\left[\mathrm{CaCl}_{2} \cdot 2 \mathrm{H}_{2} \mathrm{O}\right]$

\section{PROCEDURE}

A. Reagent Preparation

1. $1.0 \mathrm{M}$ calcium chloride stock solution

a. Dissolve $147 \mathrm{~g}$ of $\mathrm{CaCl}_{2} \cdot 2 \mathrm{H}_{2} \mathrm{O}$ in DDI water in a 1-liter volumetric flask

b. Dilute to 1-liter with DDI water

2. $0.01 \mathrm{M}$ calcium chloride solution
a. Pipet $10 \mathrm{ml}$ of $1.0 \mathrm{M}$ calcium chloride stock solution into a 1-liter volumetric flask.
b. Dilute to I-liter with DDI water
c. The $\mathrm{pH}$ of the solution should be between 5 and 7 .

B. Sample Preparation

1. Air dry sample approximately $50 \mathrm{~g}$ of soil sample.

2. Dry stiff the sample through a $\# 10(2-\mathrm{mm})$ sieve, saving the portion that passes through the sieve.

3. Weigh out two $10 \mathrm{~g}$ portions of soil into clean, labeled $50 \mathrm{ml}$ beakers.

4. Add $20 \mathrm{ml}$ of DDI water to one of the beakers. 
5. Add $20 \mathrm{ml}$ of $0.01 \mathrm{M}$ calcium chloride solution to the other beaker.

6 Periodically stir mixtures for 30 minutes.

7. Let the samples settle for 1 hour.

C. $\mathrm{pH}$ Meter Calibration

1. Thoroughly rinse electrode with DDI water and blot dry.

2. Place electrode in a secondary standard solution of $\mathrm{pH}=2.0$. Record meter reading in $\mathrm{mV}$ in lab notebook.

3. Rinse the electrode with DDI water and blot dry.

4. Place electrode in a secondary standard solution of $\mathrm{pH}=4.0$. Record meter reading in $\mathrm{mV}$ in lab notebook.

5. Rinse the electrode with DDI water and blot dry.

6. Place electrode in a secondary standard solution of $\mathrm{pH}=7.0$. Record meter reading in $\mathrm{mV}$ in lab notebook.

7. Rinse the electrode with DDI water and blot dry.

8. Place electrode in a secondary standard solution of $\mathrm{pH}=10.0$. Record meter reading in $\mathrm{mV}$ in lab notebook.

9. Measure and record $\mathrm{pH}$ of the type II water being used in logbook.

10. Perform a linear regression of $\mathrm{pH}$ against recorded $\mathrm{mV}$. Record the slope, intercept and correlation value of the regression in the lab notebook.

a. Slope of the line should be $-59.2 \pm 3.0$. If not, replace or refill the electrode.

D. Analysis of samples

1. Thoroughly rinse electrode with DDI water.

2. Insert electrode far enough into the clear supernatant to make good contact with the solution, but not the soil. Minimize shaking of the mixture to avoid resuspending the soil particles.

3. Record meter reading (in $\mathrm{mV}$ ) in the lab notebook. Calculate $\mathrm{pH}$ on the regression results obtained in section $B$.

4. Measure and record temperature of the supernatant in logbook.

5. Report measured $\mathrm{pH}$ value of the DDI water sample as "Soil $\mathrm{pH}$ measured in water" $\left(\mathrm{pH}_{\mathrm{w}}\right)$.

a. Record the $\mathrm{pH}$ value measured with the $0.01 \mathrm{M} \mathrm{CaCl}_{2}$ electrolyte as "Soil $\mathrm{pH}$ measured in $0.01 \mathrm{M}$ calcium chloride $\left(\mathrm{pH}_{\mathrm{e}}\right)$.

\section{UNCERTAINTIES}

A. $\mathrm{pH}$ meter calibration and inherent uncertainties.

B. Temperature correction of $\mathrm{pH}$

1. This procedure is based on maintaining a constant temperature or using a temperature compensated $\mathrm{pH}$ meter.

C. $\mathrm{pH}$ of water and $\mathrm{CaCl}_{2}$ solution being used 


\section{REFERENCES}
A. EPA. 1986. Method $9045-$ Soil pH.
B. $\mathrm{pH}$ meter technical manual
C. ASTM-D4972-89 


\subsubsection{Soil Particle Size Analysis}

\section{PURPOSE}

This procedure provides an analytical method for determining the size distribution of particles in a soil sample. It employs a sieve method to separate the sand fraction and the pipet method to measure the particle size distribution of the clay and silt fraction.

\section{REQUIRED EQUIPMENT/REAGENTS}

\section{A. Equipment}

1. Balance sensitive to $0.1 \mathrm{~g}$

2. Balance sensitive to $0.01 \mathrm{~g}$

3. Centrifuges-low speed $(1500 \mathrm{rpm})$ and high speed $(12,000 \mathrm{rpm})$ with $250 \mathrm{ml}$ bottles

4. Orbital reciprocating shaker

5. Wrist action shaker

6. Electric and hand stirrers

7. Constant temperature water bath capable of maintaining temperature $\pm 0.5^{\circ} \mathrm{C}$

8. 1000-ml cylinders

9. Set of brass sieves down to $53 \mu \mathrm{m}$ in size. See ASTM-D422 step 3.6 a) for a listing of suggested sizes.)

10. Sieve shaker

11. Pipets and pipet holding apparatus that will allow precision placement of pipet at a given depth in a 1000-ml cylinder.

12. $1000 \mathrm{ml}$ beakers

13. Clock or watch with second hand

14. Wooden or rubber rolling pin

15. Stainless steel trays for drying and mixing of sample.

16. Conventional constant temperature drying oven

17. $\mathrm{pH}$ meter with combination electrode

18. Aluminum drying dishes

19. 4" watch glass capable of covering 1000-ml cylinders

20. 2-1 LDPE bottle

21. 1-liter reagent bottles

22. 250-ml reagent bottle

23. 1-liter wide-mouth LDPE bottles

24. 250-ml drying dishes 
25. Stirring rods

26. Laboratory film

27. Desiccator

28. 1-liter volumetric flasks

29. Brass sieve receiver and cover

B. Reagents

1. Distilled water

2. Sodium hexametaphosphate $\left[\left(\mathrm{NaPO}_{3}\right)_{6}\right]$

3. Sodium carbonate $\left[\mathrm{Na}_{2} \mathrm{CO}_{3}\right]$

4. $30 \%$ Hydrogen peroxide $\left[\mathrm{H}_{2} \mathrm{O}_{2}\right]$ (store in refrigerator)

5. Sodium acetate $\left[\mathrm{NaC}_{2} \mathrm{H}_{3} \mathrm{O}_{2} \cdot 3 \mathrm{H}_{2} \mathrm{O}\right]$

6. Sodium bicarbonate $\left[\mathrm{NaHCO}_{3}\right]$

7. Sodium citrate $\left[\mathrm{Na}_{3} \mathrm{C}_{6} \mathrm{H}_{5} \mathrm{O}_{7} \cdot 2 \mathrm{H}_{2} \mathrm{O}\right]$

8. Sodium dithionite $\left[\mathrm{Na}_{2} \mathrm{~S}_{2} \mathrm{O}_{4}\right]$

9. Calcium chloride $\left[\mathrm{CaCl}_{2}\right.$ or $\left.\mathrm{CaCl}_{2} \cdot 2 \mathrm{H}_{2} \mathrm{O}\right]$

10. Sodium chloride $[\mathrm{NaCl}]$

11. $36 \%$ Hydrochloric acid

12. Acetone

13. Acetic acid, glacial $(99.5 \%)\left[\mathrm{HC}_{2} \mathrm{H}_{3} \mathrm{O}_{2}\right]$

\section{PROCEDURES}

\section{A. Reagent Preparation}

1. Preparation of dispersing agent

a. Mix $25 \mathrm{~g}$ of $\left(\mathrm{NaPO}_{3}\right)_{6}$ with distilled water to make a 1 liter solution.

b. Ensure that the pH of solution is between 8 and 9 .

1) Add $\mathrm{Na}_{2} \mathrm{CO}_{3}$ if necessary to adjust $\mathrm{pH}$.

c. Bring temperature of solution to temperature of procedure $\left(\approx 20^{\circ} \mathrm{C}\right)$ before using.

2. $1 \mathrm{M} \mathrm{NaOAc}$

a. Add $136 \mathrm{~g}$ of $\mathrm{NaC}_{2} \mathrm{H}_{3} \mathrm{O}_{2} \cdot 3 \mathrm{H}_{2} \mathrm{O}$ to a 1 -liter volumetric flask

b. Dilute to the mark by adding distilled water

c. Adjust solution $\mathrm{pH}$ to 5 by adding acetic acid.

3. Citrate-bicarbonate buffer solution

a. Add $21.0 \mathrm{~g}$ of $\mathrm{NaHCO}_{3}$ to a 1-liter volumetric flask.

b. Add a small amount of water to the flask and shake to dissolve the $\mathrm{NaHCO}_{3}$.

c. Add $176.5 \mathrm{~g}$ of $\mathrm{Na}_{3} \mathrm{C}_{6} \mathrm{H}_{5} \mathrm{O}_{7} \cdot 2 \mathrm{H}_{2} \mathrm{O}$ to the flask.

d. Dilute to 11 by adding distilled water to the flask.

e. Transfer solution to a 2-1 LDPE bottle.

f. Add an additional 11 of distilled water to the LDPE bottle and stir to allow adequate mixing.

4. $1 \mathrm{M} \mathrm{CaCl}_{2}$ stock solution 
a. Add $111 \mathrm{~g}$ of $\mathrm{CaCl}_{2}$ (or $147 \mathrm{~g}$ of $\mathrm{CaCl}_{2} \cdot 2 \mathrm{H}_{2} \mathrm{O}$ ) to a 1-liter volumetric flask.

b. Dilute to 11 with distilled water.

\section{5. $1 \mathrm{M} \mathrm{HCl}$}

a. Add $100 \mathrm{ml}$ of distilled water to a $250-\mathrm{ml}$ reagent bottle.

b. In a fume hood, pipet $9 \mathrm{ml}$ of $36 \% \mathrm{HCl}$ into the reagent bottle.

c. Store solution in the acid storage area.

6. Saturated $\mathrm{NaCl}$ solution

a. Add $360 \mathrm{~g}$ of $\mathrm{NaCl}$ to a 1-liter reagent bottle.

b. Add $800 \mathrm{ml}$ of distilled water to the reagent bottle and stir for 15 minutes.

c. At the end of the stirring time, undissolved $\mathrm{NaCl}$ should remain in the bottle. If not, add more $\mathrm{NaCl}$ and continue stirring.

7. $10 \% \mathrm{NaCl}$ solution
a. Thoroughly stir the saturated $\mathrm{NaCl}$ solution.
b. Pour $100 \mathrm{ml}$ of saturated $\mathrm{NaCl}$ solution into a 1-liter volumetric flask.
c. Dilute solution to 11 by adding distilled water to the flask.

B. Sample Preparation and Determination of Gravel Fraction.

1. Oven dry about $150 \mathrm{~g}$ of sample at $110 \pm 5^{\circ} \mathrm{C}$ for at least 12 hours (overnight).

2. Weigh the sample and record the total oven-dried weight in the lab notebook.

3. Thinly spread the sample out in a stainless steel tray.

4. Gently grind and mix the sample with a wooden rolling pin to break up the clods.

5. Dry-sift all the samples through the sieve nest with a $2 \mathrm{~mm}$ sieve being the smallest.

6. Weigh and record the weights of the samples retained on each sieve and that passing through all sieves, to the nearest $0.001 \mathrm{~g}$.

7. Calculate the total percentage of sample material retained on the top of each screen and record mass as "greater than (the sieve size from which it was collected)", as well as that portion which is less than $2 \mathrm{~mm}$ in size (not retained by the smallest sieve).

C. Pretreatment Of Sample Portion Less Than $2 \mathrm{~mm}$.

NOTE: Care should be taken when decanting solution in this procedure to ensure that loss of the fine portion of the sample is minimized.

1. Weigh and record the mass of two portions of the $<2 \mathrm{~mm}$ sample fraction according to the following table:

\begin{tabular}{|l|c|}
\hline Main Constituent of Sample & Amount Required \\
\hline Clays & $10 \mathrm{~g}$ \\
Loams & $20 \mathrm{~g}$ \\
Sandy Loams & $40 \mathrm{~g}$ \\
Loamy Sands & $40 \mathrm{~g}$ \\
Sands & $80 \mathrm{~g}$ \\
\hline
\end{tabular}


2. Place the weighted samples into $250-\mathrm{ml}$ bottles and add $100 \mathrm{ml}$ of distilled water to each bottle.

a. Shake bottles for about 1 minute.

3. Add $10 \mathrm{ml}$ of $1 \mathrm{M} \mathrm{NaOAc}$ to each bottle and shake for approximately 5 minutes.

4. Centrifuge mixture until the supernate is clear (15 minutes at 3000 RPM).

5. Carefully pour off the supernate.

6. Add $50 \mathrm{ml}$ of distilled water and shake tube for about 1 minute to wash sample.

7. Centrifuge the sample for 15 minutes at 3000 RPM and pore off the supernate if it is clear.

a. If the supernate is not clear, further washing may be necessary before centrifuging.

8. Repeat steps 6 and 7.

9. Add $25 \mathrm{ml}$ of distilled water to the bottle and shake on a wrist action shaker for 1 minute.

10. If the sample appears to have a large amount of organic matter in it $(>5 \%)$, transfer the mixture to a $1000 \mathrm{ml}$ bottle.

11. Add $5 \mathrm{ml}$ of $\mathrm{H}_{2} \mathrm{O}_{2}$ to mixture and stir. Cover the sample and observe closely for several minutes.

a. If excessive frothing is present, cool in a water bath

12. Add more $\mathrm{H}_{2} \mathrm{O}_{2}$ when the reaction subsides, rinsing the sides if necessary.

a. For samples with little or no organic matter present, no frothing will be observed and the use of additional $\mathrm{H}_{2} \mathrm{O}_{2}$ is unnecessary. Skip to step 15 .

13. When the frothing has ceased, remove cover from bottle and heat to $90^{\circ} \mathrm{C}$ in a constant-temperature water bath.

14. Repeat steps 12 and 13 until most organic matter has been destroyed, as determined by color of sample and rate of reaction.

15. With the cover removed, heat at $90^{\circ} \mathrm{C}$ for one hour after final addition of $\mathrm{H}_{2} \mathrm{O}_{2}$.

16. If a $1000 \mathrm{ml}$ bottle was used, transfer contents back into a clean $250 \mathrm{ml}$ bottle.

17. Add citrate-bicarbonate buffer to bring volume to $150 \mathrm{ml}$.

18. Shake the bottle for 1 minute to disperse the soil.

Caution: Rapid addition of $\mathrm{Na}_{2} \mathrm{~S}_{2} \mathrm{O}_{4}$ to samples with a high iron content may cause extensive heat and a possible boiling/steam hazard.

19. In a fume hood, slowly add $3 \mathrm{~g}$ of $\mathrm{Na}_{2} \mathrm{~S}_{2} \mathrm{O}_{4}$ to the bottle while stirring. When foaming has subsided, shake for approximately 30 seconds.

20. Place in a $80^{\circ} \mathrm{C}$ water bath and stir intermittently for 20 minutes.

a. Occasionally loosen the top of the bottle to vent gases in samples with a high iron content.

21. Remove from bath and add $10 \mathrm{ml}$ of saturated $\mathrm{NaCl}$ solution. Mix thoroughly.

22. Centrifuge and decant off the supernate. 
23. Repeat steps 17 through 22 until the sample color changes to gray.

24. Wash sample with $50 \mathrm{ml}$ of citrate-bicarbonate buffer and $20 \mathrm{ml}$ of saturated $\mathrm{NaCl}$ solution.

a. Shake for 5 minutes.

b. Centrifuge at $3000 \mathrm{RPM}$ for 15 minutes

c. Decant off the supernate.

25. Wash the sample with $50 \mathrm{ml}$ of $10 \% \mathrm{NaCl}$.

a. Shake for 5 minutes.

b. Centrifuge at $3000 \mathrm{RPM}$ for 15 minutes

c. Decant off the supernate

26. Repeat step 25.

27. Wash with $50 \mathrm{ml}$ of distilled water.

a. Shake for 5 minutes.

b. Centrifuge at $3000 \mathrm{RPM}$ for 15 minutes

c. Decant off the supernate

1). If the supernate is not clear, transfer to a plastic centrifuge bottle and then centrifuge at a higher speed before decanting.

b. If the supernate is still not clear, add several drops of acetone, warm in water bath, and then centrifuge.

28. Add $150 \mathrm{ml}$ of water, shake for 5 minutes and then measure and record the solution $\mathrm{pH}$.

a. pH should be approximately 8 if the soil is Na saturated.

29. Transfer the solution to a 1 liter shaker bottle and add $400 \mathrm{ml}$ of distilled water and $10 \mathrm{ml}$ of dispersing agent solution.

30. Shake overnight.

D. Determination Of Sand Fraction.

1. Wet the screen of a 53- $\mu \mathrm{m}$ sieve with water.

2. Slowly pour the solution through the sieve. Collect the solution passing through the sieve in a $1000-1$ beaker.

3. Collect the sand particles remaining on the 53- $\mu \mathrm{m}$ sieve into a dish. Using a distilled water rinse stream, pass this fraction through a stack of sieves with sizes between 53- $\mu \mathrm{m}$ and 2-m (arranged coarsest on top, finest on the bottom).

a. Shake the sieves for approximately 3 minutes.

b. Add any solution passing through the $53 \mu \mathrm{m}$ sieve to the $1000-\mathrm{ml}$ beaker containing the rinsing from step 2.

1) If rinsing exceed $1000 \mathrm{ml}$, all the solution to evaporate to less than $1000 \mathrm{ml}$ by placing the beaker (lightly covered with aluminum foil) in a oven or water bath set to about $40^{\circ} \mathrm{C}$.

4. Label, weight, and record the mass of a aluminum drying dish for each sieve used.

a. Transfer the soil particles retained on each sieve to the appropriate weighting dish. Rinse sieve to ensure allow particles are transferred.

5. Oven dry the sand fraction captured on each sieve. 
6. Weigh and record the mass of the oven-dried sand fraction and dishes.

7. Calculate the mass of soil collected on each sieve.

E. Measure the specific gravity of the clay and silt portion in accordance with procedure 3.3.1.5.a).

F. Analysis of the Clay and Silt Fraction

1. Label, weight, and record the weight three aluminum drying dishes.

2. Place the dispersed mixture which has been allowed to soak to the $1000-\mathrm{ml}$ graduated cylinder. Rinse beaker thoroughly with distilled water to ensure that all soil particles are in the cylinder.

3. Add $10 \mathrm{ml}$ of dispersing agent to the cylinder.

4. Add sufficient distilled water to bring the volume in the solution to $1000 \mathrm{ml}$.

5. Cover the cylinder with lab film and briefly mix solution by shaking the cylinder.

6. Allow the solution to equilibrate overnight.

7. Mark the point which is $10 \mathrm{~cm}$ below the top of the liquid surface.

8. Shake the cylinder for at least one minute to ensure that the soil is dispersed throughout the solution.

a) Record the time that stirring is completed.

8. At the three appropriate times, as specified in Gee (1986), tables 15-1, 15-2, $15-3$ (attached) for the $20 \mathrm{~mm}, 5 \mu \mathrm{m}$, and $2 \mu \mathrm{m}$ size fractions perform the following: For soil with a specific gravity of $2.60 \mathrm{Mg} \mathrm{m}^{-3}$ and a room temperature of $22^{\circ} \mathrm{C}$, use times of 4.6 mimutes, 73.2 mimutes and 7.63 hours respectively.)

a) Gently insert the $25-\mathrm{ml}$ pipet to the marked depth of $10 \mathrm{~cm}$ in the cylinder's liquid.

b) Using a controlled vacuum, withdraw $25 \mathrm{ml}$ of solution in approximately

12 seconds.

c) Gradually remove the full pipet from the solution.

d) Discharge the liquid from the pipet into a previously weighted and numbered aluminum drying dish.

e) Using a small amount of distilled water, rinse the pipet into the drying dish.

9. Oven-dry the samples at $105 \pm 5^{\circ} \mathrm{C}$ until the water evaporates.

10. Cool the drying dishes in a desiccator and then weigh and record each weight of the sample contained in each.

11. Add $10 \mathrm{ml}$ of $1 \mathrm{M} \mathrm{CaCl}_{2}$ and $1 \mathrm{ml}$ of $1 \mathrm{M} \mathrm{HCl}$ to the solution remaining in the cylinder.

12. After allowing flocculation to occur overnight, carefully pour off the clear portion of solution.

13. Clean, dry, label and weigh a 250-ml drying dish. Record the weight in the lab notebook.

14. Transfer the remaining soil into the 250-ml drying dish, thoroughly rinsing the graduated cylinder with distilled water to ensure that all soil particles are transferred. 
15. Oven dry the sample at $110 \pm 5^{\circ} \mathrm{C}$.

16. Allow the beaker to cool in a desiccator and then weigh and record the weight of the remaining soil.

G. Calculation of \% in Suspension Using the Pipet Method.

1. Subtract $0.013 \mathrm{~g}$ from each weight recorded in step F.10 to account for the weight of the dispersing agent.

2. Divide the weight calculated in step G.1 by 0.025 to obtain the total of soil particles less than a given fraction.

3. Subtract $0.463 \mathrm{~g}$ from the soil weight calculated in step F.15 to account for the weight of the dispersing agent.

4. Add the weights calculated in steps G.1 and G.3 to obtain the total weight of the silt and clay fraction.

5. Calculate the total weight of the treated soil by summing the fraction weights calculated in steps D.7 and G.4.

H. Report Contents

1. Maximum size of particles

2. Percentage of particles greater than a given size (or fraction of sand, silt and clay portion greater than a given size).

a. Plot of percentage of particles greater than a given size versus particle size.

b. List any large particles were removed prior to testing.

3. Description of the sand and gravel particles
a. Shape - rounded or angular
b. Hardness - hard and durable, soft, weathered and friable
c. Color and texture

4. The weight loss of the sample due to treating (Step C.1 - Step G.5).

5. Any difficulty encountered in dispersing the particles smaller than $2 \mathrm{~mm}$.

\section{UNCERTAINTIES}

A. Measurement

1. Weights

2. Depth of pipet upon withdrawing sample.

3. Sieve sizes

4. Settling time measurements (time, viscosity, temp)

B. Procedural

1. Specific gravity of soil

a. If not within $\pm 0.05 \mathrm{~g} \mathrm{~cm}^{-1}$, there will be a settling time error of up to $2 \%$.

2. Specific gravity of the water

a. If assumed to be equal to 1 and the dispersing agent is $<0.5 \mathrm{~g}^{-1}$, there will be an up to $0.3 \%$ error.

b. If dispersing agent has a concentration of up to $5.0 \mathrm{~g} \mathrm{l}^{-1}$, error will be up to 
$2 \%$ in settling time.

3. Deviation of settling rate from Stoke's Law

4. Speed at which particles are pipeted from settling column.

5. Losses/particle break down during agitat. jn, treatment and separation.

\section{REFERENCES}

\section{ASTM-D422}

Gee, G. W. and J.W. Bauder. 1986. Particle-size analysis. In: Klute, A ed. Methods of soil analysis, Part 1. Madison, WI: American Society of Agronomy, $383-412$.

Procedure 3.3.1.5.a. Soil Specific Gravity 
Sampling times for $5-\mu \mathrm{m}$ and $20-\mu \mathrm{m}$ size fractions at a $10-\mathrm{cm}$ sampling depth for pipet in $0.5 \mathrm{~g} / \mathrm{L}$ HMP solution. over the temperature range 20 to $30^{\circ} \mathrm{C}$ for selected particle densities.

\begin{tabular}{|c|c|c|c|c|c|c|}
\hline \multirow{3}{*}{$\begin{array}{c}\text { Tempera. } \\
\text { ture }\end{array}$} & \multicolumn{3}{|c|}{ 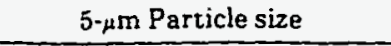 } & \multicolumn{3}{|c|}{$20-\mu \mathrm{m}$ Particle size } \\
\hline & \multicolumn{3}{|c|}{ Particle density $\left(\mathrm{Mg} / \mathrm{m}^{3}\right)$} & \multicolumn{3}{|c|}{ Particle density $\left(\mathrm{M} \mathrm{g} / \mathrm{m}^{\prime}\right)$} \\
\hline & 2.4 & 2.6 & 2.8 & 2.4 & 2.6 & 2.8 \\
\hline${ }^{\circ} \mathrm{C}$ & . & - & $-\operatorname{tir}$ & 2) & & \\
\hline 20 & 87.7 & 76.8 & 68.3 & 5.5 & 4.8 & 4.3 \\
\hline 21 & 85.7 & 75.0 & 66.7 & 5.4 & 4.7 & 4.2 \\
\hline 22 & 83.7 & 73.2 & 65.1 & 5.2 & 4.6 & 4.1 \\
\hline 23 & 81.7 & 71.5 & 63.6 & 5.1 & 4.5 & 4.0 \\
\hline 24 & 79.9 & 69.9 & 62.1 & 5.0 & 4.4 & 3.9 \\
\hline 25 & 78.0 & 68.3 & 60.7 & 4.9 & 4.3 & 3.8 \\
\hline 26 & 76.3 & 66.8 & 59.3 & 4.8 & 4.2 & 37 \\
\hline 27 & 74.6 & 65.3 & 58.0 & 4.7 & 4.1 & 3.6 \\
\hline 28 & 73.0 & 63.9 & 56.8 & 4.6 & 4.0 & 3.5 \\
\hline 29 & 71.4 & 62.5 & 55.6 & 4.5 & 3.9 & 3.5 \\
\hline 30 & 69.9 & 61.2 & 54.4 & 4.4 & 3.8 & 3.4 \\
\hline
\end{tabular}

Settling times for 2- $\mu \mathrm{m}$ clay at various temperatures. Calculated for a $10-\mathrm{cm}$ sampling depth in distilled water. $0.5 \mathrm{~g} / \mathrm{L}$, and $5 \mathrm{~g} / \mathrm{L}$ HMP solutions: with a particle density equal to $2.60 \mathrm{Mg} / \mathrm{m}^{3}$.

\begin{tabular}{|c|c|c|c|c|c|c|}
\hline \multirow[b]{2}{*}{$\begin{array}{l}\text { Tempera- } \\
\text { ture }\end{array}$} & \multicolumn{3}{|c|}{ Viscosity } & \multicolumn{3}{|c|}{ Settling time } \\
\hline & $\begin{array}{c}\text { Distilled } \\
\mathrm{H}_{2} \mathrm{O} \\
\end{array}$ & $\begin{array}{c}0.5 \mathrm{~g} / \mathrm{L} \\
\text { HMP }\end{array}$ & $\begin{array}{c}5.0 \mathrm{~g} / \mathrm{L} \\
\mathrm{HMP} \\
\end{array}$ & $\begin{array}{c}\text { Distilled } \\
\mathrm{H}_{2} \mathrm{O} \\
\end{array}$ & $\begin{array}{l}0.5 \mathrm{~g} / \mathrm{L} \\
\mathrm{HMP}\end{array}$ & $\begin{array}{c}5.0 \mathrm{~g} / \mathrm{L} \\
\mathrm{HMP} \\
\end{array}$ \\
\hline${ }^{\circ} \mathrm{C}$ & \multicolumn{3}{|c|}{$\longrightarrow 10^{-3} \mathrm{~kg} \mathrm{~m}^{-1} \mathrm{~s}^{-1} \longrightarrow$} & 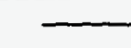 & $-h$ & - \\
\hline $\begin{array}{l}18 \\
20 \\
22 \\
24 \\
26 \\
28 \\
30\end{array}$ & $\begin{array}{l}1.0530 \\
1.0020 \\
0.9548 \\
0.9111 \\
0.8705 \\
0.8327 \\
0.7975\end{array}$ & $\begin{array}{l}1.0553 \\
1.0042 \\
0.9569 \\
0.9131 \\
0.8724 \\
0.8345 \\
0.7992\end{array}$ & $\begin{array}{l}1.0759 \\
1.0238 \\
0.9756 \\
0.9310 \\
0.8895 \\
0.8508 \\
0.8149\end{array}$ & $\begin{array}{l}8.39 \\
7.99 \\
7.61 \\
7.26 \\
6.94 \\
6.64 \\
6.36\end{array}$ & $\begin{array}{l}8.41 \\
8.00 \\
7.63 \\
7.28 \\
6.95 \\
6.65 \\
6.37\end{array}$ & $\begin{array}{l}8.58 \\
8.16 \\
7.78 \\
7.42 \\
7.09 \\
6.78 \\
6.50\end{array}$ \\
\hline
\end{tabular}

Selected depths for 2- $\mathrm{m}$ clay at specified times and temperatures. assuming a particle density of $2.60 \mathrm{Mg} / \mathrm{m}^{2}$ and dispersion with $0.5 \mathrm{~g} / \mathrm{L}$ HMP solution.

\begin{tabular}{cccccc}
\hline & & \multicolumn{5}{c}{ Sampling depth } \\
\cline { 4 - 6 } Temperature & Viscosity & $4.5 \mathrm{~h}$ & $5.0 \mathrm{~h}$ & $5.5 \mathrm{~h}$ & $6.0 \mathrm{~h}$ \\
\hline${ }^{\circ} \mathrm{C}$ & $10^{-\mathrm{J}} \mathrm{kg} \mathrm{m}^{-1} \mathrm{~s}^{-1}$ & & & & \\
20 & 1.0042 & 5.6 & 6.2 & 6.9 & 7.5 \\
21 & 0.9800 & 5.8 & 6.4 & 7.0 & 7.7 \\
22 & 0.9569 & 5.9 & 6.5 & 7.2 & 7.9 \\
23 & 0.9345 & 6.0 & 6.7 & 7.4 & 8.1 \\
24 & 0.9131 & 6.2 & 6.9 & 7.6 & 8.2 \\
25 & 0.8923 & 6.3 & 7.0 & 7.7 & 8.4 \\
26 & 0.8724 & 6.5 & 7.2 & 7.9 & 8.6 \\
27 & 0.8532 & 6.6 & 7.4 & 8.1 & 8.8 \\
28 & 0.8345 & 6.8 & 7.5 & 8.3 & 9.0 \\
29 & 0.8166 & 6.9 & 7.7 & 8.4 & 9.2 \\
30 & 0.7992 & 7.1 & 7.8 & 8.6 & 9.4 \\
\hline
\end{tabular}




\subsubsection{5.a Soil Specific Gravity}

\section{PURPOSE}

This procedure provides an analytical method of determining the specific gravity of the silt and clay fraction of a soil sample.

\section{REQUIRED EQUIPMENT/REAGENTS}

A. Equipment

1. 100 -ml volumetric flask with stopper

2. Balance sensitive to $0.001 \mathrm{~g}$

3. Thermometer accurate to $0.5^{\circ} \mathrm{C}$

4. 50-ml beaker

5. Sample drying tray

6. Wooden rolling pin

7. $53-\mu \mathrm{m}$ sieve

8. Desiccator

9. Magnetic stirrer/hot plate

B. Reagents

1. Distilled water

\section{PROCEDURE}

A. Sample Preparation

1. Thinly spread about $100 \mathrm{~g}$ of sample out on a drying tray and allow it air dry.

2. Mix and roll the sample with a wooden rolling pin to break up the clods.

3. Using distilled water, wash sample through a 53- $\mu \mathrm{m}$ sieve.

4. Oven-dry both the portion of the sample retained on the sieve, and that portion passing through it, at $110 \pm 5^{\circ} \mathrm{C}$.

5. Dry-sieve the previously retained portion through the $53-\mu \mathrm{m}$ sieve.

6. Thoroughly mix both portions of the sample that passed through the sieve.

7. Repeat steps 1 through 6 as necessary until about $35 \mathrm{~g}$ of sample $<53 \mathrm{~mm}$ is obtained.

8. Oven dry the $35 \mathrm{~g}$ sample that passed through the $53-\mu \mathrm{m}$ sieve overnight or until the sample weight changes by less than $0.05 \%$ between successive weighings. 
a) Cool sample in a desiccator before weighing.

9. Record final sample weight.

10. Place sample in a clean 50-ml beaker and add enough distilled water to cover the sample. Soak for at least 12 hours (overnight).

B. Calibration of Pycnometer

1. Wash, dry, weigh, and record the mass a $100-\mathrm{ml}$ volumetric flask.

2. Fill the volumetric flask to the mark with distilled water.

3. Wipe dry the outside of the flask and then weigh, and record the weight of the flask with the water in it.

4. Measure and record the water temperature.

5. Record the difference of weights between steps 3 and 1 as the weight of the water.

6. Record the volume of water in the flask $(100 \mathrm{ml})$

C. Measurement

1. Transfer the sample slurry from the sample beaker (from step A.10, above) to the volumetric flash, thoroughly rinsing the sample from the beaker with distilled water.

2. Add enough distilled water to bring the volume of the water in the flask to slightly cover the soil.

3. Place a stir bar into the flask and while slowly stirring, gently boil the solution for ten minutes to remove entrapped air.

4. Cool the flask to room temperature and retrieve stir bar, rinsing off into flask.

5. Fill the flask with distilled water to the mark.

6. Clean and dry the outside of the flask.

7. Weight and record the mass of the flask.

8. Measure and record the temperature of the contents of the flask.

D. Calculations

1. Calculate the expected mass of the pycnometer (100-ml volumetric flask) the experimental temperature $\left(\mathrm{W}_{I_{x}}\right)$ as follows:

$$
\mathrm{W}_{\text {TX }}=\left(\frac{\rho_{\text {Tcal }}}{\rho T} \mathrm{~W}_{\text {water }}\right)+\mathrm{W}_{\text {reak }}
$$

where: $\quad \mathrm{W}_{\text {wate }}$ is the weight of the water recorded in step C.5

$\mathrm{W}_{\text {flack }}$ is the weight of the flask recorded in step C.1

$\rho_{\text {Teal }}$ is the density of water at the temperature recorded in step C.4 $\rho_{\mathrm{Tx}}$ is the density of water at the temperature recorded in step D.8 (See attached tables for water densities.) 
2. Calculate the specific gravity of the soil at the experimental temperature as follows:

$$
\mathrm{G}_{\mathrm{TX}}=\frac{\mathrm{W}_{\text {semple }}}{\mathrm{W}_{\text {semple }}+\mathrm{W}_{\mathrm{TX}}-\mathrm{W}_{\text {Fillod }}}
$$

where: $\mathrm{G}_{\mathrm{Tx}}$ is the specific gravity of the slit and clay at the experimental temperature

$\mathrm{W}_{\text {sumple }}$ is the oven-dried sample weight recorded in step B.9

$\mathrm{W}_{\text {filled }}$ is the weight of the flask recorded in step D.7.

3. If the specific gravity of the soil is desired at some temperature other than $T_{x}$, it may be obtained by multiplying $G$ by the ratio the density of water at $T_{x}$ to the density of water at the desired temperature.

\section{UNCERTAINTIES}

A. Measurement

1. Weights

2. Temperature

3. Volume errors

B. Procedural

1. Recorded water densities at given temperature

\section{REFERENCES}

ASTM-D854-91. Standard test method for specific gravity of soils. 
TABLE 1 Density of Water and Correction Factor $K$ for Various Temperatures

\begin{tabular}{ccc}
\hline Temperature. ${ }^{\circ} \mathrm{C}$ & Density of Water $(\mathrm{g} / \mathrm{mL})$ & Correction Facto $K$ \\
\hline 16.0 & 0.99897 & 1.0007 \\
16.5 & 0.99889 & 1.0007 \\
17.0 & 0.99880 & 1.0006 \\
17.5 & 0.99871 & 1.0005 \\
18.0 & 0.99862 & 1.0004 \\
18.5 & 0.99853 & 1.0003 \\
19.0 & 0.99843 & 1.0002 \\
19.5 & 0.99833 & 1.0001 \\
20.0 & 0.99823 & 1.0000 \\
20.5 & 0.99812 & 0.9999 \\
21.0 & 0.99802 & 0.9998 \\
21.5 & 0.99791 & 0.9997 \\
22.0 & 0.99780 & 0.9996 \\
22.5 & 0.99768 & 0.9995 \\
23.0 & 0.99757 & 0.9993 \\
23.5 & 0.99745 & 0.9992 \\
24.0 & 0.99732 & 0.9991 \\
24.5 & 0.99720 & 0.9990 \\
25.0 & 0.99707 & 0.9988 \\
25.5 & 0.99694 & 0.9987 \\
26.0 & 0.99681 & 0.9986 \\
26.5 & 0.99668 & 0.9984 \\
27.0 & 0.99654 & 0.9983 \\
27.5 & 0.99640 & 0.9982 \\
28.0 & 0.99626 & 0.9980 \\
28.5 & 0.99612 & 0.9979 \\
29.0 & 0.99597 & 0.9977 \\
29.5 & 0.99582 & 0.9976 \\
30.0 & 099567 & 0.9974 \\
\hline & & \\
\hline & & 0
\end{tabular}




\subsubsection{Soil Cation Exchange Capacity}

\section{PURPOSE}

This procedure provides an analytical method of determining the cation exchange capacity per unit mass of an acidic soil. The effects of $\mathrm{pH}$ and ionic strength of the pore water on cation exchange capacity may also be examined.

\section{REQUIRED EQUIPMENT/REAGENTS}

A. Equipment

1. High speed centrifuge

2. $50 \mathrm{ml}$ Oak Ridge centrifuge tubes with caps

3. Vortex shaker

4. Wrist action shaker

5. Balance sensitive to $0.001 \mathrm{~g}$

6. $30 \mathrm{ml}$ sample bottles

7. $10 \mathrm{ml}$ test tubes for conductivity checks

8. Conductivity meter

9. $\mathrm{pH}$ meter

10. 1-liter volumetric flasks

B. Reagents

1. $0.1 \mathrm{M}$ barium chloride dihydrate $\left[\mathrm{BaCl}_{2} \cdot 2 \mathrm{H}_{2} \mathrm{O}\right]$

2. Barium hydroxide $\left[\mathrm{Ba}(\mathrm{OH})_{2} \cdot 8 \mathrm{H}_{2} \mathrm{O}\right]$

3. Hydrochloric acid [ $\mathrm{HCl}]$

4. Magnesium sulfate heptahydrate $\left[\mathrm{MgSO}_{4} \cdot 7 \mathrm{H}_{2} \mathrm{O}\right]$

5. Distilled, deionized (DDI) water

6. $\mathrm{pH}$ secondary standard solutions ( $\mathrm{pH}$ of 2.0, 4.0, 7.0 and 10.0)

\section{PROCEDURE}

NOTE: Soil $\mathrm{pH}$ and hygroscopic water content and porewater conductivity must first be measured in accordance with procedures 3.3.1.1 and 3.3.1.6 and applicable field test procedures. 


\section{A. Reagent Preparation}

CAUTION: Do not dispose of barium compounds or solutions down drains. Decant solutions into a holding beaker and transfer to an appropriate satellite waste area as necessary or at the end of shift.

1. Saturating solution

a. Dissolve $24.4 \mathrm{~g}$ of $\mathrm{BaCl}_{2} \cdot 2 \mathrm{H}_{2} \mathrm{O}$ in enough DDI water to make 1 liter of $0.1 \mathrm{M}$ solution.

2. Equilibrating solution

a. Dissolve $0.4889 \mathrm{~g}$ of $\mathrm{BaCl}_{2} \cdot 2 \mathrm{H}_{2} \mathrm{O}$ in enough DDI water to make 1 liter of $0.002 \mathrm{M}$ solution.

b. Adjust the $\mathrm{pH}$ of the equilibrating solution to that of the soil's normal $\mathrm{pH}$ through the addition of $\mathrm{HCl}$ and $\mathrm{Ba}(\mathrm{OH})_{2} \bullet 8 \mathrm{H}_{2} \mathrm{O}$.

c. If it is desired to study the effect of $\mathrm{pH}$ on cation exchange capacity, prepare additional equilibrating solutions of the desired study $\mathrm{pH}$.

3. Reactant Solution

a. Dissolve $1.232 \mathrm{~g}$ of $\mathrm{MgSO}_{4} \cdot 7 \mathrm{H}_{2} \mathrm{O}$ in enough DDI water to make 1 liter of $0.005 \mathrm{M}$ solution

4. Ionic strength reference solution

a. Dissolve $0.3700 \mathrm{~g}$ of $\mathrm{MgSO}_{4} \cdot 7 \mathrm{H}_{2} \mathrm{O}$ in enough DDI water to make 1 liter of a $0.0015 \mathrm{M}$ solution.

b. If the actual ionic strength of the pore water in equilibrium with the soil is known or the cation exchange capacity at some other ionic strength is desired, the ionic strength reference solution can be adjusted to that strength if desired.

B. Perform pH meter check in accordance with procedure 3.3.1.1 section $\mathrm{C}$.

C. Sample Analysis

1. Clean, dry and weigh a centrifuge tube. Record the weight of the tube.

2. Add approximately 2 grams of air-dried soil to the tube. Weight the tube and calculate and record the soil's weight.

3. Add $20 \mathrm{ml}$ of saturating solution to the tube.

4. Stopper and shake the tube for 2 hours.

5. Centrifuge the tubes at $10,000 \mathrm{RPM}$ for 30 minutes.

6. Decant the solution.

CAUTION: Do not dispose of barium solutions down drains. Decant solutions into a holding beaker and transfer to an appropriate satellite waste area as necessary or at the end of shift.

7. Add $20 \mathrm{ml}$ of equilibrating solution to the tube.

8. Vortex the tube for 5 to 10 seconds to disperse the sediment.

9. Stopper and shake the tube for 1 hour. 
10. Centrifuge the tubes at 10,000 RPM for 30 minutes.

11. Decant the solution.

12. Repeat steps 7 through 11 twice.

13. Dry the outside of the tube and weigh it. Record the combined weight of the tube and the mixture, then calculate and record the weight of the soil contained in the tube.

14. Add $10 \mathrm{ml}$ of reactant solution to the tube.

a. Save approximately $10 \mathrm{ml}$ of reactant solution for $\mathrm{Mg}$ analysis.

15. Weigh and record the weight of the tube. Calculate the actual amount of reactant solution added to the tube.

16. Vortex the tube for 5 to 10 seconds to fully disperse the sediment.

17. Shake for 1 hour.

18. Match the conductance of the solution to that of the ionic strength reference solution by adding DDI water or additional reactant solution.

a. Record if additions were necessary, and if so, how much and of what.

b. Consult the operating procedure of the conductivity instrument being used for proper measurement procedures.

19. Dry the outside of the tube and weight. Calculate and record the weight of $\mathrm{Mg}$ solution or DDI water added.

20. Gently shake the solution overnight to allow time for the $\mathrm{Mg}$ to fully react.

21. Measure the conductance of the solution.

22. Centrifuge the mixture at 10,000 RPM for 10 minutes.

23. Filter the supernate from each tube through $0.45 \mu \mathrm{m}$ filters and into a $30-\mathrm{ml}$ sample bottle.

24. Pour approximately $2 \mathrm{ml}$ of solution into a small test suitable for $\mathrm{pH}$ measurement.

25. Measure the $\mathrm{pH}$ of the supernatant

a. Complete the $\mathrm{pH}$ meter calibration in accordance with procedure 3.3.1.1.III section $\mathrm{C}$

b. Thoroughly rinse electrode with type II water and blot dry.

c. Insert electrode into the solution.

d. Record meter reading in $\mathrm{mV}$ in the lab notebook.

e. Using the calculated calibration curve, calculate the solution $\mathrm{pH}$.

26. Determine $\mathrm{Mg}$ concentration in the samples and the blank prepared in step 14.a by ICP-ES.

D. Calculation of Cation Exchange Capacity

1. If no additional reactant solution was added in step C. 18 and $\mathrm{Mg}$ concentrations are reported in $\mathrm{mg} \mathrm{l}^{-1}$, the CEC is given as follows:

$\mathrm{CEC}=\frac{[\mathrm{Mg}]_{\text {blemk }} * \mathrm{Wt}_{\text {Reactant }}-[\mathrm{Mg}]_{\text {sample }} * \mathrm{Wt}_{\text {Total }} * 0.08226}{\mathrm{Wt}_{\text {soil }}}$ 
where: Cation exchange capacity $(C E C)$ is given in milliequivelents per kilogram of soil;

$[\mathrm{Mg}]_{\text {blenk }}$ and $[\mathrm{Mg}]_{\text {sumple }}$ are the magnesium concentrations of the reagent blank and the sample reported in $\mathrm{mg} \mathrm{I}^{-1}$; and

$\mathrm{Wt}_{\text {reactent }}, \mathrm{W}_{\text {sample, }}$ and $\mathrm{Wt}_{\text {soil }}$ are the weights calculated in steps $\mathrm{C} .15$, C. 19 and C. 2 respectively. The soil mass may be corrected for hygroscopic moisture content, if significant.

2. If additional reactant solution was added in step C.18:

$$
\mathrm{CEC}=\frac{[\mathrm{Mg}]_{\text {bimk }}-[\mathrm{Mg}]_{\text {sempte }} * \mathrm{Wt}_{\text {Toal }} * 0.08226}{\mathrm{Wt}_{\text {soil }}}
$$

3. Report CEC in meq $\mathrm{kg}^{-1}$ at the $\mathrm{pH}$ and ionic strength measured in steps $\mathrm{D} .25$ and $D .21$ respectively.

\section{UNCERTAINTIES}

A. Measurement

1. Weights

2. Precision of the ICP-ES

3. Ionic strength measurement

4. $\mathrm{pH}$ measurement

B. Procedural

1. Concentrations of reagents

a. Are they strong enough for the CEC of the soil?

2. Completeness of processes

\section{REFERENCES}

A. Rhoades, J. D. 1982. Cation Exchange Capacity. In: Page, A. L., Miller, R. H., and Keeney, D. R. eds. Methods of soil analysis, part 2. Madison, WI: Soil Science Society of America, 149-157.

Procedure 3.3.1.6.a. Hygroscopic moisture content of soil

Procedure 3.3.1.1. Soil pH 


\subsubsection{Soil Hygroscopic Moisture Content}

\section{PURPOSE}

This procedure provides a simple method of determining the hygroscopic moisture content of a soil sample.

\section{REQUIRED EQUIPMENT/REAGENTS}

A. Equipment

1. Conventional constant temperature oven

2. Desiccator

3. 50-ml pyrex beakers

4. Balance accurate to $0.001 \mathrm{~g}$

B. Reagents

1. None

\section{PROCEDURE}

A. Analysis

1. Clean, dry, weigh, and record the mass of a $50 \mathrm{ml}$ Pyrex beaker.

2. Add approximately $15 \mathrm{~g}$ of the air-dried sample to the beaker.

3. Weight the beaker with the sample. Calculate and record initial sample mass.

4. Oven dry the sample at $110 \pm 5^{\circ} \mathrm{C}$.

5. Remove sample from oven and allow it to cool in a desiccator.

6 . Weigh and record the mass of the sample.

7. Repeat steps 4,5 , and 6 until the difference in sample mass between successive readings is $\leq 0.05 \mathrm{~g}$.

8. Calculate the mass of the oven-dried sample.

9. Calculate hygroscopic moisture content $(\theta)$ as follows:

$\theta \rho=\frac{\text { Air - dried soil mass recorded in step A.3 }}{\text { Oven - dried soil mass recorded in step A.8 }}-1$ 


\section{UNCERTAINTLES}
A. Measurements
1. Weights
B. Procedural
1. Sample dryness
2. Temperature of weighing.

\section{REFERENCES}

Gerdner, W. H. 1986. Water content. In Klute, A. ed. Methods of soil analysis, part 1. Madison, WI: Soil Science of America, 493-544.

\section{ASTM-D422}




\subsubsection{Soil Sequential Extraction}

\section{PURPOSE}

This procedure provides a method of identifying the trace metals and contaminates sorbed on to specific soil phases of a sample. Through selective chemistry, 8 different soil phases are identified and removed utilizing a sequential extraction procedure as outlined by Miller (1986). Samples extracted from this procedure can be analyzed by ICP-MS.

\section{REQUIRED EQUIPMENT/REAGENTS}

A. Equipment

1. 50-ml Oak Ridge centrifuge tubes

2. \#10 (2-mm) soil sieve

3. $0.45-\mu \mathrm{m}$ cellulose acetate or cellulose nitrate syringe filters

4. $0.45-\mu \mathrm{m}$ PTFE syringe filters
a. or \#42 Whatman filter paper and plastic filtering funnels

5. 30-ml syringes

6. 1-liter volumetric flasks

7. 1-liter reagent bottles

8. 125-ml glass reagent bottle

9. 50-ml plastic volumetric flasks

10. 30-ml sample bottles

11. 60-ml sample bottles

12. Hot acid digestion bombs with PTFE inserts

13. Disposable transfer pipets

14. Test tube racks for 50-ml Oak Ridge tubes

15. Alumimum foil

16. aluminum drying dishes

17. High-speed centrifuge

18. 25-ml graduated cylinders

19. 50-ml graduated cylinders

B. Reagents

1. Ultra-pure $72 \%$ nitric acid $\left[\mathrm{HNO}_{3}\right]$

2. Ultra-pure $48 \%$ hydrofluoric acid [HF]

3. Ultra-pure $36 \%$ hydrochloric acid $[\mathrm{HCl}]$ 
4. Hydroxylamine-hydrochloride $\left[\mathrm{NH}_{2} \mathrm{OH} \cdot \mathrm{HCl}\right]$

5. Ammonium oxalate $\left[\left(\mathrm{NH}_{4}\right)_{2} \mathrm{C}_{2} \mathrm{O}_{4} \cdot \mathrm{H}_{2} \mathrm{O}\right]$

6. Calcium nitrate $\left[\mathrm{Ca}\left(\mathrm{NO}_{3}\right)_{2} \cdot 4 \mathrm{H}_{2} \mathrm{O}\right]$

7. $99.5 \%$ Glacial acetic acid $\left[\mathrm{CH}_{3} \mathrm{COOH}\right]$

8. Sodium pyrophosphate $\left[\mathrm{NaP}_{2} \mathrm{O}_{2} \cdot 1 \mathrm{OH}_{2} \mathrm{O}\right]$

9. Oxalic acid [C2 $\left.2 \mathrm{H}_{2} \mathrm{O} 4\right]$ or oxalic acid dihydrate [C2 $\left.2 \mathrm{H}_{2} \mathrm{O} 4 \cdot 2 \mathrm{H}_{2} \mathrm{O}\right]$

10. Citric acid monohydrate $\left[\mathrm{C}_{6} \mathrm{H}_{8} \mathrm{O}_{7} \cdot \mathrm{H}_{2} \mathrm{O}\right.$ or $\left.\mathrm{HOC}\left(\mathrm{CH}_{2} \mathrm{CO}_{2} \mathrm{H}_{2}\right)_{2} \mathrm{CO}_{2} \mathrm{H} \cdot \mathrm{H}_{2} \mathrm{O}\right]$

11. Sodium citrate dihydrate $\left[\mathrm{NaC}_{6} \mathrm{H}_{5} \mathrm{O}_{7} \cdot 2 \mathrm{H}_{2} \mathrm{O}\right]$

12. Sodium hydrosulfite or hyposulfite dithionite $\left[\mathrm{Na}_{2} \mathrm{~S}_{2} \mathrm{O}_{4} \cdot \mathrm{H}_{2} \mathrm{O}\right]$

13. Distilled, deionized (DDI) water

\section{PROCEDURE}

A. Preparation of Reagents

1. Exchangeable Reagent

a. Add $118 \mathrm{~g}$ of calcium nitrate to a 1-liter volumetric flask.

b. Fill the flask about half full with DDI water and swirl to dissolve calcium nitrate.

c. Dilute to the mark with DDI water.

2. Acid Soluble Reagent

a. Add $23.6 \mathrm{~g}$ of calcium nitrate and $25.4 \mathrm{ml}$ of glacial acetic acid into a 1-liter volumetric flask

b. Dilute up to the mark with DDI water to make a $0.44 \mathrm{M}$ acetic acid $0.1 \mathrm{M}$ calcium nitrate solution.

3. Manganese Oxide Occluded Reagent

a. Add $0.694 \mathrm{~g}$ of hydroxylamine-hydrochloride and $6.3 \mathrm{ml}$ of $72 \%$ nitric acid into a 1-liter volumetric flask.

b. Dilute up to the mark with DDI water to obtain a $0.01 \mathrm{M}$ hydroxylaminehydrochloride/0.1 M nitric acid solution.

4. Organically Bound Reagent

a. Add $44.6 \mathrm{~g}$ of sodium pyrophosphate into a 1-liter volumetric flask.

b. Dilute to the mark with DDI water to make a $0.1 \mathrm{M}$ solution.

5. Amorphous Iron Oxide Reagent:

a. $24.9 \mathrm{~g}$ of ammonium oxalate and $9.00 \mathrm{~g}$ of oxalic acid (or $12.6 \mathrm{~g}$ of oxalic acid dihydrate) into a 1-liter volumetric flask

b Dilute to the mark with DDI water to make a $0.175 \mathrm{M}$ ammonium oxate/0.1 M oxalic acid solution.

6. Crystalline Fe Oxide Reagent:

a. Add $44.1 \mathrm{~g}$ of sodium citrate dihydrate and $10.51 \mathrm{~g}$ of citric acid hydrate to a 1-liter volumetric flask.

b. Dilute to the mark with DDI water to make a $0.15 \mathrm{M}$ sodium citrate/ $0.05 \mathrm{M}$ citric acid solution.

7. Aqua Regia

a. In a fume hood add $20 \mathrm{ml}$ of $72 \%$ nitric acid and $60 \mathrm{ml}$ of $36 \%$ 
hydrochloric acid in a 125-ml labeled storage bottle.

b. Leave the bottle uncovered overnight to allow gas from reaction to escape.

c. Store in an approved acid storage area.

8. Calcium Nitrate Wash Solution

a. Add $3 \mathrm{~g}$ of calcium nitrate to a 1-liter volumetric flask.

b. Dilute to the mark with DDI water to make a $0.0127 \mathrm{M}$ solution.

B. Sample Preparation

1. Sieve the air-dried soil sample through a $2-\mathrm{mm}$ sieve.

2. Record the weight of clean, dry, 50-ml Oak Ridge centrifuge tubes. 4 tubes are required for each sample (three repetitions and one blank).

3. Add approximately $0.750 \mathrm{~g}$ of the air-dried, sieved soil into each pre-weighed centrifuge tube (except the blank).

C. Soluble Fraction

1. Add 30-ml of DDI water to each tube

2. Record the weight of the tube and added water. Calculate and record the actual volume of water added.

3. Shake for 16 hours on a wrist-action shaker at approximately 90 cycles per minute

4. Centrifuge at $10,000 \mathrm{RPM}$ for 30 minutes.

NOTE: During this entire procedure, use a transfer pipet to carefully remove the supernate from the tubes and transfer it to the syringe barrel or discard it.

5. Filter supernate from each tube through a $0.45-\mu \mathrm{m}$ cellulose-acetate filter and into a 30-ml sample bottle.

6. Acidify the supernate with $150 \mu \mathrm{l}$ of $72 \%$ nitric acid and save for analysis.

7. Weigh and record the weight of each tube and extract. Record the actual volume of extract removed from each tube.

8. Add $20 \mathrm{ml}$ of DDI water to each tube.

9. Vortex each tube for 5 to 10 seconds to loosen soil from the tube.

10. Centrifuge for 30 minutes at $10,000 \mathrm{rpm}$.

11. Discard the supernate.

D. Easily Exchangeable Fraction

1. Record the weight of tube and residue.

2. Add $30 \mathrm{ml}$ of exchangeable reagent to each tube.

3. Record the weight of the tube and added reagent. Calculate and record the actual volume of reagent added.

4. Shake for 16 hours on a wrist-action shaker at approximately 90 cycles per minute.

5. Centrifuge for 30 minutes at $10,000 \mathrm{rpm}$.

6. Filter supernate from each tube through a $0.45-\mu \mathrm{m}$ cellulose-acetate filter 
and into a 30-ml sample bottle.

7. Acidify supernate with $150 \mu \mathrm{l}$ of $72 \%$ nitric acid and save for analysis.

8. Record the weight of tube and residue. Calculate the actual amount of supernate extracted.

9. Add $20 \mathrm{ml}$ of calcium nitrate wash solution to each tube.

10. Vortex each tube for 5 to 10 seconds to loosen soil from the tube.

11. Centrifuge for 30 minutes at $10,000 \mathrm{rpm}$.

12. Discard the supernate.

E. Acid Soluble Fraction

1. Record the weight of tube and residue.

2. Add $30 \mathrm{ml}$ of the acid soluble reagent to each tube.

3. Record the weight tube and residue. Calculate the amount of reagent added.

4. Shake for 8 hours on a wrist-action shaker at approximately 90 cycles per minute.

5. Centrifuge for 30 minutes at $10,000 \mathrm{rpm}$.

6. Filter supernate from each tube through a $0.45-\mu \mathrm{m}$ cellulose-acetate filter and into a 30-ml sample bottle.

7. Acidify supernate with $150 \mu l$ of $72 \%$ nitric acid and save for analysis.

8. Record the weight of tube and residue. Calculate the actual amount of supernate extracted.

9. Add $20 \mathrm{ml}$ of calcium nitrate wash solution to each tube.

10. Vortex each tube for 5 to 10 seconds to loosen soil from the tube.

11. Centrifuge for 30 minutes at $10,000 \mathrm{rpm}$.

12. Discard the supernate.

F. Manganese Oxide

1. Record weight of tube and residue.

2. Add $30 \mathrm{ml}$ of the acid soluble reagent to each tube.

3. Record the weight tube and residue. Calculate the amount of reagent added.

4. Shake for 30 minutes on a wrist-action shaker at approximately 90 cycles per minute.

5. Centrifuge for $30 \mathrm{~min}$. at $10,000 \mathrm{rpm}$.

6. Filter the supernate through a $0.45-\mu \mathrm{m}$ cellulose acetate filter and into a 30-ml sample bottle.

7. Acidify supernate with $150 \mu l$ of $72 \%$ nitric acid and save for analysis.

8. Record the weight of tube and residue. Calculate the actual amount supernate extracted.

9. Add $20 \mathrm{ml}$ of calcium nitrate wash solution to each tube.

10. Vortex each tube for 5 to 10 seconds to loosen soil from the tube.

11. Centrifuge for 3 to minutes at $10,000 \mathrm{rpm}$.

12. Discard the supernate.

G. Organically Bound Fraction: 
1. Record the weight of tube and residue.

2. Add $30 \mathrm{ml}$ of organically bound reagent to each tube.

3. Record the weight of each tube and reagent. Calculate and record the actual volume of reagent added.

4. Shake for 24 hours on a wrist-action shaker at approximately 90 cycles per minute.

5. Centrifuge for $30 \mathrm{~min}$. at $10,000 \mathrm{rpm}$.

6. Filter the supernate through a $0.45-\mu \mathrm{m}$ cellulose-acetate filter and into a 30-ml sample bottle.

7. Acidify the supernate with $150 \mu \mathrm{l}$ of $72 \%$ nitric acid and save for analysis.

8. Record the weight of each tube and residue. Calculate the actual amount supernate extracted.

9. Add $20 \mathrm{ml}$ of calcium nitrate wash solution to each tube.

10. Vortex each tube for 5 to 10 seconds to loosen soil from the tube.

11. Centrifuge for 30 minutes at $10,000 \mathrm{rpm}$.

12. Discard the supernate.

H. Noncystalline Aluminosilcates and Hydrous Oxides

NOTE: Steps 2 through 10 must be completed in such a manner to prevent ultra-violet light from influencing the sample. Steps 2, 3, and 7, and when loading or unloading tubes from the centrifuge should be performed under red light if possible. The presence of ultra-violet light cause the removal of the crystalline as well as the non-crystalline phases.

1. Record the weight of the tube and residue.

2. Label and record the weight of a $15 \mathrm{~cm}$ by $15 \mathrm{~cm}$ sheet of aluminum foil for each tube

3. Add $30 \mathrm{ml}$ of amorphous iron oxide reagent to each tube and immediately warp the tube in aluminum foil.

4. Record the weight of each tube, reagent and foil. Calculate and record the actual volume of reagent used.

5. Shake for 4 hours on a wrist-action shaker at approximately 90 cycles per minute.

6. Centrifuge the tubes for 30 minutes at $10,000 \mathrm{rpm}$.

7. Filter the supernate through a $0.45-\mu \mathrm{m}$ cellulose-acetate filter and into a $30-\mathrm{ml}$ sample bottle.

8. Record the weight of the each tube, residue and foil. Calculate the actual volume of supernate extracted.

9. Add $20 \mathrm{ml}$ of calcium nitrate wash solution to each tube.

10. Vortex each tube for 5 to 10 seconds to loosen soil from the tube.

11. Discard the foil and centrifuge each tube for 30 minutes at $10,000 \mathrm{rpm}$.

12. Discard the supernate.

I. Crystalline Iron Oxides and Aluminum Oxides 
1. Record the weight of the tubes and residue.

2. Add $30 \mathrm{ml}$ of crystalline oxide reagent to each tube.

3. Add $0.75 \mathrm{~g}$ of sodium hydrosulfite to each tube.

4. Record the weight of each tube and reagent. Calculate and record the actual volume of reagent added.

5. Shake for 0.5 hours in a water bath at $50^{\circ} \mathrm{C}$ at approximately 90 cycles per minute.

6. Centrifuge for 30 minutes at $10,000 \mathrm{rpm}$.

7. Filter the supernate through a $0.45-\mu \mathrm{m}$ cellulose-acetate filter and into a 30-ml sample bottle.

8. Acidify the supernate with $150 \mu l$ of $72 \%$ nitric acid and save for analysis.

9. Record the weight of tube and residue. Calculate the actual volume supernate extracted.

10. Add 20 nil of calcium nitrate wash solution to each tube.

11. Vortex each tube for 5 to 10 seconds to loosen soil from the tube.

12. Centrifuge for 30 minutes at $10,000 \mathrm{rpm}$

13. Discard the supernate.

J. Total and Partial Digestions of Samples

1. Label and weigh an aluminum drying dish for each tube.

2. Spray $\approx 5 \mathrm{ml}$ of DDI water into each tube and vortex the tube long enough to break up the pellet at the bottom.

3. With the aid of a DDI water rinse, transfer the tube's contents to a drying dish.

4. Dry the soil residue at $105^{\circ} \mathrm{C}$ in an oven until a constant weight is reached.

5. Place approximately $200 \mathrm{mg}$ of oven-dried soil residue from each tube into an acid digestion bomb insert.

6. Add approximately $200 \mathrm{mg}$ of original soil which has been air-dried and passed through a \#10 sieve into an acid digestion bomb insert.

DANGER: Steps $7,11,12$, and 13 must be performed inside of a flume hood.

7. Add to each $1 \mathrm{ml}$ of aqua regia and $10 \mathrm{ml}$ of $48 \% \mathrm{HF}$ to each vessel.

8. Place the bomb inserts into the digestion bomb shells and tighten until just hand tight.

9. Place bombs into a $105^{\circ} \mathrm{C}$ oven for 3 hours.

10. Remove the bombs from the oven and allow to cool for at least 15 minutes.

11. Filter the extract from the bomb inserts flasks through either PTFE syringe filters or \#42 Whatman filter paper into a plastic 50-ml volumetric flask.

12. Dilute to $50 \mathrm{ml}$ with DDI water.

13. Transfer to a $60-\mathrm{ml}$ sample bottle for storage and transfer.

\section{UNCERTAINTTES}

A. Actual volume and concentration of trace metals in sample extracted. Influenced 
by:

1. Initial sample size.

2. Density of reactant (deviation from unity)

3. Amount of and trace metal concentration in the washing reagent remaining in the tube from previous step.

B. Masking of metal concentrations during detection by the sample matrix.

\section{REFERENCES}

Miller, W. P.; Martens, D. C.; Zelanzy, L. W. 1986. Effect of the sequence in extraction of trace minerals from soils. Soil Sci. Soc. Am. 50:598-601.

Jackson, M. L.; Lim, C. H.; Zelazny, L. W. 1986. Oxides, hydroxides, and aluminosilicates. In: Klute, A. ed. Methods of soil analysis, part 1: Physical and mineralogical methods. 2nd ed. Madison, WI: American Society of Agronomy; 101-150. 
Appendix B

\section{RESULTS OF F AND H AREA BASIN SOIL ANALYSES}


Table B-1. Chemical and physical properties of soil samples in the A-transect (F-Area).

\begin{tabular}{|c|c|c|c|c|c|c|c|c|c|}
\hline $\begin{array}{c}\text { Sample } \\
\text { ID }\end{array}$ & $\begin{array}{l}\text { pH in } \\
\text { water }\end{array}$ & $\begin{array}{c}\mathrm{pH} \text { in } \\
0.01 \mathrm{M} \mathrm{CaCl}\end{array}$ & $\begin{array}{c}\text { Carbon } \\
\text { Content (\%) }\end{array}$ & $\begin{array}{c}\text { Carbon } \\
\text { Std. Dev. }\end{array}$ & $\%$ Sand & $\%$ Silt & $\%$ Clay & $\begin{array}{c}\text { CEC } \\
\left.(\operatorname{meg~kg})^{-1}\right)\end{array}$ & $\begin{array}{c}\text { CEC } \\
\text { Std. Dev. }\end{array}$ \\
\hline A11S & 4.28 & NA & NA & NA & 88.3 & 2.7 & 9.0 & 9.97 & 0.14 \\
\hline A12S & 3.79 & NA & NA & NA & 99.5 & 0 & 0.5 & 1.11 & 0.16 \\
\hline A13S & 4.23 & 4.06 & 0.00870 & 0.00048 & 95.0 & 1.6 & 3.3 & 1.82 & 0.20 \\
\hline A13SR & 4.53 & NA & 0.00208 & 0.00079 & 93.6 & 3.4 & 3.0 & 3.74 & 0.16 \\
\hline A21S & 3.96 & 3.91 & NA & NA & 89.9 & 5.1 & 5.0 & 5.94 & 0.17 \\
\hline A22S & 3.59 & NA & NA & NA & 95.7 & 2.8 & 1.5 & 1.39 & 0.16 \\
\hline A23S & 3.85 & 3.95 & NA & NA & 95.3 & 0.2 & 4.5 & 1.40 & 0.17 \\
\hline A31S & 3.63 & 3.61 & NA & NA & 95.6 & 0 & 4.4 & 7.92 & 0.17 \\
\hline A32S & 3.41 & NA & NA & NA & 89.5 & 7.4 & 3.1 & $<1$ & \\
\hline A41S & 4.67 & NA & 0.00149 & 0.00103 & 92.2 & 2.9 & 4.9 & 4.03 & 0.20 \\
\hline A42S & 3.79 & 3.76 & NA & NA & 91.7 & 3.6 & 4.7 & 2.10 & 0.15 \\
\hline A51S & 4.74 & 3.86 & 0.00702 & 0.00239 & 76.5 & 10.0 & 13.5 & 12.5 & 0.17 \\
\hline A52S & 5.07 & 4.27 & 0.00414 & 0.00255 & 92.6 & 4.8 & 2.5 & 0.68 & 0.18 \\
\hline A53S & 5.12 & 4.93 & 0.00732 & 0.00268 & 91.7 & 6.3 & 2.0 & 0.42 & 0.18 \\
\hline
\end{tabular}

NA - Not analyzed for. 
Table B-2. Chemical and physical properties of soil samples in the B-transect (F-Area).

\begin{tabular}{|c|c|c|c|c|c|c|c|c|c|}
\hline $\begin{array}{c}\text { Sample } \\
\text { ID }\end{array}$ & $\begin{array}{l}\text { pH in } \\
\text { water }\end{array}$ & $\begin{array}{c}\mathrm{pH} \text { in } \\
0.01 \mathrm{M} \mathrm{CaCl}_{2}\end{array}$ & $\begin{array}{c}\text { Carbon } \\
\text { Content (\%) }\end{array}$ & $\begin{array}{c}\text { Carbon } \\
\text { Std. Dev. }\end{array}$ & $\%$ Sand & $\%$ Silt & $\%$ Clay & $\begin{array}{c}\text { CEC } \\
\left(\operatorname{meq~} \mathbf{k g}^{-1}\right)\end{array}$ & $\begin{array}{c}\text { CEC } \\
\text { Std. Dev. }\end{array}$ \\
\hline B11S & 4.69 & NA & 0.0126 & 0.0014 & 80.4 & 4.4 & 15.2 & 10.8 & 0.12 \\
\hline B12S & 4.83 & NA & NA & NA & 89.6 & 4.1 & 6.2 & 11.9 & 0.15 \\
\hline B13S & 4.29 & 4.20 & 0.00283 & 0.00167 & 89.6 & 7.6 & 2.8 & 4.71 & 0.15 \\
\hline B14S & 6.04 & 5.47 & NA & NA & 93.2 & 3.0 & 3.9 & 3.06 & 0.18 \\
\hline B21S & 4.79 & 4.18 & NA & NA & 85.3 & 1.8 & 12.9 & 10.3 & 0.22 \\
\hline B22S & 4.20 & 4.05 & NA & NA & 92.3 & 1.2 & 6.4 & & \\
\hline B23S & 4.02 & 4.12 & NA & NA & 90.8 & 4.3 & 4.9 & & \\
\hline B23SR & 4.64 & NA & NA & NA & 93.5 & 2.4 & 2.5 & 3.80 & 0.12 \\
\hline B24S & 4.41 & 3.98 & 0.00795 & 0.00230 & 89.9 & 2.7 & 7.5 & 5.69 & 0.22 \\
\hline B31S & 4.46 & NA & NA & NA & 76.7 & 3.9 & 19.4 & 17.3 & 0.17 \\
\hline B32S & 4.71 & 4.22 & 0.00369 & 0.00157 & 92.3 & 1.5 & 6.2 & 2.5 & 0.19 \\
\hline B33S & 4.16 & 3.93 & 0.00519 & 0.00447 & 89.2 & 5.3 & 5.5 & 8.42 & 0.198 \\
\hline B41S & 4.91 & 4.91 & NA & NA & 83.7 & 4.0 & 12.3 & 12.1 & 0.15 \\
\hline B42S & 4.08 & NA & 0.00507 & 0.00091 & 85.3 & 2.1 & 12.6 & 21.4 & 0.14 \\
\hline B43S & 4.28 & NA & 0.00365 & 0.00032 & 98.8 & 0.0 & 1.2 & 3.02 & 0.16 \\
\hline B51S & 4.72 & NA & 0.00642 & 0.00124 & 87.3 & 4.5 & 8.2 & 15.1 & 0.14 \\
\hline B52S & 5.23 & 4.88 & NA & NA & NA & NA & NA & $\mathrm{NA}$ & NA \\
\hline B52SR & 5.27 & NA & 0.00198 & 0.00026 & 91.8 & 4.9 & 3.3 & 2.39 & 0.11 \\
\hline
\end{tabular}

NA-Not analyzed for 
Table B-3. Chemical and physical properties of soil samples in the C-transect (F-Area).

\begin{tabular}{|c|c|c|c|c|c|c|c|c|c|}
\hline $\begin{array}{c}\text { Sample } \\
\text { ID }\end{array}$ & $\begin{array}{l}\text { pH in } \\
\text { water }\end{array}$ & $\begin{array}{c}\mathrm{pH} \text { in } \\
0.01 \mathrm{M} \mathrm{CaCl}_{2}\end{array}$ & $\begin{array}{c}\text { Carbon } \\
\text { Content }(\%) \\
\end{array}$ & $\begin{array}{c}\text { Carbon } \\
\text { Std. Dev. }\end{array}$ & $\%$ Sand & $\%$ Silt & $\%$ Clay & $\begin{array}{c}\text { CEC } \\
\left(\operatorname{meq~kg} g^{-1}\right)\end{array}$ & $\begin{array}{c}\text { CEC } \\
\text { Std. Dev. }\end{array}$ \\
\hline C11S & 5.68 & NA & NA & NA & 84.8 & 6.9 & 8.3 & 4.13 & 0.11 \\
\hline C12S & 4.80 & NA & 0.00731 & 0.00237 & 80.2 & 5.7 & 14.1 & 13.3 & 0.13 \\
\hline C13S & 4.45 & NA & NA & NA & NA & NA & NA & NA & $\mathrm{NA}$ \\
\hline C14S & 4.83 & NA & 0.00632 & 0.00008 & 84.2 & 9.4 & 6.4 & NA & NA \\
\hline C21S & 4.80 & 4.14 & NA & NA & 82.2 & 5.8 & 12.0 & 11.4 & 0.15 \\
\hline C21SR & 5.29 & 4.13 & 0.0186 & 0.0030 & 80.9 & 6 & 13.1 & 6.82 & 0.11 \\
\hline C22S & NA & NA & 0.00445 & 0.00125 & NA & NA & $\mathrm{NA}$ & 1.28 & 0.19 \\
\hline C23S & 4.63 & 4.12 & 0.00528 & 0.00117 & 84.2 & 9.4 & 6.4 & 6.12 & 0.14 \\
\hline C31S & 4.81 & 4.26 & 0.0148 & 0.0047 & 93.5 & 1.4 & 5.0 & 5.87 & 0.14 \\
\hline C32S & 4.81 & 4.26 & 0.00431 & 0.00014 & 85.7 & 8.8 & 5.5 & 2.54 & 0.12 \\
\hline C33S & 4.68 & 4.09 & NA & NA & 85.9 & 8.0 & 6.1 & 8.54 & 0.12 \\
\hline C41S & 5.04 & 4.20 & NA & NA & 81.0 & 2.6 & 16.4 & 11.5 & 0.13 \\
\hline C42S & 4.89 & 4.19 & NA & NA & 90.3 & 1.9 & 7.9 & 11.4 & 0.12 \\
\hline C43S & 5.10 & 4.24 & NA & NA & 91.0 & 6.0 & 3.0 & 5.04 & 0.12 \\
\hline
\end{tabular}

NA-Not analyzed for. 
Table B-4. Chemical and physical properties of soil samples in the D-transect (F-Area).

\begin{tabular}{|c|c|c|c|c|c|c|c|}
\hline $\begin{array}{c}\text { Sample } \\
\text { ID }\end{array}$ & $\begin{array}{l}\mathrm{pH} \text { in } \\
\text { water }\end{array}$ & $\begin{array}{c}\mathrm{pH} \text { in } \\
0.01 \mathrm{M} \mathrm{CaCl}\end{array}$ & $\%$ Sand & $\%$ Silt & $\%$ Clay & $\begin{array}{c}\text { CEC } \\
(\mathrm{meq} \mathrm{kg})\end{array}$ & $\begin{array}{c}\text { CEC } \\
\text { Std. Dev. }\end{array}$ \\
\hline D11S & 5.07 & 4.66 & 73.3 & 10.7 & 16 & 8.07 & 0.19 \\
\hline D12S & 6.35 & 6.01 & 83.2 & 6.5 & 10.3 & 4.86 & 0.12 \\
\hline D13S & 4.95 & 4.25 & 86.7 & 8 & 5.3 & 1.96 & 0.10 \\
\hline D13SRA & 5.00 & NA & NA & NA & NA & NA & NA \\
\hline D13SRB & 4.53 & NA & 96.7 & 1.3 & 2.0 & 2.55 & 0.10 \\
\hline D21S & 5.04 & 4.52 & 86.1 & 3.2 & 10.7 & 8.96 & 0.14 \\
\hline D41S & 4.69 & 4.11 & 78.6 & 4.3 & 17.1 & 11.0 & 0.12 \\
\hline DS1S & 4.60 & NA & 86.3 & 3.0 & 10.7 & 12.1 & 0.14 \\
\hline
\end{tabular}

NA- Not analyzed for. 
Table B-5. Chemical and physical properties of soil samples in the E-transect (H-Area).

\begin{tabular}{|c|c|c|c|c|c|c|c|c|c|}
\hline $\begin{array}{c}\text { Sample } \\
\text { ID }\end{array}$ & $\begin{array}{l}\mathrm{pH} \text { in } \\
\text { water }\end{array}$ & $\begin{array}{c}\mathrm{pH} \text { in } \\
0.01 \mathrm{M} \mathrm{CaCl}{ }_{2}\end{array}$ & $\begin{array}{c}\text { Carbon } \\
\text { Content (\%) }\end{array}$ & $\begin{array}{l}\text { Carbon } \\
\text { Std. Dev. }\end{array}$ & $\%$ Sand & $\%$ Silt & $\%$ Clay & $\begin{array}{c}\text { CEC } \\
\left.(\operatorname{meq~kg})^{-1}\right)\end{array}$ & $\begin{array}{c}\text { CEC } \\
\text { Std. Dev. }\end{array}$ \\
\hline E11S & 4.76 & 4.08 & $\mathrm{NA}$ & NA & 70.4 & 3.7 & 25.9 & 29.3 & 0.12 \\
\hline E12S & 5.05 & 4.18 & NA & NA & 86.4 & 4.4 & 9.2 & 7.42 & 0.09 \\
\hline E12SR & 4.55 & NA & NA & NA & 97.9 & 0.0 & 2.1 & 5.05 & 0.10 \\
\hline E13S & 4.62 & 4.09 & 0.0128 & 0.0036 & 84.6 & 4.9 & 10.5 & 11.4 & 0.08 \\
\hline E14S & 4.34 & 3.99 & NA & NA & 94.2 & 1.3 & 4.5 & 8.50 & 0.07 \\
\hline E21S & 4.71 & 4.05 & NA & NA & 69.4 & 5.9 & 24.3 & 15.7 & 0.11 \\
\hline E22S & 4.64 & 4.11 & NA & NA & 70.8 & 10.1 & 19.1 & & \\
\hline E23S & 4.82 & 4.05 & NA & NA & 91.1 & 2.5 & 6.4 & 15.5 & 0.17 \\
\hline E23SR & 4.15 & NA & $\mathrm{NA}$ & NA & 94.4 & 1.7 & 3.9 & 13.3 & 0.11 \\
\hline E24S & 4.54 & 4.05 & NA & NA & 87.9 & 4.8 & 7.3 & 13.8 & 0.10 \\
\hline E31S & 5.14 & 4.20 & NA & NA & 58.1 & 8.5 & 33.4 & 13.0 & 0.09 \\
\hline E32S & 4.87 & 4.06 & NA & NA & 72.6 & 3.2 & 24.2 & 17.3 & 0.11 \\
\hline E33S & 4.38 & 3.96 & NA & NA & 90.5 & 3.0 & 6.5 & 11.5 & 0.12 \\
\hline E34S & 4.50 & 3.94 & NA & $N A$ & 87.2 & 9.1 & 3.7 & 10.5 & 0.12 \\
\hline E41S & 4.43 & 4.10 & 0.0492 & 0.0047 & 66.6 & 1.6 & 31.8 & 20.6 & 0.11 \\
\hline E42S & 4.67 & 4.08 & 0.0132 & 0.0016 & 80.2 & 5.3 & 14.5 & 20.6 & 0.17 \\
\hline
\end{tabular}

NA-Not analyzed for. 
Table B-6. Chemical and physical properties of soil samples in the F-transect (H-Area).

\begin{tabular}{|c|c|c|c|c|c|c|c|c|c|}
\hline $\begin{array}{c}\text { Sample } \\
\text { ID }\end{array}$ & $\begin{array}{l}\mathrm{pH} \text { in } \\
\text { Water }\end{array}$ & $\begin{array}{c}\mathrm{pH} \text { in } \\
0.01 \mathrm{M} \mathrm{CaCl} \\
\end{array}$ & $\begin{array}{c}\text { Carbon } \\
\text { Content }(\%)\end{array}$ & $\begin{array}{l}\text { Carbon } \\
\text { Std. Dev. }\end{array}$ & $\%$ Sand & $\%$ Silt & $\%$ Clay & $\begin{array}{c}\text { CEC } \\
\left(\mathrm{meq} \mathrm{kg}^{-1}\right)\end{array}$ & $\begin{array}{c}\text { CEC } \\
\text { Std. Dev. }\end{array}$ \\
\hline F11S & 4.75 & 3.92 & NA & NA & 81 & 5.2 & 13.8 & 14.6 & 0.13 \\
\hline F12S & 4.85 & 4.20 & NA & NA & 81 & 3.5 & 15.5 & 16.1 & 0.15 \\
\hline F13S & NA & NA & NA & NA & NA & NA & NA & 8.51 & 0.17 \\
\hline F21S & 4.47 & 4.12 & NA & NA & 84.5 & 7.4 & 8.1 & 11.1 & 0.14 \\
\hline F21SR & 4.39 & NA & NA & NA & 85.9 & 4.5 & 8.1 & 9.39 & 0.10 \\
\hline F22S & 5.50 & 4.12 & NA & NA & 86.7 & 5.2 & 8.1 & 7.48 & 0.10 \\
\hline F23S & 6.58 & 4.27 & 0.00558 & 0.00042 & 82.9 & 4.1 & 13 & 11.6 & 0.18 \\
\hline F31S & 4.89 & 4.09 & 0.0187 & 0.0018 & 61.3 & 5.6 & 33.1 & 14.3 & 0.23 \\
\hline F32S & 4.85 & 4.22 & NA & NA & 82.8 & 2.9 & 14.2 & 15.1 & 0.16 \\
\hline F33S & 5.37 & 4.16 & NA & NA & 80.7 & 0 & 18.3 & 13.6 & 0.15 \\
\hline F41S & 4.81 & 4.03 & NA & NA & 59.1 & 4.7 & 36.1 & 26.7 & 0.23 \\
\hline F42S & 4.77 & 4.08 & NA & NA & 84.2 & 0 & 17.2 & 11.8 & 0.14 \\
\hline F43S & 5.05 & 4.22 & NA & NA & 89.2 & 0 & 14.2 & NA & NA \\
\hline F51S & 5.21 & 4.08 & NA & NA & 70.9 & 0 & 29.6 & 17.4 & 0.16 \\
\hline F52S & 5.39 & 4.12 & NA & NA & 62.5 & 0 & 42.2 & 19.9 & 0.15 \\
\hline F53S & 4.82 & 4.13 & NA & NA & 86.3 & 0 & 16.3 & 13.3 & 0.15 \\
\hline
\end{tabular}

NA-Not analyzed for. 
Table B-7. Chemical and physical properties of soil samples from the Little Grand Canyon area (F-Area).

\begin{tabular}{c|ccccccc}
\hline $\begin{array}{c}\text { Sample } \\
\text { ID }\end{array}$ & $\begin{array}{c}\text { pH in } \\
\text { Water }\end{array}$ & $\begin{array}{c}\text { pH in } \\
\text { 0.01 } \mathbf{M ~ C a C l}_{\mathbf{2}}\end{array}$ & \% Sand & \% Silt & \% Clay & $\begin{array}{c}\text { CEC } \\
\text { (meq } \mathbf{~ k g}^{-1} \text { ) }\end{array}$ & $\begin{array}{c}\text { CEC } \\
\text { Std. Dev. }\end{array}$ \\
\hline GC4S & 5.25 & 4.50 & 92.2 & 0.5 & 7.3 & NA & NA \\
GC5S & 4.87 & 4.27 & 96.1 & 0.0 & 3.8 & 3.9 & 0.24 \\
\hline
\end{tabular}

NA-Not analyzed for 
Table B-8.a. Particle size analysis results of soil samples from the A-transect (F-Area).

Percentage of soil particles with diameters greater than

\begin{tabular}{|c|c|c|c|c|c|c|c|c|c|c|c|}
\hline $\begin{array}{l}\text { Sample } \\
\text { ID }\end{array}$ & $\begin{array}{l}2000 \\
\mu \mathrm{m}\end{array}$ & $\begin{array}{l}600 \\
\mu \mathrm{m}\end{array}$ & $\begin{array}{l}425 \\
\mu \mathrm{m}\end{array}$ & $\begin{array}{l}300 \\
\mu \mathrm{m}\end{array}$ & $\begin{array}{l}180 \\
\mu \mathrm{m}\end{array}$ & $\begin{array}{l}150 \\
\mu \mathrm{m}\end{array}$ & $\begin{array}{l}75 \\
\mu \mathrm{m} \\
\end{array}$ & $\begin{array}{l}53 \\
\mu \mathrm{m}\end{array}$ & $\begin{array}{l}20 \\
\mu \mathrm{m}\end{array}$ & $\begin{array}{l}5 \\
\mu \mathrm{m}\end{array}$ & $\begin{array}{l}2 \\
\mu \mathrm{m}\end{array}$ \\
\hline A11S & 0.03 & 14.1 & 28.3 & 47.2 & 75.9 & 81.9 & 87.8 & 88.3 & 89.4 & 89.9 & 91.0 \\
\hline A12S & 0.0 & 29.7 & 60.6 & 82.4 & 91.6 & 93.3 & 95.1 & 99.5 & 99.5 & 99.5 & 99.5 \\
\hline A13S & 3.2 & 41.5 & 57.1 & 73.5 & 89.2 & 91.3 & 94.4 & 95.2 & 95.6 & 96.7 & 96.8 \\
\hline A13SR & 3.7 & 26.1 & 41.3 & 56.3 & 83.7 & 87.9 & 92.5 & 93.6 & 95.0 & 96.6 & 97.0 \\
\hline A21S & 0.08 & 64.1 & 79.8 & 84.7 & 87.5 & 88.1 & 89.5 & 89.9 & 90.1 & 92.8 & 95.0 \\
\hline A22S & 0.02 & 38.1 & 50.0 & 62.3 & 88.3 & 92.2 & 95.0 & 95.7 & 95.7 & 97.4 & 98.5 \\
\hline A23S & 0.31 & 20.7 & 30.6 & 63.6 & 89.2 & 91.4 & 94.4 & 95.4 & 95.4 & 95.5 & 95.5 \\
\hline A31S & 0.04 & 39.3 & 59.7 & 74.3 & 92.3 & 94.2 & 95.4 & 95.6 & 95.6 & 95.6 & 95.6 \\
\hline A32S & 0.0 & 0.09 & 2.2 & 35.8 & 76.0 & 80.1 & 87.1 & 89.5 & 93.5 & 96.1 & 96.9 \\
\hline A42S & 0.0 & 5.0 & 31.7 & 79.3 & 89.8 & 90.4 & 91.4 & 91.7 & 95.3 & 95.3 & 95.3 \\
\hline A51S & 0.2 & 32.4 & 46.3 & 55.6 & 72.6 & 74.4 & 76.2 & 76.6 & 80.1 & 84.5 & 86.6 \\
\hline A52S & 0.0 & 2.0 & 22.9 & 75.1 & 86.5 & 88.0 & 91.5 & 92.6 & 64.6 & 96.8 & 97.5 \\
\hline A53S & 0.6 & 21.2 & 37.1 & 71.6 & 82.1 & 84.6 & 90.0 & 91.7 & 94.8 & 96.7 & 98.0 \\
\hline
\end{tabular}

Table B-8.b. Particle size analysis results of soil sample A41S (F-Area).

Percentage of soil particles with diameters greater than

\begin{tabular}{l|lllllllllll}
\hline Sample & $\mathbf{8 4 0}$ & $\mathbf{4 2 5}$ & $\mathbf{2 5 0}$ & $\mathbf{1 8 0}$ & $\mathbf{1 5 0}$ & $\mathbf{7 5}$ & $\mathbf{5 3}$ & $\mathbf{1 4}$ & $\mathbf{5}$ & $\mathbf{3}$ & 1.4 \\
$\mathrm{ID}$ & $\mu \mathrm{m}$ & $\mu \mathrm{m}$ & $\mu \mathrm{m}$ & $\mu \mathrm{m}$ & $\mu \mathrm{m}$ & $\mu \mathrm{m}$ & $\mu \mathrm{m}$ & $\mu \mathrm{m}$ & $\mu \mathrm{m}$ & $\mu \mathrm{m}$ & $\mu \mathrm{m}$ \\
\hline A41S & 36.5 & 56.2 & 68.1 & 86.5 & 89.6 & 92.2 & 94.3 & 95.1 & 95.1 & 95.1 & 97.5 \\
\hline
\end{tabular}


Table B-9.a. Particle size analysis results of soil samples from the B-transect (F-Area).

Percentage of soil particles with diameters greater than

\begin{tabular}{|c|c|c|c|c|c|c|c|c|c|c|c|}
\hline $\begin{array}{l}\text { Sample } \\
\text { ID }\end{array}$ & $\begin{array}{l}2000 \\
\mu \mathrm{m}\end{array}$ & $\begin{array}{l}600 \\
\mu \mathrm{m}\end{array}$ & $\begin{array}{l}425 \\
\mu \mathrm{m}\end{array}$ & $\begin{array}{l}300 \\
\mu \mathrm{m}\end{array}$ & $\begin{array}{l}180 \\
\mu \mathrm{m}\end{array}$ & $\begin{array}{l}150 \\
\mu \mathrm{m}\end{array}$ & $\begin{array}{l}75 \\
\mu \mathrm{m}\end{array}$ & $\begin{array}{l}53 \\
\mu \mathrm{m}\end{array}$ & $\begin{array}{l}20 \\
\mu \mathrm{m}\end{array}$ & $\begin{array}{l}5 \\
\mu \mathrm{m}\end{array}$ & $\begin{array}{l}2 \\
\mu \mathrm{m}\end{array}$ \\
\hline B12S & 0.05 & 25.6 & 47.8 & 64.8 & 82.2 & 85.6 & 88.8 & 89.6 & 91.8 & 92.4 & 93.8 \\
\hline B14S & 0.0 & 14.2 & 38.2 & 52.7 & 78.1 & 83.5 & 91.0 & 93.2 & 94.3 & 96.1 & 96.1 \\
\hline B21S & 0.0 & 7.7 & 18.7 & 36.5 & 72.1 & 78.8 & 84.6 & 85.3 & 85.3 & 85.3 & 87.1 \\
\hline B22S & 0 & 46.9 & 62.7 & 77.2 & 88.6 & 89.7 & 91.3 & 92.3 & 92.8 & 93.2 & 93.6 \\
\hline B23SR & 1.6 & 23.9 & 48.3 & 67.8 & 88.7 & 91.0 & 94.2 & 95.1 & 95.9 & 97.3 & 97.5 \\
\hline B41S & 0.0 & 10.2 & 23.0 & 37.7 & 62.7 & 70.7 & 82.9 & 83.7 & 86.6 & 87.1 & 87.7 \\
\hline B42S & 0.0 & 37.3 & 53.0 & 63.1 & 81.2 & 84.0 & 85.1 & 85.3 & 87.2 & 87.4 & 87.4 \\
\hline B43S & 0.0 & 0.0 & 2.0 & 51.0 & 94.3 & 96.3 & 98.3 & 98.7 & 98.7 & 98.7 & 98.7 \\
\hline B52SR & 0.0 & 9.1 & 15.4 & 58.4 & 82.7 & 85.3 & 90.1 & 91.8 & 94.4 & 96.6 & 96.7 \\
\hline
\end{tabular}


Table B-9.b. Particle size analysis results of soil samples from the B-transect (F-Area).

Percentage of soil particles with diameters greater than

\begin{tabular}{|c|c|c|c|c|c|c|c|c|c|c|c|}
\hline $\begin{array}{l}\text { Sample } \\
\text { ID }\end{array}$ & $\begin{array}{l}840 \\
\mu m \\
\end{array}$ & $\begin{array}{l}425 \\
\mu \mathrm{m}\end{array}$ & $\begin{array}{l}250 \\
\mu \mathrm{m} \\
\end{array}$ & $\begin{array}{l}180 \\
\mu \mathrm{m}\end{array}$ & $\begin{array}{l}150 \\
\mu \mathrm{m}\end{array}$ & $\begin{array}{l}75 \\
\mu \mathrm{m} \\
\end{array}$ & $\begin{array}{l}53 \\
\mu \mathrm{m} \\
\end{array}$ & $\begin{array}{l}14 \\
\mu \mathrm{m}\end{array}$ & $\begin{array}{l}5 \\
\mu \mathrm{m}\end{array}$ & $\begin{array}{l}3 \\
\mu \mathrm{m}\end{array}$ & $\begin{array}{l}1.4 \\
\mu \mathrm{m}\end{array}$ \\
\hline B11S & 1.1 & 15.3 & 39.6 & 66.8 & 71.7 & 80.4 & 82.7 & 84.8 & 84.8 & 84.8 & 88.9 \\
\hline B13S & 12.3 & 31.7 & 65.0 & 79.8 & 82.1 & 89.6 & 92.2 & 94.3 & 97.2 & 96.5 & 97.2 \\
\hline B23S & 4.3 & 34.1 & 66.0 & 83.7 & 85.8 & 90.7 & 91.6 & 93.7 & 94.4 & 95.1 & 96.5 \\
\hline B24S & 0.1 & 4.3 & 42.5 & 83.1 & 85.9 & 89.8 & 90.5 & 93.2 & 92.5 & 92.5 & 94.6 \\
\hline B31S & 0.8 & 9.9 & 22.0 & 38.7 & 47.7 & 76.7 & 77.8 & 79.2 & 79.9 & 80.6 & 82.6 \\
\hline B32S & 4.6 & 44.2 & 78.8 & 88.5 & 89.7 & 92.3 & 93.1 & 93.1 & 93.8 & 93.8 & 94.5 \\
\hline B33S & 1.5 & 20.6 & 59.8 & 79.0 & 81.8 & 89.2 & 90.4 & 92.4 & 93.8 & 94.5 & 95.2 \\
\hline B51S & 5.8 & 13.3 & 30.4 & 67.9 & 76.5 & 87.3 & 89.7 & 91.8 & 91.8 & 91.8 & 93.1 \\
\hline
\end{tabular}


Table B-10.a Particle size analysis results of soil samples from the C-transect (F-Area).

Percentage of soil particles with diameters greater than

\begin{tabular}{l|lllllllllll} 
Sample & $\mathbf{2 0 0 0}$ & $\mathbf{6 0 0}$ & $\mathbf{4 2 5}$ & $\mathbf{3 0 0}$ & $\mathbf{1 8 0}$ & $\mathbf{1 5 0}$ & $\mathbf{7 5}$ & $\mathbf{5 3}$ & $\mathbf{2 0}$ & $\mathbf{5}$ & $\mathbf{2}$ \\
$\mathrm{ID}$ & $\boldsymbol{\mu m}$ & $\boldsymbol{\mu m}$ & $\boldsymbol{\mu m}$ & $\boldsymbol{\mu m}$ & $\boldsymbol{\mu m}$ & $\boldsymbol{\mu m}$ & $\boldsymbol{\mu m}$ & $\boldsymbol{\mu m}$ & $\boldsymbol{\mu m}$ & $\boldsymbol{\mu m}$ & $\boldsymbol{\mu m}$ \\
\hline C31S & 0.0 & 21.0 & 44.0 & 65.0 & $\mathbf{8 5 . 7}$ & 90.6 & 93.2 & 93.5 & 93.6 & 94.2 & 95.0 \\
C33S & 0.0 & 0.4 & 1.8 & 15.2 & 69.4 & 75.1 & 83.2 & 85.9 & 85.8 & 92.6 & 93.9 \\
& & & & & & & & & & & \\
C42S & 0.0 & 20.2 & 36.2 & 61.2 & 84.7 & 87.3 & 89.7 & 90.3 & 90.3 & 91.5 & 92.1 \\
C43S & 0.6 & 10.2 & 15.7 & 29.5 & 75.3 & 80.9 & 88.6 & 91.0 & 95.3 & 96.3 & 97.0 \\
\hline
\end{tabular}

Table B-10.b. Particle size analysis results of soil samples from the B-transect (F-Area).

Percentage of soil particles with diameters greater than

\begin{tabular}{|c|c|c|c|c|c|c|c|c|c|c|c|}
\hline $\begin{array}{l}\text { Sample } \\
\text { ID }\end{array}$ & $\begin{array}{l}840 \\
\mu \mathrm{m}\end{array}$ & $\begin{array}{l}425 \\
\mu \mathrm{m}\end{array}$ & $\begin{array}{l}250 \\
\mu \mathrm{m}\end{array}$ & $\begin{array}{l}180 \\
\mu \mathrm{m}\end{array}$ & $\begin{array}{l}150 \\
\mu \mathrm{m}\end{array}$ & $\begin{array}{l}75 \\
\mu \mathrm{m}\end{array}$ & $\begin{array}{l}53 \\
\mu \mathrm{m}\end{array}$ & $\begin{array}{l}14 \\
\mu \mathrm{m}\end{array}$ & $\begin{array}{l}5 \\
\mu \mathrm{m}\end{array}$ & $\begin{array}{l}3 \\
\mu \mathrm{m}\end{array}$ & $\begin{array}{l}1.4 \\
\mu \mathrm{m}\end{array}$ \\
\hline C11S & 1.1 & 22.1 & 62.0 & 76.4 & 79.1 & 84.8 & 88.4 & 90.0 & 90.9 & 91.7 & 94.2 \\
\hline C12S & 0.3 & 13.8 & 46.0 & 68.8 & 73.2 & 80.2 & 82.7 & 84.3 & 85.9 & 85.9 & 89.0 \\
\hline C14S & 0.06 & 5.3 & 29.5 & 70.0 & 75.0 & 82.3 & 88.6 & 91.8 & 94.3 & 94.3 & 95.9 \\
\hline C21S & 3.3 & 29.5 & 51.8 & 70.1 & 74.2 & 82.2 & 85.5 & 86.3 & 88.0 & 88.0 & 90.4 \\
\hline C21SR & 2.7 & 22.2 & 48.5 & 69.4 & 74.6 & 80.9 & 84.8 & 84.8 & 86.2 & 86.9 & 87.6 \\
\hline C23S & 0.03 & 3.3 & 29.4 & 74.1 & 78.7 & 84.2 & 89.7 & 92.1 & 92.9 & 93.6 & 95.2 \\
\hline $\mathrm{C} 32 \mathrm{~S}$ & 4.9 & 21.5 & 44.3 & 71.5 & 75.5 & 85.7 & 87.7 & 91.8 & 93.8 & 94.5 & 95.2 \\
\hline C41S & 0.4 & 8.4 & 23.2 & 43.6 & 51.1 & 81.0 & 80.9 & 82.9 & 82.9 & 83.6 & 83.6 \\
\hline
\end{tabular}


Table B-11.a Particle size analysis results of soil samples from the D-transect (F-Area).

Percentage of soil particles with diameters greater than

\begin{tabular}{l|lllllllllll}
\hline Sample & $\mathbf{2 0 0 0}$ & $\mathbf{6 0 0}$ & $\mathbf{4 2 5}$ & $\mathbf{3 0 0}$ & $\mathbf{1 8 0}$ & $\mathbf{1 5 0}$ & $\mathbf{7 5}$ & $\mathbf{5 3}$ & $\mathbf{2 0}$ & $\mathbf{5}$ & $\mathbf{2}$ \\
ID & $\boldsymbol{\mu m}$ & $\boldsymbol{\mu m}$ & $\boldsymbol{\mu m}$ & $\boldsymbol{\mu m}$ & $\boldsymbol{\mu m}$ & $\boldsymbol{\mu m}$ & $\boldsymbol{\mu m}$ & $\boldsymbol{\mu m}$ & $\boldsymbol{\mu m}$ & $\boldsymbol{\mu m}$ & $\boldsymbol{\mu m}$ \\
\hline D13SRB & 0.0 & 43.5 & 68.5 & 86.6 & 93.8 & 94.7 & 96.3 & 96.7 & 97.7 & 97.8 & 98.0 \\
D21S & 0.06 & 12.4 & 25.1 & 38.2 & 62.7 & 79.7 & 85.6 & 86.1 & 86.3 & 87.9 & 87.3 \\
D51S & 0.2 & 17.8 & 32.9 & 47.9 & 66.6 & 74.8 & 85.6 & 86.3 & 86.5 & 87.7 & 89.3 \\
\hline
\end{tabular}

Table B-11. b. Particle size analysis results of soil samples from the D-transect (F-Area).

Percentage of soil particles with diameters greater than

\begin{tabular}{l|lllllllllll}
\hline Sample & $\mathbf{8 4 0}$ & $\mathbf{4 2 5}$ & $\mathbf{2 5 0}$ & $\mathbf{1 8 0}$ & $\mathbf{1 5 0}$ & $\mathbf{7 5}$ & $\mathbf{5 3}$ & $\mathbf{1 4}$ & $\mathbf{5}$ & $\mathbf{3}$ & $\mathbf{1 . 4}$ \\
D & $\boldsymbol{\mu m}$ & $\boldsymbol{\mu m}$ & $\boldsymbol{\mu m}$ & $\boldsymbol{\mu m}$ & $\boldsymbol{\mu m}$ & $\boldsymbol{\mu m}$ & $\boldsymbol{\mu m}$ & $\boldsymbol{\mu m}$ & $\boldsymbol{\mu m}$ & $\boldsymbol{\mu m}$ & $\boldsymbol{\mu m}$ \\
\hline D11S & 0.6 & 27.9 & 60.1 & 70.6 & 72.2 & 73.3 & $\mathbf{7 9 . 5}$ & $\mathbf{8 1 . 4}$ & $\mathbf{8 3 . 4}$ & $\mathbf{8 4 . 0}$ & $\mathbf{8 7 . 2}$ \\
D12S & 5.3 & 22.5 & 46.1 & 66.4 & 71.8 & 83.2 & 86.5 & 88.4 & $\mathbf{8 9 . 0}$ & $\mathbf{8 9 . 7}$ & 90.3 \\
D13S & 5.8 & 44.7 & 71.2 & 79.0 & $\mathbf{8 0 . 0}$ & $\mathbf{8 6 . 7}$ & 89.5 & 92.8 & $\mathbf{9 4 . 1}$ & 94.7 & 94.7 \\
& & & & & & & & & & & \\
D41S & 0.7 & 12.4 & 33.1 & 51.3 & 57.2 & $\mathbf{7 8 . 6}$ & $\mathbf{8 0 . 9}$ & $\mathbf{8 1 . 6}$ & $\mathbf{8 2 . 9}$ & $\mathbf{8 2 . 9}$ & $\mathbf{8 4 . 2}$ \\
\hline
\end{tabular}


Table B-12.a. Particle size analysis results of soil samples from the E-transect (H-Area).

Percentage of soil particles with diameters greater than

\begin{tabular}{l|lllllllllll}
\hline Sample & $\mathbf{2 0 0 0}$ & $\mathbf{6 0 0}$ & $\mathbf{4 2 5}$ & $\mathbf{3 0 0}$ & $\mathbf{1 8 0}$ & $\mathbf{1 5 0}$ & $\mathbf{7 5}$ & $\mathbf{5 3}$ & $\mathbf{2 0}$ & $\mathbf{5}$ & $\mathbf{2}$ \\
$\mathbf{I D}$ & $\boldsymbol{\mu m}$ & $\boldsymbol{\mu m}$ & $\boldsymbol{\mu m}$ & $\boldsymbol{\mu m}$ & $\boldsymbol{\mu m}$ & $\boldsymbol{\mu m}$ & $\boldsymbol{\mu m}$ & $\boldsymbol{\mu m}$ & $\boldsymbol{\mu m}$ & $\boldsymbol{\mu m}$ & $\boldsymbol{\mu m}$ \\
\hline E12SR & 0.0 & 34.3 & 62.8 & $\mathbf{8 7 . 7}$ & 93.3 & 95.0 & 97.4 & 97.9 & 97.9 & 97.9 & 97.9 \\
E14S & 0.8 & 43.3 & 61.8 & 70.3 & 83.0 & 87.2 & 92.7 & 94.2 & 94.2 & 94.2 & 95.6 \\
& & & & & & & & & & & \\
E23SR & 0.1 & 52.1 & 69.3 & 77.6 & $\mathbf{8 5 . 8}$ & $\mathbf{8 8 . 4}$ & $\mathbf{9 2 . 6}$ & 94.4 & 94.6 & 95.0 & 96.1 \\
\hline
\end{tabular}


Table B-12.b. Particle size analysis results of soil samples from the E-transect (H-Area).

Percentage of soil particles with diameters greater than

\begin{tabular}{|c|c|c|c|c|c|c|c|c|c|c|c|}
\hline $\begin{array}{l}\text { Sample } \\
\text { ID }\end{array}$ & $\begin{array}{l}840 \\
\mu \mathrm{m}\end{array}$ & $\begin{array}{l}425 \\
\mu \mathrm{m}\end{array}$ & $\begin{array}{l}250 \\
\mu \mathrm{m}\end{array}$ & $\begin{array}{l}180 \\
\mu \mathrm{m}\end{array}$ & $\begin{array}{l}150 \\
\mu \mathrm{m}\end{array}$ & $\begin{array}{l}75 \\
\mu \mathrm{m}\end{array}$ & $\begin{array}{l}53 \\
\mu \mathrm{m}\end{array}$ & $\begin{array}{l}14 \\
\mu \mathrm{m}\end{array}$ & $\begin{array}{l}5 \\
\mu \mathrm{m}\end{array}$ & $\begin{array}{l}3 \\
\mu \mathbf{m}\end{array}$ & $\begin{array}{l}1.4 \\
\mu \mathrm{m}\end{array}$ \\
\hline E11S & 1.2 & 15.9 & 44.1 & 57.2 & 62.4 & 70.4 & 72.1 & 72.8 & 74.1 & 74.1 & 74.1 \\
\hline E12S & 6.4 & 46.3 & 76.7 & 82.6 & 83.5 & 86.4 & 86.8 & 88.8 & 90.8 & 90.8 & 90.8 \\
\hline E13S & 5.4 & 19.5 & 44.9 & 76.4 & 80.0 & 84.6 & 86.3 & 88.9 & 89.5 & 89.5 & 91.5 \\
\hline E21S & 2.1 & 23.4 & 38.1 & 50.2 & 53.2 & 69.8 & 72.4 & 74.9 & 75.7 & 74.9 & 77.3 \\
\hline E22S & 29.7 & 52.5 & 63.2 & 69.3 & 70.8 & 76.3 & 77.1 & 78.7 & 79.4 & 80.9 & 81.7 \\
\hline E23S & 5.8 & 23.8 & 46.4 & 82.4 & 86.8 & 91.1 & 91.1 & 92.0 & 92.0 & 93.6 & 93.6 \\
\hline E24S & 15.1 & 50.9 & 69.1 & 80.1 & 82.1 & 87.7 & 88.6 & 90.3 & 91.9 & 92.7 & 92.7 \\
\hline E31S & 0.5 & 13.1 & 28.6 & 40.6 & 44.3 & 58.1 & 60.1 & 64.2 & 66.6 & 65.8 & 65.8 \\
\hline E32S & 3.8 & 31.0 & 60.8 & 68.3 & 69.6 & 72.6 & 73.0 & 74.3 & 75.8 & 75.8 & 75.8 \\
\hline E33S & 1.3 & 17.9 & 53.8 & 81.8 & 85.5 & 90.5 & 91.1 & 92.7 & 93.5 & 93.5 & 93.5 \\
\hline E34S & 11.7 & 48.0 & 64.8 & 73.1 & 75.8 & 87.2 & 92.0 & 95.7 & 96.3 & 96.3 & 96.3 \\
\hline E41S & 0.04 & 17.9 & 41.8 & 61.0 & 63.1 & 66.6 & 65.0 & 66.1 & 66.1 & 68.2 & 70.3 \\
\hline E42S & 12.3 & 27.6 & 43.7 & 68.8 & 74.4 & 80.2 & 80.6 & 83.0 & 85.5 & 85.5 & 85.5 \\
\hline
\end{tabular}


Table B-13.a. Particle size analysis results of soil samples from the F-transect (H-Area).

Percentage of soil particles with diameters greater than

\begin{tabular}{l|lllllllllll}
\hline Sample & $\mathbf{2 0 0 0}$ & $\mathbf{6 0 0}$ & $\mathbf{4 2 5}$ & $\mathbf{3 0 0}$ & $\mathbf{1 8 0}$ & $\mathbf{1 5 0}$ & $\mathbf{7 5}$ & $\mathbf{5 3}$ & $\mathbf{2 0}$ & $\mathbf{5}$ & $\mathbf{2}$ \\
ID & $\boldsymbol{\mu m}$ & $\boldsymbol{\mu m}$ & $\boldsymbol{\mu m}$ & $\boldsymbol{\mu m}$ & $\boldsymbol{\mu m}$ & $\boldsymbol{\mu m}$ & $\boldsymbol{\mu m}$ & $\boldsymbol{\mu m}$ & $\boldsymbol{\mu m}$ & $\boldsymbol{\mu m}$ & $\boldsymbol{\mu m}$ \\
\hline F21SR & 12.2 & 65.8 & 77.0 & 81.7 & 85.4 & 86.2 & 88.2 & 88.7 & 89.4 & 90.3 & 92.7 \\
F32S & 0.9 & 21.4 & 34.3 & 47.9 & 67.0 & 73.9 & 81.9 & 82.8 & 83.1 & 84.2 & 85.8 \\
F41S & 3.9 & 23.8 & 31.4 & 37.8 & 48.1 & 52.9 & 59.7 & 60.7 & 64.1 & 64.6 & 65.3 \\
\hline
\end{tabular}


Table B-13.b. Particle size analysis results of soil samples from the F-transect (H-Area).

Percentage of soil particles with diameters greater than

\begin{tabular}{|c|c|c|c|c|c|c|c|c|c|c|c|}
\hline $\begin{array}{l}\text { Sample } \\
\text { m }\end{array}$ & $\begin{array}{l}840 \\
\mu \mathrm{m}\end{array}$ & $\begin{array}{l}425 \\
\mu \mathrm{m}\end{array}$ & $\begin{array}{l}250 \\
\mu \mathrm{m}\end{array}$ & $\begin{array}{l}180 \\
\mu \mathrm{m}\end{array}$ & $\begin{array}{l}150 \\
\mu \mathrm{m}\end{array}$ & $\begin{array}{l}75 \\
\mu \mathrm{m}\end{array}$ & $\begin{array}{l}53 \\
\mu \mathrm{m}\end{array}$ & $\begin{array}{l}14 \\
\mu \mathrm{m}\end{array}$ & $\begin{array}{l}5 \\
\mu \mathrm{m}\end{array}$ & $\begin{array}{l}3 \\
\mu \mathrm{m}\end{array}$ & $\begin{array}{l}1.4 \\
\mu \mathrm{m}\end{array}$ \\
\hline F11S & 4.0 & 24.9 & 47.1 & 62.5 & 69.3 & 81.0 & 83.0 & 85.4 & 85.4 & 86.2 & 88.7 \\
\hline F12S & 0.3 & 10.3 & 40.2 & 60.8 & 65.3 & 81.0 & 81.2 & 82.8 & 84.5 & 84.5 & 86.1 \\
\hline F21S & 25.6 & 62.5 & 75.4 & 79.5 & 80.3 & 84.5 & 84.6 & 87.8 & 91.9 & 91.9 & 93.5 \\
\hline F22S & 24.5 & 68.8 & 80.9 & 83.7 & 84.3 & 86.7 & 85.4 & 88.6 & 91.9 & 91.9 & 95.1 \\
\hline F23S & 0.1 & 6.2 & 29.9 & 49.1 & 54.1 & 82.9 & 81.3 & 84.5 & 87.0 & 87.0 & 88.6 \\
\hline F31S & & & & & & 61.3 & 59.8 & 62.1 & 65.3 & 66.1 & 66.9 \\
\hline F33S & & & & & & 80.7 & 78.3 & 80.0 & 80.8 & 80.8 & 81.7 \\
\hline F42S & & & & & & 84.2 & 80.5 & 81.3 & 82.8 & 82.0 & 82.8 \\
\hline F43S & & & & & & 89.2 & 81.0 & 84.2 & 85.0 & 85.8 & 85.8 \\
\hline F51S & & & & & & 70.9 & 66.0 & 67.4 & 68.9 & 69.7 & 70.4 \\
\hline F52S & & & & & & 62.5 & 53.8 & 55.8 & 55.8 & 57.8 & 57.8 \\
\hline F53S & & & & & & 86.3 & 79.1 & 81.4 & 82.2 & 83.7 & 83.7 \\
\hline
\end{tabular}


Table B-14. Particle size analysis results of soil samples from the Little Grand Canyon area (F-Area).

Percentage of soil particles with diameters greater than

\begin{tabular}{l|lllllllllll}
\hline Sample & $\mathbf{2 0 0 0}$ & $\mathbf{6 0 0}$ & $\mathbf{4 2 5}$ & $\mathbf{3 0 0}$ & $\mathbf{1 8 0}$ & $\mathbf{1 5 0}$ & $\mathbf{7 5}$ & $\mathbf{5 3}$ & $\mathbf{2 0}$ & $\mathbf{5}$ & $\mathbf{2}$ \\
$\mathbf{I D}$ & $\boldsymbol{\mu m}$ & $\boldsymbol{\mu m}$ & $\boldsymbol{\mu m}$ & $\boldsymbol{\mu m}$ & $\boldsymbol{\mu m}$ & $\boldsymbol{\mu m}$ & $\boldsymbol{\mu m}$ & $\boldsymbol{\mu m}$ & $\boldsymbol{\mu m}$ & $\boldsymbol{\mu m}$ & $\boldsymbol{\mu m}$ \\
\hline GC4S & 0 & 25.9 & 30.6 & 38.6 & 85.6 & 90.6 & 92.1 & 92.2 & 92.2 & 92.3 & 92.7 \\
GCSS & 0.1 & 15.9 & 27.6 & 48.2 & 87.9 & 92.5 & 94.7 & 96.2 & 96.2 & 96.2 & 96.2 \\
\hline
\end{tabular}


Table B-15. Radionuclide Concentrations determined by gamma ray spectroscopy for selected soil samples.

\begin{tabular}{|c|c|c|c|c|c|}
\hline Sample ID & ${ }^{137} \mathrm{Cs}\left(\mu \mathrm{Bg} g^{-1}\right)$ & ${ }^{60} \mathrm{Co}\left(\mu \mathrm{B} q \mathrm{~g}^{-1}\right)$ & ${ }^{238} \mathrm{U}\left(\mathrm{mBgg}^{-1}\right)$ & ${ }^{226} \mathrm{Ra}\left(\mathrm{mBqg} \mathrm{g}^{-1}\right)^{1}$ & ${ }^{228} \mathrm{Ra}\left(\mathrm{mBg}^{-1}\right)$ \\
\hline A11S & $<00$ & $<300$ & 24 & 18 & 16 \\
\hline A12S & 850 & $<190$ & 25 & 14 & 8 \\
\hline A21S & 160,000 & $<150$ & 37 & 13 & 7 \\
\hline A31S & $<220$ & $<220$ & 56 & 8 & 10 \\
\hline A41S & $<460$ & $<470$ & 50 & 54 & 37 \\
\hline A51S & $<220$ & $<200$ & 28 & 17 & 23 \\
\hline A.53S & $<110$ & $<110$ & 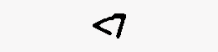 & $<4$ & $<4$ \\
\hline B11S & $<480$ & $<480$ & 21 & 35 & 17 \\
\hline B12S & $<300$ & $<300$ & 18 & 21 & 19 \\
\hline B21S & $<00$ & $<300$ & 18 & 21 & 19 \\
\hline B22S & 370 & $<330$ & 18 & 18 & 12 \\
\hline B24S & $<720$ & $<560$ & 44 & 24 & 24 \\
\hline B31S & $<330$ & $<330$ & 44 & 33 & 37 \\
\hline B32S & $<460$ & $<460$ & 39 & 62 & 62 \\
\hline B33S & $<470$ & $<460$ & 22 & NA & NA \\
\hline C12S & $<330$ & $<330$ & 27 & 21 & 21 \\
\hline C21S & $<330$ & $<330$ & $<$ & 17 & 18 \\
\hline C41S & $<330$ & $<330$ & 35 & 27 & 31 \\
\hline D11S & $<220$ & $<220$ & 26 & 13 & 19 \\
\hline D13S(R)(B) & $<190$ & $<190$ & 14 & 6 & $<4$ \\
\hline E12S(R) & $<300$ & $<300$ & 16 & 9 & 8 \\
\hline E23S(R) & $<150$ & 3552 & 19 & 17 & 16 \\
\hline F21S(R) & $<220$ & $<220$ & 8 & 10 & 10 \\
\hline
\end{tabular}

${ }^{1} \mathrm{Ra}^{226}$ concentration is based on radon daughters which may not be at equilibrium NA-Not reported. 


\section{Transect A Soil Concentrations: Aluminum}

\begin{tabular}{|c|c|c|c|c|c|}
\hline Semple id. & botope & $\begin{array}{l}\text { Concentration } \\
\text { (ug/kg soli) }\end{array}$ & $\begin{array}{l}\text { Std.Doviation } \\
\text { (4o/Kg soil) }\end{array}$ & $\begin{array}{l}\text { Eloment Avg. Conc. } \\
\text { (ug/Kg soll) }\end{array}$ & $\begin{array}{c}\text { Element Avg. Std. Dov. } \\
\text { (ug/Kg soli) }\end{array}$ \\
\hline A11 & A & 17700000 & 156000 & 17700000 & 156000 \\
\hline A12 & $\mathbf{A}$ & 3580000 & 44100 & 3580000 & 44100 \\
\hline A13 & $\bar{A}$ & 2640000 & 45400 & 2640000 & 45400 \\
\hline A13R & $\mathbf{A}$ & 2840000 & 85100 & 2840000 & 85100 \\
\hline A21 & A & 3540000 & 78900 & 3540000 & 78900 \\
\hline$A 22$ & $\mathbf{A}$ & 3470000 & 24000 & 3470000 & 24000 \\
\hline A23 & A & 3530000 & 25500 & 3530000 & 25500 \\
\hline$A 31$ & A & 10700000 & 154000 & 10700000 & 154000 \\
\hline A32 & A & 2400000 & 73800 & 2400000 & 73800 \\
\hline A41 & $\mathbf{A}$ & 4690000 & 41100 & 4690000 & 41100 \\
\hline A42 & A & 2060000 & 16700 & 2060000 & 16700 \\
\hline A51 & A & 7730000 & 170000 & 7730000 & 170000 \\
\hline A52 & $\mathbf{A}$ & 1540000 & 41400 & 1540000 & 41400 \\
\hline$A 53$ & A & 970000 & 14300 & 970000 & 14300 \\
\hline A55V & $\mathbf{A}$ & & & & \\
\hline
\end{tabular}

Note: The actual concentration of asmples for which no standard deviation b reported be less than the lower limit of detection. The reported concentration is the lower limit of quantification. 


\section{Transect A Soil Concentrations: Arsenic}

Sample Id. Isotope

Concentratton

(ug/Kg soll)
Sid.Doviation

(ug/Kg soll)
Ekment Avg. Conc.

(ug/Kg soll)
Element Avg. Std. Dov. (ug/Kg soll)

\begin{tabular}{|c|c|c|c|c|c|}
\hline A11 & As & & & & \\
\hline A12 & As & & & & \\
\hline A13 & As & 1140 & & 1140 & \\
\hline A13R & As & 689 & & 689 & \\
\hline A21 & As & 5040 & 1420 & 5040 & 1420 \\
\hline A22 & As & & & & \\
\hline A23 & As & 284 & & 284 & \\
\hline A31 & As & 1450 & 675 & 1450 & 675 \\
\hline$A 32$ & As & 547 & & 547 & \\
\hline A41 & As & 643 & & 643 & \\
\hline A42 & As & 225 & & 225 & \\
\hline A51 & As & 2740 & 2310 & 2740 & 2310 \\
\hline $\mathrm{A} 52$ & As & 458 & & 458 & \\
\hline $\mathrm{A} 53$ & As & 1690 & 1430 & 1690 & 1430 \\
\hline A55V & As & & & & \\
\hline
\end{tabular}

Note: The actual concentration of amples for which no standard deviation ls reported is leas than the lower limit of detection. The reported concentration be the lower limit of quantification. 


\section{Transect A Soil Concentrations: Barium}

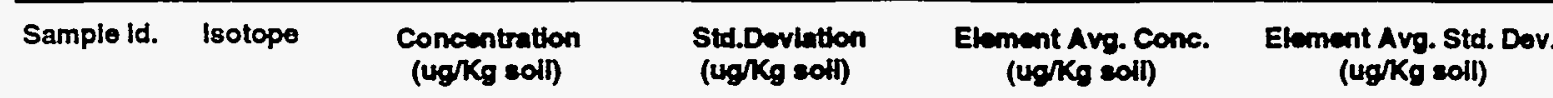

\begin{tabular}{|c|c|c|c|c|c|}
\hline A11 & Ba135 & 38500 & 1890 & & \\
\hline A11 & Ba137 & 42000 & 1730 & & \\
\hline A11 & Ba138 & 43600 & 473 & 41400 & 1360 \\
\hline A12 & Ba135 & 276000 & 2870 & & \\
\hline A12 & Ba137 & 336000 & 4450 & & \\
\hline A12 & Ba138 & 376000 & 2410 & 329000 & 3240 \\
\hline$A 13$ & Ba135 & 378000 & 3280 & & \\
\hline A13 & Ba137 & 459000 & 6610 & & \\
\hline A13 & Ba138 & 371000 & 2700 & 403000 & 4200 \\
\hline A13R & Ba135 & 241000 & 3180 & & \\
\hline A13R & Ba137 & 241000 & 5030 & & \\
\hline$A 13 R$ & Ba138 & 231000 & 1590 & 238000 & 3270 \\
\hline A21 & Ba135 & 63400 & 1270 & & \\
\hline$A 21$ & Ba137 & 56500 & 915 & & \\
\hline$A 21$ & 82138 & 60100 & 708 & 60000 & 964 \\
\hline A22 & Ba135 & 17200 & 1060 & & \\
\hline A22 & Ba137 & 15700 & 408 & & \\
\hline A22 & Ba138 & 20200 & 123 & 17700 & 530 \\
\hline A23 & Ba135 & 169000 & 3150 & & \\
\hline A23 & Ba 137 & 163000 & 1640 & & \\
\hline A23 & Ba138 & 154000 & 453 & 162000 & 1750 \\
\hline$A 31$ & Ba135 & 109000 & 1810 & & \\
\hline A31 & Ba137 & 104000 & 1440 & & \\
\hline A.31 & Ba138 & 111000 & 433 & 108000 & 1230 \\
\hline A32 & Ba135 & 68200 & 436 & & \\
\hline A32 & Ba137 & 75600 & 1520 & & \\
\hline A32 & Ba138 & 74700 & 713 & 72800 & 890 \\
\hline A41 & Ba135 & 63100 & 1060 & & \\
\hline A41 & Ba137 & 79100 & 2750 & & \\
\hline A41 & Ba138 & 67500 & 921 & 69900 & 1580 \\
\hline A42 & Ba135 & 16800 & 277 & & \\
\hline A42 & Ba137 & 23100 & 297 & & \\
\hline A42 & Ba138 & 19000 & 339 & 19600 & 304 \\
\hline A51 & Ba135 & 116000 & 2540 & & \\
\hline A51 & Ba137 & 121000 & 1340 & & \\
\hline A51 & Ba138 & 124000 & 1290 & 120000 & 1720 \\
\hline $\mathrm{A} 52$ & Ba135 & 69500 & 1640 & & \\
\hline A52 & Ba137 & 51800 & 636 & & \\
\hline A52 & Ba138 & 63000 & 334 & 61400 & 870 \\
\hline A53 & Ba135 & 12200 & 562 & & \\
\hline A53 & Ba137 & 9580 & 134 & & \\
\hline A53 & Ba138 & 11900 & 266 & 11200 & 321 \\
\hline A55V & Ba135 & & & & \\
\hline A55V & Ba137 & & & & \\
\hline A55V & Ba138 & & & & \\
\hline
\end{tabular}

Note: The actual concentration of eamples for which no standard deviation ls reported be lese than the lower limit of detection. The reported concentration ts the lower llmit of quantification. 


\section{Transect A Soil Concentrations: Cadmium}

\begin{tabular}{llccc}
\hline Sample ld. Letope & $\begin{array}{c}\text { Concentration } \\
\text { (ug/Kg soll) }\end{array}$ & $\begin{array}{c}\text { Std.Doviation } \\
\text { (Ug/Kg soil) }\end{array}$ & $\begin{array}{c}\text { Element Avg. Conc. } \\
\text { (ug/Kg soll) }\end{array}$ & $\begin{array}{c}\text { Element Avg. Std. Dov. } \\
\text { (Ug/Kg soll) }\end{array}$
\end{tabular}

\begin{tabular}{|c|c|c|c|c|c|}
\hline A11 & Cd111 & 176 & & & \\
\hline A11 & Cod114 & 176 & & 176 & \\
\hline A12 & $\operatorname{cod} 111$ & 171 & 143 & & \\
\hline A12 & $\operatorname{cod} 114$ & 349 & 102 & 260 & 153 \\
\hline A13 & $\operatorname{cod} 111$ & 232 & 170 & & \\
\hline A13 & $\operatorname{Cd114}$ & 151 & 30.8 & 192 & 100 \\
\hline A13R & Cod111 & 220 & 57.4 & & \\
\hline A13R & $\operatorname{cod114}$ & 181 & 85.6 & 201 & 71.5 \\
\hline A21 & $\operatorname{Cd} 111$ & 508 & 25.4 & & \\
\hline$A 21$ & Cd114 & 182 & 402 & 345 & 32.8 \\
\hline$A 22$ & $\operatorname{cod} 111$ & 154 & & & \\
\hline$A 22$ & $\operatorname{cod} 114$ & 154 & & 154 & \\
\hline$A 23$ & Cd111 & 142 & & & \\
\hline A23 & Cod114 & 142 & & 142 & \\
\hline$A 31$ & $\operatorname{cod} 111$ & 104 & & & \\
\hline$A 31$ & Cd114 & 60.3 & 37.5 & 82.2 & 37.5 \\
\hline $\mathrm{A} 32$ & Cod111 & 148 & 171 & & \\
\hline$A 32$ & Cod114 & 140 & 101 & 144 & 136 \\
\hline A41 & Cod111 & 132 & 75.4 & & \\
\hline A41 & $\operatorname{Cod114}$ & 111 & 42.5 & 122 & 59 \\
\hline$A 42$ & Cd111 & 146 & 88.4 & & \\
\hline$\overline{A 42}$ & $\operatorname{cod} 114$ & 112 & & 129 & 88.4 \\
\hline A51 & Cd111 & 144 & 169 & & \\
\hline$A 51$ & Cd114 & 138 & 55.1 & 141 & 112 \\
\hline A52 & $\operatorname{Cod111}$ & 279 & 223 & & \\
\hline A52 & $\operatorname{Cod114}$ & 220 & & 254 & 223 \\
\hline$A 53$ & Cd111 & 191 & & & \\
\hline A53 & $\operatorname{Cod} 114$ & 449 & 210 & 320 & 210 \\
\hline A55V & $\operatorname{Cod} 111$ & & & & \\
\hline A55V & $\operatorname{cd114}$ & & & & \\
\hline
\end{tabular}

Note: The ectual concentration of samples for which no stendard deviatton is reported lo leas than the lower llmit of detection. The reported concentration be the fower Hmit of quantincation. 


\section{Transect A Soil Concentrations: Calcium}

\begin{tabular}{|c|c|c|c|c|c|}
\hline Sample Id. & Isotope & $\begin{array}{l}\text { Concentration } \\
\text { (ug/Kg soll) }\end{array}$ & $\begin{array}{l}\text { Std.Doviation } \\
\text { (ug/Kg soif) }\end{array}$ & $\begin{array}{l}\text { Element Avg. Conc. } \\
\text { (Ug/Kg soil) }\end{array}$ & $\begin{array}{c}\text { Element Avg. Std. Dov. } \\
\text { (ug/Kg soll) }\end{array}$ \\
\hline A11 & $\mathrm{Ca} 44$ & & & & \\
\hline A12 & Ca44 & & & & \\
\hline A13 & Ca44 & 375000 & 39000 & 375000 & 39000 \\
\hline A13R & Ca44 & 13800 & & 13800 & \\
\hline A21 & $\mathrm{Ca} 44$ & 253000 & 154000 & 253000 & 154000 \\
\hline A22 & $\mathrm{Ca} 44$ & & & & \\
\hline A23 & Ca44 & & & & \\
\hline$A 31$ & $\mathrm{Ca} 44$ & 63400 & 9710 & 63400 & 9710 \\
\hline $\mathrm{A} 32$ & $\mathrm{Ca} 44$ & 522000 & 24600 & 522000 & 24600 \\
\hline A41 & Ca44 & 490000 & 73300 & 490000 & 73300 \\
\hline A42 & Ca44 & 90400 & 8920 & 90400 & 8920 \\
\hline A51 & $\mathrm{Ca} 44$ & 484000 & 89200 & 484000 & 89200 \\
\hline A52 & Ca44 & 769000 & 87900 & 769000 & 87900 \\
\hline A53 & Ca44 & 194000 & 62000 & 194000 & 62000 \\
\hline A55V & Ca44 & & & & \\
\hline
\end{tabular}

Note: The actual concentration of samples for which no standard deviation is reported is leas than the lower llmit of detection. The reported concentration is the lower limit of quantfication. 


\section{Transect A Soil Concentrations: Cesium}

\begin{tabular}{|c|c|c|c|c|c|}
\hline Sample ld. & teotope & $\begin{array}{l}\text { Concentration } \\
\text { (ug/Kg soll) }\end{array}$ & $\begin{array}{l}\text { Sid.Dovintion } \\
\text { (ug/Ka soll) }\end{array}$ & $\begin{array}{l}\text { Element Avg. Conc. } \\
\text { (ug/Kg soll) }\end{array}$ & $\begin{array}{c}\text { Element Avg. Std. Dov } \\
\text { (ug/Kg soll) }\end{array}$ \\
\hline A11 & $\mathrm{Cs}$ & 160 & 32.1 & $1 \oplus$ & 32.1 \\
\hline A12 & $\mathrm{C}_{8}$ & 158 & 23.1 & 158 & 23.1 \\
\hline A13 & Cs & 175 & 14.4 & 175 & 14.4 \\
\hline A13R & C. & 2003 & 20.9 & 203 & 20.8 \\
\hline A21 & $\mathrm{Cs}$ & 465 & 46.1 & 465 & 46.1 \\
\hline$A 22$ & Cs & 257 & 30.6 & 257 & 30.6 \\
\hline A23 & Cs & 111 & 43.8 & 111 & 43.8 \\
\hline$A 31$ & Cs & 556 & 21.8 & 556 & 21.8 \\
\hline A32 & Cs & 101 & 26.6 & 101 & 26.6 \\
\hline A41 & $\mathrm{Cs}$ & 252 & 42 & 252 & 42 \\
\hline A42 & $\mathrm{C}_{8}$ & 562 & & 562 & \\
\hline A51 & Cs & 578 & 43.8 & 578 & 43.8 \\
\hline A52 & $\mathrm{Cs}$ & 97 & 30.3 & 97 & 30.3 \\
\hline$A 53$ & Cs & 953 & & 953 & \\
\hline A55V & Cs & & & & \\
\hline
\end{tabular}




\section{Transect A Soil Concentrations: Chromium}

\begin{tabular}{|c|c|c|c|c|c|}
\hline Sample Id. & Isotope & $\begin{array}{l}\text { Concentration } \\
\text { (ug/Kg soll) }\end{array}$ & $\begin{array}{l}\text { Std.Deviation } \\
\text { (ug/Kg soll) }\end{array}$ & $\begin{array}{c}\text { Ekment Avg. Conc. } \\
\text { (ug/Kg soll) }\end{array}$ & $\begin{array}{c}\text { Element Avg. Std. Dov. } \\
\text { (ug/Kg soll) }\end{array}$ \\
\hline A11 & Gr52 & 14000 & 265 & & \\
\hline A11 & Cr53 & 13900 & 264 & 14000 & 265 \\
\hline A12 & $\mathrm{Cr} 52$ & 4040 & 198 & & \\
\hline A12 & $\mathrm{Cr} 53$ & 4030 & 196 & 4040 & 197 \\
\hline A13 & Cr52 & 7000 & 773 & & \\
\hline A13 & Cr53 & 6560 & 484 & 6780 & 629 \\
\hline A13R & Cr52 & 4620 & 204 & & \\
\hline A13R & Cr53 & 4530 & 196 & 4580 & 200 \\
\hline$A 21$ & Cr52 & 3810 & 496 & & \\
\hline$A 21$ & Cr53 & 3810 & 490 & 3810 & 493 \\
\hline A22 & Cr52 & 9360 & 167 & & \\
\hline A22 & Cr53 & 9400 & 167 & 9380 & 167 \\
\hline A23 & Cr52 & 736 & 1230 & & \\
\hline A23 & Cr53 & 656 & 1220 & 696 & 1230 \\
\hline$A 31$ & Cr52 & 7120 & 175 & & \\
\hline$A 31$ & Cr53 & 7120 & 175 & 7120 & 175 \\
\hline A32 & Cr52 & 2080 & 305 & & \\
\hline$A 32$ & Cr53 & 2050 & 299 & 2070 & 302 \\
\hline A41 & $\mathrm{Cr} 52$ & 8520 & 141 & & \\
\hline A41 & $\mathrm{Cr} 53$ & 8620 & 130 & 8570 & 136 \\
\hline A42 & $\mathrm{Cr} 52$ & 1450 & 323 & & \\
\hline A42 & Cr53 & 1460 & 322 & 1460 & 323 \\
\hline$A 51$ & Cr52 & 12100 & 708 & & \\
\hline$A 51$ & $\mathrm{Cr} 53$ & 12000 & 699 & 12100 & 704 \\
\hline A52 & $\mathrm{Cr} 52$ & 2080 & 84.3 & & \\
\hline A52 & G.53 & 2070 & 81.9 & 2080 & 83.1 \\
\hline A53 & $\mathrm{C}-52$ & 2160 & 183 & & \\
\hline$A 53$ & $\mathrm{Cr} 53$ & 2130 & 178 & 2150 & 181 \\
\hline A55V & $\mathrm{Cr} 52$ & & & & \\
\hline A55V & Cr53 & & & & \\
\hline
\end{tabular}

Note: The actual concentration of eamples for which no standard deviation te reported be leas than the lower ilmit of detection. The reported concentration is the fower limit of quantification. 


\section{Transect A Soil Concentrations: Cobalt}

Sample Id. Isotope
Concentration

(ug/Ko soll)
Std.Dovintion

(ug/Ko soll)
Element Avg. Conc. (ug/Kg soH)
Eloment Avg. Std. Dov. (ug/Kg soll)

\begin{tabular}{|c|c|c|c|c|c|}
\hline A11 & Co & 1270 & 71.2 & 1270 & 712 \\
\hline A12 & Co & 919 & 54.6 & 919 & 54.6 \\
\hline$A 13$ & Co & 1720 & 76.5 & 1720 & 76.5 \\
\hline A13R & $C_{0}$ & 330 & 65.4 & 350 & 65.4 \\
\hline A21 & Co & 393 & 82.1 & 393 & 82.1 \\
\hline A22 & $C_{0}$ & 575 & 52.9 & 575 & 52.9 \\
\hline A23 & Co & 142 & & 142 & \\
\hline$A 31$ & $\infty$ & 302 & 17.6 & 302 & 17.6 \\
\hline A32 & Co & 148 & 22 & 148 & 22 \\
\hline A41 & $c_{0}$ & 454 & 322 & 454 & 322 \\
\hline A42 & Co & 460 & 223 & 400 & 223 \\
\hline A51 & Co & 710 & 160 & 710 & 160 \\
\hline A52 & Co & 614 & 27.4 & 614 & 27.4 \\
\hline $\mathrm{A53}$ & Co & 9530 & & 9530 & \\
\hline A55V & Co & & & & \\
\hline
\end{tabular}




\section{Transect A Soil Concentrations: Copper}

\begin{tabular}{|c|c|c|c|c|c|}
\hline Sample ld. & leotope & $\begin{array}{l}\text { Concentration } \\
\text { (ug/Kg soli) }\end{array}$ & $\begin{array}{l}\text { Std.Dovlation } \\
\text { (4G/Kg sold) }\end{array}$ & $\begin{array}{l}\text { Element Avg. Conc. } \\
\text { (ug/Kg soll) }\end{array}$ & $\begin{array}{c}\text { Element Avg. Std. Dov. } \\
\text { (ug/Kg soll) }\end{array}$ \\
\hline A11 & Cu63 & & & & \\
\hline A11 & 0.65 & 5010 & 144 & 5010 & 144 \\
\hline A12 & 0.63 & & & & \\
\hline A12 & Cu65 & 1970 & 571 & 1970 & 571 \\
\hline A13 & Cu63 & 15200 & 729 & & \\
\hline A13 & Cu65 & 5640 & 246 & 10400 & 488 \\
\hline A13R & 0.63 & 2510 & 296 & & \\
\hline A13R & Cu65 & 3000 & 315 & 2760 & 306 \\
\hline A21 & Cu63 & 1220 & 377 & & \\
\hline A21 & Cu65 & 1190 & 678 & 1210 & 528 \\
\hline$A 22$ & cu63 & & & & \\
\hline$A 22$ & Cu65 & 2710 & 376 & 2710 & 376 \\
\hline A23 & 0,63 & & & & \\
\hline A23 & Cu65 & 755 & 381 & 755 & 381 \\
\hline$A 31$ & Cu63 & & & & \\
\hline$A 31$ & 0.65 & 2770 & 102 & 2770 & 162 \\
\hline$A 32$ & Cu63 & 1860 & 160 & & \\
\hline A32 & Cu65 & 4480 & 243 & 3170 & 202 \\
\hline A41 & Cu63 & 7400 & 166 & & \\
\hline A41 & cu6s & 5100 & 731 & 6300 & 449 \\
\hline $\mathrm{A42}$ & Cu63 & & & & \\
\hline A42 & Cu65 & 1120 & & 1120 & \\
\hline A51 & Cu63 & 6310 & 614 & & \\
\hline A51 & Cu65 & 6590 & 610 & 6450 & 612 \\
\hline$A 52$ & Cu63 & & & & \\
\hline A52 & Cu6s & 4880 & 1870 & 4880 & 1870 \\
\hline $\mathrm{A} 53$ & Cu63 & & & & \\
\hline$A 53$ & Cu65 & 16000 & 3080 & 16000 & 3080 \\
\hline A55V & cus3 & & & & \\
\hline$A 55 \mathrm{~V}$ & Cu65 & & & & \\
\hline
\end{tabular}

Note: The actual concentration of samples for which no standard deviation be reported ba lase than the lower limit of detection. The reported concentration is the lower limit of quantification. 


\section{Transect A Soil Concentrations: Iron}

\begin{tabular}{|c|c|c|c|c|c|}
\hline Samplo Id. & Isotope & $\begin{array}{l}\text { Concentration } \\
\text { (ug/Kg sodI) }\end{array}$ & $\begin{array}{l}\text { Std.Deviation } \\
\text { (ug/Kg solf) }\end{array}$ & $\begin{array}{l}\text { Element Avg. Conc. } \\
\text { (ug/Kg solI) }\end{array}$ & $\begin{array}{c}\text { Etement Avg. Std. Dov. } \\
\text { (ug/Kg soll) }\end{array}$ \\
\hline A11 & $\mathrm{Fe}_{\theta}$ & 13800000 & 60800 & 13800000 & 60800 \\
\hline A12 & $\mathrm{F \theta}$ & 2810000 & 15000 & 2810000 & 15000 \\
\hline A13 & $F_{\theta}$ & 3450000 & 32100 & 3450000 & 32100 \\
\hline A13R & $F_{\theta}$ & 6630000 & 81000 & 6630000 & 81000 \\
\hline A21 & $F_{\theta}$ & 6940000 & 125000 & 6940000 & 125000 \\
\hline A22 & $\mathrm{Fe}$ & 5780000 & 41800 & 5780000 & 41800 \\
\hline A23 & $\mathrm{F}_{\theta}$ & 3740000 & 15700 & 3740000 & 15700 \\
\hline$A 31$ & $\mathrm{Fo}$ & 8100000 & 76500 & 8100000 & 76500 \\
\hline$A 32$ & $\mathrm{Fe}_{\theta}$ & 3450000 & 57700 & 3450000 & 57700 \\
\hline A41 & $\mathrm{F}_{\theta}$ & 6220000 & 132000 & 6220000 & 132000 \\
\hline A42 & $F_{\theta}$ & 623000 & 6270 & 623000 & 6270 \\
\hline$A 51$ & $F_{\theta}$ & 5810000 & 71800 & 5810000 & 71800 \\
\hline A52 & $\mathrm{Fe}$ & 800000 & 11100 & 800000 & $11100^{\circ}$ \\
\hline $\mathrm{A} 53$ & $F_{\theta}$ & 544000 & 4290 & 544000 & 4290 \\
\hline $\mathrm{A} 55 \mathrm{~V}$ & $F_{\theta}$ & & & & \\
\hline
\end{tabular}

Note: The actual concentration of eamptes for which no standard deviation is reported is leas than the lower limit of detection. The reported concentration be the lower llmit of quantification. 


\section{Transect A Soil Concentrations: Lead}

\begin{tabular}{|c|c|c|c|c|c|}
\hline Sample Id. & Isotope & $\begin{array}{l}\text { Concentration } \\
\text { (ug/Kg sodi) }\end{array}$ & $\begin{array}{l}\text { Sid.Doviation } \\
\text { (ug/Kg soil) }\end{array}$ & $\begin{array}{l}\text { Elument Avg. Conc. } \\
\text { (ug/Kg eoll) }\end{array}$ & $\begin{array}{l}\text { Element Avg. Std. Dov. } \\
\text { (ug/Kg soll) }\end{array}$ \\
\hline A11 & Pb206 & 12600 & 119 & & \\
\hline A11 & Pb207 & 12500 & 222 & & \\
\hline A11 & Pb208 & 11700 & 199 & 12300 & 180 \\
\hline A12 & Pb206 & 1480 & 86.7 & & \\
\hline A12 & $\mathrm{Pb} 207$ & 1290 & 59.1 & & \\
\hline A12 & Pb208 & 1690 & 62.9 & 1490 & 69.6 \\
\hline A13 & Pb206 & 15900 & 806 & & \\
\hline$A 13$ & Pb207 & 18600 & 1050 & & \\
\hline$A 13$ & Pb208 & 17200 & 602 & 17200 & 819 \\
\hline$A 13 R$ & Pb206 & 26600 & 154 & & \\
\hline$A 13 R$ & $\mathrm{~Pb} 207$ & 17900 & 462 & & \\
\hline A13R & Pb208 & 12900 & 242 & 19100 & 286 \\
\hline A21 & $\mathrm{Pb} 206$ & 17100 & 524 & & \\
\hline A21 & Pb207 & 17100 & 671 & & \\
\hline A21 & $\mathrm{Pb} 208$ & 19000 & 505 & 17700 & 567 \\
\hline A22 & Pb206 & 1190 & 178 & & \\
\hline A22 & Pb207 & 1410 & 168 & & \\
\hline A22 & $\mathrm{Pb} 208$ & 1320 & 76.9 & 1310 & 141 \\
\hline A23 & $\mathrm{Pb} 206$ & 2700 & 143 & & \\
\hline A23 & $\mathrm{Pb} 207$ & 2550 & 139 & & \\
\hline A23 & $\mathrm{Pb} 208$ & 2490 & 152 & 2580 & 145 \\
\hline A31 & $\mathrm{Pb} 206$ & 2990 & 95.9 & & \\
\hline A31 & $\mathrm{Pb} 207$ & 3160 & 832 & & \\
\hline A31 & Pb208 & 2810 & 72.1 & 2990 & 83.7 \\
\hline A32 & Pb206 & 12500 & 91.6 & & \\
\hline A32 & Pb207 & 10700 & 394 & & \\
\hline A32 & Pb208 & 8770 & 330 & 10700 & 272 \\
\hline A41 & Pb206 & 21400 & 519 & & \\
\hline A41 & Pb207 & 24600 & 626 & & \\
\hline A41 & Pb208 & 17100 & 317 & 21000 & 487 \\
\hline A42 & Pb206 & 225 & & & \\
\hline A42 & $\mathrm{Pb} 207$ & 225 & & & \\
\hline A42 & $\mathrm{Pb} 208$ & 225 & & 225 & \\
\hline A51 & $\mathrm{Pb} 206$ & 21700 & 872 & & \\
\hline A51 & Pb207 & 21600 & 200 & & \\
\hline A51 & $\mathrm{Pb} 208$ & 16600 & 218 & 20000 & 433 \\
\hline A52 & $\mathrm{Pb} 206$ & 54000 & 992 & & \\
\hline A52 & $\mathrm{Pb} 207$ & 51800 & 636 & & \\
\hline A52 & Pb208 & 63000 & 334 & 56300 & 654 \\
\hline A53 & $\mathrm{Pb} 206$ & 16900 & 2220 & & \\
\hline A53 & $\mathrm{Pb} 207$ & 15500 & 1840 & & \\
\hline A53 & $\mathrm{Pb208}$ & 14600 & 1930 & 15700 & 2000 \\
\hline $\mathrm{A} 55 \mathrm{~V}$ & $\mathrm{~Pb} 206$ & & & & \\
\hline A55V & Pb207 & & & & \\
\hline A55V & $\mathrm{Pb} 208$ & & & & \\
\hline
\end{tabular}

Note: The actual concentratton of eamples for which no stenderd deviation ts reported is laes than the lower limit of detection. The reported concentration is the lower limit of quantification. 


\section{Transect A Soil Concentrations: Magnesium}

\begin{tabular}{|c|c|c|c|c|c|}
\hline Sample Id. & Isotope & $\begin{array}{l}\text { Concentration } \\
\text { (ug/Kg soll) }\end{array}$ & $\begin{array}{l}\text { StdDovition } \\
\text { (ug/Ka edi) }\end{array}$ & $\begin{array}{l}\text { Ekment Avg. Conc. } \\
\text { (ug/Kg soll) }\end{array}$ & $\begin{array}{c}\text { Element Avg. Std. Dov. } \\
\text { (ug/Kg soll) }\end{array}$ \\
\hline A11 & $M g 24$ & 196000 & 1800 & & \\
\hline A11 & Mg25 & 198000 & 4380 & 197000 & 3090 \\
\hline A12 & $M g 24$ & 75100 & 2590 & & \\
\hline A12 & Mges & 56500 & 1330 & 65200 & 1960 \\
\hline$A 13$ & $M g 24$ & 51100 & 1880 & & \\
\hline$A 13$ & $\mathrm{Mg} 25$ & 57300 & 5280 & 54200 & 3580 \\
\hline A13R & $\mathrm{Mg}_{24}$ & 44000 & 1970 & & \\
\hline A13R & Mg25 & 42900 & 6400 & 43500 & 4190 \\
\hline A21 & Mg24 & 54700 & 2790 & & \\
\hline A21 & Mg25 & 57600 & 2870 & 56200 & 2830 \\
\hline A22 & Mg24 & 91600 & 1330 & & \\
\hline A22 & $M g 25$ & 89300 & 3660 & 90500 & 2500 \\
\hline$A 23$ & $M g 24$ & 63100 & 612 & & \\
\hline A23 & $M و 25$ & 66900 & 897 & 65000 & 755 \\
\hline A31 & Mg24 & 186000 & 4410 & & \\
\hline A31 & Mg25 & 196000 & 3160 & 191000 & 3790 \\
\hline A32 & Mg24 & 38500 & 1700 & & \\
\hline A32 & Mg25 & 45000 & 5560 & 41800 & 3630 \\
\hline A41 & $M g 24$ & 69100 & 2960 & & \\
\hline A41 & $M g 25$ & 67400 & 3670 & 68300 & 3320 \\
\hline A42 & $M g 24$ & 22500 & 3310 & & \\
\hline A42 & Mg25 & 35500 & 568 & 29000 & 1940 \\
\hline$A 51$ & Mg24 & 144000 & 7050 & & \\
\hline A51 & Mg25 & 159000 & 7480 & 152000 & 7270 \\
\hline$A 52$ & $\mathrm{Mg}_{24}$ & 49500 & 2820 & & \\
\hline A52 & Mg25 & 48200 & 4920 & 48900 & 3870 \\
\hline A53 & Mg24 & 19100 & & & \\
\hline$A 53$ & Mg25 & 19100 & & 19100 & \\
\hline A55V & Mg24 & & & & \\
\hline A55V & Mg25 & & & & \\
\hline
\end{tabular}

Note: The actual concentration of samples for which no standard deviation is reported is less than the lower limit of detection. The reported concentration to the lower llmit of quantification. 


\begin{tabular}{|c|c|c|c|c|c|}
\hline Sample Id. & Isotope & $\begin{array}{l}\text { Concentration } \\
\text { (ug/Kg soll) }\end{array}$ & $\begin{array}{l}\text { Sid.Doviation } \\
\text { (ug/Kg soll) }\end{array}$ & $\begin{array}{l}\text { Element Avg. Conc. } \\
\text { (ug/Kg soll) }\end{array}$ & $\begin{array}{c}\text { Element Avg. Std. Dov. } \\
\text { (ug/Kg soll) }\end{array}$ \\
\hline A11 & $\mathrm{Mn}$ & 49700 & 548 & 49700 & 548 \\
\hline $\mathrm{A} 12$ & $\mathrm{Mn}$ & 7270 & 301 & 7270 & 301 \\
\hline A13 & $M n$ & 107000 & 6390 & 107000 & 6390 \\
\hline A13R & $\mathrm{Mn}$ & 159000 & 9240 & 159000 & 9240 \\
\hline$A 21$ & $\mathrm{Mn}$ & 79000 & 2660 & 79000 & 2660 \\
\hline A22 & $\mathrm{Mn}$ & 15700 & 595 & 15700 & 595 \\
\hline A23 & $\mathrm{Mn}$ & 24900 & 316 & 24900 & 316 \\
\hline $\mathrm{A} 31$ & $M n$ & 12800 & 283 & 12800 & 283 \\
\hline A32 & $\mathrm{Mn}$ & 92300 & 1440 & 92300 & 1440 \\
\hline$A 41$ & $\mathrm{Mn}$ & 144000 & 5440 & 144000 & 5440 \\
\hline A42 & $\mathrm{Mn}$ & 8910 & 326 & 8910 & 326 \\
\hline$A 51$ & $\mathrm{Mn}$ & 154000 & 7820 & 154000 & 7820 \\
\hline $\mathrm{A} 52$ & $\mathrm{Mn}$ & 5420 & 592 & 5420 & $592^{\circ}$ \\
\hline A53 & $\mathrm{Mn}$ & 8880 & 232 & 8880 & 232 \\
\hline A55V & $\mathrm{Mn}$ & & & & \\
\hline
\end{tabular}




\section{Transect A Soil Concentrations: Nickel}

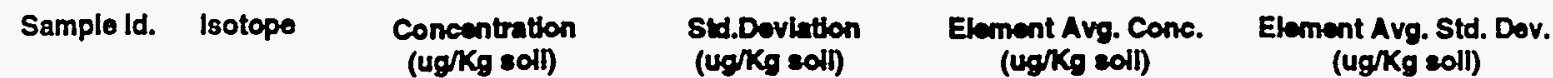

\begin{tabular}{|c|c|c|c|c|c|}
\hline A11 & Ni60 & 3150 & 464 & & \\
\hline A11 & Ni62 & 1220 & 97.8 & 2190 & 281 \\
\hline $\mathrm{A} 12$ & Ni60 & 1570 & 234 & & \\
\hline A12 & Ni62 & 2390 & & 1980 & 234 \\
\hline A13 & Ni60 & 13500 & 1080 & & \\
\hline A13 & Ni62 & 29700 & 5630 & 21600 & 3360 \\
\hline $\mathrm{A} 13 \mathrm{R}$ & Ni60 & 12000 & 659 & & \\
\hline $\mathrm{A} 13 \mathrm{R}$ & Ni62 & 12600 & 4820 & 12300 & 2740 \\
\hline A21 & Ni60 & 18800 & 1280 & & \\
\hline A21 & Ni62 & 26600 & 405 & 22700 & 843 \\
\hline A22 & Ni60 & 2370 & 550 & & \\
\hline$A 22$ & Ni62 & 7990 & 4450 & 5180 & 2500 \\
\hline A23 & Ni60 & & & & \\
\hline A23 & Ni62 & 213 & & 213 & \\
\hline A31 & Ni6o & 1560 & & & \\
\hline A31 & Ni62 & & & 1560 & \\
\hline A32 & Ni60 & 9060 & 604 & & \\
\hline A32 & Ni62 & 8640 & 2820 & 8850 & 1710 \\
\hline A41 & Ni60 & 12100 & 760 & & \\
\hline A41 & Ni62 & 15400 & 3990 & 13800 & 2380 \\
\hline A42 & Ni60 & 1530 & 782 & & \\
\hline A42 & Ni62 & & & 1530 & 782 \\
\hline A51 & Ni60 & 10700 & 836 & & \\
\hline A51 & Ni62 & 9110 & 4460 & 9910 & 2650 \\
\hline A52 & Ni60 & 2650 & 893 & & \\
\hline A52 & Ni62 & & & 2650 & 893 \\
\hline A53 & Ni60 & 2860 & & & \\
\hline A53 & Ni62 & & & 2860 & \\
\hline A55V & Ni6o & & & & \\
\hline A55V & Ni62 & & & & \\
\hline
\end{tabular}

Note: The actual concentration of samples for which no standard deviation is reported is lese than the fower llmit of detection. The reported concentration bs the lower limit of quantfication. 


\section{Transect A Soil Concentrations: Selenium}

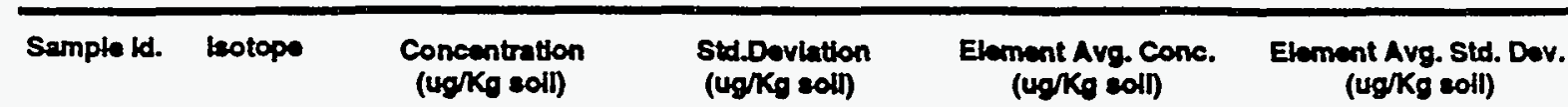

\begin{tabular}{|c|c|c|c|c|c|}
\hline A11 & So77 & 1910 & 6420 & & \\
\hline A11 & Se82 & 1720 & 6280 & 1820 & 6350 \\
\hline A12 & S०77 & 1590 & & & \\
\hline A12 & So82 & 1590 & & 1590 & \\
\hline A13 & So77 & 9260 & 6530 & & \\
\hline A13 & So82 & 8620 & 6070 & 8940 & 6300 \\
\hline A13R & Se77 & 10700 & 4240 & & \\
\hline A13R & Se82 & 9700 & 3880 & 10200 & 4060 \\
\hline$A 21$ & Se77 & 2430 & 3230 & & \\
\hline A21 & So82 & 1820 & 2950 & 2130 & 3110 \\
\hline A22 & So77 & 23000 & 14700 & & \\
\hline A22 & So82 & 22000 & 14300 & 22500 & 14500 \\
\hline$A 23$ & SeT & 7110 & & & \\
\hline A23 & Se82 & 7110 & & 7110 & \\
\hline$A 31$ & Se77 & & & & \\
\hline A31 & Se82 & 20800 & & 20800 & \\
\hline A32 & So77 & 7750 & 1290 & & \\
\hline$A 32$ & So82 & 7300 & 1250 & 7530 & 1270 \\
\hline A41 & SeT7 & 3220 & 311 & & \\
\hline A41 & So82 & 3220 & 311 & 3220 & 311 \\
\hline$A 42$ & Se77 & & & & \\
\hline A42 & Se82 & 22500 & & 22500 & \\
\hline A51 & SeT7 & 17300 & 5350 & & \\
\hline A51 & So82 & 16300 & 5040 & 16800 & 5200 \\
\hline A52 & So77 & & & & \\
\hline A52 & Se82 & 11400 & & 11400 & \\
\hline A53 & Se77 & & & & \\
\hline$A 53$ & Se82 & 13100 & 5920 & 13100 & 5920 \\
\hline A55V & Se77 & & & & \\
\hline A55V & Se82 & & & & \\
\hline
\end{tabular}

Note: The sctual concentration of eamples for which no stenderd doviation be reported is leas than the lower llmit of detection. The reported concentration is the lower Imit of quantification. 


\section{Transect A Soil Concentrations: Silver}

\begin{tabular}{|c|c|c|c|c|c|}
\hline Sample id. & Isotope & $\begin{array}{l}\text { Concentration } \\
\text { (Ug/Kg soll) }\end{array}$ & $\begin{array}{l}\text { Std.Dovintion } \\
\text { (ug/Kg coll) }\end{array}$ & $\begin{array}{l}\text { Element Avg. Conc. } \\
\text { (ug/Kg soll) }\end{array}$ & $\begin{array}{c}\text { Element Avg. Std. Dov. } \\
\text { (ug/Kg soli) }\end{array}$ \\
\hline A11 & Ag107 & 4410 & 575 & & \\
\hline A11 & Ag109 & 24800 & 1060 & 14600 & 818 \\
\hline A12 & Ag107 & 23600 & 430 & & \\
\hline A12 & Ag109 & 22500 & 1090 & 23100 & 760 \\
\hline A13 & Ag107 & 1140 & & & \\
\hline A13 & Ag109 & 1080 & & 1110 & \\
\hline A13R & Ag107 & 1590 & 796 & & \\
\hline A13R & Ag109 & 2410 & 1270 & 2000 & 1030 \\
\hline A21 & Ag107 & 737 & & & \\
\hline A21 & Agt09 & 737 & & 737 & \\
\hline$A 22$ & Ag107 & 29900 & 1350 & & \\
\hline$A 22$ & Ag109 & 33100 & 1540 & 31500 & 1450 \\
\hline$A 23$ & Ag107 & & & & \\
\hline A23 & Ag109 & & & & \\
\hline A31 & Ag107 & 138 & 118 & & \\
\hline$A 31$ & Ag109 & 208 & & 173 & 118 \\
\hline A32 & Ag107 & 848 & 360 & & \\
\hline A32 & Ag109 & 547 & & 696 & 360 \\
\hline A41 & Ag107 & 643 & & & \\
\hline$A 41$ & Ag109 & 643 & & 643 & \\
\hline$A 42$ & Ag107 & 562 & & & \\
\hline$A 42$ & Ag109 & 562 & & 562 & \\
\hline A51 & Ag107 & 2240 & 1230 & & \\
\hline$A 51$ & Ag109 & 2030 & 415 & 2140 & 823 \\
\hline A52 & Ag107 & 9150 & & & \\
\hline A52 & Ag109 & 9150 & & 9150 & \\
\hline$A 53$ & Ag107 & 7620 & & & \\
\hline A53 & Ag109 & 7620 & & 7620 & \\
\hline A55V & Ag107 & & & & \\
\hline A55V & Ag109 & & & & \\
\hline
\end{tabular}




\section{Transect A Soil Concentrations: Sodium}

\begin{tabular}{|c|c|c|c|c|c|}
\hline Sample Id. & Isotope & $\begin{array}{l}\text { Concentration } \\
\text { (ug/Kg soll) }\end{array}$ & $\begin{array}{l}\text { St.Devintion } \\
\text { (ug/Kg eoll) }\end{array}$ & $\begin{array}{l}\text { Element Avg. Conc. } \\
\text { (ug/Kg soli) }\end{array}$ & $\begin{array}{c}\text { Element Avg. Std. Dov. } \\
\text { (ug/Kg soll) }\end{array}$ \\
\hline A11 & $\mathrm{Na}$ & 22400 & 11700 & 22400 & 11700 \\
\hline A12 & $\mathrm{Na}$ & 15900 & & 15900 & \\
\hline A13 & $\mathrm{Na}$ & 97600 & 29000 & 97600 & 29000 \\
\hline A13R & $\mathrm{Na}$ & 6890 & & 6890 & \\
\hline A21 & $\mathrm{Na}$ & 7370 & & 7370 & \\
\hline$A 22$ & $\mathrm{Na}$ & 15400 & & 15400 & \\
\hline A23 & $\mathrm{Na}$ & 443000 & 30700 & 443000 & 30700 \\
\hline$A 31$ & $\mathrm{Na}$ & 98500 & 8170 & 98500 & 8170 \\
\hline A32 & $\mathrm{Na}$ & 25100 & 6240 & 25100 & 6240 \\
\hline A41 & $\mathrm{Na}$ & 6430 & & 6430 & \\
\hline A42 & $\mathrm{Na}$ & 11200 & & 11200 & \\
\hline A51 & $\mathrm{Na}$ & 92000 & 50400 & 92000 & 50400 \\
\hline A52 & $\mathrm{Na}$ & 458000 & & 458000 & \\
\hline$A 53$ & $\mathrm{Na}$ & 820000 & 35100 & 820000 & 35100 \\
\hline A55V & $\mathrm{Na}$ & & & & \\
\hline
\end{tabular}

Note: The actual concentration of samples for whlch no standard deviation is reported be lees than the lower limit of detection. The reported concentration is the lower limit of quantification. 
Transect A Soil Concentrations: Strontium

\begin{tabular}{|c|c|c|c|c|c|}
\hline Sample Id. & Isotope & $\begin{array}{l}\text { Concentration } \\
\text { (ug/Kg soll) }\end{array}$ & $\begin{array}{l}\text { Sid.Doviation } \\
\text { (ug/Kg soll) }\end{array}$ & $\begin{array}{l}\text { Element Avg. Conc. } \\
\text { (ug/Kg soll) }\end{array}$ & $\begin{array}{c}\text { Element Avg. Std. Dov. } \\
\text { (ug/Kg soll) }\end{array}$ \\
\hline A11 & $\mathrm{Sr}$ & 19200 & 341 & 19200 & 341 \\
\hline A12 & $\mathrm{Sr}$ & 59900 & 693 & 59900 & 693 \\
\hline A13 & $\mathrm{Sr}$ & 68900 & 332 & 68900 & 332 \\
\hline A13R & Sr & 63100 & 717 & 63100 & 717 \\
\hline A21 & $\mathrm{Sr}$ & 13300 & 320 & 13300 & 320 \\
\hline$A 22$ & $\mathrm{Sr}$ & 5710 & 216 & 5710 & 216 \\
\hline A23 & Si & 55400 & 663 & 55400 & 663 \\
\hline$A 31$ & $\mathrm{Sr}$ & 25300 & 191 & 25300 & 191 \\
\hline$A 32$ & $\mathrm{Sr}$ & 15000 & 220 & 15000 & 229 \\
\hline A41 & Sr & 11700 & 227 & 11700 & 227 \\
\hline $\mathrm{A} 42$ & $\mathrm{Sr}$ & 6970 & 11.5 & 6970 & 11.5 \\
\hline A51 & $\mathrm{Sr}$ & 40900 & 410 & 40900 & 410 \\
\hline A52 & $\mathrm{Sr}$ & 12300 & 141 & 12300 & 141 \\
\hline A53 & $\mathrm{Sr}$ & 3170 & 95 & 3170 & 95 \\
\hline A55V & $\mathrm{Sr}$ & & & & \\
\hline
\end{tabular}




\section{Transect A Soil Concentrations: Thorium}

\begin{tabular}{|c|c|c|c|c|c|}
\hline Sample Id. & Isotope & $\begin{array}{l}\text { Concentration } \\
\text { (ug/Kg soll) }\end{array}$ & $\begin{array}{l}\text { Std.Doviation } \\
\text { (ug/Kg soll) }\end{array}$ & $\begin{array}{l}\text { Element Avg. Conc. } \\
\text { (ug/Kg soll) }\end{array}$ & $\begin{array}{c}\text { Element Avg. Std. Dov. } \\
\text { (Ug/Kg soll) }\end{array}$ \\
\hline A11 & Th232 & 79.6 & 76.8 & 79.6 & 76.8 \\
\hline $\mathrm{A} 12$ & Th232 & 79.7 & & 79.7 & \\
\hline A13 & Th232 & 114 & & 114 & \\
\hline A13R & Th232 & 68.9 & & 68.9 & \\
\hline A21 & Th232 & 73.7 & & 73.7 & \\
\hline$A 22$ & Th232 & 55.5 & 35.4 & 55.5 & 35.4 \\
\hline A23 & Th232 & 71.1 & & 71.1 & \\
\hline$A 31$ & Th232 & 52.1 & & 52.1 & \\
\hline $\mathrm{A} 32$ & Th232 & 54.7 & & 54.7 & \\
\hline A41 & Th232 & 64.3 & & 64.3 & \\
\hline$A 42$ & Th232 & 562 & & 562 & \\
\hline$A 51$ & Th232 & 96.5 & 20.5 & 96.5 & 20.5 \\
\hline $\mathrm{A} 52$ & Th232 & 114 & & 114 & \\
\hline$A 53$ & Th232 & 95.3 & & 95.3 & \\
\hline A55V & Th232 & & & & \\
\hline
\end{tabular}

Note: The actual concentration of eamples for which no stendard deviation is reported is lees than the lower limit of detection. The reported concentration bs the lower limit of quantification. 


\section{Transect A Soil Concentrations: Tin}

\begin{tabular}{|c|c|c|c|c|c|}
\hline Sample Id. & Isotope & $\begin{array}{l}\text { Concentration } \\
\text { (ug/Kg soil) }\end{array}$ & $\begin{array}{l}\text { Std.Devintion } \\
\text { (ug/Kg soll) }\end{array}$ & $\begin{array}{l}\text { Element Avg. Conc. } \\
\text { (ug/Kg soll) }\end{array}$ & $\begin{array}{c}\text { Element Avg. Std. Dov. } \\
\text { (ug/Kg soll) }\end{array}$ \\
\hline A11 & Sn118 & 87.8 & & & \\
\hline A11 & Sn120 & 87.8 & & 87.8 & \\
\hline$\overline{\mathrm{A} 12}$ & Sn118 & 79.7 & & & \\
\hline $\mathrm{A} 12$ & Sn120 & 79.7 & & 79.7 & \\
\hline $\mathrm{A} 13$ & Sn118 & 365 & 116 & & \\
\hline A13 & Sn120 & 428 & 60.9 & 397 & 88.5 \\
\hline A13R & Sn118 & 168 & 31.5 & & \\
\hline A13R & Sn120 & 189 & 60.3 & 179 & 45.9 \\
\hline A21 & Sn118 & 305 & 39.3 & & \\
\hline$A 21$ & Sn120 & 306 & 51.5 & 306 & 45.4 \\
\hline$A 22$ & Sn118 & $\pi$ & & & \\
\hline$A 22$ & Sn120 & $\pi$ & & 77 & \\
\hline A23 & Sn118 & 71.1 & & & $\cdot$ \\
\hline$A 23$ & Sn120 & 71.1 & & 71.1 & \\
\hline$A 31$ & Sn118 & 263 & 40.7 & & \\
\hline$A 31$ & Sn120 & 226 & 39.3 & 245 & 40 \\
\hline A32 & Sn118 & 349 & 72.6 & & \\
\hline$A 32$ & Sn120 & 232 & 62.7 & 291 & 67.7 \\
\hline A41 & Sn118 & 293 & 45.1 & & \\
\hline A41 & Sn120 & 435 & 912 & 364 & 682 \\
\hline A42 & Sn118 & 562 & & & \\
\hline$A 42$ & $5 n 120$ & 562 & & 562 & \\
\hline A51 & Sn118 & 1670 & 200 & & \\
\hline A51 & Sn120 & 3470 & 249 & 2570 & 225 \\
\hline A52 & Sn118 & 1140 & & & \\
\hline A52 & Sn120 & 1140 & & 1140 & \\
\hline $\mathrm{A} 53$ & Sn118 & 17900 & 1130 & & \\
\hline A53 & $\operatorname{Sn} 120$ & 17000 & 162 & 17500 & 646 \\
\hline A55V & Sn118 & & & & \\
\hline A55V & Sn120 & & & & \\
\hline
\end{tabular}

Note: The actual concentration of amples for whlch no standard deviation bs reorted be leas than the lower llmit of detection. The reported concentration bs the lower IImit of quantification. 


\section{Transect A Soil Concentrations: Uranium-235}

\begin{tabular}{|c|c|c|c|c|c|}
\hline Sample Id. & Isotope & $\begin{array}{l}\text { Concentration } \\
\text { (ug/Kg soll) }\end{array}$ & $\begin{array}{l}\text { Sid.Doviation } \\
\text { (ug/Kg soll) }\end{array}$ & $\begin{array}{c}\text { Element Avg. Conc. } \\
\text { (ug/Kg soll) }\end{array}$ & $\begin{array}{l}\text { Element Avg. Std. Dov. } \\
\text { (ug/Kg coll) }\end{array}$ \\
\hline A11 & U235 & 19 & 2.71 & 19 & 2.71 \\
\hline A12 & U235 & 14.3 & 2.39 & 14.3 & 2.39 \\
\hline A13 & U235 & 372 & 7.37 & 372 & 7.37 \\
\hline A13R & U235 & 51.9 & 2.72 & 51.9 & 2.72 \\
\hline$A 21$ & U235 & 38.9 & 4.65 & 38.9 & 4.65 \\
\hline A22 & U235 & 11.6 & 1.54 & 11.6 & 1.54 \\
\hline A23 & U235 & 12.5 & .84 & 12.5 & .84 \\
\hline$A 31$ & U235 & 20.6 & 2.72 & 20.6 & 2.72 \\
\hline$A 32$ & U235 & 20 & 2.48 & 20 & 2.48 \\
\hline$A 41$ & U235 & 21.1 & 5.1 & 21.1 & 5.1 \\
\hline$A 42$ & U235 & 4.67 & 3.73 & 4.67 & 3.73 \\
\hline $\mathrm{A} 51$ & U235 & 11.6 & 5.34 & 11.6 & 5.34 \\
\hline $\mathrm{A} 52$ & U235 & 5.87 & .43 & 5.87 & .43 \\
\hline $\mathrm{A} 53$ & U235 & 6.61 & 8.15 & 6.61 & 8.15 \\
\hline A55V & U235 & & & & \\
\hline
\end{tabular}

Note: The actual concentration of eamples for which no standard deviatton be raported le bes than the fower limit of detection. The reported concentration is the lower limit of quantification. 


\section{Transect A Soil Concentrations: Uranium-238}

\begin{tabular}{|c|c|c|c|c|c|}
\hline Sample Id. & Isotope & $\begin{array}{l}\text { Concentration } \\
\text { (ug/Kg soil) }\end{array}$ & $\begin{array}{l}\text { Std.Devintion } \\
\text { (ug/Kg soll) }\end{array}$ & $\begin{array}{l}\text { Element Avg. Cone. } \\
\text { (ug/Kg eoll) }\end{array}$ & $\begin{array}{c}\text { Element Avg. Std. Dev. } \\
\text { (ug/Kg soli) }\end{array}$ \\
\hline A11 & U238 & 2300 & 21.6 & 2300 & 21.6 \\
\hline A12 & U238 & 3140 & 29.1 & 3140 & 29.1 \\
\hline A13 & U238 & 5550 & 41.4 & 5550 & 41.4 \\
\hline A13R & U238 & 7560 & 66.4 & 7560 & 66.4 \\
\hline$A 21$ & U238 & 7380 & 23 & 7380 & 23 \\
\hline A22 & U238 & 3250 & 35.3 & 3250 & 35.3 \\
\hline A23 & U238 & 5600 & 117 & 5600 & 117 \\
\hline A31 & $\mathrm{U} 238$ & 7880 & 138 & 7880 & 138 \\
\hline A32 & $\mathrm{U} 238$ & 4080 & 61.4 & 4080 & 61.4 \\
\hline$A 41$ & U238 & 3700 & 27.3 & 3700 & 27.3 \\
\hline A42 & U238 & 1390 & 19.5 & 1390 & 19.5 \\
\hline A51 & U238 & 1830 & 212 & 1830 & 212 \\
\hline A52 & U23B & 542 & 23.4 & 542 & 23.4 \\
\hline$A 53$ & U238 & 817 & 7.77 & 817 & 7.77 \\
\hline A55V & U238 & & & & \\
\hline
\end{tabular}




\section{Transect A Soil Concentrations: Vanadium}

\begin{tabular}{|c|c|c|c|c|c|}
\hline Sample Id. & Isotope & $\begin{array}{l}\text { Concentratton } \\
\text { (ug/Kg soll) }\end{array}$ & $\begin{array}{l}\text { Std.Dovlation } \\
\text { (ug/Kg soil) }\end{array}$ & $\begin{array}{l}\text { Eloment Avg. Conc. } \\
\text { (ug/Kg soil) }\end{array}$ & $\begin{array}{c}\text { Element Avg. Std. Dov. } \\
\text { (ug/Kg soll) }\end{array}$ \\
\hline A11 & $V$ & 24600 & 600 & 24600 & 600 \\
\hline A12 & $V$ & 8820 & 419 & 8820 & 419 \\
\hline A13 & $v$ & 3400 & 1470 & 3400 & 1470 \\
\hline A13R & $\mathrm{V}$ & 2320 & 443 & 2320 & 443 \\
\hline A21 & $V$ & 9940 & 1060 & 9940 & 1060 \\
\hline A22 & $V$ & 18700 & 818 & 18700 & 818 \\
\hline A23 & $v$ & 16700 & 1090 & 16700 & 1090 \\
\hline A31 & 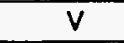 & 20300 & 503 & 20300 & 503 \\
\hline $\mathrm{A} 32$ & $v$ & 109 & & 109 & \\
\hline A41 & $\mathrm{v}$ & 7230 & 1230 & 7230 & 1230 \\
\hline$A 42$ & $\mathrm{~V}$ & 2330 & 192 & 2330 & 192 \\
\hline A51 & $V$ & 3120 & 1670 & 3120 & 1670 \\
\hline $\mathrm{A} 52$ & $V$ & 2100 & 418 & 2100 & $418^{\circ}$ \\
\hline A53 & $V$ & 1360 & 372 & 1360 & 372 \\
\hline A55V & $\mathrm{V}$ & & & & \\
\hline
\end{tabular}

Noto: The actual concentration of samples for which no stenderd deviation ts reported bs lose than the lower limit of detection. The reported concentration th the lower limit of quantincation. 


\section{Transect A Soil Concentrations: Yttrium}

\begin{tabular}{|c|c|c|c|c|c|}
\hline Sample Id. & Isotope & $\begin{array}{l}\text { Concentration } \\
\text { (ug/Kg soll) }\end{array}$ & $\begin{array}{l}\text { Std.Deviation } \\
\text { (ug/Kg solf) }\end{array}$ & $\begin{array}{l}\text { Element Avg. Conc. } \\
\text { (ug/Kg soll) }\end{array}$ & $\begin{array}{c}\text { Element Avg. Std. Dov. } \\
\text { (ug/Kg soil) }\end{array}$ \\
\hline A11 & $Y$ & 710 & 24.4 & 710 & 24.4 \\
\hline A12 & $Y$ & 121 & 21.9 & 121 & 21.9 \\
\hline A13 & $Y$ & 228 & & 228 & \\
\hline A13R & $Y$ & 142 & 51.3 & 142 & 51.3 \\
\hline$A 21$ & $\bar{Y}$ & 90.9 & 925 & 90.9 & 925 \\
\hline A22 & $Y$ & 214 & 43.4 & 214 & 43.4 \\
\hline A23 & $Y$ & 128 & 24.3 & 128 & 24.3 \\
\hline$A 31$ & $Y$ & 59.9 & 5.62 & 59.9 & 5.62 \\
\hline A32 & $Y$ & 100 & & 100 & \\
\hline A41 & $Y$ & 100 & 36.9 & 180 & 36.9 \\
\hline A42 & $Y$ & 272 & 35.1 & 272 & 35.1 \\
\hline A51 & $Y$ & 404 & 41.9 & 404 & 41.9 \\
\hline A52 & $Y$ & 459 & 45.9 & 459 & 45.9 \\
\hline A53 & $\bar{Y}$ & 42.3 & 1.59 & 42.3 & 1.59 \\
\hline A55V & $Y$ & & & & \\
\hline
\end{tabular}

Note: The actual concentratton of samples for which no standerd deviation te reported is leas than the lower limit of detection. The reported concentration ls the lower limit of quantification. 


\section{Transect A Soil Concentrations: Zinc}

\begin{tabular}{|c|c|c|c|c|c|}
\hline Sample Id. & Isotope & $\begin{array}{l}\text { Concentration } \\
\text { (ug/Kg soll) }\end{array}$ & $\begin{array}{l}\text { Std.Doviation } \\
\text { (ug/ } \mathrm{Kg} \text { soll) }\end{array}$ & $\begin{array}{l}\text { Element Avg. Conc. } \\
\text { (ug/Kg soll) }\end{array}$ & $\begin{array}{c}\text { Element Avg. Std. Dov. } \\
\text { (ug/Kg soll) }\end{array}$ \\
\hline A11 & Zn66 & 17700 & 963 & 17700 & 963 \\
\hline A12 & Zn66 & 5990 & 322 & 5990 & 322 \\
\hline A13 & Zn66 & 10300 & 1990 & 10300 & 1990 \\
\hline A13R & Zn66 & 15200 & 1210 & 15200 & 1210 \\
\hline A21 & Zn66 & 11100 & 660 & 11100 & 669 \\
\hline A22 & Zn66 & 5890 & 1740 & 5890 & 1740 \\
\hline A23 & Zn66 & 21400 & 860 & 21400 & 860 \\
\hline A31 & $2 \pi 66$ & 5010 & 474 & 5010 & 474 \\
\hline A32 & $Z n 66$ & 7200 & 825 & 7200 & 825 \\
\hline A41 & Zn66 & 14800 & 479 & 14800 & 479 \\
\hline A42 & Zn66 & 11000 & 2240 & 11000 & 2240 \\
\hline A51 & Zn66 & 9100 & 286 & 9100 & 286 \\
\hline A52 & Zn66 & 12700 & 554 & 12700 & 554 \\
\hline A53 & Zn66 & 12500 & 188 & 12500 & 188 \\
\hline $\mathrm{A} 55 \mathrm{~V}$ & Zn66 & & & & \\
\hline
\end{tabular}

Note: The actual concentration of samples for which no standard deviation is reported le lees than the lower IImIt of detection. The reported concentration is the bwer limit of quantification. 


\section{Transect A Soil Concentrations: Zirconium}

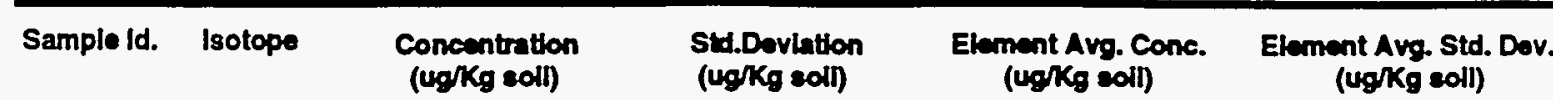

\begin{tabular}{|c|c|c|c|c|c|}
\hline A11 & $Z 990$ & 20100 & 914 & & \\
\hline A11 & Zr91 & 19400 & 1160 & 19800 & 1040 \\
\hline A12 & Zr9o & 2670 & 427 & & \\
\hline A12 & Zr91 & 3440 & 1140 & 3060 & 784 \\
\hline $\mathrm{A} 13$ & $Z \mathbf{z} 90$ & 19000 & 784 & & \\
\hline$A 13$ & $\mathrm{Zr} 91$ & 21200 & 1280 & 20100 & 1030 \\
\hline A13R & Zr9o & 27100 & 642 & & \\
\hline A13R & Z 91 & 30500 & 558 & 28800 & 600 \\
\hline A21 & Zrso & 28200 & 627 & & \\
\hline A21 & $\mathrm{Zrg1}$ & 26200 & 2660 & 27200 & 1640 \\
\hline A22 & Zr9o & 6530 & 268 & & \\
\hline A22 & Z91 & 11000 & 514 & 8770 & 391 \\
\hline A23 & $\mathrm{Zr} 90$ & 23200 & 386 & & \\
\hline A23 & Zr91 & 19200 & 2970 & 21200 & 1680 \\
\hline A31 & Zr9o & 16700 & 337 & & \\
\hline A31 & Zra1 & 17000 & 431 & 16900 & 384 \\
\hline A32 & Zr9o & 15600 & 628 & & \\
\hline A32 & Zr91 & 16200 & 921 & 15900 & 775 \\
\hline A41 & $Z r 90$ & 19200 & 340 & & \\
\hline A41 & Zra1 & 31100 & 831 & 25200 & 586 \\
\hline A42 & Zr9o & 5530 & 37.9 & & \\
\hline A42 & $\mathrm{Zr91}$ & 6760 & 555 & 6150 & 296 \\
\hline A51 & Zr9o & 23600 & 761 & & \\
\hline$A 51$ & Zr91 & 23600 & 2330 & 23600 & 1550 \\
\hline A52 & Zr9o & 8740 & 676 & & \\
\hline A52 & Zr91 & 8070 & 594 & 8410 & 635 \\
\hline A53 & Zrso & 10200 & 106 & & \\
\hline A53 & Zr91 & 5370 & 754 & 7790 & 430 \\
\hline A55V & Zr9o & & & & \\
\hline A55V & $Z 991$ & & & & \\
\hline
\end{tabular}

Note: The actual concentration of samples for which no standard deviation bs reported is leas than the lower limit of detection. The reported concentration te the lower llmit of quantification. 


\section{Transect B Soil Concentrations: Aluminum}

\begin{tabular}{|c|c|c|c|c|c|}
\hline Sample Id. & leotope & $\begin{array}{l}\text { Concentration } \\
\text { (ug/kg soil) }\end{array}$ & $\begin{array}{l}\text { Std.Dovlation } \\
\text { (ug/Ka soil) }\end{array}$ & $\begin{array}{l}\text { Element Avg. Conc. } \\
\text { (ug/Kg coll) }\end{array}$ & $\begin{array}{c}\text { Element Avg. Std. Dav. } \\
\text { (ug/Kg soll) }\end{array}$ \\
\hline B11 & $\mathbf{N}$ & 13400000 & 481000 & 13400000 & 481000 \\
\hline $\mathrm{B} 12$ & $\mathbf{A}$ & 8870000 & 32700 & 8870000 & 32700 \\
\hline $\mathrm{B} 13$ & $\mathbf{A}$ & 3230000 & 21400 & 3230000 & 21400 \\
\hline B14 & A & 1150000 & 24300 & 1150000 & 24300 \\
\hline $\mathrm{B21}$ & $\mathbf{A}$ & 20600000 & 255000 & 20500000 & 255000 \\
\hline$B 22$ & A & 8390000 & 322000 & 8390000 & 322000 \\
\hline B23 & $\mathbf{A}$ & 4030000 & 52300 & 4030000 & 52300 \\
\hline B23R & $\mathbf{A}$ & 4810000 & 1440 & 4810000 & 1440 \\
\hline B24 & A & 6930000 & 94700 & 6930000 & 94700 \\
\hline 831 & A & 19600000 & 111000 & 19600000 & 111000 \\
\hline $\mathrm{B} 32$ & A & 4310000 & 20300 & 4310000 & 20300 \\
\hline $\mathbf{B 3 3}$ & $\boldsymbol{A}$ & 8210000 & 78500 & 8210000 & 78500 \\
\hline B41 & $\mathbf{A}$ & 12200000 & 228000 & 12200000 & 228000 \\
\hline $\mathrm{B} 42$ & A & 25700000 & 137000 & 25700000 & 137000 \\
\hline $\mathrm{B} 43$ & A & 4560000 & 40500 & 4560000 & 40600 \\
\hline B45V & A & & & & \\
\hline 851 & $\mathbf{N}$ & 3440000 & 60500 & 3440000 & 60500 \\
\hline $\mathrm{B} 52$ & A & 3830000 & 92000 & 3830000 & 92000 \\
\hline B52R & $\mathbf{A}$ & 2190000 & 24100 & 2190000 & 24100 \\
\hline
\end{tabular}




\section{Transect B Soil Concentrations: Arsenic}

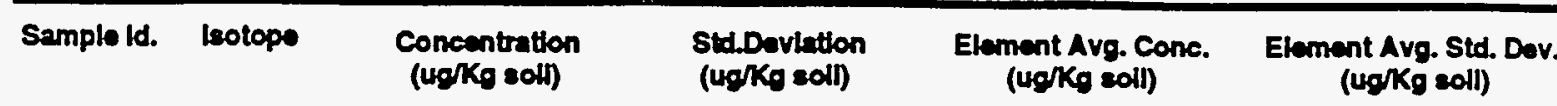

\begin{tabular}{|c|c|c|c|c|c|}
\hline B11 & As & 5690 & 3740 & 5690 & 3740 \\
\hline B12 & As & 1160 & 1440 & 1160 & 1440 \\
\hline 813 & As & 4170 & 482 & 4170 & 492 \\
\hline$B 14$ & As & 349 & & 349 & \\
\hline B21 & As & 4900 & 875 & 4900 & 875 \\
\hline$B 22$ & As & 3080 & & 3080 & \\
\hline$B 23$ & As & 1580 & 1110 & 1580 & 1110 \\
\hline B23R & As & 394 & & 394 & \\
\hline B24 & As & 1770 & 1440 & 1770 & 1440 \\
\hline B31 & As & 292 & & 292 & \\
\hline B32 & As & 2260 & 1480 & 2260 & 1480 \\
\hline $\mathrm{B} 33$ & As & 1200 & & 1200 & \\
\hline$B 41$ & As & 4670 & 1370 & 4670 & 1370 \\
\hline$B 42$ & As & 22400 & 211 & 22400 & 211 \\
\hline$B 43$ & As & 10100 & 877 & 10100 & 877 \\
\hline B45V & As & & & & \\
\hline B51 & As & 18700 & & 18700 & \\
\hline $\mathrm{B} 52$ & As & 1770 & 521 & 1770 & 521 \\
\hline B52R & As & 35600 & & 35600 & \\
\hline
\end{tabular}




\section{Transect B Soil Concentrations: Barium}

\begin{tabular}{|c|c|c|c|c|c|}
\hline Sample Id. & Eotope & $\begin{array}{l}\text { Concentration } \\
\text { (ug/Kg soli) }\end{array}$ & $\begin{array}{l}\text { Stdovilaton } \\
\text { (ug/Ko adi) }\end{array}$ & $\begin{array}{l}\text { Element Avo, Cone. } \\
\text { (ug/kg coll) }\end{array}$ & $\begin{array}{c}\text { Ekment Avg. Std. Dov. } \\
\text { (ug/Kg soll) }\end{array}$ \\
\hline B11 & Ba135 & 32000 & 785 & & \\
\hline B11 & Ba137 & 28600 & 445 & & \\
\hline $\mathrm{B11}$ & $\mathrm{Ba138}$ & 31300 & 112 & 30600 & 451 \\
\hline $\mathrm{B} 12$ & $\mathrm{Ba135}$ & 123000 & 3100 & & \\
\hline B12 & Ba137 & 126000 & 802 & & \\
\hline B12 & Ba138 & 124000 & 1050 & 124000 & 1670 \\
\hline 813 & Ba135 & 115000 & 1010 & & \\
\hline B13 & Ba137 & 104000 & 1290 & & \\
\hline $\mathrm{B} 13$ & Ba138 & 97600 & 700 & 105000 & 1000 \\
\hline Bi4 & $\mathrm{Ba} 135$ & 34500 & 1970 & & \\
\hline B14 & $\mathrm{Ba137}$ & 35700 & 555 & & \\
\hline B14 & $\mathrm{Ba138}$ & 39500 & 425 & 36600 & 983 \\
\hline B21 & Ba135 & 24800 & 482 & & \\
\hline B21 & Ba137 & 25500 & 738 & & \\
\hline B21 & Ba138 & 26400 & 403 & 25600 & 541 \\
\hline$B 22$ & $\mathrm{Ba} 135$ & 90500 & 1500 & & \\
\hline$B 22$ & Ba137 & 137000 & 865 & & \\
\hline$B 22$ & Ba138 & 77400 & 974 & 102000 & 1110 \\
\hline $\mathrm{B23}$ & $\mathrm{Ba135}$ & 85800 & 504 & & \\
\hline 823 & Ba137 & 125000 & 1080 & & \\
\hline $\mathrm{B23}$ & $\mathrm{Ba} 138$ & 111000 & 457 & 107000 & 680 \\
\hline B23R & Ba135 & 114000 & 3000 & & \\
\hline B23A & Ba137 & 122000 & 838 & & \\
\hline B23R & $\mathrm{Ba138}$ & 96100 & 1080 & 111000 & 1670 \\
\hline B24 & $\mathrm{Ba} 135$ & 43500 & 1150 & & \\
\hline B24 & Ba137 & 51300 & 1610 & & \\
\hline 824 & Ba138 & 40500 & 597 & 45100 & 1120 \\
\hline $\mathbf{8 3 1}$ & Ba135 & 27500 & 3010 & & \\
\hline B31 & Ba137 & 23600 & 1670 & & \\
\hline B31 & $\mathrm{Ba} 138$ & 25500 & 921 & 25500 & 1870 \\
\hline B32 & Ba135 & 53200 & 1490 & & \\
\hline $\mathbf{B} 32$ & Ba137 & 53500 & 1030 & & \\
\hline $\mathrm{B} 32$ & $\mathrm{Ba} 138$ & 51800 & 371 & 52800 & 964 \\
\hline 833 & $\mathrm{Ba} 135$ & 11600 & 381 & & \\
\hline $\mathbf{B 3 3}$ & $\mathrm{Ba} 137$ & 11600 & 347 & & \\
\hline 833 & $\mathrm{Ba} 138$ & 12400 & 78.9 & 11900 & 269 \\
\hline 841 & $\mathrm{Ba} 135$ & 20000 & 2040 & & \\
\hline B41 & Ba137 & 18400 & 956 & & \\
\hline B41 & Ba138 & 21500 & 424 & 20000 & 1140 \\
\hline$B 42$ & Ba135 & 245000 & 1510 & & \\
\hline 842 & $\mathrm{Ba} 137$ & 255000 & 955 & & \\
\hline 842 & $\mathrm{Ba138}$ & 272000 & 2000 & 257000 & 1490 \\
\hline$B 43$ & Ba135 & 32800 & 1050 & & \\
\hline$B 43$ & Ba137 & 32300 & 781 & & \\
\hline
\end{tabular}

Note: The sctual concentration of amples for which no stenderd doviation beported be leas than the lower limit of detection. The reported concentration is the lower limit of quantification. 


\section{Transect B Soil Concentrations: Barium}

\begin{tabular}{|c|c|c|c|c|c|}
\hline Sample Id. & leotope & $\begin{array}{l}\text { Concentration } \\
\text { (ug/Kg soll) }\end{array}$ & $\begin{array}{l}\text { Std.Doviation } \\
\text { (lug/Kg soll) }\end{array}$ & $\begin{array}{l}\text { Element Avg. Conc. } \\
\text { (ug/Ko soll) }\end{array}$ & $\begin{array}{c}\text { Element Avg. Std. Dov. } \\
\text { (ug/Kg soll) }\end{array}$ \\
\hline$B 43$ & Ba138 & 31700 & 298 & 32300 & 710 \\
\hline B45V & Ba135 & & & & \\
\hline B45V & Ba137 & & & & \\
\hline $\mathrm{B} 45 \mathrm{~V}$ & Ba138 & & & & \\
\hline$B 51$ & Ba135 & 14600 & 1120 & & \\
\hline$B 51$ & Ba137 & 17200 & 256 & & \\
\hline 851 & Ba138 & 16900 & 872 & 16200 & 488 \\
\hline $\mathrm{B} 52$ & Ba135 & 19800 & 1580 & & \\
\hline$B 52$ & Ba137 & 20100 & 1170 & & \\
\hline$B 52$ & Ba138 & 22200 & 487 & 20700 & 1000 \\
\hline B52R & Ba135 & 23600 & 1000 & & \\
\hline B52R & Ba137 & 22400 & 264 & & \\
\hline B52A & Ba138 & 21700 & 296 & 22600 & 520. \\
\hline
\end{tabular}




\section{Transect B Soil Concentrations: Cadmium}

\begin{tabular}{|c|c|c|c|c|c|}
\hline Sample ld. & leotope & $\begin{array}{l}\text { Concentration } \\
\text { (ug/Kg eoll) }\end{array}$ & $\begin{array}{l}\text { Sid.Doviation } \\
\text { (ug/Kg soll) }\end{array}$ & $\begin{array}{l}\text { Element Avg. Conc. } \\
\left(\log / \mathrm{K}_{\mathrm{g}} \mathrm{sol}\right)\end{array}$ & $\begin{array}{c}\text { Element Avg. Std. Dov. } \\
\text { (ug/Kg soll) }\end{array}$ \\
\hline B11 & $\operatorname{cod} 111$ & 136 & & & \\
\hline B11 & Cd114 & 136 & & 136 & \\
\hline 812 & $\operatorname{Cod} 111$ & 127 & 407 & & \\
\hline B12 & $\operatorname{cod114}$ & 112 & & 120 & 407 \\
\hline$B 13$ & $\operatorname{Cod111}$ & 160 & & & \\
\hline 813 & $\operatorname{Col114}$ & 160 & & 160 & \\
\hline B14 & Cd111 & 174 & & & \\
\hline B14 & Cd114 & 174 & & 174 & \\
\hline B21 & $\operatorname{Cd111}$ & 106 & & & \\
\hline B21 & Cd114 & 106 & & 106 & \\
\hline$B 22$ & Cod111 & 330 & 92.5 & & \\
\hline$B 22$ & Cd114 & 61.7 & & 196 & 92.5 \\
\hline $\mathrm{B} 23$ & $\cos 111$ & 145 & & & \\
\hline $\mathrm{B} 23$ & Cod114 & 145 & & 145 & \\
\hline$B 23 R$ & Cod111 & 37 & .99 & & \\
\hline$B 23 \mathrm{~A}$ & Cod14 & 311 & 38.8 & 174 & 19.9 \\
\hline B24 & Cd111 & 141 & & & \\
\hline$B 24$ & $\operatorname{Cod114}$ & 141 & & 141 & \\
\hline 831 & Cd111 & 146 & & & \\
\hline 831 & Cd114 & 146 & & 146 & \\
\hline 832 & Cd111 & 116 & 44.7 & & \\
\hline $\mathrm{B} 32$ & $\operatorname{Cd} 114$ & 177 & 28.3 & 147 & 36.5 \\
\hline$B 33$ & $\operatorname{cod} 111$ & 120 & & & \\
\hline$B 33$ & Cd114 & 120 & & 120 & \\
\hline$B 41$ & Cd111 & 127 & & & \\
\hline$B 41$ & Cd114 & 383 & 38.9 & 255 & 38.9 \\
\hline B42 & Cod111 & 135 & & & \\
\hline B42 & Cd114 & 135 & & 135 & \\
\hline$B 43$ & Cd111 & 123 & & & \\
\hline 843 & Cd114 & 123 & & 123 & \\
\hline$B 45 \mathrm{~V}$ & Cd111 & & & & \\
\hline$B 45 \mathrm{~V}$ & Col114 & & & & \\
\hline B51 & Cd111 & 187 & & & \\
\hline$B 51$ & Cd114 & 187 & & 187 & \\
\hline 852 & Cd111 & 301 & 260 & & \\
\hline 852 & Cd114 & 66.3 & & 184 & 260 \\
\hline B52R & Cd111 & 178 & & & \\
\hline $852 R$ & Cd114 & 71 & 26.6 & 125 & 26.6 \\
\hline
\end{tabular}




\section{Transect B Soil Concentrations: Calcium}

\begin{tabular}{|c|c|c|c|c|c|}
\hline Sample id. & leotope & $\begin{array}{l}\text { Concentration } \\
\text { (ug/Kg soil) }\end{array}$ & $\begin{array}{l}\text { Sid.Doviation } \\
\text { (ug/Kg soll) }\end{array}$ & $\begin{array}{l}\text { Element Avg. Conc. } \\
\text { (ug/Kg sold) }\end{array}$ & $\begin{array}{c}\text { Element Avg. Std. Dov. } \\
\text { (ug/Kg soil) }\end{array}$ \\
\hline B11 & Ca44 & 849000 & 79500 & 848000 & 79600 \\
\hline $\mathrm{B} 12$ & Ca44 & 1250000 & 74700 & 1250000 & 74700 \\
\hline $\mathrm{B} 13$ & Ca44 & 970000 & 61800 & 970000 & 61900 \\
\hline$B 14$ & $\operatorname{Ca44}$ & & & & \\
\hline $\mathrm{B21}$ & Ca44 & 503000 & 114000 & 503000 & 114000 \\
\hline 822 & Ca44 & 257000 & 75900 & 257000 & 75900 \\
\hline$B 23$ & Ca44 & 458000 & 34800 & 458000 & 34800 \\
\hline B23R & Ca44 & 378000 & 57000 & 378000 & 57000 \\
\hline$B 24$ & Ca44 & 537000 & 56200 & 537000 & 56200 \\
\hline 831 & Ca44 & 2110000 & 260000 & 2110000 & 260000 \\
\hline B32 & Ca44 & 126000 & & 126000 & \\
\hline$B 33$ & Ca44 & 238000 & 130000 & 238000 & 130000 \\
\hline 841 & Co44 & 493000 & 71300 & 493000 & 71300 \\
\hline$B 42$ & Ca44 & & & & \\
\hline$B 43$ & Ca44 & & & & \\
\hline B45V & $\cos 44$ & & & & \\
\hline B51 & $\mathrm{Ca} 44$ & 530000 & 139000 & 530000 & 139000 \\
\hline B52 & $\mathrm{Ca} 44$ & 352000 & 38700 & 352000 & 38700 \\
\hline B52R & $\mathrm{Ca} 44$ & 682000 & 125000 & 682000 & 125000 \\
\hline
\end{tabular}

Note: The setual concentration of eamples for which no stendard deviation be reported be leas than the lower limit of dotection. The reported concentration be the lower limit of quantification. 


\begin{tabular}{|c|c|c|c|c|c|}
\hline Sample id. & leotope & $\begin{array}{l}\text { Concentration } \\
\text { (ug/Kg soll) }\end{array}$ & $\begin{array}{l}\text { Std.Doviation } \\
\text { (ug/Kg soll) }\end{array}$ & $\begin{array}{l}\text { Element Avg. Conc. } \\
\text { (ug/Kg eoll) }\end{array}$ & $\begin{array}{c}\text { Element Avg. Std. Dov. } \\
\text { (ug/Kg soil) }\end{array}$ \\
\hline B11 & Cs & 722 & & 722 & \\
\hline 812 & Cs & 515 & 92.3 & 515 & 92.3 \\
\hline $\mathrm{B} 13$ & $\mathrm{Cs}$ & 129 & 6.38 & 120 & 6.38 \\
\hline B14 & $\mathrm{Cs}$ & 84.5 & 71.5 & 84.5 & 71.5 \\
\hline$B 21$ & Cs & 320 & 4.96 & 320 & 4.96 \\
\hline$B 22$ & Cs & 570 & 652 & 570 & 652 \\
\hline B23 & Cs & 75.5 & 17.8 & 75.5 & 17.8 \\
\hline B23R & Cs & 128 & 1.12 & 128 & 1.12 \\
\hline 824 & Cs & 249 & 18.6 & 240 & 18.6 \\
\hline 831 & Cs & 73.1 & & 73.1 & \\
\hline 832 & Cs & 181 & 15.3 & 181 & 15.3 \\
\hline 833 & Cs & 50.5 & 12.1 & 50.5 & 12.1 \\
\hline B41 & Cs & 263 & 55.4 & 263 & 55.4 \\
\hline 842 & Cs & 1790 & 33.7 & 1790 & 33.7 \\
\hline$B 43$ & Cs & 169 & 17.6 & 160 & 17.6 \\
\hline B45V & Cs & & & & \\
\hline $\mathrm{B} 51$ & Cs & 613 & 93.3 & 613 & 93.3 \\
\hline $\mathrm{B} 52$ & Cs & 171 & 173 & 171 & 173 \\
\hline B52R & Cs & 138 & 9.88 & 138 & 9.88 \\
\hline
\end{tabular}




\section{Transect B Soil Concentrations: Chromium}

\begin{tabular}{|c|c|c|c|c|c|}
\hline Sample ld. & leotope & $\begin{array}{l}\text { Concentration } \\
\text { (ug/Kg soll) }\end{array}$ & $\begin{array}{l}\text { Sid.Dovintion } \\
\text { (ug/Ko soll) }\end{array}$ & $\begin{array}{c}\text { Element Avg. Conc. } \\
\text { (ug/Kg soll) }\end{array}$ & $\begin{array}{c}\text { Element Avg. Std. Dev. } \\
\text { (ug/Kg soll) }\end{array}$ \\
\hline B11 & Cr52 & 6290 & 134 & & \\
\hline B11 & 9.53 & 6360 & 146 & 6330 & 140 \\
\hline $\mathrm{B} 12$ & a.52 & 8120 & 289 & & \\
\hline B12 & 953 & 8160 & 280 & 8140 & 289 \\
\hline $\mathrm{B} 13$ & C.52 & 7240 & 36.1 & & \\
\hline B13 & C.53 & 7240 & 36.1 & 7240 & 36.1 \\
\hline B14 & Cr52 & 349 & & & \\
\hline B14 & C.53 & 349 & & 349 & \\
\hline B21 & 0.52 & 11200 & 479 & & \\
\hline $\mathrm{B} 21$ & 0.53 & & & 11200 & 479 \\
\hline B22 & 952 & 61.7 & & & \\
\hline$B 22$ & $C .53$ & 61.7 & & 61.7 & \\
\hline $\mathrm{B} 23$ & $a 52$ & 2510 & 94.5 & & \\
\hline $\mathrm{B} 23$ & 0.53 & 2000 & 103 & 2260 & 98.8 \\
\hline B23A & Cr52 & 3150 & 161 & & \\
\hline B23R & Cr53 & 3150 & 161 & 3150 & 161 \\
\hline B24 & C.52 & 7160 & 334 & & \\
\hline$B 24$ & Cr53 & 6630 & 902 & 6900 & 618 \\
\hline $\mathrm{B} 31$ & C.52 & 7570 & 1000 & & \\
\hline $\mathrm{B} 31$ & $C \times 53$ & 7580 & 1000 & 7580 & 1000 \\
\hline $\mathrm{B} 32$ & Cr52 & 10700 & 292 & & \\
\hline $\mathrm{B} 32$ & 0.53 & & & 10700 & 292 \\
\hline $\mathrm{B} 33$ & 0.52 & 3600 & 166 & & \\
\hline 833 & Cr53 & & & 3600 & 166 \\
\hline 841 & a52 & 9970 & 600 & & \\
\hline$B 41$ & 0.53 & 9870 & 59 & 9920 & 604 \\
\hline$B 42$ & 0.52 & 14100 & 152 & & \\
\hline$B 42$ & $C 53$ & 14100 & 148 & 14100 & 150 \\
\hline 843 & C.52 & 2870 & 47.7 & & \\
\hline$B 43$ & C.53 & 2870 & 47.8 & 2870 & 47.8 \\
\hline $\mathrm{B} 45 \mathrm{~V}$ & Cr52 & & & & \\
\hline B45V & $\mathrm{Cr53}$ & & & & \\
\hline 851 & Cr52 & 3200 & 149 & & \\
\hline B51 & Cr53 & 3150 & 144 & 3180 & 147 \\
\hline$B 52$ & C752 & 2090 & 194 & & \\
\hline $\mathrm{B} 52$ & Cr53 & 2070 & 194 & 2080 & 194 \\
\hline B52R & Gr52 & 6970 & 272 & & \\
\hline B52A & $C .53$ & 6970 & 271 & 6970 & 272 \\
\hline
\end{tabular}




\section{Transect B Soil Concentrations: Cobalt}

\begin{tabular}{|c|c|c|c|c|c|}
\hline Sample ld. & Lotope & $\begin{array}{l}\text { Concentration } \\
\text { (ug/Kg soil) }\end{array}$ & $\begin{array}{l}\text { Sid.Doviation } \\
\text { (ug/Kg soll) }\end{array}$ & $\begin{array}{c}\text { Element Avg. Conc. } \\
\text { (ug/Kg soll) }\end{array}$ & $\begin{array}{c}\text { Element Avg. Std. Dov. } \\
\text { (ug/Kg soil) }\end{array}$ \\
\hline B11 & $\infty$ & 531 & 40.5 & 531 & 40.5 \\
\hline$B 12$ & Co & 371 & 38.8 & 371 & 38.8 \\
\hline$B 13$ & $C_{0}$ & 645 & 45.9 & 645 & 45.9 \\
\hline 814 & $\infty$ & 174 & & 174 & \\
\hline 821 & $\infty$ & 569 & 25.1 & 560 & 25.1 \\
\hline 822 & Co & 186 & 138 & 186 & 138 \\
\hline $\mathrm{B23}$ & Co & 143 & 44.1 & 143 & 44.1 \\
\hline B23R & $\infty$ & 1200 & 370 & 1200 & 370 \\
\hline B24 & Co & 349 & 73.2 & 349 & 732 \\
\hline B31 & Co & 812 & 260 & 912 & 260 \\
\hline $\mathrm{B} 32$ & $\infty$ & 175 & 39.2 & 175 & 392 \\
\hline $\mathrm{B} 33$ & co & 1050 & 119 & 1050 & 119 \\
\hline B41 & $\infty$ & 637 & & 637 & \\
\hline$B 42$ & $\infty$ & 20700 & 467 & 20700 & 457 \\
\hline$B 43$ & Co & 4280 & 90.6 & 4280 & 90.6 \\
\hline B45V & $\infty$ & & & & \\
\hline B51 & Co & 293 & 34.7 & 283 & 34.7 \\
\hline $\mathrm{B} 52$ & Co & 17700 & 870 & 17700 & 870 \\
\hline B52R & $\infty$ & 8250 & 218 & 8250 & 218 \\
\hline
\end{tabular}

Note: The ectual concentration of samples for which no atundard deviation le reported to leas than the lower limit of detection. The reported concentration is the lower Hinit of quantification. 


\section{Transect B Soil Concentrations: Copper}

\begin{tabular}{|c|c|c|c|c|c|}
\hline Samplo ld. & botope & $\begin{array}{l}\text { Concentration } \\
\text { (ug/Kg soll) }\end{array}$ & $\begin{array}{l}\text { Std.Deviation } \\
\text { (ug/Ko soll) }\end{array}$ & $\begin{array}{l}\text { Element Avg. Conc. } \\
\text { (ug/Ko soll) }\end{array}$ & $\begin{array}{c}\text { Element Avg. Std. Dov. } \\
\text { (ug/Kg soll) }\end{array}$ \\
\hline
\end{tabular}

\begin{tabular}{|c|c|c|c|c|c|}
\hline B11 & Qu63 & & & & \\
\hline B11 & Cu65 & 6480 & 222 & 6480 & 222 \\
\hline$B 12$ & au63 & & & & \\
\hline$B 12$ & Cu65 & 4740 & 391 & 4740 & 391 \\
\hline B13 & Cu63 & & & & \\
\hline$B_{13}$ & Cu65 & 5890 & 838 & 5890 & 838 \\
\hline$B 14$ & Cu63 & & & & \\
\hline B14 & Cu65 & 411 & 210 & 411 & 210 \\
\hline B21 & Cu63 & 3310 & 550 & & \\
\hline B21 & Cu65 & 4580 & 361 & 3950 & 456 \\
\hline 822 & Qu63 & 4880 & 642 & & \\
\hline 822 & Cu65 & 5550 & 1200 & 5220 & 921 \\
\hline$B 23$ & Cu63 & & & & \\
\hline $\mathrm{B} 23$ & $a 465$ & 1210 & 311 & 1210 & 311 \\
\hline B23A & Cu63 & & & & \\
\hline B23R & Cu65 & 6940 & 2750 & 6940 & 2750 \\
\hline B24 & Cu63 & 2570 & 180 & & \\
\hline 824 & cu65 & 528 & 196 & 1550 & 188 \\
\hline$B 31$ & cu63 & & & & \\
\hline 831 & Cu65 & 4130 & 863 & 4130 & 863 \\
\hline $\mathrm{B} 32$ & Cu63 & 2790 & 128 & & \\
\hline 832 & Qu65 & 3480 & 448 & 3140 & 288 \\
\hline $\mathrm{B} 33$ & Cu63 & 1610 & 45.2 & & \\
\hline $\mathrm{B} 33$ & Cu65 & 2100 & $\infty 2$ & 1860 & 68.6 \\
\hline$B 41$ & cu63 & 2600 & 618 & & \\
\hline$B 41$ & Cu65 & 4990 & 778 & 3800 & 698 \\
\hline$B 42$ & 0,63 & & & & \\
\hline B42 & Cu65 & 10800 & 648 & 10800 & 648 \\
\hline$B 43$ & cu63 & & & & \\
\hline$B 43$ & Cu65 & 1610 & 133 & 1610 & 133 \\
\hline $845 \mathrm{~V}$ & Cu63 & & & & \\
\hline $\mathrm{B} 45 \mathrm{~V}$ & Cu65 & & & & \\
\hline B51 & Cu63 & & & & \\
\hline B51 & Cu65 & 9320 & 534 & 9320 & 534 \\
\hline B52 & Cu63 & 9540 & 2820 & & \\
\hline $\mathrm{B} 52$ & Cu65 & 13100 & 6910 & 11300 & 4870 \\
\hline$B 52 R$ & Cu63 & & & & \\
\hline B52R & Cu65 & 6930 & 565 & 6930 & 565 \\
\hline
\end{tabular}

Note: The actual concentration of eamples for whlch no stendard deviation ts reported be leas than the lower limit of detection. The reported concentration bs the lower limit of quantification. 


\section{Transect B Soil Concentrations: Iron}

\begin{tabular}{|c|c|c|c|c|c|}
\hline Sample ld. & Letope & $\begin{array}{l}\text { Concentration } \\
\text { (ug/Kg soil) }\end{array}$ & $\begin{array}{l}\text { Std.boviation } \\
\text { (ug/Ko solf) }\end{array}$ & $\begin{array}{l}\text { Element Ayg Conc. } \\
\text { (ug/Ko soll) }\end{array}$ & $\begin{array}{c}\text { Element Avg. Std. Dov. } \\
\text { (ug/Kg soll) }\end{array}$ \\
\hline 811 & Fo & 9260000 & 69800 & 9260000 & 69800 \\
\hline $\mathrm{B} 12$ & $F$ & 8340000 & 32000 & 8340000 & 32000 \\
\hline $\mathrm{B} 13$ & Fo & 5120000 & 24900 & 5120000 & 24900 \\
\hline B14 & Fo & 2010000 & 12400 & 2010000 & 12400 \\
\hline B21 & $F$ & 32400000 & 77200 & 32400000 & 77200 \\
\hline $\mathrm{B} 22$ & Fo & 8320000 & 22900 & 8320000 & 22900 \\
\hline $\mathrm{B23}$ & $F$ & 5800000 & 44000 & 5800000 & 44000 \\
\hline B23R & Fo & 2600000 & 5320 & 2600000 & 5320 \\
\hline B24 & Fo & $\$ 330000$ & 89100 & 9330000 & 89100 \\
\hline $\mathrm{B} 31$ & $F_{\theta}$ & 13900000 & 101000 & 13900000 & 101000 \\
\hline $\mathrm{B} 32$ & Fo & 9830000 & 65000 & 9830000 & 65000 \\
\hline $\mathrm{B} 33$ & Fo & 6180000 & 31500 & 6180000 & 31500 \\
\hline B41 & $\mathrm{Fe}$ & 6030000 & 48000 & 6030000 & 48000 \\
\hline$B 42$ & $F_{\theta}$ & 24500000 & 152000 & 24500000 & 152000 \\
\hline $\mathrm{B} 43$ & $F$ & 5140000 & 43700 & 5140000 & 43700 \\
\hline $845 V$ & Fo & & & & \\
\hline 851 & $F_{0}$ & 3130000 & 17500 & 3130000 & 17500 \\
\hline $\mathrm{B} 52$ & $F_{0}$ & 2470000 & 29000 & 2470000 & 29600 \\
\hline B52R & $F 0$ & 2560000 & 8130 & 2560000 & 8130 \\
\hline
\end{tabular}

Note: The actual concentration of samples for which no standerd deviation be reported to lees than the lower limit of dotection. The roported concentration is the lower limit of quantification. 
Transect B Soil Concentrations: Lead

\begin{tabular}{|c|c|c|c|c|c|}
\hline Sampto id. & botopo & $\begin{array}{l}\text { Concentration } \\
\text { (ug/Kg \&oll) }\end{array}$ & $\begin{array}{l}\text { Sit.Deviation } \\
\text { (ug/Kg soll) }\end{array}$ & $\begin{array}{l}\text { Element Avg. Conc. } \\
\text { (ug/Kg soll) }\end{array}$ & $\begin{array}{l}\text { Element Avg. Std. Dov. } \\
\text { (ug/Kg soll) }\end{array}$ \\
\hline$B 11$ & Pt206 & 3460 & 143 & & \\
\hline B11 & Pt207 & 4030 & 377 & & \\
\hline B11 & $\mathrm{Pb} 208$ & 4260 & 153 & 3920 & 224 \\
\hline$B 12$ & Pb206 & 14700 & 127 & & \\
\hline 812 & Pb207 & 15800 & 164 & & \\
\hline$B 12$ & Pb208 & 13000 & 215 & 14500 & 160 \\
\hline$B 13$ & Pb206 & 17400 & 1140 & & \\
\hline$B 13$ & Pb207 & 16800 & 942 & & \\
\hline B13 & $\mathrm{Pb} 208$ & 15700 & 854 & 16600 & 1010 \\
\hline B14 & Pb206 & 2410 & 198 & & \\
\hline B14 & Po207 & 1140 & 141 & & \\
\hline B14 & Pb208 & 1740 & 157 & 1760 & 165 \\
\hline B21 & Pb206 & 8740 & 52.6 & & \\
\hline B21 & Pb207 & 9650 & 92.4 & & \\
\hline B21 & Pb208 & 8200 & 145 & 8860 & 96.7 \\
\hline$B 22$ & Pb206 & 8370 & 514 & & \\
\hline$B 22$ & Pb207 & 11800 & 331 & & \\
\hline$B 22$ & Pb208 & 9300 & 126 & 9820 & 324 \\
\hline$B 23$ & Pb206 & 1630 & 350 & & \\
\hline 823 & Pb207 & 3270 & 124 & & \\
\hline 823 & $\mathrm{~Pb} 208$ & 2290 & 306 & 2400 & 263 \\
\hline $\mathrm{B} 23 \mathrm{R}$ & Pb206 & 19700 & & & \\
\hline$B 23 R$ & Po207 & 18700 & & & \\
\hline$B 23 R$ & Pb208 & 19700 & & 19700 & \\
\hline 824 & $\mathrm{~Pb} 206$ & 1860 & 184 & & \\
\hline 824 & Pb207 & 1480 & 161 & & \\
\hline$B 24$ & Pb208 & 501 & 42.5 & 1280 & 120 \\
\hline$B 31$ & $\mathrm{~Pb} 206$ & 3220 & 573 & & \\
\hline$B 31$ & $\mathrm{Pb207}$ & 2530 & 1330 & & \\
\hline$B 31$ & $\mathrm{Pb208}$ & 2810 & 348 & 2850 & 750 \\
\hline$B 32$ & Pb206 & 3290 & 118 & & \\
\hline B32 & Pb207 & 2700 & 25.9 & & \\
\hline$B 32$ & Pb208 & 2900 & 502 & 2960 & 64.7 \\
\hline$B 33$ & Pb206 & 4610 & 105 & & \\
\hline$B 33$ & Pb207 & 410 & 78.7 & & \\
\hline$B 33$ & Pb208 & 4320 & 47.3 & 4450 & $\pi$ \\
\hline$B 41$ & Pb206 & 23900 & 521 & & \\
\hline$B 41$ & Pb207 & 25700 & 1150 & & \\
\hline 841 & Pb208 & 20600 & 443 & 23400 & 705 \\
\hline$B 42$ & PD206 & 5410 & 168 & & \\
\hline B42 & Pb207 & 4560 & 181 & & \\
\hline B42 & Pb208 & 4440 & 91.9 & 4800 & 147 \\
\hline$B 43$ & Pb206 & 1980 & $\pi .4$ & & \\
\hline$B 43$ & Pb207 & 1920 & $\$ 6$ & & \\
\hline
\end{tabular}

Note: The actual concentration of eamples for which no standerd doviation is reported is lese than the lower limit of detection. The reported concentration ts the lower limit of quantincation. 
Transect B Soil Concentrations: Lead

\begin{tabular}{|c|c|c|c|c|c|}
\hline Sample ld. & botope & $\begin{array}{l}\text { Concentration } \\
\text { (ug/kg soli) }\end{array}$ & $\begin{array}{l}\text { Sid.Deviation } \\
\text { (ug/Kg soll) }\end{array}$ & $\begin{array}{l}\text { Element Avg. Conc. } \\
\text { (ug/Ko soll) }\end{array}$ & $\begin{array}{c}\text { Element Avg. Std. Dov. } \\
\text { (Ug/Kg soll) }\end{array}$ \\
\hline$B 43$ & $P_{b 208}$ & 1620 & 30.6 & 1840 & 68 \\
\hline B45V & Pb206 & & & & \\
\hline B45V & $\mathrm{Pb} 207$ & & & & \\
\hline B45V & Pb208 & & & & \\
\hline $\mathrm{B} 51$ & Pb206 & 3550 & 898 & & \\
\hline 851 & Pb207 & 3190 & 223 & & \\
\hline 851 & Pb208 & 2340 & 65.3 & 3030 & 395 \\
\hline $\mathrm{B} 52$ & Pb206 & 4270 & 383 & & \\
\hline 852 & $\mathrm{~Pb} 207$ & 6600 & 45.8 & & \\
\hline $\mathrm{B} 52$ & Pb208 & 2530 & 147 & 4470 & 192 \\
\hline B52R & Pb206 & 1370 & 148 & & \\
\hline B52A & Pb207 & 1100 & 140 & & \\
\hline B52R & Pb208 & 1120 & 71.9 & 1200 & 120 \\
\hline
\end{tabular}

Note: The actual concentration of eamples for which no standard doviation le reported is leas than the lower limit of detection. The reported concentration is the lower limit of quandincation. 


\section{Transect B Soil Concentrations: Magnesium}

\begin{tabular}{ccccc}
\hline Samplo id. leotopo & $\begin{array}{c}\text { Concentration } \\
\text { (ug/Kg soll) }\end{array}$ & $\begin{array}{c}\text { Std.Doviation } \\
\text { (Ug/Kg soll) }\end{array}$ & $\begin{array}{c}\text { Element Avg. Conc. } \\
\text { (ug/Kgeoll) }\end{array}$ & $\begin{array}{c}\text { Element Avg. Std. Dov. } \\
\text { (Ug/Kg soll) }\end{array}$
\end{tabular}

\begin{tabular}{|c|c|c|c|c|c|}
\hline B11 & $\mathrm{Mg} 24$ & 165000 & 6680 & & \\
\hline$B 11$ & Mges & 146000 & 6730 & 156000 & 6710 \\
\hline$B 12$ & Mg24 & 186000 & 2000 & & \\
\hline B12 & Mge25 & 176000 & 3070 & 181000 & 3030 \\
\hline B13 & Mg24 & 53200 & 1400 & & \\
\hline B13 & $M 925$ & 53800 & 2420 & 53500 & 1910 \\
\hline B14 & Mg24 & 13000 & 778 & & \\
\hline B14 & Mg25 & 14500 & 3870 & 13800 & 2320 \\
\hline B21 & Mg24 & 174000 & 2320 & & \\
\hline B21 & Mg25 & 171000 & 2000 & 173000 & 2160 \\
\hline$B 22$ & Mg24 & 149000 & 1030 & & \\
\hline$B 22$ & Mg25 & 156000 & 1570 & 153000 & 1300 \\
\hline 823 & Mg24 & 67700 & 1640 & & \\
\hline$B 23$ & Mg25 & 68700 & 2650 & 68200 & 2150 \\
\hline B23R & Mg24 & 107000 & 1470 & & \\
\hline B23A & Mg25 & 94000 & 1660 & 101000 & 1570 \\
\hline B24 & $\mathrm{Mg24}$ & 193000 & 200 & & \\
\hline 824 & M925 & 118000 & 5230 & 156000 & 2720 \\
\hline$B 31$ & Mg24 & 233000 & 10500 & & \\
\hline$B 31$ & Mg25 & 225000 & 3920 & 229000 & 7210 \\
\hline B32 & $M 924$ & 71300 & 863 & & \\
\hline$B 32$ & M925 & 74300 & 2000 & 72800 & 1430 \\
\hline$B 33$ & $M 924$ & 46800 & 520 & & \\
\hline 833 & Mg25 & 51400 & 3580 & 49100 & 2050 \\
\hline B41 & M924 & 124000 & 2860 & & \\
\hline$B 41$ & Mg25 & 136000 & 12400 & 130000 & 7630 \\
\hline$B 42$ & $M g 24$ & 991000 & 5890 & & \\
\hline B42 & Mg25 & 1050000 & 6840 & 1020000 & 6370 \\
\hline$B 43$ & $M 924$ & 111000 & 1530 & & \\
\hline$B 43$ & Mg25 & 107000 & 305 & 109000 & 918 \\
\hline B45V & $M 924$ & & & & \\
\hline $\mathrm{B} 45 \mathrm{~V}$ & Mg25 & & & & \\
\hline B51 & Mg24 & 46500 & 2540 & & \\
\hline B51 & $M g 25$ & 58100 & 1950 & 52300 & 2250 \\
\hline B52 & $M 924$ & 72200 & 1180 & & \\
\hline $\mathrm{B} 52$ & M925 & 70400 & 4000 & 71300 & 2630 \\
\hline B52R & $M 924$ & 66500 & 2280 & & \\
\hline B52R & Mg25 & 66500 & 611 & 66500 & 1450 \\
\hline
\end{tabular}




\section{Transect B Soil Concentrations: Manganese}

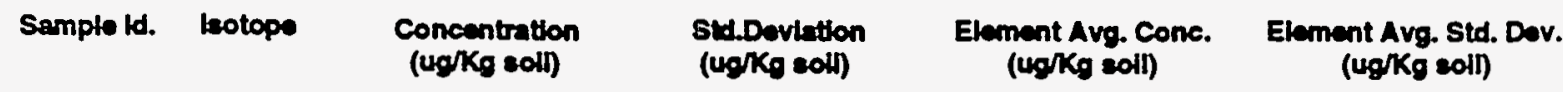

\begin{tabular}{|c|c|c|c|c|c|}
\hline B11 & $\mathrm{Mn}$ & 23500 & 423 & 23500 & 423 \\
\hline $\mathrm{B} 12$ & $\mathrm{Mn}$ & 24000 & 265 & 24000 & 265 \\
\hline B13 & $\mathrm{Mn}$ & 21800 & 245 & 21800 & 245 \\
\hline 814 & $\mathrm{Mn}$ & 11500 & 585 & 11500 & 585 \\
\hline$B 21$ & $\mathrm{Mn}$ & 31200 & 222 & 31200 & 222 \\
\hline$B 22$ & $\mathrm{Mn}$ & 124000 & 3150 & 124000 & 3150 \\
\hline $\mathrm{B23}$ & $\mathrm{Mn}$ & 36000 & 465 & 36000 & 465 \\
\hline 823R & $\mathrm{Mn}$ & 14000 & 405 & 14000 & 405 \\
\hline$B 24$ & $\mathrm{Mn}$ & 18800 & 343 & 18800 & 343 \\
\hline 831 & $\mathrm{Mn}$ & 129000 & 474 & 129000 & 474 \\
\hline B32 & $\mathrm{Mn}$ & 65600 & 4240 & 65600 & 4240 \\
\hline $\mathrm{B} 33$ & $\mathrm{Mn}$ & 62300 & 2250 & 62300 & 2250 \\
\hline $\mathrm{B} 41$ & $\mathrm{Mn}$ & 140000 & 2870 & 140000 & 2870 \\
\hline 842 & $\mathrm{Mn}$ & 303000 & 1290 & 303000 & 1290 \\
\hline 843 & $\mathrm{Mn}$ & 62400 & 657 & 62400 & 657 \\
\hline B45V & $\mathrm{Mn}$ & & & & \\
\hline B51 & Mn & 10600 & 184 & 10600 & 194 \\
\hline B52 & $\mathrm{Mn}$ & 234000 & 3080 & 234000 & 3080 \\
\hline B52R & $M n$ & 116000 & 426 & 116000 & 426 \\
\hline
\end{tabular}




\section{Transect B Soil Concentrations: Nickel}

\begin{tabular}{|c|c|c|c|c|c|}
\hline Semple id. & botope & $\begin{array}{l}\text { Concentration } \\
\text { (ug/Ka eoll) }\end{array}$ & $\begin{array}{l}\text { Std.Dovinton } \\
\text { (ug/Ko eoll) }\end{array}$ & $\begin{array}{l}\text { Element Avg. Conc. } \\
\text { (ug/Ko soll) }\end{array}$ & $\begin{array}{c}\text { Element Avg. Std. Dev. } \\
\text { (ug/Kg soll) }\end{array}$ \\
\hline B11 & Ni6o & & & & \\
\hline B11 & Ni62 & 18800 & 7720 & 19800 & 7720 \\
\hline $\mathrm{B12}$ & Ni60 & & & & \\
\hline $\mathrm{B12}$ & N:62 & 5290 & 4170 & 5290 & 4170 \\
\hline B13 & Niso & 1230 & 131 & & \\
\hline $\mathrm{B13}$ & NiE2 & & & 1230 & 131 \\
\hline 814 & Ni60 & & & & \\
\hline$B 14$ & Ni62 & $2 \approx 2$ & & 202 & \\
\hline$B 21$ & Ni60 & 2600 & 331 & & \\
\hline$B 21$ & Ni62 & 1580 & 1690 & 2090 & 1010 \\
\hline$B 22$ & Ni 60 & 28600 & 985 & & \\
\hline B22 & Ni62 & 31200 & 3490 & 29900 & 2240 \\
\hline $\mathrm{B} 23$ & Ni60 & 1490 & 297 & & \\
\hline $\mathrm{B23}$ & Ni62 & & & 1490 & 297 \\
\hline B23R & Ni60 & 2470 & 561 & & \\
\hline B23R & Ni62 & & & 2470 & 561 \\
\hline$B 24$ & Ni60 & 2790 & 938 & & \\
\hline 824 & Ni62 & 2570 & 1310 & 2680 & 1120 \\
\hline B31 & Ni60 & & & & \\
\hline B31 & Ni62 & 219 & & 218 & \\
\hline B32 & Ni60 & 3260 & 318 & & \\
\hline $\mathrm{B} 32$ & Ni62 & 19000 & & 11100 & 318 \\
\hline $\mathrm{B} 33$ & Ni60 & 4680 & 552 & & \\
\hline $\mathrm{B} 33$ & Ni62 & 1710 & 633 & 3200 & 598 \\
\hline B41 & Ni60 & 9860 & 852 & & \\
\hline 841 & Ni62 & 21300 & 6750 & 15600 & 3850 \\
\hline 842 & Ni60 & & & & \\
\hline$B 42$ & $\mathrm{Ni62}$ & 10800 & 1410 & 10800 & 1410 \\
\hline$B 43$ & Ni60 & & & & \\
\hline$B 43$ & Ni62 & 1850 & & 1850 & \\
\hline$B 45 V$ & Ni60 & & & & \\
\hline$B 45 \mathrm{~V}$ & Ni62 & & & & \\
\hline 851 & Ni60 & & & & \\
\hline 851 & Ni62 & 3530 & 1190 & 3550 & 1190 \\
\hline $\mathrm{B} 52$ & Ni60 & 34700 & 1540 & & \\
\hline 852 & Ni62 & 29900 & 4010 & 32300 & 2780 \\
\hline B52R & Ni6o & 13000 & 973 & & \\
\hline B52R & N162 & 8610 & 4200 & 10000 & 2590 \\
\hline
\end{tabular}




\section{Transect B Soil Concentrations: Selenium}

\begin{tabular}{|c|c|c|c|c|c|}
\hline Sample k. & kotope & $\begin{array}{l}\text { Concentration } \\
\text { (ug/Ko eoll) }\end{array}$ & $\begin{array}{l}\text { Std.Dovintion } \\
\text { (ug/ko soll) }\end{array}$ & $\begin{array}{l}\text { Element Avg Conc. } \\
\text { (ug/Ko soll) }\end{array}$ & $\begin{array}{l}\text { Element Avg. Std. Dov. } \\
\text { (ug/Kg soll) }\end{array}$ \\
\hline B11 & So77 & 2220 & 5610 & & \\
\hline B11 & 5082 & 2000 & 5520 & 2210 & 5570 \\
\hline $\mathrm{B12}$ & So77 & 4370 & 10800 & & \\
\hline 812 & So82 & 4130 & 10500 & 4250 & 10700 \\
\hline 813 & So77 & & & & \\
\hline 813 & So82 & 19000 & 1610 & 19000 & 1610 \\
\hline$B 14$ & Se77 & 48300 & 9640 & & \\
\hline 814 & So82 & 45800 & 9440 & 47100 & 8540 \\
\hline 821 & Se77 & & & & \\
\hline 821 & So82 & 5280 & & 5280 & \\
\hline 822 & So77 & 3080 & & & \\
\hline 822 & 5082 & 3080 & & 3080 & \\
\hline$B 23$ & Se77 & & & & . \\
\hline 823 & Sa82 & 7270 & & 7270 & \\
\hline B23R & So77 & & & & \\
\hline $823 R$ & So82 & 9850 & & 9850 & \\
\hline 824 & So77 & 7640 & 4610 & & \\
\hline 824 & Se82 & 7750 & 4600 & 7700 & 4610 \\
\hline $\mathrm{B} 31$ & Se77 & 7310 & & & \\
\hline 831 & So82 & 7310 & & 7310 & \\
\hline B32 & Se77 & 22500 & & & \\
\hline $\mathrm{B} 32$ & So82 & 25300 & & 23900 & \\
\hline $\mathrm{B} 33$ & Se77 & & & & \\
\hline $\mathrm{B33}$ & So82 & 24000 & & 24000 & \\
\hline 841 & Se77 & 6370 & & & \\
\hline B41 & So82 & 6370 & & 6370 & \\
\hline$B 42$ & Se77 & 53800 & 20500 & & \\
\hline$B 42$ & Sos2 & 55400 & 21200 & 54600 & 20900 \\
\hline$B 43$ & Se77 & 6170 & & & \\
\hline$B 43$ & So82 & 6170 & & 6170 & \\
\hline B45V & $\mathrm{SeT} 7$ & & & & \\
\hline B45V & So82 & & & & \\
\hline $\mathrm{B} 51$ & $\mathrm{~S} \theta 77$ & 18700 & & & \\
\hline 851 & So82 & 18700 & & 18700 & \\
\hline 852 & Se77 & 5570 & 1650 & & \\
\hline 852 & So82 & 5280 & 1620 & 5430 & 1640 \\
\hline B52R & Se77 & 19900 & 6210 & & \\
\hline B52R & So82 & 19400 & 6120 & 19700 & 6170 \\
\hline
\end{tabular}




\begin{tabular}{|c|c|c|c|c|c|}
\hline \multicolumn{6}{|c|}{ Transect B Soil Concentrations: Silver } \\
\hline Sample ld. & leotope & $\begin{array}{l}\text { Concentration } \\
\text { (ug/Kg soll) }\end{array}$ & $\begin{array}{l}\text { Std.Deviation } \\
\text { (ug/Kg soll) }\end{array}$ & $\begin{array}{c}\text { Element Avg. Conc. } \\
\text { (ug/ } / \mathrm{Kg} \text { soil) }\end{array}$ & $\begin{array}{c}\text { Elament Avg. Std. Dov. } \\
\text { (ug/Kg soll) }\end{array}$ \\
\hline 811 & Ag107 & 6580 & 2060 & & \\
\hline$B 11$ & Ag109 & 3570 & & 5080 & 2060 \\
\hline$B 12$ & Ag107 & 3530 & 448 & & \\
\hline 812 & Ag109 & 2240 & & 2890 & 448 \\
\hline$B 13$ & A0107 & 6380 & & & \\
\hline$B 13$ & Ag100 & 6380 & & 6390 & \\
\hline$B 14$ & Ag107 & 3490 & & & \\
\hline$B 14$ & Ad109 & 3490 & & 3490 & \\
\hline 821 & Ag107 & 211 & & & \\
\hline 821 & Ag109 & 1060 & & 636 & \\
\hline$B 22$ & Ag107 & 30.8 & & & \\
\hline 822 & $A 0109$ & 30.8 & & 30.8 & \\
\hline $\mathrm{B} 23$ & Ag107 & 5820 & & & \\
\hline B23 & Ag109 & 5820 & & 5820 & \\
\hline B23R & Ag107 & 7880 & & & \\
\hline B23R & Ag109 & 7880 & & 7880 & \\
\hline 824 & A9107 & 2010 & & & \\
\hline$B 24$ & Ag109 & 2310 & & 2160 & \\
\hline B31 & Ag107 & 2750 & 1650 & & \\
\hline B31 & Ag109 & 2920 & & 2840 & 1650 \\
\hline B32 & Ag107 & 1260 & & & \\
\hline $\mathrm{B} 32$ & Ag109 & 1260 & & 1260 & \\
\hline $\mathrm{B} 33$ & Ag107 & 1200 & & & \\
\hline$B 33$ & Ag109 & 1200 & & 1200 & \\
\hline$B 41$ & Ag107 & 1270 & & & \\
\hline$B 41$ & Ag109 & 1270 & & 1270 & \\
\hline$B 42$ & Ag107 & 2700 & & & \\
\hline$B 42$ & Ag109 & 2700 & & 2700 & \\
\hline 843 & Ag107 & 2470 & & & \\
\hline$B 43$ & Ag109 & 2470 & & 2470 & \\
\hline B45V & Ag107 & & & & \\
\hline B45V & Ag109 & & & & \\
\hline B51 & Ag107 & 1870 & & & \\
\hline$B 51$ & Ag109 & 1870 & & 1870 & \\
\hline$B 52$ & Ag107 & 33.1 & 248 & & \\
\hline B52 & Ag109 & 33.1 & & 33.1 & 248 \\
\hline B52R & Ag107 & 356 & & & \\
\hline B52R & Ag109 & 35.6 & & 196 & \\
\hline
\end{tabular}




\section{Transect B Soil Concentrations: Sodium}

\begin{tabular}{|c|c|c|c|c|c|}
\hline Samplo ld. & botope & $\begin{array}{l}\text { Concentration } \\
\text { (ug/K@ soll) }\end{array}$ & $\begin{array}{l}\text { 5td.Doviation } \\
\text { (ug/Ko eoil) }\end{array}$ & $\begin{array}{l}\text { Element Avg. Conc. } \\
\text { (ug/Kg soll) }\end{array}$ & $\begin{array}{c}\text { Element Avg. Std. Dov. } \\
\text { (ug/Kg soil) }\end{array}$ \\
\hline B11 & $\mathrm{Na}$ & 150000 & 10000 & 150000 & 10000 \\
\hline $\mathrm{B} 12$ & $\mathrm{Na}$ & 112000 & & 112000 & \\
\hline$B 13$ & $\mathrm{Na}$ & 838000 & 31100 & 838000 & 31100 \\
\hline B14 & $\mathrm{Na}$ & 1740 & & 1740 & \\
\hline$B 21$ & $\mathrm{Na}$ & 1060 & & 1060 & \\
\hline$B 22$ & $\mathrm{Na}$ & 123000 & & 123000 & \\
\hline$B 23$ & $\mathrm{Na}$ & 291000 & & 291000 & \\
\hline B23R & $\mathrm{Na}$ & 394000 & & 324000 & \\
\hline B24 & $\mathrm{Na}$ & 34900 & 10100 & 34800 & 10100 \\
\hline B31 & $\mathrm{Na}$ & 146000 & & 146000 & \\
\hline $\mathrm{B} 32$ & $\mathrm{Na}$ & 126000 & & 126000 & \\
\hline $\mathrm{B} 33$ & $\mathrm{Na}$ & 120000 & & 120000 & \\
\hline$B 41$ & $\mathrm{Na}$ & 41000 & 46800 & 41000 & 46900 \\
\hline$B 42$ & $\mathrm{Na}$ & 64800 & 6120 & 64800 & 6120 \\
\hline $\mathrm{B} 43$ & $\mathrm{Na}$ & 1230 & & 1230 & \\
\hline B45V & $\mathrm{Na}$ & & & & \\
\hline B51 & $\mathrm{Na}$ & 187000 & & 187000 & \\
\hline $\mathrm{B} 52$ & $\mathrm{Na}$ & 906000 & 120000 & 906000 & 120000 \\
\hline B52R & $\mathrm{Na}$ & 17800 & & 17800 & \\
\hline
\end{tabular}




\section{Transect B Soil Concentrations: Strontium}

\begin{tabular}{|c|c|c|c|c|c|}
\hline Semple id. & Isotope & $\begin{array}{l}\text { Concentration } \\
\text { (ug/kg aoll) }\end{array}$ & $\begin{array}{l}\text { Std.Dovition } \\
\text { (ug/Kg eoll) }\end{array}$ & $\begin{array}{l}\text { Element Avg. Conc. } \\
\text { (ug/Ko woll) }\end{array}$ & $\begin{array}{c}\text { Element Avg. Std. Dov. } \\
\text { (ug/Kg soll) }\end{array}$ \\
\hline $\mathrm{B11}$ & Sp & 9220 & 150 & 9220 & 150 \\
\hline$B 12$ & Si & 59500 & 334 & 59500 & 334 \\
\hline B13 & Sr & 24700 & 352 & 24700 & 352 \\
\hline 814 & $\mathrm{Sr}$ & 11300 & 90.6 & 11300 & 90.6 \\
\hline 821 & Si & 5890 & 48.3 & 5890 & 48.3 \\
\hline$B 22$ & Sr & 6580 & 542 & 6580 & 542 \\
\hline $\mathrm{B} 23$ & $\mathrm{Sr}$ & 34100 & 176 & 34100 & 176 \\
\hline B23R & $\mathrm{Sr}$ & 20400 & 367 & 20400 & 367 \\
\hline$B 24$ & Si & 12600 & 322 & 12600 & 322 \\
\hline $\mathrm{B} 31$ & $\mathrm{Sr}$ & 7320 & 716 & 7320 & 716 \\
\hline 832 & $\mathrm{Sr}$ & 17800 & 180 & 17000 & 180 \\
\hline $\mathrm{B} 33$ & Sr & 7850 & 792 & 7850 & 792 \\
\hline 841 & Sr & 3530 & 123 & 3550 & 123 \\
\hline$B 42$ & $\mathbf{S r}$ & 78200 & 33.3 & 78200 & 33.3 \\
\hline 843 & Sr & 12400 & 112 & 12400 & 112 \\
\hline $\mathrm{B} 45 \mathrm{~V}$ & Sr & & & & \\
\hline $\mathrm{B} 51$ & Sr & 6880 & 189 & 6880 & 189 \\
\hline $\mathrm{B} 52$ & $\mathrm{Sr}$ & & & & \\
\hline B52R & $\mathrm{Sr}$ & 6380 & 73.2 & 6380 & 732 \\
\hline
\end{tabular}

Note: The ectual concentration of eamples for which no stinderd cleviation be reporticd be lees than the lower limit of detection. The reported concentration is the lower limit of quantifeation. 


\begin{tabular}{|c|c|c|c|c|c|}
\hline Sample ld. & botope & $\begin{array}{l}\text { Concentration } \\
\text { (ug/Kg soll) }\end{array}$ & $\begin{array}{l}\text { Std.Deviation } \\
\text { (40/Kg soll) }\end{array}$ & $\begin{array}{l}\text { Element Avg. Conc. } \\
\text { (ug/Kg soll) }\end{array}$ & $\begin{array}{c}\text { Element Avg. Std. Dov. } \\
\text { (ug/Kg soll) }\end{array}$ \\
\hline$B 11$ & Th232 & 268000 & 12600 & 268000 & 12600 \\
\hline 812 & Th232 & 55.9 & & 55.9 & \\
\hline$B 13$ & Th232 & 79.8 & & 79.8 & \\
\hline$B 14$ & Th232 & 872 & & 872 & \\
\hline$B 21$ & Th232 & 568 & 122 & 568 & 12.2 \\
\hline$B 22$ & Th232 & 61.7 & & 61.7 & \\
\hline $\mathrm{B} 23$ & Th232 & 72.7 & & 72.7 & \\
\hline B23R & Th232 & 205 & 37 & 205 & 37 \\
\hline 824 & Th232 & 70.7 & & 70.7 & \\
\hline 831 & Th232 & 232000 & 30200 & 232000 & 30200 \\
\hline 832 & Th232 & 632 & & 632 & \\
\hline $\mathrm{B} 33$ & Th232 & 63.3 & 7.26 & 63.3 & 726 \\
\hline B41 & Th232 & 651 & 29.5 & 651 & 29.5 \\
\hline$B 42$ & Th232 & 99.6 & 6.89 & 89.6 & 6.89 \\
\hline$B 43$ & Th232 & 61.7 & & 61.7 & \\
\hline B45V & Th232 & & & & \\
\hline 851 & Th232 & 144 & 7.56 & 144 & 7.56 \\
\hline 852 & Th232 & 66.3 & & 66.3 & \\
\hline B52R & Th232 & 150 & 14.3 & 150 & 14.3 \\
\hline
\end{tabular}

Note: The actual concentration of eamples for which no standard deviation ts reported ts leas than the lower llmit of detection. The reported concentration bs the lower imit of quantification. 
Transect B Soil Concentrations: Tin

\begin{tabular}{|c|c|c|c|c|c|}
\hline Semple Id. & Lotop & $\begin{array}{l}\text { Concentration } \\
\text { (ug/Ka coll) }\end{array}$ & $\begin{array}{l}\text { Std.Dovitution } \\
\text { (ug/Ko roil) }\end{array}$ & $\begin{array}{l}\text { Ekement Avg. Cone. } \\
\text { (ug/Kg soil) }\end{array}$ & $\begin{array}{c}\text { Element Avg. Std. Dev. } \\
\text { (ug/Kg soll) }\end{array}$ \\
\hline$B 11$ & Sn118 & 681 & & & \\
\hline $\mathbf{B 1 1}$ & Sn120 & 681 & & 681 & \\
\hline 312 & Sn118 & 550 & & & \\
\hline $\mathrm{B12}$ & Sn120 & 550 & & 550 & \\
\hline 813 & Sn118 & 67300 & 40200 & & \\
\hline 813 & Sn120 & 65700 & 39300 & 66500 & 39800 \\
\hline 814 & Sn118 & 872 & & & \\
\hline 814 & Sn120 & 872 & & 872 & \\
\hline 821 & Sn118 & 745 & 40.6 & & \\
\hline 821 & Sn120 & 782 & 51.5 & 764 & 46.1 \\
\hline Be2 & Sn118 & 870 & 46.8 & & \\
\hline B22 & Sn120 & 950 & 62.9 & 915 & 54.9 \\
\hline B23 & Sn118 & 341 & 93 & & \\
\hline $\mathrm{B23}$ & Sn120 & 465 & 75.9 & 403 & 84.5 \\
\hline B23R & Sn118 & 1460 & 211 & & \\
\hline B23R & Sn120 & 445 & 562 & 953 & 134 \\
\hline$B 24$ & Sn118 & 426 & 105 & & \\
\hline$B 24$ & Sn120 & 337 & 21.7 & 382 & 63.4 \\
\hline $\mathrm{B31}$ & Sn118 & 731 & & & \\
\hline B31 & Sn120 & 731 & & 731 & \\
\hline $\mathrm{B} 32$ & Sn118 & 224 & 18.6 & & \\
\hline B32 & Sn120 & 188 & 11.3 & 206 & 15.5 \\
\hline B33 & Sn118 & 316 & 472 & & \\
\hline 833 & Sn120 & 417 & 36.6 & 367 & 41.9 \\
\hline$B 41$ & Sn118 & 755 & 108 & & \\
\hline B41 & Sn120 & 815 & 23.9 & 785 & 66 \\
\hline B42 & Sn118 & 550 & 45.6 & & \\
\hline$B 42$ & Sn120 & 206 & 53.9 & 378 & 49.8 \\
\hline$B 43$ & Sn118 & 478 & 37.4 & & \\
\hline $\mathrm{B} 43$ & $\operatorname{Sn} 120$ & 724 & 77.7 & 601 & 57.6 \\
\hline B45V & Sn118 & & & & \\
\hline B45V & $\operatorname{Sn} 120$ & & & & \\
\hline $\mathbf{B 5 1}$ & $\operatorname{Sn} 118$ & 1070 & 195 & & \\
\hline $\mathrm{B} 51$ & $\operatorname{Sn} 120$ & 1250 & 116 & 1160 & 156 \\
\hline $\mathrm{B} 52$ & Sn118 & & & & \\
\hline $\mathrm{B} 52$ & $\operatorname{Sn} 120$ & & & & \\
\hline B52R & Sn118 & 131 & 71.3 & & \\
\hline B52R & Sn120 & 83.7 & 78.3 & 112 & 74.8 \\
\hline
\end{tabular}

Note: The actual concentratton of eamples for which no stendard devlation be reported ts leses than the lowne limit of detection. The reported concentration b the lower limit of quantification. 


\section{Transect B Soil Concentrations: Uranium-235}

\begin{tabular}{|c|c|c|c|c|c|}
\hline Sample ld. & teotope & $\begin{array}{l}\text { Concentration } \\
\text { (ug/Kg soll) }\end{array}$ & $\begin{array}{l}\text { Std.Doviation } \\
\text { (ug/Kg soil) }\end{array}$ & $\begin{array}{l}\text { Element Avg. Conc. } \\
\text { (ug/Kg solI) }\end{array}$ & $\begin{array}{c}\text { Element Avg. Std. Dev. } \\
\text { (ug/Kg soll) }\end{array}$ \\
\hline$B 11$ & U235 & 7.07 & 1.41 & 7.07 & 1.41 \\
\hline $\mathrm{B} 12$ & U235 & 18.9 & 3.75 & 18.9 & 3.75 \\
\hline$B 13$ & U235 & 16.4 & 4.01 & 16.4 & 4.01 \\
\hline B14 & U235 & 17.4 & 28 & 17.4 & 28 \\
\hline B21 & U235 & 19.3 & 2.63 & 19.3 & 2.63 \\
\hline 822 & U235 & 17.5 & .49 & 17.5 & .49 \\
\hline $\mathrm{B} 23$ & U235 & 17 & 2.97 & 17 & 2.97 \\
\hline B23R & U235 & 11.5 & 3.55 & 11.5 & 3.55 \\
\hline$B 24$ & U235 & 29.4 & 5.53 & 29.4 & 5.53 \\
\hline $\mathrm{B} 31$ & U235 & 13.5 & 8.77 & 13.5 & 8.77 \\
\hline $\mathrm{B} 32$ & U235 & 32 & .84 & 32 & .84 \\
\hline$B 33$ & U235 & 16.9 & .93 & 16.9 & .93 \\
\hline$B 41$ & U235 & 12.7 & 4.77 & 12.7 & 4.77 \\
\hline$B 42$ & U235 & 115 & 7.84 & 115 & 7.84 \\
\hline$B 43$ & U235 & 17.3 & 227 & 17.3 & 227 \\
\hline $\mathrm{B} 45 \mathrm{~V}$ & $\mathbf{v 2 3 5}$ & & & & \\
\hline $\mathrm{B} 51$ & U235 & 7.17 & 2.89 & 7.17 & 2.99 \\
\hline 852 & U235 & 5.17 & 27 & 5.17 & 27 \\
\hline B52R & U235 & 7.55 & 4.97 & 7.55 & 4.97 \\
\hline
\end{tabular}

Note: The actual concentration of eamples for which no standerd deviation b reported be lees than the lower limit of dotection. The reported concentration is the lower limit of quantification. 


\section{Transect B Soil Concentrations: Uranium-238}

\begin{tabular}{|c|c|c|c|c|c|}
\hline Sample ld. & leotope & $\begin{array}{l}\text { Concentratton } \\
(\text { (ug/Kg soll) }\end{array}$ & $\begin{array}{l}\text { Sid.Dovintion } \\
\text { (40/Kg soil) }\end{array}$ & $\begin{array}{l}\text { Element Avg. Conc. } \\
\text { (ug/Kg soll) }\end{array}$ & $\begin{array}{c}\text { Element Avg. Std. Dov. } \\
\text { (Ug/Kg soll) }\end{array}$ \\
\hline B11 & U238 & 1950 & 13.3 & 1850 & 13.3 \\
\hline B12 & U238 & 2640 & 62.2 & 2640 & 622 \\
\hline 813 & U238 & 3710 & 39.4 & 3710 & 39.4 \\
\hline 814 & U238 & 2170 & 59.8 & 2170 & 59.8 \\
\hline$B 21$ & U238 & 2260 & 42.7 & 2260 & 42.7 \\
\hline$B 22$ & U238 & 2810 & 31.1 & 2810 & 31.1 \\
\hline 823 & U238 & & & & \\
\hline$B 23 R$ & U238 & 3010 & 45.4 & 3010 & 45.4 \\
\hline $\mathrm{B24}$ & U238 & 2790 & 112 & 2790 & 112 \\
\hline $\mathrm{B} 31$ & U238 & 2660 & 78.9 & 2660 & 78.9 \\
\hline $\mathrm{B} 32$ & U238 & 5290 & 131 & 5290 & 131 \\
\hline 833 & U238 & 2720 & 44 & 2720 & 44 \\
\hline B41 & U238 & 2080 & 422 & 2000 & 42.2 \\
\hline B42 & U238 & 19400 & 141 & 19400 & 141 \\
\hline 843 & U238 & 2580 & 22.3 & 2580 & 22.3 \\
\hline $\mathrm{B} 45 \mathrm{~V}$ & U238 & & & & \\
\hline$B 51$ & U238 & 3110 & 86.4 & 3110 & 86.4 \\
\hline$B 52$ & U238 & 1370 & 69.4 & 1370 & 69.4 \\
\hline B52R & U238 & 1360 & 20.6 & 1360 & 20.6 \\
\hline
\end{tabular}

Note: The ectual concentration of samples for which no standard doviation be reported bs leas than the lower limit of detection. The reported concentratton is the lower limit of quantification. 


\section{Transect B Soil Concentrations: Vanadium}

\begin{tabular}{|c|c|c|c|c|c|}
\hline Sample Id. & Eotope & $\begin{array}{l}\text { Concentration } \\
\text { (ug/kg eoll) }\end{array}$ & $\begin{array}{l}\text { Stdobvintion } \\
\text { (ug/Ko eoll) }\end{array}$ & $\begin{array}{l}\text { Element Avg. Conc. } \\
\text { (ug/Kg soll) }\end{array}$ & $\begin{array}{c}\text { Element Avg. Std. Dev. } \\
\text { (ug/Kg sold) }\end{array}$ \\
\hline B11 & V & 24500 & 336 & 24500 & 356 \\
\hline $\mathbf{B 1 2}$ & V & 28800 & 216 & 28800 & 216 \\
\hline B13 & $\mathbf{v}$ & 12000 & 128 & 12900 & 128 \\
\hline 814 & $\mathrm{~V}$ & 3890 & 909 & 3890 & 909 \\
\hline B21 & $V$ & 29900 & 503 & 29900 & 503 \\
\hline 822 & $\mathbf{V}$ & 617 & & 617 & \\
\hline$B 23$ & $\mathbf{V}$ & 10100 & 271 & 10100 & 271 \\
\hline B23R & $V$ & 11500 & 435 & 11500 & 436 \\
\hline 824 & $\mathbf{V}$ & 12300 & 536 & 12300 & 536 \\
\hline 831 & $\mathbf{V}$ & 39800 & 1120 & 39800 & 1120 \\
\hline B32 & $V$ & 16600 & 775 & 16600 & 775 \\
\hline 833 & $\mathbf{V}$ & 6850 & 476 & 6850 & 476 \\
\hline B41 & $\mathbf{V}$ & 1060 & 4250 & 1060 & 4250 \\
\hline 842 & $\mathbf{V}$ & 68800 & 726 & 68800 & 726 \\
\hline$B 43$ & $\mathbf{V}$ & 18300 & 576 & 18300 & 576 \\
\hline B45V & $V$ & & & & \\
\hline B51 & $\mathbf{V}$ & 6730 & 70.8 & 6730 & 70.8 \\
\hline 852 & $V$ & 477 & & 477 & \\
\hline B52R & $V$ & 4780 & 586 & 4780 & 586 \\
\hline
\end{tabular}




\begin{tabular}{|c|c|c|c|c|c|}
\hline Samplo Id. & leotopo & $\begin{array}{l}\text { Concentration } \\
\text { (ug/Kg soll) }\end{array}$ & $\begin{array}{l}\text { Std.Doviation } \\
\text { (ur/Kg soll) }\end{array}$ & $\begin{array}{l}\text { Element Avg. Conc. } \\
\text { (ug/Kg soli) }\end{array}$ & $\begin{array}{c}\text { Element Avg. Std. Dov. } \\
\text { (ug/Kg soll) }\end{array}$ \\
\hline$B 11$ & $Y$ & 146 & 20.4 & 146 & 20.4 \\
\hline$B 12$ & $Y$ & 82.6 & 9.68 & 82.6 & 9.68 \\
\hline B13 & $Y$ & 184 & 14.7 & 194 & 14.7 \\
\hline B14 & $Y$ & 93.8 & 472 & 93.8 & 472 \\
\hline$B 21$ & $Y$ & 733 & 42.3 & 733 & 42.3 \\
\hline$B 22$ & $Y$ & 105 & 36.6 & 105 & 36.6 \\
\hline $\mathrm{B} 23$ & $Y$ & 152 & 28.3 & 152 & 28.3 \\
\hline B23R & $Y$ & 292 & 11 & 292 & 11 \\
\hline$B 24$ & $\bar{Y}$ & 101 & 222 & 101 & 222 \\
\hline B31 & $Y$ & 538 & 135 & 538 & 135 \\
\hline$B 32$ & $Y$ & 103 & 18.9 & 100 & 19.9 \\
\hline 833 & $\bar{Y}$ & 287 & 20.9 & 287 & 20.9 \\
\hline 841 & $Y$ & $37 \infty$ & 372 & 3700 & 372 \\
\hline$B 42$ & $Y$ & 3410 & 41.4 & 3410 & 41.4 \\
\hline$B 43$ & $Y$ & 2940 & 5.8 & 2940 & 5.9 \\
\hline $\mathrm{B} 45 \mathrm{~V}$ & $Y$ & & & & \\
\hline $\mathrm{B} 51$ & $Y$ & 192 & 8.21 & 182 & 9.21 \\
\hline $\mathrm{B} 52$ & $Y$ & 1510 & 264 & 1510 & 264 \\
\hline B52R & $Y$ & 4160 & 128 & 4160 & 128 \\
\hline
\end{tabular}




\section{Transect B Soil Concentrations: Zinc}

\begin{tabular}{|c|c|c|c|c|c|}
\hline Sample ld. & leotope & $\begin{array}{l}\text { Concentration } \\
\text { (4rg/Kg soll) }\end{array}$ & $\begin{array}{l}\text { Std.Doviation } \\
\text { (ug/Kg soll) }\end{array}$ & $\begin{array}{c}\text { Element Avg. Conc. } \\
\text { (ug/Ko eoll) }\end{array}$ & $\begin{array}{c}\text { Element Avg. Std. Dov. } \\
\text { (Ug/Kg soll) }\end{array}$ \\
\hline B11 & Zn66 & 10900 & 2230 & 10900 & 2230 \\
\hline $\mathrm{B} 12$ & Zn66 & 17900 & 864 & 17900 & 864 \\
\hline$B 13$ & Zn66 & 13800 & 413 & 13800 & 413 \\
\hline B14 & Zn66 & 11700 & 506 & 11700 & 506 \\
\hline 821 & Zn66 & 2900 & 1030 & 2900 & 1030 \\
\hline $\mathrm{B} 22$ & Zn66 & & & & \\
\hline $\mathrm{B} 23$ & Zn66 & 8010 & 506 & 8010 & 536 \\
\hline B23R & Zn66 & 59100 & & 59100 & \\
\hline 824 & Zn66 & 31700 & & 31700 & \\
\hline 831 & Zn66 & 22100 & 1130 & 22100 & 1130 \\
\hline 832 & Zn66 & 11400 & 630 & 11400 & 639 \\
\hline 833 & Zn66 & 10300 & 170 & 10500 & 170 \\
\hline$B 41$ & Zn66 & 10400 & 741 & 10400 & 741 \\
\hline$B 42$ & Zn66 & 21900 & 615 & 21900 & 615 \\
\hline$B 43$ & Zn66 & 4100 & 431 & 4100 & 431 \\
\hline B45V & $2 \mathrm{n} 66$ & & & & \\
\hline 851 & Zn66 & 4860 & 600 & 4860 & 603 \\
\hline 852 & Zn66 & & & & \\
\hline B52R & Zn66 & 13700 & & 13700 & \\
\hline
\end{tabular}

Note: The actual concentration of eamples for which no standard deviation is reported is laes than the lowrer limit of dotection. The reported concentration is the lower limit of quantiffeation. 


\section{Transect B Soil Concentrations: Zirconium}

\begin{tabular}{|c|c|c|c|c|c|}
\hline Sumplo id. & botope & $\begin{array}{l}\text { Concentration } \\
\text { (ug/kg soll) }\end{array}$ & $\begin{array}{l}\text { StdDovintion } \\
\text { (ug/Ko eoln) }\end{array}$ & $\begin{array}{l}\text { Elament Avo. Cone. } \\
\text { (ug/ko soll) }\end{array}$ & $\begin{array}{c}\text { Element Avg. Std. Dev. } \\
\text { (ug/Kg soll) }\end{array}$ \\
\hline $\mathbf{B 1 1}$ & 280 & 22800 & 518 & & \\
\hline B11 & Z191 & 20800 & 1280 & 21800 & 899 \\
\hline 812 & 2890 & 18000 & 357 & & \\
\hline B12 & 201 & 19900 & 641 & 18000 & 504 \\
\hline 813 & 280 & 18600 & 388 & & \\
\hline $\mathrm{B13}$ & $2 \sqrt{91}$ & 18400 & 401 & 18500 & 395 \\
\hline 814 & 2890 & 17500 & 653 & & \\
\hline B14 & 2891 & 21700 & 2270 & 19000 & 1450 \\
\hline$B 21$ & 280 & 30500 & 421 & & \\
\hline$B 21$ & 291 & 32300 & 383 & 31400 & 407 \\
\hline B22 & $2 r 90$ & 26600 & 876 & & \\
\hline B22 & 2991 & 24800 & 1870 & 25700 & 1370 \\
\hline 823 & 2890 & 20500 & 669 & & \\
\hline$B 23$ & 2891 & 19900 & 639 & 20200 & 654 \\
\hline B23R & 2890 & 34300 & 638 & & \\
\hline B23A & 291 & 58300 & 397 & 46300 & 518 \\
\hline B24 & 280 & 24600 & 479 & & \\
\hline B24 & 2191 & 21000 & 1430 & 22800 & 955 \\
\hline B31 & Zroo & 54700 & 1080 & & \\
\hline $\mathrm{B31}$ & 2991 & 54700 & 4030 & 54700 & 2560 \\
\hline $\mathrm{B} 32$ & 789 & 17500 & 610 & & \\
\hline $\mathrm{B} 32$ & 281 & 17600 & 751 & 17600 & 685 \\
\hline $\mathrm{B} 33$ & 290 & 28000 & 267 & & \\
\hline $\mathrm{B} 33$ & Zr91 & 31600 & 700 & 29800 & 488 \\
\hline B41 & 780 & 23300 & 1240 & & \\
\hline$B 41$ & 2191 & 25300 & 2160 & 24300 & 1700 \\
\hline $\mathrm{B} 42$ & 2890 & 22700 & 168 & & \\
\hline $\mathrm{B} 42$ & $2 r 91$ & 30700 & 1160 & 26700 & 664 \\
\hline B43 & $2 r 90$ & 23200 & 377 & & \\
\hline$B 43$ & Zr91 & 23200 & 529 & 23200 & 453 \\
\hline B45V & Zr9o & & & & \\
\hline B45V & 2591 & & & & \\
\hline $\mathrm{B} 51$ & 290 & 9290 & 186 & & \\
\hline 851 & Zr91 & 7670 & 506 & 8480 & 346 \\
\hline $\mathrm{B} 52$ & $7 r s 0$ & 28800 & 893 & & \\
\hline$B 52$ & $Z 991$ & 24800 & 2610 & 26800 & 1750 \\
\hline B52R & 780 & 12200 & 253 & & \\
\hline B52R & Zr91 & 14200 & 562 & 13200 & 408 \\
\hline
\end{tabular}

Note: The ectual concentration of asmples for which no standerd dovlation to reported te lees than the lower limit of detection. The reported concentration ls the lower Umit of quantification. 
Transect C Soil Concentrations: Aluminum

\begin{tabular}{|c|c|c|c|c|c|}
\hline Semple ld. & leotope & $\begin{array}{l}\text { Concentration } \\
\text { (ug/Kg } 8 \text { oll) }\end{array}$ & $\begin{array}{l}\text { Std.Deviation } \\
\text { (ug/Kg soil) }\end{array}$ & $\begin{array}{c}\text { Element Avg. Conc. } \\
\text { (ug/Kg e्oll) }\end{array}$ & $\begin{array}{c}\text { Element Avg. Std. Dov. } \\
\text { (ug/Kg soll) }\end{array}$ \\
\hline C11 & A & 23300000 & 113000 & 23300000 & 113000 \\
\hline $\mathrm{C} 12$ & $\mathbf{A}$ & 13000000 & 345000 & 13000000 & 345000 \\
\hline$C_{13}$ & $\mathbf{A}$ & 2650000 & 87400 & 2650000 & 87400 \\
\hline $\mathrm{C}_{14}$ & $\mathbf{N}$ & 7140000 & 88700 & 7140000 & 88700 \\
\hline C21 & $\mathbf{A}$ & 24500000 & 631000 & 24500000 & 631000 \\
\hline C21R & $A$ & 18400000 & 289000 & 18400000 & 289000 \\
\hline $\mathrm{C} 22$ & A & 4600000 & 48800 & 4600000 & 48800 \\
\hline $\mathrm{C23}$ & $\mathbf{A}$ & 4660000 & 74700 & 4660000 & 74700 \\
\hline C.31 & $\overline{\mathbf{N}}$ & 7420000 & 195000 & 7420000 & 195000 \\
\hline $\cos 2$ & $\mathbf{A}$ & 2740000 & 54300 & 2740000 & 54300 \\
\hline $\mathrm{CO33}$ & $A$ & 3770000 & 98100 & 3770000 & 98100 \\
\hline CA1 & $N$ & 14600000 & 638000 & 14600000 & 638000 \\
\hline $\mathrm{CA2}$ & A & 8640000 & 164000 & 8640000 & 164000 \\
\hline $\mathrm{CA3}_{4}$ & A & 4880000 & 64200 & 4880000 & 64200 \\
\hline
\end{tabular}




\section{Transect C Soil Concentrations: Arsenic}

\begin{tabular}{|c|c|c|c|c|c|}
\hline Sampte ld. & Isotope & $\begin{array}{l}\text { Concentratton } \\
\text { (ug/Kg soll) }\end{array}$ & $\begin{array}{l}\text { Std.Daviation } \\
\text { (ug/Kg soll) }\end{array}$ & $\begin{array}{c}\text { Element Avg. Conc. } \\
\text { (ug/Kg soll) }\end{array}$ & $\begin{array}{c}\text { Element Avg. Std. Dov. } \\
\text { (ug/Kg soll) }\end{array}$ \\
\hline C11 & As & 215 & & 215 & \\
\hline $\mathrm{C12}$ & As & 1670 & 464 & 1670 & 464 \\
\hline $\mathrm{C13}$ & As & 635 & & 635 & \\
\hline C14 & As & 1440 & & 1440 & \\
\hline C21 & As & 9300 & 2110 & 9300 & 2110 \\
\hline C21R & As & 12300 & 891 & 12300 & 891 \\
\hline $\cos$ & As & 682 & & 682 & \\
\hline$c 23$ & As & 2930 & 1170 & 2930 & 1170 \\
\hline$\infty 31$ & As & 4660 & 1820 & 4660 & 1820 \\
\hline $\mathrm{CO} 32$ & As & 635 & & 635 & \\
\hline $\cos 3$ & As & 5360 & 1860 & 5360 & 1860 \\
\hline $\mathrm{CA1}$ & As & 4650 & 1130 & 4650 & 1130 \\
\hline $\mathrm{C} 42$ & As & 4490 & 945 & 4490 & 945 \\
\hline $\mathrm{C} 43$ & As & 267 & & 257 & \\
\hline
\end{tabular}

Note: The actual concentration of samples for which no standard deviation le reported be hes than the lower limit of detection. The reported concentration is the lower limit of quantification. 
Transect C Soil Concentrations: Barium

\begin{tabular}{|c|c|c|c|c|c|}
\hline Semple id. & Isotope & $\begin{array}{l}\text { Concentration } \\
\text { (ug/Kg eoli) }\end{array}$ & $\begin{array}{l}\text { Std.Doviation } \\
\text { (ug/Kg aoll) }\end{array}$ & $\begin{array}{c}\text { Element Avg. Conc. } \\
\text { (ug/Kg soll) }\end{array}$ & $\begin{array}{c}\text { Element Avg. Std. Dov. } \\
\text { (ug/Kg soll) }\end{array}$ \\
\hline C11 & Ba135 & 11500 & 237 & & \\
\hline C11 & Ba137 & 9720 & 129 & & \\
\hline C11 & $\mathrm{Ba138}$ & 10800 & 266 & 10700 & 211 \\
\hline $\mathrm{C} 12$ & $8 a 135$ & 27100 & 1110 & & \\
\hline $\mathrm{C12}$ & 80137 & 24200 & 500 & & \\
\hline $\mathrm{C} 12$ & Ba138 & 28900 & 577 & 26700 & 729 \\
\hline $\mathrm{C} 13$ & $\mathrm{Ba135}$ & 175000 & 3430 & & \\
\hline $\mathrm{C13}$ & Ba137 & 168000 & 2320 & & \\
\hline C13 & $\mathrm{Ba138}$ & 166000 & 1670 & 170000 & 2470 \\
\hline $\mathrm{C14}$ & Ba135 & 74000 & 2800 & & \\
\hline $\mathrm{C14}$ & Ba137 & 69100 & 669 & & \\
\hline C14 & Ba138 & 79400 & 1140 & 74200 & 1540 \\
\hline 021 & $B a 135$ & 13500 & 2410 & & \\
\hline 021 & Ba137 & 19100 & 1150 & & \\
\hline C21 & 89138 & 18400 & 918 & 17000 & 1490 \\
\hline C21R & $\mathrm{Ba} 135$ & 18600 & 1550 & & \\
\hline C21R & Ba137 & 16600 & 1760 & & \\
\hline C21R & $\mathrm{Ba138}$ & 17400 & 995 & 17500 & 1440 \\
\hline C22 & $\mathrm{Ba135}$ & 219000 & 953 & & \\
\hline$c 22$ & $\mathrm{Ba137}$ & 214000 & 2250 & & \\
\hline$c 2$ & $\mathrm{Ba138}$ & 189000 & 1040 & 207000 & 1410 \\
\hline $\mathrm{C23}$ & Ba135 & 56300 & 198 & & \\
\hline$C_{23}$ & $\mathrm{Ba} 137$ & 57200 & 1400 & & \\
\hline $\mathrm{C}_{23}$ & Ba138 & 56900 & 25.1 & 56800 & 541 \\
\hline C31 & Ba135 & 260000 & 4340 & & \\
\hline C31 & Ba137 & 311000 & 3540 & & \\
\hline C31 & Ba138 & 305000 & 1920 & 293000 & 3270 \\
\hline 032 & 89135 & 207000 & 2860 & & \\
\hline C32 & Ba137 & 200000 & 3360 & & \\
\hline 0,32 & Ba138 & 186000 & 935 & 198000 & 2390 \\
\hline C.33 & Ba135 & 246000 & 3820 & & \\
\hline C.33 & $\mathrm{Ba} 137$ & 243000 & 3650 & & \\
\hline $\cos 3$ & Ba138 & 231000 & 2150 & 240000 & 3210 \\
\hline $\mathrm{CA1}$ & Ba135 & 20900 & 784 & & \\
\hline$C_{41}$ & $\mathrm{Ba137}$ & 34500 & 1600 & & \\
\hline C41 & $\mathrm{Ba138}$ & 18700 & 514 & 24700 & 966 \\
\hline $\mathrm{CA2}$ & $\mathrm{Ba135}$ & 132000 & 3030 & & \\
\hline$C_{42}$ & Ba137 & 147000 & 2100 & & \\
\hline$C 42$ & Ba138 & 136000 & 603 & 138000 & 1910 \\
\hline $\mathrm{CA3}$ & Ba135 & 148000 & 3050 & & \\
\hline$C_{43}$ & $\mathrm{Ba137}$ & 144000 & 1650 & & \\
\hline $\mathrm{CA3}$ & Ba138 & 136000 & 385 & 143000 & 1700 \\
\hline
\end{tabular}

Note: The ectual concentration of eamples for which no standard deviation is reported bs loas than the fower limit of detection. The reported concentration is the fower limit of quantification. 


\section{Transect C Soil Concentrations: Cadmium}

\begin{tabular}{|c|c|c|c|c|c|}
\hline Semple id. & Lotope & $\begin{array}{l}\text { Concentration } \\
\text { (ug/Kg soll) }\end{array}$ & $\begin{array}{l}\text { Strd.Doviation } \\
\text { (ug/Kg soll) }\end{array}$ & $\begin{array}{l}\text { Element Avg. Conc. } \\
\text { (ug/Kg soll) }\end{array}$ & $\begin{array}{c}\text { Element Avg. Std. Dov. } \\
\text { (ug/Kg soll) }\end{array}$ \\
\hline C11 & Cd111 & 80.7 & 422 & & \\
\hline$C_{11}$ & $\operatorname{cod114}$ & 107 & & 93.9 & 42.2 \\
\hline $\mathrm{C}_{12}$ & Cd111 & 116 & 98.1 & & \\
\hline $\mathrm{C}_{12}$ & Cd114 & 148 & 76.3 & 132 & 872 \\
\hline C13 & Cod111 & 122 & 47.8 & & \\
\hline $\mathrm{C} 13$ & Cd114 & 135 & 137 & 129 & 92.4 \\
\hline $\mathrm{C} 14$ & Cd111 & 94.4 & 332 & & \\
\hline C14 & $\operatorname{Cd114}$ & 169 & 35.3 & 132 & 34.3 \\
\hline C21 & $\operatorname{cod} 111$ & 81.6 & & & \\
\hline C21 & Cod114 & 81.6 & & 81.6 & \\
\hline C2IR & $\cot 111$ & 164 & 7.03 & & \\
\hline C21R & Cd114 & 221 & 105 & 193 & 56 \\
\hline $\mathrm{CO2}$ & Cod111 & 185 & 66.4 & & \\
\hline $\cos 2$ & Cod114 & 722 & 24.6 & 129 & 45.5 \\
\hline $\mathrm{C23}$ & Cod111 & 144 & & & \\
\hline$c 23$ & $\operatorname{Cd114}$ & 144 & & 144 & \\
\hline C31 & Cd111 & 124 & & & \\
\hline C31 & Cd114 & 124 & & 124 & \\
\hline C32 & Cd111 & 63.5 & & & \\
\hline $\cos 2$ & Cd114 & 129 & 21.8 & 96.3 & 21.8 \\
\hline C33 & Cd111 & 76.5 & & & \\
\hline C33 & Cd114 & 76.5 & & 76.5 & \\
\hline $\mathrm{C}_{41}$ & Cd111 & 63.5 & & & \\
\hline CA1 & Cd114 & 63.5 & & 63.5 & \\
\hline $\mathrm{CA2}$ & Cd111 & 258 & 64.5 & & \\
\hline $\mathrm{C}_{42}$ & Cd114 & 102 & 21.3 & 180 & 42.9 \\
\hline $\mathrm{C} 43$ & Cod111 & 134 & & & \\
\hline $\mathrm{CA3}_{43}$ & Cd114 & 134 & & 134 & \\
\hline
\end{tabular}




\begin{tabular}{|c|c|c|c|c|c|}
\hline Sample ld. & lsotope & $\begin{array}{l}\text { Concentration } \\
\text { (ug/Kg soil) }\end{array}$ & $\begin{array}{l}\text { Std.Deviation } \\
\text { (ug/Kg soil) }\end{array}$ & $\begin{array}{l}\text { Element Avg. Conc. } \\
\text { (ug/Ko soll) }\end{array}$ & $\begin{array}{c}\text { Element Avg. Std. Dov. } \\
\text { (ug/Kg soll) }\end{array}$ \\
\hline $\mathrm{C} 11$ & Ca44 & 87300 & 15500 & 87300 & 15500 \\
\hline $\mathrm{C}_{12}$ & Co44 & 386000 & 40500 & 386000 & 40600 \\
\hline $\mathrm{C} 13$ & Ca44 & 12700 & & 12700 & \\
\hline C14 & Ces4 & 366000 & 195000 & 366000 & 195000 \\
\hline C21 & Co44 & 4010000 & 295000 & 4010000 & 295000 \\
\hline C21R & Ca44 & 4970000 & 238000 & 4970000 & 238000 \\
\hline C22 & Ced4 & 271000 & 101000 & 271000 & 101000 \\
\hline$C_{23}$ & $\operatorname{Co} 44$ & 144000 & & 144000 & \\
\hline C1 & Ca44 & 668000 & 47300 & 668000 & 47300 \\
\hline C32 & $\cos 4$ & 432000 & 48900 & 432000 & 48900 \\
\hline $\mathrm{C33}$ & Ca44 & 681000 & 223000 & 681000 & 223000 \\
\hline$C 41$ & Co44 & 872000 & 155000 & 872000 & 155000 \\
\hline $\mathrm{C42}$ & $\operatorname{Ca} 44$ & 546000 & 67300 & 546000 & 67300 \\
\hline $\mathrm{CA3}_{43}$ & $\operatorname{Ca} 44$ & & & & \\
\hline
\end{tabular}

Note: The actual concentration of eamples for which no standard deviation be reported la leas than the lower limit of detection. The reported concentration is the fower llmit of quantification. 


\begin{tabular}{|c|c|c|c|c|c|}
\hline Semple id. & lsotope & $\begin{array}{l}\text { Concentration } \\
\text { (ug/Kg soll) }\end{array}$ & $\begin{array}{l}\text { Std.Doviation } \\
\text { (ug/Kg soil) }\end{array}$ & $\begin{array}{l}\text { Element Avg. Conc. } \\
\text { (ug/Kg soll) }\end{array}$ & $\begin{array}{c}\text { Element Avg. Std. Dov. } \\
\text { (Ug/Kg soll) }\end{array}$ \\
\hline$C_{11}$ & $\mathrm{C}_{8}$ & 53.7 & & 53.7 & \\
\hline $\mathrm{C12}_{12}$ & $\mathrm{Cs}_{8}$ & 259 & 23.8 & 259 & 23.8 \\
\hline $\mathrm{C}_{13}$ & $\mathrm{C}_{8}$ & 155 & 31.9 & 155 & 31.9 \\
\hline $\mathrm{C} 14$ & $\mathrm{Cs}$ & 71.8 & & 71.8 & \\
\hline C21 & Cs & 299 & 50.1 & 299 & 50.1 \\
\hline C21A & $\mathrm{Cs}_{3}$ & 322 & 33.1 & 322 & 33.1 \\
\hline C22 & Cs & 187 & 15 & 187 & 15 \\
\hline $\mathrm{COS}_{3}$ & Cs & 290 & 26.4 & 290 & 26.4 \\
\hline c31 & $\mathrm{C}_{8}$ & 345 & 42.6 & 345 & 42.6 \\
\hline C32 & $\mathrm{Cs}_{3}$ & 773000 & 14.6 & 773000 & 14.6 \\
\hline C33 & Cs & 342 & 18 & 342 & 18 \\
\hline $\mathrm{CA1}$ & Cs & 175 & 13.8 & 175 & 13.8 \\
\hline $\mathrm{C42}$ & Cs & 490 & 64.7 & 490 & 64.7 \\
\hline $\mathrm{C}_{43}$ & Cs & 149 & 29 & 149 & 29 \\
\hline
\end{tabular}

Note: The actual concentration of eamples for which no atandard deviation te reported is leas than the lower limit of detection. The reported concentration ts the fower limit of quantification. 


\section{Transect C Soil Concentrations: Chromium}

$\begin{array}{ccccc}\text { Sample ld. leotope } & \begin{array}{c}\text { Concentration } \\ \text { (ug/Kg soll) }\end{array} & \begin{array}{c}\text { Std.Doviation } \\ \text { (ug/Kg soll) }\end{array} & \begin{array}{c}\text { Eloment Avg. Conc. } \\ \text { (ug/Kg soll) }\end{array} & \begin{array}{c}\text { Element Avg. Std. Dov. } \\ \text { (ug/Kg soll) }\end{array}\end{array}$

\begin{tabular}{|c|c|c|c|c|c|}
\hline$C_{11}$ & C.52 & 5690 & 250 & & \\
\hline C11 & Cr53 & 5680 & 249 & 5690 & 250 \\
\hline $\mathrm{C}_{12}$ & C.52 & 4630 & 364 & & \\
\hline $\mathrm{C}_{12}$ & $a 53$ & 4600 & 357 & 4620 & 361 \\
\hline $\mathrm{C}_{13}$ & C.52 & 1040 & 219 & & \\
\hline$C_{13}$ & C.53 & 1030 & 216 & 1040 & 218 \\
\hline $\mathrm{C14}$ & C.52 & 5540 & 230 & & \\
\hline $\mathrm{C}_{14}$ & C.53 & & & 5540 & 230 \\
\hline$C_{21}$ & cr52 & 16000 & 305 & & \\
\hline C21 & 953 & 16100 & 306 & 16100 & 306 \\
\hline C21R & C.52 & 16200 & 247 & & \\
\hline C21R & C.53 & 16400 & 252 & 16300 & 250 \\
\hline c22 & C.52 & 2080 & 379 & & \\
\hline c22 & C.53 & 2080 & 380 & 2080 & 380 \\
\hline$C_{23}$ & C.52 & 4010 & 246 & & \\
\hline$C_{23}$ & G.53 & 4010 & 248 & 4010 & 247 \\
\hline C31 & Cr52 & 7670 & 484 & & \\
\hline C31 & $a 53$ & 7750 & 463 & 7710 & 474 \\
\hline $\cos 2$ & C.52 & 63.5 & & & \\
\hline $\cos 2$ & Cr53 & 63.5 & & 63.5 & \\
\hline $\mathrm{C} 33$ & $G 52$ & 2830 & 239 & & \\
\hline C33 & C.53 & 2830 & 240 & 2830 & 240 \\
\hline$C_{41}$ & C52 & 7010 & 128 & & \\
\hline $\mathrm{CA1}$ & 9.53 & 7000 & 123 & 7010 & 126 \\
\hline$C_{42}$ & C.52 & 14400 & 672 & & \\
\hline$C 42$ & C.53 & 14400 & 654 & 14400 & 663 \\
\hline $\mathrm{C43}$ & C552 & 267 & & & \\
\hline $\mathrm{C}_{43}$ & Cr53 & 267 & & 267 & \\
\hline
\end{tabular}




\section{Transect C Soil Concentrations: Cobalt}

\begin{tabular}{|c|c|c|c|c|c|}
\hline Sample ld. & botope & $\begin{array}{l}\text { Concentration } \\
\text { (ug/Kg soli) }\end{array}$ & $\begin{array}{l}\text { Std.Doviation } \\
\text { (ug/Kg soll) }\end{array}$ & $\begin{array}{c}\text { Ekment Avg. Conc. } \\
\text { (ug/Kg soll) }\end{array}$ & $\begin{array}{l}\text { Element Avg. Std. Dov. } \\
\text { (ug/Kg soll) }\end{array}$ \\
\hline C11 & Co & 2510 & 123 & 2510 & 123 \\
\hline $\mathrm{C}_{12}$ & Co & 544 & 892 & 544 & 892 \\
\hline $\mathrm{C} 13$ & $\infty$ & 417 & 52.8 & 417 & 52.8 \\
\hline C14 & Co & 225 & 57.3 & 225 & 57.3 \\
\hline 21 & Co & 1980 & 107 & 1980 & 107 \\
\hline C21R & Co & 1590 & 472 & 1590 & 472 \\
\hline 022 & Co & 1840 & 178 & 1840 & 178 \\
\hline C23 & Co & 435 & 34.9 & 435 & 34.9 \\
\hline C31 & Co & 408 & 93.9 & 408 & 93.9 \\
\hline C32 & Co & 228 & 26.8 & 228 & 26.8 \\
\hline $\mathrm{cos} 3$ & $\infty$ & 239 & 23 & 239 & 23 \\
\hline $\mathrm{CA1}$ & Co & 536 & 36.3 & 536 & 36.3 \\
\hline$C_{42}$ & Co & 2600 & 110 & 2600 & 110 \\
\hline C43 & Co & 90.9 & 27.7 & 90.9 & 27.7 \\
\hline
\end{tabular}




\section{Transect C Soil Concentrations: Copper}

\begin{tabular}{|c|c|c|c|c|c|}
\hline Sample Id. & lsotope & $\begin{array}{l}\text { Concentration } \\
\text { (ugho soll) }\end{array}$ & $\begin{array}{l}\text { Sta.Dovintion } \\
\text { (ug/Kg soll) }\end{array}$ & $\begin{array}{l}\text { Element Avo. Conc. } \\
\text { (ug/Kg eoll) }\end{array}$ & $\begin{array}{c}\text { Element Avg. Std. Dev. } \\
\text { (ug/Kg soll) }\end{array}$ \\
\hline C11 & Cu63 & & & & \\
\hline $\mathrm{C} 11$ & 0465 & 4070 & 442 & 4070 & 442 \\
\hline $\mathrm{C} 12$ & 0.63 & 4640 & 487 & & \\
\hline $\mathrm{C}_{12}$ & Ou65 & 5560 & 587 & 5100 & 537 \\
\hline$C_{13}$ & Cu63 & 2700 & 209 & & \\
\hline C13 & Cu65 & 3040 & 421 & 2870 & 315 \\
\hline $\mathrm{C} 14$ & 0463 & 2620 & 118 & & \\
\hline C14 & au65 & 3930 & 607 & 3280 & 363 \\
\hline C21 & Cu63 & 1570 & 21.3 & & \\
\hline C21 & 0465 & 8010 & 207 & 4790 & 114 \\
\hline C21R & Cu63 & 3520 & 174 & & \\
\hline C21R & Cu65 & 8300 & 133 & 5910 & 154 \\
\hline $\mathrm{C22}$ & 0463 & & & & \\
\hline $\mathrm{C22}$ & Ou65 & 30200 & 1260 & 30200 & 1260 \\
\hline $\mathrm{C23}$ & 0.63 & 1040 & 852 & & \\
\hline $\mathrm{C23}$ & 0.65 & 1510 & 471 & 1280 & 278 \\
\hline c31 & Ou63 & 8140 & 553 & & \\
\hline C31 & Cu65 & 11400 & 872 & 9770 & 713 \\
\hline C.32 & Cu63 & 890 & 17.7 & & \\
\hline C32 & Cu65 & 568 & 137 & 729 & 77.4 \\
\hline C33 & Cu63 & 1960 & 535 & & \\
\hline C33 & Cu65 & 2320 & 409 & 2140 & 472 \\
\hline $\mathrm{CA}_{4}$ & Cu63 & 3860 & 242 & & \\
\hline$C_{41}$ & Cu65 & 7820 & 451 & 5840 & 347 \\
\hline$C_{42}$ & Cu63 & 6350 & 371 & & \\
\hline$C_{42}$ & Cu65 & 7250 & 355 & 6800 & 363 \\
\hline $\mathrm{C}_{43}$ & 0,63 & & & & \\
\hline$C_{43}$ & Cu65 & 3110 & 190 & 3110 & 190 \\
\hline
\end{tabular}




\section{Transect C Soil Concentrations: Iron}

\begin{tabular}{|c|c|c|c|c|c|}
\hline Sample ld. & Isotope & $\begin{array}{l}\text { Concentration } \\
\text { (ug/Kg soil) }\end{array}$ & $\begin{array}{l}\text { Std.Doviation } \\
\text { (ug/Kg soil) }\end{array}$ & $\begin{array}{l}\text { Element Avg. Conc. } \\
\text { (Ug/Kg soll) }\end{array}$ & $\begin{array}{c}\text { Element Avg. Std. Dov. } \\
\text { (ug/Kg soll) }\end{array}$ \\
\hline C11 & $F_{0}$ & 6280000 & 24900 & 6280000 & 24900 \\
\hline C12 & $F_{0}$ & 11200000 & 139000 & 11200000 & 139000 \\
\hline $\mathrm{C13}$ & $\mathrm{Fo}_{0}$ & 3270000 & 66100 & 3270000 & 66100 \\
\hline C14 & Fo & 17400000 & 179000 & 17400000 & 179000 \\
\hline C21 & $\mathrm{Fo}$ & 21600000 & 229000 & 21600000 & 229000 \\
\hline C21R & Fo & 27200000 & 77500 & 27200000 & 77500 \\
\hline C22 & Fo & 4470000 & 48000 & 4470000 & 48000 \\
\hline$c_{23}$ & Fo & 4780000 & 31100 & 4780000 & 31100 \\
\hline C31 & $F_{0}$ & 11400000 & 220000 & 11400000 & 220000 \\
\hline $\cos 2$ & $F_{0}$ & 2670000 & 4120 & 2670000 & 4120 \\
\hline C33 & $F_{0}$ & 2940000 & 46300 & 2940000 & 46300 \\
\hline $\mathrm{C} 41$ & Fo & 12400000 & 244000 & 12400000 & 244000 \\
\hline $\mathrm{C} 42$ & $F_{0}$ & 21700000 & 417000 & 21700000 & 417000 \\
\hline $\mathrm{CA3}_{43}$ & $F_{0}$ & 4750000 & 49200 & 4750000 & 49200 \\
\hline
\end{tabular}




\section{Transect C Soil Concentrations: Lead}

\begin{tabular}{|c|c|c|c|c|c|}
\hline Sampla ld. & leotope & $\begin{array}{l}\text { Concentration } \\
\text { (ug/Kg soll) }\end{array}$ & $\begin{array}{l}\text { Strd.Dovination } \\
\text { (ug/Kg soil) }\end{array}$ & $\begin{array}{l}\text { Element Avg. Conc. } \\
\text { (ug/kg eoli) }\end{array}$ & $\begin{array}{l}\text { Element Avg. Std. Dov. } \\
\text { (ug/Kg eoll) }\end{array}$ \\
\hline C11 & Pb206 & 2000 & 138 & & \\
\hline$C_{11}$ & $P b 207$ & 1440 & 251 & & \\
\hline C11 & P6208 & 1570 & 117 & 1700 & 169 \\
\hline C12 & Pt206 & 19900 & 588 & & \\
\hline C12 & Po207 & 18300 & 432 & & \\
\hline C12 & $P 2208$ & 19300 & 246 & 19200 & 422 \\
\hline C13 & P6206 & 9920 & 708 & & \\
\hline C13 & $P b 207$ & 8720 & 206 & & \\
\hline C13 & $P b 208$ & 9820 & 220 & 9490 & 378 \\
\hline C14 & $P b 206$ & 3690 & 102 & & \\
\hline C14 & Pb207 & 3360 & 31.5 & & \\
\hline C14 & $P \$ 208$ & 3510 & 53.7 & 3520 & 62.4 \\
\hline$C_{21}$ & Pb206 & 19200 & 2250 & & \\
\hline$C_{21}$ & $P 5207$ & 17800 & 2090 & & \\
\hline$C_{21}$ & $P 5208$ & 17700 & 994 & 18200 & 1780 \\
\hline C21R & Pb206 & 24700 & 715 & & \\
\hline C21R & $P b 207$ & 21700 & 1710 & & \\
\hline C21R & Pb208 & 21800 & 1010 & 22700 & 1150 \\
\hline$c 2$ & Po206 & 3660 & 2710 & & \\
\hline $\mathrm{C} 22$ & Po207 & 4250 & 1650 & & \\
\hline$c 2$ & Pb208 & 3630 & 2230 & 3850 & 2200 \\
\hline$C_{23}$ & Po206 & 4070 & 207 & & \\
\hline $\mathrm{C}_{23}$ & Po207 & 3990 & 68.4 & & \\
\hline C23 & $\mathrm{Pb} 208$ & 4000 & 128 & 4020 & 134 \\
\hline C31 & Pt206 & 67000 & $17 \infty$ & & \\
\hline C31 & Po207 & 66600 & 1140 & & \\
\hline C31 & $\mathrm{Pb208}$ & 61500 & 800 & 65000 & 1210 \\
\hline C32 & Pb206 & 10700 & 440 & & \\
\hline C32 & $\mathrm{Pb} 207$ & 10400 & 361 & & \\
\hline C32 & $P 6208$ & 8460 & 43.8 & 9850 & 282 \\
\hline C33 & $\mathrm{Pb} 206$ & 13300 & 278 & & \\
\hline C33 & $\mathrm{Pb} 207$ & 12100 & 346 & & \\
\hline C33 & Pb208 & 12900 & 338 & 12800 & 321 \\
\hline$C_{41}$ & $\mathrm{~Pb} 206$ & 35100 & 2150 & & \\
\hline$C_{41}$ & Pb207 & 31700 & 1250 & & \\
\hline$C 41$ & Pb208 & 31000 & 1340 & 32600 & 1580 \\
\hline $\mathrm{C} 42$ & Pb206 & 37600 & 815 & & \\
\hline C42 & $\mathrm{Pb207}$ & 34000 & 688 & & \\
\hline$C_{42}$ & Pb208 & 36800 & 452 & 36100 & 652 \\
\hline$C_{43}$ & $\mathrm{~Pb} 206$ & 3850 & 125 & & \\
\hline$C_{43}$ & Po207 & 3060 & 52 & & \\
\hline $\mathrm{C} 43$ & Pb208 & 2810 & 462 & 3240 & 74.4 \\
\hline
\end{tabular}

Note: The ectual concentration of eamples for which no standard deviation is reported is leas than the lower limit of detection. The reported concentration is the lower limit of quantification. 


\begin{tabular}{|c|c|c|c|c|c|}
\hline Sample ld. & leotope & $\begin{array}{l}\text { Concentration } \\
\text { (ug/Kg soil) }\end{array}$ & $\begin{array}{l}\text { Std.Doviation } \\
\text { (ug/Kg sodI) }\end{array}$ & $\begin{array}{l}\text { Element Avg. Conc. } \\
\text { (ug/Kg soll) }\end{array}$ & $\begin{array}{c}\text { Element Avg. Std. Dov. } \\
\text { (ug/Kg soli) }\end{array}$ \\
\hline$C 11$ & Mge4 & 45100 & 617 & & \\
\hline C11 & Mges & 40700 & 2040 & 42900 & 1330 \\
\hline $\mathrm{C} 12$ & $\mathrm{Mg} 24$ & 115000 & 1640 & & \\
\hline $\mathrm{C} 12$ & Mges & 125000 & 1640 & 120000 & 1640 \\
\hline $\mathrm{C13}$ & Mge 4 & 38500 & 2290 & & \\
\hline $\mathrm{C} 13$ & Moes & 29600 & 2090 & 34100 & 2190 \\
\hline$C_{14}$ & Mged & 105000 & 3770 & & \\
\hline C14 & Mges & 95600 & 4610 & 100000 & 4190 \\
\hline C21 & Mge4 & 12800000 & 2430 & & \\
\hline C21 & Mges & 202000 & 3280 & 6500000 & 2860 \\
\hline C21R & Mge24 & 203000 & 1430 & & \\
\hline C21R & MgOSS & 226000 & 2280 & 215000 & 1860 \\
\hline c22 & Mog4 & 71300 & 2580 & & \\
\hline$\infty 2$ & Mg25 & 84900 & 3990 & 78100 & 3290 \\
\hline c23 & $M g 24$ & 86600 & 935 & & \\
\hline $\mathrm{C}_{23}$ & MgO25 & 90800 & 3560 & 88700 & 2250 \\
\hline C31 & Mg24 & 103000 & 3030 & & \\
\hline C31 & Mg25 & 103000 & 8510 & 103000 & 5770 \\
\hline C32 & $\mathrm{Mg}_{24}$ & 50700 & 1030 & & \\
\hline C32 & Mg25 & 48100 & 2510 & 49400 & 1770 \\
\hline CO33 & $M 224$ & 76900 & 3240 & & \\
\hline C33 & Mg25 & 80400 & 2870 & 78700 & 3060 \\
\hline $\mathrm{C} 41$ & Mge24 & 141000 & 3820 & & \\
\hline $\mathrm{CA1}$ & Mg25 & 144000 & 6340 & 143000 & 5080 \\
\hline $\mathrm{C} 42$ & Mged & 176000 & 4900 & & \\
\hline $\mathrm{C}_{42}$ & Mg25 & 191000 & 5330 & 184000 & 5120 \\
\hline $\mathrm{C} 43$ & Mge24 & 112000 & 650 & & \\
\hline $\mathrm{C} 43$ & Mgas & 114000 & 7670 & 113000 & 4160 \\
\hline
\end{tabular}

Note: The actual concentratton of eamples for which no standard deviation b reported is lees than the lower limit of detection. The reported concentration is the lower limit of quantincation. 


\section{Transect C Soil Concentrations: Manganese}

Semple id. botope
Concentration

(ug/Kg soll)
Std.Deviation (ug/Kg soll)
Element Avg. Conc. (wg/Kg soll)
Element Avg. Std. Dov. (ug/Kg soll)

\begin{tabular}{|l|c|c|c|c|c|}
\hline$C_{11}$ & $M n$ & 15800 & 431 & 15800 & 431 \\
\hline$C_{12}$ & $M n$ & 287000 & 12200 & 287000 & 12300 \\
\hline$C_{13}$ & $M n$ & 84100 & 4030 & 84100 & 4030 \\
\hline$C_{14}$ & $M n$ & 19000 & 484 & 19000 & 484 \\
\hline$C_{21}$ & $M n$ & 489000 & 8610 & 489000 & 17000 \\
\hline$C_{11}$ & $M n$ & 350000 & 17000 & 350000 & 731 \\
\hline$C_{22}$ & $M n$ & 45600 & 731 & 45600 & 633 \\
\hline$C_{23}$ & $M n$ & 23000 & 633 & 23800 & 8730 \\
\hline$C_{31}$ & $M n$ & 192000 & 8730 & 192000 & 3000 \\
\hline$C_{32}$ & $M n$ & 74200 & 3000 & 74200 & 1900 \\
\hline$C_{33}$ & $M n$ & 50300 & 1900 & 50300 & 4650 \\
\hline$C_{41}$ & $M n$ & 123000 & 4650 & 123000 & 22100 \\
\hline$C_{42}$ & $M n$ & 430000 & 22100 & 430000 & 513 \\
\hline$C_{43}$ & $M n$ & 31000 & 513 & 31000 & \\
\hline
\end{tabular}

Note: The actual concentration of samples for whlch no standerd deviation is reported is lese than the lower limit of detection. The reported concentration is the lower llmit of quantification. 
Transect C Soil Concentrations: Nickel

$\begin{array}{llccc}\text { Semple ld. lsotope } & \begin{array}{c}\text { Concentration } \\ \text { (ug/Kg soil) }\end{array} & \begin{array}{c}\text { Std.Deviation } \\ \text { (ug/Kg soil) }\end{array} & \begin{array}{c}\text { Element Avg. Conc. } \\ \text { (ug/Kg soll) }\end{array} & \begin{array}{c}\text { Element Avg. Std. Dov. } \\ \text { (ug/Kg soll) }\end{array}\end{array}$

\begin{tabular}{|c|c|c|c|c|c|}
\hline C11 & Ni6o & 3390 & 364 & & \\
\hline C11 & $\mathrm{Ni} 62$ & & & 3390 & 364 \\
\hline $\mathrm{C}_{12}$ & Ni60 & 14000 & 789 & & \\
\hline $\mathrm{C}_{12}$ & Nia2 & 19400 & 2940 & 16700 & 1860 \\
\hline $\mathrm{C}_{13}$ & Niso & 5560 & 1280 & & \\
\hline $\mathrm{C}_{13}$ & Ni62 & 7710 & 1710 & 6640 & 1500 \\
\hline $\mathrm{C}_{14}$ & Ni60 & 3380 & 563 & & \\
\hline $\mathrm{C}_{14}$ & Ni62 & 2830 & 345 & 3110 & 454 \\
\hline$C_{21}$ & NiEO & 10300 & 394 & & \\
\hline$C_{21}$ & Ni62 & 12200 & 183 & 11300 & 289 \\
\hline C21R & Niso & 9130 & 290 & & \\
\hline C21R & Ni62 & 11000 & 1710 & 10100 & 1000 \\
\hline C22 & Ni60 & 5330 & 636 & & \\
\hline C22 & Ni62 & & & 5330 & 636 \\
\hline C23 & Ni60 & 4420 & 847 & & \\
\hline$C 23$ & N162 & 4550 & 882 & 4490 & 865 \\
\hline C31 & Ni60 & 32300 & 440 & & \\
\hline C31 & $\mathrm{Ni} 62$ & 39300 & 551 & 35800 & 496 \\
\hline C32 & Ni60 & 1250 & 550 & & \\
\hline C32 & Ni62 & 6320 & 2210 & 3790 & 1380 \\
\hline $\mathrm{CO} 33$ & N160 & 2240 & 1010 & & \\
\hline $\mathrm{CO3}$ & Ni62 & 4610 & 1870 & 3430 & 1440 \\
\hline $\mathrm{C}_{41}$ & Ni6o & 3430 & 200 & & \\
\hline$C_{41}$ & Ni62 & 4660 & 242 & 4050 & 226 \\
\hline $\mathrm{C} 42$ & $\mathrm{Ni60}$ & 24900 & 1390 & & \\
\hline C42 & Ni62 & 25100 & 3320 & 25000 & 2360 \\
\hline$C_{43}$ & Ni6o & & & & \\
\hline$C_{43}$ & Ni62 & 201 & & 201 & \\
\hline
\end{tabular}


Transect C Soil Concentrations: Selenium

\begin{tabular}{|c|c|c|c|c|c|}
\hline Sample Id. & botope & $\begin{array}{l}\text { Concentration } \\
\text { (ug/Ko soin) }\end{array}$ & $\begin{array}{l}\text { Stdonition } \\
\text { (ug/Ko eoll) }\end{array}$ & $\begin{array}{l}\text { Eloment Ave. Cone. } \\
\text { (ug/Ko soil) }\end{array}$ & $\begin{array}{c}\text { Element Avg. Std. Dov. } \\
\text { (ug/Kg sold) }\end{array}$ \\
\hline C11 & So77 & & & & \\
\hline $\mathrm{C11}$ & So82 & 12100 & 2610 & 12100 & 2610 \\
\hline $\mathrm{C12}$ & So77 & 3250 & 4100 & & \\
\hline C12 & So82 & 3260 & 3840 & 3250 & 3970 \\
\hline C13 & So77 & 7240 & 3480 & & \\
\hline C13 & Sos2 & 6670 & 3280 & 6900 & 3380 \\
\hline $\mathrm{C14}$ & So77 & & & & \\
\hline C14 & So82 & 7180 & & 7180 & \\
\hline C21 & So77 & 22700 & 2380 & & \\
\hline C21 & Se82 & 22600 & 2450 & 22700 & 2420 \\
\hline C21R & Se77 & 17300 & 4510 & & \\
\hline G21R & Se82 & 5360 & 593 & 11300 & 2550 \\
\hline 62 & Se77 & & & & \\
\hline $\cos$ & So82 & 17000 & & 17000 & \\
\hline $\mathrm{CO3}$ & S๑77 & 4440 & 6660 & & \\
\hline $\mathrm{C} 3$ & Se82 & 4090 & 6370 & 4270 & 6520 \\
\hline C31 & So77 & 6200 & & & \\
\hline $\mathbf{C 3 1}$ & Sos2 & 6200 & & 6200 & \\
\hline C32 & Se77 & 3560 & 2680 & & \\
\hline C32 & Sa82 & 3400 & 2550 & 3480 & 2620 \\
\hline $\mathbf{C 3 3}$ & So77 & 8670 & 8710 & & \\
\hline $\mathbf{C 3 3}$ & So82 & 7750 & 7870 & 8210 & 8290 \\
\hline CA1 & Se77 & 4580 & & & \\
\hline$C_{41}$ & Sa82 & 4590 & & 4590 & \\
\hline $\mathrm{C} 42$ & $\mathrm{Se} 77$ & 3120 & & & \\
\hline $\mathrm{C} 42$ & Se82 & 3120 & & 3120 & \\
\hline $\mathrm{CA3}_{4}$ & So77 & 30800 & 20700 & & \\
\hline $\mathrm{CA3}$ & So82 & 29300 & 20100 & 30100 & 20400 \\
\hline
\end{tabular}




\section{Transect C Soil Concentrations: Silver}

\begin{tabular}{|c|c|c|c|c|c|}
\hline Sample Id. & Eotope & $\begin{array}{l}\text { Concentration } \\
\text { (ug/kg soll) }\end{array}$ & $\begin{array}{l}\text { StdDevition } \\
\text { (ug/Ko coll) }\end{array}$ & $\begin{array}{l}\text { Element Avg. Conc. } \\
\text { (ugnk woil) }\end{array}$ & $\begin{array}{c}\text { Element Avg. Std. Dav. } \\
\text { (ug/Kg soll) }\end{array}$ \\
\hline C11 & Ag107 & 400 & & & \\
\hline C11 & Ag109 & 430 & & 430 & \\
\hline $\mathrm{C} 12$ & A0107 & 957 & 137 & & \\
\hline $\mathrm{C}_{12}$ & Ag100 & 661 & & 800 & 137 \\
\hline C13 & Ag107 & 635 & & & \\
\hline C13 & Ag109 & 635 & & 635 & \\
\hline C14 & Ag107 & 287 & & & \\
\hline C14 & Ag109 & 1440 & & 864 & \\
\hline 221 & Ag107 & 1880 & 540 & & \\
\hline C21 & Ag100 & 1250 & 207 & 1570 & 374 \\
\hline OIA & A9107 & 1690 & 502 & & \\
\hline C21R & Ag109 & 1010 & 317 & 1350 & 410 \\
\hline$c 22$ & Ag107 & 13600 & & & \\
\hline 022 & $\operatorname{Ag} 109$ & 13600 & & 13000 & \\
\hline C23 & Ag107 & 1440 & & & \\
\hline $\mathrm{C23}$ & Ag109 & 1440 & & 1440 & \\
\hline 031 & Ag107 & 1240 & & & \\
\hline C31 & Ag109 & 1240 & & 1240 & \\
\hline c32 & Ag107 & 866 & 585 & & \\
\hline C32 & Ag109 & 635 & & 751 & 585 \\
\hline $\mathbf{c 3 3}$ & Ag107 & 928 & 808 & & \\
\hline $\mathrm{C33}$ & Ag109 & 2070 & 800 & 1500 & 859 \\
\hline C41 & Ap107 & 540 & 502 & & \\
\hline$C_{41}$ & Ag109 & 938 & 118 & 739 & 310 \\
\hline$C 42$ & A9107 & 624 & & & \\
\hline$C 42$ & Ag109 & 624 & & 624 & \\
\hline $\mathrm{CA3}_{4}$ & Ag107 & 2670 & & & \\
\hline $\mathrm{CA3}$ & Ag109 & 2670 & & 2670 & \\
\hline
\end{tabular}

Note: The actual concentration of aamples for which no stendard deviation b reported le leas than the lower limit of detection. The reported concentratton is the lower limit of quentification. 
Transect C Soil Concentrations: Sodium

\begin{tabular}{|c|c|c|c|c|c|}
\hline Sampte Kd. & botope & $\begin{array}{l}\text { Concantration } \\
\text { (ug/Kg soli) }\end{array}$ & $\begin{array}{l}\text { Std.Dovitution } \\
\text { (ug/kg soll) }\end{array}$ & $\begin{array}{l}\text { Element Avg. Conc. } \\
\text { (ug/Ko soil) }\end{array}$ & $\begin{array}{c}\text { Element Avg. Std. Dov. } \\
\text { (ug/Kg soll) }\end{array}$ \\
\hline $\mathrm{C}_{11}$ & $\mathrm{Na}$ & 41800 & 5070 & 41800 & 5070 \\
\hline $\mathrm{C}_{12}$ & $\mathrm{Na}$ & 55200 & 8730 & 55200 & 8730 \\
\hline $\mathrm{C}_{13}$ & $\mathrm{Na}$ & 6350 & & 6350 & \\
\hline $\mathrm{C14}$ & $\mathrm{Na}$ & 1440 & & 1440 & \\
\hline C21 & $\mathrm{Na}$ & 113000 & 10500 & 113000 & 10500 \\
\hline C21R & $\mathrm{Na}$ & 82500 & 19600 & 82300 & 19600 \\
\hline $\cos$ & $\mathrm{Na}$ & 682000 & & 682000 & \\
\hline$C_{23}$ & $\mathrm{Na}$ & 1440 & & 1440 & \\
\hline C31 & $\mathrm{Na}$ & 12400 & & 12400 & \\
\hline C32 & $\mathrm{Na}$ & 6350 & & 6350 & \\
\hline $\cos 3$ & $\mathrm{Na}$ & 7650 & & 7650 & \\
\hline $\mathrm{C41}$ & $\mathrm{Na}$ & 81600 & 53000 & 81600 & 53000 \\
\hline $\mathrm{C} 42$ & $\mathrm{Na}$ & 22200 & 10600 & 22200 & 10600 \\
\hline$C 43$ & $\mathrm{Na}$ & 1340 & & 1340 & \\
\hline
\end{tabular}




\section{Transect C Soil Concentrations: Strontium}

\begin{tabular}{|c|c|c|c|c|c|}
\hline Semple id. & Esotope & $\begin{array}{l}\text { Concentration } \\
\text { (ug/Ko soll) }\end{array}$ & $\begin{array}{l}\text { Std.Deviation } \\
\text { (ug/Kg soll) }\end{array}$ & $\begin{array}{l}\text { Element Avg. Conc. } \\
\text { (ug/Kg soll) }\end{array}$ & $\begin{array}{c}\text { Elament Avg. Std. Dov. } \\
\text { (ug/Kg soll) }\end{array}$ \\
\hline C11 & Sr & 4490 & 36.8 & 4490 & 36.8 \\
\hline $\mathrm{C} 12$ & $\mathrm{Sr}$ & 3610 & 201 & 3610 & 201 \\
\hline $\mathrm{C13}$ & $\mathrm{Sr}$ & 90500 & 1080 & 90500 & 1080 \\
\hline C14 & $\mathrm{Sr}$ & 31800 & 431 & 31800 & 431 \\
\hline C21 & $\mathrm{Sr}$ & 3700 & 147 & 3700 & 147 \\
\hline CR1R & $\mathrm{Sr}$ & 5430 & 171 & 5430 & 171 \\
\hline Cen & Sr & 38100 & 880 & 38100 & 889 \\
\hline$C_{23}$ & $\mathrm{Sr}$ & 21100 & 654 & 21100 & 654 \\
\hline C31 & $\mathrm{Sr}$ & 84100 & 1020 & 84100 & 1020 \\
\hline $\mathrm{C} 32$ & St & 69300 & 874 & 69300 & 874 \\
\hline $\mathrm{C} 33$ & $\mathbf{S r}$ & 49300 & 751 & 49300 & 751 \\
\hline $\mathrm{CA1}$ & Sr & 3980 & 38.4 & 3980 & 38.4 \\
\hline $\mathrm{C} 42$ & Sr & 27200 & 523 & 27200 & 523 \\
\hline $\mathrm{C} 43$ & $\mathrm{Sr}$ & 85100 & 1320 & 85100 & 1320 \\
\hline
\end{tabular}




\section{Transect C Soil Concentrations: Thorium}

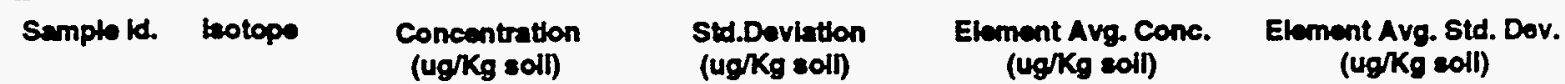

\begin{tabular}{|c|c|c|c|c|c|}
\hline C11 & Th232 & 287 & 23.1 & 287 & 23.1 \\
\hline $\mathrm{C}_{12}$ & Th232 & 1260 & 95.5 & 1260 & 95.5 \\
\hline$C_{13}$ & Th232 & 63.5 & & 63.5 & \\
\hline$C_{14}$ & Th232 & 30.4 & 6.17 & 30.4 & 6.17 \\
\hline$Q_{21}$ & Th232 & 1310 & 121 & 1310 & 121 \\
\hline C21R & Th232 & 576 & 45.6 & 576 & 45.6 \\
\hline $\cos 2$ & Th232 & 170 & & 170 & \\
\hline$C_{23}$ & Th232 & 59.3 & 16.6 & 59.3 & 16.6 \\
\hline C.31 & Th232 & 124 & & 124 & \\
\hline $\cos 2$ & Th232 & 63.5 & & 63.5 & \\
\hline $\cos 3$ & Th232 & 123 & 35.2 & 123 & 352 \\
\hline$C_{41}$ & Th232 & 518 & 19.7 & 518 & 19.7 \\
\hline $\mathrm{CA} 2$ & Th232 & 92.3 & 20.7 & 92.3 & 20.7 \\
\hline $\mathrm{CA3}$ & Th232 & 66.9 & & 66.9 & \\
\hline
\end{tabular}




\section{Transect C Soil Concentrations: Tin}

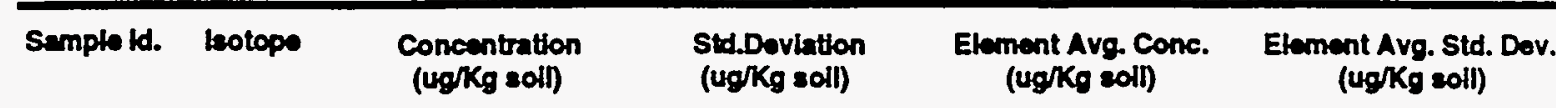

\begin{tabular}{|c|c|c|c|c|c|}
\hline C11 & Sn118 & 53.7 & & & \\
\hline C11 & $\operatorname{Sn} 120$ & 53.7 & & 53.7 & \\
\hline$C_{12}$ & Sn118 & 265 & 22.6 & & \\
\hline $\mathrm{C}_{12}$ & Sn120 & 443 & 23.4 & 354 & 23 \\
\hline$C_{13}$ & Sn118 & 175 & 81.4 & & \\
\hline $\mathrm{C13}$ & Sn120 & 113 & 60.5 & 144 & 71 \\
\hline $\mathrm{C}_{14}$ & Sn118 & 300 & 43.7 & & \\
\hline $\mathrm{C}_{14}$ & Sn120 & 344 & 11.6 & 322 & 27.7 \\
\hline $\mathrm{C}_{21}$ & Sn118 & 225 & 34.5 & & \\
\hline C21 & Sn120 & 218 & 35 & 222 & 34.8 \\
\hline C21R & Snt18 & 121 & 75.9 & & \\
\hline C21R & Sn120 & 238 & 118 & 180 & 97 \\
\hline $\cos 2$ & Sn118 & 487 & 88.5 & & \\
\hline$\infty 2$ & Sn120 & 522 & 196 & 500 & 142 \\
\hline$c_{23}$ & Sn118 & 227 & 49.7 & & \\
\hline$c 23$ & $\sin 120$ & 202 & 17 & 215 & 33.4 \\
\hline C31 & Sn118 & 1230 & 129 & & \\
\hline C31 & Sn120 & 616 & 73.5 & 923 & 101 \\
\hline C32 & Sn118 & 199 & 45.6 & & \\
\hline c32 & $\sin 120$ & 168 & 16.1 & 184 & 30.9 \\
\hline $\cos 3$ & Sn118 & 501 & 41.8 & & \\
\hline C33 & $\operatorname{sn} 120$ & 347 & 31.5 & 424 & 36.7 \\
\hline$C_{41}$ & Sn118 & 443 & 56.5 & & \\
\hline$C_{41}$ & $\operatorname{Sn} 120$ & 418 & 16.3 & 431 & 36.4 \\
\hline $\mathrm{C} 42$ & $\operatorname{Sn} 118$ & 345 & 79.3 & & \\
\hline $\mathrm{C} 42$ & $\operatorname{sn} 120$ & 370 & 73.4 & 358 & 76.4 \\
\hline $\mathrm{C} 43$ & $\operatorname{Sn} 118$ & 503 & 129 & & \\
\hline$C 43$ & Sn120 & 66.9 & & 285 & 129 \\
\hline
\end{tabular}

Note: The actual concentratton of eamples for which no standard doviation ts reported is lose than the lower limit of detection. The reported concontration is the lower limit of quantification. 


\section{Transect C Soil Concentrations: Uranium-235}

\begin{tabular}{|c|c|c|c|c|c|}
\hline Semple id. & Eotope & $\begin{array}{l}\text { Concentration } \\
\text { (ug/Kg soll) }\end{array}$ & $\begin{array}{l}\text { Std.Devintion } \\
\text { (ug/Kg soll) }\end{array}$ & $\begin{array}{l}\text { Elament Avg. Conc. } \\
\text { (ug/Kg soll) }\end{array}$ & $\begin{array}{c}\text { Element Avg. Std. Dov. } \\
\text { (Ug/Kg soll) }\end{array}$ \\
\hline $\mathrm{C} 11$ & U235 & 3.87 & & 3.87 & \\
\hline $\mathrm{C} 12$ & U235 & 10.7 & 326 & 10.7 & 326 \\
\hline C13 & U235 & 48.3 & 4.51 & 48.3 & 4.51 \\
\hline C14 & U235 & 44.9 & 3.08 & 44.9 & 3.08 \\
\hline$\infty 21$ & U235 & 29.4 & 3.56 & 29.4 & 3.56 \\
\hline C21R & U235 & 16.3 & 8.1 & 16.3 & 8.1 \\
\hline$c 22$ & $\mathbf{v 2 3 5}$ & 11.3 & 1.59 & 11.3 & 1.59 \\
\hline $\mathrm{COS3}_{3}$ & U235 & 36.3 & 4.66 & 36.3 & 4.66 \\
\hline C31 & U235 & 54 & 7.9 & 54 & 7.9 \\
\hline C.32 & U235 & 18.6 & 4.03 & 18.6 & 4.03 \\
\hline C33 & U235 & 25 & 4.44 & 25 & 4.44 \\
\hline $\mathrm{C} 41$ & U235 & 13.3 & 2.24 & 13.3 & 224 \\
\hline $\mathrm{C} 42$ & 4235 & 47.1 & 1.07 & 47.1 & 1.07 \\
\hline $\mathrm{CA3}$ & U235 & 16.9 & 5.84 & 16.9 & 5.84 \\
\hline
\end{tabular}




\section{Transect C Soil Concentrations: Uranium-238}

\begin{tabular}{|c|c|c|c|c|c|}
\hline Semple id. & botope & $\begin{array}{l}\text { Concentratton } \\
\text { (ug/Kg soll) }\end{array}$ & $\begin{array}{l}\text { Sted.Doviation } \\
\text { (ug/Kg soil) }\end{array}$ & $\begin{array}{l}\text { Element Avg. Conc. } \\
\text { (ug/Kg soll) }\end{array}$ & $\begin{array}{c}\text { Element Avg. Std. Dav. } \\
\text { (ugg/Kg soll) }\end{array}$ \\
\hline C11 & U238 & 1020 & 49.1 & 1020 & 49.1 \\
\hline $\mathrm{C}_{12}$ & U238 & 1870 & 25.1 & 1870 & 25.1 \\
\hline $\mathrm{C}_{13}$ & U238 & 8170 & 71 & 8170 & 71 \\
\hline $\mathrm{C}_{14}$ & U238 & 5960 & 61.4 & 5960 & 61.4 \\
\hline C21 & U238 & 2010 & 167 & 2010 & 167 \\
\hline C21A & U238 & 2610 & 184 & 2610 & 184 \\
\hline ces2 & U238 & 1810 & 23 & 1810 & 23 \\
\hline $\mathrm{CO}_{23}$ & U238 & 5430 & 65.7 & 5430 & 65.7 \\
\hline C31 & U238 & 7150 & 92 & 7150 & 92 \\
\hline C.32 & U238 & 1570 & 31.5 & 1570 & 31.5 \\
\hline $\mathrm{CO33}$ & U238 & 3210 & 40.8 & 3210 & 40.8 \\
\hline$C_{41}$ & U238 & 1930 & 57.7 & 1930 & 57.7 \\
\hline $\mathrm{C}_{42}$ & U238 & 7610 & 40.6 & 7610 & 40.6 \\
\hline $\mathrm{CA3}_{4}$ & U238 & 2280 & 27.5 & 2280 & 27.5 \\
\hline
\end{tabular}

Note: The setual concentration of samples for which no standard deviation le reported is leas than the lower limit of detection. The reported concentration ts the lower limit of quantification. 


\section{Transect C Soil Concentrations: Vanadium}

\begin{tabular}{|c|c|c|c|c|c|}
\hline Semple ld. & beotope & $\begin{array}{l}\text { Concentration } \\
\text { (ug/Kg soli) }\end{array}$ & $\begin{array}{l}\text { Std.Deviation } \\
\text { (ug/Kg edil) }\end{array}$ & $\begin{array}{l}\text { Element Avg. Conc. } \\
\text { (ug/Kg soll) }\end{array}$ & $\begin{array}{c}\text { Element Avg. Std. Dov. } \\
\text { (ugg/Kg soll) }\end{array}$ \\
\hline $\mathrm{C} 11$ & V & 13300 & 487 & 13300 & 487 \\
\hline$C_{12}$ & $v$ & 132 & & 132 & \\
\hline $\mathrm{C}_{13}$ & $v$ & 8220 & 542 & 8220 & 542 \\
\hline C14 & $v$ & 16000 & 323 & 16000 & 323 \\
\hline $\mathrm{C}_{21}$ & $V$ & 20100 & 3400 & 20100 & 3400 \\
\hline C21R & $\mathrm{v}$ & 20600 & 2720 & 20600 & 2720 \\
\hline $\mathrm{CO2}$ & $V$ & 12400 & 658 & 12400 & 658 \\
\hline$C_{23}$ & $V$ & 11400 & 332 & 11400 & 332 \\
\hline$C 31$ & $\mathrm{~V}$ & 13000 & 1450 & 13000 & 1450 \\
\hline C32 & $v$ & 12500 & 515 & 12500 & 515 \\
\hline $\mathrm{CO33}$ & $V$ & 8060 & 644 & 8060 & 644 \\
\hline$C_{41}$ & v & 127 & & 127 & \\
\hline $\mathrm{CA2}$ & $v$ & 125 & & 125 & \\
\hline $\mathrm{C} 43$ & $V$ & 17800 & 159 & 17800 & 159 \\
\hline
\end{tabular}

Note: The actual concentration of samples for which no standard deviation b reported be leas than the bwer limit of detection. The reported concentration is the lower limit of quantification. 


\section{Transect C Soil Concentrations: Yttrium}

\begin{tabular}{|c|c|c|c|c|c|}
\hline Semple ld. & teotope & $\begin{array}{l}\text { Concentratton } \\
\text { (ug/Kg soli) }\end{array}$ & $\begin{array}{l}\text { Std.Dovlation } \\
\text { (ug/Kg soll) }\end{array}$ & $\begin{array}{l}\text { Element Avg. Conc. } \\
\text { (ug/Kg soll) }\end{array}$ & $\begin{array}{c}\text { Element Avg. Std. Dov. } \\
\text { (ug/Kg soll) }\end{array}$ \\
\hline $\mathrm{C} 11$ & $Y$ & 346 & 24.5 & 346 & 24.5 \\
\hline C12 & $\bar{Y}$ & 458 & 23.7 & 458 & 23.7 \\
\hline $\mathrm{C}_{13}$ & $Y$ & 313 & 39.8 & 313 & 39.8 \\
\hline$C 14$ & $\mathbf{Y}$ & 204 & 22.6 & 204 & 22.6 \\
\hline C21 & $\bar{Y}$ & 552 & 32.3 & 552 & 32.3 \\
\hline C21R & $\bar{Y}$ & 393 & 39.7 & 393 & 39.7 \\
\hline $\mathrm{CO2}$ & $Y$ & 102 & 322 & 102 & 32.2 \\
\hline $\mathrm{C23}$ & $\mathbf{Y}$ & 300 & 37.4 & 300 & 37.4 \\
\hline C31 & $Y$ & 335 & 45.7 & 335 & 45.7 \\
\hline C32 & $Y$ & 219 & 12.7 & 219 & 12.7 \\
\hline C33 & $Y$ & 4070 & 96.3 & 4070 & 96.3 \\
\hline$C_{41}$ & $Y$ & 634 & 36.5 & 634 & 36.5 \\
\hline$C_{42}$ & $Y$ & 351 & 22.4 & 351 & 22.4 \\
\hline $\mathrm{C}_{43}$ & $Y$ & 857 & 39.9 & 857 & 39.9 \\
\hline
\end{tabular}

Note: The ectual concentratton of eamples for whlch no standard doviation te reported is leas than the lower llmit of detection. The reported concentration is the lower IImit of quantification. 
Transect C Soil Concentrations: Zinc

\begin{tabular}{|c|c|c|c|c|c|}
\hline Semple Id. & Lotope & $\begin{array}{l}\text { Concentration } \\
\text { (ug/Ko soll) }\end{array}$ & $\begin{array}{l}\text { Std.Dovintion } \\
\text { (ug/Kg soll) }\end{array}$ & $\begin{array}{l}\text { Element Avg. Conc. } \\
\text { (ug/Kg soll) }\end{array}$ & $\begin{array}{c}\text { Element Avg. Std. Dev. } \\
\text { (ug/Kg soll) }\end{array}$ \\
\hline C11 & Zn66 & 3220 & & 3220 & \\
\hline C12 & Zn66 & 11100 & 339 & 11100 & 339 \\
\hline C13 & Zns6 & 4290 & 1180 & 4290 & 1180 \\
\hline C14 & Zn66 & 5850 & 399 & 5850 & 399 \\
\hline C21 & Zns6 & 3410 & 1160 & 3410 & 1160 \\
\hline C21R & Zn66 & 1720 & & 1720 & \\
\hline $\mathrm{Cr2}$ & Zn66 & 102000 & & 102000 & \\
\hline C23 & Zns6 & 4580 & 409 & 4580 & 400 \\
\hline C31 & Zn66 & 4900 & 1700 & 4900 & 1700 \\
\hline $\mathrm{CO} 2$ & Zns6 & 1060 & 398 & 1060 & 398 \\
\hline C.33 & Zn66 & 13100 & 4310 & 13100 & 4310 \\
\hline C41 & Zns6 & 16300 & 1500 & 16300 & 1500 \\
\hline $\mathrm{C} 42$ & Zn66 & 14200 & 481 & 14200 & 481 \\
\hline $\mathrm{C} 43$ & ZnE6 & 16500 & 670 & 16500 & 670 \\
\hline
\end{tabular}




\section{Transect C Soil Concentrations: Zirconium}

\begin{tabular}{|c|c|c|c|c|c|}
\hline Semple ld. & botope & $\begin{array}{l}\text { Concentration } \\
\text { (ug/Kg soli) }\end{array}$ & $\begin{array}{l}\text { Std.Deviation } \\
\text { (ug/Kg soll) }\end{array}$ & $\begin{array}{l}\text { Element Avg. Conc. } \\
\text { (ug/Kg soll) }\end{array}$ & $\begin{array}{c}\text { Element Avg. Std. Dov. } \\
\text { (ug/Kg soll) }\end{array}$ \\
\hline $\mathrm{C} 11$ & 280 & 37300 & 821 & & \\
\hline C11 & 2591 & 38900 & 1230 & 38100 & 1030 \\
\hline $\mathrm{C} 12$ & Z90 & 20000 & 389 & & \\
\hline $\mathrm{C12}$ & 291 & 23800 & 887 & 21900 & 638 \\
\hline C13 & 2590 & 12000 & 505 & & \\
\hline $\mathrm{C13}$ & 291 & 17600 & 435 & 14800 & 470 \\
\hline C14 & $Z 90$ & 22300 & 527 & & \\
\hline C14 & $2 r 91$ & 21400 & 1030 & 21900 & 779 \\
\hline C1 & 2890 & 39900 & 603 & & \\
\hline C21 & 791 & 33700 & 898 & 36800 & 751 \\
\hline C21R & 290 & 24900 & 375 & & \\
\hline C21R & $2 r 91$ & 34400 & 587 & 29700 & 481 \\
\hline cos & 2500 & 17300 & 479 & & \\
\hline $\mathrm{C22}$ & 2991 & 12700 & 1220 & 15000 & 850 \\
\hline$C 23$ & Zrso & 24300 & 251 & & \\
\hline 023 & Zr91 & 21900 & 1710 & 23100 & 981 \\
\hline C31 & 2590 & 12900 & 808 & & \\
\hline C31 & 291 & 16100 & 1080 & 14500 & 944 \\
\hline C32 & 290 & 21000 & 526 & & \\
\hline C32 & 2891 & 20500 & 1080 & 20800 & 803 \\
\hline C.33 & 2590 & 32000 & 1120 & & \\
\hline $\cos 3$ & Zr91 & 38900 & 3500 & 35500 & 2310 \\
\hline $\mathrm{C}_{41}$ & 2950 & 43700 & 919 & & \\
\hline $\mathrm{C}_{41}$ & 2991 & 35600 & 1360 & 39700 & 1140 \\
\hline $\mathrm{CA2}$ & 7890 & 26600 & 526 & & \\
\hline $\mathrm{CA2}$ & 201 & 23500 & 778 & 25100 & 652 \\
\hline $\mathrm{CA3}$ & 7800 & 26200 & 593 & & \\
\hline$C_{43}$ & Zr91 & 30900 & 1850 & 28600 & 1220 \\
\hline
\end{tabular}

Note: The ectunl concentration of amples for which no standerd deviation is reported bs leas than the lower limit of detection. The reported concentration bs the lower IImit of quantification. 


\begin{tabular}{|c|c|c|c|c|c|}
\hline Sample ld. & Leotope & $\begin{array}{l}\text { Concentration } \\
\text { (ug/Kg soll) }\end{array}$ & $\begin{array}{l}\text { Std.Dovilation } \\
\text { (ug/Kg soil) }\end{array}$ & $\begin{array}{l}\text { Element Avg. Conc. } \\
\text { (ug/Kg soll) }\end{array}$ & $\begin{array}{c}\text { Element Avg. Std. Dov. } \\
\text { (ug/Kg soll) }\end{array}$ \\
\hline D11 & $\mathbf{A}$ & 8720000 & 199000 & 8720000 & 199000 \\
\hline D12 & $\mathbf{A}$ & 9580000 & 154000 & 9580000 & 154000 \\
\hline D13 & A & 3290000 & 19800 & 3290000 & 19800 \\
\hline D13RA & $\mathbf{A}$ & 5640000 & 27000 & 5640000 & 27000 \\
\hline D13RB & $\mathbf{A}$ & 3130000 & 13000 & 3130000 & 13000 \\
\hline$D 21$ & $\mathbf{A}$ & 10800000 & 114000 & 10800000 & 114000 \\
\hline D41 & $\mathbf{A}$ & 13200000 & 315000 & 13200000 & 315000 \\
\hline DS1 & $\mathbf{A}$ & 11000000 & 320000 & 11000000 & 320000 \\
\hline
\end{tabular}


Transect D Soil Concentrations: Arsenic

\begin{tabular}{|c|c|c|c|c|c|}
\hline Semplo ld. & leotope & $\begin{array}{l}\text { Concentration } \\
\text { (ug/Kg soll) }\end{array}$ & $\begin{array}{l}\text { Sid.Doviation } \\
\text { (ug/Kg soll) }\end{array}$ & $\begin{array}{l}\text { Element Avg. Conc. } \\
\text { (ug/Kg soll) }\end{array}$ & $\begin{array}{c}\text { Element Avg. Std. Dov. } \\
\text { (ug/Kg soll) }\end{array}$ \\
\hline D11 & As & 2660 & 555 & 2660 & 555 \\
\hline D12 & As & 5290 & & 5290 & \\
\hline D13 & As & 3640 & & 3640 & \\
\hline D13RA & As & 13100 & & 13100 & \\
\hline D13RB & As & 13100 & & 13100 & \\
\hline D21 & As & 6810 & 482 & 6810 & 482 \\
\hline D41 & As & 6720 & 819 & 6720 & 819 \\
\hline D51 & As & 5450 & 2500 & 5450 & 2500 \\
\hline
\end{tabular}

Note: The setual concentration of eamplea for which no standard deviation le reported is leas than the lower IImit of detection. The reported concentration is the lower limit of quantification. 


\section{Transect D Soil Concentrations: Barium}

\begin{tabular}{|c|c|c|c|c|c|}
\hline Sumple Id. & botope & $\begin{array}{l}\text { Concentration } \\
\text { (ug/Kg soil) }\end{array}$ & $\begin{array}{l}\text { Stu. ov vition } \\
\text { (ug/Kg soll) }\end{array}$ & $\begin{array}{l}\text { Element Avg. Conc. } \\
\text { (ug/Kg eoll) }\end{array}$ & $\begin{array}{c}\text { Element Avg. Std. Dov. } \\
\text { (ug/Kg soli) }\end{array}$ \\
\hline D11 & $\mathrm{Ba135}$ & 19800 & 1030 & & \\
\hline D11 & Ba137 & 25000 & 183 & & \\
\hline D11 & Ba138 & 18400 & 203 & 21100 & 472 \\
\hline 012 & Ba135 & 8010 & 1840 & & \\
\hline D12 & $\mathrm{Ba137}$ & 4160 & 1460 & & \\
\hline D12 & $\mathrm{Ba138}$ & 15200 & 313 & 9120 & 1200 \\
\hline$\overline{D 13}$ & $\mathrm{Ba135}$ & 43900 & 2490 & & \\
\hline D13 & $8 a 137$ & 45100 & 659 & & \\
\hline D13 & Ba138 & 36800 & 351 & 41900 & 1170 \\
\hline D13RA & Ba135 & 88500 & 1340 & & \\
\hline D13RA & Ba137 & 96700 & 1360 & & \\
\hline D13RA & Ba138 & 97200 & 772 & 94100 & 1160 \\
\hline D13RB & Ba135 & 14800 & 743 & & \\
\hline D13RB & $\mathrm{Ba} 137$ & 11900 & 451 & & \\
\hline D13RB & $\mathrm{Ba138}$ & 14200 & 33.1 & 13600 & 409 \\
\hline D21 & Ba135 & 32100 & 774 & & \\
\hline D21 & Ba137 & 32900 & 318 & & \\
\hline D21 & $\mathrm{Ba138}$ & 33900 & 136 & 33000 & 409 \\
\hline 041 & Ba135 & 203000 & 916 & & \\
\hline D41 & $\mathrm{Ba137}$ & 151000 & 1390 & & \\
\hline D41 & Ba138 & 219000 & 325 & 191000 & 877 \\
\hline D51 & Ba135 & 77900 & 11600 & & \\
\hline D51 & Ba137 & 66500 & 15100 & & \\
\hline D51 & $\mathrm{Ba138}$ & 84600 & 16800 & 76300 & 14500 \\
\hline
\end{tabular}




\section{Transect D Soil Concentrations: Cadmium}

\begin{tabular}{|c|c|c|c|c|c|}
\hline Sample Id. & teotope & $\begin{array}{l}\text { Concentration } \\
\text { (ug/Kg soll) }\end{array}$ & $\begin{array}{l}\text { Std.Deviation } \\
\text { (ug/Kg soll) }\end{array}$ & $\begin{array}{l}\text { Element Avg. Conc. } \\
\text { (ug/Kg soil) }\end{array}$ & $\begin{array}{c}\text { Element Avg. Std. Dov. } \\
\text { (ug/Kg soll) }\end{array}$ \\
\hline D11 & Cd111 & 69.3 & & & \\
\hline D11 & Cd114 & 55.1 & 7.87 & 622 & 7.87 \\
\hline D12 & Cd111 & 106 & & & \\
\hline D12 & Cod114 & 106 & & 106 & \\
\hline D13 & $\operatorname{cod111}$ & 72.7 & & & \\
\hline D13 & Cd114 & 93.7 & 202 & 83.2 & 202 \\
\hline D13RA & Cd111 & 211 & 61.7 & & \\
\hline D13AA & Cd114 & 148 & 275 & 180 & 168 \\
\hline D13AB & Cd111 & 131 & & & \\
\hline D13RB & Cd114 & 131 & & 131 & \\
\hline D21 & Cod111 & 64.7 & & & \\
\hline D21 & Cd114 & 64.7 & & 64.7 & \\
\hline D41 & Co1111 & 69.5 & & & \\
\hline D41 & $\operatorname{Cod114}$ & 69.5 & & 69.5 & \\
\hline D51 & Cod111 & 88.1 & & & \\
\hline D51 & Cod114 & 246 & 92.4 & 167 & 92.4 \\
\hline
\end{tabular}

Note: The ectual concentration of samples for which no standard deviation is reported is teas than the lower limit of detection. The reported concentratton is the lower llmit of quantification. 


\section{Transect D Soil Concentrations: Calcium}

Sample ld. leotops
\begin{tabular}{|c|c|c|c|c|c|}
\hline & $\begin{array}{c}\text { Concentration } \\
\text { (ug/Kg soll) }\end{array}$ & $\begin{array}{c}\text { Std.Deviation } \\
\text { (Ug/Kg soil) }\end{array}$ & $\begin{array}{c}\text { Element Avg. Conc. } \\
\text { (ug/Kg soll) }\end{array}$ & $\begin{array}{c}\text { Element Avg. Std. Dov. } \\
\text { (Ug/Kg soll) }\end{array}$ \\
\hline D11 & Ca44 & 869000 & 226000 & 869000 & 226000 \\
\hline D12 & Ca44 & 1860000 & 73700 & 1860000 & 73700 \\
\hline D13 & Ca44 & 704000 & 95800 & 704000 & 95800 \\
\hline D13RA & Ca44 & 3810000 & 233000 & 3810000 & 233000 \\
\hline D13RB & Ca44 & 1390000 & 171000 & 1390000 & 171000 \\
\hline D21 & Ca44 & 3640000 & 417000 & 3640000 & 417000 \\
\hline D41 & Ca44 & 3150000 & 101000 & 3150000 & 101000 \\
\hline D51 & Ca44 & 564000 & 295000 & 564000 & 295000 \\
\hline
\end{tabular}




\section{Transect D Soil Concentrations: Cesium}

\begin{tabular}{|c|c|c|c|c|c|}
\hline Sample ld. & Inotope & $\begin{array}{l}\text { Concentratton } \\
\text { (ug/Kg soll) }\end{array}$ & $\begin{array}{l}\text { Std.Deviation } \\
\text { (ug/Kg sodl) }\end{array}$ & $\begin{array}{l}\text { Element Avg. Conc. } \\
\text { (ug/Kg soll) }\end{array}$ & $\begin{array}{c}\text { Element Avg. Std. Dev. } \\
\text { (ug/Kg soll) }\end{array}$ \\
\hline 011 & Cs & 622 & 25.6 & 622 & 25.6 \\
\hline D12 & C8 & 428 & 14.7 & 428 & 14.7 \\
\hline D13 & $\mathrm{Cs}_{\mathrm{s}}$ & 82.7 & 1.94 & 82.7 & 1.94 \\
\hline D13RA & Cs & 4760 & 342 & 4760 & 342 \\
\hline D13RB & $\mathrm{Cs}_{8}$ & 2270 & 61.8 & 2270 & 61.8 \\
\hline D21 & Cs & 186 & 22.6 & 186 & 22.6 \\
\hline 041 & Cs & 167 & 9.75 & 167 & 9.75 \\
\hline D51 & Cs & 88.1 & & 88.1 & \\
\hline
\end{tabular}




\section{Transect D Soil Concentrations: Chromium}

\begin{tabular}{|c|c|c|c|c|c|}
\hline Semplo Id. & leotope & $\begin{array}{l}\text { Concentration } \\
\text { (ug/Kg soll) }\end{array}$ & $\begin{array}{l}\text { Std.Devintion } \\
\text { (ug/Kg soll) }\end{array}$ & $\begin{array}{l}\text { Element Avg. Conc. } \\
\text { (ug/Kg soll) }\end{array}$ & $\begin{array}{c}\text { Element Avg. Std. Dev. } \\
\text { (ug/Kg soll) }\end{array}$ \\
\hline D11 & Cr52 & 4460 & 241 & & \\
\hline D11 & $\mathrm{G} 53$ & 4450 & 243 & 4460 & 242 \\
\hline D12 & C.52 & 14700 & 45.7 & & \\
\hline D12 & C553 & 14600 & 552 & 14700 & 50.5 \\
\hline 013 & C.52 & 3310 & 249 & & \\
\hline D13 & G53 & 3260 & 246 & 3300 & 248 \\
\hline D13RA & Cr52 & 7690 & 75.9 & & \\
\hline D13RA & C553 & 7740 & 76 & 7720 & 76 \\
\hline D13RB & Cr52 & 2800 & 15.9 & & \\
\hline D13RB & Cr53 & 2790 & 19.7 & 2800 & 17.8 \\
\hline D21 & G52 & 11800 & 335 & & \\
\hline $\mathrm{D} 21$ & C.53 & 11700 & 332 & 11800 & 334 \\
\hline$D 41$ & Cr52 & 6380 & 122 & & \\
\hline 041 & $\mathrm{C} 53$ & 6410 & 124 & 6400 & 123 \\
\hline D51 & C.52 & 5510 & 594 & & \\
\hline 051 & C.53 & 5510 & 594 & 5510 & 594 \\
\hline
\end{tabular}

Note: The sctual concentration of samples for which no stendard deviation is reported b lees than the lower limit of detection. The reported concentration be the lower limit of quantification. 


\section{Transect D Soil Concentrations: Cobalt}

\begin{tabular}{lcccc}
\hline Sample ld. botope & $\begin{array}{c}\text { Concentration } \\
\text { (ug/Kg soil) }\end{array}$ & $\begin{array}{c}\text { Std.Dovlation } \\
\text { (ug/Kg soil) }\end{array}$ & $\begin{array}{c}\text { Element Avg. Conc. } \\
\text { (ug/Kg soll) }\end{array}$ & $\begin{array}{c}\text { Element Avg. Std. Dov. } \\
\text { (ug/Kg soll) }\end{array}$
\end{tabular}

\begin{tabular}{|c|c|c|c|c|c|}
\hline \multicolumn{7}{|c|}{ D11 } & Co & 812 & 29.7 & 812 & 29.7 \\
\hline D12 & Co & 1740 & 82.8 & 1740 & 82.8 \\
\hline D13 & Co & 217 & 20.3 & 217 & 20.3 \\
\hline D13RA & Co & 682 & 14.5 & 682 & 14.5 \\
\hline D13RB & C & 236 & 28.9 & 236 & 28.9 \\
\hline D21 & Co & 522 & 7.95 & 522 & 7.95 \\
\hline D41 & Co & 651 & 50.8 & 651 & 50.8 \\
\hline D51 & Co & 810 & 370 & 810 & 370 \\
\hline
\end{tabular}




\section{Transect D Soil Concentrations: Copper}

\begin{tabular}{|c|c|c|c|c|c|}
\hline Sample Id. & botope & $\begin{array}{l}\text { Concentration } \\
\text { (ug/Kg soll) }\end{array}$ & $\begin{array}{l}\text { Std.Deviation } \\
\text { (ug/Kg soll) }\end{array}$ & $\begin{array}{l}\text { Element Avg. Conc. } \\
\text { (ug/Kg soil) }\end{array}$ & $\begin{array}{c}\text { Element Avg. Std. Dev. } \\
\text { (ug/Kg soll) }\end{array}$ \\
\hline D11 & 0.63 & 2810 & 264 & & \\
\hline D11 & Cu65 & 3610 & 147 & 3210 & 206 \\
\hline 012 & Cu63 & 8070 & 225 & & \\
\hline D12 & av65 & 7830 & 361 & 7950 & 293 \\
\hline D13 & Cuss & 1830 & 64.6 & & \\
\hline D13 & Cu65 & 1510 & 114 & 1670 & 89.3 \\
\hline D13RA & 0,63 & & & & \\
\hline D13RA & 0.65 & 4010 & 152 & 4010 & 152 \\
\hline D13RB & 0463 & & & & \\
\hline D13RB & 0.65 & 4490 & 172 & 4490 & 172 \\
\hline D21 & 0463 & 3710 & 130 & & \\
\hline 021 & Cu65 & 5350 & 263 & 4530 & 197 \\
\hline$D 41$ & Cuas & 5690 & 97.9 & & I \\
\hline D41 & Qu65 & 7960 & 237 & 6830 & 167 \\
\hline D51 & Cu63 & 5340 & 2030 & & \\
\hline D51 & Cu65 & 3400 & 2780 & 4370 & 2410 \\
\hline
\end{tabular}

Note: The ectual concentration of eamples for which no standard deviation ls reported is leas than the lower llmit of detection. The reportied concentration is the lower limit of quantification. 


\section{Transect D Soil Concentrations: Iron}

\begin{tabular}{|c|c|c|c|c|c|}
\hline Sempto Id. & leotope & $\begin{array}{l}\text { Concentration } \\
\text { (ug/Kg soll) }\end{array}$ & $\begin{array}{l}\text { Std.Doviation } \\
\text { (ug/Kg soll) }\end{array}$ & $\begin{array}{l}\text { Element Avg. Conc. } \\
\text { (ug/Kg 2oil) }\end{array}$ & $\begin{array}{c}\text { Element Avg. Std. Dov. } \\
\text { (ug/Kg soll) }\end{array}$ \\
\hline 011 & Fo & 3000000 & 76400 & 3900000 & 76400 \\
\hline D12 & $F_{0}$ & 10600000 & 127000 & 10600000 & 127000 \\
\hline D13 & $\mathrm{Fo}$ & 3350000 & 34600 & 3350000 & 34600 \\
\hline D13RA & $F_{0}$ & 4010000 & 13500 & 4010000 & 13300 \\
\hline D13AB & $F_{0}$ & 2550000 & 3050 & 2550000 & 3050 \\
\hline $\mathrm{D} 21$ & $F_{0}$ & 7350000 & 24300 & 7350000 & 24300 \\
\hline D41 & $\mathrm{Fo}$ & 7990000 & 133000 & 7990000 & 133000 \\
\hline D51 & $F_{\theta}$ & 6280000 & & 6280000 & \\
\hline
\end{tabular}

Note: The ectual concentration of samples for which no standard deviation ls reported bs leas than the lower limit of dotection. The reported concentration is the lower limit of quantification. 


\section{Transect D Soil Concentrations: Lead}

\begin{tabular}{|c|c|c|c|c|c|}
\hline Semple ld. & Eotope & $\begin{array}{l}\text { Concentration } \\
\text { (ug/Kg :oil) }\end{array}$ & $\begin{array}{l}\text { Std.Doviatton } \\
\text { (ug/Kg \&oll) }\end{array}$ & $\begin{array}{l}\text { Element Avg. Conc. } \\
\text { (ug/Kg soll) }\end{array}$ & $\begin{array}{c}\text { Element Avg. Std. Dov. } \\
\text { (Ug/Kg soll) }\end{array}$ \\
\hline D11 & Pb206 & 20200 & 1030 & & \\
\hline D11 & $P b 207$ & 17200 & 496 & & \\
\hline D11 & Pto208 & 19400 & 463 & 18900 & 663 \\
\hline D12 & $P 0206$ & 21000 & 573 & & \\
\hline D12 & $P 6207$ & 20700 & 1350 & & \\
\hline D12 & Pb208 & 17400 & 456 & 19700 & 793 \\
\hline D13 & Pb206 & 14300 & 776 & & \\
\hline D13 & Pb207 & 14600 & 625 & & \\
\hline D13 & Pb208 & 14700 & 810 & 14500 & 737 \\
\hline D13RA & Po206 & 3760 & 252 & & \\
\hline D13RA & Pb207 & 2530 & 240 & & \\
\hline D13RA & Pb208 & 2760 & 205 & 3020 & 236 \\
\hline D13RB & $\mathrm{Pb200}$ & 3390 & 618 & & \\
\hline D13RB & Pb207 & 1810 & 126 & & \\
\hline D13RB & Pb208 & 1750 & 52.1 & 2320 & 265 \\
\hline D21 & Pb206 & 26300 & 785 & & \\
\hline D21 & $\mathrm{Pb} 207$ & 25100 & 258 & & \\
\hline D21 & Pb208 & 25900 & 517 & 25800 & 520 \\
\hline$D 41$ & Pb206 & 47600 & 960 & & \\
\hline D41 & Pb207 & 40800 & 731 & & \\
\hline$D 41$ & Pbaos & 39200 & 244 & 42500 & 645 \\
\hline D51 & $\mathrm{Pb206}$ & 4400 & & & \\
\hline D51 & $\mathrm{Pb} 207$ & 4400 & & & \\
\hline D.51 & $\mathrm{Pb208}$ & 4400 & & 4400 & \\
\hline
\end{tabular}




\section{Transect D Soil Concentrations: Magnesium}

\begin{tabular}{|c|c|c|c|c|c|}
\hline Semple ld. & Eotope & $\begin{array}{l}\text { Concentration } \\
\text { (ug/kg soil) }\end{array}$ & $\begin{array}{l}\text { Std.Deviatton } \\
\text { (ug/Kg soll) }\end{array}$ & $\begin{array}{l}\text { Element Avg. Conc. } \\
\text { (ug/Kg soll) }\end{array}$ & $\begin{array}{c}\text { Element Avg. Std. Dov. } \\
\text { (ug/Kg soll) }\end{array}$ \\
\hline D11 & Mged & 40300 & 1970 & & \\
\hline D11 & Mgs5 & 33600 & 2510 & 37000 & 2240 \\
\hline D12 & Mge 4 & 118000 & 3800 & & \\
\hline D12 & Mges & 119000 & 2930 & 119000 & 3370 \\
\hline D13 & Mge4 & 56200 & 1710 & & \\
\hline D13 & Mges & 62000 & 2670 & 59100 & 2190 \\
\hline D13RA & Mge 4 & 69200 & 483 & & \\
\hline D13RA & Mges & 79000 & 1050 & 74100 & 767 \\
\hline D13RB & $M 024$ & 40400 & 381 & & \\
\hline D13RB & Mg25 & 45200 & 1060 & 42800 & 721 \\
\hline 021 & Mg24 & 160000 & 3100 & & \\
\hline D21 & Mges & 153000 & 2710 & 157000 & 2910 \\
\hline 041 & Mog4 & 118000 & 695 & & \\
\hline D41 & Mges & 140000 & 2320 & 129000 & 1510 \\
\hline D51 & Mge 4 & 93300 & 1640 & & \\
\hline D51 & Mo25 & 104000 & 20100 & 98700 & 10900 \\
\hline
\end{tabular}

Note: The actual concentration of samples for which no standard deviation be reported bs leas than the lower limit of detection. The reported concentration is the lower limit of quantification. 


\section{Transect D Soil Concentrations: Manganese}

\begin{tabular}{|c|c|c|c|c|c|}
\hline Sample ld. & leotope & $\begin{array}{l}\text { Concentration } \\
\text { (ug/Kg soll) }\end{array}$ & $\begin{array}{l}\text { Std.Deviation } \\
\text { (ug/Kg sodl) }\end{array}$ & $\begin{array}{l}\text { Element Avg. Conc. } \\
\text { (ug/Kg soil) }\end{array}$ & $\begin{array}{c}\text { Element Avg. Std. Dov. } \\
\text { (ug/Kg soll) }\end{array}$ \\
\hline D11 & $\mathrm{Mn}$ & 55800 & 3150 & 55800 & 3150 \\
\hline D12 & $\mathrm{Mn}$ & 101000 & 1750 & 101000 & 1750 \\
\hline D13 & $\mathrm{Mn}$ & 14600 & 231 & 14600 & 231 \\
\hline DI3RA & $\mathrm{Mn}$ & 11400 & 59.5 & 11400 & 59.5 \\
\hline DI3RB & $\mathrm{Mn}$ & 5540 & 147 & 5540 & 147 \\
\hline 021 & $\mathrm{Mn}$ & 183000 & 14400 & 183000 & 14400 \\
\hline$D 41$ & $\mathrm{Nn}$ & 228000 & 10700 & 228000 & 10700 \\
\hline D51 & $\mathrm{Mn}$ & 27500 & 2720 & 27500 & 2720 \\
\hline
\end{tabular}

Note: The ectual concentration of samples for which no standard doviation b reported be loes than the lower limit of detection. The reported concentration is the lower limit of quantification. 


\begin{tabular}{|c|c|c|c|c|c|}
\hline Sample ld. & teotope & $\begin{array}{l}\text { Concentration } \\
\text { (ug/Kg soli) }\end{array}$ & $\begin{array}{l}\text { Std.Deviation } \\
\text { (ug/Kg soil) }\end{array}$ & $\begin{array}{l}\text { Element Avg. Conc. } \\
\text { (ug/Kg soil) }\end{array}$ & $\begin{array}{c}\text { Element Avg. Std. Dov. } \\
\text { (ug/Kg soll) }\end{array}$ \\
\hline D11 & N160 & 6390 & 318 & & \\
\hline D11 & Ni62 & 5270 & 769 & 5830 & 544 \\
\hline D12 & Ni60 & 3360 & 160 & & \\
\hline D12 & Ni62 & 8560 & 595 & 5960 & 378 \\
\hline D13 & Ni60 & 1110 & 139 & & \\
\hline D13 & N62 & 727 & & 919 & 139 \\
\hline D13RA & $N 160$ & & & & \\
\hline D13RA & Ni62 & 5140 & 739 & 5140 & 739 \\
\hline D13AB & Niso & & & & \\
\hline D13RB & N162 & 2210 & 235 & 2210 & 235 \\
\hline D21 & Ni60 & 7660 & 367 & & \\
\hline D21 & Ni62 & 7730 & 87 & 7700 & 227 \\
\hline$D 41$ & $\mathrm{Ni} 6 \mathrm{O}$ & 2460 & 379 & & \\
\hline D41 & N162 & 4520 & 727 & 3490 & 553 \\
\hline D51 & Ni60 & 11100 & 3350 & & \\
\hline D51 & Ni62 & 16800 & 9490 & 14000 & 6420 \\
\hline
\end{tabular}




\section{Transect D Soil Concentrations: Selenium}

\begin{tabular}{|c|c|c|c|c|c|}
\hline Sample kd. & botope & $\begin{array}{l}\text { Concentration } \\
\text { (ug/Ko soll) }\end{array}$ & $\begin{array}{l}\text { Std.Doviation } \\
\text { (ug/Kg soll) }\end{array}$ & $\begin{array}{l}\text { Element Avg. Conc. } \\
\text { (ug/Kg soll) }\end{array}$ & $\begin{array}{c}\text { Element Avg. Std. Dov. } \\
\text { (ug/Kg soll) }\end{array}$ \\
\hline D11 & S०77 & 5300 & 2500 & & \\
\hline D11 & So82 & 4670 & 2340 & 4990 & 2420 \\
\hline D12 & So77 & 4960 & 2670 & & \\
\hline 012 & So82 & 4980 & 2640 & 4970 & 2660 \\
\hline D13 & So77 & 3640 & & & \\
\hline D13 & Sase & 3640 & & 3640 & \\
\hline D13RA & So77 & 15500 & 2000 & & \\
\hline D13RA & So82 & 15400 & 1930 & 15500 & 1970 \\
\hline D13RB & 5077 & 8250 & 978 & & \\
\hline D13RB & Se82 & 8100 & 872 & 8180 & 924 \\
\hline D21 & So $\pi$ & 6620 & 753 & & \\
\hline$D 21$ & So82 & 6410 & 1830 & 6520 & 1290 \\
\hline D41 & So77 & 8100 & 3000 & & \\
\hline D41 & Se82 & 7600 & 2830 & 7850 & 2920 \\
\hline D51 & So77 & 8810 & & & \\
\hline D51 & So82 & 8810 & & 8810 & \\
\hline
\end{tabular}

Note: The ectual concentration of samples for which no stendard deviation be reported be leas than the fowner limit of detection. The reportiod concentration is the lower limit of quantification. 


\begin{tabular}{|c|c|c|c|c|c|}
\hline Sample kd. & lsotope & $\begin{array}{l}\text { Concentration } \\
\text { (ug/Kg soll) }\end{array}$ & $\begin{array}{l}\text { Std.Deviation } \\
\text { (ug/Kg soli) }\end{array}$ & $\begin{array}{l}\text { Element Avg. Conc. } \\
\text { (ug/Kg soli) }\end{array}$ & $\begin{array}{c}\text { Element Avg. Std. Dov. } \\
\text { (ug/Kg soil) }\end{array}$ \\
\hline D11 & Ag107 & 693 & & & \\
\hline D11 & Ag109 & 653 & & 693 & \\
\hline D12 & Ag107 & $4 \infty 0$ & 235 & & \\
\hline D12 & Ag109 & 323 & 559 & 392 & 397 \\
\hline D13 & Ag107 & 364 & & & \\
\hline 013 & $A g 109$ & 364 & & 364 & \\
\hline D13RA & Ag107 & 4400 & 493 & & \\
\hline D13RA & $A 9109$ & 3390 & 183 & 3900 & 338 \\
\hline D13RB & Ag107 & 2830 & 1810 & & \\
\hline D13RB & Ag109 & 2000 & 948 & 2420 & 1380 \\
\hline D21 & Ag107 & 1160 & 178 & & \\
\hline$D 21$ & Ag109 & 647 & & 904 & 178 \\
\hline 041 & Ag107 & 1140 & 214 & & . \\
\hline D41 & Ag109 & 2690 & & 1920 & 214 \\
\hline D51 & Ag107 & 4400 & & & \\
\hline D51 & Ag109 & 4400 & & 4400 & \\
\hline
\end{tabular}

Note: The actual concentration of eamples for which no standard deviation bs reported is leas than the lower limit of dotection. The reported concentration ls the fower limit of quantification. 
Transect D Soil Concentrations: Sodium

\begin{tabular}{|c|c|c|c|c|c|}
\hline Sampte ld. & leotope & $\begin{array}{l}\text { Concantration } \\
\text { (ug/kg soll) }\end{array}$ & $\begin{array}{l}\text { StdDoviation } \\
\text { (ug/Kg soll) }\end{array}$ & $\begin{array}{l}\text { Element Avg. Conc. } \\
\text { (ug/Kg soll) }\end{array}$ & $\begin{array}{l}\text { Element Avg- std. Dov. } \\
\text { (ug/Kg soll) }\end{array}$ \\
\hline D11 & $\mathrm{Na}$ & 6930 & & 6930 & \\
\hline D12 & $\mathrm{Na}$ & 212000 & & 212000 & \\
\hline D13 & $\mathrm{Na}$ & 145000 & & 145000 & \\
\hline DI3RA & $\mathrm{Na}$ & 86500 & 26400 & 86900 & 26400 \\
\hline D13RB & $\mathrm{Na}$ & 131000 & & 131000 & \\
\hline D21 & $\mathrm{Na}$ & 6470 & & 6470 & \\
\hline D41 & $\mathrm{Na}$ & 6950 & & 6950 & \\
\hline D51 & $\mathrm{Na}$ & 176000 & & 176000 & \\
\hline
\end{tabular}

Note: The wetual concentration of eamples for which no stenderd deviation is reported ts leas than the lower llmit of detection. The reported concentration b the lower linit of quantification. 


\section{Transect D Soil Concentrations: Strontium}

\begin{tabular}{|c|c|c|c|c|c|}
\hline Sample ld. & botope & $\begin{array}{l}\text { Concentration } \\
\text { (ug/Kg soll) }\end{array}$ & $\begin{array}{l}\text { Std.Dovlation } \\
\text { (ug/Kg soll) }\end{array}$ & $\begin{array}{l}\text { Element Avg. Conc. } \\
\text { (ug/Kg soll) }\end{array}$ & $\begin{array}{c}\text { Element Avg. Std. Dov. } \\
\text { (ug/Kg soll) }\end{array}$ \\
\hline D11 & Sr & 6510 & 128 & 6510 & 128 \\
\hline D12 & $\mathrm{Sr}$ & 6480 & 156 & 6480 & 156 \\
\hline D13 & St & 6540 & 129 & 6540 & 129 \\
\hline DI3RA & $\mathrm{St}$ & 14300 & 100 & 14300 & 109 \\
\hline D13RB & Sr & 4260 & 31.4 & 4260 & 31.4 \\
\hline D21 & Sr & 8810 & 119 & 8810 & 119 \\
\hline D41 & $\mathrm{Sr}$ & 59000 & 717 & 59000 & 717 \\
\hline D51 & Sr & 15100 & 520 & 15100 & 520 \\
\hline
\end{tabular}




\section{Transect D Soil Concentrations: Thorium}

\begin{tabular}{|c|c|c|c|c|c|}
\hline Semple ld. & Lotope & $\begin{array}{l}\text { Concentratton } \\
\text { (ug/Kg soil) }\end{array}$ & $\begin{array}{l}\text { Stri.Deviation } \\
\text { (ug/Kg soll) }\end{array}$ & $\begin{array}{l}\text { Element Avg. Conc. } \\
\text { (Ug/Kg soll) }\end{array}$ & $\begin{array}{c}\text { Element Avg. Std. Dov. } \\
\text { (ug/Kg soll) }\end{array}$ \\
\hline D11 & Th232 & 622 & 9.77 & 622 & 9.77 \\
\hline D12 & Th232 & 298 & 11.9 & 298 & 11.9 \\
\hline D13 & Th232 & 52.9 & 6.8 & 52.9 & 6.8 \\
\hline D13RA & Th232 & 65.4 & & 65.4 & \\
\hline D13RB & Th232 & 107 & 11.9 & 107 & 11.9 \\
\hline D21 & Th232 & 55.7 & 132 & 55.7 & 13.2 \\
\hline D41 & Th232 & 69.5 & & 69.5 & \\
\hline D51 & Th232 & 85.8 & 61 & 85.8 & 61 \\
\hline
\end{tabular}

Note: The actual concentration of samples for which no standard deviation is reported is hes than the lower limlt of detection. The reported concentration is the fower limit of quantification. 


\section{Transect D Soil Concentrations: Tin}

\begin{tabular}{|c|c|c|c|c|c|}
\hline Sample ld. & Lotope & $\begin{array}{l}\text { Concentration } \\
\text { (ug/Kg soll) }\end{array}$ & $\begin{array}{l}\text { Std.Doviation } \\
\text { (ug/Kg soil) }\end{array}$ & $\begin{array}{l}\text { Element Avg. Conc. } \\
\text { (ug/Kg soll) }\end{array}$ & $\begin{array}{c}\text { Element Avg. Std. Dov. } \\
\text { (ug/Kg soll) }\end{array}$ \\
\hline D11 & $\sin 118$ & 430 & 77.4 & & \\
\hline D11 & Sn 120 & 484 & 27.1 & 457 & 52.3 \\
\hline D12 & $\sin 118$ & 222 & 79.1 & & \\
\hline D12 & $\sin 120$ & 381 & 101 & 305 & 90.1 \\
\hline D13 & $\operatorname{Sn} 118$ & 151 & 43.3 & & \\
\hline D13 & $\operatorname{sn} 120$ & 246 & 33.9 & 199 & 38.6 \\
\hline D13RA & Sn118 & 1170 & 43.8 & & \\
\hline D13RA & $\sin 120$ & 1490 & 862 & 1350 & 65 \\
\hline D13RB & $\sin 118$ & 886 & 235 & & \\
\hline D13RB & $\operatorname{sn} 120$ & 536 & 146 & 711 & 191 \\
\hline$D 21$ & $\operatorname{Sn} 118$ & 274 & 32.8 & & \\
\hline$D 21$ & $\sin 120$ & 64.7 & & 169 & 32.8 \\
\hline D41 & $\operatorname{sn} 118$ & 313 & 812 & & \\
\hline D41 & $\operatorname{Sn} 120$ & 332 & 76.8 & 323 & 79 \\
\hline D51 & Sn118 & 626 & 182 & & \\
\hline D51 & Sn120 & 179 & 92.6 & 403 & 137 \\
\hline
\end{tabular}

Note: The ectual concantration of samples for which no standard doviation ls reported is leas than the lower limlt of detection. The reported concentration is the lower limit of quantification. 


\section{Transect D Soil Concentrations: Uranium-235}

Semple k. Lotope $\begin{gathered}\text { Concentration } \\
\text { (Ug/Kg soli) }\end{gathered}$
\begin{tabular}{|c|c|c|c|c|c|}
\hline (Ug/Kg soil) & $\begin{array}{c}\text { Std.Deviation } \\
\text { (ug/Kg soil) }\end{array}$ & $\begin{array}{c}\text { Element Avg. Std. Dov. } \\
\text { (Ug/Kg soil) }\end{array}$ \\
\hline D11 & U235 & 25 & & 25 & 5.21 \\
\hline D12 & U235 & 38.4 & 5.21 & 38.4 & 4.41 \\
\hline D13 & U235 & 23.5 & 4.41 & 23.5 & 2.71 \\
\hline D13RA & U235 & 9.58 & 2.71 & 9.58 & 2.45 \\
\hline D13RB & U235 & 16.9 & 2.45 & 16.9 & 3.22 \\
\hline D21 & U235 & 10.8 & 3.22 & 10.8 & 1.8 \\
\hline D41 & U235 & 15.9 & 1.8 & 15.9 & \\
\hline D51 & U235 & & & & \\
\hline
\end{tabular}




\section{Transect D Soil Concentrations: Uranium-238}

\begin{tabular}{|c|c|c|c|c|c|}
\hline Semple id. & leotope & $\begin{array}{l}\text { Concentration } \\
\text { (ug/Kg soll) }\end{array}$ & $\begin{array}{l}\text { Std.Devlation } \\
\text { (ug/Kg soil) }\end{array}$ & $\begin{array}{l}\text { Element Avg. Conc. } \\
\text { (ug/Kg soll) }\end{array}$ & $\begin{array}{c}\text { Element Avg. Std. Dev. } \\
\text { (ug/Kg soll) }\end{array}$ \\
\hline D11 & U238 & 894 & 172 & 894 & 172 \\
\hline D12 & U238 & 9370 & 104 & 9370 & 104 \\
\hline D13 & U238 & 2930 & 64.3 & 2930 & 64.3 \\
\hline D13RA & U238 & 3520 & 137 & 3520 & 137 \\
\hline D13RB & 4238 & 2710 & 94.5 & 2710 & 94.5 \\
\hline $\mathrm{D} 21$ & U238 & 1690 & 562 & 1690 & 562 \\
\hline D41 & U238 & 2100 & 462 & 2100 & 462 \\
\hline D51 & U238 & 1900 & 122 & 1900 & 122 \\
\hline
\end{tabular}

Noto: The actual concentration of eamples for which no standard doviation b reported is leas than the fower limit of detection. The reported concentration le the lower limit of quantification. 


\section{Transect D Soil Concentrations: Vanadium}

\begin{tabular}{|c|c|c|c|c|c|}
\hline Semple ld. & Eotope & $\begin{array}{l}\text { Concentration } \\
\text { (ug/Ko soll) }\end{array}$ & $\begin{array}{l}\text { Std.Doviation } \\
\text { (Uakko soll) }\end{array}$ & $\begin{array}{l}\text { Element Avg. Conc. } \\
\text { (ug/kg eoll) }\end{array}$ & $\begin{array}{c}\text { Element Avg. Std. Dov. } \\
\text { (ug/Kg soil) }\end{array}$ \\
\hline D11 & $V$ & 8350 & 630 & 8350 & 639 \\
\hline D12 & $\mathbf{V}$ & 16300 & 228 & 16300 & 228 \\
\hline 013 & $v$ & 6830 & 281 & 6850 & 281 \\
\hline D13RA & $v$ & 11400 & 91.8 & 11400 & 91.9 \\
\hline D13RB & $\mathrm{v}$ & 8000 & 160 & 8000 & 169 \\
\hline D21 & $V$ & 9520 & $17 \oplus 0$ & 9520 & 1760 \\
\hline D41 & V & 14600 & 1670 & 14600 & 1670 \\
\hline$D 51$ & $V$ & 13200 & 1090 & 13200 & 1080 \\
\hline
\end{tabular}




\begin{tabular}{|c|c|c|c|c|c|}
\hline Sample ld. & leotope & $\begin{array}{l}\text { Concentration } \\
\text { (Lg/Kg soli) }\end{array}$ & $\begin{array}{l}\text { Std.Doviation } \\
\text { (ug/Kg soil) }\end{array}$ & $\begin{array}{l}\text { Element Avg. Conc. } \\
\text { (ug/Kg soll) }\end{array}$ & $\begin{array}{c}\text { Element Avg. Std. Dov. } \\
\text { (ug/Kg soll) }\end{array}$ \\
\hline D11 & $Y$ & 866 & 44.6 & 866 & 44.6 \\
\hline D12 & $Y$ & 684 & 18.7 & 684 & 18.7 \\
\hline D13 & $Y$ & 138 & 10.6 & 138 & 10.6 \\
\hline D13RA & $Y$ & 279 & 15.8 & 279 & 15.8 \\
\hline D13RB & $Y$ & 144 & 10.9 & 144 & 10.9 \\
\hline D21 & $Y$ & 374 & 14.5 & 374 & 14.5 \\
\hline D41 & $Y$ & 292 & 33.1 & 292 & 33.1 \\
\hline D51 & $\bar{Y}$ & 228 & 127 & 228 & 127 \\
\hline
\end{tabular}

Note: The sctural concentration of eamples for which no standard deviatton is reported is leas than the lower limit of detection. The reported concentration is the lower limit of quantification. 


\section{Transect D Soil Concentrations: Zinc}

\begin{tabular}{|c|c|c|c|c|c|}
\hline Sempte ld. & botope & $\begin{array}{l}\text { Concentration } \\
\text { (ug/Kg soll) }\end{array}$ & $\begin{array}{l}\text { Std.Deviation } \\
\text { (ug/Kg soil) }\end{array}$ & $\begin{array}{l}\text { Element Avg. Conc. } \\
\text { (ug/Kg soil) }\end{array}$ & $\begin{array}{c}\text { Element Avg. Std. Dov. } \\
\text { (ug/Kg eoll) }\end{array}$ \\
\hline D19 & Zn66 & 18000 & 897 & 18000 & 897 \\
\hline$\overline{D 12}$ & Zn66 & 4330 & 440 & 4330 & 440 \\
\hline D13 & Zn66 & 782 & 555 & 782 & 555 \\
\hline D13RA & Zn66 & 6290 & 141 & 6290 & 141 \\
\hline D13RB & Zn66 & 4880 & 96.1 & 4880 & 96.1 \\
\hline D21 & $2 n 66$ & 7640 & 353 & 7640 & 353 \\
\hline D41 & 206 & 7500 & 382 & 7500 & 382 \\
\hline D51 & Zn66 & 5290 & 1180 & 5290 & 1180 \\
\hline
\end{tabular}

Note: The actual concentration of samples for which no standard deviation bs reported is leas than the fower limit of detection. The reported concentration is the lower limit of quantification. 


\begin{tabular}{|c|c|c|c|c|c|}
\hline Sample id. & botope & $\begin{array}{l}\text { Concentration } \\
\text { (ug/Kg soll) }\end{array}$ & $\begin{array}{l}\text { Sid.Doviation } \\
\text { (ug/Kg soil) }\end{array}$ & $\begin{array}{l}\text { Element Avg. Conc. } \\
\text { (ug/Kg sold) }\end{array}$ & $\begin{array}{c}\text { Element Avg. Std. Dov. } \\
\text { (Ug/Kg soll) }\end{array}$ \\
\hline D11 & 2500 & 33400 & 1190 & & \\
\hline D11 & 291 & 38800 & 1080 & 36100 & 1140 \\
\hline D12 & $Z 190$ & 13100 & 316 & & \\
\hline D12 & 2991 & 25400 & 733 & 19300 & 525 \\
\hline D13 & Zrso & 15600 & 232 & & \\
\hline D13 & Zr91 & 13900 & 524 & 14800 & 378 \\
\hline D13RA & $2 r 90$ & 19200 & 270 & & \\
\hline D13RA & Zro1 & 18200 & 1180 & 18700 & 725 \\
\hline D13RB & Zrso & 10300 & 231 & & \\
\hline D13RB & Zr91 & 9470 & 457 & 9890 & 344 \\
\hline$D 21$ & 280 & 28100 & 606 & & \\
\hline D21 & Z91 & 29700 & 443 & 28900 & 525 \\
\hline 041 & Zr9o & 33400 & 469 & & $\cdot$ \\
\hline 041 & 299 & 40100 & 741 & 36800 & 605 \\
\hline D51 & 2590 & 24400 & 4420 & & \\
\hline DS1 & Zr91 & 31600 & 9070 & 28000 & 6750 \\
\hline
\end{tabular}




\section{Transect E Soil Concentrations: Aluminum}

\begin{tabular}{|c|c|c|c|c|c|}
\hline Sample ld. & leotope & $\begin{array}{l}\text { Concentration } \\
\text { (ug/kg soll) }\end{array}$ & $\begin{array}{l}\text { Std.Doviation } \\
\text { (ug/Ko soll) }\end{array}$ & $\begin{array}{l}\text { Element Avg. Conc. } \\
\text { (ug/Kg soll) }\end{array}$ & $\begin{array}{c}\text { Element Avg. Std. Dov. } \\
\text { (ug/Kg soil) }\end{array}$ \\
\hline E11 & A & 48800000 & 542000 & 48800000 & 542000 \\
\hline E12 & $\overline{\mathbf{A}}$ & 5940000 & 34900 & 5940000 & 34900 \\
\hline E12R & $\mathbf{A}$ & 18700000 & 37200 & 18700000 & 37200 \\
\hline E13 & $\mathbf{A}$ & 8530000 & 115000 & 8530000 & 115000 \\
\hline E14 & $\mathbf{A}$ & 5040000 & 68200 & 5040000 & 68200 \\
\hline E21 & $\mathbf{N}$ & 30100000 & 427000 & 30100000 & 427000 \\
\hline E22 & $\mathbf{A}$ & 27700000 & 320000 & 27700000 & 320000 \\
\hline E23 & A & 12900000 & 448000 & 12900000 & 448000 \\
\hline E23R & A & 6180000 & 46500 & 6180000 & 46500 \\
\hline E24 & $\mathbf{A}$ & 4310000 & 207000 & 4310000 & 207000 \\
\hline E31 & $\mathbf{A}$ & 65200000 & 298000 & 65200000 & 298000 \\
\hline$E 32$ & $\mathbf{A}$ & 56100000 & 818000 & 56100000 & 818000 \\
\hline$E 33$ & $\mathbf{A}$ & 3410000 & 66300 & 3410000 & 86300 \\
\hline E34 & $\mathbf{A}$ & 2590000 & 46700 & 2590000 & 46700 \\
\hline E41 & $\mathbf{A}$ & 44300000 & 487000 & 44300000 & 487000 \\
\hline$E 42$ & $\mathbf{A}$ & 29600000 & 373000 & 29600000 & 373000 \\
\hline
\end{tabular}

Note: The ectual concentration of samples for which no standerd doviation is reported be kes than the fowrer limit of detection. The reported concentration is the lower limit of quantification. 


\section{Transect E Soil Concentrations: Arsenic}

\begin{tabular}{|c|c|c|c|c|c|}
\hline Samplo ld. & botope & $\begin{array}{l}\text { Concentration } \\
\text { (ug/Kg soil) }\end{array}$ & $\begin{array}{l}\text { Std.Dovlation } \\
\text { (ug/Kg soll) }\end{array}$ & $\begin{array}{l}\text { Element Avg. Conc. } \\
\text { (ug/Kg soll) }\end{array}$ & $\begin{array}{c}\text { Element Avg. Std. Dov. } \\
\text { (ug/Kg soll) }\end{array}$ \\
\hline E11 & As & 7210 & 4000 & 7210 & 4000 \\
\hline E12 & As & 2660 & & 2660 & \\
\hline E12R & As & 58800 & & 58800 & \\
\hline E13 & As & 4970 & 790 & 4970 & 790 \\
\hline E14 & As & 2680 & 152 & 2680 & 152 \\
\hline E21 & As & 2120 & 787 & 2120 & 787 \\
\hline E22 & As & 6300 & 292 & 6300 & 292 \\
\hline E23 & As & 4140 & 371 & 4140 & 371 \\
\hline E23A & As & 29400 & & 29400 & \\
\hline E24 & As & 3080 & & 3080 & \\
\hline E31 & As & 6450 & 1790 & 6450 & 1790 \\
\hline E32 & As & 9040 & 742 & 9040 & 742 \\
\hline E33 & As & 22600 & 2190 & 22600 & 2190 \\
\hline E34 & As & 3330 & & 3330 & \\
\hline E41 & As & 20900 & 428 & 20900 & 428 \\
\hline E42 & As & 2960 & 746 & 2960 & 746 \\
\hline
\end{tabular}

Note: The actual concentration of eamptes for which no standard deviation be reported is leas than the lower limit of detection. The reported concentration is the lower limlt of quantification. 


\section{Transect E Soil Concentrations: Barium}

\begin{tabular}{|c|c|c|c|c|c|}
\hline Sample ld. & botope & $\begin{array}{l}\text { Concentration } \\
\text { (ug/Kg soll) }\end{array}$ & $\begin{array}{l}\text { Std.Doviation } \\
\text { (4g/Kg soll) }\end{array}$ & $\begin{array}{l}\text { Element Avg. Conc. } \\
\text { (ug/Kg eoll) }\end{array}$ & $\begin{array}{c}\text { Element Avg. Std. Dov. } \\
\text { (ug/Kg soll) }\end{array}$ \\
\hline
\end{tabular}

\begin{tabular}{|c|c|c|c|c|c|}
\hline E11 & Ba135 & 366000 & 6230 & & \\
\hline E11 & Ba137 & 493000 & 5020 & & \\
\hline E11 & Ba138 & 563000 & 4010 & 474000 & 5090 \\
\hline E12 & Ba135 & 33200 & 3200 & & \\
\hline E12 & Ba137 & 25300 & 968 & & \\
\hline E12 & Ba138 & 37300 & 985 & 31900 & 1720 \\
\hline E12R & Ba135 & 41200 & 890 & & \\
\hline E12R & Ba137 & 41300 & 945 & & \\
\hline E12R & Ba138 & 41300 & 384 & 41300 & 740 \\
\hline E13 & Ba135 & 244000 & 10300 & & \\
\hline E13 & Ba137 & 265000 & 4480 & & \\
\hline E13 & Ba138 & 258000 & 1860 & 256000 & 5550 \\
\hline E14 & Ba135 & 45500 & 836 & & . \\
\hline E14 & Ba137 & 45400 & 795 & & \\
\hline E14 & Ba138 & 57100 & 522 & 49300 & 718 \\
\hline E21 & Ba135 & 23300 & 373 & & \\
\hline E21 & Ba137 & 26200 & 1270 & & \\
\hline E21 & Ba138 & 27000 & 166 & 25500 & 600 \\
\hline E22 & Ba135 & 22900 & 2290 & & \\
\hline E22 & Ba137 & 26500 & 2690 & & \\
\hline E22 & Ba138 & 25500 & 306 & 25000 & 1760 \\
\hline E23 & Ba135 & 145000 & 2450 & & \\
\hline E23 & Ba137 & 142000 & 1670 & & \\
\hline$E 23$ & Ba138 & 150000 & 1810 & 146000 & 1980 \\
\hline E23R & Ba135 & 144000 & 2390 & & \\
\hline E23R & Ba137 & 133000 & 250 & & \\
\hline E23R & Ba138 & 131000 & 868 & 136000 & 1170 \\
\hline E24 & Ba135 & 182000 & 3000 & & \\
\hline E24 & Ba137 & 151000 & 3710 & & \\
\hline E24 & Ba138 & 148000 & 1330 & 160000 & 2680 \\
\hline E31 & Ba135 & 48100 & 1470 & & \\
\hline E31 & Ba137 & 154000 & 1490 & & \\
\hline E31 & Ba138 & 54900 & 397 & 85700 & 1120 \\
\hline E32 & Ba135 & 34800 & 1700 & & \\
\hline$E 32$ & Ba137 & 39100 & 1220 & & \\
\hline E32 & Ba138 & 43000 & 478 & 39000 & 1130 \\
\hline$E 33$ & Ba135 & 150000 & 3870 & & \\
\hline E33 & Ba137 & 136000 & 1480 & & \\
\hline$E 33$ & Ba138 & 121000 & 944 & 136000 & 2100 \\
\hline E34 & Ba135 & 69500 & 302 & & \\
\hline E34 & Ba137 & 91900 & 717 & & \\
\hline E34 & Ba138 & 81500 & 916 & 81000 & 645 \\
\hline$E 41$ & Be135 & 60500 & 541 & & \\
\hline E41 & $8 a 137$ & 82000 & 423 & & \\
\hline
\end{tabular}

Note: The setual concentration of eamples for which no standard deviation te reported bs leas than the lower llmit of dotection. The reported concentration is the lower limit of quantincation. 


\section{Transect E Soil Concentrations: Barium}

\begin{tabular}{|c|c|c|c|c|c|}
\hline Sampte ld. & Eotope & $\begin{array}{l}\text { Concentration } \\
\text { (ug/Kg soll) }\end{array}$ & $\begin{array}{l}\text { Stu.Doviation } \\
\text { (ug/Kg soil) }\end{array}$ & $\begin{array}{l}\text { Element Avg. Conc. } \\
\text { (ug/Kg soll) }\end{array}$ & $\begin{array}{c}\text { Element Avg. Std. Dov. } \\
\text { (ug/Kg soll) }\end{array}$ \\
\hline E41 & Ba138 & 54800 & 29.5 & 65800 & 331 \\
\hline E42 & Ba135 & 407000 & 29000 & & \\
\hline E42 & Ba137 & 393000 & 23300 & & \\
\hline E42 & Ba138 & 470000 & 31800 & 423000 & 28000 \\
\hline
\end{tabular}

Note: The ectual concentration of samples for which no standard deviatton is reported be leas than the lower limit of detection. The reported concentration is the lower limit of quantification. 


\section{Transect E Soil Concentrations: Cadmium}

\begin{tabular}{|c|c|c|c|c|c|}
\hline Sample ld. & leotope & $\begin{array}{l}\text { Concentration } \\
\text { (ug/Kg roll) }\end{array}$ & $\begin{array}{l}\text { Std.Doviation } \\
\text { (ug/Ko soll) }\end{array}$ & $\begin{array}{l}\text { Element Avg. Conc. } \\
\text { (ug/Ko edr) }\end{array}$ & $\begin{array}{l}\text { Element Avg. Std. Dov. } \\
(\mathrm{\omega g} / \mathrm{Kg} g \mathrm{eOll})\end{array}$ \\
\hline E11 & Cd111 & 124 & & & \\
\hline E11 & $\operatorname{Cod114}$ & 124 & & 124 & \\
\hline E12 & Cd111 & 532 & & & \\
\hline E12 & $\operatorname{cod114}$ & 53.2 & & 532 & \\
\hline E12R & Cod111 & 399 & 216 & & \\
\hline E12R & Cod114 & 230 & 43.6 & 315 & 130 \\
\hline E13 & Cod111 & 77.3 & & & \\
\hline E13 & $\operatorname{Cd114}$ & 77.3 & & 77.3 & \\
\hline E14 & Col119 & 207 & 110 & & \\
\hline E14 & Cod114 & 81.7 & 61.1 & 140 & 85.6 \\
\hline E21 & Cod111 & 71.3 & & & \\
\hline E21 & Cd114 & 71.3 & & 71.3 & \\
\hline$E 22$ & Cod111 & 126 & & & \\
\hline$E 22$ & Cd114 & 126 & & 126 & \\
\hline$E 23$ & Cod111 & 398 & 104 & & \\
\hline E23 & Co114 & 272 & 482 & 335 & 76.1 \\
\hline E23R & $\operatorname{Col111}$ & 356 & 8.68 & & \\
\hline E23R & $\operatorname{Cot114}$ & 239 & 120 & 298 & 64.3 \\
\hline E24 & Col111 & 61.6 & & & \\
\hline E24 & $\operatorname{Col114}$ & 94.9 & 322 & 78.3 & 322 \\
\hline E31 & $\operatorname{Col111}$ & 151 & 65.4 & & \\
\hline E31 & $\operatorname{Cd114}$ & 124 & 84.6 & 138 & 75 \\
\hline E32 & Cd111 & 85.4 & .97 & & \\
\hline E32 & Cd114 & 148 & 36.6 & 117 & 18.8 \\
\hline E33 & Cd111 & 236 & 27 & & \\
\hline$E 33$ & Cd114 & 381 & 105 & 300 & 53.9 \\
\hline E34 & Cd111 & 66.6 & & & \\
\hline E34 & Cd114 & 66.6 & & 66.6 & \\
\hline E41 & Cd111 & 30.6 & 7.58 & & \\
\hline E41 & Cd114 & 101 & 35.8 & 65.8 & 21.7 \\
\hline$E 42$ & Cd111 & 271 & 89.8 & & \\
\hline$E_{42}$ & $\operatorname{Cd} 114$ & 86.9 & 3.75 & 179 & 46.8 \\
\hline
\end{tabular}

Note: The ectual concentration of samples for which no standard deviation be reported bs leas than the lowner limit of detection. The reported concentratton bs the lower Himlt of quantification. 


\section{Transect E Soil Concentrations: Calcium}

\begin{tabular}{|c|c|c|c|c|c|}
\hline Sample kd. & leotope & $\begin{array}{l}\text { Concentration } \\
\text { (ug/kg soll) }\end{array}$ & $\begin{array}{l}\text { Std.Doviation } \\
\text { (4g/Ko soll) }\end{array}$ & $\begin{array}{l}\text { Elemment Avg. Conc. } \\
\text { (ug/Kg soil) }\end{array}$ & $\begin{array}{c}\text { Element Avg. Std. Dov. } \\
\text { (4g/Kg soll) }\end{array}$ \\
\hline E11 & $\operatorname{Ca} 44$ & 1590000 & 132000 & 1590000 & 132000 \\
\hline E12 & $\cos 44$ & 403000 & 85900 & 493000 & 85900 \\
\hline E12R & Ca44 & 294000 & & 294000 & \\
\hline E13 & CeA44 & 155000 & & 155000 & \\
\hline E14 & $\cos 44$ & 268000 & & 268000 & \\
\hline E21 & CaA4 & 867000 & 112000 & 867000 & 112000 \\
\hline$E 22$ & $C_{344}$ & 935000 & 145000 & 935000 & 145000 \\
\hline$E 23$ & $\operatorname{Ca} 44$ & 5250000 & 203000 & 5250000 & 203000 \\
\hline E23R & Cas4 & 977000 & 85100 & 977000 & 95100 \\
\hline E24 & Co44 & 61600 & & 61600 & \\
\hline E31 & Ca44 & 5300000 & 340000 & 5300000 & 340000 \\
\hline E32 & $\mathrm{Ca} 44$ & 3720000 & 336000 & 3720000 & 336000 \\
\hline E33 & $\mathrm{C}_{844}$ & 1230000 & 301000 & 1230000 & 301000 \\
\hline E34 & Co44 & 1030000 & 72200 & 1030000 & 72200 \\
\hline E41 & Ca44 & 1660000 & 119000 & 1660000 & 119000 \\
\hline E42 & Cas4 & 116000 & & 116000 & \\
\hline
\end{tabular}

Note: The actual concentration of eamples for which no standard deviation le reported bs lese than the lower limit of detection. The reported concentration is the lower umit of quantification. 


\section{Transect E Soil Concentrations: Cesium}

\begin{tabular}{|c|c|c|c|c|c|}
\hline Sample ld. & botope & $\begin{array}{l}\text { Concentration } \\
\text { (ug/Ko soll) }\end{array}$ & $\begin{array}{l}\text { StdDoviation } \\
\text { (ug/Ko soll) }\end{array}$ & $\begin{array}{l}\text { Ehament Avg. Cone. } \\
\text { (ug/Kg soll) }\end{array}$ & $\begin{array}{l}\text { Element Avg. Std. Dev. } \\
\text { (ug/Kg soll) }\end{array}$ \\
\hline E11 & Cs & 1540 & 61.7 & 1540 & 61.7 \\
\hline E12 & Cs & 350 & 78.8 & 350 & 78.8 \\
\hline E12R & $\mathrm{Cs}$ & 205 & 23.6 & 205 & 23.6 \\
\hline E13 & Cs & 556 & 17.7 & 556 & 17.7 \\
\hline E14 & Cs & 188 & 24.4 & 188 & 24.4 \\
\hline E21 & Cs & 778 & 30.5 & 778 & 30.5 \\
\hline E22 & Cs & 1020 & 46.9 & 1020 & 46.9 \\
\hline E23 & $\mathrm{Cs}_{8}$ & 503 & 52.1 & 503 & 52.1 \\
\hline E23R & $\mathrm{Cs}$ & 376 & 8.73 & 376 & 8.73 \\
\hline E24 & Cs & 425 & 25.9 & 425 & 25.9 \\
\hline E31 & $\mathrm{Cs}$ & 1830 & 41.5 & 1830 & 41.5 \\
\hline$E 32$ & Cs & 1180 & 64 & 1180 & 64 \\
\hline E33 & Cs & 1320 & 92.6 & 1320 & 92.6 \\
\hline E34 & $\mathrm{Cs}$ & 400 & 32.8 & 400 & 32.8 \\
\hline E41 & Cs & 2240 & 66.6 & 2240 & 66.6 \\
\hline$E 42$ & Cs & 1840 & 157 & 1840 & 157 \\
\hline
\end{tabular}




\section{Transect E Soil Concentrations: Chromium}

\begin{tabular}{|c|c|c|c|c|c|}
\hline Sampte ld. & botope & $\begin{array}{l}\text { Concentration } \\
\text { (ug/Kg soil) }\end{array}$ & $\begin{array}{l}\text { Str.Doviation } \\
\text { (ug/Kg soil) }\end{array}$ & $\begin{array}{l}\text { Element Avg. Cons. } \\
\text { (ug/Kg soli) }\end{array}$ & $\begin{array}{c}\text { Element Avg. Std. Dov. } \\
\text { (ug/Kg soll) }\end{array}$ \\
\hline E11 & C.52 & 37300 & 916 & & \\
\hline$E 11$ & Cr53 & 36900 & 920 & 37100 & 918 \\
\hline$E 12$ & C.52 & 4500 & 428 & & \\
\hline E12 & C.53 & 4460 & 429 & 4480 & 429 \\
\hline E12R & C.52 & 4160 & 142 & & \\
\hline E12R & G53 & 4170 & 142 & 4170 & 142 \\
\hline E13 & C.52 & 5410 & 226 & & \\
\hline $\mathrm{E} 13$ & C.53 & 5340 & 223 & 5380 & 225 \\
\hline E14 & C.52 & 3540 & 138 & & \\
\hline E14 & C.53 & 3540 & 139 & 3540 & 139 \\
\hline E21 & C.52 & 15800 & 295 & & \\
\hline E21 & C.53 & 15700 & 295 & 15800 & 295 \\
\hline E22 & 9.52 & 22400 & 719 & & \\
\hline E22 & 953 & 22300 & 707 & 22400 & 713 \\
\hline E23 & Cr52 & 12200 & 142 & & \\
\hline E23 & C.53 & 12300 & 143 & 12300 & 143 \\
\hline E23R & Cr52 & 6410 & 31.1 & & \\
\hline E23R & 953 & 6430 & 31.1 & 6420 & 31.1 \\
\hline E24 & Cr52 & 6570 & 262 & & \\
\hline E24 & C.53 & 6570 & 260 & 6570 & 261 \\
\hline E31 & C.52 & 41400 & 43.8 & & \\
\hline E31 & Cr53 & 49900 & 192 & 45700 & 118 \\
\hline E32 & C.52 & 41400 & 447 & & \\
\hline E32 & $a 53$ & 41800 & 451 & 41600 & 449 \\
\hline E33 & C.52 & 17500 & 600 & & \\
\hline E33 & C.53 & 16700 & 598 & 17100 & 599 \\
\hline E34 & C.52 & 6570 & 515 & & \\
\hline E34 & 9.53 & 6570 & 510 & 6570 & 513 \\
\hline E41 & C.52 & 48700 & 719 & & \\
\hline E41 & C.53 & & & 48700 & 719 \\
\hline E42 & C.52 & 24000 & 1070 & & \\
\hline E42 & C.53 & 24000 & 1070 & 24000 & 1070 \\
\hline
\end{tabular}

Note: The ectual concentration of samples for which no standard deviabion la reported is lose than the lower llmit of detection. The reported concentration to the lower limit of quantification. 
Transect E Soil Concentrations: Cobalt

\begin{tabular}{|c|c|c|c|c|c|}
\hline Semple kd. & Eotope & $\begin{array}{l}\text { Concentration } \\
\text { (ug/Kg soil) }\end{array}$ & $\begin{array}{l}\text { Str.Deviation } \\
\text { (ug/Kg soll) }\end{array}$ & $\begin{array}{l}\text { Element Avg. Conc. } \\
\text { (ug/Kg coll) }\end{array}$ & $\begin{array}{c}\text { Element Avg. Std. Dov. } \\
\text { (4g/Kg soll) }\end{array}$ \\
\hline E11 & Co & 1880 & 280 & 1880 & 280 \\
\hline E12 & Co & 378 & 146 & 378 & 146 \\
\hline E12R & Co & 272 & 34.1 & 272 & 34.1 \\
\hline E13 & $C_{0}$ & 536 & 26.6 & 536 & 26.6 \\
\hline E14 & $\infty$ & 389 & 97.8 & 389 & 97.8 \\
\hline E21 & $C_{0}$ & 1470 & 30.7 & 1470 & 30.7 \\
\hline$E 22$ & Co & 1370 & 88.3 & 1370 & 88.3 \\
\hline E23 & Co & 3200 & 61.3 & 3200 & 61.3 \\
\hline E23R & $\infty$ & 400 & $\pi$ & 400 & $\pi$ \\
\hline E24 & $\infty$ & 1240 & 98.4 & 1240 & 98.4 \\
\hline E31 & Co & 2950 & 66.7 & 2950 & 66.7 \\
\hline E32 & Co & 1590 & 13 & 1590 & 12 \\
\hline E33 & $C_{0}$ & 420 & 291 & 420 & $\bar{z}$ \\
\hline E34 & Co & 271 & 72.2 & 271 & $?$ \\
\hline E41 & Co & 1970 & 75.8 & 1970 & 75 \\
\hline E42 & Co & 1120 & 81.6 & 1120 & 81.6 \\
\hline
\end{tabular}




\section{Transect E Soil Concentrations: Copper}

\begin{tabular}{|c|c|c|c|c|c|}
\hline Sample kd. & ketope & $\begin{array}{l}\text { Concentration } \\
\text { (ug/kg soll) }\end{array}$ & $\begin{array}{l}\text { StdDoviation } \\
\text { (Lg/kg edll) }\end{array}$ & $\begin{array}{l}\text { Element Avg. Conc. } \\
\text { (ug/Kg goll) }\end{array}$ & $\begin{array}{l}\text { Element Avg. Std. Dov. } \\
\text { (ug/Kg soll) }\end{array}$ \\
\hline E11 & 0,63 & 12100 & 867 & & \\
\hline$E 11$ & 0,65 & 8630 & 804 & 10400 & 886 \\
\hline$E 12$ & Cu63 & 2230 & 156 & & \\
\hline E12 & 0.65 & 1950 & 760 & 2000 & 458 \\
\hline E12R & Cu63 & & & & \\
\hline E12R & Cu65 & 5930 & 1790 & 5930 & 1790 \\
\hline$E 13$ & Cu63 & 77.3 & & & \\
\hline$E 13$ & 0465 & 77.3 & & 77.3 & \\
\hline E14 & $a \sqrt{63}$ & & & & \\
\hline E14 & Cu65 & 2260 & 710 & 2260 & 710 \\
\hline E21 & Cu63 & 149000 & 296 & & \\
\hline E21 & Cu65 & 5110 & 246 & 77100 & 271 \\
\hline E22 & Cu63 & 7120 & 683 & & . \\
\hline E22 & Cu65 & 8310 & 586 & 7720 & 635 \\
\hline E23 & Cu63 & 2900 & 123 & & \\
\hline $\mathrm{E} 23$ & Qu65 & 5980 & 63.8 & 4440 & 93.4 \\
\hline E23A & Cu63 & & & & \\
\hline E23R & Cu65 & 31600 & 281 & 31600 & 281 \\
\hline E24 & Cu63 & 2980 & 44.1 & & \\
\hline E24 & Cu65 & 4940 & 155 & 3960 & 99.6 \\
\hline E31 & Cu63 & 8320 & 150 & & \\
\hline E31 & Cu65 & 22700 & 978 & 15500 & 568 \\
\hline E32 & Cu63 & 10600 & 702 & & \\
\hline E32 & Cu65 & 20900 & 804 & 15800 & 437 \\
\hline E33 & Cu63 & 18800 & 640 & & \\
\hline E33 & Cu65 & 10000 & 757 & 14400 & 690 \\
\hline E34 & Cu63 & 66.6 & & & \\
\hline E34 & Cu65 & 66.6 & & 66.6 & \\
\hline E41 & Cu63 & 8320 & 248 & & \\
\hline E41 & Cu65 & 9680 & 880 & 8000 & 469 \\
\hline E42 & Cu63 & 5080 & 450 & & \\
\hline $\mathrm{E} 42$ & Cu65 & 4340 & 375 & 4710 & 413 \\
\hline
\end{tabular}




\section{Transect E Soil Concentrations: Iron}

\begin{tabular}{|c|c|c|c|c|c|}
\hline Sample ld. & botope & $\begin{array}{l}\text { Concentration } \\
\text { (Ug/Kg soll) }\end{array}$ & $\begin{array}{l}\text { Std.Doviation } \\
\text { (4q/Ko soll) }\end{array}$ & $\begin{array}{l}\text { Element Avg. Conc. } \\
\text { (ug/Ko coil) }\end{array}$ & $\begin{array}{c}\text { Element Avg. Std. Dov. } \\
\text { (Ug/Kg soll) }\end{array}$ \\
\hline E11 & Fo & 28600000 & 420000 & 28600000 & 420000 \\
\hline$E 12$ & Fo & 5940000 & 117000 & 5940000 & 117000 \\
\hline E12R & Fo & 7380000 & 22200 & 7380000 & 22200 \\
\hline E13 & Fo & 6140000 & 42900 & 6140000 & 42900 \\
\hline E14 & $\mathrm{Fo}$ & 4660000 & 41700 & 4660000 & 41700 \\
\hline E21 & Fo & 7710000 & 100000 & 7710000 & 100000 \\
\hline E22 & Fo & 11000000 & 184000 & 11000000 & 184000 \\
\hline E23 & Fo & 11000000 & 133000 & 11000000 & 133000 \\
\hline E23R & $F_{\theta}$ & 4670000 & 48400 & 4670000 & 48400 \\
\hline E24 & Fo & 4010000 & 17900 & 4010000 & 17900 \\
\hline E31 & $F_{\theta}$ & 23300000 & 247000 & 23300000 & 247000 \\
\hline E32 & Fo & 24800000 & 486000 & 24800000 & 496000 \\
\hline E33 & Fo & 2400000 & 15400 & 2400000 & 15400 \\
\hline E34 & $F_{0}$ & 1160000 & 31600 & 1160000 & 31600 \\
\hline E41 & $F_{0}$ & 90400000 & 3120000 & 90400000 & 3120000 \\
\hline$E 42$ & $F_{0}$ & 8580000 & 89700 & 8580000 & 89700 \\
\hline
\end{tabular}

Note: The actual concentration of samples for which no stendard doviation is reported be leas than the fower limit of dotection. The reported concentration b the lower Hinit of quantification. 
Transect E Soil Concentrations: Lead

\begin{tabular}{|c|c|c|c|c|c|}
\hline Semple id. & botopes & $\begin{array}{l}\text { Concentration } \\
\text { (ug/Kg eoll) }\end{array}$ & $\begin{array}{l}\text { Std.Devlatton } \\
\text { (ug/Kg soll) }\end{array}$ & $\begin{array}{l}\text { Element Avg. Conc. } \\
\text { (ug/Kg coll) }\end{array}$ & $\begin{array}{c}\text { Element Avg. Std. Dov. } \\
\text { (ug/Kg soll) }\end{array}$ \\
\hline E11 & Pb206 & 78600 & 2190 & & \\
\hline E11 & Pb207 & 75100 & 1760 & & \\
\hline E11 & Pb208 & 77000 & 302 & 76900 & 1420 \\
\hline E12 & Pto206 & 15800 & 545 & & \\
\hline E12 & Pb207 & 11800 & 236 & & \\
\hline E12 & Pb208 & 11200 & 340 & 12900 & 374 \\
\hline E12R & Pb206 & 2210 & 155 & & \\
\hline E12R & Pb207 & 2180 & 154 & & \\
\hline E12R & Pb208 & 1190 & 97.7 & 1860 & 136 \\
\hline E13 & Pb206 & 12100 & 720 & & \\
\hline E13 & Pb207 & 10200 & $\pi 74$ & & \\
\hline E13 & Pb208 & 7310 & 122 & 9870 & 539 \\
\hline E14 & Pb206 & 1450 & 44.1 & & \\
\hline E14 & Pb207 & 2480 & 232 & & \\
\hline E14 & Pb208 & 1660 & 45.9 & 1860 & 107 \\
\hline E21 & Pb206 & 46200 & 975 & & \\
\hline E21 & Pb207 & 46700 & 738 & & \\
\hline E21 & Pb208 & 46900 & 388 & 46600 & 700 \\
\hline E22 & Pb206 & 40500 & 1150 & & \\
\hline E22 & Pb207 & 44000 & 1350 & & \\
\hline E22 & Pb208 & 45200 & 930 & 43200 & 1140 \\
\hline E23 & Pb206 & 9390000 & 915 & & \\
\hline E23 & Pb207 & 18800 & 768 & & \\
\hline E23 & Pb208 & 16800 & 763 & 3140000 & 815 \\
\hline E23R & Pb206 & 4200 & 226 & & \\
\hline E23R & Pb207 & 3910 & 74.9 & & \\
\hline E23R & Pb208 & 2890 & 62.4 & 3670 & 121 \\
\hline E24 & Pb206 & 6160 & & & \\
\hline E24 & Pb207 & 6160 & & & \\
\hline E24 & $P_{0208}$ & 6160 & & 6160 & \\
\hline E31 & Pb206 & 66300 & 1460 & & \\
\hline E31 & Pb207 & 61600 & 1690 & & \\
\hline E31 & Pb208 & 59400 & 1930 & 62400 & 1690 \\
\hline E32 & Pb206 & 52900 & 1220 & & \\
\hline E32 & Pb207 & 58300 & 1770 & & \\
\hline E32 & Pb208 & 57500 & 599 & 56200 & 1200 \\
\hline E33 & Pb206 & 21900 & 321 & & \\
\hline E33 & Pb207 & 26300 & 1350 & & \\
\hline E33 & Pb208 & 17600 & 654 & 21900 & 775 \\
\hline E34 & Pb206 & 12100 & 979 & & \\
\hline E34 & Pt207 & 10800 & 597 & & \\
\hline E34 & Pb208 & 8410 & 172 & 10400 & 583 \\
\hline E41 & Pb206 & 21300 & 320 & & \\
\hline E41 & Pb207 & 20600 & 163 & & \\
\hline
\end{tabular}

Note: The actual concentratton of samples for which no standard doviation ts reported le leas than the lower limit of detection. The reported concentratton is the lower limit of quantification. 


\section{Transect E Soil Concentrations: Lead}

\begin{tabular}{|c|c|c|c|c|c|}
\hline Semple id. & botope & $\begin{array}{l}\text { Concentration } \\
\text { (ug/Kg soll) }\end{array}$ & $\begin{array}{l}\text { Std.Dovintion } \\
\text { (ug/Kg soll) }\end{array}$ & $\begin{array}{l}\text { Elmment Avg. Conc. } \\
\text { (ug/Kg soll) }\end{array}$ & $\begin{array}{c}\text { Element Avg. Std. Dov. } \\
\text { (ug/Kg soll) }\end{array}$ \\
\hline E41 & Pb208 & 19100 & 98.8 & 20300 & 194 \\
\hline E42 & Pb206 & 49100 & 6120 & & \\
\hline E42 & Po207 & 42400 & 5570 & & \\
\hline E42 & Pb208 & 43800 & 5500 & 45100 & 5730 \\
\hline
\end{tabular}


Transect E Soil Concentrations: Magnesium

\begin{tabular}{|c|c|c|c|c|c|}
\hline Sempto ld. & kotopo & $\begin{array}{l}\text { Concentration } \\
\text { (ug/Kg soil) }\end{array}$ & $\begin{array}{l}\text { Std.Dovinton } \\
\text { (ug/Kg soll) }\end{array}$ & $\begin{array}{c}\text { Element Avg- Conc. } \\
\text { (ug/Kg coll) }\end{array}$ & $\begin{array}{c}\text { Element Avg. Std. Dov. } \\
\text { (ug/Kg soll) }\end{array}$ \\
\hline E11 & Mg24 & 384000 & 9390 & & \\
\hline E11 & Mg25 & 460000 & 10900 & 422000 & 10100 \\
\hline E12 & Mg24 & 101000 & 2040 & & \\
\hline E12 & Mges & 102000 & 6710 & 102000 & 4380 \\
\hline E12R & Mg24 & 118000 & 483 & & \\
\hline E12R & Mges & 128000 & 3340 & 123000 & 1910 \\
\hline E13 & Mge4 & 196000 & 1050 & & \\
\hline E13 & Mges & 246000 & 6810 & 221000 & 3930 \\
\hline E14 & Mge24 & 126000 & 1910 & & \\
\hline E14 & Mges & 132000 & 2540 & 129000 & 2230 \\
\hline E21 & Mg24 & 176000 & 2100 & & \\
\hline E21 & Mges & 167000 & 3330 & 172000 & 2720 \\
\hline E22 & Mg24 & 212000 & 3300 & & \\
\hline E22 & Mges & 253000 & 4210 & 233000 & 3760 \\
\hline E23 & Mge 4 & 736000 & 5050 & & \\
\hline E23 & Mges & 586000 & 4010 & 661000 & 4530 \\
\hline E23R & Mg24 & 409000 & 3720 & & \\
\hline E23R & Mg25 & 477000 & 1900 & 443000 & 2810 \\
\hline E24 & Mge4 & 215000 & 7070 & & \\
\hline E24 & Mgas & 236000 & 10300 & 226000 & 8690 \\
\hline E31 & $\mathrm{Mge} 4$ & 193000 & 404 & & \\
\hline E31 & Mges & 218000 & 875 & 206000 & 640 \\
\hline E32 & Mg24 & 238000 & 1880 & & \\
\hline E32 & Mges & 237000 & 2210 & 238000 & 2050 \\
\hline E33 & $M g 24$ & 148000 & 3300 & & \\
\hline E33 & Mges & 428000 & 5820 & 288000 & 4560 \\
\hline E34 & Mg24 & 97100 & 1680 & & \\
\hline E34 & Mges & 105000 & 1470 & 102000 & 1580 \\
\hline E41 & Mg24 & 167000 & 4040 & & \\
\hline E41 & Mges & 159000 & 4700 & 163000 & 4370 \\
\hline E42 & $M g 24$ & 214000 & 1440 & & \\
\hline E42 & Mg25 & 207000 & 8620 & 211000 & 5030 \\
\hline
\end{tabular}




\section{Transect E Soil Concentrations: Manganese}

\begin{tabular}{|c|c|c|c|c|c|}
\hline Sample ld. & leotope & $\begin{array}{l}\text { Concentration } \\
\text { (ug/Kg soll) }\end{array}$ & $\begin{array}{l}\text { Sid.Doviation } \\
\text { (ug/Kg solf) }\end{array}$ & $\begin{array}{l}\text { Eloment Avg. Conc. } \\
\text { (ug/Kg soll) }\end{array}$ & $\begin{array}{c}\text { Element Avg. Std. Dov. } \\
\text { (ugg/Kg soll) }\end{array}$ \\
\hline E11 & $\mathrm{Mn}$ & 354000 & 17700 & 354000 & 17700 \\
\hline E12 & $\mathrm{Mn}$ & 80000 & 4770 & 80000 & 4770 \\
\hline E12R & $\mathrm{Mn}$ & 14100 & 269 & 14100 & 260 \\
\hline E13 & $\mathrm{Mn}$ & 172000 & 976 & 172000 & 976 \\
\hline E14 & $\mathrm{Mn}$ & 25300 & 543 & 25300 & 543 \\
\hline E21 & $\mathrm{Mn}$ & 54300 & 621 & 54300 & 621 \\
\hline E22 & $\mathrm{Mn}$ & 30700 & 1170 & 30700 & 1170 \\
\hline E23 & $\mathrm{Mn}$ & 515000 & 8430 & 515000 & 8430 \\
\hline E23R & $\mathrm{Mn}$ & 47700 & 491 & 47700 & 491 \\
\hline E24 & $\mathrm{Mn}$ & 143000 & 4620 & 143000 & 4620 \\
\hline E31 & $\mathrm{Mn}$ & 503000 & 23000 & 503000 & 23000 \\
\hline E32 & $\mathrm{Mn}$ & 344000 & 25300 & 344000 & 25300 \\
\hline E33 & $M n$ & 35400 & 2500 & 35400 & 2500 \\
\hline E34 & $\mathrm{Mn}$ & 28900 & 1510 & 28900 & 1510 \\
\hline E41 & $\mathrm{Mn}$ & 52500 & 577 & 52500 & 577 \\
\hline$E 42$ & $\mathrm{Mn}$ & 39600 & 625 & 39600 & 625 \\
\hline
\end{tabular}


Transect E Soil Concentrations: Nickel

\begin{tabular}{|c|c|c|c|c|c|}
\hline Sample id. & teotope & $\begin{array}{l}\text { Concentration } \\
\text { (ug/Kg soil) }\end{array}$ & $\begin{array}{l}\text { Std.Dovintlon } \\
\text { (ug/Kg soll) }\end{array}$ & $\begin{array}{l}\text { Element Avg. Conc. } \\
\text { (ug/Kg woll) }\end{array}$ & $\begin{array}{c}\text { Element Avg. Std. Dov. } \\
\text { (ug/Kg soll) }\end{array}$ \\
\hline E11 & Ni60 & 9450 & 1910 & & \\
\hline E11 & Ni62 & 9060 & 5370 & 9260 & 3640 \\
\hline E12 & N160 & 5320 & & & \\
\hline E12 & Ni62 & 5320 & & 5320 & \\
\hline E12R & N160 & 4410 & & & \\
\hline E12A & Ni62 & 4410 & & 4410 & \\
\hline E13 & Ni60 & 6020 & 1140 & & \\
\hline E13 & NiE2 & 11100 & 1650 & 8560 & 1400 \\
\hline E14 & Niso & 3390 & 385 & & \\
\hline E14 & Ni62 & & & 3390 & 385 \\
\hline E21 & Ni60 & 6600 & 137 & & \\
\hline E21 & Ni62 & 4370 & 942 & 5490 & 540 \\
\hline E22 & Ni6o & 7670 & 769 & & \\
\hline E22 & Ni62 & 7040 & 2030 & 7360 & 1400 \\
\hline E23 & Ni60 & 8500 & 182 & & \\
\hline E23 & Ni62 & 9900 & 676 & 9200 & 429 \\
\hline E23R & Niso & 5120 & 556 & & \\
\hline E23R & Nis2 & 8720 & 4910 & 6920 & 2730 \\
\hline E24 & Ni60 & 3670 & 35.4 & & \\
\hline E24 & Ni62 & 3800 & 2120 & 3740 & 1080 \\
\hline E31 & Ni6o & 15300 & 342 & & \\
\hline E31 & Ni62 & 30500 & 1170 & 22900 & 756 \\
\hline E32 & Niso & 14600 & 389 & & \\
\hline E32 & Nis2 & 18700 & 665 & 16700 & 527 \\
\hline E33 & N160 & 38300 & 1070 & & \\
\hline E33 & Ni62 & 29800 & 1630 & 34100 & 1350 \\
\hline E34 & Ni60 & 1300 & 495 & & \\
\hline E34 & Ni62 & 6310 & 3380 & 3810 & 1940 \\
\hline E41 & $\mathrm{Ni60}$ & 11700 & 575 & & \\
\hline E41 & Ni62 & 12100 & 1100 & 11900 & 838 \\
\hline E42 & Ni60 & 40700 & 5430 & & \\
\hline E42 & Ni62 & 42300 & 5540 & 41500 & 5490 \\
\hline
\end{tabular}




\section{Transect E Soil Concentrations: Selenium}

\begin{tabular}{|c|c|c|c|c|c|}
\hline Sampte Id. & kotope & $\begin{array}{l}\text { Concentration } \\
\text { (ug/Ko eoll) }\end{array}$ & $\begin{array}{l}\text { Stadoviation } \\
\text { (ug/Ko soll) }\end{array}$ & $\begin{array}{c}\text { Element Avg. Conc. } \\
\text { (ug/Kg coll) }\end{array}$ & $\begin{array}{c}\text { Element Avg. Std. Dev. } \\
\text { (ug/Kg soll) }\end{array}$ \\
\hline E11 & 5077 & 12400 & & & \\
\hline E11 & So82 & 12400 & & 12400 & \\
\hline E12 & so77 & 5320 & & & \\
\hline E12 & So82 & 5320 & & 5320 & \\
\hline E12R & So77 & 2940 & & & \\
\hline E12R & 5082 & 2940 & & 2940 & \\
\hline$E 13$ & So77 & 3870 & & & \\
\hline E13 & So82 & 3870 & & 3870 & \\
\hline E14 & SeT7 & & & & \\
\hline E14 & 5082 & 26800 & & 26800 & \\
\hline E21 & Se77 & 3570 & & & \\
\hline E21 & So82 & 3570 & & 3570 & \\
\hline E22 & Se77 & 6300 & & & \\
\hline E22 & Sos2 & 6300 & & 6300 & \\
\hline E23 & Se77 & 13400 & 402 & & \\
\hline E23 & Se82 & 13600 & 421 & 13500 & 412 \\
\hline E23R & Se77 & 1470 & & & \\
\hline E23R & Se82 & 1470 & & 1470 & \\
\hline E24 & SeT7 & 2180 & 1590 & & \\
\hline E24 & So82 & 1970 & 1580 & 2080 & 1590 \\
\hline E31 & 5077 & 12700 & 2840 & & \\
\hline E31 & So82 & 13100 & 2900 & 12900 & 2870 \\
\hline E32 & So77 & 10400 & 2160 & & \\
\hline E32 & Se82 & 10500 & 2210 & 10500 & 2190 \\
\hline E33 & So77 & 17900 & 2590 & & \\
\hline E33 & Ses2 & 16400 & 2390 & 17200 & 2490 \\
\hline E34 & Se77 & 13700 & 3300 & & \\
\hline E34 & $S 082$ & 12400 & 2980 & 13100 & 3140 \\
\hline E41 & Se77 & & & & \\
\hline E41 & Se82 & 10100 & 2770 & 10100 & 2770 \\
\hline E42 & So77 & 5820 & & & \\
\hline E42 & So82 & 5820 & & 5820 & \\
\hline
\end{tabular}




\begin{tabular}{|c|c|c|c|c|c|}
\hline Semple kd. & Eotope & $\begin{array}{l}\text { Concentration } \\
\text { (ug/kg sold) }\end{array}$ & $\begin{array}{l}\text { 8tuboviation } \\
\text { (ug/kg soll) }\end{array}$ & $\begin{array}{l}\text { Element Avg- Conc. } \\
\text { (ug/Kg eoll) }\end{array}$ & $\begin{array}{c}\text { Element Avg. Std. Dov } \\
\text { (u\&/Kg coll) }\end{array}$ \\
\hline E11 & A0107 & 1680 & 336 & & \\
\hline E11 & A0100 & 645 & 414 & 1160 & 375 \\
\hline$E 12$ & A0107 & 266 & & & \\
\hline E12 & A0100 & 266 & & 268 & \\
\hline E12R & Ag107 & 1640 & 363 & & \\
\hline E12R & $A 0100$ & 58.8 & & 849 & 363 \\
\hline E13 & Ad107 & 527 & 170 & & \\
\hline E13 & Ad100 & 7580 & 313 & 4050 & 242 \\
\hline E14 & Ag107 & 268 & & & \\
\hline E14 & $A 9100$ & 268 & & 268 & \\
\hline E21 & A0107 & 357 & & & \\
\hline E21 & $A 0109$ & 357 & & 357 & \\
\hline E22 & Ag107 & 1460 & & & \\
\hline Ex2 & Ag100 & 1460 & & 1460 & \\
\hline $\mathrm{E23}$ & Ag107 & 2050 & 430 & & \\
\hline E23 & Ag109 & 1260 & 296 & 1660 & 363 \\
\hline E23R & Ag107 & 294 & & & \\
\hline E23R & $A 0109$ & 29.4 & & 162 & \\
\hline E24 & Ag107 & 488 & 15.6 & & \\
\hline E24 & Ag109 & 642 & 267 & 565 & 141 \\
\hline E31 & A0107 & 3550 & 268 & & \\
\hline E31 & A9109 & 2150 & 218 & 2850 & 243 \\
\hline E32 & Ag107 & 1870 & 281 & & \\
\hline E32 & Ag109 & 1880 & 331 & 1880 & 306 \\
\hline$E 33$ & Ag107 & 1460 & 1650 & & \\
\hline$E 33$ & Ad109 & 1410 & 1060 & 1440 & 1360 \\
\hline E34 & Ag107 & 333 & & & \\
\hline E34 & Ag109 & 333 & & 333 & \\
\hline E41 & Ad107 & 251 & & & \\
\hline E41 & Ag109 & 330 & 163 & 291 & 163 \\
\hline$E 42$ & Ag107 & 2910 & & & \\
\hline$E 42$ & Ag109 & 2910 & & 2910 & \\
\hline
\end{tabular}

Note: The ectual concentration of eamples for which no stenderd dovkation be reported is loes than the lower limit of detection. The reported concentration ts the bwe limit of quantification. 


\section{Transect E Soil Concentrations: Sodium}

\begin{tabular}{|c|c|c|c|c|c|}
\hline Sample Id. & leotope & $\begin{array}{l}\text { Concentration } \\
\text { (ug/Kg soll) }\end{array}$ & $\begin{array}{l}\text { Stiogviation } \\
\text { (ug/Kg eoll) }\end{array}$ & $\begin{array}{l}\text { Element Avg. Conc. } \\
\text { (ug/Kg soll) }\end{array}$ & $\begin{array}{l}\text { Element Avg. Std. Dov. } \\
\text { (ug/Kg soli) }\end{array}$ \\
\hline E11 & $\mathrm{Na}$ & 68400 & 48000 & 68400 & 48000 \\
\hline E12 & $\mathrm{Na}$ & 106000 & & 106000 & \\
\hline E12R & $\mathrm{Na}$ & 29400 & & 29400 & \\
\hline E13 & $N_{a}$ & 155000 & & 155000 & \\
\hline E14 & $\mathrm{Na}$ & 13400 & & 13400 & \\
\hline E21 & $\mathrm{Na}$ & 194000 & 22300 & 194000 & 22300 \\
\hline E22 & $\mathrm{Na}$ & 64400 & 27800 & 64400 & 27900 \\
\hline E23 & $\mathrm{Na}$ & 12200 & 9570 & 12200 & 9570 \\
\hline E23R & $\mathrm{Na}$ & 14700 & & 14700 & \\
\hline E24 & $\mathrm{Na}$ & 6160 & & 6160 & \\
\hline E31 & $\mathrm{Na}$ & 1700 & & 1700 & \\
\hline E32 & $\mathrm{Na}$ & 1590 & & 1590 & \\
\hline E33 & $\mathrm{Na}$ & 494000 & & 484000 & \\
\hline E34 & $\mathrm{Na}$ & 133000 & & 133000 & \\
\hline E41 & $\mathrm{Na}$ & 111000 & 12700 & 111000 & 12700 \\
\hline $\mathrm{E} 42$ & $\mathrm{Na}$ & 116000 & & 116000 & \\
\hline
\end{tabular}


Transect E Soil Concentrations: Strontium

\begin{tabular}{|c|c|c|c|c|c|}
\hline Sample ld. & botope & $\begin{array}{l}\text { Concentration } \\
\text { (ug/Kg soll) }\end{array}$ & $\begin{array}{l}\text { Sid.Deviation } \\
\text { (ug/Kg soil) }\end{array}$ & $\begin{array}{l}\text { Element Avg. Conc. } \\
\text { (ug/kg soll) }\end{array}$ & $\begin{array}{c}\text { Element Avg- Std. Dov. } \\
\text { (ug/Kg soll) }\end{array}$ \\
\hline E11 & $S_{i}$ & 100000 & 1470 & 108000 & 1470 \\
\hline E12 & Sr & 10200 & 350 & 10200 & 350 \\
\hline E12R & Sr & 22800 & 324 & 22800 & 324 \\
\hline E13 & $\mathrm{Sr}$ & 297000 & 3710 & 297000 & 3710 \\
\hline E14 & $\mathrm{Sr}_{\mathrm{r}}$ & 17000 & 200 & 17000 & 200 \\
\hline E21 & Sr & 6610 & 142 & 6810 & 142 \\
\hline $\mathrm{E} 22$ & S? & 7810 & 219 & 7810 & 219 \\
\hline $\mathrm{E23}$ & $\mathrm{Sr}$ & 132000 & 1240 & 132000 & 1240 \\
\hline E23R & $S_{p}$ & 122000 & 41 & 122000 & 441 \\
\hline E24 & $\mathrm{Sr}$ & 268000 & 4000 & 268000 & 4090 \\
\hline E31 & Sr & 9510 & 43.8 & 9510 & 43.8 \\
\hline E32 & $\mathrm{Sr}$ & 12200 & 162 & 12200 & 102 \\
\hline E33 & $\mathrm{Sr}$ & 40400 & 892 & 40400 & 998 \\
\hline E34 & Sr & 58600 & 1420 & 58600 & 1420 \\
\hline E41 & $\mathrm{Sr}$ & 53500 & 454 & 53500 & 454 \\
\hline E42 & Sr & 191000 & 1540 & 191000 & 1540 \\
\hline
\end{tabular}

Note: The ectual concentratton of aumples for which no standerd deviation te reported bs leas than the lower llmit of detection. The reported concentration is the lower Hintt of quantification. 


\section{Transect E Soil Concentrations: Thorium}

\begin{tabular}{|c|c|c|c|c|c|}
\hline Semplo id. & leotope & $\begin{array}{l}\text { Concentration } \\
\text { (ug/Kg soli) }\end{array}$ & $\begin{array}{l}\text { Std.Devintion } \\
\text { (ug/Kg soll) }\end{array}$ & $\begin{array}{l}\text { Element Avg. Conc. } \\
\text { (ug/Kg eoll) }\end{array}$ & $\begin{array}{c}\text { Element Avg. Std. Dov. } \\
\text { (ug/Kg soll) }\end{array}$ \\
\hline E11 & Th232 & 1260 & 19.6 & 1260 & 19.6 \\
\hline E12 & Th232 & 128 & 31.5 & 128 & 31.5 \\
\hline E12R & Th232 & 147 & & 147 & \\
\hline E13 & Th232 & 77.3 & & 77.3 & \\
\hline E14 & Th232 & 42.9 & 1.4 & 42.9 & 1.4 \\
\hline E21 & Th232 & 1380 & 35.4 & 1380 & 35.4 \\
\hline E22 & Th232 & 918 & 60.9 & 918 & 60.9 \\
\hline $\mathrm{E} 23$ & Th232 & 199 & 47 & 199 & 47 \\
\hline E23R & Th232 & 284 & 24.4 & 284 & 24.4 \\
\hline E24 & Th232 & 352 & 222 & 352 & 222 \\
\hline E31 & Th232 & 3200 & 103 & 3200 & 103 \\
\hline E32 & Th232 & 2210 & 49 & 2210 & 49 \\
\hline E33 & Th232 & 362 & 19.7 & 362 & 19.7 \\
\hline E34 & Th232 & 289 & 11.9 & 289 & 11.9 \\
\hline E41 & Th232 & 1140 & 15.7 & 1140 & 15.7 \\
\hline$E 42$ & Th232 & 59.4 & 31.7 & 59.4 & 31.7 \\
\hline
\end{tabular}

Note: The actual concentratton of samples for which no standard deviation b reported is lees than the lower limit of detection. The reported concentratton ts the lower limit of quantification. 


\section{Transect E Soil Concentrations: Tin}

\begin{tabular}{|c|c|c|c|c|c|}
\hline Sempto Id. & leotope & $\begin{array}{l}\text { Concentration } \\
\text { (4 } / \mathrm{Kg} \text { soll) }\end{array}$ & $\begin{array}{l}\text { Std.Doviation } \\
\text { (ug/Kg soil) }\end{array}$ & $\begin{array}{l}\text { Element Avg. Conc. } \\
\text { (ug/ } \mathrm{K}_{\sigma} \text { soll) }\end{array}$ & $\begin{array}{c}\text { Element Avg. Std. Dov. } \\
\text { (ug/Kg soll) }\end{array}$ \\
\hline E11 & Sn118 & 1270 & 215 & & \\
\hline E11 & Sn120 & 1420 & 102 & 1350 & 159 \\
\hline E12 & Sn118 & 253 & 86.2 & & \\
\hline E12 & $\operatorname{Sn} 120$ & 263 & 49.4 & 258 & 67.8 \\
\hline E12R & $\sin 118$ & 147 & & & \\
\hline E12R & Sn120 & 147 & & 147 & \\
\hline$E 13$ & $\operatorname{Sn} 118$ & 528 & 33.6 & & \\
\hline E13 & $\sin 120$ & 430 & 217 & 479 & 125 \\
\hline E14 & $\operatorname{Sn} 118$ & 328 & 32.5 & & \\
\hline E14 & Sn120 & 283 & 49.5 & 306 & 41 \\
\hline E21 & $\operatorname{Sn} 118$ & 773 & 121 & & \\
\hline E21 & $\sin 120$ & 1150 & 26.8 & 902 & 73.9 \\
\hline E22 & $\operatorname{Sn} 118$ & 908 & 83.9 & & \\
\hline E22 & $\operatorname{sn} 120$ & 805 & 79.5 & 857 & 81.7 \\
\hline E23 & $\operatorname{Sn} 118$ & 286 & 45.5 & & \\
\hline$E_{23}$ & Sn120 & 334 & 23.9 & 310 & 34.7 \\
\hline E23R & Sn118 & 321 & 54.4 & & \\
\hline E23R & $\operatorname{sn} 120$ & 290 & 13 & 306 & 33.7 \\
\hline$E 24$ & Sn118 & 415 & 35.9 & & \\
\hline E24 & Sn120 & 217 & 20.3 & 316 & 28.1 \\
\hline E31 & Sn118 & 1240 & 66.5 & & \\
\hline E31 & Sn120 & 1300 & 72.3 & 1270 & 69.4 \\
\hline E32 & Sn118 & 1370 & 123 & & \\
\hline E32 & Sn 120 & 1190 & 35.3 & 1280 & 792 \\
\hline E33 & Sn118 & 1970 & 157 & & \\
\hline E33 & Sn120 & 2080 & 165 & 2030 & 161 \\
\hline E34 & Sn118 & 157 & 50.9 & & \\
\hline E34 & Sn120 & 145 & 10.5 & 151 & 30.7 \\
\hline E41 & Sn118 & 1610 & 111 & & \\
\hline E41 & Sn120 & 1410 & 52.8 & 1510 & 81.9 \\
\hline E42 & Sn118 & 1000 & 81.1 & & \\
\hline$E 42$ & $\operatorname{sn} 120$ & 1200 & 7.04 & 1100 & 44.1 \\
\hline
\end{tabular}

Note: The ectual concentration of samples for which no standard deviation is reported is lees than the lower limit of detectlon. The reported concentration ts the lower limit of quantification. 


\section{Transect E Soil Concentrations: Uranium-235}

\begin{tabular}{|c|c|c|c|c|c|}
\hline Sample ld. & leotope & $\begin{array}{l}\text { Concentration } \\
\text { (ug/kg soil) }\end{array}$ & $\begin{array}{l}\text { Std.Deviation } \\
\text { (ug/Kg soil) }\end{array}$ & $\begin{array}{l}\text { Element Avg. Conc. } \\
\text { (ug/Kg soll) }\end{array}$ & $\begin{array}{c}\text { Element Avg. Std. Dov. } \\
\text { (ug/Kg soll) }\end{array}$ \\
\hline E11 & U235 & 36.4 & 15.3 & 36.4 & 15.3 \\
\hline E12 & U235 & 19.1 & 184 & 19.1 & 184 \\
\hline E12R & U235 & 11.4 & 1.32 & 11.4 & 1.32 \\
\hline E13 & U235 & 23.4 & 3.42 & 23.4 & 3.42 \\
\hline E14 & U235 & 15 & 2.76 & 15 & 2.76 \\
\hline E21 & U235 & 16.7 & 6.69 & 16.7 & 6.69 \\
\hline$E 22$ & U235 & 26.6 & 72 & 26.6 & 72 \\
\hline $\mathrm{E} 23$ & U235 & 26.7 & & 26.7 & \\
\hline E23R & U235 & 7.7 & 1.44 & 7.7 & 1.44 \\
\hline E24 & U235 & 11.4 & 6.13 & 11.4 & 6.13 \\
\hline E31 & U235 & 17.3 & 4.13 & 17.3 & 4.13 \\
\hline E32 & U235 & 14.1 & 3.76 & 14.1 & 3.76 \\
\hline E33 & U235 & 22.5 & 3.87 & 22.5 & 3.87 \\
\hline E34 & U235 & 9.62 & 4.81 & 9.62 & 4.81 \\
\hline E41 & U235 & 312 & 6.63 & 312 & 6.63 \\
\hline$E 42$ & U235 & 26.9 & 1.5 & 26.9 & 1.5 \\
\hline
\end{tabular}

Note: The actus concentration of samples for which no standard deviation is reported ts hes than the lower limit of detection. The reported concentration is the fower limit of quantincation. 


\section{Transect E Soil Concentrations: Uranium-238}

\begin{tabular}{|c|c|c|c|c|c|}
\hline Semple ld. & botope & $\begin{array}{l}\text { Concentration } \\
\text { (ug/Kg soil) }\end{array}$ & $\begin{array}{l}\text { Std.Doviation } \\
\text { (ug/Kg soil) }\end{array}$ & $\begin{array}{l}\text { Element Avg. Conc. } \\
\text { (4g/Kg soll) }\end{array}$ & $\begin{array}{c}\text { Element Avg. Std. Dov. } \\
\text { (ug/Kg soll) }\end{array}$ \\
\hline E11 & U238 & 5810 & 89.9 & 5810 & 89.9 \\
\hline E12 & U238 & 1350 & 30.8 & 1350 & 30.8 \\
\hline E12R & U238 & 1390 & 5.25 & 1390 & 5.25 \\
\hline E13 & U238 & 1660 & 76.5 & 1660 & 76.5 \\
\hline E14 & v238 & 1330 & 51.8 & 1330 & 51.8 \\
\hline E21 & U238 & 1560 & 51.3 & 1560 & 51.3 \\
\hline E22 & U238 & 1840 & 54.6 & 1840 & 54.6 \\
\hline$E 23$ & U238 & 3160 & 212 & 3160 & 212 \\
\hline E23R & U238 & 1930 & 16.8 & 1930 & 16.8 \\
\hline E24 & U238 & 1850 & 40.8 & 1850 & 40.8 \\
\hline E31 & U238 & 1810 & 25 & 1810 & 25 \\
\hline$E 32$ & U238 & 1660 & 47.7 & 1660 & 47.7 \\
\hline$E 33$ & U238 & 2480 & 70.3 & 2480 & 70.3 \\
\hline E34 & U238 & 1050 & 28.1 & 1050 & 28.1 \\
\hline E41 & U238 & 4420 & 572 & 4420 & 572 \\
\hline$E 42$ & U238 & 3080 & 247 & 3080 & 247 \\
\hline
\end{tabular}

Noto: The actual concentration of eamples for which no standard deviation is reported is lees than the lower limit of detection. The reported concentration ls the lower limit of quantification. 


\section{Transect E Soil Concentrations: Vanadium}

\begin{tabular}{|c|c|c|c|c|c|}
\hline Sample ld. & leotope & $\begin{array}{l}\text { Concentration } \\
\text { (ug/Kg soll) }\end{array}$ & $\begin{array}{l}\text { Std.Deviatton } \\
\text { (ug/Kg soll) }\end{array}$ & $\begin{array}{l}\text { Element Avg. Conc. } \\
\text { (ug/Kg soll) }\end{array}$ & $\begin{array}{c}\text { Element Avg. Std. Dov. } \\
\text { (ug/Kg soll) }\end{array}$ \\
\hline E11 & V & 30200 & 2780 & 30200 & 2780 \\
\hline$E 12$ & $v$ & 7170 & 1690 & 7170 & 1690 \\
\hline E12R & $V$ & 22200 & 682 & 22200 & 682 \\
\hline E13 & $\mathrm{v}$ & 21200 & 541 & 21200 & 541 \\
\hline$E 14$ & $v$ & 10700 & 397 & 10700 & 397 \\
\hline E21 & $v$ & 26900 & 481 & 26900 & 481 \\
\hline$E 22$ & V & 29400 & 609 & 29400 & 600 \\
\hline E23 & $v$ & 12600 & 1320 & 12600 & 1320 \\
\hline E23R & $v$ & 18700 & 107 & 18700 & 107 \\
\hline E24 & $\mathrm{v}$ & 4120 & 5390 & 4120 & 5390 \\
\hline E31 & $v$ & 46900 & 2740 & 46900 & 2740 \\
\hline$E 32$ & v & 36500 & 2780 & 36500 & 2780 \\
\hline$E 33$ & V & 9190 & 551 & 9190 & 551 \\
\hline E34 & $v$ & 6160 & 1130 & 6160 & 1130 \\
\hline E41 & $\mathrm{V}$ & 90500 & 891 & 90500 & 891 \\
\hline E42 & $\mathrm{v}$ & 35100 & 775 & 35100 & 775 \\
\hline
\end{tabular}




\begin{tabular}{|c|c|c|c|c|c|}
\hline Sample ld. & botope & $\begin{array}{l}\text { Concentratton } \\
\text { (ug/kg sold) }\end{array}$ & $\begin{array}{l}\text { Std.Doviation } \\
\text { (ug/Kg solf) }\end{array}$ & $\begin{array}{l}\text { Element Avg. Conc. } \\
\text { (ug/Kg soll) }\end{array}$ & $\begin{array}{c}\text { Element Avg. Std. Dov. } \\
\text { (ug/Kg soll) }\end{array}$ \\
\hline E11 & $Y$ & 1200 & 78.7 & 1200 & 78.7 \\
\hline E12 & $Y$ & 106 & 242 & 106 & 242 \\
\hline E12R & $Y$ & 260 & 172 & 260 & 172 \\
\hline E13 & $\mathbf{Y}$ & 400 & 42.9 & 409 & 42.9 \\
\hline E14 & $Y$ & 249 & 45.6 & 249 & 45.6 \\
\hline E21 & $Y$ & 1130 & 74.4 & 1130 & 74.4 \\
\hline $\mathrm{E22}$ & $Y$ & 1050 & 44.6 & 1050 & 44.6 \\
\hline E23 & $Y$ & 2790 & 344 & 2790 & 344 \\
\hline E23R & $Y$ & 2350 & 26.4 & 2350 & 26.4 \\
\hline E24 & $Y$ & 1080 & 222 & 1080 & 222 \\
\hline E31 & $Y$ & 2440 & 68.8 & 2440 & 68.8 \\
\hline$E 32$ & $Y$ & 1460 & 27.6 & 1460 & 27.6 \\
\hline$E 33$ & $Y$ & 3070 & 70.7 & 3070 & 70.7 \\
\hline E34 & $Y$ & 2320 & 129 & 2320 & 129 \\
\hline E41 & $Y$ & 1400 & 75.1 & 1400 & 75.1 \\
\hline$E 42$ & $Y$ & 903 & 143 & 903 & 143 \\
\hline
\end{tabular}




\section{Transect E Soil Concentrations: Zinc}

\begin{tabular}{|c|c|c|c|c|c|}
\hline Semple kd. & kotope & $\begin{array}{l}\text { Concentratton } \\
\text { (ugKg soll) }\end{array}$ & $\begin{array}{l}\text { Std.Dovintion } \\
\text { (ug/Kg soll) }\end{array}$ & $\begin{array}{l}\text { Ekment Avg. Conc. } \\
\text { (ug/Kg coll) }\end{array}$ & $\begin{array}{c}\text { Element Avg. Std. Dov. } \\
\text { (ug/Kg soll) }\end{array}$ \\
\hline E11 & Zne6 & 2050000 & 2420 & 2050000 & 2420 \\
\hline E12 & Zn66 & 2460 & 291 & 2460 & 291 \\
\hline E12R & Zn66 & 4410 & & 4410 & \\
\hline E13 & Zn66 & 7920 & 845 & 7920 & 845 \\
\hline E14 & Zn66 & 8860 & 1620 & 8860 & 1620 \\
\hline E21 & Zn66 & 10500 & 131 & 10500 & 131 \\
\hline$E 22$ & Zn66 & 13700 & 388 & 13700 & 388 \\
\hline E23 & Zn66 & 32900 & 598 & 32900 & 590 \\
\hline E23R & Zn66 & 4670 & 895 & 4670 & 895 \\
\hline$E 24$ & Zn66 & 22500 & 375 & 22500 & 375 \\
\hline E31 & Zn66 & 28500 & 1030 & 28500 & 1030 \\
\hline E32 & Zn66 & 28200 & 754 & 28200 & 754 \\
\hline E33 & Zns6 & 48200 & 1190 & 48200 & 1190 \\
\hline E34 & Zn66 & 9210 & 7620 & 9210 & 7620 \\
\hline E41 & Zn66 & 19300 & 724 & 19300 & 724 \\
\hline E42 & Zn66 & 4000 & 1990 & 4000 & 1990 \\
\hline
\end{tabular}

Note: The actual concentration of samples for which no stendard devlation be reported be lees than the lower limlt of detection. The reported concentration be the lower IImit of quantification. 


\section{Transect E Soil Concentrations: Zirconium}

\begin{tabular}{llccc}
\hline Semple ld. keotope & $\begin{array}{c}\text { Concentration } \\
\text { (ug/Kg soll) }\end{array}$ & $\begin{array}{c}\text { Std.Dovlation } \\
\text { (Ug/Kg soll) }\end{array}$ & $\begin{array}{c}\text { Element Avg. Conc. } \\
\text { (Ug/Kg soll) }\end{array}$ & $\begin{array}{c}\text { Element Avg. Std. Dev. } \\
\text { (Ug/Kg soll) }\end{array}$
\end{tabular}

\begin{tabular}{|c|c|c|c|c|c|}
\hline E11 & Zr9o & 61700 & 332 & & \\
\hline E11 & Z91 & 64800 & 1930 & 63300 & 1130 \\
\hline E12 & Z990 & 12200 & 590 & & \\
\hline E12 & Zr91 & 13000 & 970 & 12600 & 785 \\
\hline E12R & 290 & 8410 & 237 & & \\
\hline$\overline{E 12 R}$ & Zr91 & 10200 & 693 & 9310 & 465 \\
\hline$E 13$ & Zr90 & 21200 & 688 & & \\
\hline$E 13$ & Zr91 & 18900 & 890 & 20100 & 789 \\
\hline$E 14$ & Zr9o & 25300 & 500 & & \\
\hline E14 & $2 r 91$ & 31100 & 407 & 28200 & 458 \\
\hline E21 & Zr9o & 64100 & 543 & & \\
\hline E21 & Zra1 & 3000000 & 1310 & 1530000 & 927 \\
\hline$E 22$ & $Z 990$ & 23400 & 419 & & \\
\hline$E 22$ & $Z 991$ & 27700 & 267 & 25600 & 343 \\
\hline$E 23$ & Zr90 & 25400 & 603 & & \\
\hline$E 23$ & $2 r 91$ & 24600 & 731 & 25000 & 667 \\
\hline E23R & Zr9o & 19200 & 282 & & \\
\hline E23R & Zr91 & 21900 & 890 & 20600 & 586 \\
\hline E24 & $Z 90$ & 43100 & 832 & & \\
\hline$E 24$ & Zr91 & 31700 & 1250 & 37400 & 1040 \\
\hline E39 & Zr9o & 78300 & 226 & & \\
\hline E31 & Zr91 & 73500 & 449 & 75900 & 338 \\
\hline E32 & Zr9o & 50600 & 607 & & \\
\hline E32 & Zr91 & 49900 & 77.4 & 50300 & 342 \\
\hline E33 & $Z 90$ & 25300 & 713 & & \\
\hline E33 & Zr91 & 25400 & 4150 & 25400 & 2430 \\
\hline E34 & 2590 & 52100 & 2560 & & \\
\hline E34 & $Z 991$ & 54900 & 1350 & 53500 & 1960 \\
\hline E41 & $Z 900$ & 61400 & 345 & & \\
\hline E41 & $Z 991$ & 58900 & 223 & 60200 & 284 \\
\hline$E 42$ & Zr9o & 99900 & 1130 & & \\
\hline$E 42$ & Zr91 & 96400 & 1070 & 97700 & 1100 \\
\hline
\end{tabular}

Note: The actual concentration of eamples for which no standard deviation ts reported bs leas than the lower limlt of detection. The reported concentration is the lower limit of quantification. 


\begin{tabular}{|c|c|c|c|c|c|}
\hline Semple ld. & leotope & $\begin{array}{l}\text { Concentration } \\
\text { (ug/Kg soll) }\end{array}$ & $\begin{array}{l}\text { Std.Deviation } \\
\text { (ug/Kg soll) }\end{array}$ & $\begin{array}{l}\text { Element Avg. Conc. } \\
\text { (ug/Kg soll) }\end{array}$ & $\begin{array}{c}\text { Element Avg. Std. Dov. } \\
\text { (ug/Kg soll) }\end{array}$ \\
\hline F11 & $\mathbf{A}$ & 12300000 & 484000 & 12300000 & 484000 \\
\hline $\mathrm{F} 12$ & A & 14200000 & 401000 & 14200000 & 401000 \\
\hline F13 & A & 4980000 & 51300 & 4980000 & 51300 \\
\hline F21 & $\mathbf{A}$ & 27500000 & 87800 & 27500000 & 87800 \\
\hline F21R & $\mathbf{A}$ & 11500000 & 158000 & 11500000 & 158000 \\
\hline$F 22$ & $\boldsymbol{A}$ & 21100000 & 195000 & 21100000 & 195000 \\
\hline F23 & $\mathbf{A}$ & 16200000 & 201000 & 16200000 & 201000 \\
\hline F31 & $\mathbf{A}$ & 46900000 & 662000 & 46900000 & 662000 \\
\hline F32 & $\overline{\mathbf{A}}$ & 13000000 & 533000 & 13000000 & 533000 \\
\hline$F 33$ & $\mathbf{A}$ & 16200000 & 94800 & 16200000 & 94800 \\
\hline F41 & $\mathbf{A}$ & 30900000 & 145000 & 30900000 & 145000 \\
\hline$F 42$ & A & 14600000 & 375000 & 14600000 & 375000 \\
\hline$F 43$ & $\mathbf{A}$ & 6620000 & 10800 & 6620000 & 10800 \\
\hline F51 & $\mathbf{A}$ & 21500000 & 31600 & 21500000 & 31600 \\
\hline F52 & $\bar{A}$ & 26500000 & 566000 & 26500000 & 566000 \\
\hline$F 53$ & A & 9760000 & 362000 & 9760000 & 362000 \\
\hline
\end{tabular}




\section{Transect F Soil Concentrations: Arsenic}

\begin{tabular}{|c|c|c|c|c|c|}
\hline Sample ld. & leotope & $\begin{array}{l}\text { Concentration } \\
\text { (ug/Kg soll) }\end{array}$ & $\begin{array}{l}\text { Std.Deviation } \\
\text { (ug/Kg soll) }\end{array}$ & $\begin{array}{l}\text { Element Avg. Conc. } \\
\text { (ug/Kg soll) }\end{array}$ & $\begin{array}{c}\text { Element Avg. Std. Dov. } \\
\text { (ug/Kg soll) }\end{array}$ \\
\hline$F 11$ & As & 8970 & 690 & 8970 & 603 \\
\hline $\mathbf{F 1 2}$ & As & 5710 & 402 & 5710 & 400 \\
\hline$F 13$ & As & 17500 & 664 & 17500 & 664 \\
\hline F21 & As & 1770 & 231 & 1770 & 231 \\
\hline F21R & As & 7840 & & 7840 & \\
\hline $\mathrm{F} 22$ & As & 2350 & 432 & 2350 & 432 \\
\hline F23 & As & 2270 & 1800 & 2270 & 1800 \\
\hline F31 & As & 8170 & 1330 & 8170 & 1330 \\
\hline F32 & As & 8780 & 921 & 8780 & 921 \\
\hline$F 33$ & As & 2300 & 740 & 2300 & 740 \\
\hline F41 & As & 15500 & 700 & 15500 & 700 \\
\hline F42 & As & 10500 & 325 & 10500 & 325 \\
\hline F43 & As & 11300 & 1020 & 11300 & 1020 \\
\hline F51 & As & 1260 & 721 & 1260 & 721 \\
\hline F52 & As & 7470 & 243 & 7470 & 243 \\
\hline F53 & As & 3280 & 617 & 3280 & 617 \\
\hline
\end{tabular}




\section{Transect F Soil Concentrations: Barium}

\begin{tabular}{|c|c|c|c|c|c|}
\hline Semple ld. & wotopo & $\begin{array}{l}\text { Concantration } \\
\text { (ug/Kg soil) }\end{array}$ & $\begin{array}{l}\text { Std.Doviation } \\
\text { (ug/Kg soll) }\end{array}$ & $\begin{array}{l}\text { Etement Avg. Conc. } \\
\text { (ug/Kg eoll) }\end{array}$ & $\begin{array}{l}\text { Element Avg. Std. Dov. } \\
\text { (ug/Kg soil) }\end{array}$ \\
\hline
\end{tabular}

\begin{tabular}{|c|c|c|c|c|c|}
\hline$F 11$ & Ba135 & 42700 & 286 & & \\
\hline$F 11$ & Ba137 & 43800 & 780 & & \\
\hline F11 & Ba138 & 35000 & 610 & 40500 & 559 \\
\hline$F_{12}$ & Ba135 & 53400 & 67 & & \\
\hline F12 & Ba137 & 54800 & 202 & & \\
\hline$F 12$ & Ba138 & 55900 & 362 & 54700 & 210 \\
\hline F13 & Ba135 & 28500 & 372 & & \\
\hline F13 & Ba137 & 23800 & 321 & & \\
\hline$F 13$ & Ba138 & 24600 & 141 & 25600 & 278 \\
\hline F21 & Ba135 & 21900 & 271 & & \\
\hline F21 & Ba137 & 21000 & 516 & & \\
\hline F21 & 8 8a138 & 20200 & 171 & 21000 & 319 \\
\hline F21R & Ba135 & 26600 & 2580 & & \\
\hline F21R & Ba137 & 18600 & 1820 & & \\
\hline F21R & Ba138 & 20700 & 999 & 22000 & 1800 \\
\hline$F 22$ & Ba135 & 29900 & 1200 & & \\
\hline$F 22$ & Ba137 & 26000 & 1080 & & \\
\hline$F 22$ & Ba138 & 23300 & 263 & 26400 & 848 \\
\hline$F 23$ & Ba135 & 46900 & 665 & & \\
\hline $\mathrm{F} 23$ & Ba137 & 46200 & 1070 & & \\
\hline$F 23$ & Ba138 & 54900 & 521 & 49300 & 752 \\
\hline F31 & Ba135 & 90200 & 1540 & & \\
\hline F31 & Ba137 & 89400 & 2040 & & \\
\hline F31 & Ba138 & 87900 & 595 & 89200 & 1390 \\
\hline $\mathrm{F} 32$ & Ba135 & 36700 & 907 & & \\
\hline$F_{32}$ & Ba137 & 39300 & 917 & & \\
\hline F32 & Ba138 & 41200 & 295 & 39100 & 706 \\
\hline$F 33$ & Ba135 & 19700 & 335 & & \\
\hline F33 & Ba137 & 19500 & 311 & & \\
\hline F33 & Ba138 & 21500 & 75 & 20200 & 240 \\
\hline F41 & Ba135 & 41000 & 1310 & & \\
\hline F41 & $8 a 137$ & 32500 & 422 & & \\
\hline$F 41$ & Ba138 & 44600 & 378 & 39400 & 703 \\
\hline$F 42$ & Ba135 & 93400 & 1790 & & \\
\hline F 42 & Ba137 & 93500 & 695 & & \\
\hline $\mathrm{F} 42$ & Ba138 & 74500 & 255 & 87100 & 913 \\
\hline$F 43$ & Ba135 & 10600 & 422 & & \\
\hline $\mathrm{F} 43$ & Ba137 & 12100 & 401 & & \\
\hline $\mathrm{F} 43$ & Ba138 & 11100 & 192 & 11300 & 338 \\
\hline$F 51$ & Ba135 & 36400 & 490 & & \\
\hline$F 51$ & Ba137 & 39300 & 1450 & & \\
\hline F51 & Ba138 & 40700 & 104 & 38800 & 681 \\
\hline$F 52$ & Ba135 & 53200 & 1800 & & \\
\hline$F 52$ & Ba137 & 52900 & 643 & & \\
\hline
\end{tabular}

\footnotetext{
Note: The actual concentration of samples for which no standerd deviation be reported b lees than the fower IImit of detection. The reported concentration is the lower limit of quantification.
} 


\begin{tabular}{|c|c|c|c|c|c|}
\hline Sampte ld. & leotope & $\begin{array}{l}\text { Concentration } \\
\text { (ug/Kg soll) }\end{array}$ & $\begin{array}{l}\text { Std.Doviation } \\
\text { (ug/Kg soil) }\end{array}$ & $\begin{array}{l}\text { Element Avg. Conc. } \\
\text { (ug/Kg soll) }\end{array}$ & $\begin{array}{c}\text { Element Avg. Std. Dov. } \\
\text { (ug/Kg soll) }\end{array}$ \\
\hline F52 & Ba138 & 49600 & 816 & 51900 & 1090 \\
\hline $\mathrm{F53}$ & Ba135 & 29900 & 785 & & \\
\hline F53 & Ba137 & 43900 & 1820 & & \\
\hline F53 & Ba138 & 49800 & 349 & 41200 & 985 \\
\hline
\end{tabular}




\section{Transect F Soil Concentrations: Cadmium}

\begin{tabular}{|c|c|c|c|c|c|}
\hline Sampte ld. & leotope & $\begin{array}{l}\text { Concentration } \\
\text { (ug/Kg soil) }\end{array}$ & $\begin{array}{l}\text { Std.Deviation } \\
\text { (ug/Kg soll) }\end{array}$ & $\begin{array}{c}\text { Element Avg. Conc. } \\
\text { (ug/Kg soll) }\end{array}$ & $\begin{array}{c}\text { Element Avg. Std. Dov. } \\
\text { (ug/Kg soll) }\end{array}$ \\
\hline$F 11$ & Cd111 & 183 & 105 & & \\
\hline F11 & Cd114 & 129 & & 156 & 105 \\
\hline$F 12$ & Co1111 & 86.1 & 68.3 & & \\
\hline$F_{12}$ & Cd114 & 109 & 94 & 97.6 & 812 \\
\hline$F_{13}$ & Cd111 & 209 & & & \\
\hline$F 13$ & $\operatorname{cod} 114$ & 200 & & 209 & \\
\hline F21 & $\operatorname{cod} 111$ & 122 & 28.8 & & \\
\hline F21 & Cd114 & 13 & 14.5 & 67.5 & 21.7 \\
\hline F21R & Cd111 & 629 & 277 & & \\
\hline F21R & $\operatorname{cod114}$ & 1250 & 696 & 940 & 487 \\
\hline$F 22$ & Cd111 & 61.2 & & & \\
\hline$F 22$ & Cd114 & 612 & & 612 & \\
\hline F23 & Co1111 & 114 & & & $\cdot$ \\
\hline F23 & Co114 & 114 & & 114 & \\
\hline F31 & Cd111 & 80.7 & & & \\
\hline F31 & Co1114 & 80.7 & & 80.7 & \\
\hline F32 & Cd111 & 64.1 & & & \\
\hline$F_{32}$ & Cd114 & 64.1 & & 64.1 & \\
\hline$F 33$ & $\operatorname{cod} 111$ & 130 & 295 & & \\
\hline$F 33$ & Cdt14 & 122 & 127 & 126 & 211 \\
\hline $\mathrm{F} 41$ & Cod111 & 130 & 30.7 & & \\
\hline F41 & Cod114 & 38 & 51 & 84 & 40.9 \\
\hline$F 42$ & $\cot 111$ & 64.2 & 39.3 & & \\
\hline$F_{42}$ & Cd114 & 65.3 & 21.8 & 64.8 & 30.6 \\
\hline$F_{43}$ & Col111 & 105 & 217 & & \\
\hline$F 43$ & Co1114 & 105 & & 105 & 217 \\
\hline$F 51$ & Cod111 & 78.4 & 47.8 & & \\
\hline$F 51$ & Cd114 & $\$ 0.3$ & & 84.4 & 47.8 \\
\hline$F 52$ & $\operatorname{cod} 111$ & 63.1 & & & \\
\hline F52 & $\operatorname{Cod114}$ & 63.1 & & 63.1 & \\
\hline F53 & Cd111 & 78.3 & & & \\
\hline $\mathrm{F}_{53}$ & $\operatorname{cod} 114$ & 104 & 61.8 & 912 & 61.8 \\
\hline
\end{tabular}

Note: The actual concentration of eamplea for which no standard deviation is reported is loes than the lower Ilmit of detection. The reported concentration is the lower IImit of quantification. 


\begin{tabular}{|c|c|c|c|c|c|}
\hline Sumple ld. & Eotope & $\begin{array}{l}\text { Concentration } \\
\text { (ug/kg soll) }\end{array}$ & $\begin{array}{l}\text { Std.Dovintion } \\
\text { (ug/Ko soil) }\end{array}$ & $\begin{array}{l}\text { Element Avg. Conc. } \\
\text { (ug/Ko soll) }\end{array}$ & $\begin{array}{c}\text { Element Avg. Std. Dov. } \\
\text { (ug/Kg soll) }\end{array}$ \\
\hline F11 & $\mathrm{Ca} 44$ & 1070000 & 236000 & 1070000 & 236000 \\
\hline F12 & $\operatorname{Cos4}$ & 1450000 & 361000 & 1450000 & 361000 \\
\hline F13 & Ce44 & 2110000 & 183000 & 2110000 & 183000 \\
\hline F21 & Ca44 & 1250000 & 55500 & 1250000 & 55500 \\
\hline F21A & $\operatorname{Ca44}$ & 150000 & 30800 & 150000 & 30800 \\
\hline F22 & $\operatorname{Ca} 44$ & 1030000 & 198000 & 1030000 & 198000 \\
\hline $\mathrm{F} 23$ & $\mathrm{Ca} 44$ & 176000 & & 176000 & \\
\hline F31 & $\operatorname{Co} 44$ & 161000 & & 161000 & \\
\hline F32 & Ca44 & 1610000 & 135000 & 1610000 & 135000 \\
\hline F33 & Ca44 & 883000 & 207000 & 883000 & 207000 \\
\hline$F_{41}$ & Ca44 & 1600000 & 127000 & 1600000 & 127000 \\
\hline$F_{42}$ & $\operatorname{Cos4}$ & 3300000 & 267000 & 3300000 & 267000 \\
\hline $\mathrm{F} 43$ & $\mathrm{Ca} 44$ & & & & \\
\hline$F 51$ & $\operatorname{Co} 44$ & 90300 & & 90300 & \\
\hline F52 & Co44 & 737000 & 33300 & 737000 & 33300 \\
\hline F53 & Ca44 & 2450000 & 83100 & 2450000 & 93100 \\
\hline
\end{tabular}

Note: The ectual concentration of eamples for which no standerd deviation to reported to leas than the lower limit of detection. The reported concentration is the lower Umit of quantification. 


\section{Transect F Soil Concentrations: Cesium}

\begin{tabular}{|c|c|c|c|c|c|}
\hline Semple ld. & botope & $\begin{array}{l}\text { Concentration } \\
\text { (ug/Kg soll) }\end{array}$ & $\begin{array}{l}\text { Std.Dovlation } \\
\text { (ug/Kg soll) }\end{array}$ & $\begin{array}{l}\text { Element Avg. Conc. } \\
\text { (ug/Kg soll) }\end{array}$ & $\begin{array}{c}\text { Ekement Avg. Std. Dov. } \\
\text { (ug/Kg soll) }\end{array}$ \\
\hline F11 & Cs & 202 & 5.37 & 200 & 5.37 \\
\hline F12 & Cs & 229 & 12.5 & 229 & 12.5 \\
\hline F13 & Cs & 585 & 49.1 & 585 & 49.1 \\
\hline F21 & $\mathrm{C}_{8}$ & 238 & 22.8 & 238 & 22.8 \\
\hline F21R & $\mathrm{Cs}_{8}$ & 88.6 & & 88.6 & \\
\hline $\mathrm{F} 22$ & $\mathrm{C}_{8}$ & 115 & 13.9 & 115 & 13.9 \\
\hline $\mathrm{F} 23$ & Cs & 218 & 46.6 & 218 & 46.6 \\
\hline F31 & Cs & 1030 & 72.8 & 1030 & 72.8 \\
\hline F32 & Cs & 538 & 23.3 & 538 & 23.3 \\
\hline F33 & $\mathrm{Cs}$ & 117 & 10.5 & 117 & 10.5 \\
\hline F 41 & Cs & 3210 & 136 & 3210 & 136 \\
\hline$F 42$ & $\mathrm{C}_{8}$ & 1250 & 54.9 & 1250 & 54.9 \\
\hline $\mathrm{F} 43$ & Cs & 479 & 19.6 & 479 & 19.6 \\
\hline F51 & Cs & 922 & 58.1 & 922 & 58.1 \\
\hline F52 & Cs & 1200 & 28.7 & 1200 & 28.7 \\
\hline$F 53$ & Cs & 600 & 43.7 & 600 & 43.7 \\
\hline
\end{tabular}




\section{Transect F Soil Concentrations: Chromium}

\begin{tabular}{|c|c|c|c|c|c|}
\hline Sample ld. & botope & $\begin{array}{l}\text { Concentration } \\
\text { (ug/Kg soil) }\end{array}$ & $\begin{array}{l}\text { Std.Doviation } \\
\text { (ug/Kg soll) }\end{array}$ & $\begin{array}{l}\text { Element Avg. Conc. } \\
\text { (ug/Kg soll) }\end{array}$ & $\begin{array}{c}\text { Element Avg. Std. Dov. } \\
\text { (ug/Kg soll) }\end{array}$ \\
\hline $\mathrm{F} 11$ & 952 & 3860 & 212 & & \\
\hline$F 11$ & C.53 & 3260 & 248 & 3560 & 230 \\
\hline$F 12$ & 952 & 14200 & 185 & & \\
\hline$F 12$ & C.53 & 14200 & 179 & 14200 & 182 \\
\hline $\mathrm{F} 13$ & C.52 & 22800 & 559 & & \\
\hline F13 & Cr53 & 22800 & 559 & 22800 & 559 \\
\hline$F 21$ & C.52 & 7740 & 126 & & \\
\hline F21 & 953 & 7840 & 126 & 7790 & 126 \\
\hline F21R & C.52 & 6890 & 1220 & & \\
\hline F21R & C.53 & 6890 & 1220 & 6890 & 1220 \\
\hline$F 22$ & C.52 & 5550 & 122 & & \\
\hline $\mathrm{F} 22$ & C.53 & 5510 & 123 & 5530 & 123 \\
\hline $\mathbf{F 2 3}$ & Cr52 & 6270 & 208 & & \\
\hline F23 & Cr53 & 6280 & 210 & 6280 & 200 \\
\hline $\mathrm{F31}$ & C.52 & 21300 & 295 & & \\
\hline F31 & C.53 & 21100 & 297 & 21200 & 296 \\
\hline$F 32$ & C.52 & 26300 & 356 & & \\
\hline F32 & C.53 & 24500 & 315 & 25400 & 336 \\
\hline F33 & 952 & 14400 & 53.5 & & \\
\hline F33 & $C_{553}$ & 14800 & 46 & 14600 & 49.8 \\
\hline $\mathrm{F} 41$ & C.52 & 37900 & 219 & & \\
\hline F41 & $\mathrm{Cr} 53$ & & & 37900 & 219 \\
\hline F42 & 952 & 47800 & 930 & & \\
\hline$F 42$ & C.53 & 48100 & 941 & 48000 & 936 \\
\hline $\mathrm{F} 43$ & 952 & 25700 & 469 & & \\
\hline $\mathrm{F} 43$ & G53 & 25700 & 467 & 25700 & 468 \\
\hline F51 & C.52 & 17400 & 447 & & \\
\hline F51 & 0.53 & 17300 & 438 & 17400 & 443 \\
\hline F52 & C52 & 29400 & 660 & & \\
\hline$F 52$ & C.53 & 29200 & 641 & 29300 & 651 \\
\hline F53 & G52 & 16300 & 574 & & \\
\hline F53 & Cr53 & 16200 & 57 & 16300 & 576 \\
\hline
\end{tabular}

Note: The actual concentration of eamples for which no standard deviation ls reported ts leas than the fower llmit of detection. The reported concentration is the lower limit of quantification. 


\section{Transect F Soil Concentrations: Cobalt}

\begin{tabular}{|c|c|c|c|c|c|}
\hline Semple Id. & Lnotope & $\begin{array}{l}\text { Concentration } \\
\text { (ug/Kg soll) }\end{array}$ & $\begin{array}{l}\text { Std.Deviation } \\
\text { (ug/Kg soll) }\end{array}$ & $\begin{array}{l}\text { Element Aya. Conc. } \\
\text { (ughKo woil) }\end{array}$ & $\begin{array}{c}\text { Element Avg. Std. Dev. } \\
\text { (ug/Kg soli) }\end{array}$ \\
\hline $\mathrm{F} 11$ & Co & 726 & 46 & 726 & 46 \\
\hline$F 12$ & $C_{0}$ & 721 & 32.1 & 721 & 32.1 \\
\hline F13 & $\infty$ & 10500 & & 10500 & \\
\hline F21 & $\infty$ & 1390 & 24.1 & 1390 & 24.1 \\
\hline F21R & Co & 966 & 81.9 & 966 & 81.9 \\
\hline$F 22$ & Co & 834 & 64.7 & 834 & 64.7 \\
\hline F23 & Co & 1310 & 179 & 1310 & 179 \\
\hline$F 31$ & Co & 1470 & 82.4 & 1470 & 82.4 \\
\hline F32 & $C_{0}$ & 835 & 52.4 & 835 & 52.4 \\
\hline F33 & Co & 284 & 15.9 & 284 & 15.9 \\
\hline$F 41$ & Co & 2750 & 123 & 2750 & 123 \\
\hline F42 & Co & 894 & 15.6 & 894 & 15.6 \\
\hline$F 43$ & Co & 1050 & & 1050 & \\
\hline$F 51$ & Co & 1460 & 36.8 & 1460 & 36.8 \\
\hline F52 & $C_{0}$ & 1100 & 51.7 & 1100 & 51.7 \\
\hline F53 & Co & 476 & 45.7 & 476 & 45.7 \\
\hline
\end{tabular}

Note: The sctual concentration of eamples for whlch no standerd deviation is reported bs leas than the lower limlt of detection. The reportod concentration ls the lower limit of quantification. 


\section{Transect F Soil Concentrations: Copper}

\begin{tabular}{|c|c|c|c|c|c|}
\hline Semple ld. & botope & $\begin{array}{l}\text { Concentration } \\
\text { (ug/Kg soli) }\end{array}$ & $\begin{array}{l}\text { Std.Dovintion } \\
\text { (ug/Kg sol) }\end{array}$ & $\begin{array}{l}\text { Element Avg. Conc. } \\
\text { (ug/Kg roll) }\end{array}$ & $\begin{array}{c}\text { Element Avg. Std. Dov. } \\
\text { (ugKg soli) }\end{array}$ \\
\hline F11 & av63 & 1380 & 156 & & \\
\hline F11 & Ou65 & 3980 & 337 & 2680 & 247 \\
\hline F12 & av63 & 2820 & 47.7 & & \\
\hline F12 & Cus5 & 5960 & 172 & 4390 & 110 \\
\hline F13 & Cu63 & & & & \\
\hline F13 & Cu65 & 7360 & 1040 & 7360 & 1040 \\
\hline F21 & 0463 & 4900 & 508 & & \\
\hline F21 & Cu65 & 8340 & 210 & 6620 & 359 \\
\hline F21R & au63 & 5300 & 683 & & \\
\hline F21R & Cu65 & 4590 & 1540 & 4950 & 1110 \\
\hline $\mathrm{F} 22$ & Cu63 & 3080 & 86.5 & & \\
\hline$F 22$ & Cu65 & 2590 & 327 & 2840 & 207 \\
\hline $\mathrm{F} 23$ & 0463 & 4050 & 308 & & \\
\hline F23 & Cu65 & 5060 & 864 & 4560 & 586 \\
\hline F31 & Cu63 & 867 & 514 & & \\
\hline F31 & Cu65 & 1740 & 752 & 1300 & 633 \\
\hline F32 & Cu63 & 641 & & & \\
\hline F32 & Cu65 & 641 & & 641 & \\
\hline F33 & Cu63 & 3010 & 49.5 & & \\
\hline F33 & Cu65 & 3750 & 92.8 & 3380 & 712 \\
\hline F41 & Cu63 & 13000 & 135 & & \\
\hline F41 & Cu65 & 17300 & 638 & 15200 & 387 \\
\hline F42 & Cu63 & 5710 & 113 & & \\
\hline F42 & Cu65 & 7730 & 295 & 6720 & 204 \\
\hline $\mathrm{F} 43$ & Ou63 & & & & \\
\hline$F 43$ & Cu65 & 3230 & 229 & 3230 & 229 \\
\hline $\mathrm{F51}$ & Cu63 & 4360 & 257 & & \\
\hline$F 51$ & Cu65 & 4840 & 388 & 4600 & 323 \\
\hline F52 & Cu63 & 5290 & 126 & & \\
\hline F52 & Cu65 & 4740 & 407 & 5020 & 267 \\
\hline $\mathrm{F53}$ & Cu63 & 3270 & 197 & & \\
\hline F53 & Cu65 & 1450 & 170 & 2360 & 184 \\
\hline
\end{tabular}

Note: The ectual concentration of anmples for which no standard deviation b reported b leas than the lower limit of detection. The reported concentration is the lower limit of quantification. 
Transect F Soil Concentrations: Iron

\begin{tabular}{|c|c|c|c|c|c|}
\hline Sample ld. & leotope & $\begin{array}{l}\text { Concentration } \\
\text { (ug/Kg soll) }\end{array}$ & $\begin{array}{l}\text { Std.Deviation } \\
\text { (ug/Kg soll) }\end{array}$ & $\begin{array}{l}\text { Element Avg. Conc. } \\
\text { (ug/Kg soll) }\end{array}$ & $\begin{array}{c}\text { Element Avg. Std. Dov. } \\
\text { (ug/Kg soll) }\end{array}$ \\
\hline$F 11$ & Fo & 9030000 & 179000 & 9030000 & 179000 \\
\hline$F 12$ & $F_{0}$ & 9000000 & 105000 & 9000000 & 105000 \\
\hline F13 & $F_{0}$ & 14500000 & 77000 & 14500000 & 77000 \\
\hline F21 & $\mathrm{Fo}_{0}$ & 5890000 & 37100 & 5890000 & 37100 \\
\hline F21R & $F_{0}$ & 3130000 & 8780 & 3130000 & 8780 \\
\hline$F 22$ & $F_{0}$ & 3680000 & 50000 & 3680000 & 50000 \\
\hline F23 & Fo & 11200000 & 43000 & 11200000 & 43000 \\
\hline F31 & $F_{0}$ & 16400000 & 115000 & 16400000 & 115000 \\
\hline$F 32$ & Fo & 7150000 & 165000 & 7150000 & 165000 \\
\hline F33 & $\mathrm{Fo}$ & 5660000 & 12500 & 5660000 & 12500 \\
\hline$F_{41}$ & $F_{\theta}$ & 22600000 & 26600 & 22600000 & 26600 \\
\hline$F 42$ & $F_{\theta}$ & 14000000 & 109000 & 14000000 & 109000 \\
\hline $\mathrm{F} 43$ & $F_{0}$ & 22300000 & 235000 & 22300000 & 235000 \\
\hline$F 51$ & $F_{\theta}$ & 4360000 & 25100 & 4360000 & 25100 \\
\hline$F 52$ & $F_{\theta}$ & 10200000 & 221000 & 10200000 & 221000 \\
\hline$F 53$ & $F_{\theta}$ & 19300000 & 333000 & 19300000 & 333000 \\
\hline
\end{tabular}

Noto: The actual concentration of samples for which no standard doviation is reported be lees than the lower limit of detection. The reported concentration is the lower limit of quantification. 
Transect F Soil Concentrations: Lead

\begin{tabular}{|c|c|c|c|c|c|}
\hline Sampte ld. & leotope & $\begin{array}{l}\text { Concentratton } \\
\text { (ug/Kg soll) }\end{array}$ & $\begin{array}{l}\text { Str.Dovintion } \\
\text { (ug/Kg soll) }\end{array}$ & $\begin{array}{l}\text { Element Avg. Conc. } \\
\text { (ug/Kg soil) }\end{array}$ & $\begin{array}{c}\text { Element Avg. Std. Dov. } \\
\text { (ug/Kg soll) }\end{array}$ \\
\hline F11 & Pb206 & 55100 & 521 & & \\
\hline$F 11$ & Pb207 & 24300 & 201 & & \\
\hline F11 & Pb208 & 47300 & 400 & 42200 & 377 \\
\hline F12 & Pb206 & 25900 & 490 & & \\
\hline F12 & Pb207 & 22000 & 376 & & \\
\hline$F 12$ & Pb208 & 23900 & 384 & 23900 & 417 \\
\hline F13 & $\mathrm{Pb} 206$ & 7390 & 338 & & \\
\hline F13 & $\mathrm{Pb207}$ & 7610 & 182 & & \\
\hline F13 & $\mathrm{Pb} 208$ & 6360 & 254 & 7120 & 258 \\
\hline F21 & $\mathrm{Pb} 206$ & 32300 & 520 & & \\
\hline$F_{21}$ & $\mathrm{~Pb} 207$ & 35900 & 799 & & \\
\hline F21 & $\mathrm{Pb} 208$ & 25400 & 397 & 31200 & 575 \\
\hline F21R & $\mathrm{Pb} 206$ & 83400 & 1910 & & \\
\hline F21R & $\mathrm{Pb} 207$ & 60700 & 2410 & & \\
\hline F21R & Pb208 & 78500 & 1660 & 74200 & 1990 \\
\hline$F 22$ & $\mathrm{~Pb} 206$ & 91300 & 1650 & & \\
\hline$F 22$ & Pb207 & 95100 & 2730 & & \\
\hline$F \not 22$ & Pb208 & 83400 & 1450 & 89900 & 1940 \\
\hline$F 23$ & Pb206 & 13900 & 521 & & \\
\hline$F 23$ & Pt207 & 12800 & 607 & & \\
\hline F23 & Pb208 & 13600 & 367 & 13400 & 498 \\
\hline F31 & $\mathrm{Pb} 206$ & 64000 & 572 & & \\
\hline$F 31$ & Pb207 & 72100 & 2000 & & \\
\hline F31 & $\mathrm{Pb} 208$ & 60100 & 1010 & 65400 & 1190 \\
\hline F32 & Pb206 & 40400 & 1090 & & \\
\hline F32 & Pb207 & 37900 & 861 & & \\
\hline F32 & $\mathrm{Pb} 208$ & 40100 & 542 & 39500 & 831 \\
\hline$F 33$ & Pb206 & 17200 & 298 & & \\
\hline$F 33$ & Pb207 & 19500 & 576 & & \\
\hline F33 & Pb208 & 16300 & 236 & 17700 & 370 \\
\hline F41 & Pb206 & 18600 & 433 & & \\
\hline F41 & $\mathrm{Pb} 207$ & 18400 & 375 & & \\
\hline F41 & $\mathrm{Pb} 208$ & 16500 & 181 & 17800 & 330 \\
\hline F42 & Pb206 & 51500 & 963 & & \\
\hline F42 & $\mathrm{Pb} 207$ & 45700 & 1460 & & \\
\hline F42 & $\mathrm{Pb} 208$ & 47600 & 1150 & 48300 & 1190 \\
\hline$F 43$ & Pb206 & 4840 & 184 & & \\
\hline$F 43$ & Pb207 & 3630 & 82.8 & & \\
\hline$F 43$ & P6208 & 3960 & 25.1 & 4140 & 97.3 \\
\hline F51 & Pb206 & 45000 & 363 & & \\
\hline F51 & Pb207 & 41100 & 867 & & \\
\hline F51 & $\mathrm{Pb} 208$ & 42600 & 1030 & 42900 & 753 \\
\hline$F 52$ & Pb206 & 50800 & 655 & & \\
\hline$F 52$ & $\mathrm{~Pb} 207$ & 51100 & 448 & & \\
\hline
\end{tabular}




\begin{tabular}{|c|c|c|c|c|c|}
\hline Sample ld. & leotope & $\begin{array}{l}\text { Concentration } \\
\text { (ug/Kg soli) }\end{array}$ & $\begin{array}{l}\text { Std.Devlation } \\
\text { (ug/Kg soil) }\end{array}$ & $\begin{array}{l}\text { Element Avg. Conc. } \\
\text { (ug/Kg soil) }\end{array}$ & $\begin{array}{c}\text { Element Avg. Std. Dov. } \\
\text { (ug/Kg soll) }\end{array}$ \\
\hline F52 & $\mathrm{Pb208}$ & 45400 & 271 & 49100 & 458 \\
\hline F53 & Pb206 & 26300 & 589 & & \\
\hline F53 & $\mathrm{Pb} 207$ & 22400 & 1090 & & \\
\hline F53 & Pb208 & 23100 & 864 & 23900 & 848 \\
\hline
\end{tabular}

Note: The ectual concentration of eamples for which no standard deviation is reported is leas than the fower llmit of detection. The reported concentration is the lower limit of quantification. 


\section{Transect F Soil Concentrations: Magnesium}

\begin{tabular}{|c|c|c|c|c|c|}
\hline Semple ld. & botope & $\begin{array}{l}\text { Concentration } \\
\text { (ug/Kg soli) }\end{array}$ & $\begin{array}{l}\text { Std.Dovituton } \\
\text { (ug/Kg soll) }\end{array}$ & $\begin{array}{l}\text { Element Avg. Cone. } \\
\text { (ug/Kg soll) }\end{array}$ & $\begin{array}{c}\text { Element Avg. Std. Dev. } \\
\text { (ug/Kg soll) }\end{array}$ \\
\hline F11 & $M g 24$ & 98300 & 2720 & & \\
\hline F11 & Mg25 & 161000 & 3480 & 130000 & 3100 \\
\hline F12 & $\mathrm{Mg} 24$ & 168000 & 3870 & & \\
\hline$F 12$ & Mgas & 204000 & 1180 & 186000 & 2530 \\
\hline$F 13$ & Mg24 & 299000 & 3740 & & \\
\hline F13 & Mges & 302000 & 5350 & 301000 & 4550 \\
\hline F21 & Mg24 & 94000 & 856 & & \\
\hline $\mathrm{F} 21$ & Mg2s & 76800 & 1810 & 85400 & 1330 \\
\hline F21R & Mg24 & 54800 & 1170 & & \\
\hline F21R & Mges & 58000 & 4280 & 56400 & 2730 \\
\hline $\mathrm{F} 22$ & Mge4 & 93500 & 2130 & & \\
\hline F22 & Mg25 & 89100 & 2880 & 91300 & 2510 \\
\hline$F 23$ & Mg24 & 167000 & 1970 & & \\
\hline F23 & Mg25 & 164000 & 3010 & 166000 & 2490 \\
\hline F31 & $M g 24$ & 256000 & 7330 & & \\
\hline F31 & Mges & 305000 & 6730 & 281000 & 7030 \\
\hline F32 & $\mathrm{Mg24}$ & 138000 & 3040 & & \\
\hline F32 & Mges & 133000 & 3840 & 136000 & 3440 \\
\hline F33 & Mg24 & 58100 & 864 & & \\
\hline F33 & Mges & 80100 & 1040 & 69100 & 952 \\
\hline F41 & Mg24 & 124000 & 561 & & \\
\hline F41 & Mgas & 73700 & 2470 & 98900 & 1520 \\
\hline$F 42$ & $M g 24$ & 110000 & 2970 & & \\
\hline F42 & Mges & 103000 & 3180 & 107000 & 3080 \\
\hline $\mathrm{F} 43$ & Mg24 & 218000 & 406 & & \\
\hline$F_{43}$ & Mg25 & 211000 & 1130 & 215000 & 768 \\
\hline F51 & $\mathrm{Mg} 24$ & 145000 & 2380 & & \\
\hline$F 51$ & Mgas & 150000 & 7760 & 148000 & 5070 \\
\hline$F 52$ & $M g 24$ & 198000 & 2110 & & \\
\hline$F 52$ & Mg25 & 239000 & 2640 & 219000 & 2380 \\
\hline $\mathrm{F} 53$ & Mg24 & 121000 & 3100 & & \\
\hline F53 & Mg25 & 111000 & 4080 & 116000 & 3590 \\
\hline
\end{tabular}

Note: The ectual concentration of eamples for whlch no standard deviatton be reported be leas than the lower llmit of detection. The reported concentration is the lower Ilmit of quantification. 


\section{Transect F Soil Concentrations: Manganese}

\begin{tabular}{|c|c|c|c|c|c|}
\hline Semple ld. & beotope & $\begin{array}{l}\text { Concentration } \\
\text { (ug/Kg soll) }\end{array}$ & $\begin{array}{l}\text { Std.Deviation } \\
\text { (ug/Kg soil) }\end{array}$ & $\begin{array}{l}\text { Element Avg. Conc. } \\
\text { (ug/Kg eoll) }\end{array}$ & $\begin{array}{c}\text { Element Avg. Std. Dov. } \\
\text { (Ug/Kg soll) }\end{array}$ \\
\hline$F 11$ & $M n$ & 233000 & 13100 & 233000 & 13100 \\
\hline $\mathrm{F} 12$ & $\mathrm{Mn}$ & 171000 & 13200 & 171000 & 13200 \\
\hline F13 & $\mathrm{Mn}$ & 38600 & 534 & 38600 & 534 \\
\hline F21 & $\mathrm{Mn}$ & 67800 & 3270 & 67800 & 3270 \\
\hline F21R & $\mathrm{Mn}$ & 10500 & 493 & 10500 & 493 \\
\hline$F 22$ & $\mathrm{Mn}$ & 27700 & 489 & 27700 & 489 \\
\hline$F 23$ & $\mathbf{M n}$ & 105000 & 682 & 105000 & 682 \\
\hline$F 31$ & $\mathrm{Mn}$ & 45000 & 800 & 45000 & 800 \\
\hline$F 32$ & $\mathrm{Mn}$ & 152000 & 5920 & 152000 & 5920 \\
\hline F33 & $\mathrm{Mn}$ & 82100 & 2280 & 82100 & 2280 \\
\hline $\mathrm{F} 41$ & $\mathrm{Mn}$ & 178000 & 11400 & 178000 & 11400 \\
\hline F42 & $\mathrm{Mn}$ & 225000 & 27800 & 225000 & 27800 \\
\hline $\mathrm{F} 43$ & $\mathrm{Nn}$ & 27300 & 108 & 27300 & 108 \\
\hline F51 & $\mathrm{Mn}$ & 65600 & 3030 & 65600 & 3030 \\
\hline F52 & $\mathrm{Mn}$ & 38800 & 635 & 38800 & 635 \\
\hline F53 & $M n$ & 181000 & 15300 & 181000 & 15300 \\
\hline
\end{tabular}

Note: The sctual concentration of samples for which no standard deviation ls reported is leas than the lower IImit of detection. The reported concentration is the lower limit of quantification. 
Transect F Soil Concentrations: Nickel

\begin{tabular}{|c|c|c|c|c|c|}
\hline Sampla Id. & botope & $\begin{array}{l}\text { Concentration } \\
\text { (ug/kg soll) }\end{array}$ & $\begin{array}{l}\text { Std.Deviation } \\
\text { (ug/Kg soll) }\end{array}$ & $\begin{array}{c}\text { Element Avg. Conc. } \\
\text { (ug/Kg coll) }\end{array}$ & $\begin{array}{c}\text { Element Avg. Std. Dov. } \\
\text { (ug/Kg soll) }\end{array}$ \\
\hline F11 & Niso & 3420 & 524 & & \\
\hline F11 & Nis2 & 2830 & 1070 & 3130 & 797 \\
\hline F12 & Niso & 3180 & 311 & & \\
\hline F12 & Ni62 & 5900 & 218 & 4540 & 265 \\
\hline F13 & Ni60 & 3140 & & & \\
\hline F13 & Ni62 & & & 3140 & \\
\hline F21 & Niso & 6750 & 345 & & \\
\hline F21 & Ni62 & 5140 & 731 & 5950 & 538 \\
\hline F21R & $N i \in 0$ & 20000 & 1700 & & \\
\hline F21R & Nis2 & 16800 & 5680 & 18400 & 3690 \\
\hline $\mathrm{F} 22$ & Niso & 10500 & 227 & & \\
\hline $\mathrm{F} 22$ & Ni62 & 8630 & 1480 & 9570 & 854 \\
\hline F23 & Niso & 5290 & 507 & & \\
\hline $\mathrm{F} 23$ & Ni62 & 1980 & 1050 & 3640 & 779 \\
\hline F31 & Ni6o & 6070 & 360 & & \\
\hline F31 & Ni62 & 12700 & 1210 & 9390 & 785 \\
\hline F32 & Ni60 & 11100 & 336 & & \\
\hline F32 & Ni62 & 14000 & 878 & 12600 & 607 \\
\hline F33 & Ni60 & 5640 & 207 & & \\
\hline F33 & Ni62 & 789 & 180 & 3210 & 194 \\
\hline F41 & $N i 60$ & 10400 & 412 & & \\
\hline F41 & Ni62 & 19900 & 1790 & 15200 & 1100 \\
\hline F42 & Ni6o & 9790 & 284 & & \\
\hline F42 & Ni62 & 11600 & 1040 & 10700 & 662 \\
\hline $\mathrm{F} 43$ & Ni60 & 1240 & 237 & & \\
\hline F43 & Ni62 & & & 1240 & 237 \\
\hline F51 & Ni60 & 23600 & 2720 & & \\
\hline F51 & Ni62 & 31300 & 2850 & 27500 & 2790 \\
\hline F52 & Ni60 & 44500 & 335 & & \\
\hline$F 52$ & Ni62 & 11100 & 589 & 27800 & 462 \\
\hline$F 53$ & Ni6o & 7520 & 369 & & \\
\hline$F 53$ & Ni62 & 6340 & 1060 & 6930 & 715 \\
\hline
\end{tabular}




\section{Transect F Soil Concentrations: Selenium}

\begin{tabular}{|c|c|c|c|c|c|}
\hline Semple ld. & kotope & $\begin{array}{l}\text { Concentration } \\
\text { (ug/ko soll) }\end{array}$ & $\begin{array}{l}\text { BtdDovintion } \\
\text { (ugikd soll) }\end{array}$ & $\begin{array}{l}\text { Eloment Avg. Cone. } \\
\text { (ug/Ko soln) }\end{array}$ & $\begin{array}{c}\text { Element Avg. Std. Dov. } \\
\text { (ug/Kg soll) }\end{array}$ \\
\hline F11 & Se77 & 1380 & & & \\
\hline $\mathrm{F} 11$ & 5082 & 10800 & 2010 & 6000 & 2010 \\
\hline $\mathrm{F} 12$ & so77 & 4210 & 1690 & & \\
\hline F12 & So82 & 3790 & 1580 & 4000 & 1640 \\
\hline F13 & Se77 & & & & \\
\hline F13 & So82 & 49000 & 6880 & 49000 & 6880 \\
\hline F21 & So77 & 4150 & 1040 & & \\
\hline $\mathrm{F21}$ & So82 & 4250 & 982 & 4200 & 1010 \\
\hline F21R & So77 & 7840 & & & \\
\hline F21R & SeB2 & 7840 & & 7840 & \\
\hline $\mathrm{F} 22$ & So77 & 2160 & 2600 & & \\
\hline$F 22$ & So82 & 2120 & 2580 & 2140 & 2590 \\
\hline F23 & 5077 & 8560 & 2320 & & \\
\hline F23 & Se82 & $872 C$ & 2370 & 8640 & 2350 \\
\hline F31 & Se77 & $388 x$ & 1150 & & \\
\hline F31 & So82 & $383 x$ & 1120 & 3860 & 1140 \\
\hline F32 & Se77 & 8620 & 459 & & \\
\hline F32 & Se82 & 8110 & 451 & 8370 & 455 \\
\hline F33 & Se77 & 3700 & 1030 & & \\
\hline F33 & 5082 & 3550 & 988 & 3620 & 1010 \\
\hline F41 & Se77 & & & & \\
\hline F41 & So82 & 10900 & 2800 & 10900 & 2900 \\
\hline$F 42$ & So77 & 8430 & 1110 & & \\
\hline F42 & Se82 & 8350 & 1060 & 8380 & 1090 \\
\hline$F_{43}$ & So77 & & & & \\
\hline F43 & Se82 & 12400 & 14200 & 12400 & 14200 \\
\hline F51 & Se77 & 2530 & 1760 & & \\
\hline F51 & So82 & 2340 & 1620 & 2440 & 1690 \\
\hline F52 & Se77 & 3160 & & & \\
\hline $\mathrm{F} 52$ & Se82 & 3160 & & 3160 & \\
\hline F53 & Se77 & 3910 & & & \\
\hline$F 53$ & Se82 & 3910 & & 3910 & \\
\hline
\end{tabular}




\section{Transect F Soil Concentrations: Silver}

\begin{tabular}{|c|c|c|c|c|c|}
\hline Sample Id. & botope & $\begin{array}{l}\text { Concentration } \\
\text { (ug/Kg soll) }\end{array}$ & $\begin{array}{l}\text { StdDovintion } \\
\text { (ug/Ko coll) }\end{array}$ & $\begin{array}{l}\text { Element Avg. Conc. } \\
\text { (ug/Kg soll) }\end{array}$ & $\begin{array}{l}\text { Element Avg. Std. Dov. } \\
\text { (ug/Kg soll) }\end{array}$ \\
\hline$F_{11}$ & Ag107 & 895 & & & \\
\hline F11 & Ag109 & 1910 & 629 & 1450 & 629 \\
\hline$F 12$ & Ag107 & 574 & 190 & & \\
\hline$F 12$ & Ag109 & 575 & 672 & 575 & 431 \\
\hline F13 & Ag107 & 8380 & & & \\
\hline F13 & Ag100 & 8380 & & 8380 & \\
\hline F21 & Ag107 & 341 & 20.3 & & \\
\hline F21 & A0109 & 1410 & 381 & 876 & 201 \\
\hline F21R & Ag107 & 4430 & & & \\
\hline F21R & Ag109 & 4430 & & 4430 & \\
\hline F22 & A0107 & 1270 & 476 & & \\
\hline$F 22$ & Ag100 & 306 & & 788 & 476 \\
\hline F23 & Ag107 & 22.7 & & & \\
\hline F23 & Ag109 & 22.7 & & 22.7 & \\
\hline F31 & Ag107 & 403 & & & \\
\hline F31 & Ag109 & 403 & & 403 & \\
\hline F32 & Ag107 & 2630 & 560 & & \\
\hline$F 32$ & $A \theta 100$ & 2630 & 460 & 2630 & 515 \\
\hline$F 33$ & Ag107 & 598 & & & \\
\hline F33 & Ag109 & 1310 & & 954 & \\
\hline F41 & A9107 & 1860 & 84.1 & & \\
\hline F41 & Ag109 & 1720 & 142 & 1780 & 118 \\
\hline F42 & A0107 & 1530 & 191 & & \\
\hline F42 & Ag100 & 646 & 288 & 1000 & 240 \\
\hline $\mathrm{F} 43$ & Ag107 & 4200 & & & \\
\hline $\mathrm{F} 43$ & Ag109 & 4200 & & 4200 & \\
\hline F51 & Ag107 & 667 & 153 & & \\
\hline F51 & A9109 & 236 & 113 & 452 & 133 \\
\hline$F 52$ & Ag107 & 202 & 150 & & \\
\hline$F 52$ & Ag109 & 291 & 252 & 277 & 201 \\
\hline F53 & Ag107 & 783 & & & \\
\hline F53 & Ag109 & 783 & & 783 & \\
\hline
\end{tabular}




\section{Transect F Soil Concentrations: Sodium}

\begin{tabular}{|c|c|c|c|c|c|}
\hline Semple ld. & botope & $\begin{array}{l}\text { Concentration } \\
\text { (ug/kg soli) }\end{array}$ & $\begin{array}{l}\text { SiddDoviation } \\
\text { (Lig/ko soll) }\end{array}$ & $\begin{array}{l}\text { Element Avg. Conc. } \\
\text { (ug/kg eoll) }\end{array}$ & $\begin{array}{c}\text { Ekement Avg. Std. Dov. } \\
\text { (ug/Kg soll) }\end{array}$ \\
\hline F11 & $\mathrm{Na}$ & 484000 & 16900 & 484000 & 16900 \\
\hline$F 12$ & $\mathrm{Na}$ & 36500 & 27400 & 36500 & 27400 \\
\hline F13 & $\mathrm{Na}$ & 1260000 & 41700 & 1260000 & 41700 \\
\hline F21 & $\mathrm{Na}$ & 432000 & 6710 & 432000 & 6710 \\
\hline F21A & $\mathrm{Na}$ & 157000 & & 157000 & \\
\hline$F 22$ & $\mathrm{Na}$ & 122000 & & 122000 & \\
\hline F23 & $\mathrm{Na}$ & 104000 & 8480 & 104000 & 8480 \\
\hline F31 & $\mathrm{Na}$ & 161000 & & 161000 & \\
\hline$F 32$ & $\mathrm{Na}$ & 6410 & & 6410 & \\
\hline F33 & $\mathrm{Na}$ & 128000 & 36400 & 128000 & 36400 \\
\hline F41 & $\mathrm{Na}$ & 650000 & 8470 & 650000 & 8470 \\
\hline$F 42$ & $\mathrm{Na}$ & 149000 & 15800 & 149000 & 15800 \\
\hline $\mathrm{F} 43$ & $\mathrm{Na}$ & 64700 & 13900 & 64700 & 13900 \\
\hline F51 & $\mathrm{Na}$ & 9030 & & 9030 & \\
\hline F52 & $\mathrm{Na}$ & 112000 & 10000 & 112000 & 10000 \\
\hline F53 & $\mathrm{Na}$ & 14800 & & 14800 & \\
\hline
\end{tabular}

Note: The actual concentration of eamples for which no standard deviation ts reported is has than the lower llmit of detection. The reported concentration ts the lower Umit of quantification. 


\section{Transect F Soil Concentrations: Strontium}

\begin{tabular}{|c|c|c|c|c|c|}
\hline Sample ld. & leotope & $\begin{array}{l}\text { Concentration } \\
\text { (ug/Kg soil) }\end{array}$ & $\begin{array}{l}\text { Std.Doviation } \\
\text { (Ug/Kg soli) }\end{array}$ & $\begin{array}{l}\text { Element Avg. Conc. } \\
\text { (Ug/Kg soll) }\end{array}$ & $\begin{array}{c}\text { Element Avg. Std. Dov. } \\
\text { (ug/Kg soil) }\end{array}$ \\
\hline$F 11$ & $\mathrm{Sr}$ & 14700 & 103 & 14700 & 103 \\
\hline$F 12$ & $\mathrm{Sr}$ & 14400 & 220 & 14400 & 220 \\
\hline F13 & $\mathrm{Sr}$ & 5830 & 74.7 & 5830 & 74.7 \\
\hline F21 & $\mathrm{Sr}$ & 5600 & 120 & 5600 & 120 \\
\hline F21R & $\mathrm{Sr}$ & 4510 & 276 & 4510 & 276 \\
\hline $\mathrm{F} 22$ & $\mathrm{Sr}$ & 12400 & 193 & 12400 & 193 \\
\hline$F 23$ & $\mathrm{Sr}$ & 43000 & 410 & 43000 & 410 \\
\hline F31 & $\mathrm{Sr}$ & 13600 & 324 & 13500 & 324 \\
\hline F32 & $\mathrm{Sr}$ & 8430 & 113 & 8430 & 113 \\
\hline F33 & $\mathrm{Sr}$ & 6210 & 44.7 & 6210 & 44.7 \\
\hline F41 & $\mathrm{Sr}$ & 18200 & 266 & 18200 & 266 \\
\hline F42 & $\mathrm{Sr}$ & 28800 & 234 & 28800 & 234 \\
\hline F43 & $\mathrm{Sr}$ & 4210 & 53.5 & 4210 & 53.5 \\
\hline F51 & $\mathrm{Sr}$ & 9340 & 25.3 & 9340 & 25.3 \\
\hline$F 52$ & $\mathrm{Sr}$ & 13400 & 224 & 13400 & 224 \\
\hline F53 & $\mathrm{Sr}$ & 18300 & 214 & 18300 & 214 \\
\hline
\end{tabular}




\section{Transect F Soil Concentrations: Thorium}

\begin{tabular}{cccc}
\hline Sample Id. Lotope & $\begin{array}{c}\text { Concentration } \\
\text { (ug/Kg soll) }\end{array}$ & $\begin{array}{c}\text { Std.Deviation } \\
\text { (ug/Kg soil) }\end{array}$ & $\begin{array}{c}\text { Element Avg. Conc. } \\
\text { (ug/Kg soli) }\end{array}$
\end{tabular}

\begin{tabular}{|c|c|c|c|c|c|}
\hline F11 & Th232 & 760 & 40.4 & 760 & 40.4 \\
\hline F12 & Th232 & 661 & 14.1 & 661 & 14.1 \\
\hline F13 & Th232 & 1050 & 37.9 & 1050 & 37.9 \\
\hline $\mathrm{F21}$ & Th232 & 68.6 & 6.39 & 68.6 & 6.39 \\
\hline F21R & Th232 & 45.9 & 55.7 & 45.9 & 55.7 \\
\hline$F 22$ & Th232 & 43.5 & 25.1 & 43.5 & 25.1 \\
\hline $\mathrm{F} 23$ & Th232 & 31.1 & 18.9 & 31.1 & 18.9 \\
\hline $\mathrm{F} 31$ & Th232 & 2710 & 66.9 & 2710 & 66.9 \\
\hline F32 & Th232 & 1150 & 14 & 1150 & 14 \\
\hline$F 33$ & Th232 & 862 & 18 & 862 & 18 \\
\hline F41 & Th232 & 1290 & 18.4 & 1290 & 18.4 \\
\hline F42 & Th232 & 648 & 24.5 & 648 & 24.5 \\
\hline F43 & Th232 & 1230 & 19.4 & 1230 & 19.4 \\
\hline F51 & Th232 & 1450 & 42 & 1450 & 42 \\
\hline$F 52$ & Th232 & 1160 & 24.6 & 1160 & 24.6 \\
\hline$F 53$ & Th232 & 757 & 14.9 & 757 & 14.9 \\
\hline
\end{tabular}

Note: The ectulal concentration of eamples for which no standerd deviation be reported bs leas than the lower limit of dotection. The reported concentration is the lower limit of quantification. 
Transect F Soil Concentrations: Tin

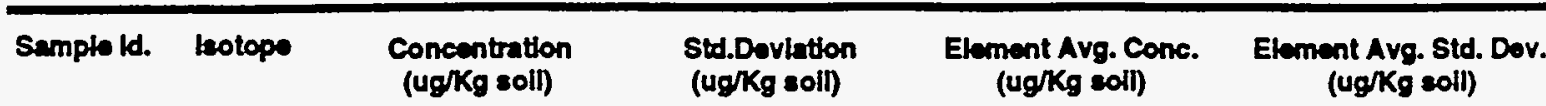

\begin{tabular}{|c|c|c|c|c|c|}
\hline$F 11$ & Sn118 & 830 & 351 & & \\
\hline$F 11$ & Sn120 & 759 & 72.3 & 795 & 212 \\
\hline F12 & Sn118 & 333 & 40.4 & & \\
\hline F12 & Sn120 & 230 & 23.4 & 282 & 31.9 \\
\hline F13 & Sn118 & 224 & 91.6 & & \\
\hline F13 & $\operatorname{Sn} 120$ & 389 & 103 & 307 & 97.3 \\
\hline F21 & Sn118 & 615 & 35.2 & & \\
\hline F21 & Sn120 & 657 & 15.6 & 636 & 25.4 \\
\hline F21R & Sn118 & 925 & 290 & & \\
\hline F21R & Sn120 & 88.6 & & 507 & 290 \\
\hline F22 & $\operatorname{Sn} 118$ & 153 & 42.7 & & \\
\hline$F 22$ & $\operatorname{Sn} 120$ & 152 & 33.2 & 153 & 38 \\
\hline F23 & Sn118 & 573 & 522 & & . \\
\hline F23 & Sn120 & 426 & 133 & 500 & 92.6 \\
\hline F31 & Sn118 & 1250 & 133 & & \\
\hline F31 & $\operatorname{Sn} 120$ & 1080 & 44.5 & 1170 & 88.8 \\
\hline F32 & Sn118 & 632 & 82.7 & & \\
\hline F32 & Sn120 & 669 & 55.8 & 651 & 69.3 \\
\hline F33 & Sn118 & 526 & 264 & & \\
\hline F33 & Sn120 & 571 & 30.7 & 549 & 147 \\
\hline$F_{41}$ & Sn118 & 1540 & 98.6 & & \\
\hline F41 & Sn120 & 1500 & 136 & 1520 & 117 \\
\hline$F_{42}$ & Sn118 & 976 & 68 & & \\
\hline$F_{42}$ & $\operatorname{sn} 120$ & 999 & 27 & 988 & 47.5 \\
\hline F43 & Sn118 & 10200 & 627 & & \\
\hline $\mathrm{F} 43$ & Sn120 & 14500 & 1630 & 12400 & 1130 \\
\hline F51 & Sn118 & 899 & 70.1 & & \\
\hline F51 & Sn120 & 1110 & 74.3 & 1000 & 72.2 \\
\hline F52 & Sn118 & 1040 & 74.3 & & \\
\hline F52 & $\operatorname{Sn} 120$ & 1100 & 57.1 & 1070 & 65.7 \\
\hline F53 & Sn118 & 401 & 35.9 & & \\
\hline F53 & Sn120 & 531 & 42.3 & 466 & 39.1 \\
\hline
\end{tabular}

Note: The actual concentration of eamplee for which no standard deviation is reported ls lees than the lower llmit of detection. The reported concentration is the lower limit of quantfication. 


\section{Transect F Soil Concentrations: Uranium-235}

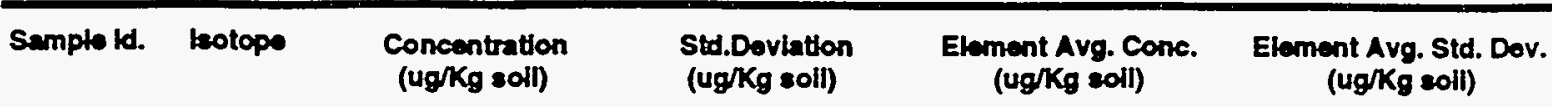

\begin{tabular}{|c|c|c|c|c|c|}
\hline F11 & U235 & 18.7 & 2.76 & 18.7 & 2.76 \\
\hline F12 & U235 & 29.6 & 4.6 & 29.6 & 4.6 \\
\hline F13 & U235 & 78.1 & 7.74 & 78.1 & 7.74 \\
\hline$F 21$ & U235 & 4.23 & 1 & 423 & 1 \\
\hline F21R & U235 & 56.4 & & 56.4 & \\
\hline $\mathrm{F} 22$ & U235 & 43.7 & 5.3 & 43.7 & 5.3 \\
\hline$F 23$ & U235 & 30.1 & 7.55 & 30.1 & 7.55 \\
\hline F31 & U235 & 29 & & 29 & \\
\hline$F 32$ & U235 & 12.7 & 2.89 & 12.7 & 2.89 \\
\hline$F 33$ & U235 & 16.5 & 3.12 & 16.5 & 3.12 \\
\hline$F_{41}$ & U235 & 24 & 1.92 & 24 & 1.92 \\
\hline$F_{42}$ & U235 & 38.4 & 3.98 & 38.4 & 3.98 \\
\hline$F_{43}$ & U235 & 20.3 & 1.11 & 20.3 & 1.11 \\
\hline F51 & U235 & 112 & 24 & 112 & 24 \\
\hline F52 & U235 & 22.8 & 5.97 & 22.8 & 5.97 \\
\hline F53 & U235 & 30.1 & 6.12 & 30.1 & 6.12 \\
\hline
\end{tabular}

Note: The ectual concentration of samples for which no standard deviation la reported is lees than the lower limit of detection. The reported concentration is the fower limit of quantification. 


\section{Transect F Soil Concentrations: Uranium-238}

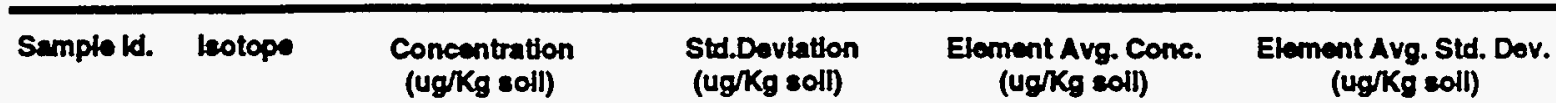

\begin{tabular}{|c|c|c|c|c|c|}
\hline F11 & U238 & 3120 & 26.7 & 3120 & 26.7 \\
\hline F12 & U238 & 3950 & 59 & 3950 & 59 \\
\hline F13 & U238 & 12300 & 141 & 12300 & 141 \\
\hline F21 & U238 & 979 & 31.7 & 979 & 31.7 \\
\hline F21R & U238 & 487 & 83.5 & 487 & 83.5 \\
\hline F22 & U238 & 5760 & 122 & 5760 & 122 \\
\hline F23 & U238 & 6400 & 732 & 6400 & 732 \\
\hline F31 & U238 & 2910 & 83.5 & 2910 & 83.5 \\
\hline F32 & U238 & 2420 & 32.6 & 2420 & 32.6 \\
\hline F33 & U238 & 2280 & 29.6 & 2280 & 29.6 \\
\hline F41 & U238 & 2630 & 70.8 & 2630 & 70.8 \\
\hline F42 & U238 & 4060 & 68.1 & 4060 & 68.1 \\
\hline F43 & U238 & 3260 & 75.9 & 3260 & 75.9 \\
\hline F51 & U238 & 1410 & 47.5 & 1410 & 47.5 \\
\hline F52 & U238 & 2810 & 86.9 & 2810 & 86.9 \\
\hline F53 & U238 & 4210 & 131 & 4210 & 131 \\
\hline
\end{tabular}




\section{Transect F Soil Concentrations: Vanadium}

\begin{tabular}{|c|c|c|c|c|c|}
\hline Sample k. & Lotope & $\begin{array}{l}\text { Concentration } \\
\text { (ug/Kg soll) }\end{array}$ & $\begin{array}{l}\text { Std.Devintion } \\
\text { (ug/Kg soll) }\end{array}$ & $\begin{array}{l}\text { Element Avg. Conc. } \\
\text { (ug/Kg eoli) }\end{array}$ & $\begin{array}{c}\text { Element Avg. Std. Dov. } \\
\text { (ug/Kg soll) }\end{array}$ \\
\hline F11 & $\bar{V}$ & 10400 & 599 & 10400 & 599 \\
\hline F12 & $V$ & 23200 & 1910 & 23200 & 1910 \\
\hline F13 & V & 29500 & 453 & 29500 & 453 \\
\hline $\mathrm{F} 21$ & $V$ & 6540 & 902 & 6540 & 902 \\
\hline F21R & $V$ & 5660 & 673 & 5660 & 673 \\
\hline$F 22$ & $\mathbf{V}$ & 8260 & 17.8 & 8260 & 17.8 \\
\hline $\mathrm{F} 23$ & $V$ & 19300 & 461 & 19300 & 461 \\
\hline F31 & V & 50200 & 1380 & 50200 & 1380 \\
\hline F32 & V & 13200 & 940 & 13200 & 940 \\
\hline F33 & $\mathbf{V}$ & 18900 & 449 & 18900 & 449 \\
\hline$F 41$ & V & 54000 & 1750 & 54000 & 1750 \\
\hline F42 & $V$ & 49900 & 1360 & 49900 & 1360 \\
\hline $\mathrm{F} 43$ & $V$ & 46200 & 568 & 46200 & 568 \\
\hline F51 & $V$ & 7940 & 2870 & 7940 & 2870 \\
\hline F52 & $V$ & 33200 & 539 & 33200 & 539 \\
\hline$F 53$ & $\mathbf{V}$ & 30600 & 3430 & 30600 & 3430 \\
\hline
\end{tabular}




\section{Transect F Soil Concentrations: Yttrium}

\begin{tabular}{ccccc}
\hline Sample ld. leotope & $\begin{array}{c}\text { Concentratton } \\
\text { (ug/Kg sold) }\end{array}$ & $\begin{array}{c}\text { Std.Doviation } \\
\text { (ug/Kg soll) }\end{array}$ & $\begin{array}{c}\text { Element Avg. Conc. } \\
\text { (ug/Kg soil) }\end{array}$ & $\begin{array}{c}\text { Element Avg. Std. Dov. } \\
\text { (ug/Kg soll) }\end{array}$
\end{tabular}

\begin{tabular}{|l|l|l|l|l|l|}
\hline F11 & $Y$ & 585 & 21.9 & 585 & 21.9 \\
\hline F12 & $Y$ & 1560 & 11.5 & 1560 & 11.5 \\
\hline F13 & $Y$ & 705 & 58.9 & 705 & 58.9 \\
\hline F21 & $Y$ & 323 & 18.6 & 323 & 18.6 \\
\hline F21R & $Y$ & 205 & 71.8 & 205 & 71.8 \\
\hline F22 & $Y$ & 234 & 34.7 & 234 & 34.7 \\
\hline F23 & $Y$ & 127 & 35 & 127 & 35 \\
\hline F31 & $Y$ & 1610 & 79.1 & 1610 & 79.1 \\
\hline F32 & $Y$ & 1780 & 67.7 & 1780 & 20 \\
\hline F33 & $Y$ & 445 & 20 & 445 & 83 \\
\hline F41 & $Y$ & 1620 & 83 & 1620 & 25.7 \\
\hline F42 & $Y$ & 846 & 25.7 & 846 & 15.5 \\
\hline F43 & $Y$ & 1140 & 15.5 & 1140 & 81.8 \\
\hline F51 & $Y$ & 1570 & 81.8 & 1570 & 77.1 \\
\hline F52 & $Y$ & 2620 & 77.1 & 2620 & 42.8 \\
\hline F53 & $Y$ & 1610 & 42.8 & 1610 & 20 \\
\hline
\end{tabular}




\section{Transect F Soil Concentrations: Zinc}

\begin{tabular}{|c|c|c|c|c|c|}
\hline Sample id. & botope & $\begin{array}{l}\text { Concentration } \\
\text { (ug/Kg soll) }\end{array}$ & $\begin{array}{l}\text { Std.Doviation } \\
\text { (ug/Kg soil) }\end{array}$ & $\begin{array}{l}\text { Element Avg. Cone. } \\
\text { (ug/Kg soll) }\end{array}$ & $\begin{array}{c}\text { Element Avg. Std. Dov. } \\
\text { (ug/Kg soil) }\end{array}$ \\
\hline F11 & Zn66 & 3840 & 3630 & 3840 & 3630 \\
\hline F12 & $2 n 66$ & 4190 & 1130 & 4190 & 1130 \\
\hline F13 & Zn66 & 62800 & & 62800 & \\
\hline F21 & Zn66 & 4540 & 530 & 4540 & 530 \\
\hline F21R & Zn66 & 15700 & & 15700 & \\
\hline$F 22$ & Zn66 & 12000 & 654 & 12000 & 654 \\
\hline F23 & Zn66 & 12900 & 371 & 12900 & 371 \\
\hline F31 & Zn66 & 2820 & 637 & 2820 & 637 \\
\hline F32 & Zn66 & 9310 & 753 & 9310 & 753 \\
\hline F33 & $2 \pi 66$ & 2560 & 2450 & 2560 & 2450 \\
\hline$F 41$ & Zn66 & 28200 & 1590 & 28200 & 1590 \\
\hline F42 & Zn66 & 18400 & 498 & 18400 & 498 \\
\hline $\mathrm{F} 43$ & Zn66 & 14500 & 1630 & 14500 & 1630 \\
\hline $\mathrm{F} 51$ & Zn66 & 5070 & 1450 & 5070 & 1450 \\
\hline F52 & Zn66 & 11000 & 318 & 11000 & 318 \\
\hline F53 & Zn66 & 15100 & 155 & 15100 & 155 \\
\hline
\end{tabular}




\section{Transect F Soil Concentrations: Zirconium}

\begin{tabular}{|c|c|c|c|c|c|}
\hline Semple id. & hotope & $\begin{array}{l}\text { Concentration } \\
\text { (ug/Kg soll) }\end{array}$ & $\begin{array}{l}\text { Std.Devintion } \\
\text { (ug/Kg soli) }\end{array}$ & $\begin{array}{l}\text { Element Avg. Conc. } \\
\text { (ug/Kg woll) }\end{array}$ & $\begin{array}{c}\text { Element Avg. Std. Dov. } \\
\text { (ug/Kg coll) }\end{array}$ \\
\hline$F 11$ & 790 & 39000 & 54.6 & & \\
\hline F11 & 291 & 51500 & 761 & 45300 & 408 \\
\hline F12 & 2900 & 37000 & 582 & & \\
\hline F12 & 291 & 38800 & 491 & 37900 & 537 \\
\hline F13 & 280 & 32600 & 568 & & \\
\hline F13 & 291 & 20500 & 304 & 26600 & 436 \\
\hline F21 & 780 & 20400 & 372 & & \\
\hline F21 & 291 & 20400 & 207 & 20400 & 290 \\
\hline F21R & $Z 190$ & 3340 & 125 & & \\
\hline F21R & Z91 & 11100 & 2660 & 7220 & 1390 \\
\hline F22 & Zrso & 15500 & 400 & & \\
\hline $\mathrm{F} 22$ & 2591 & 11300 & 426 & 13400 & 418 \\
\hline F23 & $Z 190$ & 42200 & 432 & & \\
\hline $\mathrm{F} 23$ & 291 & 40900 & 1360 & 41600 & 896 \\
\hline F31 & Zrso & 98400 & 1200 & & \\
\hline F31 & 2991 & 103000 & 1820 & 101000 & 1510 \\
\hline F32 & 2890 & 54400 & 394 & & \\
\hline F32 & Zr91 & 57600 & 1070 & 56000 & 732 \\
\hline F33 & Zr90 & 43000 & 184 & & \\
\hline F33 & 2891 & 31900 & 1120 & 37500 & 652 \\
\hline F41 & Zr9o & 79100 & 250 & & \\
\hline$F 41$ & Zr91 & 87600 & 1410 & 83400 & 830 \\
\hline$F 42$ & 2190 & 51300 & 136 & & \\
\hline F42 & 291 & 54200 & 1070 & 52800 & 603 \\
\hline F43 & Z 90 & 162000 & 772 & & \\
\hline$F 43$ & 2991 & 19400 & 881 & 90700 & 479 \\
\hline F51 & 2890 & 52800 & 129 & & \\
\hline F51 & 791 & 47800 & 1660 & 50300 & 895 \\
\hline$F 52$ & 2190 & 51800 & 357 & & \\
\hline$F 52$ & 2991 & 50000 & 1440 & 50900 & 899 \\
\hline F53 & Zr90 & 28800 & 1050 & & \\
\hline F53 & 2991 & 28400 & 724 & 28600 & 887 \\
\hline
\end{tabular}

Note: The ectual concentration of samples for which no standard deviabon ls reported bs leas than the lower limit of detection. The reported concentration is the lower limit of quantification. 


\section{Appendix C}

RESULTS OF F AND H AREA BASIN WATER ANALYSES 
Table C-1. Chemical properties and anion concentrations of water samples in the A-transect (F-Area).

\begin{tabular}{|c|c|c|c|c|c|c|c|c|c|c|c|}
\hline $\begin{array}{c}\text { Sample } \\
\text { DD }\end{array}$ & $\mathbf{p H}$ & $\begin{array}{c}\text { Eh } \\
(\mathrm{mV})\end{array}$ & $\begin{array}{l}\text { Cond. } \\
\left(\mathrm{S} \mathrm{m}^{-2}\right)\end{array}$ & $\begin{array}{l}\text { Temp } \\
\left({ }^{\circ} \mathrm{C}\right)\end{array}$ & $\begin{array}{c}\mathrm{TIC} \\
(\mathrm{ppm})\end{array}$ & $\begin{array}{c}\text { TOC } \\
\text { (ppm) }\end{array}$ & $\begin{array}{c}\mathrm{H}^{3} \\
\left(\mathrm{~Bq}^{\mathbf{r}^{-1}}\right)\end{array}$ & $\begin{array}{c}\mathrm{Cl} \\
(\mathrm{ppm})\end{array}$ & $\begin{array}{c}F \\
(\mathrm{ppm})\end{array}$ & $\begin{array}{c}\mathrm{NO}_{3}^{-} \\
(\mathrm{ppm})\end{array}$ & $\begin{array}{l}\mathrm{SO}_{4}^{2-} \\
(\mathrm{ppm})\end{array}$ \\
\hline A12W & 4.16 & 287 & 5.2 & 26 & $<1$ & 3.5 & 180,000 & 21 & $<0.84$ & 350 & 3.2 \\
\hline A13W & 4.99 & 41.3 & 2.8 & 27.2 & $<1$ & 13 & 41,000 & 10 & $<0.93$ & 196 & 4.7 \\
\hline A13WR & 3.42 & NA & NA & NA & $<1$ & 2.4 & 104,000 & 4.3 & $<1.2$ & 195 & 3.6 \\
\hline A22W & 3.19 & 378 & 13 & 24.0 & $<1$ & 14 & 630,000 & 8.8 & $<3.4$ & 1350 & 29 \\
\hline A23W & 3.01 & 442 & 17 & 25.7 & $<1$ & 8 & 955,000 & 15 & & 1600 & 30 \\
\hline A31W & 3.19 & 345 & 21 & 25.9 & $<1$ & 14 & 675,000 & 4.9 & $<1$ & 1360 & 32 \\
\hline A32W & 3.5 & 429 & 16 & 24.1 & $<1$ & 10 & $1,120,000$ & 13 & & 1130 & 25 \\
\hline A41W & 4.80 & 187 & 5.0 & 26.3 & $<1$ & 2 & 666 & 16 & $<0.2$ & 4.6 & 1.4 \\
\hline A42W & 3.29 & 404 & 35 & 28.7 & $<1$ & 16 & 79,500 & 10 & $<0.2$ & 1550 & 30.4 \\
\hline A52W & 5.42 & 113 & 13 & 22.8 & $<1$ & 6.1 & 283 & 341 & $<2$ & 241 & 3.5 \\
\hline A53W & 3.72 & NA & NA & 23.1 & $<1$ & 1.7 & 71,600 & 4.1 & $<0.2$ & 162 & 4.0 \\
\hline A5-110' & $6.33^{* *}$ & NA & NA & & $<1$ & 8.9 & 24,400 & 8.7 & $<4$ & 152 & 10.4 \\
\hline
\end{tabular}

- The water sample for A13 was brought to the surface using a bailer apparatus.

" $\mathrm{pH}$ measured in laboratory

NA - Not analyzed for. 
Table C-2. Chemical properties and anion concentrations of water samples in the B-transect (F-Area).

\begin{tabular}{|c|c|c|c|c|c|c|c|c|c|c|c|}
\hline $\begin{array}{c}\text { Sample } \\
\text { ID }\end{array}$ & $\begin{array}{c}\text { Aqueous } \\
\text { pH }\end{array}$ & $\begin{array}{c}\text { Eh } \\
(\mathrm{mV})\end{array}$ & $\begin{array}{l}\text { Cond. } \\
\left(\mathrm{S} \mathrm{m}^{-2}\right)\end{array}$ & $\begin{array}{l}\text { Temp } \\
\left({ }^{\circ} \mathrm{C}\right)\end{array}$ & $\begin{array}{c}\text { TIC } \\
\text { (ppm) }\end{array}$ & $\begin{array}{c}\text { TOC } \\
\text { (ppm) }\end{array}$ & $\begin{array}{c}\overline{\mathbf{H}}^{3} \\
\left(\mathbf{B q} \mathbf{I}^{-1}\right)\end{array}$ & $\underset{(\mathrm{ppm})}{\mathrm{Cr}}$ & $\begin{array}{c}F \\
(\mathrm{ppm})\end{array}$ & $\begin{array}{l}\mathrm{NO}_{3}^{-} \\
(\mathrm{ppm})\end{array}$ & $\begin{array}{l}\mathrm{SO}_{4}{ }^{2-} \\
(\mathrm{ppm})\end{array}$ \\
\hline B13W & 3.24 & 391 & 7.0 & 21.2 & $<1$ & 1.8 & 459,000 & 7.6 & $<1.3$ & 775 & $<2.5$ \\
\hline B14W & 3.93 & 198 & 11 & 20.6 & $<1$ & 2.9 & 149,000 & 12.8 & $<1$ & 510 & 2.66 \\
\hline B23W & 3.86 & 315 & 3.6 & 29.3 & $<1$ & 3.7 & 99,500 & 3.67 & 0.91 & 364 & 2.8 \\
\hline B23WR & $4.02^{\circ}$ & NA & NA & NA & $<1$ & 2.3 & 87,100 & 3.2 & 0.93 & 419 & 3.3 \\
\hline B24W & 3.83 & 316 & 5.0 & 30.1 & $<1$ & 2.7 & 156,000 & 8.8 & $<1$ & 382 & 4.1 \\
\hline B32W & 4.62 & 205 & 1.0 & 24.5 & $<1$ & 3.0 & 11,800 & 5.8 & $<1$ & 59 & $<2.5$ \\
\hline B33W & 4.64 & 58.6 & 0.60 & 26.8 & $<1$ & 5.1 & 17,400 & 6.1 & $<1$ & 72 & $<2.5$ \\
\hline B42W & 4.67 & NA & 0.70 & 22.5 & $<1$ & 2.1 & 550 & $<1.75$ & $<1.63$ & 31 & 5.6 \\
\hline B43W & 3.66 & NA & 2.5 & 21.6 & $<1$ & 2.4 & 688 & $<2.5$ & $<2$ & 115 & 2.7 \\
\hline B4-145' & 7.01 & NA & 1.3 & 22.1 & & & 117 & 6.7 & $<1$ & 13 & 6.6 \\
\hline B51W & $4.09^{\circ *}$ & NA & 1.4 & 26.1 & $<1$ & 1.3 & $<588$ & 4.6 & $<0.65$ & 50 & 2.8 \\
\hline B52W & 3.61 & NA & 2.1 & 21.1 & $<1$ & 1.0 & 17,400 & 4.0 & $<0.94$ & 156 & 4.8 \\
\hline B52WR & 4.69 & NA & NA & 24.1 & $<1$ & 1.4 & $<588$ & 3.7 & $<0.2$ & 29 & 3.9 \\
\hline
\end{tabular}

pH measured in laboratory

pH measured in laboratory. A pH reading of 2.57 was reported for sample B51 in the field logs.

NA - Not analyzed for 
Table C-3. Chemical properties and anion concentrations of water samples in the C-transect (F-Area).

\begin{tabular}{|c|c|c|c|c|c|c|c|c|c|c|c|}
\hline $\begin{array}{c}\text { Sample } \\
\text { ID }\end{array}$ & $\begin{array}{c}\text { Aqueous } \\
\text { pH }\end{array}$ & $\begin{array}{c}\mathbf{E h} \\
(\mathrm{mV})\end{array}$ & $\begin{array}{l}\text { Cond. } \\
\left(S \mathrm{~m}^{-2}\right)\end{array}$ & $\begin{array}{l}\text { Temp } \\
\left({ }^{\circ} \mathrm{C}\right)\end{array}$ & $\begin{array}{c}\mathrm{TIC} \\
(\mathrm{ppm})\end{array}$ & $\begin{array}{c}\text { TOC } \\
\text { (ppm) }\end{array}$ & $\begin{array}{c}\mathbf{B}^{\mathbf{3}} \\
\left(\mathrm{Bq}^{\left.\mathbf{I}^{-1}\right)}\right.\end{array}$ & $\underset{(\mathrm{ppm})}{\mathrm{Cl}^{-}}$ & $\begin{array}{c}F \\
(\mathrm{ppm})\end{array}$ & $\begin{array}{c}\mathrm{NO}_{3}^{\circ} \\
(\mathrm{ppm})\end{array}$ & $\begin{array}{l}\mathrm{SO}_{4}^{2-} \\
(\mathrm{ppm})\end{array}$ \\
\hline C13W & 3.68 & 386 & 7.0 & 27.1 & $<1$ & 3.7 & 145,000 & 6.8 & $<1$ & 827 & $<2.5$ \\
\hline C14W & 3.75 & 386 & 3.0 & 31.7 & $<1$ & 2.8 & 61,400 & 9.4 & $<1$ & 191 & $<2.5$ \\
\hline $\mathrm{C} 22 \mathrm{~W}$ & 3.96 & 225 & 0.50 & 28.7 & $<1$ & 3.0 & 1880 & 2.81 & $<1$ & 28.1 & 4.4 \\
\hline $\mathrm{C} 23 \mathrm{~W}$ & 4.17 & 280 & 0.40 & 30.6 & $<1$ & 2.5 & 283 & 6.3 & $<0.2$ & 12 & 4.0 \\
\hline C32W & 5.53 & NA & 0.30 & 22.1 & $<1$ & 1.7 & 583 & 4.4 & $<1.5$ & 3.9 & 3.7 \\
\hline C33W & 4.64 & NA & 4.0 & 23.1 & $<1$ & 2.5 & 176 & 4.4 & $<1.5$ & 9.4 & 2.8 \\
\hline $\mathrm{C} 42 \mathrm{~W}$ & 5.27 & NA & 0.90 & 23.1 & $<1$ & 3.5 & $<588$ & 14 & $<0.2$ & 4.5 & 2.6 \\
\hline C43W & 4.51 & NA & 3.8 & 23.1 & $<1$ & 1.5 & $<588$ & 14 & $<0.9$ & 3.4 & 3.0 \\
\hline
\end{tabular}

NA - Not analyzed for. 
Table C-4. Chemical properties and anion concentrations of water samples in the D-transect (F-Area).

\begin{tabular}{|c|c|c|c|c|c|c|c|c|c|c|c|}
\hline $\begin{array}{c}\text { Sample } \\
\text { m }\end{array}$ & $\begin{array}{c}\text { Aqueous } \\
\text { pH }\end{array}$ & $\begin{array}{c}\text { Eh } \\
(\mathrm{mV})\end{array}$ & $\begin{array}{l}\text { Cond. } \\
\left(\mathrm{S} \mathrm{m}^{-2}\right)\end{array}$ & $\begin{array}{l}\text { Temp } \\
\left({ }^{\circ} \mathrm{C}\right)\end{array}$ & $\begin{array}{c}\text { TIC } \\
(\mathbf{p p m})\end{array}$ & $\begin{array}{c}\text { TOC } \\
(\mathrm{ppm})\end{array}$ & $\begin{array}{c}\mathrm{H}^{3} \\
\left(\mathrm{Bg} \mathrm{l}^{-1}\right)\end{array}$ & $\underset{(\mathrm{ppm})}{\mathrm{Cl}^{-}}$ & $\begin{array}{c}F \\
(\mathrm{ppm})\end{array}$ & $\begin{array}{l}\mathrm{NO}_{3}^{-} \\
(\mathrm{ppm})\end{array}$ & $\begin{array}{l}\mathrm{SO}_{4}{ }^{2-} \\
(\mathrm{ppm})\end{array}$ \\
\hline D13W" & $6.78^{x+2}$ & 385.9 & NA & NA & $<1$ & 18.6 & 14,100 & 7.1 & $<1.1$ & 12.5 & 6.1 \\
\hline D13WR & $4.14^{\circ *}$ & 385.8 & NA & NA & $<1$ & 1.8 & 29,000 & 6.4 & $<0.2$ & 127 & 9.6 \\
\hline
\end{tabular}

- The water sample for D13 was brought to the surface using a bailer apparatus.

** pH measured in laboratory

NA - Not analyzed for. 
Table C-5. Chemical properties and anion concentrations of water samples in the E-transect (H-Area).

\begin{tabular}{|c|c|c|c|c|c|c|c|c|c|c|c|}
\hline $\begin{array}{c}\text { Sample } \\
\text { W }\end{array}$ & $\begin{array}{c}\text { Aqueous } \\
\text { pH }\end{array}$ & $\begin{array}{c}\text { Eh } \\
(\mathrm{mV})\end{array}$ & $\begin{array}{l}\text { Cond. } \\
\left(\mathrm{S} \mathrm{m}^{-2}\right)\end{array}$ & $\begin{array}{l}\text { Temp } \\
\left({ }^{\circ} \mathrm{C}\right)\end{array}$ & $\begin{array}{c}\text { TIC } \\
(\mathrm{ppm})\end{array}$ & $\begin{array}{c}\text { TOC } \\
(\mathrm{ppm})\end{array}$ & $\begin{array}{c}\mathbf{H}^{3} \\
\left(\mathrm{~Bq} \mathbf{l}^{-1}\right)\end{array}$ & $\begin{array}{c}\mathrm{Cl}^{-} \\
(\mathrm{ppm})\end{array}$ & $\begin{array}{c}F \\
(\mathrm{ppm})\end{array}$ & $\begin{array}{c}\mathrm{NO}_{3}^{-} \\
(\mathrm{ppm})\end{array}$ & $\begin{array}{l}\mathrm{SO}_{4}^{2-} \\
(\mathrm{ppm})\end{array}$ \\
\hline E13W & 4.00 & 132 & 2.0 & 28.4 & $<1$ & 3.0 & 110,000 & 11 & $<2$ & 184 & $<0.5$ \\
\hline E14W & 4.04 & 181 & 1.8 & 30.3 & $<1$ & 2.6 & 71,000 & 5.1 & $<2$ & 127 & $<0.5$ \\
\hline E23W & 5.85 & 201 & 1.1 & 29.1 & $<1$ & 1.9 & 80,300 & 3.1 & $<1$ & 110 & $<2.5$ \\
\hline E23WR & $4.32^{\circ}$ & $\mathrm{NA}$ & NA & NA & $<1$ & 2.4 & 95,800 & 5.8 & $<1.5$ & 140 & 2.4 \\
\hline E24W & 3.87 & 191 & 2.6 & 28.5 & $<1$ & 2.3 & 81,800 & 2.4 & $<1$ & 115 & $<2.5$ \\
\hline E33W & 4.27 & 278 & 0.70 & 29.4 & $<1$ & 1.6 & 19,600 & $<2$ & $<2$ & 62 & $<5$ \\
\hline E34W & 4.05 & 288 & 2.4 & 28.6 & $<1$ & 2.8 & 89,500 & 2.34 & $<2$ & 129 & $<5$ \\
\hline E41W & 5.27 & 230 & 0.50 & 32.0 & $<1$ & 1.7 & 4220 & $<2$ & $<2$ & 24 & $<5$ \\
\hline E42W & 4.87 & 272 & 0.65 & 31.8 & $<1$ & 3.7 & 4770 & 2.3 & $<0.2$ & 34 & 1.01 \\
\hline E4-89' & 7.7 & NA & 3.5 & 22.2 & NA & NA & 68,800 & 5.03 & $<0.6$ & 38 & 5.7 \\
\hline E4-107' & 7.97 & NA & 1.5 & 22.9 & NA & NA & 17,800 & 3.7 & $<1$ & 19 & 2.6 \\
\hline
\end{tabular}

pH measured in laboratory

NA - Not analyzed for. 
Table C-6. Chemical properties and anion concentrations of water samples in the F-transect (H-Area).

\begin{tabular}{|c|c|c|c|c|c|c|c|c|c|c|c|}
\hline $\begin{array}{c}\text { Sample } \\
\text { ID }\end{array}$ & $\begin{array}{c}\text { Aqueous } \\
\text { pH }\end{array}$ & $\begin{array}{c}\text { Eh } \\
(\mathrm{mV})\end{array}$ & $\begin{array}{l}\text { Cond. } \\
\left(\mathrm{S} \mathrm{m}^{-2}\right)\end{array}$ & $\begin{array}{l}\text { Temp } \\
\left({ }^{\circ} \mathrm{C}\right)\end{array}$ & $\begin{array}{c}\text { TIC } \\
(\mathrm{ppm})\end{array}$ & $\begin{array}{c}\text { TOC } \\
(\mathrm{ppm})\end{array}$ & $\begin{array}{c}\mathbf{H}^{3} \\
\left(\mathrm{~Bq}^{-1}\right)\end{array}$ & $\begin{array}{c}\mathrm{Cl}^{-} \\
(\mathrm{ppm})\end{array}$ & $\begin{array}{c}F \\
(\mathrm{ppm})\end{array}$ & $\begin{array}{c}\mathrm{NO}_{3}^{-} \\
(\mathrm{ppm})\end{array}$ & $\begin{array}{l}\mathrm{SO}_{4}^{2-} \\
(\mathrm{ppm})\end{array}$ \\
\hline F12W & 4.30 & 197 & 1.4 & 28.1 & $<1$ & 4.6 & 78,800 & 3.11 & $<2$ & 75 & $<5$ \\
\hline F13W & 4.90 & 148 & 0.40 & 29.4 & $<1$ & 4.2 & 1270 & 4.4 & $<2$ & 9.0 & 0.86 \\
\hline F22W & 4.69 & 224 & 2.7 & 31.7 & $<1$ & 5.7 & 268,000 & 2.1 & $<2$ & 271 & $<5$ \\
\hline F23W & 6.48 & 256 & 1.8 & 28.1 & $<1$ & 3.2 & 62,500 & 3.1 & $<2$ & 90 & 8.8 \\
\hline F32W & 4.85 & 237 & 0.85 & 30.3 & $<1$ & 3.2 & 466 & 5.3 & $<2$ & 13 & 0.90 \\
\hline F33W & 4.77 & 199 & 10 & 30.9 & $<1$ & 8.5 & 426,000 & 4.0 & $<2$ & 464 & 44 \\
\hline F42W & 5.20 & 211 & 2.2 & 28.6 & $<1$ & 3.4 & 22,500 & 8.4 & $<2$ & 144 & $<5$ \\
\hline F43W & 4.12 & 176 & 1.9 & 30.1 & $<1$ & 3.7 & 33,100 & 4.1 & 2.8 & 87 & $<5$ \\
\hline F52W & 5.91 & 193 & 0.20 & 27.4 & $<1$ & 3.1 & 566 & 2.0 & $<2$ & 8.0 & $<0.5$ \\
\hline F53W & 5.63 & 221 & 1.0 & 28.6 & $<1$ & 3.6 & 3430 & 8.8 & $<0.2$ & 32 & 1.2 \\
\hline
\end{tabular}




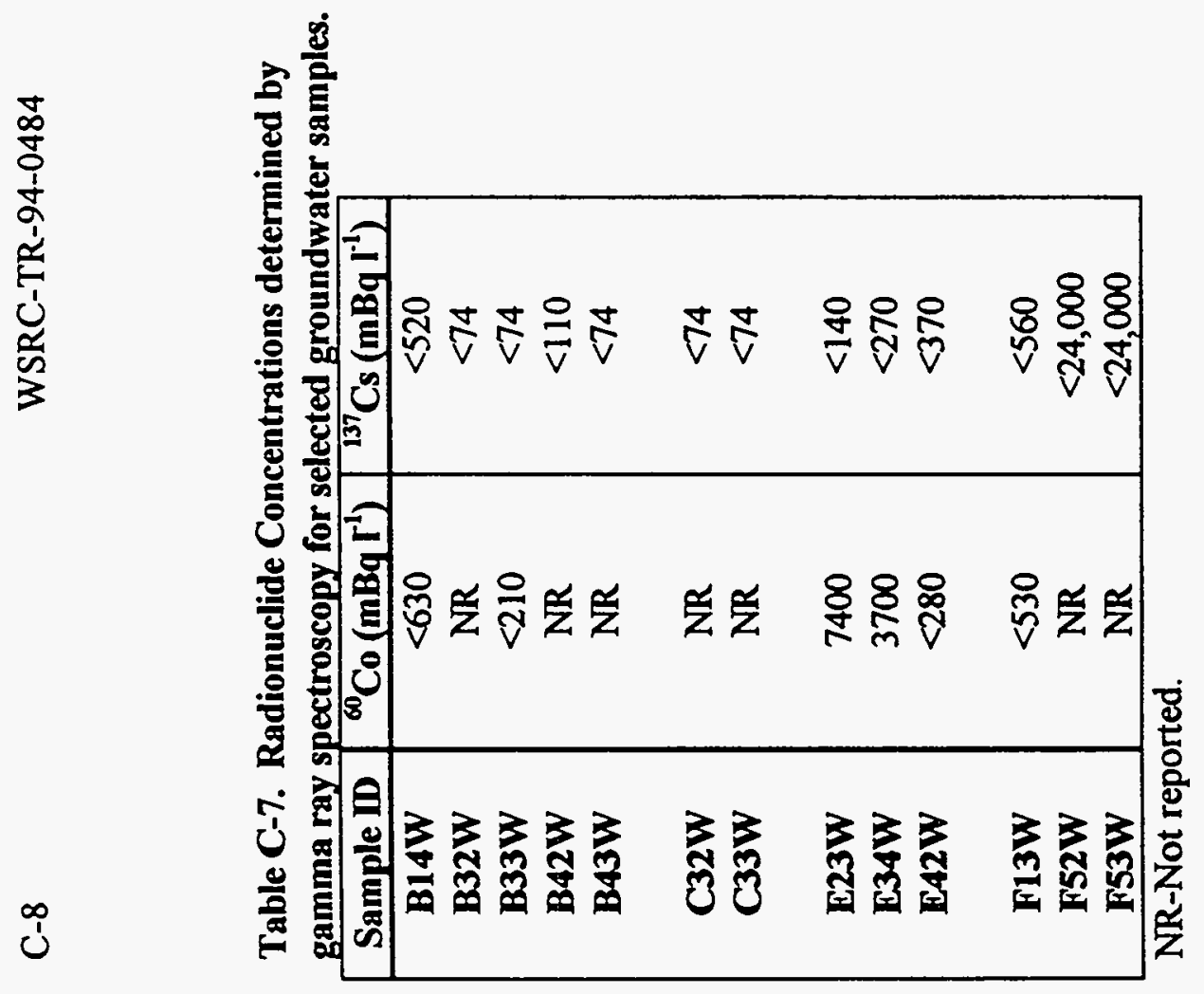




\section{Transect A Porewater Concentrations: Aluminum}

\begin{tabular}{|c|c|c|c|c|c|}
\hline sumple ld. & leotope & $\begin{array}{l}\text { Concentratton } \\
\text { (ug/L) }\end{array}$ & $\begin{array}{l}\text { Std.Daviation } \\
\text { (ugh) }\end{array}$ & $\begin{array}{l}\text { Element Avg. Conc. } \\
\text { (ugh) }\end{array}$ & $\begin{array}{c}\text { Element Avg. Std. Dov. } \\
\text { (ugh) }\end{array}$ \\
\hline A11 & $\mathbf{N}$ & & & & \\
\hline A12 & A & 5760 & 48.1 & 5760 & 48.1 \\
\hline A13 & $\mathbf{A}$ & 448 & .75 & 448 & .75 \\
\hline A13R & $\mathrm{A}$ & 8050 & 169 & 8050 & 169 \\
\hline A21 & $\mathbf{A}$ & & & & \\
\hline$A 22$ & $\bar{A}$ & 71400 & 1400 & 71400 & 1400 \\
\hline A23 & A & 105000 & 223 & 105000 & 223 \\
\hline$A 31$ & A & 75800 & 598 & 75800 & 598 \\
\hline A32 & $\mathbf{A}$ & 106000 & 4750 & 106000 & 4750 \\
\hline A41 & Al & & & & \\
\hline A42 & $\mathbf{A}$ & 77300 & 936 & 77300 & 936 \\
\hline A51 & A & & & & \\
\hline A52 & A & 25 & & 25 & \\
\hline$A 53$ & A & 4410 & 107 & 4410 & 107 \\
\hline A55V & $\mathbf{A}$ & 25 & & 25 & \\
\hline
\end{tabular}




\section{Transect A Porewater Concentrations: Arsenic}

\begin{tabular}{|c|c|c|c|c|c|}
\hline Sampte ld. & botope & $\begin{array}{l}\text { Concentration } \\
\text { (ug/ })\end{array}$ & $\begin{array}{l}\text { Std.Daviation } \\
\text { (ug/ })\end{array}$ & $\begin{array}{l}\text { Element Avg. Conc. } \\
\text { (ugL) }\end{array}$ & $\begin{array}{c}\text { Element Avg. Std. Dov. } \\
\text { (ugh) }\end{array}$ \\
\hline A11 & As & & & & \\
\hline A12 & As & 24.6 & 17.3 & 24.6 & 17.3 \\
\hline A13 & As & 39.2 & 19.6 & 392 & 19.6 \\
\hline A13R & As & 14.4 & 16.4 & 14.4 & 16.4 \\
\hline A21 & As & & & & \\
\hline$A 22$ & As & 10.4 & 1.66 & 10.4 & 1.66 \\
\hline A23 & As & 8.27 & 2.49 & 8.27 & 2.49 \\
\hline$A 31$ & As & 3.58 & 3.15 & 3.58 & 3.15 \\
\hline$A 32$ & As & 5.01 & 1.66 & 5.01 & 1.66 \\
\hline A41 & As & & & & \\
\hline A42 & As & 9.29 & 4.38 & 9.29 & 4.38 \\
\hline A51 & As & & & & \\
\hline $\mathrm{A} 52$ & As & 2.97 & 3.37 & 2.97 & 3.37 \\
\hline $\mathrm{A} 53$ & As & 13.1 & 152 & 13.1 & 15.2 \\
\hline A55V & As & 75 & & 75 & \\
\hline
\end{tabular}




\section{Transect A Porewater Concentrations: Barium}

\begin{tabular}{ccccc}
\hline Sample ld. Leotope & $\begin{array}{c}\text { Concentration } \\
\text { (ugh })\end{array}$ & $\begin{array}{c}\text { Std.Devlation } \\
\text { (ugh) }\end{array}$ & $\begin{array}{c}\text { Element Avg. Conc. } \\
\text { (ugh })\end{array}$ & $\begin{array}{c}\text { Element Avg. Std. Dov. } \\
\text { (ugh) }\end{array}$ \\
\hline
\end{tabular}

\begin{tabular}{|c|c|c|c|c|c|}
\hline A11 & Ba135 & & & & \\
\hline A11 & Ba137 & & & & \\
\hline A11 & Ba138 & & & & \\
\hline A12 & Ba135 & 456 & 6.08 & & \\
\hline A12 & Ba137 & 464 & 5.51 & & \\
\hline A12 & $8 a 138$ & 467 & 3.83 & 462 & 5.14 \\
\hline$\overline{\mathrm{A} 13}$ & Ba135 & 526 & 8.77 & & \\
\hline A13 & Ba137 & 526 & 6.23 & & \\
\hline A13 & Ba138 & 528 & 3.33 & 527 & 6.11 \\
\hline A13R & Ba135 & 408 & 54.9 & & \\
\hline A13R & Ba137 & 396 & 8.39 & & \\
\hline$A 13 R$ & Ba138 & 395 & 5.71 & 400 & 23 \\
\hline A21 & Ba135 & & & & \\
\hline A21 & Ba137 & & & & \\
\hline A21 & Ba138 & & & & \\
\hline A22 & Ba135 & 876 & 4.87 & & \\
\hline$A 22$ & Ba137 & 863 & 5.88 & & \\
\hline A22 & Ba138 & 874 & 6.18 & 871 & 5.64 \\
\hline A23 & Ba135 & 1050 & 27.3 & & \\
\hline A23 & Ba137 & 1050 & 8.41 & & \\
\hline A23 & Ba138 & 1050 & 727 & 1050 & 14.3 \\
\hline A31 & Ba135 & 227 & 7.7 & & \\
\hline A31 & Ba137 & 220 & 9.07 & & \\
\hline A31 & Ba138 & 219 & 321 & 222 & 6.66 \\
\hline A32 & Ba135 & 986 & 37.6 & & \\
\hline A32 & Ba137 & 976 & 9.65 & & \\
\hline A32 & Ba138 & 1010 & 4.15 & 991 & 17.1 \\
\hline A41 & Ba135 & & & & \\
\hline A41 & Ba137 & & & & \\
\hline A41 & Ba138 & & & & \\
\hline A42 & Ba135 & 393 & 10.4 & & \\
\hline A42 & Ba137 & 402 & 3.47 & & \\
\hline A42 & Ba138 & 396 & 7.8 & 397 & 722 \\
\hline A51 & Ba135 & & & & \\
\hline A51 & Ba137 & & & & \\
\hline A51 & Ba138 & & & & \\
\hline A52 & Ba135 & 39.1 & 2.96 & & \\
\hline A52 & $8 a 137$ & 40.1 & 2.56 & & \\
\hline A52 & Ba138 & 38 & .75 & 39.1 & 2.09 \\
\hline A53 & Ba135 & 138 & 28.2 & & \\
\hline A53 & Ba137 & 158 & 10.4 & & \\
\hline A53 & $B a 138$ & 127 & 421 & 141 & 14.3 \\
\hline A55V & Ba135 & 41.7 & 2.24 & & \\
\hline A55V & Ba137 & 42.5 & 1.58 & & \\
\hline
\end{tabular}

Note: The actual concentration of samples for which no standard deviation la reported is less than the lower limit of detection. The reported concentration is the bwor llmit of quantification. 


\section{Transect A Porewater Concentrations: Barium}

\begin{tabular}{|c|c|c|c|c|c|}
\hline Sample Id. & Isotope & $\begin{array}{l}\text { Concentration } \\
\text { (ug/L) }\end{array}$ & $\begin{array}{l}\text { Std.Devlation } \\
\text { (ug/l) }\end{array}$ & $\begin{array}{l}\text { Element Avg. Conc. } \\
\text { (ug/L) }\end{array}$ & $\begin{array}{c}\text { Element Avg. Std. Dov. } \\
\text { (ugh) }\end{array}$ \\
\hline $\mathrm{A} 55 \mathrm{~V}$ & $\mathrm{Ba} 138$ & 43 & .82 & 42.4 & 1.55 \\
\hline
\end{tabular}

Note: The actual concentration of samples for which no standard deviation is reported is less than the lower IImIt of detection. The reported concentration is the lower IImit of quantification. 


\section{Transect A Porewater Concentrations: Cadmium}

$\begin{array}{cccccc}\text { Sample ld. leotope } & \begin{array}{c}\text { Concentration } \\ \text { (ugh) }\end{array} & \begin{array}{c}\text { Std.Doviation } \\ \text { (ugh) }\end{array} & \begin{array}{c}\text { Element Avg. Conc. } \\ \text { (ugh } L)\end{array} & \begin{array}{c}\text { Eloment Avg. Std. Dov. } \\ \text { (ugh })\end{array}\end{array}$

\begin{tabular}{|c|c|c|c|c|c|}
\hline A11 & Cd111 & & & & \\
\hline A11 & Cd114 & & & & \\
\hline A12 & Cd111 & 428 & 1 & & \\
\hline A12 & Cd114 & 5.12 & 29 & 4.7 & .65 \\
\hline A13 & Cd111 & 3.15 & .62 & & \\
\hline A13 & Cd114 & 4.13 & .02 & 3.64 & .32 \\
\hline A13R & Cd111 & .96 & .08 & & \\
\hline A13R & Cd114 & 1.22 & 1.48 & 1.09 & .78 \\
\hline A21 & Cd111 & & & & \\
\hline A21 & Cd114 & & & & \\
\hline$A 22$ & Cd111 & 1.31 & 24 & & \\
\hline$A 22$ & Cd114 & 1.57 & .45 & 1.44 & .35 \\
\hline A23 & Cd111 & .67 & .11 & & \\
\hline A23 & Cd114 & 1.39 & 25 & 1.03 & .18 \\
\hline$A 31$ & Cd111 & 1.91 & .83 & & \\
\hline A31 & Cd114 & 19 & .16 & 10.5 & .5 \\
\hline A32 & Cod111 & 1.13 & 27 & & \\
\hline $\mathrm{A} 32$ & Cod114 & 1.66 & .38 & 1.4 & .33 \\
\hline A41 & Cod111 & & & & \\
\hline$A 41$ & $\operatorname{Cd114}$ & & & & \\
\hline $\mathrm{A} 42$ & Cd111 & .16 & .19 & & \\
\hline A42 & Cd114 & 2.06 & 1.82 & 1.11 & 1.01 \\
\hline$A 51$ & Cd111 & & & & \\
\hline$A 51$ & Cod114 & & & & \\
\hline$A 52$ & $\operatorname{Cod} 111$ & .48 & 2 & & \\
\hline A52 & Cd114 & .14 & .15 & .31 & .18 \\
\hline$A 53$ & Cod111 & 1.17 & 3.58 & & \\
\hline A53 & $\operatorname{Cod114}$ & 21 & .85 & .69 & 222 \\
\hline A55V & $\operatorname{cod111}$ & .62 & .13 & & \\
\hline A55V & Cod114 & .62 & 21 & .62 & .17 \\
\hline
\end{tabular}




\section{Transect A Porewater Concentrations: Calcium}

\begin{tabular}{|c|c|c|c|c|c|}
\hline Sample ld. & Isotope & $\begin{array}{l}\text { Concentration } \\
\text { (ugh) }\end{array}$ & $\begin{array}{l}\text { Std.Doviation } \\
\text { (ugh) }\end{array}$ & $\begin{array}{c}\text { Element Avg. Conc. } \\
\text { (ug } L \text { ) }\end{array}$ & $\begin{array}{c}\text { Element Avg. Std. Dov. } \\
\text { (Ug })\end{array}$ \\
\hline A11 & Ca44 & & & & \\
\hline A12 & Ca44 & 37700 & 288 & 37700 & 288 \\
\hline A13 & $\mathrm{Ca} 44$ & 20200 & 145 & 20200 & 145 \\
\hline A13R & Ca44 & 5340 & 683 & 5340 & 683 \\
\hline A21 & Ca44 & & & & \\
\hline A22 & Ca44 & 759 & 23.1 & 759 & 23.1 \\
\hline A23 & Ca44 & 2660 & 762 & 2660 & 76.2 \\
\hline A31 & Ca44 & 3950 & 46.9 & 3950 & 46.9 \\
\hline A32 & $\operatorname{Ca} 44$ & 50 & & 50 & \\
\hline A41 & $\mathrm{Ca} 44$ & & & & \\
\hline A42 & Ca44 & 437 & 22.4 & 437 & 22.4 \\
\hline A51 & Ca44 & & & & \\
\hline A52 & Ca44 & 392 & 7.12 & 392 & 7.12 \\
\hline A53 & Ca44 & 403 & 146 & 403 & 146 \\
\hline A55V & Ca44 & & & & \\
\hline
\end{tabular}

Note: The actual concentration of samples for which no standard deviation is reported is leas than the lower limit of detection. The reported concentration is the lower limit of quantincation. 


\section{Transect A Porewater Concentrations: Cesium}

\begin{tabular}{|c|c|c|c|c|c|}
\hline Sample ld. & Lotope & $\begin{array}{l}\text { Concentration } \\
\text { (ugh) }\end{array}$ & $\begin{array}{c}\text { Std.Deviation } \\
\text { (ug/l) }\end{array}$ & $\begin{array}{c}\text { Element Avg. Conc. } \\
\text { (ugh) }\end{array}$ & $\begin{array}{c}\text { Element Avg. Std. Dov. } \\
\text { (ugh) }\end{array}$ \\
\hline A11 & Cs & & & & \\
\hline A12 & Cs & .32 & .02 & .32 & .02 \\
\hline A13 & Cs & .45 & .07 & .45 & .07 \\
\hline A13R & $\mathrm{Cs}_{8}$ & 1.31 & .6 & 1.31 & .6 \\
\hline A21 & $\mathrm{C}_{8}$ & & & & \\
\hline A22 & $\mathrm{Cs}$ & 2.42 & .37 & 2.42 & .37 \\
\hline A23 & $\mathrm{C}_{8}$ & 3.08 & .03 & 3.08 & .03 \\
\hline$A 31$ & Cs & 2.88 & .06 & 2.88 & .06 \\
\hline A32 & $\mathrm{Co}$ & 4.42 & 27 & 4.42 & 27 \\
\hline A41 & $\mathrm{Cs}$ & & & & \\
\hline A42 & Cs & 3.31 & 28 & 3.31 & 28 \\
\hline A51 & Cs & & & & \\
\hline A52 & Cs & .41 & .05 & .41 & .05 \\
\hline $\mathrm{A53}$ & Cs & 1.05 & .67 & 1.05 & .67 \\
\hline A55V & $\mathrm{Cs}_{\mathrm{s}}$ & 24 & .05 & 24 & .05 \\
\hline
\end{tabular}

Note: The ectual concentratton of eamples for which no standard dovition bs reported bs leas than the lower limit of detection. The reported concentration is the lower llmit of quantincation. 


\section{Transect A Porewater Concentrations: Chromium}

\begin{tabular}{|c|c|c|c|c|c|}
\hline Sample ld. & Isotope & $\begin{array}{l}\text { Concentrattion } \\
\text { (ugL) }\end{array}$ & $\begin{array}{l}\text { Std.Doviation } \\
\text { (ugh) }\end{array}$ & $\begin{array}{l}\text { Element Avg. Conc. } \\
\text { (ugh) }\end{array}$ & $\begin{array}{c}\text { Element Avg. Std. Dov. } \\
\text { (ugl) }\end{array}$ \\
\hline
\end{tabular}

\begin{tabular}{|c|c|c|c|c|c|}
\hline A11 & Cr52 & & & & \\
\hline A11 & C.53 & & & & \\
\hline A12 & C.52 & 5.14 & .06 & & \\
\hline A12 & C.53 & 5.12 & .06 & 5.13 & .06 \\
\hline A13 & $C_{552}$ & 3 & & & \\
\hline A13 & C.53 & 3 & & 3 & \\
\hline A13R & C.52 & 10.3 & 1.52 & & \\
\hline A13R & $\mathrm{Cr}_{53}$ & 10.4 & 1.53 & 10.4 & 1.53 \\
\hline A21 & C.52 & & & & \\
\hline A21 & Cr53 & & & & \\
\hline A22 & Cr52 & 202 & .47 & & \\
\hline A22 & $\mathrm{Cr} 53$ & 20 & .47 & 20.1 & .47 \\
\hline A23 & Cr52 & 24.5 & 1.07 & & \\
\hline A23 & Cr53 & 24.4 & 1.06 & 24.5 & 1.07 \\
\hline$A 31$ & Cr52 & 32.6 & .39 & & \\
\hline A31 & $C_{553}$ & 32.5 & .4 & 32.6 & .4 \\
\hline $\mathrm{A} 32$ & Cr52 & 29.5 & 1.38 & & \\
\hline A32 & Cr53 & 29.4 & 1.39 & 29.5 & 1.38 \\
\hline A41 & Cr52 & & & & \\
\hline A41 & C.53 & & & & \\
\hline A42 & Cr52 & 51.7 & .47 & & \\
\hline A42 & C.53 & 51 & .47 & 51.4 & .47 \\
\hline A51 & Cr52 & & & & \\
\hline A51 & Cr53 & & & & \\
\hline A52 & Cr52 & 1.22 & .06 & & \\
\hline A52 & C.53 & 123 & .06 & 123 & .06 \\
\hline $\mathrm{A} 53$ & C.52 & 9.51 & 1.61 & & \\
\hline A53 & C.53 & 9.57 & 1.62 & 9.54 & 1.62 \\
\hline $\mathrm{A} 55 \mathrm{~V}$ & Cr52 & .94 & 28 & & \\
\hline $\mathrm{A} 55 \mathrm{~V}$ & Cr53 & .85 & 28 & .95 & 28 \\
\hline
\end{tabular}

Note: The actual concentration of eamples for which no standard doviation is reported le leas than the lower limit of detection. The reported concentration is the lower limit of quantification. 


\section{Transect A Porewater Concentrations: Cobalt}

Sample ld. Lotope

Concentration

(ugh)

Std.Doviation

(ugh)

Element Avg. Conc. (ugh)

Element Avg. Std, Dov. (ugh)

\begin{tabular}{|c|c|c|c|c|c|}
\hline A11 & Co & & & & \\
\hline A12 & $C_{0}$ & 10.5 & .15 & 10.5 & .15 \\
\hline A13 & Co & 13.5 & .17 & 13.5 & .17 \\
\hline A13R & Co & 152 & 122 & 152 & 122 \\
\hline A21 & Co & & & & \\
\hline A22 & $C_{0}$ & 64 & .89 & 64 & .89 \\
\hline A23 & $\infty$ & 53.6 & 1.19 & 53.6 & 1.18 \\
\hline A31 & Co & 15 & .51 & 15 & .51 \\
\hline A32 & Co & 52.6 & .76 & 52.6 & .76 \\
\hline A41 & Co & & & & \\
\hline A42 & Co & 18.1 & .66 & 18.1 & .66 \\
\hline A51 & Co & & & & \\
\hline A52 & Co & 1.34 & 24 & 1.34 & 24 \\
\hline A53 & Co & 8.83 & .16 & 8.83 & .16 \\
\hline A55V & $c_{0}$ & 2.53 & 23 & 2.53 & 23 \\
\hline
\end{tabular}




\section{Transect A Porewater Concentrations: Copper}

\begin{tabular}{|c|c|c|c|}
\hline Sample Id. & lsotope & $\begin{array}{l}\text { Concontration } \\
(\operatorname{ug} / 2)\end{array}$ & $\begin{array}{l}\text { Std.Deviation } \\
\text { (ug/L) }\end{array}$ \\
\hline
\end{tabular}

\begin{tabular}{|c|c|c|c|c|c|}
\hline A11 & Cuts & & & & \\
\hline A11 & Cu65 & & & & \\
\hline A12 & Cu63 & 8.97 & .59 & & \\
\hline A12 & Cu65 & 7.04 & .6 & 8.01 & 6 \\
\hline A13 & Cu63 & 14 & 1.33 & & \\
\hline A13 & Cu65 & 14.5 & 1.52 & 14.3 & 1.43 \\
\hline A13R & Cu63 & & & & \\
\hline$A 13 R$ & Cu65 & 322 & 1.59 & 32.2 & 1.59 \\
\hline A21 & Cu63 & & & & \\
\hline$A 21$ & Cu65 & & & & \\
\hline$A 22$ & Cu63 & 342 & 1.61 & & \\
\hline A22 & Cu65 & 34.5 & 2.3 & 34.4 & 1.96 \\
\hline A23 & Cu63 & 141 & 5.03 & & \\
\hline A23 & Cu65 & 138 & 3.32 & 140 & 4.18 \\
\hline A31 & Cu63 & 56.7 & 2.47 & & \\
\hline A31 & Cu65 & 582 & .73 & 57.5 & 1.6 \\
\hline A32 & Cu63 & 231 & 6.52 & & \\
\hline A32 & Cu65 & 224 & 2.3 & 228 & 4.41 \\
\hline A41 & Cu63 & & & & \\
\hline A41 & Cu65 & & & & \\
\hline A42 & Cu63 & 53 & .62 & & \\
\hline $\mathrm{A} 42$ & Cu65 & 52.3 & 1.90 & 52.7 & 1.31 \\
\hline A51 & Cu63 & & & & \\
\hline A51 & Cu65 & & & & \\
\hline $\mathrm{A} 52$ & Cu63 & 7.12 & .68 & & \\
\hline A52 & Cu65 & 7.03 & .73 & 7.08 & .71 \\
\hline $\mathrm{A} 53$ & Cu63 & & & & \\
\hline$A 53$ & Cu65 & 43.6 & 4.06 & 43.6 & 4.06 \\
\hline A55V & Cu63 & 2.87 & .59 & & \\
\hline A55V & Cu65 & 3.5 & .36 & 3.19 & .48 \\
\hline
\end{tabular}


Transect A Porewater Concentrations: Iron

\begin{tabular}{|c|c|c|c|c|c|}
\hline Sample Id. & Isotope & $\begin{array}{l}\text { Concantration } \\
\text { (ugh) }\end{array}$ & $\begin{array}{l}\text { Std.Doviation } \\
\text { (ug/L) }\end{array}$ & $\begin{array}{l}\text { Element Avg. Conc. } \\
\text { (ugh) }\end{array}$ & $\begin{array}{c}\text { Element Avg. Std. Dov. } \\
\text { (ug/) }\end{array}$ \\
\hline A11 & Fo & & & & \\
\hline A12 & $F_{\theta}$ & 4720 & 47.6 & 4720 & 47.6 \\
\hline A13 & $F_{0}$ & 36600 & 355 & 36600 & 355 \\
\hline A13A & $F 0$ & 993 & 26.3 & 993 & 26.3 \\
\hline A21 & Fo & & & & \\
\hline A22 & $F_{0}$ & 912 & 5.56 & 912 & 5.56 \\
\hline A23 & Fo & 429 & 10.4 & 429 & 10.4 \\
\hline A31 & $F_{0}$ & 2040 & 45.5 & 2040 & 45.5 \\
\hline$A 32$ & $F_{0}$ & 1210 & 43.1 & 1210 & 43.1 \\
\hline A41 & Fo & & & & \\
\hline A42 & $F_{\theta}$ & 4430 & 67.3 & 4430 & 67.3 \\
\hline A51 & $\mathrm{F}_{\theta}$ & & & & \\
\hline A52 & $F_{\theta}$ & 169 & 24.4 & 169 & 24.4 \\
\hline$A 53$ & $F_{\theta}$ & 1210 & 31 & 1210 & 31 \\
\hline A55V & $F_{0}$ & 2020 & 88.8 & 2020 & 88.8 \\
\hline
\end{tabular}




\section{Transect A Porewater Concentrations: Lead}

\begin{tabular}{|c|c|c|c|c|c|}
\hline Sample id. & lsotope & $\begin{array}{l}\text { Concentration } \\
\text { (ug/L) }\end{array}$ & $\begin{array}{l}\text { Std.Dovlation } \\
\text { (ug })\end{array}$ & $\begin{array}{l}\text { Element Avg. Conc. } \\
(u g /)\end{array}$ & $\begin{array}{c}\text { Element Avg. Std. Dov. } \\
\text { (ugg) }\end{array}$ \\
\hline A11 & $\mathrm{Pb} 206$ & & & & \\
\hline A11 & $\mathrm{Pb} 207$ & & & & \\
\hline A11 & $\mathrm{Pb} 208$ & & & & \\
\hline A12 & Pb206 & 37.7 & .51 & & \\
\hline A12 & $\mathrm{Pb} 207$ & 33 & .51 & & \\
\hline A12 & $\mathrm{Pb} 208$ & 34.7 & .72 & 35.1 & .58 \\
\hline A13 & $\mathrm{Pb} 206$ & 11.3 & & & \\
\hline$A 13$ & $\mathrm{~Pb} 207$ & 11.3 & & & \\
\hline A13 & $\mathrm{Pb208}$ & 11.3 & & \begin{tabular}{|l|l}
11.3 \\
\end{tabular} & \\
\hline A13R & Pb206 & 1 & & & \\
\hline A13R & $\mathrm{Pb207}$ & 1 & & & \\
\hline A13R & Pb208 & 1 & & 1 & \\
\hline A21 & $\mathrm{Pb206}$ & & & & \\
\hline A21 & Pb207 & & & & \\
\hline A21 & $\mathrm{Pb208}$ & & & & \\
\hline$A 22$ & $\mathrm{Pb206}$ & 33.5 & 1.79 & & \\
\hline A22 & $\mathrm{Pb207}$ & 30.8 & 2.31 & & \\
\hline A22 & $\mathrm{Pb} 208$ & 30.4 & .07 & 31.6 & 1.39 \\
\hline A23 & $\mathrm{Pb} 206$ & 15.1 & .46 & & \\
\hline A23 & Pb207 & 13.6 & .95 & & \\
\hline A23 & $\mathrm{Pb208}$ & 152 & 1.62 & 14.6 & 1.01 \\
\hline$A 31$ & $\mathrm{~Pb} 206$ & 4.78 & .72 & & \\
\hline $\mathrm{A} 31$ & Pb207 & 3.1 & .93 & & \\
\hline$A 31$ & P6208 & 4.97 & .73 & 4.28 & .79 \\
\hline A32 & $\mathrm{Pb} 206$ & 4.15 & .68 & & \\
\hline $\mathrm{A} 32$ & $\mathrm{~Pb} 207$ & 2.21 & .53 & & 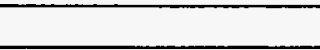 \\
\hline A32 & $\mathrm{Pb208}$ & 2.81 & 26 & 3.06 & .49 \\
\hline A41 & Pb206 & & & & \\
\hline A41 & $\mathrm{Pb207}$ & & & & \\
\hline A41 & Pb208 & & & & \\
\hline A42 & Pb206 & 8.27 & .33 & & \\
\hline A42 & $\mathrm{Pb} 207$ & 6.95 & .13 & & \\
\hline A42 & $\mathrm{Pb} 208$ & 7.58 & .16 & 7.6 & 21 \\
\hline A51 & Pb206 & & & & \\
\hline A51 & Pb207 & & & & \\
\hline A51 & $\mathrm{Pb} 208$ & & & & \\
\hline A52 & Pb206 & .75 & & & \\
\hline A52 & Pb207 & .75 & & & \\
\hline A52 & $\mathrm{Pb} 208$ & .75 & & .75 & \\
\hline A53 & Pb206 & 1 & & & \\
\hline A53 & Pb207 & 1 & & & \\
\hline$A 53$ & $\mathrm{~Pb} 208$ & 1 & & $\frac{1}{1}$ & \\
\hline A55V & $\mathrm{Pb} 206$ & 7.5 & & & \\
\hline A55V & Pb207 & 7.5 & & & \\
\hline
\end{tabular}

Note: The actual concentration of samples for which no standard deviation la reported lo leas than the lower limit of detection. The reported concentration is the lower limit of quantification. 


\begin{tabular}{|c|c|c|c|c|c|}
\hline \multicolumn{6}{|c|}{ Transect A Porewater Concentrations: Lead } \\
\hline Sample Id. & Leotope & $\begin{array}{l}\text { Concentration } \\
\text { (ug/L) }\end{array}$ & $\begin{array}{l}\text { Std.Deviation } \\
\text { (ug/) }\end{array}$ & $\begin{array}{l}\text { Element Avg. Conc. } \\
\text { (ug/L) }\end{array}$ & $\begin{array}{l}\text { Eloment Avg. Std. Dov. } \\
\text { (ugh) }\end{array}$ \\
\hline A55V & $P 6208$ & 7.5 & & 7.5 & \\
\hline
\end{tabular}




\section{Transect A Porewater Concentrations: Magnesium}

\begin{tabular}{ccccc}
\hline Samplo ld. Isotope & $\begin{array}{c}\text { Concontration } \\
\text { (ug/ } / \text { ) }\end{array}$ & $\begin{array}{c}\text { Std.Dovlation } \\
\text { (ug/ })\end{array}$ & $\begin{array}{c}\text { Element Avg. Conc. } \\
\text { (ug/L) }\end{array}$ & $\begin{array}{c}\text { Element Avg. Std. Dov. } \\
\text { (ug } / \text { ) }\end{array}$ \\
\hline
\end{tabular}

\begin{tabular}{|c|c|c|c|c|c|}
\hline A11 & Mg24 & & & & \\
\hline A11 & Mops & & & & \\
\hline A12 & Mq24 & 18100 & 222 & & \\
\hline $\mathrm{A} 12$ & Mg25 & 20000 & 357 & 19100 & 290 \\
\hline A13 & $M g 24$ & 4360 & 33.1 & & \\
\hline A13 & $M g 25$ & 4300 & 142 & 4330 & 23.7 \\
\hline A13R & Mg24 & 1850 & 15.8 & & \\
\hline A13R & Mg25 & 1810 & 24.5 & 1830 & 202 \\
\hline A21 & $M 924$ & & & & \\
\hline A21 & $M g=5$ & & & & \\
\hline A22 & Mg24 & 910 & 14.1 & & \\
\hline A22 & M925 & 949 & 19.8 & 930 & 17 \\
\hline A23 & M924 & 845 & 7.6 & & \\
\hline$A 23$ & M925 & 885 & 19.1 & 865 & 13.4 \\
\hline A31 & $M 924$ & 699 & 8.98 & & \\
\hline A31 & Mg25 & 719 & 8.97 & 709 & 8.98 \\
\hline $\mathrm{A} 32$ & Mg24 & 1310 & 27.3 & & \\
\hline $\mathrm{A} 32$ & Mg25 & 1100 & 17.4 & 1210 & 22.4 \\
\hline A41 & Mg24 & & & & \\
\hline A41 & $M 925$ & & & & \\
\hline A42 & M924 & 460 & 7.63 & & \\
\hline A42 & Mg25 & 409 & 13.4 & 465 & 10.5 \\
\hline A51 & Mg24 & & & & \\
\hline A51 & $M 925$ & & & & \\
\hline $\mathrm{A} 52$ & $M 924$ & 135 & 7.05 & & \\
\hline A52 & Mg25 & 136 & 5.73 & 136 & 6.39 \\
\hline $\mathrm{A} 53$ & M924 & 186 & 4.05 & & \\
\hline $\mathrm{A} 53$ & Mg25 & 180 & 38 & 183 & 21 \\
\hline A55V & Mg24 & 4120 & 72.6 & & \\
\hline A55V & Mg25 & 3390 & 45.5 & 3760 & 59.1 \\
\hline
\end{tabular}

Note: The actual concentration of samples for which no standard deviation is reported is lees than the lower limit of detection. The reported concentration is the lower limit of quantification. 


\section{Transect A Porewater Concentrations: Manganese}

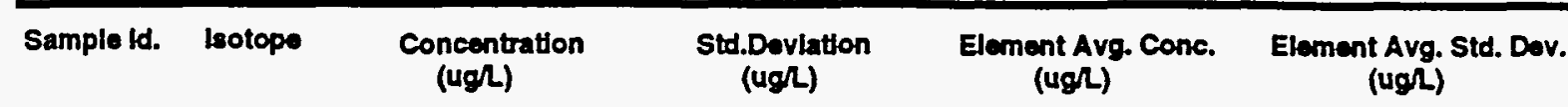

\begin{tabular}{|c|c|c|c|c|c|}
\hline A11 & $M n$ & & & & \\
\hline A12 & $\mathrm{Mn}$ & 826 & 3.85 & 826 & 3.85 \\
\hline A13 & $\mathrm{Mn}$ & 2390 & 14.6 & 2390 & 14.6 \\
\hline A13R & $\mathrm{Mn}$ & 167 & 6.91 & 167 & 6.91 \\
\hline A21 & Mn & & & & \\
\hline A22 & $\mathrm{Mn}$ & 22000 & 90.8 & 22000 & 90.8 \\
\hline A23 & $\mathrm{Mn}$ & 23300 & 176 & 23300 & 176 \\
\hline$A 31$ & $\mathrm{Mn}$ & 10300 & 141 & 10300 & 141 \\
\hline$A 32$ & $\mathrm{Mn}$ & 29500 & 188 & 29500 & 188 \\
\hline A41 & $\mathrm{Mn}$ & & & & \\
\hline$A 42$ & $\mathrm{Mn}$ & 5420 & 28.3 & 5420 & 28.3 \\
\hline$A 51$ & $M n$ & & & & \\
\hline A52 & $\mathrm{Mn}$ & 35.7 & 129 & 35.7 & 129 \\
\hline$A 53$ & $\mathrm{Mn}$ & 211 & 7.54 & 211 & 7.54 \\
\hline A55V & $\mathrm{Mn}$ & 1940 & 32 & 1940 & 32 \\
\hline
\end{tabular}


Transect A Porewater Concentrations: Nickel

\begin{tabular}{|c|c|c|c|c|c|}
\hline Sample Id. & Lsotope & $\begin{array}{l}\text { Concentration } \\
\text { (ug/n) }\end{array}$ & $\begin{array}{l}\text { Std.Deviation } \\
\text { (ugh) }\end{array}$ & $\begin{array}{c}\text { Element Avg. Cone. } \\
\text { (ugh) }\end{array}$ & $\begin{array}{c}\text { Element Avg. Std. Dov. } \\
\text { (ugh) }\end{array}$ \\
\hline A11 & Ni60 & & & & \\
\hline A11 & Ni62 & & & & \\
\hline A12 & Ni60 & 118 & 2.86 & & \\
\hline A12 & Ni62 & 144 & 4.69 & 131 & 3.78 \\
\hline A13 & Ni6o & 255 & 3.54 & & \\
\hline A13 & Ni62 & 282 & 3.87 & 200 & 3.71 \\
\hline A13R & Ni60 & 96.4 & 10.4 & & \\
\hline A13R & Ni62 & 79.7 & 672 & 88.1 & 38.8 \\
\hline$A 21$ & Ni6o & & & & \\
\hline$A 21$ & Ni62 & & & & \\
\hline A22 & Ni6o & 183 & 4.15 & & \\
\hline A22 & Ni62 & 198 & 11.6 & 191 & 7.88 \\
\hline A23 & Ni6o & 143 & 5.82 & & \\
\hline A23 & Ni62 & 153 & 19.6 & 148 & 12.7 \\
\hline$A 31$ & $\mathrm{Ni} 6 \mathrm{O}$ & 230 & 2.18 & & \\
\hline$A 31$ & $\mathrm{Ni62}$ & 268 & 18.4 & 249 & 10.3 \\
\hline A32 & Nico & 181 & 4.16 & & \\
\hline A32 & $\mathrm{Ni62}$ & 205 & 10.8 & 193 & 7.48 \\
\hline A41 & Ni6o & & & & \\
\hline A41 & Ni62 & & & & \\
\hline A42 & Ni6o & 348 & .15 & & \\
\hline A42 & $\mathrm{Ni62}$ & 385 & 38.9 & 367 & 19.5 \\
\hline A51 & Ni6o & & & & \\
\hline A51 & Ni62 & & & & \\
\hline A52 & Ni60 & 29.5 & 1.09 & & \\
\hline A52 & $\mathrm{Ni62}$ & 25.7 & 3.54 & 27.6 & 2.32 \\
\hline$A 53$ & Ni60 & 123 & 21.9 & & \\
\hline$A 53$ & Ni62 & 141 & 42.4 & 132 & 322 \\
\hline $\mathrm{A} 55 \mathrm{~V}$ & $\mathrm{Ni} 6 \mathrm{O}$ & 54.1 & 4.18 & & \\
\hline A55V & Ni62 & 54.3 & 426 & 542 & 422 \\
\hline
\end{tabular}

Note: The actual concentration of samples for which no standard doviation is reported is lese than the lower limit of detection. The reported concentration is the lower llmit of quantification. 


\begin{tabular}{|c|c|c|c|c|c|}
\hline Sample ld. & leotope & $\begin{array}{l}\text { Concentration } \\
\text { (Ug/L) }\end{array}$ & $\begin{array}{l}\text { Sudbovintion } \\
\text { (ugl) }\end{array}$ & $\begin{array}{l}\text { Element Avg- Conc. } \\
\text { (ugh) }\end{array}$ & $\begin{array}{c}\text { Element Avg. Std. Dav. } \\
\text { (Ug/) }\end{array}$ \\
\hline A11 & So77 & & & & \\
\hline A11 & 5082 & & & & \\
\hline A12 & So77 & 7.5 & & & \\
\hline A12 & Sege & 7.5 & & 7.5 & \\
\hline A13 & so77 & 18.3 & 18.7 & & \\
\hline A13 & 5082 & 19.3 & 18.4 & 19.3 & 18.5 \\
\hline A13R & so7 & .5 & & & \\
\hline A13A & 5082 & .5 & & .5 & \\
\hline$A 21$ & 5077 & & & & \\
\hline$\overline{A 21}$ & Se82 & & & & \\
\hline$A 22$ & so77 & 12.7 & & & \\
\hline$A 22$ & 5082 & 13.2 & & 13 & \\
\hline$A 23$ & So77 & 19.6 & 3.57 & & \\
\hline A23 & So82 & 19.9 & 3.62 & 19.8 & 3.6 \\
\hline$A 31$ & so77 & 14.9 & 6.51 & & \\
\hline A31 & Se82 & 15.3 & 6.52 & 15.1 & 6.52 \\
\hline A32 & so77 & 8.88 & 4.62 & & \\
\hline A32 & So82 & 9.6 & 4.72 & 9.24 & 4.67 \\
\hline$\overline{A 41}$ & So77 & & & & \\
\hline A41 & 5082 & & & & \\
\hline$A 42$ & So77 & 15.8 & 6.42 & & \\
\hline A42 & S082 & 16.4 & 6.4 & 16.1 & 6.41 \\
\hline$A 51$ & Se77 & & & & \\
\hline$A 51$ & Se82 & & & & \\
\hline$A 52$ & so77 & 37.5 & & & \\
\hline$A 52$ & 5082 & 37.5 & & 37.5 & \\
\hline$A 53$ & So77 & 25 & & & \\
\hline$A 53$ & Se82 & 25 & & 25 & \\
\hline $\mathrm{A} 55 \mathrm{~V}$ & se77 & 37.5 & & & \\
\hline A55V & Se82 & 37.5 & & 37.5 & \\
\hline
\end{tabular}

Note: The actual concentration of eamples for wheh no standard deviation be reported b has than the lower limit of detection. The reported concentration is the bower Himit of quantification. 
Transect A Porewater Concentrations: Silver

\begin{tabular}{|c|c|c|c|c|c|}
\hline Sample ld. & Lsotope & $\begin{array}{l}\text { Concentration } \\
\text { (ughL) }\end{array}$ & $\begin{array}{l}\text { Std.Doviation } \\
\text { (ug/L) }\end{array}$ & $\begin{array}{l}\text { Element Avg. Conc. } \\
\text { (ugh) }\end{array}$ & $\begin{array}{c}\text { Element Avg. Std. Dov. } \\
\text { (Ug/) }\end{array}$ \\
\hline A11 & Ag107 & & & & \\
\hline A11 & Ag109 & & & & \\
\hline $\mathrm{A} 12$ & Ag107 & 10.8 & .59 & & \\
\hline A12 & Ag109 & 9.7 & .51 & 10.3 & .55 \\
\hline A13 & Ag107 & 151 & 9.69 & & \\
\hline A13 & Ag109 & 166 & 15 & 159 & 12.3 \\
\hline A13R & Ag107 & 882 & 6.1 & & \\
\hline A13R & Ag109 & 95.7 & 10.7 & 92 & 8.4 \\
\hline A21 & Ag107 & & & & \\
\hline A21 & Ag109 & & & & \\
\hline$A 22$ & Ag107 & 111 & 1.5 & & \\
\hline A22 & Ag109 & 119 & 1.38 & 115 & 1.44 \\
\hline A23 & Ag107 & 70.7 & 3.01 & & \\
\hline $\mathrm{A} 23$ & Ag109 & $\pi$ & & 73.9 & 3.01 \\
\hline A31 & Ag107 & 90.7 & 5.57 & & \\
\hline$A 31$ & Ag109 & 86.6 & 4.39 & 88.7 & 4.98 \\
\hline A32 & Ag107 & 79.7 & 8.14 & & \\
\hline A32 & Ag109 & 70.1 & 429 & 74.9 & 6.22 \\
\hline A41 & Ag107 & & & & \\
\hline A41 & Ag109 & & & & \\
\hline A42 & Ag107 & 55.1 & 4.86 & & \\
\hline A42 & Ag109 & 62.7 & 6.48 & 58.9 & 5.67 \\
\hline $\mathrm{A} 51$ & Ag107 & & & & \\
\hline A51 & Ag109 & & & & \\
\hline A52 & Ag107 & 131 & 17.6 & & \\
\hline A52 & Ag109 & 145 & 6.18 & 138 & 11.9 \\
\hline A53 & Ag107 & 69.1 & 13.5 & & \\
\hline$A 53$ & Ag109 & 81.3 & 4.06 & 75.2 & 8.78 \\
\hline A55V & Ag107 & 44.7 & 3.96 & & \\
\hline A55V & Ag109 & 56.1 & 12 & 50.4 & 2.58 \\
\hline
\end{tabular}




\section{Transect A Porewater Concentrations: Sodium}

\begin{tabular}{|c|c|c|c|c|c|}
\hline Sample ld. & leotope & $\begin{array}{l}\text { Concentration } \\
\text { (ugh) }\end{array}$ & $\begin{array}{l}\text { Std.Devlation } \\
\text { (ug/) }\end{array}$ & $\begin{array}{l}\text { Element Avg. Conc. } \\
\text { (ug/l) }\end{array}$ & $\begin{array}{c}\text { Element Avg. Std. Dov. } \\
\text { (ugh) }\end{array}$ \\
\hline A11 & $\mathrm{Na}$ & & & & \\
\hline A12 & $\mathrm{Na}$ & 3090 & 106 & 3090 & 105 \\
\hline A13 & $\mathrm{Na}$ & 17400 & 221 & 17400 & 221 \\
\hline A13R & $\mathrm{Na}$ & 18400 & 373 & 18400 & 373 \\
\hline$A 21$ & $\mathrm{Na}$ & & & & \\
\hline A22 & $\mathrm{Na}$ & 65200 & 1820 & 65200 & 1820 \\
\hline A23 & $\mathrm{Na}$ & 43500 & 1570 & 43500 & 1570 \\
\hline$A 31$ & $\mathrm{Na}$ & 38500 & 3670 & 38600 & 3670 \\
\hline A32 & $\mathrm{Na}$ & 66400 & 4610 & 66400 & 4610 \\
\hline A41 & $\mathrm{Na}$ & & & & \\
\hline A42 & Na & 27800 & 5040 & 27800 & 5040 \\
\hline$A 51$ & $\mathrm{Na}$ & & & & \\
\hline$A 52$ & $\mathrm{Na}$ & 2130 & 107 & 2130 & 107 \\
\hline$A 53$ & $\mathrm{Na}$ & 30200 & 352 & 30200 & 352 \\
\hline A55V & $\mathrm{Na}$ & 24300 & 325 & 24300 & 325 \\
\hline
\end{tabular}




\section{Transect A Porewater Concentrations: Strontium}

Sample Id. Lotope

Concentration

Std.Doviation

(ugh)

Element Avg. Conc.

(ugh)

Element Avg. Std. Dov.

(ugh)

\begin{tabular}{l|l}
$\mathrm{Sr}$ & \\
\hline $\mathrm{Sr}$ & \\
\hline $\mathrm{Sr}$ & \\
\hline $\mathrm{Sr}$ &
\end{tabular}

\begin{tabular}{l|l} 
A13R & Sr
\end{tabular}

\begin{tabular}{l|l} 
A21 & $\mathrm{Sr}$
\end{tabular}

\begin{tabular}{l|l} 
A22 & \\
A23 & Sr \\
\hline
\end{tabular}

A31 13

\begin{tabular}{l|l} 
A32 & \\
A41 &
\end{tabular}

\begin{tabular}{l|l} 
A42 & \\
A51 &
\end{tabular}

\begin{tabular}{l|l|r}
$\mathrm{A} 52$ & $\mathrm{Sr}$ & 12 \\
\hline
\end{tabular}

\begin{tabular}{l|l|l}
$\mathrm{A} 53$ & $\mathrm{Sr}$ & 25.8 \\
\hline
\end{tabular}

\begin{tabular}{l|l|l} 
A55V & Sr & 38.7
\end{tabular}

\begin{tabular}{|l|l}
\hline 479 & \\
\hline 140 & \\
\hline 912 & \\
\hline 34.9 & \\
\hline 39.9 & \\
\hline 25.7 & \\
\hline 35.6 & \\
\hline 19.4 & \\
\hline 12 & \\
\hline 25.8 & \\
\hline 38.7 & \\
\hline
\end{tabular}

\begin{tabular}{|l|c|l|}
\hline 1.96 & & \\
\hline 1.49 & 149 & 1.96 \\
\hline 4.18 & 912 & 1.49 \\
\hline 1.1 & & 4.18 \\
\hline .49 & 34.9 & \\
\hline .59 & 39.9 & 1.1 \\
\hline .38 & 25.7 & .49 \\
\hline .74 & 35.6 & .59 \\
\hline .12 & & .38 \\
\hline 2.6 & 19.4 & .74 \\
\hline .51 & 12 & .12 \\
\hline
\end{tabular}




\section{Transect A Porewater Concentrations: Thorium}

\begin{tabular}{|c|c|c|c|c|c|}
\hline Sample Id. & Isotope & $\begin{array}{l}\text { Concentratton } \\
\text { (ug/L) }\end{array}$ & $\begin{array}{l}\text { Std.Devlation } \\
\text { (ug/ })\end{array}$ & $\begin{array}{l}\text { Element Avg. Conc. } \\
\text { (ugh) }\end{array}$ & $\begin{array}{c}\text { Element Avg. Std. Dov. } \\
\text { (ugh) }\end{array}$ \\
\hline A11 & Th232 & & & & \\
\hline$\overline{A 12}$ & Th232 & 5 & & 5 & \\
\hline A13 & Th232 & .13 & .03 & .13 & .03 \\
\hline A13R & Th232 & 25 & & 25 & \\
\hline A21 & Th232 & & & & \\
\hline$A 22$ & Th232 & .5 & & .5 & \\
\hline A23 & Th232 & .5 & & .5 & \\
\hline A31 & Th232 & & & & \\
\hline A32 & Th232 & .5 & & .5 & \\
\hline A41 & Th232 & & & & \\
\hline A42 & Th232 & .5 & & .5 & \\
\hline A51 & Th232 & & & & \\
\hline A52 & Th232 & .5 & & .5 & \\
\hline A53 & Th232 & 25 & & 25 & \\
\hline A55V & Th232 & .5 & & .5 & \\
\hline
\end{tabular}




\section{Transect A Porewater Concentrations: Tin}

\begin{tabular}{|c|c|c|c|c|c|}
\hline Sample Id. & Isotope & $\begin{array}{l}\text { Concentration } \\
\text { (ugl) }\end{array}$ & $\begin{array}{l}\text { Stcd.Doviation } \\
\text { (ug/) }\end{array}$ & $\begin{array}{l}\text { Element Avg. Conc. } \\
\text { (ugh) }\end{array}$ & $\begin{array}{c}\text { Element Avg. Std. Dov. } \\
\text { (ugh) }\end{array}$ \\
\hline A11 & $\operatorname{Sn} 118$ & & & & \\
\hline A11 & $\sin 120$ & & & & \\
\hline A12 & Sn118 & 2.5 & & & \\
\hline A12 & $\sin 120$ & 2.5 & & 2.5 & \\
\hline A13 & Sn118 & 30.2 & 11.3 & & \\
\hline A13 & Sn 120 & 32.3 & 10.9 & 31.3 & 11.1 \\
\hline A13R & $\sin 118$ & 25 & & & \\
\hline A13R & $\sin 120$ & 25 & & 25 & \\
\hline A21 & $\operatorname{Sn} 118$ & & & & \\
\hline A21 & $\operatorname{Sn} 120$ & & & & \\
\hline$A 22$ & $\operatorname{Sn} 118$ & 12.8 & 1.12 & & \\
\hline$A 22$ & $\operatorname{Sn} 120$ & 13.6 & 1.45 & 132 & 1.29 \\
\hline A23 & Sn118 & 10.7 & .49 & & \\
\hline A23 & $\operatorname{Sn} 120$ & 8.73 & .49 & 9.72 & .49 \\
\hline$A 31$ & Sn118 & 4.54 & .3 & & \\
\hline A31 & Sn120 & 4.68 & .42 & 4.61 & .36 \\
\hline A32 & $\operatorname{sn} 118$ & 4.98 & .66 & & \\
\hline A32 & Sn120 & 423 & .19 & 4.61 & .43 \\
\hline A41 & Sn118 & & & & \\
\hline A41 & Sn120 & & & & \\
\hline$A 42$ & Sn118 & 2.61 & .61 & & \\
\hline$A 42$ & Sn120 & 1.61 & 22 & 2.11 & .42 \\
\hline A51 & Sn118 & & & & \\
\hline A51 & Sn120 & & & & \\
\hline A52 & $\sin 118$ & .79 & 25 & & \\
\hline A52 & $\operatorname{Sn} 120$ & 1.15 & 25 & .97 & 25 \\
\hline$A 53$ & Sn118 & 2.5 & & & \\
\hline $\mathrm{A} 53$ & $\operatorname{Sn} 120$ & 2.5 & & 2.5 & \\
\hline $\mathrm{A} 55 \mathrm{~V}$ & Sn118 & 1.91 & .7 & & \\
\hline A55V & Sn120 & 1.34 & .55 & 1.63 & .63 \\
\hline
\end{tabular}




\section{Transect A Porewater Concentrations: Uranium-235}

\begin{tabular}{|c|c|c|c|c|c|}
\hline Sample Id. & botope & $\begin{array}{l}\text { Concentration } \\
\text { (Uerl) }\end{array}$ & $\begin{array}{l}\text { Siddowiation } \\
\text { (ugh) }\end{array}$ & $\begin{array}{l}\text { Element Avg. Conc. } \\
\text { (u\&h) }\end{array}$ & $\begin{array}{c}\text { Element Avg, Std. Dov. } \\
\text { (ugh) }\end{array}$ \\
\hline A11 & U235 & & & & \\
\hline A12 & U235 & .05 & .01 & .05 & .01 \\
\hline $\mathrm{A13}$ & U235 & .17 & .03 & .17 & .03 \\
\hline A13R & U235 & 4.9 & .55 & 4.8 & .55 \\
\hline A21 & U235 & & & & \\
\hline$A 22$ & U235 & 427 & .14 & 427 & .14 \\
\hline A23 & U235 & 11.3 &.$\infty$ & 11.3 & .09 \\
\hline A31 & U235 & 4.52 & 29 & 4.52 & 29 \\
\hline A32 & U235 & 13.9 & .53 & 13.9 & .53 \\
\hline A41 & U235 & & & & \\
\hline A42 & U235 & 3.63 & .32 & 3.63 & .32 \\
\hline A51 & U235 & & & & \\
\hline A52 & U235 & .02 & & .02 & $\cdot$ \\
\hline A53 & U235 & .85 & .31 & .85 & .31 \\
\hline A55V & U235 & .02 & & .02 & \\
\hline
\end{tabular}




\section{Transect A Porewater Concentrations: Uranium-238}

\begin{tabular}{|c|c|c|c|c|c|}
\hline Sample ld. & kotope & $\begin{array}{l}\text { Concentration } \\
\text { (ugl) }\end{array}$ & $\begin{array}{l}\text { Std.Devintion } \\
\text { (ugh) }\end{array}$ & $\begin{array}{l}\text { Element Avg. Cons. } \\
\text { (ug/L) }\end{array}$ & $\begin{array}{c}\text { Element Avg. Std. Dov. } \\
\text { (ugh) }\end{array}$ \\
\hline A11 & U238 & & & & \\
\hline A12 & U238 & 13.8 & 25 & 13.8 & 25 \\
\hline A13 & U238 & 35.3 & .7 & 35.3 & .7 \\
\hline A13R & U238 & 1410 & 41.8 & 1410 & 41.8 \\
\hline A21 & U238 & & & & \\
\hline A22 & U238 & 1500 & 482 & 1500 & 482 \\
\hline A23 & U238 & 3210 & 56.9 & 3210 & 56.9 \\
\hline$A 31$ & U238 & 1980 & 86 & 1980 & 86 \\
\hline A32 & U238 & 2990 & 186 & 2990 & 186 \\
\hline A41 & U238 & & & & \\
\hline A42 & U238 & 975 & 27.6 & 975 & 27.6 \\
\hline$A 51$ & U238 & & & & \\
\hline $\mathrm{A52}$ & U238 & .42 & 28 & .42 & 28 \\
\hline A53 & U238 & 232 & 1.33 & 232 & 1.33 \\
\hline $\mathrm{A} 55 \mathrm{~V}$ & U238 & .08 & .03 & .08 & .03 \\
\hline
\end{tabular}

Note: The actual concentration of amples for whlch no standard deviation is reported ls leas than the lower limit of detection. The reported concentration is the lower limit of quantification. 


\section{Transect A Porewater Concentrations: Vanadium}

\begin{tabular}{|c|c|c|c|c|c|}
\hline Sample ld. & Isotope & $\begin{array}{l}\text { Concentration } \\
\text { (ugh) }\end{array}$ & $\begin{array}{l}\text { Std.Dovlation } \\
\text { (ugl) }\end{array}$ & $\begin{array}{l}\text { Element Avg. Cons. } \\
\text { (ugh) }\end{array}$ & $\begin{array}{c}\text { Element Avg. Std. Dov. } \\
\text { (ugh) }\end{array}$ \\
\hline A11 & $\mathbf{v}$ & & & & \\
\hline A12 & V & 2.5 & & 2.5 & \\
\hline A13 & $v$ & .61 & 23 & .61 & 23 \\
\hline A13R & $v$ & .13 & & .13 & \\
\hline A21 & $v$ & & & & \\
\hline A22 & $v$ & 25 & & 25 & \\
\hline A23 & $v$ & 1.39 & .45 & 1.39 & .45 \\
\hline$A 31$ & $v$ & 22 & .89 & 22 & .89 \\
\hline A32 & $V$ & 1.55 & .92 & 1.55 & .92 \\
\hline A41 & $v$ & & & & \\
\hline A42 & $v$ & 25 & & 25 & \\
\hline $\mathrm{A51}$ & $v$ & & & & \\
\hline A52 & $\mathbf{v}$ & 25 & .06 & 25 & .06 \\
\hline A53 & $\mathbf{v}$ & 6.25 & & 6.25 & \\
\hline A55V & $v$ & .38 & .15 & .38 & .15 \\
\hline
\end{tabular}

Note: The actual concentration of eamples for which no standard deviatton be reported ts leas than the lower limit of idetection. The reported concentration ls the lower limit of quantification. 


\section{Transect A Porewater Concentrations: Yttrium}

Sample ld. leotope

Concentration

(ugh)

Std.Deviation

(ugh)

Element Avg. Conc.

(ugh)

Element Avg. Std. Dov. (ugl)

\begin{tabular}{|c|c|c|c|c|c|}
\hline A11 & $Y$ & & & & \\
\hline A12 & $\bar{Y}$ & 50.9 & .15 & 50.9 & .15 \\
\hline A13 & $Y$ & .53 & .02 & .53 & .02 \\
\hline A13R & $Y$ & 6.32 & .54 & 6.32 & .54 \\
\hline A21 & $Y$ & & & & \\
\hline$A 22$ & $Y$ & 3.54 & .07 & 3.54 & .07 \\
\hline A23 & $Y$ & 14.8 & .38 & 14.8 & .38 \\
\hline A31 & $Y$ & 15.8 & .86 & 15.8 & .86 \\
\hline A32 & $\mathbf{Y}$ & 16.3 & .32 & 16.3 & .32 \\
\hline A41 & $Y$ & & & & \\
\hline A42 & $Y$ & 6.16 & .08 & 6.16 & .08 \\
\hline A51 & $Y$ & & & & \\
\hline A52 & $Y$ & 28 & .07 & 28 & .07 \\
\hline$A 53$ & $Y$ & 4.93 & .67 & 4.93 & $.67^{\circ}$ \\
\hline $\mathrm{A} 55 \mathrm{~V}$ & $Y$ & & & & \\
\hline
\end{tabular}

Note: The actual concentration of samples for which no standard deviation bs reported is leces than the lower llmit of detection. The reported concentration is the lower limit of quantification. 


\section{Transect A Porewater Concentrations: Zinc}

\begin{tabular}{|c|c|c|c|c|c|}
\hline Samplo id. & Lotope & $\begin{array}{l}\text { Concentration } \\
\text { (ugl) }\end{array}$ & $\begin{array}{l}\text { Std.Dovintion } \\
\text { (ugh) }\end{array}$ & $\begin{array}{l}\text { Element Avg. Conc. } \\
\text { (Ugh) }\end{array}$ & $\begin{array}{c}\text { Element Avg. Std. Dov. } \\
\text { (ug/) }\end{array}$ \\
\hline A11 & Zn66 & & & & \\
\hline A12 & Zn66 & 174 & 4.9 & 174 & 4.9 \\
\hline A13 & Zn66 & 3770 & 39.8 & 3770 & 39.8 \\
\hline A13R & Zn66 & 90.7 & 7.79 & 90.7 & 7.79 \\
\hline A21 & Zn66 & & & & \\
\hline A22 & Zn66 & 100 & 5.6 & 100 & 5.6 \\
\hline A23 & Zn66 & 347 & 2.51 & 347 & 2.51 \\
\hline$A 31$ & Zn66 & 381 & 6.82 & 381 & 6.82 \\
\hline A32 & Zn66 & 629 & 7.78 & 629 & 7.78 \\
\hline A41 & Zn66 & & & & \\
\hline A42 & Zn66 & 250 & 9.74 & 250 & 9.74 \\
\hline$A 51$ & Zn66 & & & & \\
\hline A52 & Zn66 & 32.3 & 2.32 & 32.3 & 2.32 \\
\hline$A 53$ & Zn66 & 190 & 58.6 & 190 & 58.6 \\
\hline A55V & Zn66 & 1380 & .56 & 1380 & .56 \\
\hline
\end{tabular}


Transect A Porewater Concentrations: Zirconium

\begin{tabular}{|c|c|c|c|c|c|}
\hline Samplo id. & leotope & $\begin{array}{l}\text { Concontration } \\
(u g / L)\end{array}$ & $\begin{array}{l}\text { Std.Dovlation } \\
\text { (ug/) }\end{array}$ & $\begin{array}{l}\text { Element Avg. Conc. } \\
\text { (ug/) }\end{array}$ & $\begin{array}{c}\text { Element Avg. Std. Dev. } \\
\text { (Ug } \Omega \text {. }\end{array}$ \\
\hline
\end{tabular}

\begin{tabular}{|c|c|c|c|c|c|}
\hline A11 & Zrso & & & & \\
\hline A11 & $Z 991$ & & & & \\
\hline A12 & $Z 90$ & 5 & & & \\
\hline$\overline{A 12}$ & $Z 91$ & 5 & & 5 & \\
\hline A13 & $Z 900$ & 2.75 & & & \\
\hline A13 & $Z 991$ & 2.75 & & 2.75 & \\
\hline A13R & $\mathrm{Z}=0$ & 25 & & & \\
\hline A13R & Z 91 & 25 & & 25 & \\
\hline A21 & $\mathrm{Z} \times 0$ & & & & \\
\hline A21 & $\mathrm{ZP1}$ & & & & \\
\hline$A 22$ & $Z \mathbf{z} 90$ & .5 & & & \\
\hline A22 & $Z 91$ & .5 & & .5 & \\
\hline A23 & $Z r 90$ & .06 & .08 & & \\
\hline $\mathrm{A} 23$ & Zra1 & .05 & 0 & .06 & .04 \\
\hline A31 & $Z r 90$ & .5 & & & \\
\hline A31 & $Z 91$ & .5 & & .5 & \\
\hline A32 & $Z r 90$ & .13 & .02 & & \\
\hline A32 & $Z 991$ & .05 & .14 & .09 & .08 \\
\hline A41 & $Z r 90$ & & & & \\
\hline A41 & Zr91 & & & & \\
\hline A42 & $Z 90$ & .5 & & & \\
\hline A42 & Zr91 & .5 & & .5 & \\
\hline A51 & $Z r 90$ & & & & \\
\hline A51 & Zr91 & & & & \\
\hline $\mathrm{A} 52$ & Zr9o & .5 & & & \\
\hline A52 & Zr91 & .5 & & .5 & \\
\hline$A 53$ & Zr90 & 2.5 & & & \\
\hline A53 & Zr91 & 2.5 & & 2.5 & \\
\hline A55V & Zr9o & .5 & & & \\
\hline$\overline{\mathrm{A} 55 \mathrm{~V}}$ & Zr91 & $\begin{array}{l}.5 \\
\end{array}$ & & .5 & \\
\hline
\end{tabular}




\section{Transect B Porewater Concentrations: Aluminum}

Sample ld. leotope

Concentration

(ugh)
Std.Doviation

(ugh)
Element Avg. Conc.

(ugl)
Element Avg. Std. Dov. (ug/)

\begin{tabular}{|c|c|c|c|c|c|}
\hline B11 & A & & & & \\
\hline $\mathrm{B} 12$ & $\overline{\mathbf{A}}$ & & & & \\
\hline B13 & $\mathbf{A}$ & 95200 & 839 & 95200 & 839 \\
\hline B14 & A & 14500 & 69.4 & 14500 & 69.4 \\
\hline$B 21$ & $\mathbf{A}$ & & & & \\
\hline$B 22$ & $\mathbf{A}$ & & & & \\
\hline$B 23$ & $\mathbf{A}$ & 15800 & 151 & 15800 & 151 \\
\hline B23R & $\mathbf{A}$ & 13000 & 176 & 13000 & 176 \\
\hline B24 & $\mathbf{A}$ & 28900 & 142 & 28900 & 142 \\
\hline 831 & $\mathbf{A}$ & & & & \\
\hline $\mathrm{B} 32$ & $\mathbf{N}$ & 612 & 5.61 & 612 & 5.61 \\
\hline B33 & $\mathbf{A}$ & 775 & 8.08 & 775 & 8.08 \\
\hline B41 & $\mathrm{A}$ & & & & \\
\hline B42 & $\mathbf{N}$ & 4100 & 402 & 4100 & 40.2 \\
\hline$B 43$ & A & 15400 & 190 & 15400 & 190 \\
\hline B45V & $\mathbf{A}$ & 25 & & 25 & \\
\hline $\mathrm{B} 51$ & $\mathbf{A}$ & 3310 & 47.5 & 3310 & 47.5 \\
\hline B52 & $\mathbf{A}$ & 6640 & 55.3 & 6640 & 55.3 \\
\hline B52R & $\mathbf{A}$ & 777 & 12.1 & $\pi$ & 12.1 \\
\hline
\end{tabular}

Note: The actual concentration of eamples for which no standerd deviation is reported b leas than the lower limit of detection. The reported concentration is the lower llmit of quantfication. 


\section{Transect B Porewater Concentrations: Arsenic}

\begin{tabular}{|c|c|c|c|}
\hline Sample ld. & leotope & $\begin{array}{l}\text { Concentration } \\
\text { (ug/ })\end{array}$ & $\begin{array}{l}\text { Std.Doviation } \\
\text { (ugh) }\end{array}$ \\
\hline
\end{tabular}

\begin{tabular}{|c|c|c|c|c|c|}
\hline 811 & As & & & & \\
\hline$B 12$ & $A_{3}$ & & & & \\
\hline$B 13$ & As & 75 & & 75 & \\
\hline B14 & $A_{s}$ & 75 & & 75 & \\
\hline$B 21$ & As & & & & \\
\hline 822 & $A_{s}$ & & & & \\
\hline$B 23$ & As & 38 & & 38 & \\
\hline$B 23 R$ & As & 8.69 & 18.7 & 8.69 & 18.7 \\
\hline$B 24$ & As & 7.67 & 1.35 & 7.67 & 1.35 \\
\hline$B 31$ & As & & & & \\
\hline B32 & As & 45 & & 45 & \\
\hline 833 & As & 12 & 5.04 & 12 & 5.04 \\
\hline$B 41$ & As & & & & \\
\hline$B 42$ & As & 75 & & 75 & \\
\hline$B 43$ & As & 75 & & 75 & \\
\hline$B 45 V$ & As & 75 & & 75 & \\
\hline $\mathrm{B} 51$ & As & 1 & & 1 & \\
\hline$B 52$ & As & 1.43 & 1.7 & 1.43 & 1.7 \\
\hline B52R & As & 1 & & 1 & \\
\hline
\end{tabular}




\section{Transect B Porewater Concentrations: Barium}

\begin{tabular}{ccccc}
\hline Sample ld. leotope & $\begin{array}{c}\text { Concentration } \\
(u g /)\end{array}$ & $\begin{array}{c}\text { Std.Devlation } \\
\text { (ug/L) }\end{array}$ & $\begin{array}{c}\text { Element Avg. Conc. } \\
(u g /)\end{array}$ & $\begin{array}{c}\text { Element Avg. Std. Dev. } \\
(u g /)\end{array}$
\end{tabular}

\begin{tabular}{|c|c|c|c|c|c|}
\hline B11 & $8 a 135$ & & & & \\
\hline B11 & Ba137 & & & & \\
\hline$B 11$ & Ba138 & & & & \\
\hline$B 12$ & Ba135 & & & & \\
\hline B12 & Ba137 & & & & \\
\hline$B 12$ & Ba138 & & & & \\
\hline$B 13$ & Ba135 & 654 & 13 & & \\
\hline$B 13$ & $8 a 137$ & 665 & 7.31 & & \\
\hline$B 13$ & Ba138 & 677 & 12.3 & 665 & 10.9 \\
\hline 814 & Ba135 & 162 & 5.78 & & \\
\hline$B 14$ & Ba137 & 165 & 3.42 & & \\
\hline$B 14$ & Ba138 & 166 & 2.74 & 164 & 3.98 \\
\hline$B 21$ & Ba135 & & & & \\
\hline 821 & Ba137 & & & & \\
\hline$B 21$ & Ba138 & & & & \\
\hline$B 22$ & Ba135 & & & & \\
\hline 822 & Ba137 & & & & \\
\hline 822 & Ba138 & & & & \\
\hline 823 & Ba135 & 229 & 2.89 & & \\
\hline$B 23$ & Ba137 & 217 & 3.55 & & \\
\hline$B 23$ & Ba138 & 221 & 1.75 & 2222 & 2.73 \\
\hline B23A & Ba135 & 245 & 11.1 & & \\
\hline B23A & Ba137 & 245 & 9.78 & & \\
\hline B23R & Ba138 & 237 & 2.8 & 242 & 7.89 \\
\hline 824 & Ba135 & 141 & 3.01 & & \\
\hline$B 24$ & Ba137 & 145 & 3.51 & & \\
\hline$B 24$ & Ba138 & 147 & 1.94 & 144 & 2.82 \\
\hline $\mathrm{B} 31$ & Ba135 & & & & \\
\hline$B 31$ & Ba137 & & & & \\
\hline 831 & Ba138 & & & & \\
\hline 832 & Ba135 & 36.6 & 2.76 & & \\
\hline$B 32$ & Ba137 & 392 & 1.76 & & \\
\hline 832 & Ba138 & 40 & .54 & 38.6 & 1.69 \\
\hline 833 & Ba135 & 59.5 & .52 & & \\
\hline 833 & Ba137 & 552 & 2.9 & & \\
\hline B33 & Ba138 & 58.1 & .65 & 57.6 & 1.36 \\
\hline$B 41$ & Ba135 & & & & \\
\hline$B 41$ & Ba137 & & & & \\
\hline 841 & Ba138 & & & & \\
\hline B42 & Ba135 & 59.5 & 1.57 & & \\
\hline B42 & Ba137 & 63 & 5.04 & & \\
\hline$B 42$ & Ba138 & 60.4 & .37 & 61 & 2.33 \\
\hline$B 43$ & Ba135 & 166 & 7.56 & & \\
\hline 843 & Ba137 & 161 & 5.93 & & \\
\hline
\end{tabular}

iNote: The sctual concentration of eamples for which no standard doviation ls reported ls lees than the lower limit of detection. The reported concentration la the lower limit of quantilicatton. 


\section{Transect B Porewater Concentrations: Barium}

\begin{tabular}{|c|c|c|c|c|c|}
\hline Sampie Id. & Isotope & $\begin{array}{l}\text { Concentration } \\
\text { (ugh) }\end{array}$ & $\begin{array}{l}\text { Std.Devlation } \\
\text { (Ugl) }\end{array}$ & $\begin{array}{l}\text { Element Avg. Conc. } \\
(u g L)\end{array}$ & $\begin{array}{c}\text { Element Avg. Std. Dov. } \\
\text { (Ug } \Omega \text { ) }\end{array}$ \\
\hline$B 43$ & Ba138 & 165 & .19 & 164 & 4.56 \\
\hline B45V & Ba135 & 33.6 & 1.41 & & \\
\hline B45V & Ba137 & 32.6 & 1.33 & & \\
\hline $845 \mathrm{~V}$ & Ba138 & 31.3 & .91 & 32.5 & 122 \\
\hline $\mathrm{B} 51$ & Ba135 & 148 & 9.59 & & \\
\hline $\mathrm{B} 51$ & Ba137 & 156 & 4.68 & & \\
\hline 851 & Ba138 & 153 & 2.21 & 152 & 5.49 \\
\hline B52 & Ba135 & 261 & 2.59 & & \\
\hline $\mathrm{B} 52$ & Ba137 & 249 & 4.79 & & \\
\hline 852 & Ba138 & 237 & 3.1 & 249 & 3.49 \\
\hline B52R & Ba135 & 104 & 10 & & \\
\hline$B 52 R$ & Ba137 & 116 & 4.87 & & \\
\hline B52R & Ba138 & 117 & 129 & 112 & 5.39 \\
\hline
\end{tabular}




\section{Transect B Porewater Concentrations: Cadmium}

\begin{tabular}{|c|c|c|c|c|c|}
\hline Sample id. & Isotope & $\begin{array}{l}\text { Concentration } \\
\text { (ugh) }\end{array}$ & $\begin{array}{l}\text { Std.Dovlation } \\
\text { (ugh) }\end{array}$ & $\begin{array}{l}\text { Element Avg. Conc. } \\
\text { (ug/ })\end{array}$ & $\begin{array}{c}\text { Element Avg. Std. Dov. } \\
\text { (ug })\end{array}$ \\
\hline
\end{tabular}

\begin{tabular}{|c|c|c|c|c|c|}
\hline B11 & Cod111 & & & & \\
\hline$B 11$ & $\operatorname{col} 114$ & & & & \\
\hline B12 & $\operatorname{Cod111}$ & & & & \\
\hline B12 & $\operatorname{Cod} 114$ & & & & \\
\hline B13 & $\operatorname{Cod} 111$ & 11.9 & .5 & & \\
\hline$B 13$ & Cd114 & 12.5 & .89 & 12.2 & .7 \\
\hline B14 & $\operatorname{cod} 111$ & 5.66 & 1.36 & & \\
\hline B14 & Cod114 & 6.05 & 25 & 5.86 & .81 \\
\hline B21 & Cd111 & & & & \\
\hline B21 & Col114 & & & & \\
\hline $\mathrm{B} 22$ & Cod111 & & & & \\
\hline$B 22$ & Cod114 & & & & \\
\hline $\mathrm{B} 23$ & Cd111 & 7.78 & .35 & & \\
\hline $\mathrm{B} 23$ & Cd114 & 7.17 & .66 & 7.48 & .51 \\
\hline B23R & Cd111 & 5.7 & 125 & & \\
\hline B23R & Cod114 & 7.07 & 1.16 & 6.39 & 121 \\
\hline 824 & Cod111 & 6.07 & 1.46 & & \\
\hline$B 24$ & $\operatorname{col} 114$ & 4.44 & .7 & 5.26 & 1.08 \\
\hline B31 & Cod111 & & & & \\
\hline B31 & $\operatorname{Cod} 114$ & & & & \\
\hline B32 & Cd111 & 2.4 & .06 & & \\
\hline B32 & $\operatorname{cot114}$ & 1.53 & 22 & 1.97 & .14 \\
\hline $\mathrm{B} 33$ & Cod11 & .91 & 26 & & \\
\hline $\mathrm{B} 33$ & $\operatorname{Cod} 114$ & .56 & .1 & .74 & .18 \\
\hline$B 41$ & $\operatorname{Cd} 111$ & & & & \\
\hline B41 & $\operatorname{Cod114}$ & & & & \\
\hline$B 42$ & Cod111 & 1.64 & .14 & & \\
\hline$B 42$ & $\operatorname{Cd} 114$ & 124 & .43 & 1.44 & 29 \\
\hline$B 43$ & Cod111 & 1.4 & 26 & & \\
\hline 843 & $\operatorname{Cd114}$ & .98 & .06 & 1.18 & .16 \\
\hline$B 45 \mathrm{~V}$ & Cd111 & .75 & & & \\
\hline $\mathrm{B} 45 \mathrm{~V}$ & $\operatorname{cod} 114$ & .75 & & .75 & \\
\hline 851 & Cod111 & 4.08 & $\infty$ & & \\
\hline 851 & $\operatorname{cod} 114$ & 4.1 & .36 & 4.09 & 22 \\
\hline$B 52$ & Cd111 & 2.03 & .76 & & \\
\hline $\mathrm{B} 52$ & Cd114 & 2.07 & .11 & 2.05 & .44 \\
\hline B52R & Cod111 & 1.4 & .57 & & \\
\hline B52R & Cd114 & 1.09 & .47 & 125 & .52 \\
\hline
\end{tabular}




\begin{tabular}{|c|c|c|c|c|c|}
\hline Sample ld. & Eotope & $\begin{array}{l}\text { Concentration } \\
\text { (ugh) }\end{array}$ & $\begin{array}{l}\text { Sid.Doviation } \\
\text { (ugh) }\end{array}$ & $\begin{array}{l}\text { Element Avg. Conc. } \\
\text { (ugh) }\end{array}$ & $\begin{array}{c}\text { Element Avg. Std. Dov. } \\
\text { (ug/L) }\end{array}$ \\
\hline B11 & $\mathrm{Ce} 44$ & & & & \\
\hline$B 12$ & $\cos 4$ & & & & \\
\hline 813 & $\operatorname{Cos} 4$ & 5850 & 97.9 & 5850 & 97.9 \\
\hline B14 & Co44 & 3650 & 21.6 & 3650 & 21.6 \\
\hline B21 & Ca44 & & & & \\
\hline$B 22$ & Ces4 & & & & \\
\hline$B 23$ & $\operatorname{cos4}$ & 4180 & 47.3 & 4180 & 47.3 \\
\hline B23R & $\operatorname{Co} 44$ & 1490 & 214 & 1490 & 214 \\
\hline$B 24$ & $\mathrm{Ca44}$ & 4150 & 2.9 & 4150 & 2.9 \\
\hline B31 & Ca44 & & & & \\
\hline B32 & $\mathrm{Ca} 44$ & 889 & 2.33 & 889 & 2.33 \\
\hline B33 & Ca44 & 2020 & 31.5 & 2020 & 31.5 \\
\hline B41 & $\operatorname{Ca} 44$ & & & & \\
\hline 842 & Co44 & 1600 & 7.02 & 1680 & 7.02 \\
\hline$B 43$ & Ca44 & 1550 & 52 & 1550 & 52 \\
\hline B45V & Ca44 & 23200 & 17 & 23200 & 177 \\
\hline $\mathrm{B} 51$ & Ca44 & 1210 & 70.4 & 1210 & 70.4 \\
\hline $\mathrm{B} 52$ & $\mathrm{Ca} 44$ & 2470 & 101 & 2470 & 101 \\
\hline $\mathrm{B} 52 \mathrm{~A}$ & $\mathrm{Ca} 44$ & 500 & & 500 & \\
\hline
\end{tabular}

Note: The actual concentration of eamples for which no stunderd deviation be reported is laes than the lower llmit of dotection. The reported concentration is the lower kmit of quantification. 


\section{Transect B Porewater Concentrations: Cesium}

\begin{tabular}{|c|c|c|c|c|c|}
\hline Sample ld. & leotope & $\begin{array}{l}\text { Concentration } \\
\text { (ugh) }\end{array}$ & $\begin{array}{l}\text { Std.Deviation } \\
\text { (ugL) }\end{array}$ & $\begin{array}{c}\text { Element Avg. Conc. } \\
(u g / L)\end{array}$ & $\begin{array}{c}\text { Element Avg. Std. Dov. } \\
\text { (ugh) }\end{array}$ \\
\hline B11 & Cs & & & & \\
\hline $\mathrm{B} 12$ & Cs & & & & \\
\hline $\mathrm{B} 13$ & Cs & 1.67 & .08 & 1.67 & .08 \\
\hline 814 & Cs & 26 & .03 & 26 & .03 \\
\hline B21 & Cs & & & & \\
\hline 822 & Cs & & & & \\
\hline B23 & Cs & .07 & .01 & .07 & .01 \\
\hline B23R & Cs & 28 & 21 & 28 & 21 \\
\hline $\mathrm{B} 24$ & Cs & .11 & .06 & .11 & .06 \\
\hline 831 & Cs & & & & \\
\hline 832 & Cs & 3 & & 3 & \\
\hline $\mathrm{B} 33$ & Cs & .11 & .05 & .11 & .05 \\
\hline$B 41$ & Cs & & & & \\
\hline 842 & Cs & & & & \\
\hline$B 43$ & Cs & .11 & .05 & .11 & .05 \\
\hline B45V & Cs & 5 & & 5 & \\
\hline $\mathrm{B51}$ & $\mathrm{C}_{8}$ & .58 & .18 & .58 & .18 \\
\hline B52 & Cs & 2 & .01 & 2 & .01 \\
\hline B52R & Cs & 25 & & 25 & \\
\hline
\end{tabular}




\section{Transect B Porewater Concentrations: Chromium}

\begin{tabular}{|c|c|c|c|c|c|}
\hline Sample Id. & Isotope & $\begin{array}{l}\text { Concentration } \\
\text { (ugh) }\end{array}$ & $\begin{array}{l}\text { Std.Dovlation } \\
\text { (ug/L) }\end{array}$ & $\begin{array}{l}\text { Element Avg. Conc. } \\
\text { (ug/L) }\end{array}$ & $\begin{array}{c}\text { Element Avg. Std. Dov. } \\
\text { (ug/L) }\end{array}$ \\
\hline 811 & Cr52 & & & & \\
\hline B11 & Cr53 & & & & \\
\hline $\mathrm{B} 12$ & $\mathrm{Cr} 52$ & & & & \\
\hline $\mathrm{B} 12$ & $\mathrm{C} 5 \mathrm{53}$ & & & & \\
\hline B13 & $\mathrm{Cr} 52$ & 8.92 &.$\infty$ & & \\
\hline$B 13$ & Cr53 & 8.91 &.$\infty$ & 8.91 & .09 \\
\hline$B 14$ & Cr52 & 925 & .46 & & \\
\hline B14 & Cr53 & 924 & .46 & 925 & .46 \\
\hline $\mathrm{B} 21$ & C.52 & & & & \\
\hline $\mathrm{B} 21$ & Cr53 & & & & \\
\hline $\mathrm{B} 22$ & Cr52 & & & & \\
\hline $\mathrm{B} 22$ & $\mathrm{Cr} 53$ & & & & \\
\hline $\mathrm{B} 23$ & Cr52 & 5.41 & .15 & & \\
\hline $\mathrm{B23}$ & $\mathrm{C.53}$ & 5.41 & .15 & 5.41 & .15 \\
\hline B23R & Cr52 & 5.76 & .88 & & \\
\hline B23R & $\mathrm{Cr} 53$ & 5.77 & .88 & 5.77 & .88 \\
\hline$B 24$ & Cr52 & 8.9 & .84 & & \\
\hline $\mathrm{B} 24$ & C.53 & 8.89 & .84 & 8.9 & .84 \\
\hline B31 & Cr52 & & & & \\
\hline B31 & Cr53 & & & & \\
\hline $\mathrm{B} 32$ & Cr52 & 2.5 & .3 & & \\
\hline$B 32$ & $\mathrm{Cr53}$ & 2.5 & .3 & 2.5 & .3 \\
\hline $\mathrm{B} 33$ & Cr52 & 2.74 & .4 & & \\
\hline $\mathrm{B} 33$ & Cr53 & 2.73 & .4 & 2.74 & .4 \\
\hline B41 & C.52 & & & & \\
\hline$B 41$ & $\mathrm{C} 553$ & & & & \\
\hline $\mathrm{B} 42$ & Cr52 & 2.8 & 24 & & \\
\hline 842 & $\mathrm{Cr} 53$ & 2.8 & 24 & 2.8 & 24 \\
\hline$B 43$ & Cr52 & 10.4 & 1.24 & & \\
\hline 843 & $\mathrm{Cr} 53$ & 10.4 & 124 & 10.4 & 124 \\
\hline $\mathrm{B} 45 \mathrm{~V}$ & $\mathrm{C} 552$ & 1.41 & 27 & & \\
\hline $\mathrm{B} 45 \mathrm{~V}$ & $\mathrm{Cr} 53$ & 1.41 & 27 & 1.41 & 27 \\
\hline 851 & C.52 & 2.3 & .16 & & \\
\hline 851 & $\mathrm{C} 53$ & 2.3 & .16 & 2.3 & .16 \\
\hline 852 & Cr52 & 11.6 & .36 & & \\
\hline $\mathrm{B} 52$ & Cr53 & 11.6 & .36 & 11.6 & .36 \\
\hline B52R & Cr52 & 2.89 & .66 & & \\
\hline B52R & Cr53 & 2.89 & .66 & 2.89 & .66 \\
\hline
\end{tabular}

Note: The actual concentration of samples for whlch no standard doviation be reported be leas than the lower llmit of detection. The reported concentration is the lower limit of quantification. 


\section{Transect B Porewater Concentrations: Cobalt}

\begin{tabular}{|c|c|c|c|c|c|}
\hline Sample Id. & kotope & $\begin{array}{l}\text { Concentration } \\
\text { (ug/L) }\end{array}$ & $\begin{array}{l}\text { Std.Deviation } \\
\text { (ug/L) }\end{array}$ & $\begin{array}{l}\text { Element Avg. Conc. } \\
\text { (ugl) }\end{array}$ & $\begin{array}{c}\text { Element Avg. Std. Dev. } \\
\text { (ugh) }\end{array}$ \\
\hline B11 & $\infty$ & & & & \\
\hline 812 & $C_{0}$ & & & & \\
\hline$B 13$ & Co & 16.7 & 26 & 16.7 & 26 \\
\hline B14 & $C_{0}$ & 22.3 & .35 & 22.3 & .35 \\
\hline$B 21$ & Co & & & & \\
\hline$B 22$ & $C_{0}$ & & & & \\
\hline 823 & Co & 15.5 & .31 & 15.5 & .31 \\
\hline$B 23 R$ & Co & 15.6 & .34 & 15.6 & .34 \\
\hline $\mathrm{B} 24$ & Co & 19.3 & .88 & 19.3 & .88 \\
\hline$B 31$ & Co & & & & \\
\hline 832 & Co & 2.32 & .15 & 2.32 & .15 \\
\hline $\mathrm{B} 33$ & $C_{0}$ & 7.31 & .32 & 7.31 & .32 \\
\hline$B 41$ & Co & & & & \\
\hline$B 42$ & Co & 4.14 & 26 & 4.14 & 26 \\
\hline 843 & $C_{0}$ & 20 & .75 & 20 & .75 \\
\hline$B 45 V$ & $C_{0}$ & .79 & .06 & .79 & .06 \\
\hline 851 & $C_{0}$ & 26.9 & .41 & 26.9 & .41 \\
\hline 852 & $C_{0}$ & 164 & 2.24 & 164 & 2.24 \\
\hline B52R & Co & 14.5 & .73 & 14.5 & .73 \\
\hline
\end{tabular}

Note: The sctual concentration of eamples for which no standard deviation be reported bs laes than the lower limit of detection. The reported concentration is the lower IImit of quantification. 


\begin{tabular}{|c|c|c|c|c|c|}
\hline \multicolumn{6}{|c|}{ Transect B Porewater Concentrations: Copper } \\
\hline Sample id. & leotope & $\begin{array}{l}\text { Concentration } \\
\text { (ugh) }\end{array}$ & $\begin{array}{l}\text { Sto.Doviation } \\
\text { (Ug/) }\end{array}$ & $\begin{array}{l}\text { Element Avg. Conc. } \\
\text { (Ugh) }\end{array}$ & $\begin{array}{l}\text { Element Avg. Std. Dov. } \\
\text { (ugh) }\end{array}$ \\
\hline B11 & Cues & & & & \\
\hline$B 11$ & Cu65 & & & & \\
\hline B12 & Cuts & & & & \\
\hline B12 & Cu65 & & & & \\
\hline B13 & $c a 63$ & 41.8 & 125 & & \\
\hline B13 & Cuts & 42.7 & .98 & 42.3 & 1.12 \\
\hline B14 & Cu63 & 14.6 & .51 & & \\
\hline B14 & Cut5 & 14.8 & .8 & 14.7 & .66 \\
\hline$B 21$ & cuts3 & & & & \\
\hline B21 & Cu65 & & & & \\
\hline$B 22$ & Cubs & & & & \\
\hline$B 22$ & 0.65 & & & & \\
\hline B23 & Cu63 & 54 & 1.17 & & \\
\hline$B 23$ & Cu65 & 52.8 & 1.02 & 53.4 & 1.1 \\
\hline$B 23 R$ & Cu63 & & & & \\
\hline B23R & Cu65 & 84.2 & 5.98 & 84.2 & 5.98 \\
\hline B24 & Cu63 & 34.6 & .67 & & \\
\hline$B 24$ & Cu65 & 31.5 & .8 & 33.1 & .74 \\
\hline 831 & Cu63 & & & & \\
\hline 831 & Cu65 & & & & \\
\hline 832 & Cu63 & 7.82 & .38 & & \\
\hline $\mathrm{B} 32$ & Cu65 & 6.85 & .42 & 7.34 & .4 \\
\hline$B 33$ & Cu63 & 3.75 & & & \\
\hline$B 33$ & Cu65 & 3.75 & & 3.75 & \\
\hline B41 & Cu63 & & & & \\
\hline B41 & Cu65 & & & & \\
\hline 842 & Cu63 & 5.82 & .47 & & \\
\hline 842 & Cu65 & 6.16 & .57 & 5.99 & .52 \\
\hline$B 43$ & Cu63 & 22.4 & .66 & & \\
\hline 843 & Cu65 & 23.4 & 1.64 & 22.9 & 1.15 \\
\hline B45V & Cu63 & 2.5 & & & \\
\hline B45V & Cu65 & 2.5 & & 2.5 & \\
\hline B51 & Cu63 & 28.7 & 1.13 & & \\
\hline B51 & Cu65 & 29.8 & 2.6 & 29.3 & 1.87 \\
\hline $\mathrm{B} 52$ & Cu63 & & & & \\
\hline$B 52$ & Cu65 & 29.1 & 1.78 & 29.1 & 1.78 \\
\hline B52R & Cu63 & & & & \\
\hline B52R & Cu65 & 29 & 2.52 & 20 & 2.52 \\
\hline
\end{tabular}




\section{Transect B Porewater Concentrations: Iron}

\begin{tabular}{|c|c|c|c|c|c|}
\hline Sample Id. & leotope & $\begin{array}{l}\text { Concentration } \\
\text { (ug/L) }\end{array}$ & $\begin{array}{l}\text { Std.Dovlation } \\
\text { (ugh) }\end{array}$ & $\begin{array}{l}\text { Element Avg. Conc. } \\
\text { (ugh) }\end{array}$ & $\begin{array}{l}\text { Element Avg. Std. Dov. } \\
\text { (ugh) }\end{array}$ \\
\hline B11 & Fo & & & & \\
\hline$B 12$ & $F_{\theta}$ & & & & \\
\hline B13 & $F_{\theta}$ & 502 & 23.9 & 502 & 23.9 \\
\hline B14 & Fo & 2850 & 36.9 & 2850 & 36.9 \\
\hline B21 & $F_{0}$ & & & & \\
\hline$B 22$ & $F_{0}$ & & & & \\
\hline $\mathrm{B} 23$ & $\mathrm{Fe}$ & 734 & 1.18 & 734 & 1.18 \\
\hline B23R & Fo & 1460 & 22.1 & 1460 & 22.1 \\
\hline B24 & Fo & 4360 & 105 & 4360 & 105 \\
\hline B31 & $\mathrm{Fo}$ & & & & \\
\hline $\mathrm{B} 32$ & Fo & 569 & 18.8 & 560 & 18.8 \\
\hline $\mathrm{B} 33$ & $F_{\theta}$ & 16000 & 146 & 16000 & 146 \\
\hline$B 41$ & $F_{\theta}$ & & & & \\
\hline $\mathrm{B} 42$ & $F_{0}$ & 91.9 & 14.1 & 91.9 & 14.1 \\
\hline$B 43$ & $F_{0}$ & 2990 & 109 & 2990 & 109 \\
\hline $\mathrm{B} 45 \mathrm{~V}$ & $\mathrm{Fo}$ & 662 & 13.5 & 662 & 13.5 \\
\hline $\mathrm{B} 51$ & $\mathrm{Fo}$ & 25 & & 25 & \\
\hline $\mathrm{B} 52$ & $\mathrm{Fe}$ & 368 & 8.81 & 368 & 8.81 \\
\hline B52R & $F_{\theta}$ & 476 & 3.54 & 476 & 3.54 \\
\hline
\end{tabular}

Note: The actual concentration of eamples for which no standerd deviation ls reported is leas than the fower llmit of detection. The reported concentration is the lower limit of quantification. 


\section{Transect B Porewater Concentrations: Lead}

\begin{tabular}{|c|c|c|c|c|c|}
\hline Sample ld. & lsotope & $\begin{array}{l}\text { Concentration } \\
\text { (ug/) }\end{array}$ & $\begin{array}{l}\text { Std.Doviation } \\
\text { (ug/L) }\end{array}$ & $\begin{array}{l}\text { Element Avg. Conc. } \\
\text { (ug/) }\end{array}$ & $\begin{array}{c}\text { Element Avg. Std. Dov. } \\
\text { (ugh) }\end{array}$ \\
\hline
\end{tabular}

\begin{tabular}{|c|c|c|c|c|c|}
\hline B11 & Pb206 & & & & \\
\hline B11 & $\mathrm{Pb} 207$ & & & & \\
\hline B11 & Pb208 & & & & \\
\hline$B 12$ & Pb206 & & & & \\
\hline$B 12$ & Pb207 & & & & \\
\hline $\mathrm{B} 12$ & Pb208 & & & & \\
\hline $\mathrm{B} 13$ & Pb206 & 2.13 & .12 & & \\
\hline$B 13$ & $\mathrm{~Pb} 207$ & 2.13 & 26 & & \\
\hline$B 13$ & $\mathrm{~Pb} 208$ & 1.99 & 2 & 2.08 & .19 \\
\hline$B 14$ & Pb206 & 7.5 & & & \\
\hline B14 & $\mathrm{Pb} 207$ & 7.5 & & & \\
\hline B14 & Pb208 & 7.5 & & 7.5 & \\
\hline B21 & Pb206 & & & & \\
\hline B21 & $\mathrm{Pb} 207$ & & & & \\
\hline $\mathrm{B} 21$ & $\mathrm{~Pb} 208$ & & & & \\
\hline$B 22$ & Pb206 & & & & \\
\hline$B 22$ & $\mathrm{Pb207}$ & & & & \\
\hline $\mathrm{B} 22$ & $\mathrm{~Pb} 208$ & & & & \\
\hline 823 & Pb206 & 425 & & & \\
\hline $\mathrm{B} 23$ & $\mathrm{~Pb} 207$ & 4.25 & & & \\
\hline 823 & $\mathrm{~Pb} 208$ & 425 & & 4.25 & \\
\hline B23R & $\mathrm{Pb} 206$ & 3.65 & 1.05 & & \\
\hline B23R & $\mathrm{Pb} 207$ & 2.82 & 1.14 & & \\
\hline B23R & $\mathrm{Pb} 208$ & 2.14 & .35 & 2.87 & .85 \\
\hline $\mathrm{B} 24$ & $\mathrm{~Pb} 206$ & 85.1 & 4.87 & & \\
\hline$B 24$ & $\mathrm{~Pb} 207$ & 79.8 & 1.41 & & \\
\hline$B 24$ & $\mathrm{~Pb} 208$ & 78.2 & .77 & 81 & 2.35 \\
\hline B31 & $\mathrm{Pb} 206$ & & & & \\
\hline 831 & $\mathrm{~Pb} 207$ & & & & \\
\hline B31 & $\mathrm{Pb} 208$ & & & & \\
\hline $\mathrm{B} 32$ & $\mathrm{~Pb} 206$ & 3.2 & .08 & & \\
\hline B32 & $\mathrm{Pb} 207$ & 1.89 & .38 & & \\
\hline 832 & $\mathrm{~Pb} 208$ & 2.00 & 25 & 2.39 & 24 \\
\hline $\mathrm{B} 33$ & Pb206 & 11.3 & & & \\
\hline $\mathrm{B} 33$ & $\mathrm{~Pb} 207$ & 11.3 & & & \\
\hline 833 & Pb208 & 11.3 & & 11.3 & \\
\hline$B 41$ & $\mathrm{~Pb} 206$ & & & & \\
\hline B41 & $\mathrm{Pb} 207$ & & & & \\
\hline$B 41$ & $\mathrm{~Pb} 208$ & & & & \\
\hline 842 & $\mathrm{~Pb} 206$ & 7.5 & & & \\
\hline$B 42$ & $\mathrm{~Pb} 207$ & 7.5 & & & \\
\hline 842 & Pb208 & 7.5 & & 7.5 & \\
\hline 843 & Pb206 & 7.5 & & & \\
\hline 843 & $\mathrm{~Pb} 207$ & 7.5 & & & \\
\hline
\end{tabular}

Note: The actual concentration of samples for which no standard deviation le reported is leas than the lower limit of detection. The reported concentration is the lower limit of quantification. 


\section{Transect B Porewater Concentrations: Lead}

\begin{tabular}{|c|c|c|c|c|c|}
\hline Sample ld. & leotope & $\begin{array}{l}\text { Concentration } \\
\text { (ugh) }\end{array}$ & $\begin{array}{l}\text { Std.Doviation } \\
\text { (ug } / \text { ) }\end{array}$ & $\begin{array}{l}\text { Element Avg. Conc. } \\
\text { (ugR) }\end{array}$ & $\begin{array}{c}\text { Element Avg. Std. Dov. } \\
\text { (ugh) }\end{array}$ \\
\hline 843 & Pb208 & 7.5 & & 7.5 & \\
\hline$B 45 \mathrm{~V}$ & Pb206 & 7.5 & & & \\
\hline B45V & Pb207 & 7.5 & & & \\
\hline $845 \mathrm{~V}$ & Pb208 & 7.5 & & 7.5 & \\
\hline 851 & Pb206 & 1 & & & \\
\hline 851 & Pb207 & 1 & & & \\
\hline $\mathrm{B} 51$ & Pb208 & 1 & & 1 & \\
\hline 852 & Pb206 & 1 & & & \\
\hline 852 & Pb207 & 1 & & & \\
\hline 852 & $\mathrm{Pb208}$ & 1 & & 1 & \\
\hline B52R & Pb206 & 4.02 & .69 & & \\
\hline B52R & $\mathrm{Pb207}$ & 3.63 & .8 & & \\
\hline B52R & Pb208 & 4.3 & .46 & 3.98 & .65 \\
\hline
\end{tabular}




\section{Transect B Porewater Concentrations: Magnesium}

\begin{tabular}{|c|c|c|c|}
\hline Sample ld. & leotope & $\begin{array}{c}\text { Concentration } \\
\text { (ugh) }\end{array}$ & $\begin{array}{l}\text { Std.Doviation } \\
\text { (ugh) }\end{array}$ \\
\hline
\end{tabular}

\begin{tabular}{|c|c|c|c|c|c|}
\hline B11 & Mg24 & & & & \\
\hline B11 & $M 925$ & & & & \\
\hline B12 & Mgश4 & & & & \\
\hline$B 12$ & $M 925$ & & & & \\
\hline 813 & MgQ4 & 3760 & 31.5 & & \\
\hline B13 & $M 925$ & 3050 & 63.7 & 3410 & 47.6 \\
\hline$B 14$ & $M 924$ & 2630 & 39.9 & & \\
\hline 814 & $M 925$ & 2230 & 44 & 2430 & 42 \\
\hline B21 & Mg24 & & & & \\
\hline B21 & Mg25 & & & & \\
\hline$B 22$ & $M 924$ & & & & \\
\hline$B 22$ & Mg25 & & & & \\
\hline 823 & Mg24 & 2430 & 10.1 & & \\
\hline 823 & Mg25 & 2100 & 21.6 & 2270 & 15.9 \\
\hline B23R & $M g 24$ & 1050 & 14.2 & & \\
\hline B23A & Mg25 & 989 & 31.3 & 1020 & 22.8 \\
\hline $\mathrm{B} 24$ & $M 924$ & 2290 & 15.6 & & \\
\hline B24 & Mg25 & 1860 & 302 & 2080 & 22.9 \\
\hline B31 & $M_{924}$ & & & & \\
\hline 831 & $M 925$ & & & & \\
\hline 832 & Mg24 & 1910 & 5.93 & & \\
\hline $\mathrm{B} 32$ & $M g 25$ & 1430 & 15.3 & 1670 & 10.6 \\
\hline B33 & Mg24 & 768 & 2.56 & & \\
\hline B33 & Mges & 796 & 10.3 & 782 & 6.43 \\
\hline B41 & M924 & & & & \\
\hline$B 41$ & Mg25 & & & & \\
\hline$B 42$ & $M g 24$ & 694 & 11.1 & & \\
\hline B42 & Mg25 & 718 & 22.8 & 706 & 17 \\
\hline$B 43$ & $M g 24$ & 500 & 11.8 & & \\
\hline$B 43$ & Mg25 & 492 & 14.7 & 496 & 13.3 \\
\hline B45V & Mg24 & 736 & 14.9 & & \\
\hline B45V & M925 & 750 & 23.4 & 743 & 19.2 \\
\hline $\mathrm{B} 51$ & $M 924$ & 472 & 4.85 & & \\
\hline B51 & $M 925$ & 477 & 9.43 & 475 & 7.14 \\
\hline B52 & $M 924$ & 1160 & 17.1 & & \\
\hline $\mathrm{B} 52$ & $M 925$ & 1030 & 27.5 & 1100 & 22.3 \\
\hline$B 52 R$ & $M 924$ & 418 & 5.01 & & \\
\hline$B 52 R$ & Mg25 & 387 & 16.8 & 403 & 10.9 \\
\hline
\end{tabular}

Note: The actual concentration of eamples for which no standard deviation b reported is leas than the fower limit of detection. The reported concentration is the lower Imit of quantification. 


\begin{tabular}{|c|c|c|c|c|c|}
\hline Sample ld. & lsotope & $\begin{array}{l}\text { Concentration } \\
\text { (ug/L) }\end{array}$ & $\begin{array}{l}\text { Std.Devintion } \\
\text { (ugh) }\end{array}$ & $\begin{array}{l}\text { Element Avg. Conc. } \\
\text { (ug/) }\end{array}$ & $\begin{array}{l}\text { Element Avg. Std. Dev. } \\
\text { (ug/L) }\end{array}$ \\
\hline B11 & $\mathrm{Mn}$ & & & & \\
\hline $\mathrm{B} 12$ & $\mathrm{Mn}$ & & & & \\
\hline B13 & $\mathrm{Mn}$ & 3910 & 36.2 & 3910 & 362 \\
\hline$B 14$ & $M n$ & 3540 & 26.7 & 3540 & 26.7 \\
\hline 821 & $M n$ & & & & \\
\hline 822 & $M n$ & & & & \\
\hline$B 23$ & $M n$ & 941 & 4.46 & 941 & 4.46 \\
\hline B23R & $M n$ & 892 & 12 & 892 & 12 \\
\hline$B 24$ & $M n$ & 3940 & 38.1 & 3940 & 38.1 \\
\hline B31 & $M n$ & & & & \\
\hline$B 32$ & $\mathrm{Mn}$ & 128 & 1.75 & 128 & 1.75 \\
\hline 833 & $M n$ & 453 & 3.71 & 453 & 3.71 \\
\hline B41 & $M n$ & & & & \\
\hline$B 42$ & $M n$ & 150 & 1.61 & 159 & 1.61 \\
\hline$B 43$ & $M n$ & 2270 & 16.8 & 2270 & 16.8 \\
\hline $\mathrm{B} 45 \mathrm{~V}$ & $\mathrm{Mn}$ & 74 & 1.51 & 74 & 1.51 \\
\hline B51 & $M n$ & 504 & .51 & 504 & .51 \\
\hline $\mathrm{B} 52$ & $\mathrm{Mn}$ & 1990 & 2.81 & 1990 & 2.81 \\
\hline B52R & $\mathrm{Mn}$ & 314 & 3.42 & 344 & 3.42 \\
\hline
\end{tabular}




\begin{tabular}{|c|c|c|c|c|c|}
\hline Sample Id. & leotope & $\begin{array}{l}\text { Concentration } \\
\text { (ug/L) }\end{array}$ & $\begin{array}{l}\text { Sid.Deviation } \\
\text { (ugh) }\end{array}$ & $\begin{array}{c}\text { Element Avg. Conc. } \\
\text { (ugh) }\end{array}$ & $\begin{array}{c}\text { Element Avg. Std. Dev. } \\
\text { (ugh) }\end{array}$ \\
\hline 811 & $\mathrm{Ni} 6 \mathrm{O}$ & & & & \\
\hline 811 & Ni62 & & & & \\
\hline $\mathrm{B} 12$ & Ni60 & & & & \\
\hline $\mathrm{B} 12$ & $\mathrm{Ni62}$ & & & & \\
\hline B13 & Ni60 & 99.8 & 3.56 & & \\
\hline $\mathbf{B 1 3}$ & Ni62 & 111 & 3.92 & 105 & 3.74 \\
\hline 814 & $\mathrm{Ni} 60$ & 141 & 4.86 & & \\
\hline B14 & $\mathrm{Ni62}$ & 139 & 9.4 & 140 & 7.13 \\
\hline $\mathrm{B} 21$ & $\mathrm{Ni} 60$ & & & & \\
\hline 821 & Ni62 & & & & \\
\hline 822 & $\mathrm{Ni} 60$ & & & & \\
\hline B22 & Ni62 & & & & \\
\hline$B 23$ & Ni60 & 141 & 2.06 & & \\
\hline 823 & Ni62 & 151 & 928 & 146 & 5.67 \\
\hline $\mathrm{B} 23 \mathrm{P}$ & $\mathrm{Ni} 60$ & 432 & 3.16 & & \\
\hline B23R & Ni62 & 30 & 5.6 & 36.6 & 4.38 \\
\hline 824 & Ni6o & 131 & 2.61 & & \\
\hline 824 & Ni62 & 130 & 6.17 & 131 & 4.39 \\
\hline 831 & Ni6o & & & & \\
\hline 831 & Ni62 & & & & \\
\hline $\mathrm{B} 32$ & $\mathrm{Ni} 60$ & 42.4 & 1.75 & & \\
\hline B32 & Ni62 & 40.4 & .63 & 41.4 & 1.19 \\
\hline $\mathrm{B} 33$ & $\mathrm{Ni} 60$ & 119 & 3.34 & & \\
\hline $\mathrm{B} 33$ & Ni62 & 134 & 3.65 & 127 & 3.5 \\
\hline$B 41$ & $\mathrm{Ni} 60$ & & & & \\
\hline 341 & Ni62 & & & & \\
\hline$B 42$ & $\mathrm{Ni} 6 \mathrm{O}$ & 101 & 3.54 & & \\
\hline$B 42$ & Ni62 & 111 & 3.56 & 105 & 3.55 \\
\hline 843 & $\mathrm{Ni60}$ & 181 & .95 & & \\
\hline $\mathrm{B} 43$ & Ni62 & 191 & 18.5 & 186 & 9.73 \\
\hline $\mathrm{B} 45 \mathrm{~V}$ & $\mathrm{Ni} 60$ & 9.19 & 1.1 & & \\
\hline B45V & Ni62 & 92 & 4.98 & 92 & 3.04 \\
\hline $\mathrm{B} 51$ & $\mathrm{Ni} 60$ & 44.7 & 1.67 & & \\
\hline $\mathrm{B} 51$ & Ni62 & 42.1 & 4.96 & 43.4 & 3.32 \\
\hline 852 & Ni60 & 195 & 2.6 & & \\
\hline 852 & Ni62 & 192 & 3.35 & 194 & 2.98 \\
\hline $\mathrm{B} 52 \mathrm{R}$ & $\mathrm{Ni60}$ & 94.5 & 2.88 & & \\
\hline B52A & Ni62 & 81.4 & 16.3 & 88 & 9.59 \\
\hline
\end{tabular}

Note: The actual concentration of eamples for which no standard deviation bs reported lo hees than the lower limit of detection. The reported concentration is the lower limit of quantification. 


\section{Transect B Porewater Concentrations: Selenium}

\begin{tabular}{|c|c|c|c|c|c|}
\hline Sample ld. & leotope & $\begin{array}{l}\text { Concentration } \\
\text { (ug/ } 2)\end{array}$ & $\begin{array}{l}\text { Std.Doviation } \\
\text { (ug/ })\end{array}$ & $\begin{array}{l}\text { Element Avg Conc. } \\
\text { (Ugh) }\end{array}$ & $\begin{array}{l}\text { Element Avg. Std. Dov. } \\
\text { (ugh) }\end{array}$ \\
\hline$B 11$ & 5077 & & & & \\
\hline B11 & So82 & & & & \\
\hline 812 & so77 & & & & \\
\hline $\mathrm{B} 12$ & 5082 & & & & \\
\hline$B 13$ & S०77 & 10.8 & 5.93 & & \\
\hline$B 13$ & 5082 & 10.7 & 5.98 & 10.6 & 5.96 \\
\hline B14 & So77 & 3.89 & 8.73 & & \\
\hline 814 & So82 & 3.92 & 8.92 & 3.91 & 8.82 \\
\hline$B 21$ & $\mathrm{~s} \oplus 77$ & & & & \\
\hline 821 & So82 & & & & \\
\hline$B 22$ & 5077 & & & & \\
\hline 822 & 5082 & & & & \\
\hline $\mathrm{B} 23$ & So77 & 37.5 & & & \\
\hline 823 & So82 & 31.3 & & 34.4 & \\
\hline B23R & 5077 & 25 & & & \\
\hline$B 23 A$ & So82 & 62.5 & & 43.8 & \\
\hline 824 & $\mathrm{Se} 77$ & 16.6 & 3.97 & & \\
\hline B24 & 5082 & 16.7 & 3.91 & 16.7 & 3.94 \\
\hline 831 & Se7 & & & & \\
\hline B31 & So82 & & & & \\
\hline B32 & So77 & 22.5 & & & \\
\hline $\mathrm{B} 32$ & Soe2 & 22.5 & & 22.5 & \\
\hline $\mathrm{B} 33$ & So77 & 22.5 & & & \\
\hline $\mathrm{B} 33$ & So82 & 22.5 & & 22.5 & \\
\hline$B 41$ & So 77 & & & & \\
\hline$B 41$ & So82 & & & & \\
\hline 842 & So77 & 37.5 & & & \\
\hline$B 42$ & So82 & 37.5 & & 37.5 & \\
\hline$B 43$ & SoT & 7.89 & 8.69 & & \\
\hline 843 & 5082 & 7.98 & 8.65 & 7.94 & 8.67 \\
\hline $845 \mathrm{~V}$ & Se77 & 37.5 & & & \\
\hline $845 \mathrm{~V}$ & So82 & 37.5 & & 37.5 & \\
\hline $\mathrm{B} 51$ & So77 & 25 & & & \\
\hline 851 & So82 & 25 & & 25 & \\
\hline $\mathrm{B} 52$ & So77 & 25 & & & \\
\hline $\mathrm{B} 52$ & So82 & 25 & & 25 & \\
\hline $\mathrm{B} 52 \mathrm{R}$ & $\mathrm{SeT}$ & 25 & & & \\
\hline B52R & So82 & 25 & & 25 & \\
\hline
\end{tabular}

Note: The ectual concentratton of samples for which no standerd doviatton te reported be has than the lower limit of dotection. The reported concentration is the lower Hmit of quantification. 
Transect B Porewater Concentrations: Silver

\begin{tabular}{|c|c|c|c|c|c|}
\hline Sample id. & Isotope & $\begin{array}{l}\text { Concentration } \\
\text { (ug/L) }\end{array}$ & $\begin{array}{l}\text { Std.Doviation } \\
\text { (ug/) }\end{array}$ & $\begin{array}{l}\text { Element Avg. Conc. } \\
\text { (ug/ })\end{array}$ & $\begin{array}{c}\text { Element Avg. Std. Dav. } \\
\text { (ug/ })\end{array}$ \\
\hline B11 & Ag107 & & & & \\
\hline$B 11$ & Ag109 & & & & \\
\hline$B 12$ & Ag107 & & & & \\
\hline B12 & Ag100 & & & & \\
\hline B13 & Ag107 & 15 & & & \\
\hline$B 13$ & A9109 & 15 & & 15 & \\
\hline B14 & Ag107 & 15 & & & \\
\hline B14 & Ag109 & 15 & & 15 & \\
\hline B21 & Ag107 & & & & \\
\hline$B 21$ & Ag100 & & & & \\
\hline$B 22$ & Ag107 & & & & \\
\hline$B 22$ & Ag109 & & & & \\
\hline B23 & Ag107 & 10 & & & \\
\hline B23 & Ag109 & 10 & & 10 & \\
\hline B23R & Ag107 & 40.1 & 10.1 & & \\
\hline B23R & Ag109 & 17.3 & 6.89 & 28.7 & 8.49 \\
\hline$B 24$ & Ag107 & 15 & & & \\
\hline 824 & Ag109 & 15 & & 15 & \\
\hline 831 & Ag107 & & & & \\
\hline 831 & Ag109 & & & & \\
\hline 832 & Ag107 & 825 & & & \\
\hline 832 & Ag109 & 8.25 & & 8.25 & \\
\hline B33 & Ag107 & & & & \\
\hline B33 & Ag109 & & & & \\
\hline$B 41$ & Ag107 & & & & \\
\hline B41 & Ag109 & & & & \\
\hline$B 42$ & Ag107 & & & & \\
\hline$B 42$ & Ag109 & & & & \\
\hline 843 & Ag107 & 15 & & & \\
\hline 843 & Ag109 & 15 & & 15 & \\
\hline B45V & Ag107 & 15 & & & \\
\hline $\mathrm{B} 45 \mathrm{~V}$ & Ag109 & 15 & & 15 & \\
\hline $\mathrm{B} 51$ & Ag107 & 25.8 & 5.57 & & \\
\hline B51 & Ag109 & 45.3 & 27.5 & 35.6 & 16.5 \\
\hline$B 52$ & Ag107 & 42.9 & 22.3 & & \\
\hline $\mathrm{B} 52$ & Ag109 & 31.7 & 9.44 & 37.3 & 15.9 \\
\hline B52R & Ag107 & 80.2 & 4.57 & & \\
\hline B52R & Ag109 & 80.5 & 2.54 & 80.4 & 3.56 \\
\hline
\end{tabular}




\section{Transect B Porewater Concentrations: Sodium}

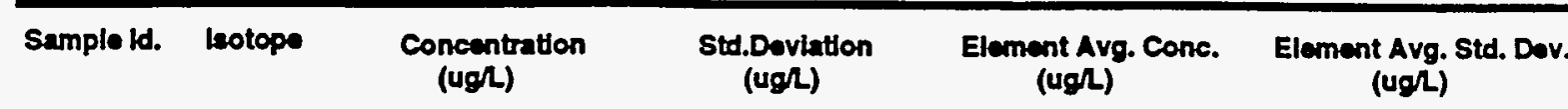

\begin{tabular}{|c|c|c|c|c|c|}
\hline $\mathrm{B} 11$ & $\mathrm{Na}$ & & & & \\
\hline$B 12$ & $\mathrm{Na}$ & & & & \\
\hline$B 13$ & $\mathrm{Na}$ & 74600 & 605 & 74600 & 605 \\
\hline$B 14$ & $\mathrm{Na}$ & 60200 & 3290 & 60200 & 3290 \\
\hline$B 21$ & $\mathrm{Na}$ & & & & \\
\hline$B 22$ & $\mathrm{Na}$ & & & & 622 \\
\hline$B 23$ & $\mathrm{Na}$ & 76000 & 622 & 76000 & 1860 \\
\hline$B 23 \mathrm{R}$ & $\mathrm{Na}$ & 81300 & 1860 & 81300 & 2550 \\
\hline$B 24$ & $\mathrm{Na}$ & 91000 & 2550 & 91000 & \\
\hline$B 31$ & $\mathrm{Na}$ & & & & 69.8 \\
\hline$B 32$ & $\mathrm{Na}$ & 18000 & 69.8 & 18000 & 28.3 \\
\hline$B 33$ & $\mathrm{Na}$ & 12000 & 28.3 & 12000 & 3.71 \\
\hline$B 41$ & $\mathrm{Na}$ & & & & 169 \\
\hline$B 42$ & $\mathrm{Na}$ & 2560 & 3.71 & 2560 & 173 \\
\hline$B 43$ & $\mathrm{Na}$ & 24300 & 169 & 24300 & 20.1 \\
\hline$B 45 \mathrm{~V}$ & $\mathrm{Na}$ & 13600 & 173 & 13600 & 64.9 \\
\hline$B 51$ & $\mathrm{Na}$ & 1870 & 20.1 & 1870 & 272 \\
\hline$B 52$ & $\mathrm{Na}$ & 24400 & 64.9 & 24400 & 6540 \\
\hline$B 52 \mathrm{R}$ & $\mathrm{Na}$ & 6540 & 272 & & \\
\hline
\end{tabular}

Note: The actual concentration of samples for which no standard deviation te reported b leas than the lower limit of 'detection. The reported concentration is the lower limit of quantification. 
Transect B Porewater Concentrations: Strontium

\begin{tabular}{|c|c|c|c|c|c|}
\hline Sample Id. & leotope & $\begin{array}{l}\text { Concentration } \\
\text { (ug/L) }\end{array}$ & $\begin{array}{l}\text { Std.Doviation } \\
\text { (ug/L) }\end{array}$ & $\begin{array}{c}\text { Element Avg. Conc. } \\
\text { (ugh) }\end{array}$ & $\begin{array}{c}\text { Element Avg. Std. Dov. } \\
\text { (ugh) }\end{array}$ \\
\hline B11 & Sr & & & & \\
\hline $\mathrm{B} 12$ & Sr & & & & \\
\hline$B 13$ & Sr & 64.3 & 1.48 & 64.3 & 1.48 \\
\hline B14 & $\mathrm{Sr}$ & 37 & 2 & 37 & 2 \\
\hline$B 21$ & $\mathrm{Sr}$ & & & & \\
\hline $\mathrm{B} 22$ & Sr & & & & \\
\hline$B 23$ & $\mathrm{Sr}$ & 39 & .43 & 39 & .43 \\
\hline B23R & $\mathrm{Sr}$ & 37 & .83 & 37 & .83 \\
\hline B24 & Sr & 31 & 1.49 & 31 & 1.49 \\
\hline 831 & $\mathrm{Sr}$ & & & & \\
\hline $\mathrm{B} 32$ & $\mathrm{Sr}$ & 22.1 & 28 & 22.1 & 28 \\
\hline $\mathrm{B} 33$ & $\mathrm{Sr}$ & 18.5 & .37 & 18.5 & .37 \\
\hline $\mathrm{B} 41$ & $\mathrm{Sr}$ & & & & \\
\hline$B 42$ & $\mathrm{Sr}$ & 14.5 & .44 & 14.5 & .44 \\
\hline 843 & $\mathrm{Sr}$ & 34.6 & .44 & 34.6 & .44 \\
\hline B45V & Sr & 19.5 & .38 & 19.5 & .38 \\
\hline $\mathrm{B} 51$ & $\mathrm{Sr}$ & 42.8 & .46 & 42.8 & .46 \\
\hline $\mathrm{B} 52$ & $\mathrm{Sr}$ & 47.4 & .82 & 47.4 & .82 \\
\hline B52R & $\mathrm{Sr}$ & 30.8 & 1.14 & 30.8 & 1.14 \\
\hline
\end{tabular}

Note: The actual concentration of samples for which no standard deviation ls reported ls leas than the lower limit of detection. The reported concentration is the lower limit of quantification. 


\section{Transect B Porewater Concentrations: Thorium}

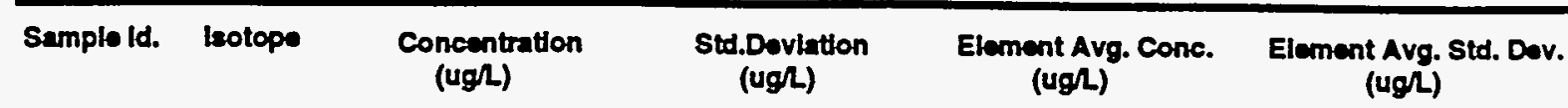

\begin{tabular}{|c|c|c|c|c|c|}
\hline B11 & Th232 & & & & \\
\hline B12 & Th232 & & & & \\
\hline B13 & Th232 & .5 & & .5 & \\
\hline 814 & Th232 & .5 & & .5 & \\
\hline B21 & Th232 & & & & \\
\hline$B 22$ & Th232 & & & & \\
\hline$B 23$ & Th232 & .38 & & .38 & \\
\hline B23R & Th232 & 25 & & 25 & \\
\hline$B 24$ & Th232 & .5 & & .5 & \\
\hline 831 & Th232 & & & & \\
\hline B32 & Th232 & & & & \\
\hline B33 & Th232 & .03 & .02 & .03 & .02 \\
\hline$B 41$ & Th232 & & & & \\
\hline$B 42$ & Th232 & .5 & & .5 & \\
\hline$B 43$ & Th232 & .5 & & .5 & \\
\hline $\mathrm{B} 45 \mathrm{~V}$ & Th232 & .5 & & .5 & \\
\hline $\mathrm{B} 51$ & Th232 & 25 & & 25 & \\
\hline $\mathrm{B} 52$ & Th232 & 25 & & 25 & \\
\hline B52R & Th232 & 25 & & 25 & \\
\hline
\end{tabular}




\begin{tabular}{|c|c|c|c|c|c|}
\hline \multirow[b]{2}{*}{ Semple ld. } & \multicolumn{5}{|c|}{ Transect B Porewater Concentrations: Tin } \\
\hline & Eotope & $\begin{array}{l}\text { Concantration } \\
\text { (ugh) }\end{array}$ & $\begin{array}{l}\text { Std.Doviation } \\
\text { (ug/) }\end{array}$ & $\begin{array}{l}\text { Element Avg. Conc. } \\
\text { (ug/) }\end{array}$ & $\begin{array}{c}\text { Element Avg. Std. Dov. } \\
\text { (ug } \Omega)\end{array}$ \\
\hline$B 11$ & $\operatorname{Sn} 118$ & & & & \\
\hline$B 11$ & $\operatorname{Sn} 120$ & & & & \\
\hline$B 12$ & Sn118 & & & & \\
\hline 812 & $\operatorname{sn} 120$ & & & & \\
\hline 813 & $\operatorname{Sn} 118$ & .72 & .16 & & \\
\hline$B 13$ & $\sin 120$ & & & .72 & .16 \\
\hline B14 & $\operatorname{Sn} 118$ & 25 & & & \\
\hline$B 14$ & $\operatorname{Sn} 120$ & 25 & & 25 & \\
\hline 821 & Sn118 & & & & \\
\hline 821 & $\sin 120$ & & & & \\
\hline $\mathrm{B} 22$ & Sn118 & & & & \\
\hline 822 & Sn120 & & & & \\
\hline E23 & Sn118 & 12.6 & & & \\
\hline E23 & $\operatorname{Sn} 120$ & 12.6 & & 12.6 & \\
\hline B23R & $\operatorname{Sn} 118$ & .07 & 0 & & \\
\hline $\mathrm{B} 23 \mathrm{R}$ & $\operatorname{Sn} 120$ & .08 & .07 & .08 & .04 \\
\hline B24 & Sn118 & 25 & & & \\
\hline B24 & $\sin 120$ & 25 & & 25 & \\
\hline$B 31$ & $\operatorname{Sn} 118$ & & & & \\
\hline 839 & Sn120 & & & & \\
\hline 832 & $\operatorname{Sn} 118$ & 13.8 & & & \\
\hline 832 & $\operatorname{Sn} 120$ & 13.8 & & 13.8 & \\
\hline 833 & $\sin 118$ & 13.8 & & & \\
\hline $\mathrm{B} 33$ & $\sin 120$ & 13.8 & & 13.8 & \\
\hline 841 & Sn118 & & & & \\
\hline$B 41$ & $\sin 120$ & & & & \\
\hline 842 & $\sin 118$ & 25 & & & \\
\hline 842 & Sn120 & 25 & & 25 & \\
\hline$B 43$ & Sn118 & 25 & & & \\
\hline 843 & $\sin 120$ & 25 & & 25 & \\
\hline B45V & $\operatorname{Sn} 118$ & 25 & & & \\
\hline B45V & $\operatorname{Sn} 120$ & 25 & & 25 & \\
\hline 851 & Sn118 & 1.38 & & & \\
\hline $\mathrm{B} 51$ & Sn120 & 1.38 & & 1.38 & \\
\hline 852 & Sn118 & 1.38 & & & \\
\hline $\mathrm{B} 52$ & Sn120 & .1 & .06 & .74 & .06 \\
\hline $\mathrm{B} 52 \mathrm{R}$ & $\operatorname{Sn} 118$ & 1.38 & & & \\
\hline $\mathrm{B} 52 \mathrm{R}$ & Sn120 & .12 & .05 & .75 & .05 \\
\hline
\end{tabular}


Transect B Porewater Concentrations: Uranium-235

\begin{tabular}{|c|c|c|c|c|c|}
\hline Sample ld. & leotope & $\begin{array}{l}\text { Concentration } \\
\text { (ug/) }\end{array}$ & $\begin{array}{l}\text { Std.Dovlation } \\
\text { (ug/L) }\end{array}$ & $\begin{array}{c}\text { Element Avg. Conc. } \\
\text { (ug/) }\end{array}$ & $\begin{array}{c}\text { Element Avg. Std. Dov. } \\
\text { (ug/L) }\end{array}$ \\
\hline B11 & U235 & & & & \\
\hline B12 & U235 & & & & \\
\hline B13 & U235 & 8.61 &.$\infty$ & 8.61 & .09 \\
\hline B14 & U235 & 1.62 & .11 & 1.62 & .11 \\
\hline B21 & U235 & & & & \\
\hline $\mathrm{B} 22$ & U235 & & & & \\
\hline $\mathrm{B} 23$ & U235 & 1.52 & .04 & 1.52 & .04 \\
\hline B23R & U235 & 3.18 & .3 & 3.19 & .3 \\
\hline B24 & U235 & 32 & .14 & 32 & .14 \\
\hline B31 & U235 & & & & \\
\hline B32 & U235 & .05 & .02 & .05 & .02 \\
\hline$B 33$ & U235 & .05 & 0 & .05 & 0 \\
\hline $\mathrm{B} 41$ & U235 & & & & \\
\hline$B 42$ & U235 & .63 & .06 & .63 & .06 \\
\hline$B 43$ & U235 & 2.43 & .17 & 2.43 & .17 \\
\hline $\mathrm{B} 45 \mathrm{~V}$ & U235 & .02 & & .02 & \\
\hline B51 & U235 & .51 & .03 & .51 & .03 \\
\hline 852 & U235 & 1.58 & .06 & 1.58 & .06 \\
\hline B52R & U235 & .02 & & .02 & \\
\hline
\end{tabular}




\section{Transect B Porewater Concentrations: Uranium-238}

\begin{tabular}{|c|c|c|c|c|c|}
\hline Sample ld. & beotope & $\begin{array}{l}\text { Concentration } \\
\text { (ugh) }\end{array}$ & $\begin{array}{l}\text { Std.Devlation } \\
\text { (ug } / \text { ) }\end{array}$ & $\begin{array}{c}\text { Eloment Avg. Conc. } \\
\text { (ugh) }\end{array}$ & $\begin{array}{c}\text { Element Avg. Std. Dav. } \\
\text { (ug/) }\end{array}$ \\
\hline B11 & U238 & & & & \\
\hline $\mathrm{B} 12$ & $\mathrm{U} 238$ & & & & \\
\hline 813 & U238 & 1290 & 19.9 & 1290 & 19.9 \\
\hline 814 & U238 & 209 & 5.02 & 269 & 5.02 \\
\hline 821 & U238 & & & & \\
\hline$B 22$ & U238 & & & & \\
\hline 823 & U238 & 150 & 1.09 & 159 & 1.09 \\
\hline B23R & U238 & 415 & 3.98 & 415 & 3.98 \\
\hline 824 & U238 & 552 & 8.71 & 520 & 8.71 \\
\hline B31 & U238 & & & & \\
\hline B32 & U238 & 7.78 & .65 & 7.78 & .65 \\
\hline $\mathrm{B} 33$ & U238 & 6.31 & .05 & 6.31 & .05 \\
\hline$B 41$ & U238 & & & & \\
\hline B42 & U238 & 992 & 4.41 & 99.2 & 4.41 \\
\hline$B 43$ & U238 & 357 & 22.4 & 367 & 22.4 \\
\hline B45V & U238 & 125 & & 125 & \\
\hline $\mathrm{B} 51$ & U238 & 89.9 & .92 & 89.9 & .92 \\
\hline $\mathrm{B} 52$ & U238 & 252 & .32 & 252 & .32 \\
\hline$B 52 R$ & U238 & 5.63 & .3 & 5.63 & 3 \\
\hline
\end{tabular}




\section{Transect B Porewater Concentrations: Vanadium}

\begin{tabular}{|c|c|c|c|c|c|}
\hline Samplo ld. & Hetope & $\begin{array}{l}\text { Concentratton } \\
(4 \mathrm{~g} /)\end{array}$ & $\begin{array}{l}\text { Sid.Doviation } \\
\text { (Ler) }\end{array}$ & $\begin{array}{c}\text { Element Avg Conc. } \\
\text { (ug/L) }\end{array}$ & $\begin{array}{l}\text { Element Avg. Std. Dev. } \\
\text { (ugh) }\end{array}$ \\
\hline
\end{tabular}

\begin{tabular}{|c|c|c|c|c|c|}
\hline B11 & $v$ & & & & \\
\hline$B 12$ & V & & & & \\
\hline B13 & V & .47 & & .47 & \\
\hline B14 & V & 25 & & 25 & \\
\hline$B 21$ & $\mathbf{v}$ & & & & \\
\hline 822 & V & & & & \\
\hline $\mathrm{B} 23$ & $\mathbf{v}$ & 28 & .42 & 28 & .42 \\
\hline B23R & $\mathbf{v}$ & 4.38 & & 4.38 & \\
\hline 824 & V & 1.5 & & 1.5 & \\
\hline B31 & $v$ & & & & \\
\hline B32 & $v$ & .34 & 24 & .34 & 24 \\
\hline$B 33$ & $\mathbf{v}$ & 1.38 & & 1.38 & \\
\hline 841 & V & & & & \\
\hline$B 42$ & $v$ & 25 & & 25 & \\
\hline$B 43$ & $v$ & 25 & & 25 & \\
\hline B45V & $\mathbf{v}$ & .75 & .18 & .75 & .18 \\
\hline B51 & $v$ & 3.25 & & 325 & \\
\hline 852 & $v$ & 3.25 & & 325 & \\
\hline B52R & $v$ & 4.38 & & 4.38 & \\
\hline
\end{tabular}




\section{Transect B Porewater Concentrations: Yttrium}

\begin{tabular}{ccccc}
\hline Sample ld. leotope & $\begin{array}{c}\text { Concentration } \\
(u g h)\end{array}$ & $\begin{array}{c}\text { Std.Doviation } \\
\text { (ugh })\end{array}$ & $\begin{array}{c}\text { Element Avg. Conc. } \\
\text { (ugh) }\end{array}$ & $\begin{array}{c}\text { Element Avg. Std. Dov. } \\
\text { (ugh) }\end{array}$
\end{tabular}

\begin{tabular}{|c|c|c|c|c|c|}
\hline$B 11$ & $Y$ & & & & \\
\hline 812 & $Y$ & & & & \\
\hline$B 13$ & $Y$ & 102 & .83 & 102 & .83 \\
\hline 814 & $Y$ & 201 & 2.71 & 201 & 2.71 \\
\hline$B 21$ & $Y$ & & & & \\
\hline $\mathrm{B} 22$ & $Y$ & & & & \\
\hline 823 & $Y$ & 10.1 & .11 & 10.1 & .11 \\
\hline B23R & $Y$ & 46.9 & 1.09 & 46.9 & 1.09 \\
\hline 824 & $Y$ & 36.8 & .3 & 36.8 & .3 \\
\hline $\mathrm{B31}$ & $\bar{Y}$ & & & & \\
\hline B32 & $Y$ & 3.91 & .09 & 3.91 & .09 \\
\hline 833 & $Y$ & 3.93 & .07 & 3.93 & .07 \\
\hline B41 & $Y$ & & & & \\
\hline 842 & $Y$ & 19.9 & 23 & 19.9 & 23 \\
\hline B43 & $\bar{Y}$ & 260 & 3.49 & 260 & 3.49 \\
\hline B45V & $Y$ & .25 & & 25 & \\
\hline B51 & $Y$ & 195 & .86 & 195 & .86 \\
\hline 852 & $\bar{Y}$ & 595 & 9.73 & 595 & 9.73 \\
\hline B52R & $\vec{Y}$ & 44.7 & .96 & 44.7 & .96 \\
\hline
\end{tabular}




\section{Transect B Porewater Concentrations: Zinc}

Sample Id. leotope

Concentration

(ug $\Omega$ )
Std.Dovlation

(ugl)
Element Avg. Conc. (ugh)
Element Avg. Std. Dov. (ugh)

\begin{tabular}{|c|c|c|c|c|c|}
\hline B11 & Zn66 & & & & \\
\hline B12 & Zn66 & & & & \\
\hline$B 13$ & Zn66 & 92.7 & 1.13 & 92.7 & 1.13 \\
\hline B14 & Zn66 & 50.2 & 1.23 & 502 & 123 \\
\hline$B 21$ & Zn66 & & & & \\
\hline$B 22$ & Zn66 & & & & \\
\hline $\mathrm{B23}$ & Zn66 & 189 & 1.74 & 189 & 1.74 \\
\hline B23R & Zn66 & 349 & 19.2 & 340 & 192 \\
\hline 824 & Zn66 & 505 & 2.94 & 505 & 2.94 \\
\hline $\mathrm{B} 31$ & Zn66 & & & & \\
\hline 832 & Zn66 & 432 & 2.44 & 432 & 2.44 \\
\hline 833 & Zn66 & 40.2 & 1.01 & 402 & 1.01 \\
\hline 841 & Zn66 & & & & \\
\hline$B 42$ & Zn66 & 102 & 1.31 & 102 & 1.31 \\
\hline$B 43$ & Zn66 & 299 & 5.16 & 299 & 5.16 \\
\hline B45V & Zn66 & 5 & & 5 & \\
\hline $\mathrm{B} 51$ & Zn66 & 101 & 2.69 & 101 & 2.69 \\
\hline $\mathrm{B} 52$ & Zn66 & 147 & 2.54 & 147 & 2.54 \\
\hline B52R & Zn66 & 126 & 34.5 & 126 & 34.5 \\
\hline
\end{tabular}

Note: The actual concentrattion of eamples for which no standard deviation bs reportad bs lees than the lower limit of detection. The reported concentration ts the lower limit of quantification. 


\section{Transect B Porewater Concentrations: Zirconium}

\begin{tabular}{ccccc}
\hline Samplo ld. Leotope & $\begin{array}{c}\text { Concentration } \\
\text { (ugh) }\end{array}$ & $\begin{array}{c}\text { Std.Devlation } \\
\text { (ugh) }\end{array}$ & $\begin{array}{c}\text { Element Avg. Conc. } \\
\text { (ug } /)\end{array}$ & $\begin{array}{c}\text { Element Avg. Std. Dov. } \\
\text { (Ug })\end{array}$ \\
\hline
\end{tabular}

\begin{tabular}{|c|c|c|c|c|c|}
\hline 811 & $Z 590$ & & & & \\
\hline 811 & $Z 91$ & & & & \\
\hline $\mathrm{B} 12$ & 2500 & & & & \\
\hline$B 12$ & 2991 & & & & \\
\hline B13 & $Z 90$ & .5 & & & \\
\hline B13 & $\mathrm{Z} 91$ & .5 & & .5 & \\
\hline B14 & $Z 990$ & .03 & .02 & & \\
\hline B14 & Zr91 & .5 & & 27 & .02 \\
\hline$B 21$ & $Z 90$ & & & & \\
\hline 821 & $Z 991$ & & & & \\
\hline 822 & Zr9o & & & & \\
\hline$B 22$ & Zr91 & & & & \\
\hline 823 & $Z 90$ & 22 & .16 & & \\
\hline 823 & Z91 & 21 & .5 & 22 & .33 \\
\hline B23R & Zr90 & 1.38 & & & \\
\hline B23R & $Z 991$ & 1.38 & & 1.38 & \\
\hline$B 24$ & $Z r 90$ & .5 & & & \\
\hline 824 & $Z 91$ & 2 & .14 & .35 & .14 \\
\hline 831 & 2590 & & & & \\
\hline 831 & $Z 591$ & & & & \\
\hline $\mathrm{B} 32$ & $Z 90$ & 2.75 & & & \\
\hline $\mathrm{B} 32$ & Zr91 & 2.75 & & 2.75 & \\
\hline$B 33$ & $Z r 90$ & 2.75 & & & \\
\hline$B 33$ & 291 & 2.75 & & 2.75 & \\
\hline B41 & Z 890 & & & & \\
\hline$B 41$ & 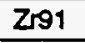 & & & & \\
\hline$B 42$ & Zr9o & .5 & & & \\
\hline$B 42$ & $\mathrm{Z} 91$ & .5 & & .5 & \\
\hline 843 & $Z r 90$ & .5 & & & \\
\hline$B 43$ & Zr91 & .5 & & .5 & \\
\hline $\mathrm{B} 45 \mathrm{~V}$ & $Z r 90$ & .5 & & & \\
\hline B45V & Zro1 & .5 & & .5 & \\
\hline 851 & $Z 590$ & 1.38 & & & \\
\hline 851 & Z991 & 1.38 & & 1.38 & \\
\hline $\mathrm{B} 52$ & $Z \times 90$ & 1.38 & & & \\
\hline $\mathrm{B} 52$ & 291 & 1.38 & & 1.38 & \\
\hline B52R & $Z 190$ & 1.38 & & & \\
\hline B52R & $Z 991$ & 1.38 & & 1.38 & \\
\hline
\end{tabular}

Note: The actual concentration of samples for which no standard deviation ts reported is lese than the lower limit of detection. The reported concentration le the lower llmit of quantification. 


\section{Transect C Porewater Concentrations: Aluminum}

\begin{tabular}{|c|c|c|c|c|c|}
\hline Semple ld. & botope & $\begin{array}{l}\text { Concentration } \\
\text { (ug/h) }\end{array}$ & $\begin{array}{l}\text { Std.Doviatton } \\
\text { (Ug/L) }\end{array}$ & $\begin{array}{l}\text { Element Avg- Conc. } \\
\text { (ual) }\end{array}$ & $\begin{array}{l}\text { Element Avg. Std. Dav. } \\
\text { (ugh) }\end{array}$ \\
\hline C11 & $\mathbf{A}$ & & & & \\
\hline $\mathrm{C}_{12}$ & $\mathbf{N}$ & & & & \\
\hline $\mathrm{C13}$ & $\overline{\mathbf{N}}$ & 42600 & 85.4 & 42600 & 95.4 \\
\hline$C 14$ & $\mathbf{A}$ & 22800 & 421 & 228000 & 421 \\
\hline C21 & $\mathbf{A}$ & & & & \\
\hline C21R & $\mathbf{N}$ & & & & \\
\hline $\cos$ & $\mathbf{A}$ & 2420 & 76.8 & 2420 & 76.8 \\
\hline C23 & $\mathbf{A}$ & 40 & 8.99 & 420 & 8.99 \\
\hline$C 31$ & A & & & & \\
\hline C32 & $\mathbf{A}$ & 34 & 15.5 & 34 & 15.5 \\
\hline $\mathbf{C 3 3}$ & A & 116 & 18.4 & 116 & 18.4 \\
\hline $\mathrm{C}_{41}$ & A & & & & \\
\hline $\mathrm{C} 42$ & A & 37.5 & & 37.5 & . \\
\hline $\mathrm{C43}$ & $\mathbf{A}$ & 50 & & 50 & \\
\hline
\end{tabular}




\section{Transect C Porewater Concentrations: Arsenic}

Sample ld. kotope

Concentration

(ugl)
Stopoviation

(ugh)
Element Avg Conc. (ugl)
Element Avg. Std. Dov.

(ugh)

\begin{tabular}{|c|c|c|c|c|c|}
\hline C11 & As & & & & \\
\hline $\mathrm{C} 12$ & As & & & & \\
\hline C13 & As & 75 & & 75 & \\
\hline $\mathrm{C14}$ & As & 75 & & 75 & \\
\hline c21 & As & & & & \\
\hline CIR & As & & & & \\
\hline $\mathrm{CO2}_{2}$ & As & 75 & & 75 & \\
\hline $\mathrm{C} 23$ & As & 3.49 & 325 & 3.49 & 325 \\
\hline C31 & As & & & & \\
\hline C32 & As & 38 & & 38 & \\
\hline C33 & As & 18.6 & 6.06 & 18.6 & 6.06 \\
\hline$C_{41}$ & As & & & & \\
\hline $\mathrm{C} 42$ & As & 31.9 & 8.63 & 31.9 & 8.63 \\
\hline $\mathrm{C}_{43}$ & As & 1 & & 1 & \\
\hline
\end{tabular}




\begin{tabular}{|c|c|c|c|c|c|}
\hline \multicolumn{6}{|c|}{ Transect C Porewater Concentrations: Barium } \\
\hline Sample ld. & beotope & $\begin{array}{l}\text { Concentration } \\
\text { (ug/L) }\end{array}$ & $\begin{array}{l}\text { Std.Doviation } \\
\text { (ugh) }\end{array}$ & $\begin{array}{l}\text { Element Avg. Conc. } \\
\text { (ugh) }\end{array}$ & $\begin{array}{l}\text { Element Avg. Std. Dov. } \\
\text { (ugh) }\end{array}$ \\
\hline$C 11$ & Ba135 & & & & \\
\hline C11 & Ba137 & & & & \\
\hline C11 & Ba138 & & & & \\
\hline $\mathrm{C} 12$ & Ba135 & & & & \\
\hline $\mathrm{C}_{12}$ & Ba137 & & & & \\
\hline C12 & Ba138 & & & & \\
\hline $\mathrm{C13}$ & Ba135 & 339 & 9.46 & & \\
\hline C13 & Ba137 & 347 & $5 . \pi 7$ & & \\
\hline C13 & Ba138 & 353 & 2.18 & 346 & 5.81 \\
\hline$C_{14}$ & Ba135 & 106 & 4.33 & & \\
\hline $\mathrm{C14}_{4}$ & Be137 & 115 & 4.65 & & \\
\hline$C_{14}$ & Ba138 & 113 & 1.34 & 111 & 3.44 \\
\hline C21 & Ba135 & & & & \\
\hline$C_{21}$ & Ba137 & & & & \\
\hline$C_{21}$ & Ba138 & & & & \\
\hline C21R & Ba135 & & & & \\
\hline C21R & Ba137 & & & & \\
\hline C21R & Ba138 & & & & \\
\hline $\mathrm{CP2}$ & Ba135 & 110 & 3.32 & & \\
\hline $\mathrm{cos}_{2}$ & Ba137 & 120 & 1.67 & & \\
\hline$C 22$ & Ba138 & 117 & .85 & 116 & 1.95 \\
\hline$C_{23}$ & Ba135 & 40.1 & 1.53 & & \\
\hline $\mathrm{C}_{23}$ & Ba137 & 39.7 & 3.02 & & \\
\hline C23 & Ba138 & 38.6 & 1.75 & 39.5 & 2.1 \\
\hline C31 & Ba135 & & & & \\
\hline C31 & Ba137 & & & & \\
\hline C31 & $8 a 138$ & & & & \\
\hline C32 & Ba135 & 17.8 & 2.14 & & \\
\hline C32 & Ba137 & 18.1 & 1.78 & & \\
\hline C32 & Ba138 & 19.3 & 24 & 18.4 & 1.39 \\
\hline C.33 & Ba135 & 39.3 & 13.6 & & \\
\hline C.33 & Ba137 & 52.9 & 8.98 & & \\
\hline Ca3 & Ba138 & 36.9 & 122 & 43 & 7.93 \\
\hline $\mathrm{C}_{41}$ & Ba135 & & & & \\
\hline$C 41$ & $8 a 137$ & & & & \\
\hline C41 & Be138 & & & & \\
\hline$C_{42}$ & Ba135 & 7.83 & 2.49 & & \\
\hline $\mathrm{C}_{42}$ & Be137 & 7.75 & 23 & & \\
\hline $\mathrm{C42}$ & Ba138 & 7.54 & .43 & 7.71 & 1.05 \\
\hline $\mathrm{C} 43$ & Ba135 & 21 & 112 & & \\
\hline $\mathrm{C}_{43}$ & Ba137 & 4 & 10.9 & & \\
\hline$C_{43}$ & Ba138 & 37.5 & 1.99 & 342 & 8.03 \\
\hline
\end{tabular}

Note: The actual concentratton of samples for whlch no standard deviation be reported is leas than the lower limit of detection. The roported concentration ts the lower limit of quantification. 


\section{Transect C Porewater Concentrations: Cadmium}

\begin{tabular}{|c|c|c|c|c|c|}
\hline Sample ld. & botope & $\begin{array}{l}\text { Concentration } \\
\text { (ugh) }\end{array}$ & $\begin{array}{c}\text { StdDevition } \\
\text { (u:Aㄴ) }\end{array}$ & $\begin{array}{l}\text { Element Avg Conc. } \\
\text { (ugh) }\end{array}$ & $\begin{array}{l}\text { Element Avg. Std. Dov } \\
\text { (ugr) }\end{array}$ \\
\hline
\end{tabular}

\begin{tabular}{|c|c|c|c|c|c|}
\hline C11 & CdI11 & & & & \\
\hline C11 & Cd114 & & & & \\
\hline $\mathrm{C} 12$ & Cd111 & & & & \\
\hline $\mathrm{C} 12$ & $\operatorname{Cd} 114$ & & & & \\
\hline$C_{13}$ & Cd111 & 23.9 & 4.48 & & \\
\hline C13 & Cd114 & 23.1 & 1.01 & 23.5 & 2.75 \\
\hline $\mathrm{Cl}_{4}$ & Col111 & 2.99 & .8 & & \\
\hline C14 & Cd114 & 3.39 & .48 & 3.19 & .64 \\
\hline C1 & Cd111 & & & & \\
\hline C21 & Cdi14 & & & & \\
\hline C21R & Col111 & & & & \\
\hline C21R & Cd114 & & & & \\
\hline$\infty 2$ & Cd111 & .75 & & & \\
\hline 022 & Cd114 & .75 & & .75 & \\
\hline$C_{23}$ & $\operatorname{cot111}$ & .63 & & & \\
\hline C23 & Cd114 & .63 & & .63 & \\
\hline C31 & Cd111 & & & & \\
\hline C31 & Cdi14 & & & & \\
\hline C32 & Cd111 & .63 & & & \\
\hline $\cos 2$ & Cd114 & .63 & & .63 & \\
\hline $\cos 3$ & Cd111 & .5 & & & \\
\hline C.33 & Cd114 & .5 & & .5 & \\
\hline C41 & Cd111 & & & & \\
\hline $\mathrm{C} 41$ & Cd114 & & & & \\
\hline$C_{42}$ & Cd111 & .63 & & & \\
\hline$C 42$ & Cd114 & .63 & & .63 & \\
\hline$C_{43}$ & Cd111 & .5 & & & \\
\hline$C_{43}$ & Cd114 & .5 & & .5 & \\
\hline
\end{tabular}




\begin{tabular}{|c|c|c|c|c|c|}
\hline Sample ld. & leotope & $\begin{array}{l}\text { Concentratton } \\
\text { (ug/L) }\end{array}$ & $\begin{array}{l}\text { Std.Deviation } \\
\text { (ugll) }\end{array}$ & $\begin{array}{l}\text { Element Avg. Conc. } \\
\text { (ugh) }\end{array}$ & $\begin{array}{l}\text { Element Avg. Std. Dov. } \\
\text { (ugh) }\end{array}$ \\
\hline $\mathrm{C11}$ & $\operatorname{Ca44}$ & & & & \\
\hline $\mathrm{C} 12$ & Ca44 & & & & \\
\hline C13 & Ca44 & 13600 & 88.7 & 13500 & 88.7 \\
\hline C14 & $\mathrm{Ca44}$ & 349 & 9.3 & 349 & 9.3 \\
\hline G1 & Ca44 & & & & \\
\hline C21R & Ca44 & & & & \\
\hline C22 & Cas4 & 124 & 4.64 & 124 & 4.64 \\
\hline $\mathrm{CO23}$ & Co44 & 109 & 3.57 & 100 & 3.57 \\
\hline 631 & Co44 & & & & \\
\hline C32 & $\mathrm{Ca} 44$ & 13.3 & 2.89 & 13.3 & 2.89 \\
\hline Ca33 & Ca44 & 468 & 230 & 468 & 230 \\
\hline $\mathrm{CA1}$ & Ca44 & & & & \\
\hline $\mathrm{C} 42$ & Ca44 & 59.1 & 23 & 59.1 & 23 \\
\hline $\mathrm{C43}$ & Ca44 & 786 & 379 & 786 & 379 \\
\hline
\end{tabular}




\section{Transect C Porewater Concentrations: Cesium}

\begin{tabular}{|c|c|c|c|c|c|}
\hline Sample Id. & Lotope & $\begin{array}{l}\text { Concentration } \\
\text { (ug/l) }\end{array}$ & $\begin{array}{l}\text { Std. Deviation } \\
\text { (ugh) }\end{array}$ & $\begin{array}{l}\text { Element Avg. Conc. } \\
\text { (ugh) }\end{array}$ & $\begin{array}{l}\text { Element Avg. Std. Dov. } \\
\text { (ug/l) }\end{array}$ \\
\hline $\mathrm{Cr} 1$ & $\mathrm{Cs}$ & & & & \\
\hline$C 12$ & $\mathrm{Cs}_{\mathrm{s}}$ & & & & \\
\hline$C_{13}$ & $\mathrm{Cs}$ & .46 &.$\infty 2$ & .46 & .02 \\
\hline $\mathrm{C} 14$ & $\mathrm{Cs}_{8}$ & .05 & .02 & .05 & .02 \\
\hline$C_{21}$ & $\mathrm{Cs}$ & & & & \\
\hline C21A & Cs & & & & \\
\hline$c_{22}$ & Cs & 5 & & 5 & \\
\hline 023 & Cs & 2.63 & & 2.63 & \\
\hline $\mathrm{C} 31$ & Cs & & & & \\
\hline c32 & $\mathrm{Cs}$ & 2.63 & & 2.63 & \\
\hline C33 & Cs & 25 & & 25 & \\
\hline C41 & Cs & & & & \\
\hline $\mathrm{C} 42$ & Cs & 2.63 & & 2.63 & . \\
\hline $\mathrm{CA3}_{4}$ & Cs & .35 & .07 & .35 & .07 \\
\hline
\end{tabular}




\section{Transect C Porewater Concentrations: Chromium}

\begin{tabular}{|c|c|c|c|c|c|}
\hline Sample ld. & leotope & $\begin{array}{l}\text { Concentration } \\
\text { (ug/) }\end{array}$ & $\begin{array}{l}\text { Std.Doviation } \\
\text { (ugh) }\end{array}$ & $\begin{array}{l}\text { Element Avg. Conc. } \\
\text { (ugh) }\end{array}$ & $\begin{array}{l}\text { Element Avg. Std. Dov. } \\
\text { (ug })\end{array}$ \\
\hline C11 & C.52 & & & & \\
\hline$C 11$ & C.53 & & & & \\
\hline $\mathrm{C}_{12}$ & c.52 & & & & \\
\hline $\mathrm{C} 12$ & $c 53$ & & & & \\
\hline $\mathrm{C} 13$ & $a 52$ & 8.87 & .53 & & \\
\hline C13 & C.53 & 8.86 & .53 & 8.86 & .53 \\
\hline C14 & C.52 & 5.24 & 1 & & \\
\hline$C_{14}$ & C.53 & 8.24 & 1 & 6.74 & 1 \\
\hline C21 & C.52 & & & & \\
\hline C21 & Cr53 & & & & \\
\hline C21R & C.52 & & & & \\
\hline C21R & C.53 & & & & \\
\hline $\mathrm{C22}$ & cr52 & 222 & .13 & & \\
\hline $\mathrm{C22}$ & cr53 & 2.22 & .13 & 222 & .13 \\
\hline$c 23$ & C.52 & 1.18 & .47 & & \\
\hline$C_{23}$ & C.53 & 1.18 & .47 & 1.18 & .47 \\
\hline C31 & cr52 & & & & \\
\hline C31 & C.53 & & & & \\
\hline C32 & C.52 & .67 & 21 & & \\
\hline C32 & $C_{53}$ & .67 & 21 & .67 & 21 \\
\hline CO3 & C.52 & 10 & & & \\
\hline C.33 & C.53 & 10 & & 10 & \\
\hline$C_{41}$ & C.52 & & & & \\
\hline C41 & Cr53 & & & & \\
\hline$C_{42}$ & C.52 & .77 & .11 & & \\
\hline $\mathrm{CA2}_{2}$ & C.53 & $\pi$ & .11 & .77 & .11 \\
\hline $\mathrm{C}_{43}$ & c.52 & 10 & & & \\
\hline$C_{43}$ & Cr53 & 10 & & 10 & \\
\hline
\end{tabular}

Note: The actual concentratton of samples for which no standard doviation be reported b lees than the lower limit of detection. The reported concentration la the lower limit of quantification. 


\section{Transect C Porewater Concentrations: Cobalt}

\begin{tabular}{|c|c|c|c|c|c|}
\hline Sample ld. & leotope & $\begin{array}{l}\text { Concentratton } \\
\text { (ugl) }\end{array}$ & $\begin{array}{l}\text { Std.Devlation } \\
\text { (ug/) }\end{array}$ & $\begin{array}{c}\text { Element Avg. Conc. } \\
\text { (ugh) }\end{array}$ & $\begin{array}{c}\text { Element Avg. Std. Dav. } \\
\text { (ug/) }\end{array}$ \\
\hline
\end{tabular}

\begin{tabular}{|c|c|c|c|c|c|}
\hline C11 & $c_{0}$ & & & & \\
\hline C12 & $C_{0}$ & & & & \\
\hline $\mathrm{C}_{13}$ & $\infty$ & 29.7 & .46 & 29.7 & .46 \\
\hline $\mathrm{C}_{14}$ & Co & 15.9 & .84 & 15.9 & .84 \\
\hline$C_{21}$ & $\omega_{0}$ & & & & \\
\hline C21R & $C_{0}$ & & & & \\
\hline$C_{22}$ & $\infty$ & 6.5 & 27 & 6.5 & 27 \\
\hline$c_{23}$ & $\infty$ & 2.9 & .45 & 2.8 & .45 \\
\hline C31 & $C_{0}$ & & & & \\
\hline C32 & $C_{0}$ & .32 & .15 & .32 & .15 \\
\hline $\mathrm{C} 33$ & Co & .5 & & .5 & \\
\hline$C_{41}$ & $\infty$ & & & & \\
\hline$C_{42}$ & Co & .75 & & .75 & \\
\hline $\mathrm{C}_{43}$ & $C_{0}$ & 14.6 & 1.97 & 14.6 & 1.97 \\
\hline
\end{tabular}




\begin{tabular}{|c|c|c|c|c|c|}
\hline \multicolumn{6}{|c|}{ Transect C Porewater Concentrations: Copper } \\
\hline Sample ld. & leotope & $\begin{array}{l}\text { Concentration } \\
\text { (ug/L) }\end{array}$ & $\begin{array}{l}\text { Ste.Doviation } \\
\text { (ug/L) }\end{array}$ & $\begin{array}{c}\text { Element Avg. Conc. } \\
\text { (ugh) }\end{array}$ & $\begin{array}{c}\text { Element Avg. Std. Dov. } \\
\text { (ugh) }\end{array}$ \\
\hline$C_{11}$ & 0,63 & & & & \\
\hline C11 & Cu65 & & & & \\
\hline $\mathrm{C}_{12}$ & Cu63 & & & & \\
\hline $\mathrm{C12}$ & Qu65 & & & & \\
\hline C13 & Cu63 & 34.7 & .58 & & \\
\hline C13 & Qu65 & 34.6 & 2.13 & 34.7 & 1.36 \\
\hline C14 & Qu63 & 192 & .59 & & \\
\hline $\mathrm{C14}_{14}$ & Qu65 & 18.9 & .76 & 18 & .68 \\
\hline$C_{21}$ & a 463 & & & & \\
\hline$C_{21}$ & Cu65 & & & & \\
\hline C21R & Cu63 & & & & \\
\hline C21R & Cu65 & & & & \\
\hline $\mathrm{CO2}$ & Cu63 & 14.6 & .45 & & \\
\hline $\mathrm{CO}_{22}$ & Cu65 & 13.4 & 29 & 14 & .37 \\
\hline $\mathrm{C} 23$ & Cu63 & 823 & 1.62 & & \\
\hline$C_{23}$ & Cu65 & 9.63 & .8 & 8.93 & 121 \\
\hline C31 & Qu63 & & & & \\
\hline C31 & Cu65 & & & & \\
\hline C32 & Cu63 & 2.5 & & & \\
\hline C32 & Cu65 & 13.8 & & 8.15 & \\
\hline CO33 & Cu63 & & & & \\
\hline C.33 & Cu65 & 25 & & 25 & \\
\hline $\mathrm{C} 41$ & Cu63 & & & & \\
\hline C41 & Cu65 & & & & \\
\hline $\mathrm{C}_{42}$ & Cu63 & 2.5 & & & \\
\hline$C_{42}$ & Cu65 & 13.8 & & 8.15 & \\
\hline$C_{43}$ & Qu63 & & & & \\
\hline$C_{43}$ & Cu65 & 25 & & 25 & \\
\hline
\end{tabular}




\begin{tabular}{|c|c|c|c|c|c|}
\hline Sample Id. & lsotope & $\begin{array}{l}\text { Concentration } \\
\text { (ug/) }\end{array}$ & $\begin{array}{l}\text { Std.Doviation } \\
\text { (ugh) }\end{array}$ & $\begin{array}{l}\text { Element Avg. Conc. } \\
\text { (ug/L) }\end{array}$ & $\begin{array}{c}\text { Element Avg. Std. Dov. } \\
\text { (ugL) }\end{array}$ \\
\hline $\mathrm{C} 11$ & $F_{0}$ & & & & \\
\hline$C_{12}$ & $F_{0}$ & & & & \\
\hline $\mathrm{C} 13$ & $F_{\theta}$ & 617 & 29.6 & 617 & 29.6 \\
\hline $\mathrm{C14}$ & Fo & 1000 & 57.1 & 1090 & 57.1 \\
\hline C21 & Fo & & & & \\
\hline G21R & $F_{0}$ & & & & \\
\hline $\mathrm{C} 22$ & $F \theta$ & 65.3 & 1.4 & 65.3 & 1.4 \\
\hline$c 23$ & Fo & 49.8 & 6.49 & 49.8 & 6.49 \\
\hline C31 & $F_{0}$ & & & & \\
\hline $\mathrm{C} 32$ & Fo & 7.85 & 1.51 & 7.85 & 1.51 \\
\hline $\mathrm{C} 33$ & Fo & 22 & 9.51 & 22 & 9.51 \\
\hline$C 41$ & $F_{\theta}$ & & & & \\
\hline $\mathrm{C} 42$ & $F_{0}$ & 14.3 & 8.62 & 14.3 & 8.62 \\
\hline$C_{43}$ & $F_{\theta}$ & 15.6 & 9.13 & 15.6 & 9.13 \\
\hline
\end{tabular}




\section{Transect C Porewater Concentrations: Lead}

\begin{tabular}{|c|c|c|c|c|c|}
\hline Sample Id. & botope & $\begin{array}{l}\text { Concantration } \\
\text { (ugh) }\end{array}$ & $\begin{array}{l}\text { 8ttidovition } \\
\text { (ugh) }\end{array}$ & $\begin{array}{l}\text { Eloment Avg. Conc. } \\
\text { (ugh) }\end{array}$ & $\begin{array}{c}\text { Element Avg. Std. Dev. } \\
\text { (ugh) }\end{array}$ \\
\hline
\end{tabular}

\begin{tabular}{|c|c|c|c|c|c|}
\hline C11 & Pb206 & & & & \\
\hline C11 & Pb207 & & & & \\
\hline C11 & Pb208 & & & & \\
\hline $\mathrm{C}_{12}$ & $P b 206$ & & & & \\
\hline$C_{12}$ & $P b 207$ & & & & \\
\hline $\mathrm{C}_{12}$ & $P_{6200}$ & & & & \\
\hline $\mathrm{C13}$ & Pt206 & 21.8 & .82 & & \\
\hline $\mathrm{C}_{13}$ & $\mathrm{Pb207}$ & 21.4 & 2.66 & & \\
\hline C13 & Pb208 & 212 & 1.04 & 21.5 & 1.51 \\
\hline $\mathrm{C}_{14}$ & Pt206 & 7.5 & & & \\
\hline $\mathrm{C}_{14}$ & $\mathrm{Pb207}$ & 7.5 & & & \\
\hline $\mathrm{C}_{14}$ & $\mathrm{~Pb} 208$ & 7.5 & & 7.5 & \\
\hline$C 21$ & Pb206 & & & & \\
\hline$C_{21}$ & $P 6207$ & & & & \\
\hline C21 & $\mathrm{Pb208}$ & & & & \\
\hline C21R & $\mathrm{Pb206}$ & & & & \\
\hline C21R & $P 6207$ & & & & \\
\hline C21R & Pb208 & & & & \\
\hline$c 22$ & $\mathrm{Pb206}$ & 7.5 & & & \\
\hline $\cos$ & $\mathrm{Pb207}$ & 7.5 & & & \\
\hline $\cos 2$ & $\mathrm{Pb208}$ & 7.5 & & 7.5 & \\
\hline$C_{23}$ & Pt206 & 18.8 & 128 & & \\
\hline$c_{23}$ & Pt207 & 17.2 & .65 & & \\
\hline$C_{23}$ & $\mathrm{~Pb} 208$ & 18.6 & .3 & 18.6 & .74 \\
\hline C31 & Pb206 & & & & \\
\hline C31 & Po207 & & & & \\
\hline C31 & Ft208 & & & & \\
\hline$c_{32}$ & $\mathrm{~Pb} 206$ & 425 & & & \\
\hline c32 & $\mathrm{Pb} 207$ & 425 & & & \\
\hline C32 & $\mathrm{Pb} 208$ & 425 & & 425 & \\
\hline C.33 & Pb206 & 58.5 & 8.1 & & \\
\hline$C_{33}$ & Pb207 & 47.7 & 4.79 & & \\
\hline CO3 & $\mathrm{Pb} 208$ & 54.6 & 2.53 & 53.6 & 5.14 \\
\hline$C_{41}$ & Pb206 & & & & \\
\hline $\mathrm{CA1}_{1}$ & Pb207 & & & & \\
\hline C41 & Pb208 & & & & \\
\hline $\mathrm{C}_{42}$ & Pb206 & 4.25 & & & \\
\hline $\mathrm{CA2}_{4}$ & $\mathrm{~Pb} 207$ & 425 & & & \\
\hline $\mathrm{C}_{42}$ & Pt208 & 4.25 & & 4.25 & \\
\hline $\mathrm{C}_{43}$ & Pb206 & 1 & & & \\
\hline $\mathrm{C}_{43}$ & Pb207 & 1 & & & \\
\hline $\mathrm{C}_{43}$ & $\mathrm{~Pb} 208$ & 1 & & 1 & \\
\hline
\end{tabular}




\section{Transect C Porewater Concentrations: Magnesium}

\begin{tabular}{|c|c|c|c|c|c|}
\hline Sample ld. & leotope & $\begin{array}{l}\text { Concentration } \\
\text { (ug/) }\end{array}$ & $\begin{array}{l}\text { Sid.Doviation } \\
\text { (ugL) }\end{array}$ & $\begin{array}{l}\text { Element Avg. Conc. } \\
\text { (ug/) }\end{array}$ & $\begin{array}{l}\text { Element Avg. Std. Dov. } \\
\text { (ugh) }\end{array}$ \\
\hline C11 & $\mathrm{Mg} 24$ & & & & \\
\hline$C_{11}$ & Mo25 & & & & \\
\hline $\mathrm{C}_{12}$ & MF24 & & & & \\
\hline $\mathrm{C} 12$ & MF25 & & & & \\
\hline C13 & $M F 24$ & 19700 & 110 & & \\
\hline $\mathrm{C} 13$ & Mg25 & 17600 & 77.6 & 18700 & 93.8 \\
\hline C14 & Mog4 & 1630 & 39.9 & & \\
\hline C14 & Mg25 & 1330 & 41.5 & 1480 & 40.7 \\
\hline$C_{1}$ & $M g 24$ & & & & \\
\hline C21 & M925 & & & & \\
\hline C21R & $M 924$ & & & & \\
\hline C21R & Mg25 & & & & \\
\hline $\mathrm{C}_{22}$ & Mge 4 & 921 & 13.6 & & \\
\hline $\mathrm{CO2}_{2}$ & M925 & 914 & 20 & 918 & 16.8 \\
\hline C23 & $M 924$ & 101 & 1.05 & & \\
\hline C23 & M925 & 95.4 & 5.14 & 982 & 3.1 \\
\hline Co31 & M924 & & & & \\
\hline Ca1 & Mg25 & & & & \\
\hline C32 & M924 & 216 & 2.85 & & \\
\hline C32 & My25 & 208 & 8.66 & 212 & 5.76 \\
\hline Cas & M924 & 141 & 622 & & \\
\hline C33 & Mg25 & 127 & 6.88 & 134 & 6.55 \\
\hline$C_{41}$ & Mg24 & & & & \\
\hline C41 & Mg25 & & & & \\
\hline$C_{42}$ & Mg24 & 797 & 13.2 & & \\
\hline$C_{42}$ & Mg:5 & 771 & 16.5 & 784 & 14.9 \\
\hline $\mathrm{C}_{43}$ & Mg24 & 932 & 68.2 & & \\
\hline$C_{43}$ & Mg25 & 804 & 19.1 & 868 & 43.7 \\
\hline
\end{tabular}




\section{Transect C Porewater Concentrations: Manganese}

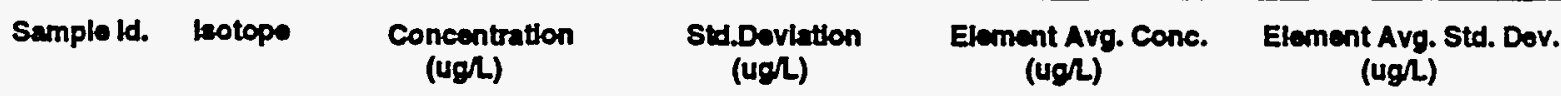

\begin{tabular}{|c|c|c|c|c|c|}
\hline C11 & $\mathrm{Mn}$ & & & & \\
\hline $\mathrm{C}_{12}$ & $\mathrm{Mn}$ & & & & \\
\hline $\mathrm{C13}$ & $\mathrm{Mn}$ & 7130 & 49 & 7130 & 49 \\
\hline C14 & $\mathrm{Mn}$ & 708 & 12.6 & 708 & 12.6 \\
\hline C21 & $\mathrm{Mn}$ & & & & \\
\hline C21R & $\mathrm{Mn}$ & & & & \\
\hline $\mathrm{CP2}$ & $\mathrm{Mn}$ & 450 & 4.63 & 450 & 4.63 \\
\hline $\mathrm{C}_{23}$ & $\mathrm{Mn}$ & 138 & .64 & 138 & .64 \\
\hline C31 & $\mathrm{Mn}$ & & & & \\
\hline $\mathrm{C} 32$ & $\mathrm{Mn}$ & 24.1 & 1.3 & 24.1 & 1.3 \\
\hline $\mathrm{C} 33$ & $\mathrm{Mn}$ & 200 & 6.59 & 200 & 6.59 \\
\hline $\mathrm{C}_{41}$ & $\mathrm{Mn}$ & & & & \\
\hline $\mathrm{C} 42$ & $\mathrm{Mn}$ & 9.72 & .65 & 9.72 & .65 \\
\hline $\mathrm{C}_{43}$ & Mn & 226 & 6.84 & 2266 & 6.84 \\
\hline
\end{tabular}

Nota: The actual concentration of aamples for which no standard devlatton bs reported bs lees than the lower ilmit of detection. The reported concentration ts the lower IImit of quantification. 


\begin{tabular}{|c|c|c|c|c|c|}
\hline Sample Id. & Esotope & $\begin{array}{l}\text { Concentration } \\
\text { (ugl) }\end{array}$ & $\begin{array}{l}\text { Std.Doviation } \\
\text { (4ag) }\end{array}$ & $\begin{array}{l}\text { Element Avg. Conc. } \\
\text { (ug })\end{array}$ & $\begin{array}{c}\text { Element Avg. Std. Dov. } \\
\text { (ugl) }\end{array}$ \\
\hline C11 & Ni60 & & & & \\
\hline$C_{11}$ & Nis2 & & & & \\
\hline $\mathrm{C}_{12}$ & Ni60 & & & & \\
\hline $\mathrm{C} 12$ & Nife & & & & \\
\hline $\mathrm{C}_{13}$ & Ni60 & 135 & 4.7 & & \\
\hline $\mathrm{C} 13$ & Ni62 & 152 & 8.74 & 144 & 6.72 \\
\hline$C 14$ & Ni6o & 71.8 & 1.97 & & \\
\hline $\mathrm{C} 14$ & $N 162$ & 96.5 & 132 & 842 & 7.59 \\
\hline C21 & Ni60 & & & & \\
\hline C21 & $\mathrm{Ni62}$ & & & & \\
\hline C21R & $\mathrm{Ni60}$ & & & & \\
\hline C21R & Ni62 & & & & \\
\hline $\cos 2$ & Ni60 & 30.1 & 1.52 & & \\
\hline $\mathrm{COS2}_{2}$ & Ni62 & 25 & 1.83 & 27.6 & 1.68 \\
\hline $\mathrm{C}_{23}$ & Ni6o & 20 & 1.78 & & \\
\hline $\mathrm{C} 23$ & NI62 & 19.7 & 2.73 & 18.9 & 2.26 \\
\hline C.31 & Ni60 & & & & \\
\hline C.31 & Ni62 & & & & \\
\hline $\mathrm{C32}$ & Ni60 & 10.7 & .57 & & \\
\hline C32 & Ni62 & 6.46 & 4.66 & 8.58 & 2.62 \\
\hline C33 & Ni6o & 30.9 & 13 & & \\
\hline 0.33 & Ni62 & & & 30.9 & 13 \\
\hline $\mathrm{C}_{41}$ & Ni60 & & & & \\
\hline $\mathrm{C} 41$ & Ni62 & & & & \\
\hline $\mathrm{C} 42$ & Ni60 & 3.99 & .64 & & \\
\hline $\mathrm{C} 42$ & Ni62 & 4.3 & 2.51 & 4.14 & 1.58 \\
\hline $\mathrm{C}_{43}$ & Ni60 & 26.7 & 9.05 & & \\
\hline $\mathrm{CA3}$ & Ni62 & .75 & & 13.7 & 9.05 \\
\hline
\end{tabular}




\section{Transect C Porewater Concentrations: Selenium}

\begin{tabular}{|c|c|c|c|c|c|}
\hline Sumple Id. & leotope & $\begin{array}{l}\text { Concentration } \\
\text { (ugh) }\end{array}$ & $\begin{array}{l}\text { Std.Dovintion } \\
\text { (ugll) }\end{array}$ & $\begin{array}{l}\text { Element Avg. Conc. } \\
\text { (ugh) }\end{array}$ & $\begin{array}{c}\text { Element Avg. Std. Dov. } \\
\text { (ugh) }\end{array}$ \\
\hline C11 & Se77 & & & & \\
\hline C11 & Seg2 & & & & \\
\hline $\mathrm{C12}$ & 5077 & & & & \\
\hline C12 & Sage & & & & \\
\hline C13 & So77 & 58.4 & 20.9 & & \\
\hline $\mathrm{C13}$ & Se82 & 56 & 20 & 572 & 20.5 \\
\hline C14 & Se77 & 37.5 & & & \\
\hline C14 & Se82 & 37.5 & & 37.5 & \\
\hline 021 & Se77 & & & & \\
\hline C21 & So82 & & & & \\
\hline C21R & $\mathrm{SeT}$ & & & & \\
\hline C21R & So82 & & & & \\
\hline C22 & Se77 & 37.5 & & & \\
\hline 022 & Se82 & 37.5 & & 37.5 & \\
\hline $\mathrm{CO23}$ & Se77 & 4.28 & 5.33 & & \\
\hline C23 & 5082 & 4.19 & 5.33 & 424 & 5.33 \\
\hline C31 & Se77 & & & & \\
\hline 631 & So82 & & & & \\
\hline $\operatorname{co3} 2$ & Se77 & 12 & 7.89 & & \\
\hline $\mathrm{C} 32$ & Se82 & 11.6 & 7.7 & 11.8 & 7.8 \\
\hline C33 & Se77 & 25 & & & \\
\hline 633 & Se82 & 25 & & 25 & \\
\hline CA1 & Se77 & & & & \\
\hline C41 & Se82 & & & & \\
\hline $\mathrm{C} 42$ & Se77 & 12.5 & 2.84 & & \\
\hline $\mathrm{C} 42$ & Se82 & 122 & 2.85 & 12.4 & 2.85 \\
\hline $\mathrm{C43}$ & Se77 & 35.5 & 3.7 & & \\
\hline $\mathrm{CA3}$ & Se82 & 35.5 & 3.5 & 35.5 & 3.6 \\
\hline
\end{tabular}




\begin{tabular}{|c|c|c|c|c|c|}
\hline \multicolumn{6}{|c|}{ Transect C Porewater Concentrations: Silver } \\
\hline Sempte id. & kotope & $\begin{array}{l}\text { Concentration } \\
(u g / L)\end{array}$ & $\begin{array}{l}\text { Stdooviation } \\
\text { (ug/L) }\end{array}$ & $\begin{array}{l}\text { Eloment Avg. Conc. } \\
\text { (ugh) }\end{array}$ & $\begin{array}{c}\text { Element Avg. Std. Dov. } \\
\text { (ugh) }\end{array}$ \\
\hline C11 & Ag107 & & & & \\
\hline C11 & Ag100 & & & & \\
\hline C12 & Ag107 & & & & \\
\hline$C 12$ & Ad109 & & & & \\
\hline $\mathrm{C}_{13}$ & Ad107 & 15 & & & \\
\hline C13 & Ag109 & 15 & & 15 & \\
\hline $\mathrm{C}_{14}$ & Ag107 & 15 & & & \\
\hline $\mathrm{C14}_{4}$ & A0100 & 15 & & 15 & \\
\hline$C_{21}$ & A9107 & & & & \\
\hline C21 & Ad100 & & & & \\
\hline C21R & Ag107 & & & & \\
\hline C21R & $A g 100$ & & & & \\
\hline$\infty 2$ & Ag107 & 15 & & & . \\
\hline$c_{22}$ & Ag109 & 15 & & 15 & \\
\hline C23 & Ag107 & 98.3 & 22.8 & & \\
\hline$C_{23}$ & Ag109 & 87.4 & 12.6 & 92.9 & 17.7 \\
\hline C31 & Ag107 & & & & \\
\hline \$1 & Ad109 & & & & \\
\hline 932 & Ag107 & 27.3 & 14.8 & & \\
\hline c32 & Ag109 & 29.5 & 16 & 28.4 & 15.4 \\
\hline C33 & Ag107 & 49 & 10.7 & & \\
\hline CO3 & $A 0100$ & 33.6 & 19.4 & 41.3 & 15.1 \\
\hline $\mathrm{C} 41$ & Ag107 & & & & \\
\hline$C 41$ & Ag109 & & & & \\
\hline$C_{42}$ & Ag107 & 972 & 22.7 & & \\
\hline $\mathrm{C}_{42}$ & Ag100 & 92.4 & 11.7 & 94.8 & 172 \\
\hline $\mathrm{C} 43$ & Ag107 & 10.2 & 2.98 & & \\
\hline$C_{43}$ & Ag109 & 10 & & 10.1 & 2.98 \\
\hline
\end{tabular}




\section{Transect C Porewater Concentrations: Sodium}

\begin{tabular}{|c|c|c|c|c|c|}
\hline Sample ld. & Eotope & $\begin{array}{l}\text { Concentration } \\
\text { (ug/L) }\end{array}$ & $\begin{array}{l}\text { Stod.Doviation } \\
\text { (ugh) }\end{array}$ & $\begin{array}{l}\text { Element Avg. Conc. } \\
\text { (ugh) }\end{array}$ & $\begin{array}{c}\text { Element Avg. Std. Dov. } \\
\text { (ugL) }\end{array}$ \\
\hline C11 & $\mathrm{Na}$ & & & & \\
\hline $\mathrm{C} 12$ & $\mathrm{Na}$ & & & & \\
\hline $\mathrm{C13}$ & $\mathrm{Na}$ & 47800 & 1360 & 47800 & 1360 \\
\hline $\mathrm{C14}$ & $\mathrm{Na}$ & 61200 & 1490 & 61200 & 1490 \\
\hline C21 & $\mathrm{Na}$ & & & & \\
\hline C21R & $\mathrm{Na}$ & & & & \\
\hline 922 & $\mathrm{Na}$ & $7 \infty$ & 156 & $77 \infty$ & 156 \\
\hline $\mathrm{C23}$ & $\mathrm{Na}$ & 6390 & 158 & 6390 & 158 \\
\hline c31 & $\mathrm{Na}$ & & & & \\
\hline $\mathbf{C 3 2}$ & $\mathrm{Na}$ & 3780 & 592 & 3780 & 592 \\
\hline $\mathrm{CO33}$ & $\mathrm{Na}$ & 1340 & 902 & 1340 & 902 \\
\hline $\mathrm{C}_{41}$ & $\mathrm{Na}$ & & & & \\
\hline $\mathrm{C} 42$ & $\mathrm{Na}$ & 4960 & 67.5 & 4960 & 67.5 \\
\hline $\mathrm{CA3}$ & $\mathrm{Na}$ & 1570 & 427 & 1570 & 427 \\
\hline
\end{tabular}

Note: The ectual concentration of samples for which no stendard dovlation be reported la lees than the lower llmit of detection. The reported concentration b the lower limit of quantification. 


\section{Transect C Porewater Concentrations: Strontium}

\begin{tabular}{|c|c|c|c|c|c|}
\hline Sample ld. & leotope & $\begin{array}{l}\text { Concentration } \\
\text { (ugl) }\end{array}$ & $\begin{array}{l}\text { Std.Dovlation } \\
\text { (ug/L) }\end{array}$ & $\begin{array}{l}\text { Element Avg. Conc. } \\
\text { (ugh) }\end{array}$ & $\begin{array}{c}\text { Element Avg. Std. Dov. } \\
(u g h)\end{array}$ \\
\hline $\mathrm{C} 11$ & Sr & & & & \\
\hline $\mathrm{C}_{12}$ & $\mathrm{Sr}$ & & & & \\
\hline $\mathrm{C}_{13}$ & $\mathrm{Sr}$ & 216 & 3.93 & 216 & 3.93 \\
\hline $\mathrm{C14}$ & Sr & 25.5 & .72 & 25.5 & .72 \\
\hline$c 21$ & $\mathrm{Sr}$ & & & & \\
\hline C21R & $\mathrm{Sr}$ & & & & \\
\hline $\mathrm{C}_{22}$ & Sr & 14.8 & 27 & 14.8 & 27 \\
\hline$C_{23}$ & $\mathrm{Sr}$ & 6.95 & 2 & 6.95 & 2 \\
\hline $\mathrm{C}_{31}$ & $\mathrm{Sr}$ & & & & \\
\hline $\mathrm{C} 32$ & $\mathrm{Sr}$ & 5.15 & 2 & 5.15 & 2 \\
\hline C.33 & $\mathrm{Sr}$ & 7.83 & 1.17 & 7.83 & 1.17 \\
\hline $\mathrm{C41}$ & $\mathrm{Sr}$ & & & & \\
\hline $\mathrm{C} 42$ & $\mathrm{Sr}$ & 10.7 & .41 & 10.7 & .41 \\
\hline $\mathrm{C}_{43}$ & $\mathrm{Sr}$ & 22.5 & 2.69 & 22.5 & 2.69 \\
\hline
\end{tabular}




\section{Transect C Porewater Concentrations: Thorium}

\begin{tabular}{|c|c|c|c|c|c|}
\hline Semple Id. & botopo & $\begin{array}{l}\text { Concentration } \\
\text { (ug/) }\end{array}$ & $\begin{array}{l}\text { Std.Devilation } \\
\text { (ug/L) }\end{array}$ & $\begin{array}{l}\text { Element Avg. Conc. } \\
\text { (ugh) }\end{array}$ & $\begin{array}{c}\text { Ejement Avg. Std. Dov. } \\
\text { (Ugh) }\end{array}$ \\
\hline C11 & Th232 & & & & \\
\hline $\mathrm{C} 12$ & Th232 & & & & \\
\hline $\mathrm{C13}$ & Th232 & .5 & & .5 & \\
\hline $\mathrm{C} 14$ & Th232 & .5 & & .5 & \\
\hline C21 & Th232 & & & & \\
\hline C21R & Th232 & & & & \\
\hline$c 22$ & Th232 & .5 & & .5 & \\
\hline $\mathrm{C} 23$ & Th232 & .38 & & .38 & \\
\hline C11 & Th232 & & & & \\
\hline C32 & Th232 & .38 & & .38 & \\
\hline C.33 & Th232 & 25 & & 25 & \\
\hline C41 & Th232 & & & & \\
\hline $\mathrm{CA2}_{2}$ & Th232 & .38 & & .38 & \\
\hline $\mathrm{C}_{43}$ & Th232 & 25 & & 25 & \\
\hline
\end{tabular}

Note: The actual concentration of samples for which no standard deviation is neported ts lese than the lower llmit of detection. The reported concentration is the fower limit of quantification. 


\section{Transect C Porewater Concentrations: Tin}

\begin{tabular}{|c|c|c|c|c|c|}
\hline Sampleft. & leotope & $\begin{array}{l}\text { Concentration } \\
(u g /)\end{array}$ & $\begin{array}{l}\text { Std.Doviation } \\
\text { (ug/ })\end{array}$ & $\begin{array}{l}\text { Element Avg. Conc. } \\
\text { (ugh) }\end{array}$ & $\begin{array}{c}\text { Element Avg. Std. Dov. } \\
\text { (ug })\end{array}$ \\
\hline$C 11$ & $\operatorname{Sn} 118$ & & & & \\
\hline C11 & $\operatorname{Sn} 120$ & & & & \\
\hline$C_{12}$ & Sn118 & & & & \\
\hline $\mathrm{C}_{12}$ & $\sin 120$ & & & & \\
\hline $\mathrm{C} 13$ & Sn118 & 25 & & & \\
\hline C13 & $\operatorname{sn} 120$ & 25 & & 25 & \\
\hline C14 & Sn118 & 25 & & & \\
\hline $\mathrm{C14}$ & $\operatorname{Sn} 120$ & 25 & & 25 & \\
\hline$C_{21}$ & Sn118 & & & & \\
\hline$C_{21}$ & Sn120 & & & & \\
\hline C21R & Sn118 & & & & \\
\hline C21R & Sn120 & & & & \\
\hline$c 22$ & Sn118 & 25 & & & \\
\hline$c 22$ & $\sin 120$ & 25 & & 25 & \\
\hline$C_{23}$ & Sn118 & 1.38 & & & \\
\hline$c_{23}$ & $\sin 120$ & 1.38 & & 1.38 & \\
\hline C31 & Sn118 & & & & \\
\hline C31 & $\operatorname{Sn} 120$ & & & & \\
\hline 032 & Sn118 & 1.38 & & & \\
\hline Cos2 & Sn 120 & 1.38 & & 1.38 & \\
\hline 0.33 & Sn118 & 2.5 & & & \\
\hline C.33 & Sn120 & 2.5 & & 2.5 & \\
\hline$C 41$ & $\sin 118$ & & & & \\
\hline$C_{41}$ & $\sin 120$ & & & & \\
\hline $\mathrm{C42}$ & Sn118 & 13.8 & & & \\
\hline$C_{42}$ & $\operatorname{Sn} 120$ & 13.8 & & 13.8 & \\
\hline $\mathrm{C} 43$ & Sn118 & 2.5 & & & \\
\hline$C_{43}$ & $\operatorname{Sn} 120$ & 2.5 & & 2.5 & \\
\hline
\end{tabular}




\section{Transect C Porewater Concentrations: Uranium-235}

\begin{tabular}{|c|c|c|c|c|c|}
\hline Sample ld. & leotope & $\begin{array}{l}\text { Concentration } \\
\text { (Ug/L) }\end{array}$ & $\begin{array}{l}\text { Std.Deviation } \\
\text { (ug/L) }\end{array}$ & $\begin{array}{l}\text { Element Avg. Cons. } \\
\text { (ugh) }\end{array}$ & $\begin{array}{c}\text { Element Avg. Std. Dov. } \\
\text { (ugh) }\end{array}$ \\
\hline C11 & U235 & & & & \\
\hline $\mathrm{C} 12$ & U235 & & & & \\
\hline $\mathrm{C13}$ & U235 & 8.02 & .67 & 8.02 & .67 \\
\hline $\mathrm{C}_{14}$ & v235 & 129 & .11 & 129 & .11 \\
\hline C21 & U235 & & & & \\
\hline C21R & U235 & & & & \\
\hline$c 22$ & U235 & .3 & .08 & .3 & .08 \\
\hline $\mathrm{C} 23$ & U235 & .03 & .02 & .03 & .02 \\
\hline C31 & U235 & & & & \\
\hline C32 & U235 & .02 & & .02 & \\
\hline C33 & U235 & 2.5 & & 2.5 & \\
\hline $\mathrm{C}_{41}$ & U235 & & & & \\
\hline $\mathrm{C} 42$ & U235 & .02 & & .02 & . \\
\hline$C 43$ & U235 & .02 & & .02 & \\
\hline
\end{tabular}


Transect C Porewater Concentrations: Uranium-238

\begin{tabular}{|c|c|c|c|c|c|}
\hline Sample ld. & Letope & $\begin{array}{l}\text { Concentration } \\
\text { (ug/L) }\end{array}$ & $\begin{array}{l}\text { Std.Doviation } \\
\text { (ug/h) }\end{array}$ & $\begin{array}{c}\text { Element Avg. Conc. } \\
\text { (Ua/L) }\end{array}$ & $\begin{array}{c}\text { Element Avg. Std. Dov. } \\
\text { (ug/) }\end{array}$ \\
\hline $\mathrm{C11}$ & U238 & & & & \\
\hline $\mathrm{C}_{12}$ & U238 & & & & \\
\hline $\mathrm{C} 13$ & U238 & 1340 & 98.8 & 1340 & 98.8 \\
\hline C14 & $\mathrm{U} 238$ & 271 & 2.19 & 271 & 2.19 \\
\hline$\infty 21$ & $\sqrt{238}$ & & & & \\
\hline C21R & U238 & & & & \\
\hline$\infty$ & U238 & 69.4 & 3.34 & 69.4 & 3.34 \\
\hline $\mathrm{CO}_{23}$ & U238 & 5.55 & .15 & 5.55 & .15 \\
\hline C31 & U238 & & & & \\
\hline $\mathrm{C32}$ & U238 & .75 & & .75 & \\
\hline C.33 & U238 & .55 & 0 & .55 & 0 \\
\hline $\mathrm{CA1}$ & U238 & & & & \\
\hline $\mathrm{C} 42$ & U238 & .75 & & .75 & \\
\hline $\mathrm{C}_{43}$ & U238 & .19 & .01 & .19 & .01 \\
\hline
\end{tabular}




\section{Transect C Porewater Concentrations: Vanadium}

Sample ld, botope

Concentration

(ugl)
Std.Devlation

(ug/)
Element Avg. Conc. (ug/)
Element Avg. Std. Dov.

(ugh)

\begin{tabular}{|c|c|c|c|c|c|}
\hline C11 & $v$ & & & & \\
\hline $\mathrm{C}_{12}$ & V & & & & \\
\hline$C_{13}$ & v & & & & \\
\hline $\mathrm{C}_{14}$ & $v$ & 25 & & 25 & \\
\hline C21 & $v$ & & & & \\
\hline C21R & $v$ & & & & \\
\hline c22 & $v$ & .57 & .31 & .57 & .31 \\
\hline $\mathrm{C23}$ & $v$ & 3.25 & & 325 & \\
\hline C.31 & $v$ & & & & \\
\hline C.32 & 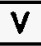 & 6.38 & & 6.38 & \\
\hline $\mathrm{CO3}$ & $v$ & 625 & & 6.25 & \\
\hline $\mathrm{C}_{41}$ & $v$ & & & & \\
\hline $\mathrm{C} 42$ & $\mathbf{v}$ & 3.25 & & 3.25 & \\
\hline$C_{43}$ & v & 6.25 & & 625 & \\
\hline
\end{tabular}

Note: The sctual concentration of samples for which no standard deviation le reported is leas than the lower limit of detection. The reported concentration bs the lower limit of quantfication. 


\section{Transect C Porewater Concentrations: Yttrium}

\begin{tabular}{|c|c|c|c|c|c|}
\hline Sample ld. & botope & $\begin{array}{l}\text { Concentration } \\
\text { (ug/L) }\end{array}$ & $\begin{array}{l}\text { Std.Daviation } \\
\text { (ugl) }\end{array}$ & $\begin{array}{l}\text { Element Avg. Conc. } \\
\text { (ugh) }\end{array}$ & $\begin{array}{c}\text { Element Avg. Std. Dov. } \\
\text { (ugL) }\end{array}$ \\
\hline C11 & $Y$ & & & & \\
\hline $\mathrm{C} 12$ & $Y$ & & & & \\
\hline $\mathrm{C} 13$ & $Y$ & 658 & 3.14 & 658 & 3.14 \\
\hline C14 & $Y$ & 90.1 & .83 & $\$ 0.1$ & .83 \\
\hline C21 & $Y$ & & & & \\
\hline C21R & $Y$ & & & & \\
\hline $\mathrm{CO2}$ & $Y$ & 4.53 & .07 & 4.53 & .07 \\
\hline $\mathrm{CO23}$ & $Y$ & .32 & .06 & .32 & .06 \\
\hline C31 & $Y$ & & & & \\
\hline $\mathrm{C} 32$ & $Y$ & .18 & .02 & .18 & .02 \\
\hline C33 & $Y$ & 11.4 & 26 & 11.4 & 26 \\
\hline $\mathrm{C}_{41}$ & $Y$ & & & & \\
\hline C42 & $Y$ & 25 & & 25 & \\
\hline $\mathrm{C} 43$ & $Y$ & 1.86 & .12 & 1.86 & .12 \\
\hline
\end{tabular}




\section{Transect C Porewater Concentrations: Zinc}

\begin{tabular}{|c|c|c|c|c|c|}
\hline Sample ld. & Isotope & $\begin{array}{l}\text { Concentration } \\
\text { (ug/t) }\end{array}$ & $\begin{array}{l}\text { Std.Dovintion } \\
\text { (ug/L) }\end{array}$ & $\begin{array}{l}\text { Element Avg. Conc. } \\
\text { (ugh) }\end{array}$ & $\begin{array}{c}\text { Element Avg. Std. Dov. } \\
\text { (ugL) }\end{array}$ \\
\hline $\mathrm{C} 11$ & Zn66 & & & & \\
\hline $\mathrm{C}_{12}$ & Zn66 & & & & \\
\hline C13 & Zn66 & 316 & 10.7 & 316 & 10.7 \\
\hline $\mathrm{C}_{14}$ & Zn66 & 85.9 & 3.03 & 85.9 & 3.03 \\
\hline C21 & Zns6 & & & & \\
\hline C21R & Zn66 & & & & \\
\hline$c 22$ & Zn66 & 35.5 & 1.43 & 35.5 & 1.43 \\
\hline 623 & Zn66 & 32.7 & 32 & 32.7 & 32 \\
\hline C31 & Zn66 & & & & \\
\hline C32 & Zn66 & 19.9 & 1.39 & 19.9 & 1.39 \\
\hline $\mathrm{CB3}$ & Zn66 & 37.5 & & 37.5 & \\
\hline 041 & Zn66 & & & & \\
\hline $\mathrm{C42}$ & Zn66 & 4.87 & 1.81 & 4.87 & 1.81 \\
\hline$C_{43}$ & Zn66 & 682 & 23.1 & 682 & 23.1 \\
\hline
\end{tabular}

Note: The actual concentration of eamples for which no atandard doviation is reported bs lese than the lower llmit of detection. The reported concentration la the lower limit of quantification. 


\begin{tabular}{|c|c|c|c|c|c|}
\hline \multicolumn{6}{|c|}{ Transect C Porewater Concentrations: Zirconium } \\
\hline Sample ld. & Eotope & $\begin{array}{l}\text { Concentration } \\
\text { (ugh) }\end{array}$ & $\begin{array}{l}\text { Std.Doviation } \\
\text { (ugh) }\end{array}$ & $\begin{array}{l}\text { Element Avg. Conc. } \\
\text { (ug/) }\end{array}$ & $\begin{array}{c}\text { Element Avg. Std. Dov. } \\
\text { (Ug })\end{array}$ \\
\hline $\mathrm{C}_{11}$ & $Z=0$ & & & & \\
\hline C11 & Zro1 & & & & \\
\hline$C_{12}$ & 2790 & & & & \\
\hline$C_{12}$ & Zra1 & & & & \\
\hline$C_{13}$ & $Z 590$ & .5 & & & \\
\hline $\mathrm{C}_{13}$ & 2991 & .5 & & .5 & \\
\hline $\mathrm{C}_{14}$ & 2590 & .5 & & & \\
\hline C14 & Zr91 & .5 & & .5 & \\
\hline$C_{21}$ & 2590 & & & & \\
\hline$C_{21}$ & $Z 91$ & & & & \\
\hline C21R & $Z 990$ & & & & \\
\hline C21R & Zr91 & & & & \\
\hline$c_{22}$ & Zr90 & .5 & & &. \\
\hline$c 22$ & Zr91 & .5 & & .5 & \\
\hline$c 23$ & $Z 90$ & 1.5 & & & \\
\hline$c 23$ & 291 & 1.5 & & 1.5 & \\
\hline C31 & $Z r 90$ & & & & \\
\hline C31 & $Z=91$ & & & & \\
\hline C32 & $Z r 90$ & 1.5 & & & \\
\hline C32 & $Z r 91$ & 1.5 & & 1.5 & \\
\hline C.33 & $Z r 90$ & 2.5 & & & \\
\hline C.33 & $Z 991$ & 2.5 & & 2.5 & \\
\hline $\mathrm{C}_{41}$ & $Z 90$ & & & & \\
\hline$C_{41}$ & Zr91 & & & & \\
\hline $\mathrm{C} 42$ & $Z 90$ & 1.5 & & & \\
\hline $\mathrm{C} 42$ & Zr91 & 1.5 & & 1.5 & \\
\hline $\mathrm{C} 43$ & $Z r 90$ & 2.5 & & & \\
\hline $\mathrm{C} 43$ & Zr91 & 2.5 & & 2.5 & \\
\hline
\end{tabular}




\section{Transect D Porewater Concentrations: Aluminum}

\begin{tabular}{|c|c|c|c|c|c|}
\hline Sample ld. & leotope & $\begin{array}{l}\text { Concentration } \\
\text { (ugh) }\end{array}$ & $\begin{array}{l}\text { Std.Doviation } \\
\text { (ug/L) }\end{array}$ & $\begin{array}{l}\text { Element Avg. Conc. } \\
\text { (U日L) }\end{array}$ & $\begin{array}{c}\text { Element Avg. Std. Dov. } \\
\text { (ug/) }\end{array}$ \\
\hline D11 & $\mathbf{A}$ & & & & \\
\hline D12 & $\mathbf{A}$ & & & & \\
\hline D13 & $\mathbf{A}$ & 19.4 & 1.19 & 19.4 & 1.19 \\
\hline D13RA & A & 1530 & 91 & 1530 & 91 \\
\hline D13RB & $\mathbf{A}$ & & & & \\
\hline D21 & $\mathbf{A}$ & & & & \\
\hline D41 & $\mathbf{N}$ & & & & \\
\hline D51 & $\mathbf{A}$ & & & & \\
\hline
\end{tabular}




\section{Transect D Porewater Concentrations: Arsenic}

Sample ld. Lotope $\begin{gathered}\text { Concentration } \\
\text { (Ugh) }\end{gathered}$
\begin{tabular}{|c|c|c|c|c|c|}
\hline D11 & As & & $\begin{array}{c}\text { Std.Doviation } \\
\text { (ugh) }\end{array}$ & $\begin{array}{c}\text { Element Avg. Conc. } \\
\text { (ugh) }\end{array}$ & $\begin{array}{c}\text { Element Avg. Std. Dov. } \\
\text { (ugh) }\end{array}$ \\
\hline D12 & As & & & & \\
\hline D13 & As & 4.17 & 3.63 & 3.17 & 3.63 \\
\hline D13RA & As & 38.3 & 12.7 & 38.3 & 12.7 \\
\hline D13RB & As & & & & \\
\hline D21 & As & & & & \\
\hline D41 & As & & & & \\
\hline D51 & As & & & & \\
\hline
\end{tabular}




\section{Transect D Porewater Concentrations: Barium}

\begin{tabular}{ccccc}
\hline Sample ld. leotopo & $\begin{array}{c}\text { Concentration } \\
\text { (ug/) }\end{array}$ & $\begin{array}{c}\text { Std.Dovlation } \\
(\mathrm{ug} / \mathrm{L})\end{array}$ & $\begin{array}{c}\text { Element Avg. Cone. } \\
(\mathrm{ug} / \mathrm{L})\end{array}$ & $\begin{array}{c}\text { Element Avg. Std. Dov. } \\
(\mathrm{ug} /)\end{array}$
\end{tabular}

\begin{tabular}{|l|l|l|l|l|l|}
\hline$D 11$ & Ba135 & & & \\
\hline$D 11$ & Ba137 & & & & \\
\hline$D 11$ & Ba138 & & & & \\
\hline$D 12$ & Ba135 & & & & \\
\hline$D 12$ & Ba137 & & & & \\
\hline$D 12$ & Ba138 & & & & \\
\hline$D 13$ & Ba135 & 26.1 & 2.44 & & \\
\hline$D 13$ & Ba137 & 25.3 & 2.55 & & \\
\hline$D 13$ & Ba138 & 26.2 & .97 & & \\
\hline$D 13 R A$ & Ba135 & 21 & 5.93 & & \\
\hline$D 13 R A$ & Ba137 & 35.6 & 13.4 & & \\
\hline$D 13 R A$ & Ba138 & 47.1 & 2.19 & & \\
\hline$D 13 R B$ & Ba135 & & & 34.6 & \\
\hline$D 13 R B$ & Ba137 & & & & \\
\hline$D 13 R B$ & Ba138 & & & & \\
\hline$D 21$ & Ba135 & & & & \\
\hline$D 21$ & Ba137 & & & & \\
\hline$D 21$ & Ba138 & & & & \\
\hline$D 41$ & Ba135 & & & & \\
\hline$D 41$ & Ba137 & & & & \\
\hline$D 41$ & Ba138 & & & & \\
\hline$D 51$ & Ba135 & & & & \\
\hline$D 51$ & Ba137 & & & & \\
\hline$D 51$ & Ba138 & & & & \\
\hline & & & & & \\
\hline
\end{tabular}

Note: The actual concentratton of eamples for which no standard deviation be reported be lees than the lowrer limit of detection. The reported concentration bs the lower llmit of quantification. 
Transect D Porewater Concentrations: Cadmium

\begin{tabular}{|c|c|c|c|c|c|}
\hline Sample ld. & leotope & $\begin{array}{l}\text { Concentration } \\
(u g / L)\end{array}$ & $\begin{array}{l}\text { Std.Doviation } \\
\text { (ugl) }\end{array}$ & $\begin{array}{c}\text { Element Avg. Conc. } \\
\text { (ug/l) }\end{array}$ & $\begin{array}{c}\text { Element Avg. Std. Dov. } \\
\text { (ugL) }\end{array}$ \\
\hline D11 & Cd111 & & & & \\
\hline D11 & Cd114 & & & & \\
\hline D12 & Cd111 & & & & \\
\hline $\mathrm{D} 12$ & Cd114 & & & & \\
\hline D13 & Cd111 & .75 & & & \\
\hline D13 & Co1114 & .75 & & .75 & \\
\hline DI3RA & Cod111 & .5 & & & \\
\hline D13RA & Cd114 & .5 & & .5 & \\
\hline D13RB & Cd111 & & & & \\
\hline D13RB & Cd114 & & & & \\
\hline D21 & $\operatorname{Cod111}$ & & & & \\
\hline 021 & Cd114 & & & & \\
\hline 041 & Cd111 & & & & \\
\hline 041 & Cd114 & & & & \\
\hline D51 & Cd111 & & & & \\
\hline D51 & Cd114 & & & & \\
\hline
\end{tabular}




\section{Transect D Porewater Concentrations: Calcium}

\begin{tabular}{|c|c|c|c|c|c|}
\hline Sample ld. & leotope & $\begin{array}{l}\text { Concentration } \\
\text { (ugl) }\end{array}$ & $\begin{array}{l}\text { Std.Deviation } \\
\text { (ugl) }\end{array}$ & $\begin{array}{l}\text { Element Avg. Conc. } \\
\text { (ugl) }\end{array}$ & $\begin{array}{c}\text { Element Avg. Std. Dov. } \\
\text { (Ugh) }\end{array}$ \\
\hline D11 & Ca44 & & & & \\
\hline D12 & Ca44 & & & & \\
\hline D13 & $\operatorname{Ca} 44$ & 11800 & 34.9 & 11800 & 34.9 \\
\hline D13RA & $\operatorname{Cos4}$ & 834 & 494 & 834 & 484 \\
\hline D13RB & $\operatorname{Ca} 44$ & & & & \\
\hline 021 & Ca44 & & & & \\
\hline D41 & Ca44 & & & & \\
\hline D51 & Ca44 & & & & \\
\hline
\end{tabular}




\section{Transect D Porewater Concentrations: Cesium}

\begin{tabular}{|c|c|c|c|c|c|}
\hline Samplo id. & botope & $\begin{array}{l}\text { Concentration } \\
\text { (ugl) }\end{array}$ & $\begin{array}{l}\text { Std.Doviation } \\
\text { (ugh }\end{array}$ & $\begin{array}{l}\text { Element Avg. Conc. } \\
\text { (ugh) }\end{array}$ & $\begin{array}{l}\text { Element Avg. Std. Dov. } \\
\text { (ugl) }\end{array}$ \\
\hline D11 & $\mathrm{Cs}$ & & & & \\
\hline D12 & Cs & & & & \\
\hline D13 & Cs & .11 & .07 & .11 & .07 \\
\hline D13RA & Cs & 25 & & 25 & \\
\hline D13RB & Cs & & & & \\
\hline $\mathrm{D} 21$ & $\mathrm{Cs}$ & & & & \\
\hline 041 & Cs & & & & \\
\hline D51 & Cs & & & & \\
\hline
\end{tabular}




\section{Transect D Porewater Concentrations: Chromium}

Semple ld. kotope

Concentration

Std.Dovlation

Element Avg. Conc.

Element Avg. Std. Dov.

(ugh)

(ugh)

(ugh)

(ug/)

\begin{tabular}{|c|c|c|c|c|c|}
\hline D11 & cr52 & & & & \\
\hline D11 & C553 & & & & \\
\hline D12 & 952 & & & & \\
\hline D12 & C.53 & & & & \\
\hline D13 & C552 & .95 & .35 & & \\
\hline D13 & C53 & .94 & .35 & .95 & .35 \\
\hline D13AA & $a 52$ & 4.97 & 3.09 & & \\
\hline D13RA & C553 & 5.01 & 3.11 & 4.99 & 3.1 \\
\hline D13RB & $C 52$ & & & & \\
\hline D13RB & $c_{53}$ & & & & \\
\hline D21 & C.52 & & & & \\
\hline D21 & C.53 & & & & \\
\hline$D 41$ & C52 & & & & \\
\hline$D 41$ & C.53 & & & & \\
\hline D51 & $C_{552}$ & & & & \\
\hline D51 & C.53 & & & & \\
\hline
\end{tabular}

Note: The ectual concentration of eamples for which no standard deviation be reported be leas than the fower limit of dotection. The reported concentration is the lower limit of quantincation. 


\begin{tabular}{|c|c|c|c|c|c|}
\hline Sample ld. & Eotope & $\begin{array}{l}\text { Concentration } \\
\text { (ugh) }\end{array}$ & $\begin{array}{l}\text { Std.Doviation } \\
\text { (ug/L) }\end{array}$ & $\begin{array}{c}\text { Element Avg. Cone. } \\
\text { (ug/) }\end{array}$ & $\begin{array}{c}\text { Element Avg. Std. Dov. } \\
\text { (ugh) }\end{array}$ \\
\hline D11 & $\infty$ & & & & \\
\hline D12 & $\infty$ & & & & \\
\hline D13 & $\infty$ & 13 & .51 & 13 & .51 \\
\hline D13RA & Co & 13.4 & 1.51 & 13.4 & 1.51 \\
\hline D13RB & $\infty$ & & & & \\
\hline D21 & $\infty$ & & & & \\
\hline D41 & Co & & & & \\
\hline D51 & Co & & & & \\
\hline
\end{tabular}




\begin{tabular}{|c|c|c|c|c|c|}
\hline Sample ld. & leotope & $\begin{array}{c}\text { Concentration } \\
\text { (ug/L) }\end{array}$ & $\begin{array}{l}\text { Std.Deviation } \\
\text { (Ug/L) }\end{array}$ & $\begin{array}{l}\text { Element Avg. Conc. } \\
\text { (ugh) }\end{array}$ & $\begin{array}{l}\text { Element Avg. Std. Dev. } \\
\text { (ugh) }\end{array}$ \\
\hline D11 & cu6s & & & & \\
\hline D11 & 0465 & & & & \\
\hline D12 & cu63 & & & & \\
\hline D12 & Cu65 & & & & \\
\hline D13 & Cu63 & 3.84 & 1.03 & & \\
\hline$D 13$ & Cu65 & 322 & 124 & 3.53 & 1.14 \\
\hline D13RA & Cu63 & & & & \\
\hline D13RA & Cu65 & 25 & & 25 & \\
\hline D13RB & Cu63 & & & & \\
\hline D13RB & Cu65 & & & & \\
\hline$D 21$ & Cu63 & & & & \\
\hline D21 & Cu65 & & & & \\
\hline D41 & Cu63 & & & & . \\
\hline D41 & Cu65 & & & & \\
\hline D51 & Cu63 & & & & \\
\hline D51 & Cu65 & & & & \\
\hline
\end{tabular}

Note: The sctula concentration of samples for which no standard deviation le reportad be lase than the lower Hmit of detection. The reported concentration b the lower ilmit of quantilication. 


\section{Transect D Porewater Concentrations: Iron}

\section{Sample ld. lsotope}

Concentration

Std.Doviation

Element Avg. Conc.

(ug/ $/$ )

(ugh)

Element Avg. Std. Dov.

(ugh)

(ugh)

(ugh)

\begin{tabular}{|c|c|c|c|c|c|}
\hline$D 11$ & $F_{0}$ & & & & \\
\hline$D 12$ & $F_{0}$ & & & & \\
\hline$D 13$ & $F_{0}$ & 357 & 13.6 & 357 & 13.6 \\
\hline$D 13 R A$ & $F_{0}$ & 408 & 16 & 408 & 16 \\
\hline$D 13 R B$ & $F_{0}$ & & & & \\
\hline$D 21$ & $F_{0}$ & & & & \\
\hline$D 41$ & $F_{0}$ & & & & \\
\hline$D 51$ & $F_{0}$ & & & & \\
\hline
\end{tabular}

Note: The actual concentration of eamples for which no standard deviation le reported is leas than the lower limit of detection. The reported concentration is the lower limit of quantification. 


\section{Transect D Porewater Concentrations: Lead}

\begin{tabular}{|c|c|c|c|c|c|}
\hline Sample ld. & botope & $\begin{array}{l}\text { Concentration } \\
\text { (ug/L) }\end{array}$ & $\begin{array}{l}\text { Std.Devlatton } \\
\text { (ugh) }\end{array}$ & $\begin{array}{l}\text { Element Avg. Conc. } \\
\text { (ug/) }\end{array}$ & $\begin{array}{l}\text { Element Avg. Std. Dav. } \\
\text { (ughL) }\end{array}$ \\
\hline D11 & Pt206 & & & & \\
\hline D11 & Pb207 & & & & \\
\hline D11 & Pb208 & & & & \\
\hline D12 & Pb206 & & & & \\
\hline D12 & P6207 & & & & \\
\hline D12 & P6208 & & & & \\
\hline$D 13$ & Pt206 & 1.5 & & & \\
\hline D13 & Pt207 & 1.5 & & & \\
\hline D13 & $P_{6208}$ & 1.5 & & 1.5 & \\
\hline D13RA & $\mathrm{Pb206}$ & 1 & & & \\
\hline D13RA & Pt207 & 1 & & & \\
\hline D13RA & Pb208 & 1 & & 1 & \\
\hline D13RB & Pb206 & & & & \\
\hline D13RB & $P b 207$ & & & & \\
\hline D13RB & $\mathrm{Pb} 208$ & & & & \\
\hline D21 & Pb206 & & & & \\
\hline D21 & Pt207 & & & & \\
\hline D21 & P6208 & & & & \\
\hline D41 & Pb206 & & & & \\
\hline$D 41$ & P6207 & & & & \\
\hline$D 41$ & Pb208 & & & & \\
\hline D51 & Pt206 & & & & \\
\hline D51 & Pb207 & & & & \\
\hline D51 & Pb208 & & & & \\
\hline
\end{tabular}




\section{Transect D Porewater Concentrations: Magnesium}

\begin{tabular}{|c|c|c|c|c|c|}
\hline Sample ld. & kotope & $\begin{array}{l}\text { Concentration } \\
\text { (LG/L) }\end{array}$ & $\begin{array}{l}\text { Stad.Deviation } \\
\text { (ugh) }\end{array}$ & $\begin{array}{l}\text { Element Avg. Conc. } \\
\text { (ugl) }\end{array}$ & $\begin{array}{c}\text { Element Avg. Std. Dov. } \\
\text { (ugh) }\end{array}$ \\
\hline D11 & $\operatorname{Mg} 24$ & & & & \\
\hline D11 & Mg25 & & & & \\
\hline D12 & $\mathrm{Mg} 24$ & & & & \\
\hline D12 & Mges & & & & \\
\hline D13 & $M g 24$ & 1470 & 15.4 & & \\
\hline D13 & Mg25 & 1220 & 31.3 & 1350 & 23.4 \\
\hline D13RA & $M g 24$ & 1080 & 19 & & \\
\hline D13RA & Mg25 & 943 & 115 & 1010 & 67 \\
\hline D13RB & $M g 24$ & & & & \\
\hline D13RB & Mg25 & & & & \\
\hline D21 & Mg24 & & & & \\
\hline D21 & Mg25 & & & & \\
\hline D41 & Mg24 & & & & \\
\hline D41 & Mg्25 & & & & \\
\hline D51 & Mg24 & & & & \\
\hline D51 & $M g 25$ & & & & \\
\hline
\end{tabular}




\section{Transect D Porewater Concentrations: Manganese}

\begin{tabular}{|c|c|c|c|c|c|}
\hline Semple ld. & Lotope & $\begin{array}{l}\text { Concentration } \\
\text { (ugh) }\end{array}$ & $\begin{array}{l}\text { Strd.Deviation } \\
\text { (ugl) }\end{array}$ & $\begin{array}{l}\text { Element Avg. Conc. } \\
\text { (ugh) }\end{array}$ & $\begin{array}{c}\text { Element Avg. Std. Dov. } \\
\text { (ugl) }\end{array}$ \\
\hline D11 & $\mathrm{Mn}$ & & & & \\
\hline D12 & $\mathrm{Mn}$ & & & & \\
\hline D13 & $\mathrm{Mn}$ & 3450 & 422 & 3450 & 422 \\
\hline D13RA & $\mathrm{Mn}$ & 128 & 326 & 128 & 326 \\
\hline D13RB & $\mathbf{M n}$ & & & & \\
\hline$D 21$ & $\mathrm{Mn}$ & & & & \\
\hline D41 & $\mathrm{Mn}$ & & & & \\
\hline$\overline{D 51}$ & $\mathrm{Mn}$ & & & & \\
\hline
\end{tabular}




\begin{tabular}{|c|c|c|c|c|c|}
\hline Sampto id. & botope & $\begin{array}{l}\text { Concentration } \\
\text { (ugl) }\end{array}$ & $\begin{array}{l}\text { Std.Doviation } \\
\text { (Ug/L) }\end{array}$ & $\begin{array}{l}\text { Element Avg. Conc. } \\
\text { (ug/L) }\end{array}$ & $\begin{array}{c}\text { Element Avg. Std. Dov. } \\
\text { (ugh) }\end{array}$ \\
\hline D11 & N60 & & & & \\
\hline D11 & NiE2 & & & & \\
\hline 012 & Ni60 & & & & \\
\hline D12 & Ni62 & & & & \\
\hline D13 & Ni60 & 666 & 5.22 & & \\
\hline D13 & Ni62 & 695 & 32.2 & 681 & 18.7 \\
\hline D13RA & $N 160$ & 39.7 & 162 & & \\
\hline D13RA & Ni62 & & & 39.7 & 162 \\
\hline D13R8 & Ni60 & & & & \\
\hline D13RB & Ni62 & & & & \\
\hline D21 & NiEO & & & & \\
\hline $\mathrm{D} 21$ & Ni62 & & & & \\
\hline$D 41$ & Ni60 & & & & \\
\hline D41 & $\mathrm{Ni62}$ & & & & \\
\hline D51 & Ni6o & & & & \\
\hline D51 & Ni62 & & & & \\
\hline
\end{tabular}




\section{Transect D Porewater Concentrations: Selenium}

\begin{tabular}{|c|c|c|c|c|c|}
\hline samplo ld. & Eotope & $\begin{array}{l}\text { Concentration } \\
\text { (ugl) }\end{array}$ & $\begin{array}{l}\text { Std.Doviation } \\
\text { (uglL) }\end{array}$ & $\begin{array}{l}\text { Element Avg. Conc. } \\
\text { (ugh) }\end{array}$ & $\begin{array}{c}\text { Element Avg. Std. Dev. } \\
\text { (ugh) }\end{array}$ \\
\hline D11 & So77 & & & & \\
\hline D11 & So82 & & & & \\
\hline D12 & So77 & & & & \\
\hline D12 & So82 & & & & \\
\hline D13 & So77 & 37.5 & & & \\
\hline D13 & So82 & 37.5 & & 37.5 & \\
\hline D13RA & Se77 & 25 & & & \\
\hline D13AA & Se82 & 25 & & $\mathbf{2 5}$ & \\
\hline D13AB & So77 & & & & \\
\hline D13AB & So82 & & & & \\
\hline $\mathrm{D} 21$ & So77 & & & & \\
\hline D21 & So82 & & & & \\
\hline D41 & SoT7 & & & & \\
\hline D41 & SeB2 & & & & \\
\hline D51 & Se77 & & & & \\
\hline D51 & So82 & & & & \\
\hline
\end{tabular}




\section{Transect D Porewater Concentrations: Silver}

\begin{tabular}{ccccc}
\hline Sempte ld. botope & $\begin{array}{c}\text { Concentration } \\
(\mathrm{ug} /)\end{array}$ & $\begin{array}{c}\text { Std.Doviation } \\
(\mathrm{ug} /)\end{array}$ & $\begin{array}{c}\text { Element Avg. Conc. } \\
(\mathrm{ug} /)\end{array}$ & $\begin{array}{c}\text { Element Avg. Std. Dov. } \\
(\mathrm{ug} / \mathrm{L})\end{array}$ \\
\hline
\end{tabular}

\begin{tabular}{|c|c|c|c|c|c|}
\hline D11 & Ag107 & & & & \\
\hline D11 & A0109 & & & & \\
\hline D12 & A0107 & & & & \\
\hline D12 & Ag100 & & & & \\
\hline D13 & A0107 & .15 & & & \\
\hline D13 & $A_{\theta 100}$ & .15 & & .15 & \\
\hline D13RA & Ag107 & 52.4 & 16.8 & & \\
\hline D13RA & Ag109 & 31.4 & & 41.9 & 16.9 \\
\hline D13RB & Ag107 & & & & \\
\hline D13RB & Ag100 & & & & \\
\hline D21 & Ag107 & & & & \\
\hline D21 & Ag109 & & & & \\
\hline D41 & Ag107 & & & & \\
\hline$D 41$ & $A g 100$ & & & & \\
\hline$D 51$ & Ag107 & & & & \\
\hline$D 51$ & Ag109 & & & & \\
\hline
\end{tabular}




\section{Transect D Porewater Concentrations: Sodium}

\begin{tabular}{|c|c|c|c|c|c|}
\hline Semple Id. & Botopo & $\begin{array}{l}\text { Concentration } \\
\text { (ug/h) }\end{array}$ & $\begin{array}{l}\text { Std.Dovintion } \\
\text { (4g/L) }\end{array}$ & $\begin{array}{l}\text { Element Avg. Conc. } \\
\text { (ugh) }\end{array}$ & $\begin{array}{c}\text { Element Avg. Std. Dav. } \\
\text { (ug/) }\end{array}$ \\
\hline D11 & $\mathrm{Na}$ & & & & \\
\hline D12 & $\mathrm{Na}$ & & & & \\
\hline D13 & $\mathrm{Na}$ & 36200 & 318 & 36200 & 318 \\
\hline DI3RA & $\mathrm{Na}$ & 28200 & 1600 & 28200 & 1600 \\
\hline D13RB & $\mathrm{Na}$ & & & & \\
\hline $\mathrm{D} 21$ & $\mathrm{Na}$ & & & & \\
\hline D41 & $\mathrm{Na}$ & & & & \\
\hline D51 & $\mathrm{Na}$ & & & & \\
\hline
\end{tabular}




\begin{tabular}{|c|c|c|c|c|c|}
\hline Semple Id. & botope & $\begin{array}{l}\text { Concentration } \\
\text { (Ug/L) }\end{array}$ & $\begin{array}{l}\text { Str. Doviation } \\
\text { (ugl) }\end{array}$ & $\begin{array}{c}\text { Element Avg- Conc. } \\
\text { (ugh) }\end{array}$ & $\begin{array}{c}\text { Element Avg. Std. Dov. } \\
\text { (Ugh) }\end{array}$ \\
\hline D11 & $S_{r}$ & & & & \\
\hline D12 & $\mathrm{Sr}$ & & & & \\
\hline D13 & Sr & 22.1 & .39 & 22.1 & .39 \\
\hline D13RA & $\mathrm{Sr}$ & 182 & 1.85 & 192 & 1.85 \\
\hline D13RB & Sr & & & & \\
\hline D21 & Sr & & & & \\
\hline$D 41$ & $\mathrm{Sr}$ & & & & \\
\hline DS1 & $\mathrm{Sr}$ & & & & \\
\hline
\end{tabular}




\section{Transect D Porewater Concentrations: Thorium}

Sample Id. Lotope $\begin{gathered}\text { Concentration } \\
\text { (Ug/L) }\end{gathered}$
\begin{tabular}{|c|c|c|c|c|c|}
\hline D11 & Th232 & & & $\begin{array}{c}\text { Std.Devlation } \\
\text { (ugh) }\end{array}$ \\
\hline D12 & Th232 & & & & \\
\hline D13 & Th232 & .5 & & .5 & \\
\hline D13RA & Th232 & 25 & & 25 & \\
\hline D13RB & Th232 & & & & \\
\hline D21 & Th232 & & & & \\
\hline D41 & Th232 & & & & \\
\hline D51 & Th232 & & & & \\
\hline
\end{tabular}

Note: The actual concentration of eamples for which no standerd doviation is reported b hes than the lower llmit of detection. The reported coneentration is the lower llmit of quantification. 


\section{Transect D Porewater Concentrations: Tin}

\begin{tabular}{|c|c|c|c|c|c|}
\hline Sample Id. & Lotope & $\begin{array}{l}\text { Concentration } \\
\text { (ug/L) }\end{array}$ & $\begin{array}{l}\text { Sid.Dovilation } \\
\text { (ug/L) }\end{array}$ & $\begin{array}{l}\text { Element Avo Cone. } \\
\text { (ugh) }\end{array}$ & $\begin{array}{l}\text { Element Avg. Std. Dov. } \\
\text { (ug/L) }\end{array}$ \\
\hline D11 & Sn118 & & & & \\
\hline D11 & Sn 120 & & & & \\
\hline D12 & Sn118 & & & & \\
\hline D12 & Sn 120 & & & & \\
\hline D13 & Sn118 & .33 & .14 & & \\
\hline D13 & Sn120 & .17 & .15 & 25 & .15 \\
\hline D13RA & Sn118 & 2.5 & & & \\
\hline D13RA & $\operatorname{Sn} 120$ & 2.5 & & 2.5 & \\
\hline D13RB & Sn118 & & & & \\
\hline D13RB & Sn120 & & & & \\
\hline$D 21$ & Sn118 & & & & \\
\hline $\mathrm{D} 21$ & Sn120 & & & & \\
\hline D41 & Sn118 & & & & \\
\hline D41 & $\operatorname{Sn} 120$ & & & & \\
\hline D51 & Sn118 & & & & \\
\hline D51 & $\operatorname{Sn} 120$ & & & & \\
\hline
\end{tabular}




\section{Transect D Porewater Concentrations: Uranium-235}

\begin{tabular}{|c|c|c|c|c|c|}
\hline Sample ld. & hotope & $\begin{array}{l}\text { Concentration } \\
\text { (ug/L) }\end{array}$ & $\begin{array}{l}\text { Std.Davlatton } \\
\text { (ugL) }\end{array}$ & $\begin{array}{l}\text { Element Avg. Cone. } \\
\cdot \text { (ugl) }\end{array}$ & $\begin{array}{l}\text { Element Avg. Std. Dov. } \\
\text { (ugh) }\end{array}$ \\
\hline D11 & U235 & & & & \\
\hline D12 & U235 & & & & \\
\hline D13 & U235 & .02 & & .02 & \\
\hline D13RA & U235 & .61 & .17 & .61 & .17 \\
\hline D13RB & U235 & & & & \\
\hline D21 & U235 & & & & \\
\hline D41 & U235 & & & & \\
\hline D51 & U235 & & & & \\
\hline
\end{tabular}

Note: The actulal concentration of samplea for which no standard deviation be reported be lees than the lower limit of detection. The reported concentration is the bwer imit of quantification. 
Transect D Porewater Concentrations: Uranium-238

\begin{tabular}{|c|c|c|c|c|c|}
\hline Sample ld. & Lotope & $\begin{array}{l}\text { Concentration } \\
\text { (ugh) }\end{array}$ & $\begin{array}{l}\text { Std.Doviation } \\
\text { (ug/L) }\end{array}$ & $\begin{array}{c}\text { Element Avg. Conc. } \\
\text { (ugh) }\end{array}$ & $\begin{array}{c}\text { Element Avg. Std. Dov. } \\
\text { (ugL) }\end{array}$ \\
\hline D11 & U238 & & & & \\
\hline D12 & U238 & & & & \\
\hline D13 & U238 & 26 & & 26 & \\
\hline D13RA & U238 & 296 & 14.7 & 296 & 14.7 \\
\hline D13RB & U238 & & & & \\
\hline 021 & U238 & & & & \\
\hline D41 & U238 & & & & \\
\hline$D 51$ & U238 & & & & \\
\hline
\end{tabular}




\section{Transect D Porewater Concentrations: Vanadium}

\begin{tabular}{|c|c|c|c|c|c|}
\hline Semple Id. & leotope & $\begin{array}{l}\text { Concentration } \\
\text { (UG/L) }\end{array}$ & $\begin{array}{l}\text { Stdideviation } \\
\text { (Lo/L) }\end{array}$ & $\begin{array}{l}\text { Element Avg. Conc. } \\
\text { (ugh) }\end{array}$ & $\begin{array}{l}\text { Element Avg. Std. Dov. } \\
\text { (ugh) }\end{array}$ \\
\hline D11 & $v$ & & & & \\
\hline D12 & $v$ & & & & \\
\hline D13 & $V$ & 25 & & 25 & \\
\hline D13RA & $V$ & 625 & & 625 & \\
\hline D13RB & $\mathbf{v}$ & & & & \\
\hline$D 21$ & $V$ & & & & \\
\hline D41 & $V$ & & & & \\
\hline D51 & $v$ & & & & \\
\hline
\end{tabular}




\section{Transect D Porewater Concentrations: Yttrium}

$\begin{array}{ccccc}\text { Sample ld. Lotope } & \begin{array}{c}\text { Concentration } \\ \text { (ug/) }\end{array} & \begin{array}{c}\text { Std.Deviation } \\ \text { (ugh) }\end{array} & \begin{array}{c}\text { Element Avg. Conc. } \\ \text { (ugh) }\end{array} & \begin{array}{c}\text { Element Avg. Std. Dov. } \\ \text { (ugh) }\end{array}\end{array}$

\begin{tabular}{|c|c|c|c|c|c|}
\hline D11 & $Y$ & & & & \\
\hline$D 12$ & $Y$ & & & & \\
\hline$D 13$ & $Y$ & 25 & & 25 & \\
\hline D13RA & $Y$ & 1.3 & .19 & 1.3 & .19 \\
\hline D13RB & $Y$ & & & & \\
\hline D21 & $Y$ & & & & \\
\hline D41 & $Y$ & & & & \\
\hline D51 & $Y$ & & & & \\
\hline
\end{tabular}




\section{Transect D Porewater Concentrations: Zinc}

\begin{tabular}{|c|c|c|c|c|c|}
\hline Sample ld. & Eotope & $\begin{array}{l}\text { Concentration } \\
\text { (ugh) }\end{array}$ & $\begin{array}{l}\text { StddDeviation } \\
\text { (ugh) }\end{array}$ & $\begin{array}{l}\text { Ekment Avg. Conc. } \\
\text { (ugR) }\end{array}$ & $\begin{array}{c}\text { Element Avg. Std. Dov. } \\
\text { (ugh) }\end{array}$ \\
\hline D11 & Zn66 & & & & \\
\hline D12 & Zn66 & & & & \\
\hline D13 & Zn66 & 7000 & .85 & 7000 & .95 \\
\hline D13RA & $Z n 66$ & 73 & 34.1 & 73 & 34.1 \\
\hline D13AB & Zn66 & & & & \\
\hline D21 & Zn66 & & & & \\
\hline$D 41$ & Zn66 & & & & \\
\hline D51 & Zn66 & & & & \\
\hline
\end{tabular}

Note: The actual concentration of eamples for which no stendard deviation is reported is leas than the lower limit of detection. The reported concentration lo the lower llmit of quantification. 


\section{Transect D Porewater Concentrations: Zirconium}

\begin{tabular}{|c|c|c|c|c|c|}
\hline Sample Id. & teotope & $\begin{array}{l}\text { Concentration } \\
\text { (ugh) }\end{array}$ & $\begin{array}{l}\text { Std.Deviation } \\
\text { (Ugl) }\end{array}$ & $\begin{array}{l}\text { Ekement Avg. Conc. } \\
\text { (ugl) }\end{array}$ & $\begin{array}{c}\text { Element Avg. Std. Dov. } \\
\text { (ugh) }\end{array}$ \\
\hline D11 & $Z 90$ & & & & \\
\hline D11 & Zr91 & & & & \\
\hline D12 & Zrso & & & & \\
\hline D12 & $2 r 91$ & & & & \\
\hline 013 & 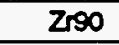 & .18 &.$\infty$ & & \\
\hline D13 & $2 \sqrt{91}$ & .57 & .49 & .38 & 29 \\
\hline D13RA & 290 & 2.5 & & & \\
\hline D13RA & 2191 & 2.5 & & 2.5 & \\
\hline D13AB & Zrso & & & & \\
\hline D13RB & Zr91 & & & & \\
\hline 021 & 2590 & & & & \\
\hline 021 & Zr91 & & & & \\
\hline D41 & 2590 & & & & \\
\hline$D 41$ & Zra1 & & & & \\
\hline D51 & 290 & & & & \\
\hline D51 & Zr91 & & & & \\
\hline
\end{tabular}


Transect E Porewater Concentrations: Aluminum

\begin{tabular}{|c|c|c|c|c|c|}
\hline Sample Id. & botope & $\begin{array}{l}\text { Concentration } \\
\text { (ugh) }\end{array}$ & $\begin{array}{l}\text { 8id.Doviation } \\
\text { (ugl) }\end{array}$ & $\begin{array}{l}\text { Element Avg. Conc. } \\
\text { (logl) }\end{array}$ & $\begin{array}{c}\text { EJement Avg. Std. Dev. } \\
\text { (Ug/) }\end{array}$ \\
\hline E11 & $\mathbf{A}$ & & & & \\
\hline E12 & $\mathbf{A}$ & & & & \\
\hline E12R & $\mathbf{A}$ & & & & \\
\hline E13 & $\mathbf{A}$ & 4760 & 37.8 & 4760 & 37.9 \\
\hline E14 & $\mathbf{A}$ & 4830 & 49 & 4850 & 49 \\
\hline E21 & $\mathbf{A}$ & & & & \\
\hline E22 & $\mathbf{A}$ & & & & \\
\hline E23 & $\mathbf{A}$ & 455 & 4.53 & 455 & 4.53 \\
\hline E23A & $\mathbf{A}$ & 3700 & 71.3 & 3700 & 71.3 \\
\hline E24 & $\mathbf{A}$ & 7450 & 115 & 7450 & 115 \\
\hline E31 & A & & & & \\
\hline E32 & $\mathbf{A}$ & & & & \\
\hline E33 & A & 2470 & 10.3 & 2470 & 10.3 \\
\hline E34 & $\mathbf{A}$ & 4380 & 20.6 & 4380 & 20.6 \\
\hline E41 & A & 103 & 2.01 & 103 & 2.01 \\
\hline E42 & $\mathbf{A}$ & 107 & 1.68 & 107 & 1.68 \\
\hline
\end{tabular}




\section{Transect E Porewater Concentrations: Arsenic}

\begin{tabular}{|c|c|c|c|c|c|}
\hline Sample ld. & botope & $\begin{array}{l}\text { Concentratton } \\
\text { (Ug/L) }\end{array}$ & $\begin{array}{l}\text { Sed.Doviation } \\
\text { (ugll) }\end{array}$ & $\begin{array}{l}\text { Element Ava. Cone. } \\
\text { (ugh) }\end{array}$ & $\begin{array}{c}\text { Element Avg. Std. Dov. } \\
\text { (ugh) }\end{array}$ \\
\hline E11 & As & & & & \\
\hline$E 12$ & As & & & & \\
\hline E12R & As & & & & \\
\hline E13 & As & 7.46 & .51 & 7.46 & .51 \\
\hline E14 & As & 75 & & 75 & \\
\hline E21 & As & & & & \\
\hline $\mathrm{E} 22$ & As & & & & \\
\hline E23 & As & 75 & & 75 & \\
\hline E23R & As & 7.54 & 1.89 & 7.54 & 1.89 \\
\hline E24 & As & 1.5 & & 1.5 & \\
\hline E31 & As & & & & \\
\hline E32 & As & & & & \\
\hline$E 33$ & As & 8.51 & .93 & 8.51 & .93 \\
\hline$E 34$ & As & 5.33 & 1.39 & 5.33 & 1.39 \\
\hline E41 & As & 5.98 & 123 & 5.88 & 1.23 \\
\hline$E 42$ & As & 2.62 & .77 & 2.62 & .77 \\
\hline
\end{tabular}




\section{Transect E Porewater Concentrations: Barium}

\begin{tabular}{|c|c|c|c|c|c|}
\hline & leotope & $\begin{array}{l}\text { Concentratlo } \\
\text { (ugh) }\end{array}$ & $\begin{array}{l}\text { Std.Doviation } \\
\text { (ug } /)\end{array}$ & $\begin{array}{l}\text { Eloment Aug. Conc. } \\
\text { (ugh) }\end{array}$ & Element Avg. Std. Dov. \\
\hline
\end{tabular}

\begin{tabular}{|c|c|c|c|c|c|}
\hline E11 & Ba135 & & & & \\
\hline E11 & Ba137 & & & & \\
\hline E11 & Ba138 & & & & \\
\hline E12 & Ba135 & & & & \\
\hline E12 & Ba137 & & & & \\
\hline E12 & Ba138 & & & & \\
\hline E12R & Ba135 & & & & \\
\hline$\overline{E 12 R}$ & Ba137 & & & & \\
\hline E12R & Be138 & & & & \\
\hline$E 13$ & Ba135 & 148 & .99 & & \\
\hline E13 & Ba137 & 155 & .92 & & \\
\hline E13 & Ba138 & 152 & .84 & 152 & .92 \\
\hline E14 & Ba135 & 268 & 3.14 & & \\
\hline E14 & Ba137 & 286 & 1.7 & & \\
\hline E14 & Ba138 & 272 & 21 & 275 & 1.68 \\
\hline$E 21$ & Ba135 & & & & \\
\hline$E 21$ & Ba137 & & & & \\
\hline$E 21$ & Ba138 & & & & \\
\hline$E 22$ & Ba135 & & & & \\
\hline$E 22$ & Ba137 & & & & \\
\hline$E 22$ & Ba138 & & & & \\
\hline$E 23$ & Ba135 & 97 & .97 & & \\
\hline E23 & Ba137 & 104 & .87 & & \\
\hline$E 23$ & Ba138 & 100 & .18 & 103 & .71 \\
\hline E23R & Ba135 & 294 & 6.15 & & \\
\hline E23R & Ba137 & 313 & 7.47 & & \\
\hline$E 23 R$ & Ba138 & 300 & 2.47 & 300 & 5.36 \\
\hline$E 24$ & Ba135 & 225 & 3.69 & & \\
\hline$E 24$ & Ba137 & 214 & 622 & & \\
\hline E24 & Ba138 & 213 & .87 & 217 & 3.59 \\
\hline E31 & Ba135 & & & & \\
\hline E31 & Ba137 & & & & \\
\hline E31 & Ba138 & & & & \\
\hline E32 & Ba135 & & & & \\
\hline E32 & Ba137 & & & & \\
\hline E32 & Ba138 & & & & \\
\hline E33 & Ba135 & 54.8 & .88 & & \\
\hline E33 & Ba137 & 53.6 & .89 & & \\
\hline$E 33$ & Be138 & 52.3 & .6 & 53.6 & .82 \\
\hline$E 34$ & Ba135 & 49.4 & .9 & & \\
\hline$E 34$ & Ba137 & 52 & .58 & & \\
\hline E34 & Ba138 & 52.5 & .34 & 51.3 & .61 \\
\hline$E 41$ & Ba135 & 20.8 & 3.45 & & \\
\hline$E 41$ & Ba13? & $2 n$ & 2 & & \\
\hline
\end{tabular}

Note: The setual concentratton of eamples for which no standerd deviation is reported is lese than the lower llmit of detection. The reported concentratton ts the lower limit of quantification. 


\section{Transect E Porewater Concentrations: Barium}

\begin{tabular}{|c|c|c|c|c|c|}
\hline Sample Id. & leotope & $\begin{array}{l}\text { Concentration } \\
\text { (ug/L) }\end{array}$ & $\begin{array}{l}\text { Std.Doviation } \\
\text { (ugl) }\end{array}$ & $\begin{array}{l}\text { Element Avg. Conse. } \\
\text { (ugh) }\end{array}$ & $\begin{array}{c}\text { Element Avg. Std. Dov. } \\
\text { (ugh) }\end{array}$ \\
\hline E41 & Ba138 & 23 & .43 & 21.9 & 1.96 \\
\hline$E 42$ & Ba135 & 192 & .56 & & \\
\hline$E 42$ & Ba137 & 20.7 & 3.08 & & \\
\hline$E 42$ & Ba138 & 19.6 & 1.06 & 19.8 & 1.57 \\
\hline
\end{tabular}

Note: The sctusl concentration of eamples for which no standard deviation be reported le kese than the lower llmit of detection. The reported concentration is the lower limit of quantification. 


\begin{tabular}{|c|c|c|c|c|c|}
\hline \multicolumn{6}{|c|}{ Transect E Porewater Concentrations: Cadmium } \\
\hline Semple kd. & betope & $\begin{array}{l}\text { Concentration } \\
\text { (ug/) }\end{array}$ & $\begin{array}{l}\text { Std.Doviation } \\
\text { (ugh) }\end{array}$ & $\begin{array}{l}\text { Element Avg. Conc. } \\
\text { (ug/) }\end{array}$ & $\begin{array}{c}\text { Element Avg. Std. Dov. } \\
\text { (ugh) }\end{array}$ \\
\hline E11 & Cd111 & & & & \\
\hline E11 & $\operatorname{Cod} 114$ & & & & \\
\hline E12 & Cod111 & & & & \\
\hline E12 & $\operatorname{Cod114}$ & & & & \\
\hline E12R & Cd111 & & & & \\
\hline E12R & $\operatorname{cod} 114$ & & & & \\
\hline$E 13$ & $\operatorname{cd111}$ & .42 & 24 & & \\
\hline$E 13$ & $\operatorname{cod} 114$ & 127 & .41 & .85 & .32 \\
\hline E14 & $\operatorname{cd} 111$ & 1.38 & 2 & & \\
\hline$E 14$ & $\operatorname{cod} 114$ & 1.11 & .4 & 125 & .3 \\
\hline E21 & $\operatorname{cod111}$ & & & & \\
\hline E21 & $\operatorname{Cod114}$ & & & & \\
\hline$E_{22}$ & $\operatorname{cod} 111$ & & & & \\
\hline$E 22$ & $\operatorname{cot114}$ & & & & \\
\hline$E_{23}$ & $\operatorname{Cod} 111$ & 1.03 & .42 & & \\
\hline$E 23$ & $\operatorname{Cod} 114$ & .95 & .31 & .99 & .37 \\
\hline E23R & Cod111 & 2.81 & .34 & & \\
\hline E23R & Cd114 & 2.03 & 29 & 2.42 & .32 \\
\hline E24 & Cd111 & 1.06 & .14 & & \\
\hline E24 & $\operatorname{cod114}$ & 1.33 & .18 & 12 & .16 \\
\hline E31 & Cd111 & & & & \\
\hline E31 & $\operatorname{cod} 114$ & & & & \\
\hline E32 & Cd111 & & & & \\
\hline E32 & Cd114 & & & & \\
\hline E33 & Cod111 & .93 & .46 & & \\
\hline$E 33$ & Cd114 & .77 & .38 & .85 & .42 \\
\hline E34 & $\operatorname{col111}$ & 126 & 2 & & \\
\hline E34 & Cd114 & 1.48 & .43 & 1.37 & .32 \\
\hline E41 & Cd111 & .75 & & & \\
\hline$E_{41}$ & Cd114 & .3 & .08 & .53 & .08 \\
\hline E42 & Cod111 & .75 & & & \\
\hline E42 & Cod114 & .75 & & .75 & \\
\hline
\end{tabular}




\section{Transect E Porewater Concentrations: Calcium}

\begin{tabular}{|c|c|c|c|c|c|}
\hline Sample tol. & botope & $\begin{array}{l}\text { Concentration } \\
\text { (ugh) }\end{array}$ & $\begin{array}{l}\text { Std.Doviation } \\
\text { (ugl) }\end{array}$ & $\begin{array}{c}\text { Element Avg. Conc. } \\
\text { (ugll) }\end{array}$ & $\begin{array}{c}\text { Element Avg. Std. Dov. } \\
\text { (ug/) }\end{array}$ \\
\hline E11 & Ca44 & & & & \\
\hline E12 & $\operatorname{Ca} 44$ & & & & \\
\hline E12R & $\cos 4$ & & & & \\
\hline E13 & Ca44 & 851 & 11.1 & 851 & 11.1 \\
\hline E14 & Ca44 & 1200 & 292 & 1200 & 29.2 \\
\hline E21 & Ca44 & & & & \\
\hline E22 & $\operatorname{Co} 44$ & & & & \\
\hline E23 & Ca44 & 6370 & 23.1 & 6970 & 23.1 \\
\hline E23R & Ca44 & 310 & 5.02 & 310 & 5.02 \\
\hline E24 & $\operatorname{Co44}$ & 7820 & 24.1 & 7820 & 24.1 \\
\hline E31 & $\mathrm{Ce44}$ & & & & \\
\hline E32 & $\mathrm{Ca} 44$ & & & & \\
\hline E33 & Ca44 & 2460 & 24 & 2460 & 24 \\
\hline E34 & $\operatorname{Cos4}$ & 2630 & 20.8 & 2630 & 20.8 \\
\hline E41 & Ces4 & 2270 & 7.81 & 2270 & 7.81 \\
\hline E42 & Ca44 & 2320 & 73.4 & 2320 & 73.4 \\
\hline
\end{tabular}




\section{Transect E Porewater Concentrations: Cesium}

Sample fd. leotope

Concentration

$($ (ugl)

Std.Deviation

(ugl)

Element Avg. Conc.

Element Avg. Sto. Dov. (ugh)

(ugh)

\begin{tabular}{|c|c|c|c|c|c|}
\hline E11 & $\mathrm{Cs}$ & & & & \\
\hline E12 & $\mathrm{Cs}$ & & & & \\
\hline E12R & $\mathrm{Cs}$ & & & & \\
\hline E13 & Cs & 23 & .07 & 23 & .07 \\
\hline E14 & Cs & 5 & & 5 & \\
\hline E21 & $\mathrm{C}_{8}$ & & & & \\
\hline E22 & Cs & & & & \\
\hline$E 23$ & Cs & .07 & .03 & .07 & .03 \\
\hline E23R & $\mathrm{C}_{8}$ & .69 & .1 & .69 & .1 \\
\hline E24 & Cs & .39 & .11 & .39 & .11 \\
\hline E31 & Cs & & & & \\
\hline E32 & Cs & & & & \\
\hline $\mathrm{E33}$ & Cs & .43 & .02 & .43 & .02 \\
\hline E34 & Cs & .47 & .09 & .47 &.$\infty 9$ \\
\hline E41 & Cs & .1 & & .1 & \\
\hline$E 42$ & $\mathrm{C}_{8}$ & .1 & & .1 & \\
\hline
\end{tabular}

Note: The actual concentration of samples for which no stundard deviation be reported te leas than the lower limit of detection. The reported concentration bs the lower limit of quantification. 


\begin{tabular}{|c|c|c|c|c|c|}
\hline \multicolumn{6}{|c|}{ Transect E Porewater Concentrations: Chromium } \\
\hline Sample ld. & Eotope & $\begin{array}{l}\text { Concentration } \\
\text { (ugl) }\end{array}$ & $\begin{array}{l}\text { Std.Doviation } \\
\text { (ugh) }\end{array}$ & $\begin{array}{l}\text { Eloment Avg. Conc. } \\
\text { (ugh) }\end{array}$ & $\begin{array}{l}\text { Element Avg. Std. Dov. } \\
\text { (ugh) }\end{array}$ \\
\hline$E 11$ & Cr52 & & & & \\
\hline E11 & C.53 & & & & \\
\hline E12 & G52 & & & & \\
\hline E12 & G53 & & & & \\
\hline E12R & C52 & & & & \\
\hline E12R & Cr53 & & & & \\
\hline E13 & C.52 & 2.81 & .19 & & \\
\hline$E_{13}$ & C.53 & 2.8 & .19 & 2.81 & .19 \\
\hline E14 & G52 & 3.39 & .55 & & \\
\hline E14 & G53 & 3.38 & .55 & 3.39 & .55 \\
\hline E21 & C.52 & & & & \\
\hline E21 & $c 53$ & & & & \\
\hline$E 22$ & Cr52 & & & & \\
\hline$E 22$ & $G 53$ & & & & \\
\hline$E 23$ & $G 52$ & 1.14 & .1 & & \\
\hline $\mathrm{E} 23$ & $C 53$ & 1.14 & .1 & 1.14 & .1 \\
\hline E23R & G.52 & 3.32 & .64 & & \\
\hline E23R & Cr53 & 3.31 & .63 & 3.32 & .64 \\
\hline$E 24$ & $G 52$ & 1.91 & 24 & & \\
\hline$E 24$ & 9.53 & 1.91 & 25 & 1.91 & 25 \\
\hline$E 31$ & $c 52$ & & & & \\
\hline E31 & $c 53$ & & & & \\
\hline E32 & C.52 & & & & \\
\hline E32 & C.53 & & & & \\
\hline E33 & $c 52$ & .68 & .1 & & \\
\hline$E 33$ & $c_{553}$ & .67 & .1 & .68 & .1 \\
\hline$E 34$ & G52 & .98 & .34 & & \\
\hline E34 & C.53 & .98 & .34 & .98 & .34 \\
\hline$E 41$ & $c 52$ & 1.5 & & & \\
\hline E41 & G53 & 1.5 & & 1.5 & \\
\hline E42 & C.52 & 1.5 & & & \\
\hline$E 42$ & 0.53 & 1.5 & & 1.5 & \\
\hline
\end{tabular}




\begin{tabular}{|c|c|c|c|c|c|}
\hline Sumple ld. & leotope & $\begin{array}{l}\text { Concentration } \\
\text { (ugh) }\end{array}$ & $\begin{array}{l}\text { Std.Deviation } \\
\text { (ugh) }\end{array}$ & $\begin{array}{l}\text { Element Avg-Conc. } \\
\text { (4ar) }\end{array}$ & $\begin{array}{c}\text { Element Avg. Std. Dav. } \\
\text { (Ug/) }\end{array}$ \\
\hline E11 & $C_{0}$ & & & & \\
\hline$E 12$ & $\infty$ & & & & \\
\hline E12R & Co & & & & \\
\hline E13 & $\infty$ & 12.8 & .53 & 12.9 & .53 \\
\hline E14 & $C_{0}$ & 27.1 & .68 & 27.1 & .68 \\
\hline E21 & $\infty$ & & & & \\
\hline $\mathrm{E} 22$ & $\infty$ & & & & \\
\hline E23 & $\infty$ & 9.76 & .3 & 9.76 & .3 \\
\hline E23R & Co & 28.7 & .62 & 28.7 & .62 \\
\hline E24 & $\infty$ & 22.8 & .84 & 22.8 & .84 \\
\hline E31 & $\mathrm{Co}$ & & & & \\
\hline E32 & Co & & & & \\
\hline E33 & Co & 2.6 & 21 & 2.6 & 21 \\
\hline E34 & $C_{0}$ & 9.09 & .38 & 9.00 & .38 \\
\hline E41 & $C_{0}$ & .49 & .11 & .49 & .11 \\
\hline E42 & $\infty$ & .84 & 23 & .84 & 23 \\
\hline
\end{tabular}




\section{Transect E Porewater Concentrations: Copper}

\begin{tabular}{|c|c|c|c|c|c|}
\hline Sample ld. & botope & $\begin{array}{l}\text { Concentration } \\
\text { (ugh) }\end{array}$ & $\begin{array}{l}\text { Std.Doviation } \\
\text { (Hg/L) }\end{array}$ & $\begin{array}{l}\text { Element Avg. Conc. } \\
\text { (ug/) }\end{array}$ & $\begin{array}{c}\text { Element Avg. Std. Dov. } \\
\text { (ugh) }\end{array}$ \\
\hline
\end{tabular}

\begin{tabular}{|c|c|c|c|c|c|}
\hline E11 & Cu63 & & & & \\
\hline E11 & 0,65 & & & & \\
\hline$E 12$ & $a+63$ & & & & \\
\hline$E 12$ & $a_{655}$ & & & & \\
\hline E12R & Cu63 & & & & \\
\hline E12R & Cu65 & & & & \\
\hline E13 & Cu63 & 6.8 & .69 & & \\
\hline E13 & Qu65 & 10.6 & 1.13 & 8.7 & .91 \\
\hline E14 & a 63 & 7.57 & .34 & & \\
\hline E14 & Cu65 & 8.96 & 1.69 & 8.27 & 1.01 \\
\hline E21 & Cu63 & & & & \\
\hline E21 & a 65 & & & & \\
\hline$E 22$ & Qu63 & & & & \\
\hline E22 & Cu65 & & & & \\
\hline E23 & Cu63 & 2.13 & .52 & & \\
\hline E23 & Cu65 & 1.93 & .48 & 2.03 & .5 \\
\hline E23R & Cu63 & 9.8 & .14 & & \\
\hline E23R & Qu65 & 8.54 & .94 & 8.67 & .54 \\
\hline E24 & cu63 & 8.14 & .3 & & \\
\hline$E 24$ & au65 & 8.90 & 1.82 & 8.56 & 1.06 \\
\hline E31 & Cur63 & & & & \\
\hline E31 & Cu65 & & & & \\
\hline$E 32$ & 0463 & & & & \\
\hline E32 & 0.65 & & & & \\
\hline$E 33$ & Cu63 & 6.17 & .86 & & \\
\hline$E 33$ & Cu65 & 6.64 & .57 & 6.4 & .72 \\
\hline$E_{34}$ & cove3 & 5.62 & 22 & & \\
\hline E34 & Cu65 & 6.01 & .78 & 5.82 & .5 \\
\hline E41 & 0463 & .74 & .3 & & \\
\hline$E 41$ & Cu65 & .99 & .86 & .87 & .58 \\
\hline$E 42$ & 0.63 & 4.82 & .86 & & \\
\hline$E 42$ & Qu65 & 3.97 & .54 & 4.4 & .7 \\
\hline
\end{tabular}




\section{Transect E Porewater Concentrations: Iron}

Sample ld. Lotope

Concentration

(ugl)
StdDoviation

(ugh)
Element Avg. Conc. (Ual)

Element Avg. Std. Dov. (ugh)

\begin{tabular}{|c|c|c|c|c|c|}
\hline E11 & $F_{0}$ & & & & \\
\hline$E 12$ & $F_{0}$ & & & & \\
\hline E12R & Fo & & & & \\
\hline E13 & $F_{0}$ & 974 & 21.3 & 974 & 21.3 \\
\hline E14 & Fo & 1370 & 152 & 1370 & 152 \\
\hline E21 & $F_{0}$ & & & & \\
\hline$E 22$ & $F_{0}$ & & & & \\
\hline$E 23$ & $F_{0}$ & 5 & & 5 & \\
\hline E23R & Fo & 5950 & 94.7 & 5930 & 94.7 \\
\hline E24 & Fo & & & & \\
\hline E31 & $F_{0}$ & & & & \\
\hline E32 & $F_{0}$ & & & & \\
\hline E33 & $F_{0}$ & 85.6 & 14.1 & 85.6 & 14.1 \\
\hline E34 & $F_{0}$ & & & & \\
\hline E41 & Fo & 5 & & 5 & \\
\hline E42 & $F_{0}$ & 68.4 & 1.01 & 68.4 & 1.01 \\
\hline
\end{tabular}




\section{Transect E Porewater Concentrations: Lead}

\begin{tabular}{|c|c|c|c|c|c|}
\hline Semple fol. & leotope & $\begin{array}{l}\text { Concentration } \\
(\log \Omega)\end{array}$ & $\begin{array}{l}\text { StdDovintion } \\
\text { (ugh) }\end{array}$ & $\begin{array}{l}\text { Element Avg. Conc. } \\
\text { (ugh) }\end{array}$ & $\begin{array}{l}\text { Element Avg. Std. Dov. } \\
\text { (Ug/) }\end{array}$ \\
\hline E11 & Pt206 & & & & \\
\hline E11 & $P 6207$ & & & & \\
\hline E11 & $P 6208$ & & & & \\
\hline$E 12$ & $P 0206$ & & & & \\
\hline E12 & Pt207 & & & & \\
\hline$E 12$ & $P_{b 208}$ & & & & \\
\hline E12R & P6206 & & & & \\
\hline$E 12 R$ & Pt207 & & & & \\
\hline E12R & PO208 & & & & \\
\hline E13 & Pt206 & 425 & & & \\
\hline E13 & Pt207 & 425 & & & \\
\hline E13 & Pt208 & 425 & & 4.25 & \\
\hline E14 & $P 6206$ & 1.5 & & & \\
\hline$E 14$ & $P b 207$ & 1.5 & & & \\
\hline E14 & Pb208 & 1.5 & & 1.5 & \\
\hline E21 & $P b 206$ & & & & \\
\hline E21 & Pb207 & & & & \\
\hline E21 & Pb208 & & & & \\
\hline$E 22$ & $\mathrm{~Pb} 206$ & & & & \\
\hline$E 22$ & P6207 & & & & \\
\hline$E 22$ & P6208 & & & & \\
\hline$E 23$ & $\mathrm{Pb206}$ & 1.5 & & & \\
\hline E23 & $P 6207$ & 1.5 & & & \\
\hline$E 23$ & Pb208 & 1.5 & & 1.5 & \\
\hline E23R & Pt206 & 7.5 & & & \\
\hline E23R & Pt207 & 7.5 & & & \\
\hline E23R & Pb208 & 7.5 & & 7.5 & \\
\hline E24 & Po206 & 1.5 & & & \\
\hline E24 & Pb207 & 1.5 & & & \\
\hline E24 & P6208 & 1.5 & & 1.5 & \\
\hline E31 & Pb206 & & & & \\
\hline E31 & $\mathrm{Pb207}$ & & & & \\
\hline E31 & $\mathrm{Pb} 208$ & & & & \\
\hline E32 & $\mathrm{Pb} 206$ & & & & \\
\hline$E 32$ & $\mathrm{~Pb} 207$ & & & & \\
\hline$E 32$ & Pb208 & & & & \\
\hline E33 & Pb206 & 1.5 & & & \\
\hline E33 & $\mathrm{Pb207}$ & 1.5 & & & \\
\hline E33 & Pb208 & 1.5 & & 1.5 & \\
\hline E34 & $\mathrm{Pb206}$ & 1.5 & & & \\
\hline E34 & Pb207 & 1.5 & & & \\
\hline E34 & $P 6200$ & 1.5 & & 1.5 & \\
\hline$E 41$ & P6206 & 1.5 & & & \\
\hline E41 & Pb207 & 1.5 & & & \\
\hline
\end{tabular}

Note: The actual concentration of eamples for which no standard doviation be reported be leas than the bower limit of detection. The reported concentration is the lower Hinit of quantification. 
Transect E Porewater Concentrations: Lead

Semplo ld. kotope

Concantration

Stdooviation

Element Avg. Cone.

Element Avg. Std. Dov. (나니)

(oㅏㄴ)

(ugh)

(ugl)

\begin{tabular}{|l|l|l|l|l|l|}
\hline E41 & Pb208 & 1.5 & & 1.5 & \\
\hline E42 & Pb206 & 1.5 & & & \\
\hline E42 & Pb207 & 1.5 & & & \\
\hline E42 & Pb208 & 1.5 & & 1.5 & \\
\hline
\end{tabular}




\section{Transect E Porewater Concentrations: Magnesium}

\begin{tabular}{|c|c|c|c|c|c|}
\hline Sample ld. & leotope & $\begin{array}{l}\text { Concentration } \\
\text { (ug/) }\end{array}$ & $\begin{array}{l}\text { Std.Devlation } \\
\text { (ugh) }\end{array}$ & $\begin{array}{l}\text { Element Avg. Conc. } \\
\text { (ugh) }\end{array}$ & $\begin{array}{c}\text { Element Avg. Std. Dev. } \\
\text { (ug/) }\end{array}$ \\
\hline E11 & $M 224$ & & & & \\
\hline E11 & Mgps & & & & \\
\hline E12 & $M g 24$ & & & & \\
\hline E12 & Mops & & & & \\
\hline E12R & $\mathrm{M} 24$ & & & & \\
\hline E12R & MF25 & & & & \\
\hline E13 & Mge 4 & 1860 & 21.9 & & \\
\hline E13 & Mges & 1870 & 24 & 1870 & 23 \\
\hline E14 & $M g 24$ & 4640 & 64.3 & & \\
\hline E14 & Mg25 & 4700 & 47.4 & 4670 & 55.9 \\
\hline E21 & Mg24 & & & & \\
\hline E21 & Mg25 & & & & \\
\hline$E 22$ & Mg24 & & & & \\
\hline$E 22$ & Mges & & & & \\
\hline E23 & $M 924$ & 836 & 9.51 & & \\
\hline E23 & Mgas & 836 & 7.54 & 896 & 8.53 \\
\hline E23R & Mg24 & 1260 & 24.7 & & \\
\hline E23R & Mges & 1270 & 22.6 & 1270 & 23.7 \\
\hline E24 & Mo24 & 3600 & 63.6 & & \\
\hline$\varepsilon 24$ & MFes & 2920 & 28.8 & 3260 & 46.2 \\
\hline E31 & $M 924$ & & & & \\
\hline E31 & Mops & & & & \\
\hline$\varepsilon 32$ & Mg24 & & & & \\
\hline E32 & Mg25 & & & & \\
\hline E33 & Mg24 & 457 & 5.36 & & \\
\hline E33 & My25 & 452 & 7.78 & 455 & 6.57 \\
\hline E34 & Mg24 & 2250 & 10.3 & & \\
\hline E34 & Mg25 & 1870 & 33.1 & 2060 & 21.7 \\
\hline E41 & Mg24 & 1420 & 11.6 & & \\
\hline E41 & MF25 & 1170 & 8.78 & 1300 & 10.7 \\
\hline$E 42$ & Mg24 & 1700 & 352 & & \\
\hline E42 & Mg25 & 1360 & 33.4 & 1530 & 34.3 \\
\hline
\end{tabular}




\section{Transect E Porewater Concentrations: Manganese}

\begin{tabular}{|c|c|c|c|c|c|}
\hline Sample ld. & Eotope & $\begin{array}{l}\text { Concentration } \\
\text { (ugl) }\end{array}$ & $\begin{array}{l}\text { Std.Deviation } \\
\text { (ug/) }\end{array}$ & $\begin{array}{l}\text { Element Avg. Conc. } \\
\text { (ugh })\end{array}$ & $\begin{array}{l}\text { Element Avg. Std. Dov. } \\
\text { (ug/) }\end{array}$ \\
\hline E11 & $\mathrm{Mn}$ & & & & \\
\hline E12 & $\mathrm{Mn}$ & & & & \\
\hline E12R & Mn & & & & \\
\hline $\mathrm{E} 13$ & $\mathrm{Mn}$ & 849 & 4.76 & 849 & 4.76 \\
\hline E14 & $\mathrm{Mn}$ & 1360 & 4.11 & 1350 & 4.11 \\
\hline E21 & $\mathrm{Mn}$ & & & & \\
\hline E22 & Mn & & & & \\
\hline$E_{23}$ & $\mathrm{Mn}$ & 3160 & 18.1 & 3160 & 18.1 \\
\hline E23R & $\mathrm{Mn}$ & 3670 & 28.9 & 3670 & 28.9 \\
\hline E24 & $\mathrm{Mn}$ & 3810 & 14.5 & 3810 & 14.5 \\
\hline E31 & $M n$ & & & & \\
\hline E32 & $\mathrm{Mn}$ & & & & \\
\hline E33 & $\mathrm{Mn}$ & 930 & 2.9 & 950 & 2.9 \\
\hline E34 & $\mathrm{Mn}$ & 2850 & 17.3 & 2830 & 17.3 \\
\hline E41 & $M n$ & 28.3 & .43 & 28.3 & .43 \\
\hline$E 42$ & $\mathrm{Mn}$ & 71.5 & .94 & 71.5 & .94 \\
\hline
\end{tabular}




\begin{tabular}{|c|c|c|c|c|c|}
\hline Sample id. & botope & $\begin{array}{l}\text { Concentration } \\
\text { (ugh) }\end{array}$ & $\begin{array}{l}\text { Std.Deviation } \\
\text { (ug/L) }\end{array}$ & $\begin{array}{c}\text { Element Avg. Conc. } \\
\text { (ug/L) }\end{array}$ & $\begin{array}{l}\text { Element Avg. Std. Dov. } \\
\text { (ugh) }\end{array}$ \\
\hline E11 & $N \in 60$ & & & & \\
\hline E11 & Ni62 & & & & \\
\hline E12 & N160 & & & & \\
\hline E12 & Ni62 & & & & \\
\hline E12R & Ni60 & & & & \\
\hline E12R & Nis2 & & & & \\
\hline E13 & Ni6o & 96.1 & 2.77 & & \\
\hline E13 & Ni62 & 79.3 & 3.39 & 87.7 & 3.08 \\
\hline E14 & $\mathrm{N} i 60$ & 972 & 2.88 & & \\
\hline E14 & Ni62 & 85.3 & 5.96 & 91.3 & 4.42 \\
\hline E21 & Ni60 & & & & \\
\hline E21 & Ni62 & & & & \\
\hline E22 & $\mathrm{Ni60}$ & & & & \\
\hline E22 & Ni62 & & & & \\
\hline E23 & Ni60 & 38.3 & 1.11 & & \\
\hline E23 & $\mathrm{Ni} 62$ & 34.6 & 1.49 & 36.5 & 1.3 \\
\hline E23R & $\mathrm{Ni60}$ & 74 & 1.12 & & \\
\hline E23R & Ni62 & 57.7 & 1.69 & 65.9 & 1.41 \\
\hline E24 & Ni6o & 79.7 & 324 & & \\
\hline E24 & Ni62 & 81.5 & 3.58 & 80.6 & 3.41 \\
\hline E31 & Ni60 & & & & \\
\hline E31 & Ni62 & & & & \\
\hline E32 & Ni60 & & & & \\
\hline E32 & Ni62 & & & & \\
\hline$E 33$ & $\mathrm{Ni} 60$ & 25.1 & .68 & & \\
\hline E33 & Ni62 & 21.6 & 3.59 & 23.4 & 2.13 \\
\hline E34 & Ni60 & 33.1 & 1.38 & & \\
\hline E34 & $\mathrm{Ni62}$ & 31.6 & 3.74 & 32.4 & 2.57 \\
\hline E41 & $\mathrm{Ni} 60$ & 11.3 & .49 & & \\
\hline E41 & Ni62 & 5.51 & .78 & 8.41 & .64 \\
\hline E42 & $\mathrm{Ni} 60$ & 21.9 & .44 & & \\
\hline E42 & Ni62 & 24.2 & 6.72 & 23 & 3.58 \\
\hline
\end{tabular}




\section{Transect E Porewater Concentrations: Selenium}

\begin{tabular}{|c|c|c|c|c|c|}
\hline Semple ld. & Lotope & $\begin{array}{l}\text { Concentration } \\
\text { (ugh) }\end{array}$ & $\begin{array}{l}\text { 8td.Dovietion } \\
\text { (ugh) }\end{array}$ & $\begin{array}{l}\text { Element Avg Conc. } \\
\text { (ugh) }\end{array}$ & $\begin{array}{c}\text { Element Avg. Std. Dov. } \\
\text { ( }(\mathrm{gg} /) \text { ) }\end{array}$ \\
\hline
\end{tabular}

\begin{tabular}{|c|c|c|c|c|c|}
\hline E11 & So77 & & & & \\
\hline E11 & Sos2 & & & & \\
\hline E12 & Se77 & & & & \\
\hline E12 & So82 & & & & \\
\hline E12A & So77 & & & & \\
\hline E12A & Sob2 & & & & \\
\hline E13 & S०77 & 37.5 & & & \\
\hline$E 13$ & Se82 & 37.5 & & 37.5 & \\
\hline E14 & Se 77 & 12.6 & 7.66 & & \\
\hline E14 & SeE2 & 124 & 7.57 & 12.5 & 7.62 \\
\hline E21 & So7 & & & & \\
\hline E21 & Se82 & & & & \\
\hline$E 22$ & so7 & & & & \\
\hline$E 22$ & Se82 & & & & \\
\hline$E 23$ & Sot7 & 37.5 & & & \\
\hline$E_{23}$ & SoE2 & 37.5 & & 37.5 & \\
\hline E23月 & So77 & 37.5 & & & \\
\hline E23R & SeB2 & 37.5 & & 37.5 & \\
\hline E24 & So77 & 37.5 & & & \\
\hline E24 & So82 & 37.5 & & 37.5 & \\
\hline E31 & So77 & & & & \\
\hline E31 & So82 & & & & \\
\hline E32 & So 7 & & & & \\
\hline$E 32$ & SeB2 & & & & \\
\hline$E 33$ & So77 & 37.5 & & & \\
\hline$E 33$ & Sog2 & 37.5 & & 37.5 & \\
\hline E34 & 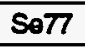 & 37.5 & & & \\
\hline$E 34$ & So82 & 37.5 & & 37.5 & \\
\hline$E 41$ & So77 & 37.5 & & & \\
\hline E41 & So82 & 37.5 & & 37.5 & \\
\hline$E 42$ & se77 & 37.5 & & & \\
\hline$E 42$ & SeB2 & 37.5 & & 37.5 & \\
\hline
\end{tabular}


Transect E Porewater Concentrations: Silver

\begin{tabular}{|c|c|c|c|c|c|}
\hline Sample ld. & botope & $\begin{array}{l}\text { Concentration } \\
\text { (ugh) }\end{array}$ & $\begin{array}{l}\text { Sudpoviation } \\
\text { (ugl) }\end{array}$ & $\begin{array}{l}\text { Element Avg. Conc. } \\
\text { (ugh) }\end{array}$ & $\begin{array}{c}\text { Element Avg. Std. Dov. } \\
\text { (ugl) }\end{array}$ \\
\hline E11 & A0107 & & & & \\
\hline E11 & Ag109 & & & & \\
\hline E12 & Ag107 & & & & \\
\hline E12 & Ad100 & & & & \\
\hline E12R & A0107 & & & & \\
\hline E12R & $A 0109$ & & & & \\
\hline E13 & Ag107 & & & & \\
\hline E13 & Ag109 & & & & \\
\hline E14 & Ag107 & & & & \\
\hline E14 & Ag109 & & & & \\
\hline E21 & Ag107 & & & & \\
\hline E21 & A0109 & & & & \\
\hline E22 & Ag107 & & & & \\
\hline E22 & Ag100 & & & & \\
\hline $\mathrm{E} 23$ & A9107 & & & & \\
\hline E23 & $A 0100$ & & & & \\
\hline E23R & Ag107 & & & & \\
\hline E23R & $A 0100$ & & & & \\
\hline E24 & Ag107 & .15 & & & \\
\hline E24 & Ad109 & .15 & & .15 & \\
\hline E31 & Ag107 & & & & \\
\hline E31 & $A 0100$ & & & & \\
\hline$E 32$ & A9107 & & & & \\
\hline E32 & A0109 & & & & \\
\hline$E 33$ & Ag107 & .15 & & & \\
\hline$E 33$ & A0109 & .15 & & .15 & \\
\hline E34 & Ag107 & .98 & .54 & & \\
\hline E34 & Ag109 & .15 & & .56 & .54 \\
\hline E41 & Ag107 & .15 & & & \\
\hline E41 & Ag109 & .15 & & .15 & \\
\hline E42 & Ag107 & 1.34 & .49 & & \\
\hline E42 & Ag109 & .15 & & .75 & .49 \\
\hline
\end{tabular}




\section{Transect E Porewater Concentrations: Sodium}

\begin{tabular}{|c|c|c|c|}
\hline Sample ld. & leotope & $\begin{array}{l}\text { Concentration } \\
(\log /)\end{array}$ & $\begin{array}{l}\text { Std.Doviation } \\
\text { (ugh) }\end{array}$ \\
\hline
\end{tabular}

\begin{tabular}{|c|c|c|c|c|c|}
\hline E11 & $\mathrm{Na}$ & & & & \\
\hline E12 & $\mathrm{Na}$ & & & & \\
\hline E12R & $\mathrm{Na}$ & & & & \\
\hline E13 & $\mathrm{Na}$ & 57200 & 417 & 57200 & 417 \\
\hline E14 & $\mathrm{Na}$ & 18200 & 122 & 18200 & 122 \\
\hline E21 & $\mathrm{Na}$ & & & & \\
\hline E22 & $\mathrm{Na}$ & & & & \\
\hline$E 23$ & $\mathrm{Na}$ & 44100 & 836 & 44100 & 836 \\
\hline E23R & $\mathrm{Na}$ & 45000 & 723 & 45000 & 723 \\
\hline E24 & $\mathrm{Na}$ & 20000 & 364 & 20000 & 364 \\
\hline E31 & $\mathrm{Na}$ & & & & \\
\hline$E 32$ & $\mathrm{Na}$ & & & & \\
\hline$E 33$ & $\mathrm{Na}$ & 24500 & 272 & 24500 & 272 \\
\hline E34 & $\mathrm{Na}$ & 44200 & 161 & 44200 & 161 \\
\hline$E 41$ & $\mathrm{Na}$ & 14200 & 40.6 & 14200 & 40.6 \\
\hline$E_{42}$ & $\mathrm{Na}$ & 8390 & 44.4 & 8390 & 44.4 \\
\hline
\end{tabular}

Note: The ectual concentration of eamples for which no stenderd deviation be reported b hase than the lower llmit of dotection. The reported concentratton bs the bwer limit of quantification. 


\section{Transect E Porewater Concentrations: Strontium}

\begin{tabular}{|c|c|c|c|c|c|}
\hline Sample ld. & Lotop & $\begin{array}{l}\text { Concantration } \\
\text { (ug/t) }\end{array}$ & $\begin{array}{l}\text { Std.Dovition } \\
\text { (ugh) }\end{array}$ & $\begin{array}{l}\text { Element Avg. Conc. } \\
\text { (uoll) }\end{array}$ & $\begin{array}{l}\text { Element Avg. Std. Dov. } \\
\text { (ugr) }\end{array}$ \\
\hline E11 & $\mathrm{Sr}$ & & & & \\
\hline E12 & Sr & & & & \\
\hline E12R & $\mathrm{Sr}$ & & & & \\
\hline E13 & Sr & 502 & .65 & 502 & .65 \\
\hline E14 & Sr & 67.9 & 1.11 & 67.8 & 1.11 \\
\hline E21 & $\mathrm{Sr}$ & & & & \\
\hline E22 & $\mathbf{S r}$ & & & & \\
\hline E23 & $\mathbf{S r}$ & 402 & .97 & 402 & .97 \\
\hline E23R & $\mathrm{Sr}$ & 49.6 & .55 & 49.6 & .55 \\
\hline E24 & $\mathrm{Sr}$ & 282 & 1.85 & 282 & 1.95 \\
\hline E31 & Sr & & & & \\
\hline E32 & Sr & & & & \\
\hline$E 33$ & Sr & 31.5 & .7 & 31.5 &.$\overline{7}$ \\
\hline E34 & $\mathrm{Sr}$ & 56 & .77 & 56 & .77 \\
\hline E41 & $\mathrm{Sr}$ & 15.7 & .76 & 15.7 & .76 \\
\hline E42 & $\mathrm{Sr}$ & 20.6 & 28 & 20.6 & 28 \\
\hline
\end{tabular}




\section{Transect E Porewater Concentrations: Thorium}

\begin{tabular}{|c|c|c|c|c|c|}
\hline Semple ld. & notope & $\begin{array}{l}\text { Concentration } \\
\text { (ug/) }\end{array}$ & $\begin{array}{l}\text { Stdidovintion } \\
\text { (ugh) }\end{array}$ & $\begin{array}{l}\text { Element Avg- Conc. } \\
\text { (ugh) }\end{array}$ & $\begin{array}{c}\text { EJement Avg. Std. Dov. } \\
\text { (ugh) }\end{array}$ \\
\hline E11 & Th232 & & & & \\
\hline E12 & Th232 & & & & \\
\hline E12R & Th232 & & & & \\
\hline E13 & Th232 & .38 & & .38 & \\
\hline E14 & Th232 & .5 & & .5 & \\
\hline E21 & The32 & & & & \\
\hline$E 22$ & Th232 & & & & \\
\hline E23 & Th232 & .5 & & .5 & \\
\hline E23R & Th232 & .5 & & .5 & \\
\hline E24 & Th232 & .5 & & .5 & \\
\hline E31 & Th232 & & & & \\
\hline E32 & Th232 & & & & \\
\hline$E 33$ & Th232 & .5 & & .5 & \\
\hline E34 & Th232 & .5 & & .5 & \\
\hline$E 41$ & Th232 & .5 & & .5 & \\
\hline$E 42$ & Th232 & .5 & & .5 & \\
\hline
\end{tabular}

Note: The actual concentration of eamples for which no etendard doviation be reported is leas than the lown llmit of detection. The reported concentration be the lower limt of quantifiestion. 


\section{Transect E Porewater Concentrations: Tin}

\begin{tabular}{|c|c|c|c|c|c|}
\hline Semple ld. & botope & $\begin{array}{l}\text { Concentration } \\
(U \mathrm{~L} / \mathrm{L})\end{array}$ & $\begin{array}{l}\text { 8ti.Dovilation } \\
\text { (uggh) }\end{array}$ & $\begin{array}{l}\text { Element Avg Conc. } \\
\text { ( } \lg h)\end{array}$ & $\begin{array}{l}\text { Element Avg. Std. Dov. } \\
\text { (ugh) }\end{array}$ \\
\hline
\end{tabular}

\begin{tabular}{|c|c|c|c|c|c|}
\hline E11 & $\operatorname{Sn} 118$ & & & & \\
\hline E11 & Sn 120 & & & & \\
\hline$E 12$ & $\operatorname{Sn} 118$ & & & & \\
\hline$E 12$ & $\operatorname{Sn} 120$ & & & & \\
\hline E12R & Sn118 & & & & \\
\hline E12R & $\sin 120$ & & & & \\
\hline$E 13$ & $\operatorname{Sn} 118$ & 12.6 & & & \\
\hline$E 13$ & $\operatorname{Sn} 120$ & 12.6 & & 12.6 & \\
\hline E14 & Sn118 & 25 & & & \\
\hline$E 14$ & Sn 120 & 25 & & 25 & \\
\hline$E_{21}$ & $\operatorname{Sn} 118$ & & & & \\
\hline$E_{21}$ & $\operatorname{Sn} 120$ & & & & \\
\hline$E 22$ & $\operatorname{Sn} 118$ & & & & \\
\hline$E 22$ & $\operatorname{Sn} 120$ & & & & \\
\hline$E_{23}$ & Sn118 & 25 & & & \\
\hline$E 23$ & $\operatorname{Sn} 120$ & .74 & 26 & .5 & 26 \\
\hline E23R & Sn118 & 25 & & & \\
\hline E23R & $\operatorname{Sn} 120$ & 25 & & 25 & \\
\hline E24 & $\operatorname{Sn} 118$ & .42 & .18 & & \\
\hline$E 24$ & $\sin 120$ & 25 & & .34 & .18 \\
\hline E31 & $\operatorname{Sn} 118$ & & & & \\
\hline$E 31$ & Sn120 & & & & \\
\hline$E 32$ & $\sin 118$ & & & & \\
\hline$E_{32}$ & $\operatorname{Sn} 120$ & & & & \\
\hline$E 33$ & $\operatorname{Sn} 118$ & .3 & .06 & & \\
\hline$E 33$ & $\operatorname{Sin} 120$ & .34 & .14 & .32 & .1 \\
\hline$E 34$ & $\operatorname{Sn} 118$ & .74 & .19 & & \\
\hline E34 & Sn120 & 25 & & .5 & .18 \\
\hline$E 41$ & Sn118 & .47 & .14 & & \\
\hline$E 41$ & Sn120 & .48 & 23 & .48 & .19 \\
\hline$E_{42}$ & $\operatorname{Sn} 118$ & .57 & .18 & & \\
\hline$E 42$ & $\operatorname{Sn} 120$ & 25 & & .41 & .18 \\
\hline
\end{tabular}




\section{Transect E Porewater Concentrations: Uranium-235}

\begin{tabular}{|c|c|c|c|c|c|}
\hline Sample id. & leotope & $\begin{array}{l}\text { Concentration } \\
\text { (ugh) }\end{array}$ & $\begin{array}{l}\text { 8id.Doviation } \\
\text { (ugh) }\end{array}$ & $\begin{array}{l}\text { Element Avg. Conc. } \\
\text { (ug/L) }\end{array}$ & $\begin{array}{c}\text { Element Avg. Std. Dov. } \\
\text { (ug/) }\end{array}$ \\
\hline E11 & U235 & & & & \\
\hline E12 & U235 & & & & \\
\hline E12R & U235 & & & & \\
\hline E13 & U235 & .02 & & .02 & \\
\hline E14 & U235 & .02 & &.$\infty 2$ & \\
\hline E21 & U235 & & & & \\
\hline E22 & U235 & & & & \\
\hline E23 & U235 & .02 & & .02 & \\
\hline E23R & U235 & .02 & &.$\infty 2$ & \\
\hline E24 & U235 & .02 & &.$\infty$ & \\
\hline E31 & U235 & & & & \\
\hline E32 & U235 & & & & \\
\hline E33 & U235 & .02 & .01 & .02 & .01 \\
\hline E34 & U235 & .02 & & .02 & \\
\hline E41 & U235 & .02 & & .02 & \\
\hline E42 & U235 & .02 & & .02 & \\
\hline
\end{tabular}




\section{Transect E Porewater Concentrations: Uranium-238}

\begin{tabular}{|c|c|c|c|c|c|}
\hline Semple ld. & botope & $\begin{array}{l}\text { Concentration } \\
\text { (ugh) }\end{array}$ & $\begin{array}{c}\text { Stdoovintion } \\
\text { (ugl) }\end{array}$ & $\begin{array}{c}\text { Ekement Avg, Cone. } \\
\text { (ugh) }\end{array}$ & $\begin{array}{l}\text { Element Avg. Std. Dev. } \\
\text { (Ug/l) }\end{array}$ \\
\hline E11 & U238 & & & & \\
\hline E12 & U238 & & & & \\
\hline E12R & U238 & & & & \\
\hline E13 & U238 & .69 & .08 & .69 & .06 \\
\hline E14 & U238 & .84 & .15 & .64 & .15 \\
\hline E21 & $\mathbf{U 2 3 8}$ & & & & \\
\hline E22 & U238 & & & & \\
\hline E23 & U238 & .02 & & .02 & \\
\hline E23R & U238 & 1.77 & .06 & 1.77 & .06 \\
\hline$E 24$ & U238 & 1.16 & .03 & 1.16 & .03 \\
\hline E31 & U238 & & & & \\
\hline E32 & U238 & & & & \\
\hline E33 & U238 & 27 & .03 & 27 & .03 \\
\hline E34 & U238 & .62 & .62 & .62 & .62 \\
\hline E41 & U238 & 25 & & 25 & \\
\hline E42 & U238 & 25 & & 25 & \\
\hline
\end{tabular}




\section{Transect E Porewater Concentrations: Vanadium}

\begin{tabular}{|c|c|c|c|c|c|}
\hline Semple ld. & leotops & $\begin{array}{l}\text { Concentration } \\
(u g /)\end{array}$ & $\begin{array}{l}\text { Std.Doviation } \\
\text { (ugL) }\end{array}$ & $\begin{array}{l}\text { Element Avg- Conc. } \\
\text { (ugh) }\end{array}$ & $\begin{array}{c}\text { Elemant Avg. Std. Dov. } \\
\text { (ugg) }\end{array}$ \\
\hline
\end{tabular}

\begin{tabular}{|c|c|c|c|c|c|}
\hline E11 & $\mathbf{v}$ & & & & \\
\hline E12 & V & & & & \\
\hline$E 12 R$ & $v$ & & & & \\
\hline E13 & v & 1.38 & & 1.38 & \\
\hline E14 & v & .52 & .17 & .52 & .17 \\
\hline E21 & V & & & & \\
\hline$E 22$ & $V$ & & & & \\
\hline$E 23$ & V & 2 & .08 & 2 & .08 \\
\hline E23R & V & 25 & & 25 & \\
\hline E24 & v & & & & \\
\hline E31 & v & & & & \\
\hline E32 & $\mathbf{v}$ & & & & \\
\hline E33 & v & 25 & & 25 & \\
\hline E34 & v & 25 & & 25 & \\
\hline E41 & $\mathbf{v}$ & 25 & & 25 & \\
\hline$E 42$ & $\bar{v}$ & 25 & & 25 & \\
\hline
\end{tabular}

Note: The actual concentration of eamples for which no atunderd doviation is reported is has than the lower limit of detection. The reported concentration th tho lower Umit of quantifiention. 


\section{Transect E Porewater Concentrations: Yttrium}

\begin{tabular}{|c|c|c|c|c|c|}
\hline Sampte ld. & Eotope & $\begin{array}{l}\text { Concentration } \\
\text { (ug/L) }\end{array}$ & $\begin{array}{l}\text { Studovitation } \\
\text { (ug/) }\end{array}$ & $\begin{array}{l}\text { Element Avg Conc. } \\
\text { (ug/L) }\end{array}$ & $\begin{array}{c}\text { Element Avg. Std. Dov. } \\
\text { (ugh) }\end{array}$ \\
\hline
\end{tabular}

\begin{tabular}{|c|c|c|c|c|c|}
\hline E11 & $Y$ & & & & \\
\hline E12 & $Y$ & & & & \\
\hline E12R & $Y$ & & & & \\
\hline E13 & $Y$ & 25.7 & .51 & 25.7 & .51 \\
\hline E14 & $Y$ & 28.6 & .51 & 28.6 & .51 \\
\hline E21 & $Y$ & & & & \\
\hline$E 22$ & $Y$ & & & & \\
\hline E23 & $Y$ & 224 & .05 & 2.24 & .05 \\
\hline E23R & $Y$ & 12.4 & .44 & 12.4 & .44 \\
\hline E24 & $Y$ & 29.1 & .49 & 29.1 & .49 \\
\hline E31 & $Y$ & & & & \\
\hline$E 32$ & $Y$ & & & & \\
\hline$E 33$ & $Y$ & 7.25 & .08 & 725 & .08 \\
\hline E34 & $Y$ & 19.7 & .36 & 18.7 & .36 \\
\hline E41 & $Y$ & .45 &.$\infty 2$ & .45 & .02 \\
\hline$E 42$ & $Y$ & 1.17 & .09 & 1.17 & .00 \\
\hline
\end{tabular}

Note: The sctual concentration of eamples for which no standerd doviation ts reportad b leces than the lower limit of detection. The reported concentration bs the lower limit of quantification. 


\section{Transect E Porewater Concentrations: Zinc}

\begin{tabular}{|c|c|c|c|c|c|}
\hline Semple $\mathrm{Cl}$. & leotope & $\begin{array}{l}\text { Coneentration } \\
\text { (4:/l) }\end{array}$ & $\begin{array}{l}\text { Stdoovituon } \\
\text { (ug/L) }\end{array}$ & $\begin{array}{l}\text { Ehment Avg. Cone. } \\
\text { (ugh) }\end{array}$ & $\begin{array}{c}\text { Ekment Avg. Std. Dov. } \\
\text { (ugh) }\end{array}$ \\
\hline E11 & Zn66 & & & & \\
\hline E12 & Zn66 & & & & \\
\hline E12R & Zn66 & & & & \\
\hline E13 & Zn66 & 140 & 2.73 & 140 & 2.73 \\
\hline E14 & Zn66 & 171 & 8.74 & 171 & 8.74 \\
\hline E21 & Zn66 & & & & \\
\hline E22 & Zn66 & & & & \\
\hline E23 & Zn66 & 88.7 & .88 & 88.7 & .98 \\
\hline E23R & Zn66 & 61.9 & 1 & 61.9 & 1 \\
\hline E24 & Zn66 & 321 & 7.43 & 321 & 7.43 \\
\hline E31 & Zn66 & & & & \\
\hline E32 & Zns6 & & & & \\
\hline E33 & Zn66 & 162 & 4 & 162 & 4 \\
\hline E34 & Zn66 & 141 & 5.7 & 141 & 5.7 \\
\hline E41 & Zns6 & 167 & 4.38 & 167 & 4.38 \\
\hline E42 & Zn66 & 140 & 4.88 & 140 & 4.88 \\
\hline
\end{tabular}




\section{Transect E Porewater Concentrations: Zirconium}

\begin{tabular}{|c|c|c|c|c|c|}
\hline Sampte ld. & ketope & $\begin{array}{l}\text { Concentration } \\
\text { (ugh) }\end{array}$ & $\begin{array}{l}\text { Std.Doviation } \\
\text { (ugh) }\end{array}$ & $\begin{array}{c}\text { Element Avg. Conc. } \\
\text { (ugh) }\end{array}$ & $\begin{array}{c}\text { Eloment Avg Std. Dav } \\
\text { (ugh) }\end{array}$ \\
\hline
\end{tabular}

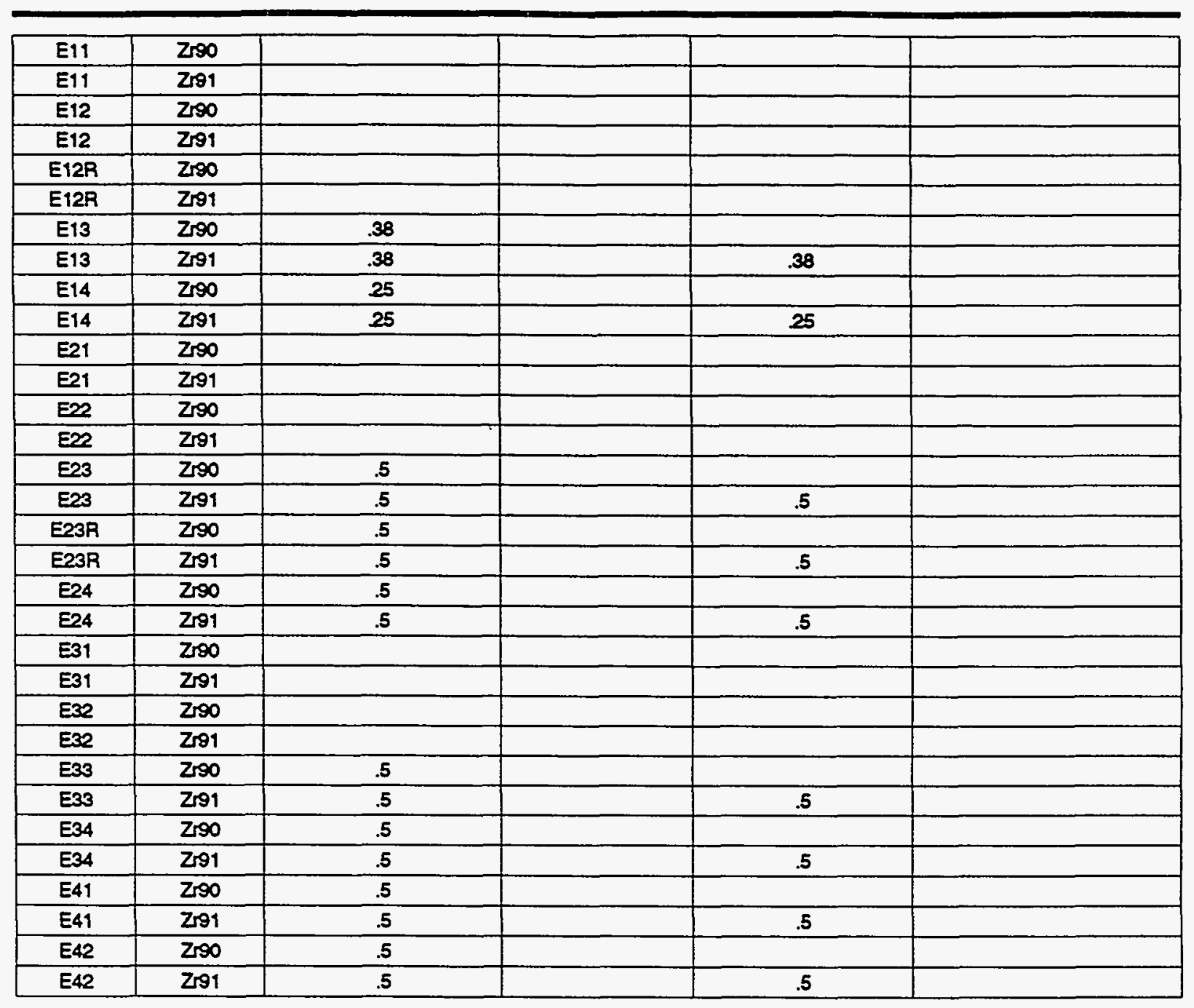




\section{Transect F Porewater Concentrations: Aluminum}

\begin{tabular}{|c|c|c|c|c|c|}
\hline Sampte id. & lsotope & $\begin{array}{l}\text { Concentration } \\
\text { (ugh) }\end{array}$ & $\begin{array}{l}\text { StdDovistion } \\
\text { (ugh) }\end{array}$ & $\begin{array}{l}\text { Element Avg. Conc. } \\
\text { (Lg/L) }\end{array}$ & $\begin{array}{c}\text { Element Avg std. Dev. } \\
\text { (ugh) }\end{array}$ \\
\hline F11 & $\mathbf{A}$ & & & & \\
\hline$F 12$ & $\mathbf{A}$ & 1980 & 41.6 & 1800 & 41.6 \\
\hline F13 & $\mathbf{A}$ & 462 & .57 & 462 & .57 \\
\hline F21 & $\mathbf{N}$ & & & & \\
\hline F21R & $\mathbf{N}$ & & & & \\
\hline F22 & $\mathbf{N}$ & 120 & 3 & 120 & 3 \\
\hline $\mathrm{P23}$ & A & 1.57 & .73 & 1.57 & .73 \\
\hline F31 & $\mathbf{N}$ & & & & \\
\hline F32 & $\mathbf{A}$ & 52.8 & 1.58 & 52.8 & 1.58 \\
\hline F33 & $\mathbf{A}$ & 21.9 & 128 & 21.8 & 128 \\
\hline F41 & $\mathbf{A}$ & & & & \\
\hline F42 & $\mathbf{A}$ & 31.3 & 2.66 & 31.3 & 2.66 \\
\hline $\mathrm{F} 43 \mathrm{I}$ & $\mathbf{A}$ & 6070 & & 6070 & \\
\hline F51 & $\mathbf{A}$ & & & & \\
\hline$F 52$ & A & 10.1 & & 10.1 & \\
\hline F53 & A & 50 & & 50 & \\
\hline
\end{tabular}

${ }^{1}$ Reported value is actual concentration. No standard deviation is reported. 


\section{Transect F Porewater Concentrations: Arsenic}

\begin{tabular}{|c|c|c|c|c|c|}
\hline Sample ld. & leotope & $\begin{array}{l}\text { Coneentration } \\
\text { (ugh) }\end{array}$ & $\begin{array}{l}\text { Std.Doviation } \\
\text { (ugh) }\end{array}$ & $\begin{array}{c}\text { Eloment Avg. Conc. } \\
\text { (ugh) }\end{array}$ & $\begin{array}{c}\text { Element Avg. Std. Dov. } \\
\text { (ug/l) }\end{array}$ \\
\hline F11 & As & & & & \\
\hline $\mathrm{F} 12$ & As & 825 & & 825 & \\
\hline F13 & As & 825 & & 825 & \\
\hline $\mathrm{F21}$ & As & & & & \\
\hline F21R & As & & & & \\
\hline F22 & As & 825 & & 825 & \\
\hline $\mathrm{P23}$ & As & 326 & 24 & 326 & 24 \\
\hline $\mathrm{F31}$ & As & & & & \\
\hline F32 & As & 825 & & 825 & \\
\hline F33 & As & 45 & & 45 & \\
\hline F41 & As & & & & \\
\hline $\mathrm{F} 42$ & As & 825 & & 825 & \\
\hline$F 43$ & As & 825 & & 825 & \\
\hline F51 & As & & & & \\
\hline $\mathrm{F}_{52}{ }^{\prime}$ & As & 3.02 & & 3.02 & \\
\hline F53 & As & 1.5 & & 1.5 & \\
\hline
\end{tabular}

${ }^{1}$ Reported value is actual concentration. No standard deviation is reported. 


\section{Transect F Porewater Concentrations: Barium}

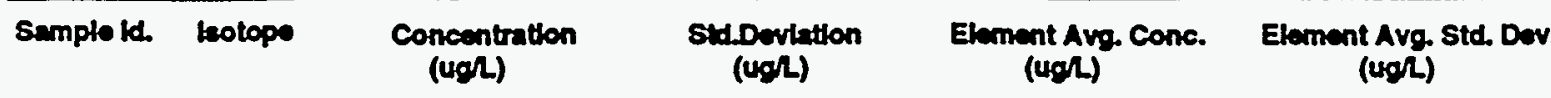

\begin{tabular}{|c|c|c|c|c|c|}
\hline$F 11$ & Ba135 & & & & \\
\hline F11 & Ba137 & & & & \\
\hline$F 11$ & Ba138 & & & & \\
\hline$F 12$ & Ba135 & 54.9 & 2.23 & & \\
\hline$F 12$ & Ba137 & 59.9 & .61 & & \\
\hline$F 12$ & Ba138 & 54.4 & .89 & 56.4 & 128 \\
\hline $\mathrm{F} 13$ & Ba135 & 23 & .83 & & \\
\hline $\mathbf{F 1 3}$ & Ba137 & 24.6 & 1.4 & & \\
\hline$F_{13}$ & Ba138 & 23.3 & .77 & 23.6 & 1 \\
\hline F21 & Ba135 & & & & \\
\hline F21 & Ba137 & & & & \\
\hline$P 21$ & Ba138 & & & & \\
\hline F21R & Ba135 & & & & \\
\hline F21R & Ba137 & & & & \\
\hline F21R & Ba138 & & & & \\
\hline$F 22$ & Ba135 & 227 & 6.05 & & \\
\hline$F 22$ & Ba137 & 225 & 288 & & \\
\hline$F 22$ & Ba138 & 219 & 4.02 & 224 & 4.32 \\
\hline$F 23$ & Ba135 & 14 & 1.48 & & \\
\hline$F 23$ & Ba137 & 15.5 & .46 & & \\
\hline$F 23$ & Ba138 & 128 & .42 & 14.1 & .79 \\
\hline$F 31$ & Ba135 & & & & \\
\hline F31 & Ba137 & & & & \\
\hline F31 & Ba138 & & & & \\
\hline$F 32$ & Ba135 & 24.9 & 3.09 & & \\
\hline F32 & Ba137 & 23.7 & 121 & & \\
\hline F32 & Ba138 & 24 & 1.34 & 242 & 1.88 \\
\hline F33 & Ba135 & 43.1 & 3.36 & & \\
\hline$F 33$ & Ba137 & 42.5 & 2.43 & & \\
\hline F33 & Ba138 & 42.9 & 1.72 & 42.8 & 2.5 \\
\hline F4t & Ba135 & & & & \\
\hline F41 & Ba137 & & & & \\
\hline$F_{41}$ & Ba138 & & & & \\
\hline F42 & Ba135 & 26.1 & 1.57 & & \\
\hline$F_{42}$ & Ba137 & 28.6 & 1.01 & & \\
\hline$F_{42}$ & Ba138 & 26.4 & 1.09 & 27 & 122 \\
\hline $\mathrm{F} 43$ & Ba135 & 88.3 & & & \\
\hline$F_{431}$ & 8a137 & 89 & & & \\
\hline $\mathrm{F}_{43^{\prime}}$ & Ba138 & 91.3 & & 89.5 & \\
\hline$F 51$ & Ba135 & & & & \\
\hline F51 & Ba137 & & & & \\
\hline F51 & Ba138 & & & & \\
\hline F52' & Ba135 & 7.3 & & & \\
\hline F52' & Ba137 & 5.61 & & & \\
\hline
\end{tabular}

${ }^{1}$ Reported value is actual concentration. No standard deviation is reported.

Note: The actual concentration of eamples for which no stendard deviation is reportad be leas than the fower limit of detection. The reported concentration be the lower lintt of quantfication. 
Transect F Porewater Concentrations: Barium

\begin{tabular}{|c|c|c|c|c|c|}
\hline Sample id. & Eotope & $\begin{array}{l}\text { Concentration } \\
\text { (ugh) }\end{array}$ & $\begin{array}{l}\text { Strd.Doviation } \\
\text { (ugl) }\end{array}$ & $\begin{array}{l}\text { Element Avg- Cons. } \\
\text { (ugh) }\end{array}$ & $\begin{array}{l}\text { Element Avg. std. Dov. } \\
\text { (ugh) }\end{array}$ \\
\hline F52 & Ba138' & 5.95 & & 629 & \\
\hline$F 53$ & Ba135' & 113 & & & \\
\hline F53 & Ba137' & 111 & & & \\
\hline F53 & Ba138 & 115 & & 113 & \\
\hline
\end{tabular}

${ }^{1}$ Reported value is actual concentration. No standard deviation is reported. 


\begin{tabular}{|c|c|c|c|c|c|}
\hline \multicolumn{6}{|c|}{ Transect F Porewater Concentrations: Cadmium } \\
\hline Sampto Id. & leotope & $\begin{array}{l}\text { Concontration } \\
\text { (ugh) }\end{array}$ & $\begin{array}{l}\text { Sid. Dowiation } \\
\text { (ugh) }\end{array}$ & $\begin{array}{l}\text { Element Aug Cone. } \\
\text { (ugh) }\end{array}$ & $\begin{array}{l}\text { Element Aug. Std. Dev. } \\
\text { (ug/L) }\end{array}$ \\
\hline F11 & $\cos 111$ & & & & \\
\hline$F 11$ & $\operatorname{cod114}$ & & & & \\
\hline$F 12$ & Cd111 & 126 & .19 & & \\
\hline$F 12$ & Cd114 & .64 & .18 & .85 & .19 \\
\hline$F 13$ & Cd111 & 4.13 & & & \\
\hline F13 & $\operatorname{cod114}$ & 4.13 & & 4.13 & \\
\hline$P 21$ & Cd111 & & & & \\
\hline $\mathrm{F} 21$ & $\operatorname{cod} 114$ & & & & \\
\hline F21R & Cd111 & & & & \\
\hline F21R & Cd114 & & & & \\
\hline $\mathrm{F} 22$ & Cd111 & .4 & .16 & & \\
\hline$P 22$ & Cd114 & .72 & 39 & .56 & 28 \\
\hline$F 23$ & Cd111 & 4.13 & & & \\
\hline$F 23$ & $\operatorname{cod114}$ & 4.13 & & 4.13 & \\
\hline F31 & $\operatorname{cod} 111$ & & & & \\
\hline F31 & $\operatorname{Cd114}$ & & & & \\
\hline F32 & Cd111 & .31 & .16 & & . \\
\hline$F 32$ & Cd114 & .16 & .02 & 24 & .09 \\
\hline$F 33$ & Cd111 & 4.13 & & & \\
\hline F33 & Cd114 & 4.13 & & 4.13 & \\
\hline$F 41$ & Cd111 & & & & \\
\hline$F_{41}$ & $\operatorname{Cd114}$ & & & & \\
\hline$F 42$ & Cod111 & 21 & .1 & & \\
\hline F42 & $\cot 114$ & .36 & .18 & 28 & .14 \\
\hline$F_{43} T$ & Cd111 & 1.16 & & & \\
\hline$F_{43}{ }^{\prime}$ & $\operatorname{cod114}$ & 1.7 & & 1.43 & \\
\hline F51 & $\operatorname{Cod111}$ & & & & \\
\hline F51 & Cd114 & & & & \\
\hline$F 52$ & Cd111 & 4.13 & & & \\
\hline$F 52$ & Cd114 & 4.13 & & 4.13 & \\
\hline F53 1 & Cd111 & .55 & & & \\
\hline F53 & Cd114 & 4.13 & & 2.34 & \\
\hline
\end{tabular}

${ }^{1}$ Reported value is actual concentration. No standard deviation is reported. 


\section{Transect F Porewater Concentrations: Calcium}

\begin{tabular}{|c|c|c|c|c|c|}
\hline Sample ld. & kotope & $\begin{array}{l}\text { Concentration } \\
\text { (배느) }\end{array}$ & $\begin{array}{c}\text { Stadowiation } \\
\text { (ug/L) }\end{array}$ & $\begin{array}{l}\text { Elmment Avg. Conc. } \\
\text { (ugh) }\end{array}$ & $\begin{array}{c}\text { Element Avg. Std. Dov. } \\
\text { (ugh) }\end{array}$ \\
\hline F11 & Cas4 & & & & \\
\hline F12 & $\operatorname{Ca} 44$ & 5140 & 45.6 & 5140 & 45.6 \\
\hline F13 & Co44 & 885 & 31.6 & 885 & 31.6 \\
\hline $\mathrm{F21}$ & Ca44 & & & & \\
\hline F21R & Ca44 & & & & \\
\hline $\mathrm{F} 22$ & Co44 & 1270 & 2.92 & 1270 & 2.92 \\
\hline$F 23$ & $\mathrm{Ca} 44$ & 40 & 11 & 40 & 11 \\
\hline F31 & $\mathrm{Ca} 44$ & & & & \\
\hline$F 32$ & Ces4 & 2250 & 58.6 & 2250 & 58.6 \\
\hline F33 & Ce44 & 1160 & 34.7 & 1160 & 34.7 \\
\hline F41 & Ce44 & & & & \\
\hline$F-42$ & Ca44 & 1380 & 71 & 1380 & 71 \\
\hline$F 431$ & Ca44 & 2020 & & 2020 & \\
\hline F51 & Ca44 & & & & \\
\hline$F_{52}$ & Ca44 & 938 & & 838 & \\
\hline $\mathrm{F}_{53} \mathrm{I}^{\mathrm{I}}$ & $\operatorname{Ca} 44$ & 794 & & 794 & \\
\hline
\end{tabular}

${ }^{1}$ Reported value is actual concentration. No standard deviation is reported. 


\section{Transect F Porewater Concentrations: Cesium}

\begin{tabular}{|c|c|c|c|c|c|}
\hline Sample ld. & Eotope & $\begin{array}{l}\text { Concentration } \\
\text { (ug/l) }\end{array}$ & $\begin{array}{l}\text { Stdovituton } \\
\text { (ug/L) }\end{array}$ & $\begin{array}{c}\text { Element Avg. Conc. } \\
\text { (ugh) }\end{array}$ & $\begin{array}{c}\text { Element Avg. Std. Dov. } \\
\text { (ugh) }\end{array}$ \\
\hline F11 & Cs & & & & \\
\hline F12 & C8 & .02 & 0 & .02 & 0 \\
\hline F13 & Cs & .55 & & .55 & \\
\hline P21 & Cs & & & & \\
\hline F21R & Cs & & & & \\
\hline$F 22$ & Cs & 25 & .11 & 25 & .11 \\
\hline F23 & Cs & 55 & & .55 & \\
\hline F31 & $\mathrm{Cs}$ & & & & \\
\hline$F 32$ & $\mathrm{Cs}$ & .55 & & .55 & \\
\hline F33 & C8 & .11 & .04 & .11 & .04 \\
\hline$F 41$ & Cs & & & & \\
\hline F42 & $\mathrm{Cs}_{8}$ & .12 & .05 & .12 & .05 \\
\hline F43 T & $\mathrm{C}_{8}$ & .3 & & .3 & \\
\hline $\mathrm{F51}$ & Cs & & & & \\
\hline F52 & C8 & .55 & & .55 & \\
\hline$F 53$ & Cs & .55 & & 55 & \\
\hline
\end{tabular}

${ }^{1}$ Reported value is actual concentration. No standard deviation is reported. 


\section{Transect F Porewater Concentrations: Chromium}

\begin{tabular}{|c|c|c|c|c|c|}
\hline Sample ld. & teotope & $\begin{array}{l}\text { Concentration } \\
\text { (ugh) }\end{array}$ & $\begin{array}{l}\text { Std.Doviation } \\
\text { (ug/L) }\end{array}$ & $\begin{array}{l}\text { Element Ava Conc. } \\
\text { (ugh) }\end{array}$ & $\begin{array}{l}\text { Element Avg. Std. Dov. } \\
\text { (ugh) }\end{array}$ \\
\hline F11 & Cr52 & & & & \\
\hline F11 & 953 & & & & \\
\hline F12 & Cr52 & .91 & .18 & & \\
\hline F12 & Cr53 & .91 & .18 & .91 & .18 \\
\hline F13 & 0.52 & 1.5 & & & \\
\hline F13 & Cr53 & 825 & & 4.88 & \\
\hline $\mathrm{F} 21$ & $c 752$ & & & & \\
\hline F21 & C.53 & & & & \\
\hline F21R & Cr52 & & & & \\
\hline F21R & C.53 & & & & \\
\hline $\mathrm{F} 22$ & $c+52$ & .61 & & & \\
\hline F22 & Cr53 & .6 & & .61 & \\
\hline $\mathrm{F23}$ & Cr52 & .16 & & & \\
\hline $\mathrm{P23}$ & C.53 & .16 & & .16 & \\
\hline $\mathrm{F31}$ & G.52 & & & & \\
\hline F31 & C.53 & & & & \\
\hline F32 & C.52 & 3.04 & & & - \\
\hline F32 & G53 & 3.03 & & 3.04 & \\
\hline F33 & $\mathrm{Cr} 52$ & .54 & .32 & & \\
\hline F33 & 9.53 & .54 & .32 & .54 & .32 \\
\hline F41 & C.52 & & & & \\
\hline F41 & $\mathrm{Cr53}$ & & & & \\
\hline $\mathrm{F} 42$ & C.52 & 8.25 & & & \\
\hline$F 42$ & C.53 & 825 & & 825 & \\
\hline$F 43$ & C.52 & 52 & & & \\
\hline F43 I & C.53 & 5.19 & & 52 & \\
\hline F51 & $\mathrm{C} 5 \mathrm{52}$ & & & & \\
\hline F51 & Cr53 & & & & \\
\hline F52 & Cr52 & .99 & & & \\
\hline$F 521$ & 0.53 & 1 & & 1 & \\
\hline$F 53$ & $\mathrm{Cr} 52$ & 8.25 & & & \\
\hline$F 53$ & C553 & 825 & & 825 & \\
\hline
\end{tabular}

${ }^{1}$ Reported value is actual concentration. No standard deviation is reported.

Note: The actual concentration of samptes for which no standard deviation is reported is feas than the fower limit of dotection. The reported concentration ts the lower Imit of quantification. 


\begin{tabular}{|c|c|c|c|c|c|}
\hline Sample Id. & leotope & $\begin{array}{l}\text { Concentration } \\
\text { (ugh) }\end{array}$ & $\begin{array}{l}\text { SidDoviation } \\
\text { (ugh) }\end{array}$ & $\begin{array}{l}\text { Element Avg. Conc. } \\
\text { (ugh) }\end{array}$ & $\begin{array}{l}\text { Element Avg Std. Dov. } \\
\text { (ug/L) }\end{array}$ \\
\hline$F 11$ & $\infty$ & & & & \\
\hline F12 & $\infty$ & 526 & .48 & 526 & .48 \\
\hline F13 & $\infty$ & 1.17 & .1 & 1.17 & .1 \\
\hline F21 & $\infty$ & & & & \\
\hline F21R & $\infty$ & & & & \\
\hline P22 & $\infty$ & 22.6 & .49 & 22.6 & .49 \\
\hline $\mathrm{F} 23$ & $\infty$ & .17 &.$\infty$ & .17 &.$\infty$ \\
\hline F31 & $\infty$ & & & & \\
\hline F32 & Co & 1.17 & 25 & 1.17 & 25 \\
\hline F33 & Co & 1.11 & .11 & 1.11 & .11 \\
\hline F41 & $\infty$ & & & & \\
\hline $\mathrm{F} 42$ & $\infty$ & 123 & .16 & 123 & .16 \\
\hline$F_{43} 1$ & Co & 6.5 & & 6.5 & \\
\hline F51 & $\infty$ & & & & \\
\hline$F 521$ & $\infty$ & .63 & & .63 & \\
\hline $\mathrm{F} 53 \mathrm{I}$ & $\infty$ & .61 & & .61 & \\
\hline
\end{tabular}

${ }^{1}$ Reported value is actual concentration. No standard deviation is reported. 


\begin{tabular}{|c|c|c|c|c|c|}
\hline \multicolumn{6}{|c|}{ Transect F Porewater Concentrations: Copper } \\
\hline Sample ld. & botope & $\begin{array}{l}\text { Concentratton } \\
\text { (ugh) }\end{array}$ & $\begin{array}{l}\text { Stad Deviation } \\
\text { (ugh) }\end{array}$ & $\begin{array}{l}\text { Element Avg. Conc. } \\
\text { (ugh) }\end{array}$ & $\begin{array}{c}\text { Element Avg. Std. Dov. } \\
\text { (ugh) }\end{array}$ \\
\hline$F 11$ & $a_{6} 63$ & & & & \\
\hline$F 11$ & Cu65 & & & & \\
\hline F12 & 0463 & 142 & 1.01 & & \\
\hline$F 12$ & Ou65 & 14.7 & 1.05 & 14.5 & 1.03 \\
\hline$F 13$ & 0463 & 6.17 & .03 & & \\
\hline$F 13$ & a 65 & 6.14 & 3 & 6.15 & .16 \\
\hline$F 21$ & Qu63 & & & & \\
\hline$F 21$ & 0,65 & & & & \\
\hline F21R & 0,63 & & & & \\
\hline F21R & a 65 & & & & \\
\hline$F 22$ & au63 & 2.51 & .33 & & \\
\hline$P 22$ & 0,65 & 1.05 & .49 & 1.78 & .41 \\
\hline$F 23$ & Cu63 & 3.75 & & & \\
\hline $\mathrm{F} 23$ & 0465 & 3.75 & & 3.75 & \\
\hline F31 & Ou63 & & & & \\
\hline$F 31$ & Qu65 & & & & \\
\hline$F 32$ & $a 663$ & 6.17 & 2.17 & & \\
\hline$F 32$ & a 65 & 6.14 & 1.05 & 6.15 & 1.61 \\
\hline$F 33$ & a 63 & 3.75 & & & \\
\hline$F_{33}$ & $a \sqrt{65}$ & 3.75 & & 3.75 & \\
\hline$F_{41}$ & 0463 & & & & \\
\hline$F_{41}$ & Cu65 & & & & \\
\hline$F 42$ & 0463 & 1.81 & .05 & & \\
\hline$F 42$ & Cu65 & 3.75 & & 278 & .05 \\
\hline$F_{43} 1$ & 0463 & 8.99 & & & \\
\hline $\mathrm{F} 431$ & 0465 & 921 & & 9.1 & \\
\hline F51 & Qu63 & & & & \\
\hline F51 & Cu65 & & & & \\
\hline$F 521$ & Cu63 & 3.04 & & & \\
\hline F52 I & Cu65 & 322 & & 3.13 & \\
\hline F53 I & Cu63 & 2.59 & & & \\
\hline$F 531$ & Cu65 & 2.79 & & 2.69 & \\
\hline
\end{tabular}

${ }^{1}$ Reported value is actual concentration. No standard deviation is reported. 


\begin{tabular}{|c|c|c|c|c|c|}
\hline Sample ld. & Leotope & $\begin{array}{l}\text { Concentration } \\
\text { (ugl) }\end{array}$ & $\begin{array}{l}\text { Std. Doviation } \\
\text { (ugh) }\end{array}$ & $\begin{array}{l}\text { Element Avg. Conc. } \\
\text { (ug/L) }\end{array}$ & $\begin{array}{l}\text { Element Avg. Std. Dov. } \\
\text { (ugh) }\end{array}$ \\
\hline F11 & $F_{0}$ & & & & \\
\hline$F 12$ & Fo & 1040 & 23.8 & 1040 & 23.8 \\
\hline F13 & $F_{0}$ & 27.5 & & 27.5 & \\
\hline F21 & $F_{0}$ & & & & \\
\hline F21R & Fo & & & & \\
\hline$F 22$ & $F_{0}$ & 149 & 16.5 & 149 & 16.5 \\
\hline F23 & $\mathrm{Fo}$ & 27.5 & & 27.5 & \\
\hline F31 & $F_{0}$ & & & & \\
\hline F32 & $F_{0}$ & 392 & 12.5 & 392 & 12.5 \\
\hline$F 33$ & $F_{0}$ & 69.8 & 2.04 & 69.8 & 204 \\
\hline F41 & $F_{0}$ & & & & \\
\hline$F 42$ & $F_{0}$ & 1210 & 152 & 1210 & 152 \\
\hline F43 & Fo & 1260 & & 1260 & \\
\hline F51 & $F_{0}$ & 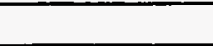 & & & \\
\hline F52 I & Fo & 20.6 & & 20.6 & \\
\hline$F 53$ & $F_{\theta}$ & 149 & & 149 & \\
\hline
\end{tabular}

${ }^{1}$ Reported value is actual concentration. No standard deviation is reported. 


\section{Transect F Porewater Concentrations: Lead}

\begin{tabular}{|c|c|c|c|c|}
\hline & & $\begin{array}{l}\text { Concentration } \\
\text { (ugh) }\end{array}$ & $\begin{array}{l}\text { std. Doviation } \\
\text { (ug/) }\end{array}$ & $\begin{array}{c}\text { Element Avg. Conc. } \\
\text { (ugh) }\end{array}$ \\
\hline
\end{tabular}

\begin{tabular}{|c|c|c|c|c|c|}
\hline F11 & $P 6206$ & & & & \\
\hline F11 & Pto207 & & & & \\
\hline F11 & Pb208 & & & & \\
\hline$\overline{F 12}$ & Pb206 & 2.43 & .76 & & \\
\hline$F 12$ & Pb207 & 2.06 & .14 & & \\
\hline F12 & Pb208 & 261 & 51 & 237 & .47 \\
\hline F13 & $P 6206$ & 8.25 & & & \\
\hline$F 13$ & $P_{b 207}$ & 8.25 & & & \\
\hline$F 13$ & Pt208 & 825 & & 825 & \\
\hline$F 21$ & $P_{b 206}$ & & & & \\
\hline$F 21$ & Po207 & & & & \\
\hline$F 21$ & Pt208 & & & & \\
\hline F21R & Po206 & & & & \\
\hline F21R & $P b 207$ & & & & \\
\hline F21R & $P \mathrm{~Pb} 208$ & & & & \\
\hline$F 22$ & Pb206 & 8.25 & & & \\
\hline$F 22$ & $\mathrm{~Pb} 207$ & 8.25 & & & \\
\hline$F 22$ & Pb208 & .88 & .14 & 5.79 & .14 \\
\hline$F 23$ & $P b 206$ & 825 & & & \\
\hline$F 23$ & $\mathrm{Pb207}$ & 8.25 & & & \\
\hline$F 23$ & Pt208 & 8.25 & & 825 & \\
\hline$F 31$ & Pt206 & & & & \\
\hline$F 31$ & $\mathrm{Pb207}$ & & & & \\
\hline F31 & Pb208 & & & & \\
\hline$F 32$ & $\mathrm{Pb206}$ & .97 & 22 & & \\
\hline F32 & $\mathrm{Pb207}$ & .47 & .52 & & \\
\hline$F 32$ & Pb208 & 1.09 & 21 & .84 & .32 \\
\hline F33 & Pto206 & 11.3 & & & \\
\hline$F 33$ & $\mathrm{~Pb} 207$ & 11.3 & & & \\
\hline F33 & $\mathrm{Pb} 208$ & 11.3 & & 11.3 & \\
\hline$F 41$ & Pb206 & & & & \\
\hline F41 & Pt207 & & & & \\
\hline $\mathrm{F} 41$ & $\mathrm{~Pb} 208$ & & & & \\
\hline$F 42$ & $P 6206$ & .38 & .34 & & \\
\hline$F 42$ & Pb207 & .73 & .4 & & \\
\hline F42 & Po208 & 1.22 & 26 & .78 & .33 \\
\hline F43I & $\mathrm{Pb} 206$ & 4.07 & & & \\
\hline F43 I & $\mathrm{Pb} 207$ & 4.07 & & & \\
\hline$F_{43} !$ & Pb208 & 4.37 & & 4.17 & \\
\hline F51 & Pb206 & & & & \\
\hline F51 & Pb207 & & & & \\
\hline F51 & Pb208 & & & & \\
\hline F52 I & $P_{b 206}$ & .62 & & & \\
\hline$F 521$ & Pb207 & .85 & & & \\
\hline
\end{tabular}

${ }^{1}$ Reported value is actual concentration. No standard deviation is reported. 


\section{Transect F Porewater Concentrations: Lead}

\begin{tabular}{|c|c|c|c|c|c|}
\hline Sample ld. & leotope & $\begin{array}{l}\text { Concentration } \\
\text { (ug/L) }\end{array}$ & $\begin{array}{l}\text { Studoviation } \\
\text { (ugh) }\end{array}$ & $\begin{array}{c}\text { Element Avg Conc. } \\
\text { (ugh) }\end{array}$ & $\begin{array}{l}\text { Elament Avg. Std. Dov. } \\
\text { (ugh) }\end{array}$ \\
\hline F52 I & $P_{b 208}$ & 121 & & .89 & \\
\hline F53 & Pb206 & 825 & & & \\
\hline$F 53$ & Pto207 & 8.25 & & & \\
\hline$F 53$ & Pb208 & 825 & & 825 & \\
\hline
\end{tabular}

${ }^{1}$ Reported value is actual concentration. No standard deviation is reported. 


\section{Transect F Porewater Concentrations: Magnesium}

\begin{tabular}{|c|c|c|c|c|c|}
\hline Sample Id. & kotope & $\begin{array}{l}\text { Concentration } \\
\text { (ugh) }\end{array}$ & $\begin{array}{l}\text { Stdpoviation } \\
\text { (ug/L) }\end{array}$ & $\begin{array}{l}\text { Element AYg Conc. } \\
\text { (Ug/나) }\end{array}$ & $\begin{array}{l}\text { Element Avg. Std. Dov. } \\
\text { (ug/l) }\end{array}$ \\
\hline F11 & Mge4 & & & & \\
\hline F11 & Mo25 & & & & \\
\hline F12 & No24 & 1840 & 44.1 & & \\
\hline F12 & No25 & 1580 & 25.4 & 1760 & 34.8 \\
\hline F13 & $\mathrm{Mg} 24$ & 515 & 3.25 & & \\
\hline F13 & Moes & 509 & 5.51 & 512 & 4.73 \\
\hline$F 21$ & $\mathrm{Mg} 24$ & & & & \\
\hline$F 21$ & Mges & & & & \\
\hline F21R & Mige4 & & & & \\
\hline F21R & Mo2s & & & & \\
\hline$F 22$ & Mge4 & 925 & 102 & & \\
\hline $\mathrm{F} 22$ & Mogs & 953 & 20.8 & $\$ 39$ & 15.5 \\
\hline $\mathrm{F} 23$ & Mget & 126 & .6 & & \\
\hline $\mathrm{F} 23$ & Mges & 14 & .39 & 13.3 & $.5 \cdot$ \\
\hline F31 & Mge4 & & & & \\
\hline F31 & Mo25 & & & & \\
\hline F32 & $\mathrm{Mg} 24$ & 2120 & 36.4 & & - \\
\hline $\mathrm{F} 32$ & Mg25 & 1720 & 30.7 & 1920 & 33.6 \\
\hline $\mathrm{F} 33$ & Mge4 & 690 & 13.1 & & \\
\hline F33 & Mges & 723 & 5.93 & 707 & 9.52 \\
\hline F41 & $M g 24$ & & & & \\
\hline F41 & Mges & & & & \\
\hline$F 42$ & Mg24 & 531 & 3.63 & & \\
\hline$F 42$ & Mges & 512 & 10.9 & 522 & 727 \\
\hline $\mathrm{F} 43$ & Mg24 & 1580 & & & \\
\hline $\mathrm{F} 431$ & Mg25 & 1540 & & 1560 & \\
\hline $\mathrm{F} 51$ & $\mathrm{Mg} 24$ & & & & \\
\hline $\mathrm{F51}$ & Mgs & & & & \\
\hline F52 1 & $\mathrm{Mg} 24$ & 538 & & & \\
\hline F52 I & Mges & 518 & & 528 & \\
\hline F53 I & $M g 24$ & 579 & & & \\
\hline$F 531$ & Mges & 586 & & 583 & \\
\hline
\end{tabular}

${ }^{1}$ Reported value is actual concentration. No standard deviation is reported. 


\section{Transect F Porewater Concentrations: Manganese}

\begin{tabular}{|c|c|c|c|c|c|}
\hline Sample Id. & botope & $\begin{array}{l}\text { Concentuation } \\
\text { (ugh) }\end{array}$ & $\begin{array}{c}\text { Stdocviation } \\
\text { (ug/L) }\end{array}$ & $\begin{array}{c}\text { Element Avg. Conc. } \\
\text { (ug/L) }\end{array}$ & $\begin{array}{l}\text { Element Avg. Std. Dov. } \\
\text { (ugh) }\end{array}$ \\
\hline F11 & $\mathrm{Mn}$ & & & & \\
\hline F12 & $\mathrm{Mn}$ & 1870 & 36.3 & 1870 & 36.3 \\
\hline F13 & $\operatorname{Mn}$ & 24 & 26 & 24 & 26 \\
\hline$F 21$ & $\mathrm{Mn}$ & & & & \\
\hline F21R & $\mathrm{Mn}$ & & & & \\
\hline $\mathrm{F} 22$ & $\operatorname{Mn}$ & 22200 & 112 & 22200 & 112 \\
\hline $\mathrm{F} 23$ & $\operatorname{Mn}$ & 19.9 & 53 & 18.9 & .53 \\
\hline F31 & $\mathrm{Mn}$ & & & & \\
\hline$F 32$ & $\operatorname{Mn}$ & 488 & 10 & 488 & 10 \\
\hline F33 & $\mathrm{Mn}$ & 136 & .82 & 136 & .82 \\
\hline F41 & $\mathrm{Mn}$ & & & & \\
\hline F42 & $\sin$ & 86.4 & .97 & 86.4 & .97 \\
\hline$F 431$ & $\mathrm{Mn}$ & 544 & & 544 & \\
\hline $\mathrm{F51}$ & $\mathrm{Mn}$ & & & & $\dot{ }$ \\
\hline$F 52$ & $\mathrm{Mn}$ & 59.5 & & 59.5 & \\
\hline F53 & $\mathrm{Mn}$ & 38.6 & & 38.6 & \\
\hline
\end{tabular}

${ }^{1}$ Reported value is actual concentration. No standard deviation is reported. 
Transect F Porewater Concentrations: Nickel

\begin{tabular}{|c|c|c|c|c|c|}
\hline Sample Id. & teotope & $\begin{array}{l}\text { Concentration } \\
\text { (ㅂy니) }\end{array}$ & $\begin{array}{c}\text { Std.owintion } \\
(u \circ / \text { L) }\end{array}$ & $\begin{array}{l}\text { Eloment Avg. Conc. } \\
\text { (ug/L) }\end{array}$ & $\begin{array}{l}\text { Element Avg. Std. Dev. } \\
\text { (ugoll) }\end{array}$ \\
\hline F11 & Niso & & & & \\
\hline F11 & Ni62 & & & & \\
\hline F12 & $N=0$ & 85 & 7.85 & & \\
\hline F12 & Nis2 & 111 & 7.61 & 103 & 7.78 \\
\hline F13 & Niso & 16.4 & 222 & & \\
\hline $\mathrm{F} 13$ & N:2 & 18.6 & .68 & 17.5 & 1.45 \\
\hline F21 & Ni60 & & & & \\
\hline P21 & Ni62 & & & & \\
\hline F21R & Niso & & & & \\
\hline F21R & Nis2 & & & & \\
\hline $\mathrm{F} 22$ & Ni60 & 37.0 & 1.15 & & \\
\hline$F 22$ & $\mathrm{Ni62}$ & 36.2 & 6.99 & 37.1 & 4.07 \\
\hline $\mathrm{F} 23$ & Ni60 & 275 & & & \\
\hline F23 & N62 & 275 & & 2.75 & \\
\hline F31 & Ni6o & & & & \\
\hline F31 & NiG2 & & & & \\
\hline F32 & Ni6o & 16.4 & 1.52 & & \\
\hline $\mathrm{F32}$ & Nis2 & 18.6 & 1.42 & 17.5 & 1.47 \\
\hline F33 & Niso & 18. & .42 & & \\
\hline F33 & Ni62 & 17.5 & 3.75 & 182 & 200 \\
\hline F41 & Ni60 & & & & \\
\hline F41 & Ni62 & & & & \\
\hline$F 42$ & Ni6o & 47.8 & 5.05 & & \\
\hline $\mathrm{F} 42$ & Nis2 & 45.4 & 528 & 46.6 & 5.17 \\
\hline$F 43$ & Ni6o & 85.6 & & & \\
\hline$F 431$ & Ni62 & 88.1 & & 91.9 & \\
\hline F51 & Ni60 & & & & \\
\hline F51 & N162 & & & & \\
\hline F52 I & Ni60 & 34.1 & & & \\
\hline $\mathrm{F} 521$ & Ni62 & 362 & & 352 & \\
\hline $\mathrm{Fs3}$ & Ni60 & 16.6 & & & \\
\hline F53 & Ni62 & 22.3 & & 19.5 & \\
\hline
\end{tabular}

${ }^{1}$ Reported value is actual concentration. No standard deviation is reported. 


\section{Transect F Porewater Concentrations: Selenium}

\begin{tabular}{|c|c|c|c|c|c|}
\hline Sample ld. & teotope & $\begin{array}{l}\text { Concentration } \\
\text { (Ug/h) }\end{array}$ & $\begin{array}{l}\text { StdDaviation } \\
\text { (ugh) }\end{array}$ & $\begin{array}{l}\text { Eloment Avg. Conc. } \\
\text { (ughl) }\end{array}$ & $\begin{array}{l}\text { Ekment Avg. Std. Dov. } \\
\text { (ugh) }\end{array}$ \\
\hline F11 & S०77 & & & & \\
\hline F11 & 5082 & & & & \\
\hline $\mathrm{F} 12$ & So77 & 225 & & & \\
\hline$F_{12}$ & 5082 & 225 & & 22.5 & \\
\hline F13 & So77 & 225 & & & \\
\hline F13 & So82 & 22.5 & & 225 & \\
\hline P21 & So77 & & & & \\
\hline$F 21$ & So82 & & & & \\
\hline F21R & So77 & & & & \\
\hline F21R & 5082 & & & & \\
\hline $\mathrm{F} 22$ & So77 & 22.5 & & & \\
\hline$F 22$ & So82 & 225 & & 22.5 & \\
\hline$F 23$ & SoT7 & 225 & & & \\
\hline F23 & So82 & 22.5 & & 225 & \\
\hline F31 & So77 & & & & \\
\hline F31 & Sese & & & & \\
\hline F32 & So77 & 22.5 & & & \\
\hline F32 & Se82 & 225 & & 225 & \\
\hline F33 & 5077 & 41.3 & & & \\
\hline F33 & SeB2 & 41.6 & & 41.5 & \\
\hline F41 & Se77 & & & & \\
\hline F41 & So82 & & & & \\
\hline F42 & So7 & 22.5 & & & \\
\hline F42 & So82 & 225 & & 22.5 & \\
\hline F43 & SeT7 & 5.44 & & & \\
\hline$F 431$ & Se82 & 5.45 & & 5.45 & \\
\hline F51 & SoT7 & & & & \\
\hline F51 & So82 & & & & \\
\hline F52 & Se77 & 225 & & & \\
\hline F52 & So82 & 22.5 & & 22.5 & \\
\hline F53 I & Se $\pi$ & 7.42 & & & \\
\hline $\mathrm{F}_{53} \mathrm{I}$ & So82 & 728 & & 7.35 & \\
\hline
\end{tabular}

${ }^{1}$ Reported value is actual concentration. No standard deviation is reported. 


\begin{tabular}{|c|c|c|c|c|c|}
\hline Samplo ld. & Eotope & $\begin{array}{l}\text { Concentration } \\
\text { (ugh) }\end{array}$ & $\begin{array}{l}\text { stdonviation } \\
\text { (ugh) }\end{array}$ & $\begin{array}{l}\text { Element Avg. Conc. } \\
\text { (ugh) }\end{array}$ & $\begin{array}{l}\text { Element Avg-Std. Dov. } \\
\text { (Ug/) }\end{array}$ \\
\hline F11 & A0107 & & & & \\
\hline F11 & $A 0100$ & & & & \\
\hline F12 & A9107 & .83 & & & \\
\hline F12 & Ag100 & .83 & & .83 & \\
\hline F13 & A0107 & .83 & & & \\
\hline F13 & $A 9109$ & .83 & & .83 & \\
\hline F21 & Ag107 & & & & \\
\hline F21 & Ag109 & & & & \\
\hline F21R & Ag107 & & & & \\
\hline F21R & Ag109 & & & & \\
\hline$F 22$ & Ag107 & 1.5 & & & \\
\hline P22 & Ag109 & 1.5 & & 1.5 & \\
\hline F23 & Ag107 & 1.5 & & & \\
\hline$F 23$ & Ag109 & 1.5 & & 1.5 & \\
\hline F31 & Ag107 & & & & \\
\hline F31 & Ag109 & & & & \\
\hline F32 & Ag107 & 1.5 & & & $\cdot$ \\
\hline$F 32$ & $A 0109$ & 1.5 & & 1.5 & \\
\hline F33 & A9107 & 8.25 & & & \\
\hline F33 & Ag109 & 825 & & 8.25 & \\
\hline F41 & Ag107 & & & & \\
\hline F41 & Ag109 & & & & \\
\hline F42 & Ag107 & 1.5 & & & \\
\hline$F 42$ & Ag109 & 1.5 & & 1.5 & \\
\hline$F 43 I$ & Ag107 & 24 & & & \\
\hline F43! & Ag100 & 23 & & 24 & \\
\hline F51 & Ag107 & & & & \\
\hline F51 & Ag100 & & & & \\
\hline F52 I & Ag107 & 24 & & & \\
\hline F52 1 & Ag109 & 23 & & 24 & \\
\hline F53 I & Ag107 & 1.5 & & & \\
\hline F53 1 & A0109 & 1.5 & & 1.5 & \\
\hline
\end{tabular}

${ }^{1}$ Reported value is actual concentration. No standard deviation is reported. 


\section{Transect F Porewater Concentrations: Sodium}

\begin{tabular}{ccccc}
\hline Sample ld. leotope & $\begin{array}{c}\text { Concentratton } \\
\text { (ugh) }\end{array}$ & $\begin{array}{c}\text { Std.Doviation } \\
\text { (ugh) }\end{array}$ & $\begin{array}{c}\text { Element Avg. Conc. } \\
\text { (ughl) }\end{array}$ & $\begin{array}{c}\text { Element Avg. Std. Dov. } \\
\text { (ugh) }\end{array}$
\end{tabular}

\begin{tabular}{|c|c|c|c|c|c|}
\hline F11 & $\mathrm{Na}$ & & & & \\
\hline$F_{12}$ & $\mathrm{Na}$ & 22700 & 524 & 22700 & 524 \\
\hline$F 13$ & $\mathrm{Na}$ & 5440 & 28.6 & 5440 & 28.6 \\
\hline$P_{21}$ & $\mathrm{Na}$ & & & & \\
\hline F21R & $\mathrm{Na}$ & & & & \\
\hline F22 & $\mathrm{Na}$ & 80300 & 2070 & 80300 & 2070 \\
\hline $\mathrm{F} 23$ & $\mathrm{Na}$ & 85500 & 1130 & 85500 & 1130 \\
\hline F31 & $\mathrm{Na}$ & & & & \\
\hline F32 & $\mathrm{Na}$ & 2530 & 55.8 & 2530 & 55.8 \\
\hline$F 33$ & $\mathrm{Na}$ & 47700 & 2200 & 47700 & 2200 \\
\hline $\mathrm{F} 41$ & $\mathrm{Na}$ & & & & \\
\hline F42 & $\mathrm{Na}$ & 85100 & 1420 & 85100 & 1420 \\
\hline $\mathrm{F} 43^{\prime}$ & $\mathrm{Na}$ & 6370 & & 6370 & \\
\hline F51 & $\mathrm{Na}$ & & & & \\
\hline$F 521$ & $\mathrm{Na}$ & 3770 & & 3770 & \\
\hline$F_{53} I^{\prime}$ & $\mathrm{Na}$ & 9750 & & 8750 & \\
\hline
\end{tabular}

${ }^{1}$ Reported value is actual concentration. No standard deviation is reported. 


\section{Transect F Porewater Concentrations: Strontium}

\begin{tabular}{|c|c|c|c|c|c|}
\hline Sample Id. & kotope & $\begin{array}{l}\text { Concentration } \\
\text { (ugh) }\end{array}$ & $\begin{array}{l}\text { Std.owviation } \\
\text { (ugh) }\end{array}$ & $\begin{array}{l}\text { Ekment Avg. Conc. } \\
\text { (ugl) }\end{array}$ & $\begin{array}{c}\text { Element Avg. Std. Dov. } \\
\text { (ugh) }\end{array}$ \\
\hline F11 & Sr & & & & \\
\hline$F 12$ & Sr & 16 & 29 & 16 & 29 \\
\hline F13 & Sr & 6.62 & .3 & 6.62 & .3 \\
\hline P21 & Sr & & & & \\
\hline F21R & Sr & & & & \\
\hline $\mathbf{F 2 2}$ & Sr & 10.6 & 31 & 10.6 & .31 \\
\hline P23 & Sr & 231 & 24 & 231 & 24 \\
\hline$F 31$ & Sr & & & & \\
\hline F32 & Sr & 17 & 36 & 17 & .36 \\
\hline F33 & $\mathrm{Sr}$ & 822 & .75 & 822 & .75 \\
\hline$F_{41}$ & Sr & & & & \\
\hline$F 42$ & $\mathbf{S r}$ & 8.09 & 34 & 8.09 & .34 \\
\hline$F 43 !$ & Sr & 31.4 & & 31.4 & \\
\hline F51 & $\mathrm{sr}$ & & & & \\
\hline$F 52^{\prime}$ & Sr & 5.04 & & 5.04 & \\
\hline $\mathrm{FS3}^{\prime}$ & Sr & 9.32 & & 9.32 & \\
\hline
\end{tabular}

${ }^{1}$ Reported value is actual concentration. No standard deviation is reported. 


\begin{tabular}{|c|c|c|c|c|c|}
\hline Sample ld. & Eotope & $\begin{array}{l}\text { Concentration } \\
\text { (ugh) }\end{array}$ & $\begin{array}{c}\text { Stidovitation } \\
\text { (4gh) }\end{array}$ & $\begin{array}{c}\text { Element Avg. Conc. } \\
\text { (ug/ })\end{array}$ & $\begin{array}{c}\text { Element Avg. Std. Dov. } \\
\text { (ugh) }\end{array}$ \\
\hline F11 & Th232 & & & & \\
\hline F12 & Th232 & 275 & & 2.75 & \\
\hline F13 & Th232 & 2.75 & & 2.75 & \\
\hline F21 & Th232 & & & & \\
\hline F21R & Th232 & & & & \\
\hline F22 & Th232 & 2.75 & & 2.75 & \\
\hline $\mathrm{F} 23$ & Th232 & 275 & & 2.75 & \\
\hline F31 & Th232 & & & & \\
\hline F32 & Th232 & 2.75 & & 2.75 & \\
\hline$F 33$ & Th232 & 2.75 & & 275 & \\
\hline$F 41$ & Th232 & & & & \\
\hline$F 42$ & Th232 & 2.75 & & 2.75 & \\
\hline$F_{43}$ & Th232 & 2.75 & & 2.75 & \\
\hline F51 & Th232 & & & & \\
\hline F52 & Th232 & 275 & & 2.75 & \\
\hline$F 53$ & Th232 & 2.75 & & 275 & \\
\hline
\end{tabular}




\section{Transect F Porewater Concentrations: Tin}

\begin{tabular}{|c|c|c|c|c|c|}
\hline Sample Id. & Eotope & $\begin{array}{l}\text { Concentration } \\
\text { (ugh) }\end{array}$ & $\begin{array}{c}\text { Stdooviation } \\
\text { (ug/L) }\end{array}$ & $\begin{array}{c}\text { Element Avg. Conc. } \\
\text { (ugl) }\end{array}$ & $\begin{array}{c}\text { Element Avg. Std. Dov. } \\
\text { (ug/L) }\end{array}$ \\
\hline F11 & Sn118 & & & & \\
\hline Fit & Sn120 & & & & \\
\hline F12 & Sn118 & 1.38 & & & \\
\hline$F 12$ & Sn120 & 1.38 & & 1.38 & \\
\hline F13 & $\operatorname{Sn} 118$ & 1.38 & & & \\
\hline F13 & Sn120 & 1.38 & & 1.38 & \\
\hline F21 & Sn118 & & & & \\
\hline$F 21$ & $\operatorname{Sn} 120$ & & & & \\
\hline F21R & Sn118 & & & & \\
\hline F21R & Sn120 & & & & \\
\hline $\mathrm{F} 2$ & Snt18 & 1,38 & & & \\
\hline $\mathrm{F} 22$ & Sn120 & 1.38 & & 1.38 & \\
\hline $\mathrm{F} 23$ & Sn118 & 1.38 & & & \\
\hline $\mathrm{F} 23$ & Sn120 & 1.38 & & 138 & \\
\hline F31 & Sn118 & & & & \\
\hline F31 & $\operatorname{Sn} 120$ & & & & \\
\hline F32 & Sn118 & .83 & 24 & & - \\
\hline F32 & Sn120 & .97 & .15 & .8 & 2 \\
\hline $\mathrm{F33}$ & Sn118 & 13.8 & & & \\
\hline F33 & Sn 120 & 13.8 & & 13.8 & \\
\hline F41 & Sn118 & & & & \\
\hline F41 & Sn120 & & & & \\
\hline F42 & Sn118 & .96 & 28 & & \\
\hline F42 & Sn120 & .91 & .1 & .94 & .19 \\
\hline F 43 l & Sn118 & 123 & & & \\
\hline$F 431$ & Sn120 & .85 & & 1.00 & \\
\hline F51 & Sn118 & & & & \\
\hline F51 & Sn120 & & & & \\
\hline$F 521$ & Sn118 & 1.36 & & & \\
\hline F52 & Sn120 & .51 & & .94 & \\
\hline $\mathrm{F} 53 \mathrm{I}$ & Sn118 & 26 & & & \\
\hline $\mathrm{F}_{53}{ }^{\prime}$ & Sn120 & .16 & & 21 & \\
\hline
\end{tabular}

${ }^{1}$ Reported value is actual concentration. No standard deviation is reported. 


\section{Transect F Porewater Concentrations: Uranium-235}

\begin{tabular}{|c|c|c|c|c|c|}
\hline Samplo ld. & leotope & $\begin{array}{l}\text { Concentration } \\
\text { (ug/L) }\end{array}$ & $\begin{array}{l}\text { Std.Deviation } \\
\text { (ugh) }\end{array}$ & $\begin{array}{l}\text { Element Avg. Cone. } \\
\text { (ughl) }\end{array}$ & $\begin{array}{c}\text { Element Avg. Std. Dov. } \\
\text { (ug })\end{array}$ \\
\hline$F 11$ & U235 & & & & \\
\hline$F 12$ & U235 & .01 & .01 & .01 & .01 \\
\hline F13 & U235 & .02 & & .02 & \\
\hline F21 & U235 & & & & \\
\hline F21R & U235 & & & & \\
\hline F22 & U235 & .05 & & .05 & \\
\hline F23 & U235 & .02 & & .02 & \\
\hline F31 & U235 & & & & \\
\hline F32 & U235 & .02 & & .02 & \\
\hline$F 33$ & U235 & .02 & & .02 & \\
\hline$F_{41}$ & U235 & & & & \\
\hline$F_{42}$ & U235 & .02 & & .02 & \\
\hline F43 & U235 & .02 & & .02 & \\
\hline F51 & U235 & & & & \\
\hline F52 I & U235 & .02 & & .02 & \\
\hline$F 53$ & U235 & .02 & & .02 & \\
\hline
\end{tabular}

${ }^{1}$ The reported value is the actual concentration. No standard deviation is reported. 


\section{Transect F Porewater Concentrations: Uranium-238}

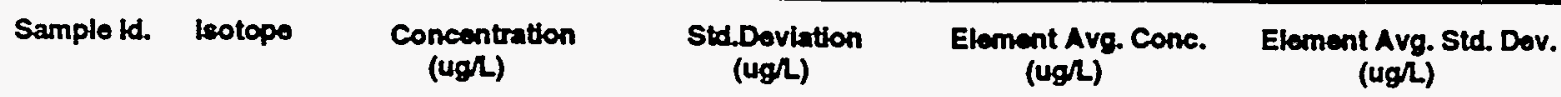

\begin{tabular}{|l|l|l|l|l|l|}
\hline$F 11$ & $U 238$ & & & & \\
\hline$F 12$ & $U 238$ & .5 & .02 & .5 & .02 \\
\hline$F 13$ & $U 238$ & 2.58 & .05 & 2.58 & .05 \\
\hline$F 21$ & $U 238$ & & & & \\
\hline$F 21 R$ & $U 238$ & & & & \\
\hline$F 22$ & $U 238$ & 4.9 & .08 & 4.9 & .08 \\
\hline$F 23$ & $U 238$ & .38 & .09 & .38 & .09 \\
\hline$F 31$ & $U 238$ & & & & \\
\hline$F 32$ & $U 238$ & 29 & & 29 & \\
\hline$F 33$ & $U 238$ & 2.5 & & 2.5 & \\
\hline$F 41$ & $U 238$ & & & .12 & \\
\hline$F 42$ & $U 238$ & .12 & & 10.3 & \\
\hline$F 431$ & $U 238$ & 10.3 & & .15 & \\
\hline$F 51$ & $U 238$ & & & .17 & \\
\hline$F 521$ & $U 238$ & .15 & & & \\
\hline$F 531$ & $U 238$ & & & & \\
\hline
\end{tabular}

${ }^{1}$ The reported value is the actual concentration. No standard deviation is reported. 


\begin{tabular}{|c|c|c|c|c|c|}
\hline Sample Id. & leotope & $\begin{array}{l}\text { Concentration } \\
\text { (ugh) }\end{array}$ & $\begin{array}{l}\text { Stadooviation } \\
\text { (ugh) }\end{array}$ & $\begin{array}{c}\text { Element Avg. Conc. } \\
\text { (ug/L) }\end{array}$ & $\begin{array}{c}\text { Element Avg. Std. Dov. } \\
\text { (ugh) }\end{array}$ \\
\hline F11 & $v$ & & & & \\
\hline F12 & $v$ & 1.38 & & 1.38 & \\
\hline $\mathrm{F} 13$ & $v$ & 1.38 & & 1.38 & \\
\hline F21 & $v$ & & & & \\
\hline F21R & $\bar{v}$ & & & & \\
\hline $\mathrm{F} 22$ & $v$ & 1.38 & & 1.38 & \\
\hline F23 & $V$ & 3.7 & .02 & 3.7 & .02 \\
\hline F31 & $v$ & & & & \\
\hline F32 & $\mathrm{v}$ & 1.38 & & 1.38 & \\
\hline F33 & $V$ & 1.38 & & 1.38 & \\
\hline F41 & $v$ & & & & \\
\hline F42 & $v$ & 1.38 & & 1.38 & $\cdot$ \\
\hline $\mathrm{F} 43 \mathrm{I}$ & $v$ & .13 & & .13 & \\
\hline F51 & $V$ & & & & \\
\hline F52 & $V$ & 1.38 & & 1.38 & \\
\hline F53 & $v$ & 1.38 & & 1.38 & \\
\hline
\end{tabular}

${ }^{1}$ The reported value is the actual concentration. No standard deviation is reported. 
Transect F Porewater Concentrations: Yttrium

Samplo ld. botope

Concentration

(ugh)

Std.Doviation

(ugh)

Element Avg. Conc. (ugh)

Element Avg. Std. Dov. (ugh)

\begin{tabular}{|c|c|c|c|c|c|}
\hline $\mathrm{F} 11$ & $Y$ & & & & \\
\hline$F 12$ & $Y$ & 6.46 & .07 & 6.46 & .07 \\
\hline$F 13$ & $Y$ & .05 & 0 & .05 & 0 \\
\hline F21 & $Y$ & & & & \\
\hline F21R & $Y$ & & & & \\
\hline $\mathrm{F} 22$ & $Y$ & .83 & .14 & .83 & .14 \\
\hline $\mathrm{F} 23$ & $Y$ & .02 & .01 & .02 & .01 \\
\hline F31 & $Y$ & & & & \\
\hline$F 32$ & $Y$ & .17 & .03 & .17 & .03 \\
\hline$F 33$ & $\bar{Y}$ & .46 & .03 & .46 & .03 \\
\hline $\mathrm{F} 41$ & $Y$ & & & & \\
\hline $\mathrm{F} 42$ & $Y$ & 21 & .04 & 21 & .04 \\
\hline$F 43$ & $Y$ & 29.2 & & 292 & \\
\hline F51 & $Y$ & & & & \\
\hline F52 & $\bar{Y}$ & 1.38 & & 1.38 & \\
\hline F53 & $\bar{Y}$ & 1.38 & & 1.38 & \\
\hline
\end{tabular}

${ }^{1}$ The reported value is the actual concentration. No standard deviation is reported. 


\section{Transect F Porewater Concentrations: Zinc}

\begin{tabular}{|c|c|c|c|c|c|}
\hline Sample ld. & Isotope & $\begin{array}{l}\text { Concentratton } \\
\text { (ug/) }\end{array}$ & $\begin{array}{c}\text { Std.Doviation } \\
\text { (ug/ })\end{array}$ & $\begin{array}{l}\text { Element Avg. Conc. } \\
\text { (ugh) }\end{array}$ & $\begin{array}{c}\text { Element Avg. Std. Dov. } \\
\text { (ugL) }\end{array}$ \\
\hline F11 & Zn66 & & & & \\
\hline$F 12$ & Zn66 & 190 & 13.4 & 190 & 13.4 \\
\hline$F 13$ & Zn66 & 17.4 & .93 & 17.4 & .93 \\
\hline F21 & Zn66 & & & & \\
\hline F21R & Zn66 & & & & \\
\hline $\mathrm{F} 22$ & $2 n 66$ & 17.1 & 1.00 & 17.1 & 1.09 \\
\hline F23 & Zn66 & 17.4 & 2.29 & 17.4 & 229 \\
\hline F31 & Zn66 & & & & \\
\hline F32 & Zn66 & 98.7 & 11.4 & 98.7 & 11.4 \\
\hline F 33 & Zn66 & 16 & .66 & 18 & .66 \\
\hline F41 & Zn66 & & & & \\
\hline F42 & Zn66 & 168 & 9.08 & 168 & 9.08 \\
\hline$F_{43} 1$ & Zn66 & 132 & & 132 & \\
\hline F51 & Zn66 & & & & \\
\hline$F 52^{\prime}$ & Zn66 & 47.5 & & 47.5 & \\
\hline F53 I & Zn66 & 26.8 & & 26.8 & \\
\hline
\end{tabular}

${ }^{1}$ The reported value is the actual concentration. No standard deviation is reported. 


\section{Transect F Porewater Concentrations: Zirconium}

\begin{tabular}{|c|c|c|c|c|c|}
\hline Sample ld. & beotope & $\begin{array}{l}\text { Concentration } \\
\text { (ug/ })\end{array}$ & $\begin{array}{l}\text { Stdideviation } \\
\text { (ugl) }\end{array}$ & $\begin{array}{c}\text { Element Avg. Conc. } \\
\text { (ug/L) }\end{array}$ & $\begin{array}{c}\text { Element Avg. Std. Dov. } \\
\text { (ugh) }\end{array}$ \\
\hline
\end{tabular}

\begin{tabular}{|c|c|c|c|c|c|}
\hline$F 11$ & Zr9o & & & & \\
\hline F11 & Z91 & & & & \\
\hline$F 12$ & $Z 990$ & .11 & .05 & & \\
\hline F12 & $Z 91$ & 2.75 & & 1.43 & .05 \\
\hline F13 & 2990 & 2.75 & & & \\
\hline$F 13$ & 2991 & 2.75 & & 2.75 & \\
\hline$F 21$ & 2890 & & & & \\
\hline$\overline{F 21}$ & Z191 & & & & \\
\hline F21R & Zr9o & & & & \\
\hline F21R & Zr91 & & & & \\
\hline$F 22$ & Zr9o & 2.75 & & & \\
\hline $\mathrm{F} 22$ & Zr91 & 2.75 & & 2.75 & \\
\hline F23 & Zr9o & 26 & .1 & & \\
\hline F23 & $Z \mathbf{7 9 1}$ & 23 & .16 & 25 & .13 \\
\hline F31 & $Z 900$ & & & & \\
\hline F31 & Zr91 & & & & \\
\hline$F 32$ & $Z 90$ & .5 & & & \\
\hline F32 & $\mathrm{Zr91}$ & .5 & & .5 & \\
\hline$F 33$ & Zr9o & 2.75 & & & \\
\hline F33 & $\mathrm{Zr91}$ & 2.75 & & 2.75 & \\
\hline $\mathrm{F} 41$ & Zr9o & & & & \\
\hline F41 & Zra1 & & & & \\
\hline$F 42$ & Zr9o & 2.75 & & & \\
\hline $\mathrm{F} 42$ & Zr91 & 2.75 & & 2.75 & \\
\hline $\mathrm{F} 43 \mathrm{I}$ & Zr9o & 27 & & & \\
\hline $\mathrm{F} 431$ & Zr91 & 23 & & 25 & \\
\hline F51 & Zr9o & & & & \\
\hline F51 & Zr91 & & & & \\
\hline $\mathrm{F} 52$ & Zr9o & 10 & & & \\
\hline F52 & $Z 991$ & 10 & & 10 & \\
\hline F53 & Zr9o & 2.75 & & & \\
\hline$F 53$ & Zr91 & 2.75 & & 2.75 & \\
\hline
\end{tabular}

${ }^{1}$ The reported value is the actual concentration. No standard deviation is reported. 
D-1

WSRC-TR-94-0484

Appendix D

DATABASE DICTIONARY 


\section{DATABASE - F\&H Area Ground Water Model Data Base - F\&HDB.MDB}

The collected data has been entered into the F\&H Area Ground Water Model Data Base. The Relational Database Management System (RDBMS) Microsoft Access version 2.0 for Windows 3.1 was used to create the database to store and perform analysis of the data. MS Access uses database objects such as forms, tables, queries, modules made up of functions and Sub procedures (MS Access Basic subprograms) and reports and to organize, analyze and present the data.

The tables that contain the data have been named according to the six transects along which data was taken, transects A, B, C, D, E, and F. Each transect is associated with three tables, a transect table, an isotope table, and a cross-section table. Transect tables contain information that is generic to all sample locations along the entire transect. The isotope tables contain the concentration and standard deviation for all runs of an experiment at each location where a sample was taken along a transect. Cross-section tables contain the X-and Y-coordinates of each sample location taken from Intergraph CADD design (.DGN) files. The Intergraph design files are graphics files that were drafted to show the vertical cross-section along each of the transect lines. The X- and Ycoordinates of the sample locations combined with the concentration data can be used to develop isoconcentration contours on the vertical cross-sections. Each of these types of tables are explained in greater detail below in the DATA AREA subsection.

There is another table, the "Dilution Factors for All Transects" table, that contains the dilution factor for any sample. Queries on this table and isotope tables were used to perform statistical analysis.

Queries of the transect and isotope tables were constructed to compile weighted averages of concentrations of isotopes at various sample locations along each transect. The weighted averages were calculated from statistical methods for analyzing error propagation, (Knoll 1989) using the Microsoft Access Basic programming language. MS Basic allows user written functions and procedures to be combined into modules. A query can then be used to call a function which returns the value for a field in the table created by the query. In this way, queries were constructed which incorporated both the original data and calls to user-written statistical functions which determined the weighted averages of the concentration and standard deviation of isotopes. The result of a query on a table for information on a particular isotope allowed the original analytical data as well as weighted averages to be presented in a report.

The database belongs to Site Geotechnical Services Department/Geo-Environmental Engineering group; Bruce Schappell, manager. The work was produced under the direction of Steve Serkiz, Task Team Leader. Use of the database is restricted to the 
following:

- Steve M. Serkiz

- William H. Johnson

- Mike McHood

- Dan Boltz

- Mira Malek

Update of the database shall be done under the direction of William $\mathrm{H}$. Johnson of Geo- Envir. Custodian of the database is Steven M. Serkiz.

\subsection{DATA AREA - F AND H GROUNDWATER DATABASE}

\section{$1.1 \mathrm{~A}, \mathrm{~B}, \mathrm{C}, \mathrm{D}, \mathrm{E}$, and F - Transect Tables}

The data tables have been divided into six transects (A, B, C, D, E, \& F). The data pertaining to samples in each transect has been incorporated into three tables, a transect table, an isotope table, and a cross-section table.

\subsubsection{Permissions}

There are no restrictions on these tables.

\subsubsection{Record}

Each record of the Transect tables contains the following information.

\subsubsection{Primary Key}

Field of Primary Key = Sample ID (e.g.: A11)

Sample ID is a alpha-numeric designator that identifies the transect $(A, B, C, D, E$, or F) the sample was taken from, the core number $(1,2,3$, etc.) the sample was taken from, and the relative depth location $(1,2,3$, etc.) in the hole. Some sample numbers $(\mathrm{R})$ indicate that repetitive samples were taken from that depth of the hole in a given transect. A listing of samples applicable to this database is given in Appendix A.

\subsubsection{Foreign $\mathrm{Key}(\mathrm{S})$}

None 


\subsubsection{Fields}

Following are the fields in the transect tables. The title gives an abbreviation, a name and the units for each given field. Below the titie is a short description of each field.

1.1.2.3.1 SAA - Soil Surface Area \# 1 (Units of m2 g-1)

Surface area of the soil as determined by the BET method (nitrogen gas exchange)

1.1.2.3.2 SAB - Soil Surface Area \#2 (units of $\mathrm{m} 2 \mathrm{~g}-1$ )

Not currently being used.

1.1.2.3.3 SA avg - Average of surface area (Units of $\mathrm{m} 2 \mathrm{~g}-1$ )

$\mathrm{SA}$ avg $=(\mathrm{SAA}+\mathrm{SAB}) / 2$

\subsection{SMA - CEC \# 1 (Units of meq kg-1)}

Cation Exchange Capacity of the soil as determined by barium-magnesium exchange using the Rhoades method.

\subsection{SMB - CEC \# 2 (Units of meq $\mathrm{kg}-1$ )}

Not currently being used.

\subsection{SM ave.- Average of CEC (units of meq $\mathrm{kg}-1$ )}

$\mathrm{SM}$ ave $=(\mathrm{SMA}+\mathrm{SMB}) / 2$

\subsection{SPA - (pH Units)}

Soil $\mathrm{pH}$ for run $\mathrm{A}$

\subsection{SPB - (pH Units)}

Soil $\mathrm{pH}$ for run $\mathrm{B}$

\subsection{SP avg - (pH Units)}

Average of $\mathrm{pH}$ for run $\mathrm{A}$ and $\mathrm{B}$

$\mathrm{SP}$ avg $=(\mathrm{SPA}+\mathrm{SPB}) / 2$ 


\subsection{SQA - Net Soil Charge (units of meq $100 \mathrm{mg}^{-1}$ )}

Net soil charge as determined by the Uehara and Gillman method using calcium chloride backings.

\subsection{Temp - Porewater Temperature (Units of ${ }^{\circ} \mathrm{C}$ )}

Temperature of a porewater subsample as measured in the field immediately upon sampling.

\subsection{Field $\mathrm{pH}-\mathrm{pH}$ units}

$\mathrm{pH}$ of a porewater subsample as measured in the field immediately upon sampling.

\subsection{WPA - Laboratory $\mathrm{pH}-\mathrm{pH}$ units}

$\mathrm{pH}$ of porewater subsample $\mathrm{A}$ as measured in the laboratory.

\subsection{WPB - Laboratory $\mathrm{pH}$ - $\mathrm{pH}$ units}

$\mathrm{pH}$ of porewater subsample $\mathrm{B}$ as measured in the laboratory.

\subsection{WP avg - Average of laboratory measured $\mathrm{pHs} \mathrm{-} \mathrm{pH}$ units}

$W$ avg $=(W P A+W P B) / 2$

\subsection{Redox - (Units of mV)}

Porewater redox potential of a subsample measured in the field immediately upon sampling using the platinum bright method.

\subsection{TIC - Porewater Inorganic Carbon Content (in ppm)}

Total inorganic carbon content of the porewater as measured using an automated carbon analyazer.

\subsection{TOC - Porewater Organic Carbon Content (in ppm)}

Total inorganic carbon content of the porewater as measured using an automated carbon analyazer.

\subsection{Fldcon - Porewater Conductivity (Units of 10-2 S m-2)}

Field conductivity of a porewater subsample as measured immediately upon sampling. 
1.1.1.2.3.20\% Sand - (Units of percent)

Percent of sand in sample

1.1.2.3.21\% Silt - (Units of percent)

Percent of silt in sample

1.1.2.3.22 \% Clay - (Units of percent)

Percent of clay in sample

\subsection{A, B, C, D, E, and F - Isotope Tables}

\subsubsection{Permissions}

There are no restrictions on these tables.

\subsubsection{Record}

Each record of the Isotope tables contains the following information.

\subsubsection{Primary Key}

Field of Primary Key = Sample ID ( e.g.: Al1)

See Section 1.1.2.1.

\subsubsection{Composite Key}

Field of Composite Key = Isotope

Data is needed from more than one field to meet the uniqueness requirement of a primary key. The Primary and Composite Keys together define a unique row on this database table.

These fields contain the concentration of a given element, anion or radionuclide. For cases where stable isotopes are listed, the concentrations are the total elemental concentration as determined by analyzing for the given isotope. Units are in $\mathrm{ppb}$ for elemental concetrations, ppm for anionic concentrations, and $\mathrm{nCi}^{-1}$ for ${ }^{3} \mathrm{H},{ }^{90} \mathrm{Sr}$, and ${ }^{137} \mathrm{Cs}$. 


\subsubsection{Fields}

Following are the fields in the isotope tables. The title gives an abbreviation, a name and the units for each given field. Below the title is a short description of each field.

\subsection{WFA - Analysis of filtered porewater - Repetition A.}

Analysis of elemental concetrations by ICP-MS, anion concentrations by IC, and ${ }^{3} \mathrm{H}$ by liquid scintillation counting.

\subsection{WFAtxt - Text field that describes the WFA field in case of special} circumstances:

$\mathrm{LDL}=$ Less than Dectection Limit; number entered in the standard deviation column is the lower limit of quanitification

LTQ $=$ Less than Quatification Limit

SS $=$ Single Standard

1.2.2.3.3 WFA sd - standard deviation of porewater data

Standard deviation of elemental data from above.

1.2.2.3.4 WFB - Analysis of filtered porewater - Repetition B.

See 1.2.2.3.1

1 2.2.3.5 WFBtxt - Text field that describes the WFB field

See 1.2.2.3.2

1.2.2.3.6 WFB sd - standard deviation of porewater data

Standard deviation of elemental data from above.)

1.2.2.3.7 WFE - Analysis of filtered porewater - Repetition E.

See 1.2.2.3.1

1.2.2.3.8 WFEtxt - Text field that describes the WFE field

See 1.2.2.3.2 
1.1.2.2.3.9 WFE sd - standard deviation of porewater data

Standard deviation of elemental data from above.

1.2.2.3.10 WFF - Analysis of filtered porewater - Repetition F.

See 1.2.2.3.1.

1.2.2.3.11 WFFtxt - Text field that describes the WFF field

See 1.2.2.3.2

1.2.2.3.12 WFFsd - standard deviation of porewater data

Standard deviation of elemental data from above.

1.2.2.3.13 SOA - Analysis of soil total digestion extracts - Repetition A (Units in ppb)

ICP-MS results of soil acid digestion extracts

1.2.2.3.14 SOAtxt - Text field that describes the SOA field

See 1.2.2.3.2

1.2.2.3.15 SOA sd - Standard deviation of soil digestion data (Units of ppb)

Standard devation of above data.

1.2.2.3.16 SOB - Analysis of soil total digestion extracts - Repetition B (Units in ppb)

ICP-MS results of soil acid digestion extracts

1.2.2.3.17 SOBtxt - Text field that describes the SOB field

See 1.2.2.3.2

1.2.2.3.18 SOB sd - Standard deviation of soil digestion data (Units of ppb)

Standard devation of above data.

1.2.2.3.19 SOE - Analysis of soil total digestion extracts - Repetition E (Units in ppb)

ICP-MS results of soil acid digestion extracts 
1.1.2.2.3.20 SOEtxt - Text field that describes the SOE field

See 1.2.2.3.2

1.2.2.3.21 SOE sd - Standard deviation of soil digestion data (Units of ppb)

Standard devation of above data.

1.2.2.3.22 SOF - Analysis of soil total digestion extracts- Repetition F (Units in ppb) ICP-MS results of soil acid digestion extracts

1.2.2.3.23 SOFtxt - Text field that describes the SOF field

See 1.2.2.3.2

1.2.2.3.24 SOF sd - Standard deviation of soil digestion data (Units of $\mathrm{ppb}$ )

Standard devation of above data.

1.2.2.3.25 SOG - Analysis of soil total digestion extracts - Repetition G (Units in ppb) ICP-MS results of soil acid digestion extracts

1.2.2.3.26 SOGtxt - Text field that describes the SOG field

See 1.2.2.3.2

1.2.2.3.27 SOG sd - Standard deviation of soil digestion data (Units of ppb)

Standard devation of above data.

1.2.2.3.28 S1A - Concentration of water souble ions - Repetition A (Units in ppb)

ICP-MS analysis of soil water souble extracts

1.2.2.3.29 S1Atxt - Text field that describes the S1A field

See 1.2.2.3.2

1.2.2.3.30 S1A sd - Standard deviaiton of water soluble data- (units of $\mathrm{ppb}$ )

Standard deviation of above data 
1.1.2.2.3.31 S1B - Concentration of water souble ions - Repetition B (Units in ppb)

ICP-MS analysis of soil water souble extracts

1.2.2.3.32 S1Btxt - Text field that describes the S1B field

See 1.2.2.3.2

1.2.2.3.33 S1B sd - Standard deviaiton of water soluble data- (units of ppb)

Standard deviation of above data

1.2.2.3.34 S1E - Concentration of water souble ions - Repetition E (Units in ppb)

ICP-MS analysis of soil water souble extracts

1.2.2.3.35 S1Etxt - Text field that describes the S1E field

See 1.2.2.3.2

1.2.2.3.36 S1E sd - Standard deviaiton of water soluble data- (units of $\mathrm{ppb}$ )

Standard deviation of above data

1.2.2.3.37 S2A - Concentration of easily exchangable ions - Repetition A (Units in ppb)

ICP-MS analysis of soil easily excahngable ion extracts

1.2.2.3.38 S2Atxt - Text field that describes the S2A field

See 1.2.2.3.2

1.2.2.3.39 S2A sd - Standard deviaiton of easily exchangable data- (units of ppb)

Standard deviation of above data

1.2.2.3.40 S2B - Concentration of easily exchangable ions - Repetition B (Units in ppb)

ICP-MS analysis of soil easily excahngable ion extracts

1.2.2.3.41 S2Btxt - Text field that describes the S2B field

See 1.2.2.3.2 
1.1.2.2.3.42 S2B sd - Standard deviaiton of easily exchangable data- (units of ppb)

Standard deviation of above data

1.2.2.3.43 S2E - Concentration of easily exchangable ions - Repetition E (Units in ppb)

ICP-MS analysis of soil easily excahngable ion extracts

1.2.2.3.44 S2Etxt - Text field that describes the S2E field

See 1.2.2.3.2

1.2.2.3.45 S2E sd - Standard deviaiton of easily exchangable data- (units of ppb)

Standard deviation of above data

1.2.2.3.46 S3A - Concentration of acid soluble ions - Repetition A (Units in ppb)

ICP-MS analysis of soil easily excahngable ion extracts

1.2.2.3.47 S3Atxt - Text field that describes the S3A field

See 1.2.2.3.2

1.2.2.3.48 S3A sd - Standard deviaiton of acid soluble data- (units of $\mathrm{ppb}$ )

Standard deviation of above data

1.2.2.3.49 S3B - Concentration of acid soluble ions - Repetition B (Units in ppb)

ICP-MS analysis of soil easily excahngable ion extracts

1.2.2.3.50 S3Btxt - Text field that describes the S3B field

See 1.2.2.3.2

1.2.2.3.51 S3B sd - Standard deviaiton of acid soluble data- (units of ppb)

Standard deviation of above data

1.2.2.3.52 S3E - Concentration of acid soluble ions - Repetition E (Units in ppb)

ICP-MS analysis of soil easily excahngable ion extracts 
1. 2.2.3.53 S3Etxt - Text field that describes the S3E field

See 1.2.2.3.2

1.2.2.3.54 S3E sd - Standard deviaiton of acid soluble data- (units of $\mathrm{ppb}$ )

Standard deviation of above data

1.2.2.3.55 S4A - Concentration of manganese oxide occluded ions - Repetition A (Units in ppb)

ICP-MS analysis of soil manganese oxide occluded ion extracts

1.2.2.3.56 S4Atxt - Text field that describes the S4A field

See 1.2.2.3.2

1.2.2.3.57 S4A sd - Standard deviaiton of manganese oxide occluded data(units of ppb)

Standard deviation of above data

1.2.2.3.58 S4B - Concentration of manganese oxide occluded ions - Repetition B (Units in ppb)

ICP-MS analysis of soil manganese oxide occluded ion extracts

1.2.2.3.59 S4Btxt - Text fieid that describes the S4B field

See 1.2.2.3.2

1.2.2.3.60 S4B sd Standard deviaiton of manganese oxide occluded data(units of ppb)

Standard deviation of above data

1.2.2.3.61 S4E - Concentration of manganese oxide occluded ions - Repetition $\mathrm{E}$ (Units in ppb)

ICP-MS analysis of soil manganese oxide occluded ion extracts

1.2.2.3.62 S4Etxt - Text field that describes the S4E field

See 1.2.2.3.2 
1.2.2.3.63 S4E sd - Standard deviaiton of manganese oxide occluded data(units of $\mathrm{ppb}$ )

Standard deviation of above data

1.2.2.3.64 S5A - Concentration of organically bound ions - Repetition A (Units in ppb)

ICP-MS analysis of soil organically bound ion extracts

1.2.2.3.65 S5Atxt - Text field that describes the S5A field

See 1.2.2.3.2

1.2.2.3.66 S5A sd - Standard deviaiton of organically bound data- (units of ppb) Standard deviation of above data

1.2.2.3.67 S5B - Concentration of organically bound ions - Repetition B (Units in ppb) ICP-MS analysis of soil organically bound ion extracts

1.2.2.3.68 S5Btxt - Text field that describes the S5B field

See 1.2.2.3.2

1.2.2.3.69 S5B sd - Standard deviaiton of organically bound data- (units of ppb) Standard deviation of above data

1.2.2.3.70 S5E - Concentration of organically bound ions - Repetition E (Units in ppb) ICP-MS analysis of soil organically bound ion extracts

1.2.2.3.71 S5Etxt - Text field that describes the S5E field

See 1.2.2.3.2

1.2.2.3.72 S5E sd - Standard deviaiton of organically bound data - (units of ppb) Standard deviation of above data 
1.2.2.3.73 S6A - Concentration of noncyrstalline iron oxide data ions - Repetition A (Units in ppb)

ICP-MS analysis of soil noncyrstalline iron oxide data ion extracts

1.2.2.3.74 S6Atxt - Text field that describes the S6A field

See 1.2.2.3.2

1.2.2.3.75 S6A sd - Standard deviaiton of noncyrstalline iron oxide data - (units of ppb) Standard deviation of above data

1.2.2.3.76 S6B - Concentration of noncyrstalline iron oxide data ions - Repetition B (Units in ppb)

ICP-MS analysis of soil noncyrstalline iron oxide data ion extracts

1.2.2.3.77 S6Btxt - Text field that describes the S6B field

See 1.2.2.3.2

1.2.2.3.78 S6B sd - Standard deviaiton of noncyrstalline iron oxide data - (units of ppb)

Standard deviation of above data

1.2.2.3.79 S6E - Concentration of noncyrstalline iron oxide ions - Repetition $\mathrm{E}$ (Units in ppb)

ICP-MS analysis of soil noncyrstalline iron oxide ion extracts

1.2.2.3.80 S6Etxt - Text field that describes the S6E field

See 1.2.2.3.2

1.2.2.3.81 S6E sd - Standard deviaiton of noncyrstalline iron oxide data - (units of ppb)

Standard deviation of above data

1.2.2.3.82 S7A - Concentration of cyrstalline iron oxide data ions - Repetition A (Units in ppb)

ICP-MS analysis of soil cyrstalline iron oxide ion extracts 
1.2.2.3.83 S7Atxt - Text field that describes the S7A field

See 1.2.2.3.2

1.2.2.3.84 S7A sd - Standard deviaiton of cyrstalline iron oxide data - (units of ppb)

Standard deviation of above data

1.2.2.3.85 STB - Concentration of cyrstalline iron oxide ions - Repetition B (Units in ppb)

ICP-MS analysis of soil cyrstalline iron oxide ion extracts

1.2.2.3.86 S7Btxt - Text field that describes the S7B field

See 1.2.2.3.2

1.2.2.3.87 S7B sd - Standard deviaiton of cyrstalline iron oxide data - (units of ppb)

Standard deviation of above data

1.2.2.3.88 S7E - Concentration of cyrstalline iron oxide ions - Repetition E (Units in ppb)

ICP-MS analysis of soil cyrstalline iron oxide ion extracts

1.2.2.3.89 S7Etxt - Text field that describes the S7E field

See 1.2.2.3.2

1.2.2.3.90 S7E sd - Standard deviaiton of cyrstalline iron oxide data - (units of ppb)

Standard deviation of above data

1.2.2.3.91 S8A - Concentration of residual soil acid digestion extracts - Repetition A (Units in ppb)

ICP-MS analysis of residual soil acid digestion extracts

1.2.2.3.92 S8Atxt - Text field that describes the S8A field

See 1.2.2.3.2 
1.2.2.3.93 S8A sd - Standard deviaiton of residual soil acid digestion data -

(units of $\mathrm{ppb}$ )

Standard deviation of above data

1.2.2.3.94 S8B - Concentration of residual soil acid digestion extracts - Repetition B (Units in ppb)

ICP-MS analysis of residual soil acid digestion extracts

1.2.2.3.95 S8Btxt - Text field that describes the S8B field

See 1.2.2.3.2

1.2.2.3.96 S8B sd - Standard deviaiton of residual soil acid digestion data (units of $\mathrm{ppb}$ )

Standard deviation of above data

1.2.2.3.97 S8E - Concentration of residual soil acid digestion extracts - Repetition $\mathrm{E}$ (Units in ppb)

ICP-MS analysis of residual soil acid digestion extracts

1.2.2.3.98 S8Etxt - Text field that describes the S8E field

See 1.2.3.2

1.2.2.3.99 S8E sd - Standard deviaiton of residual soil acid digestion data (units of ppb)

Standard deviation of above data 


\subsection{A, B, C, D, E \& F - XSECTION TABLES}

Cross-section tables contain the $\mathrm{X}$ - and $\mathrm{Y}$-coordinates of each sample location taken from Intergraph CADD design (.DGN) files. The Intergraph design files are graphics files that were drafted to show the vertical cross-section along each of the transect lines. The $\mathrm{X}$ - and $\mathrm{Y}$-coordinates of the sample locations combined with the concentration data can be used to develop isoconcentration contours on the vertical cross-sections.

\subsubsection{Permissions}

There are no restrictions for the $\mathrm{X}$-section tables.

\subsubsection{Record}

Each record of the X-section tables contains the following information.

\subsubsection{Primary Key}

Field of Primary Key = Sample ID ( e.g.: A11)

See Section 1.1.1.2.1

\subsubsection{Fields}

Following are the fields in the isotope tables. The title gives an abbreviation, a name and the units for each given field. Below the title is a short description of each field.

1.3.2.2.1 XCoord - X - coordinates of each sample location taken from Intergraph CADD design (.DGN) files.

1.3.2.2.2 YCoord - Y - coordinates of each sample location taken from Intergraph CADD design (.DGN) files.

\subsection{Dilution Factors for All Transects Table}

This table contains the dilution factor used for each sample.

\subsubsection{Permissions}

There are no restrictions for the dilution factors table. 


\subsubsection{Record}

Each record of the X-section tables contains the following information.

\subsubsection{Primary Key}

Field of Primary Key = Sample ID ( e.g.: A11)

See Section 1.1.1.2.1.

\subsubsection{Fields}

Following are the fields in the dilution factor table, the dilution factor has no units.

The field name and a short description of each field is given.

1.4.2.2.1 A_Factor_PW - Dilution factor for run A, porewater

1.4.2.2.2 B_Factor_PW - Dilution factor for run B, porewater

1.4.2.2.3 E_Factor_PW - Dilution factor for run E, porewater

1.4.2.2.4 F_Factor_PW - Dilution factor for run F, porewater

1.4.2.2.5 G_Factor_PW - Dilution factor for run G, porewater

1.4.2.2.6 A_Factor_0 - Dilution factor for run A, total digestion

1.4.2.2.7 B_Factor_0 - Dilution factor for run B, total digestion

1.4.2.2.8 E_Factor_0 - Dilution factor for run $\mathrm{E}$, total digestion

1.4.2.2.9 F_Factor_0 - Dilution factor for run $F$, total digestion

1.4.2.2.10 G_Factor_0 - Dilution factor for run $\mathrm{G}$, total digestion

1.4.2.2.11 A_Factor_1 - Dilution factor for run A, water soluble

1.4.2.2.12 B_Factor_1 - Dilution factor for run B, water soluble

1.4.2.2.13 E_Factor_1 - Dilution factor for run E, water soluble

1.4.2.2.14 A_Factor_2 - Dilution factor for run A, easily exchangeable 
1.1.4.2.2.15 B_Factor_2 - Dilution factor for run B, easily exchangeable

1.4.2.2.16 E_Factor_2 - Dilution factor for run E, easily exchangeable

1.4.2.2.17 A_Factor_3 - Dilution factor for run A, acid soluble

1.4.2.2.18 B_Factor_3 - Dilution factor for run B, acid soluble

1.4.2.2.19 E_Factor_3 - Dilution factor for run E, acid soluble

1.4.2.2.20 A_Factor_4 - Dilution factor for run A, $\mathrm{MnO}$ occluded

1.4.2.2.21 B_Factor_4 - Dilution factor for run B, MnO occluded

1.4.2.2.22 E_Factor_4 - Dilution factor for run E, MnO occluded

1.4.2.2.23 A_Factor_5 - Dilution factor for run A, organically bound

1.4.2.2.24 B_Factor_5 - Dilution factor for run B, organically bound

1.4.2.2.25 E_Factor_5 - Dilution factor for run E, organically bound

1.4.2.2.26 A_Factor_6 - Dilution factor for run A, amorphous Fe oxides

1.4.2.2.27 B_Factor_6 - Dilution factor for run B, amorphous Fe oxides

1.4.2.2.28 E_Factor_6 - Dilution factor for run E, amorphous Fe oxides

1.4.2.2.29 A_Factor_7 - Dilution factor for run A, crystalline Fe oxides

1.4.2.2.30 B_Factor_7 - Dilution factor for run B, crystalline Fe oxides

1.4.2.2.31 E_Factor_7 - Dilution factor for run E, crystalline Fe oxides

1.4.2.2.32 A_Factor_8 - Dilution factor for run A, partial digestion

1.4.2.2.33 B_Factor_8 - Dilution factor for run B, partial digestion

1.4.2.2.34 E_Factor_8 - Dilution factor for run E, partial digestion 


\subsection{APPLICATIONS THAT USE THE DATABASE TABLES}

There are several applications that use the "raw data" that has been entered into the transect, isotope, and cross-section tables. The purpose of these applications is to find an average value for both the concentration and standard deviation of isotopes at various sample locations along each transect. The applications used to achieve this result include modules, queries, forms and reports.

\subsection{Access BASIC Modules}

The Access BASIC programming language was used to create user-written functions that performed simple statistical calculations on data in tables. The functions were grouped into three modules, Generic Transect Procedures, Element Average Procedures for Concentration, and Element Average Procedures for Std Deviation. Short descriptions of the functions in each module follow. The MS Access BASIC code for the functions in each of these modules is included in Appendix E.

\subsubsection{Permissions}

There are no restrictions to the modules.

\subsubsection{Generic Transect Procedures}

In general, the purpose of these functions is to return the value for the weighted average of the input values for the concentration and the weighted average of the input values for the standard deviation for an isotope. There is one function, however, that is utilized by the report application to generate hardcopy output; it is called Elmt_Name.

\subsubsection{Element Average Procedures for Concentration}

These procedures are used for calculating the arithmetic mean of the weighted average concentration for each isotope of an element, thus giving an average value of the concentration for each element.

\subsubsection{Element Average Procedures for Standard Deviation}

These procedures are used for calculating the arithmetic mean of the weighted average standard deviation of the concentration for each isotope of an element, thus giving an average value of the standard deviation for the concentration for each element. 


\subsection{Queries}

The queries have been named according to the five transects (A, B, C, D, E, \& F). The queries used to compile the weighted averages of the concentration and standard deviation for the isotopes have been incorporated into three action queries for each transect. Action queries are used to read information from the data tables, perform statistical calculations on the data, and write the results of these calculations into a new table each time the query is executed. By creating a new table which contains the results of the calculations, the original data is not altered in any way, preserving the integrity of the original input data.

The queries must be performed in a specified order to insure that the data in the report is correct. The first query to be discussed is the query on which the other two queries are based.

The first query to be executed is called "Transect X - Wghted Conc and StDev for Expmt 0 and Porewater", (where " $X$ " is the letter A, B, E, etc designating the transect). The query is based on two tables, the "X_Isotope" table and the "Dilution Factors for All Transects" table. This query creates a new table called "Transect X Query Result: Conc and StDev for Expmt 0 and Porewat", (where " $X$ " is the letter A, B, E, etc.). The reader should take note that the query must have the "Ascending" attribute set for the SAMPLE_ID and ISOTOPE fields when executing this query.

The other two queries are based on the new table created by the first query. One query is called "Transect X - Elemental Averages for Expmt 0", the other is called "Transect X - Elemental Averages for Porewater", (where " $X$ " is the letter A, B, E, etc.). Like the first query, both of these queries are used to create new tables. In this case, however, the new tables contain data for only one element. The element is specified by the user.

A more detailed description of the queries and the new tables that are created by each query follows.

\subsubsection{Permissions}

There are no restrictions to this query.

\subsubsection{Transect $X$ - Wghted Conc and StDev for Expmt 0 and Porewater}

$$
(X=A, B, E, F \text { or } G)
$$

This query utilizes the functions in the module "Generic Transect Procedures" (see Section 2.1), to return the value for the weighted average of the input values for the concentration and the weighted average of the input values for the standard deviation for an isotope. The query creates a new table called "Transect X - Wghted Conc and StDev for Expmt 0 and Porewat" and is described below. The reader should take note that the 
query must have the "Ascending" attribute set for the SAMPLE_ID and ISOTOPE fields when executing this query.

2.2.2.1 Primary Key - There is no primary key.

\subsubsection{Fields}

Following are the fields in the table. The field name, its units, and a short description of each field is given.

\subsection{Sample_ID -}

Sample $\mathrm{D}$ is a alpha-numeric designator that identifies the transect (A, B, E, F or G) the sample was taken from, the core number $(1,2,3$, etc.) the sample was taken from, and the relative depth location $(1,2,3$, etc.) in the hole. Some sample numbers (R) indicate that repetitive samples were taken from that depth of the hole in a given transect. A listing of samples applicable to this database is given in Appendix A.

\subsection{Isotope -}

These fields contain the concentration of a given element, anion or radionuclide. For cases where stable isotopes are listed, the concentrations are the total elemental concentration as determined by analyzing for the given isotope. (Data for Th-232 is total thorium.) Units are in ppb for elemental concetrations, ppm for anionic concentrations, and $\mathrm{pCi} \mathrm{ml-1}$ for $\mathrm{H}-3$.

\subsection{Conc XPW}

$$
(\mathrm{X}=\mathrm{A}, \mathrm{B}, \mathrm{E}, \mathrm{F} \text { or } \mathrm{G})
$$

The values in this field are calculated by the function "CONC_PW". This function returns the value for the weighted average of the input values, (i.e. the values for the concentration of an isotope for a particular sample ID), for the concentration using Porewater along ANY transect.

\subsection{StDev_XPW -}

$$
(\mathrm{X}=\mathrm{A}, \mathrm{B}, \mathrm{E}, \mathrm{F} \text { or } \mathrm{G})
$$

The values in this field are calculated by the function "StanDev_PW". This function returns the weighted average of the input values for the standard deviation of the concentration using Porewater along ANY transect.

\subsection{Conc_XO -}




$$
(\mathrm{X}=\mathrm{A}, \mathrm{B}, \mathrm{E}, \mathrm{F} \text { or } \mathrm{G})
$$

The values in this field are calculated by the function "CONC_O". This function returns the value for the weighted average of the input values, (i.e. the values for the concentration of an isotope for a particular sample ID), for the concentration using Porewater along ANY transect.

\subsection{StDev_XO -}

$$
(X=A, B, E, F \text { or } G)
$$

The values in this field are calculated by the function "StanDev_0". This function returns the weighted average of the input values for the standard deviation of the concentration using Porewater along ANY transect.

\subsubsection{Transect X - Elemental Averages for Expmt 0}

$(\mathrm{X}=\mathrm{A}, \mathrm{B}, \mathrm{E}, \mathrm{F}$ or $\mathrm{G})$

This query uses the modules for calculating the arithmetic mean of the weighted average concentration for each isotope of an element, thus giving an average value of the concentration for each element. The query creates a new table called "Transect $\mathrm{X}$ - Elemental Averages for Expmt 0" and is described below.

\subsubsection{Primary Key - There is no primary key.}

\subsubsection{Fields}

Following are the fields in the table. The field name, its units, and a short description of each field is given.

\subsection{Sample_ID - See section 2.2.2.2.1}

2.2.3.2.2 Isotope - See section 2.2.2.2.2

2.2.3.2.3 Conc_AO - See section 2.2.2.2.3

2.2.3.2.4 StDev_A0 - See section 2.2.2.2.4

\subsection{Element_Avg_Conc -}

The values in this field are calculated by the function "Element_Avg". This function is used for calculating the arithmetic mean of the concentration(s) of isotope(s) for each element, thus giving an average value of the concentration for each element. 
This function reads values from a table that has the weighted average concentration for an isotope, along with the isotope name. An element average function (for example, Ag_Avg, Al_Avg, etc.) keeps tally of the total concentration of an element by keeping a sum of the concentration of each isotope at a particular sample location. The average concentration of an element is returned to the Element_Avg_Conc field after the sum of all isotopes divided by the number of occurrences of isotopes is calculated by the element average function. For example:

If $\mathrm{Ag} 107=2.5$ and $\mathrm{Ag} 109=2.9$, then $\mathrm{Ag}_{-} \mathrm{Avg}=(2.5+2.9) / 2=2.7$

NOTE: If there is a null value for an isotope, then it is ignored. For Example:

If $\mathrm{Ag} 107=\mathrm{Null}$, and $\mathrm{Ag} 109=2.9$, then Ag_Avg $=(2.9) / 1=2.9$

NOTE: The value in this field is rounded to the nearest hundredth, i.e., two (2) decimal places by the variant function, Roundoff. This function is in the module called "Generic Transect Procedures".

\subsection{Element_Avg_StDev -}

The values in this field are calculated by the function "Element_SD_Avg". This function is used for calculating the arithmetic mean of the concentration(s) of isotope(s) for each element, thus giving an average value of the standard deviation of the concentration for each element. This function operates in the same manner as the previous function, (see section 2.2.3.2.5).

\subsubsection{Transect $X$ - Elemental Averages for Porewater}

$(X=A, B, C, D, E, \& F)$

These procedures are used for calculating the arithmetic mean of the weighted average standard deviation of the concentration for each isotope of an element, thus giving an average value of the standard deviation for the concentration for each element. The query creates a new table called "Transect X - Elemental Averages for Porewater" and is described below.

\subsubsection{Primary Key - there is no primary key.}

\subsubsection{Fields}

Following are the fields in the table. The field name, its units, and a short description 
of each field is given.

2.2.4.2.1 Sample_ID - See section 2.2.3.2.1

2.2.4.2.2 Isotope - See section 2.2.3.2.2

2.2.4.2.3 Conc_A0 - See section 2.2.3.2.3

2.2.4.2.4 StDev_AO - See section 2.2.3.2.4

2.2.4.2.5 Element_Avg_Conc - See section 2.2.3.2.5

2.2.4.2.6 Element_Avg_StDev - See section 2.2.3.2.6 


\subsection{FORMS}

There are two forms that were created to be used by data entry personnel. These forms were used when entering data into the Transect and Isotope tables.

\subsection{Transect Data Input}

This form was developed to assist the data entry personnel with the input of sample data into the Transect Tables (A, B, E, F \& G). To use the forms, do the following:

3.1.1 From the database window, choose Form button, then choose the transect form desired. Choose Run button.

3.1.2 The form is displayed in View Form with the sample field in the main form and the remainder of the Transect table's fields in the subform.

3.1.3 Select the View Datasheet button on the toolbar (third button from left).

3.1.4 Highlight the Sample No.(left hand field) by clicking on the cell with the sample number you are about to enter data into. Click the View Form button (second from left) on the toolbar.

3.1.5 While in View Form, scroll through the forms fields in the field box in the toolbar. Select the desired subform field by highlighting it.

3.1.6 Enter the sample data into the cell with the blinking bar.

3.1.7 Repeat the process until all data has been entered into that table.

\subsection{Isotope Data Input}

This form was developed to assist the data entry personnel with the input of sample data into the Isotope Tables (A, B, C, D, E, \& F). To use the forms, do the following:

3.2.1 From the database window, choose Form button, then choose the Isotope form desired. Choose Run button.

3.2.2 The form is displayed in View Form with the sample field and isotope field in the main form and the remainder of the Isotope table's fields in the subform.

3.2.3 Select the View Datasheet button on the toolbar (third button from left).

3.2.4 Highlight the Sample No.(left hand field) and Isotope No. (second from left hand 
field) by clicking on the cells with the sample no. and isotope number you are about to enter data into. Click the View Form button (second from left) on the toolbar.

3.2.5 While in View Form, scroll through the forms fields in the field box in the toolbar. Select the desired subform field by highlighting it.

3.2.6 Enter the sample data into the cell with the blinking bar.

3.2.7 Repeat the process until all data has been entered into that table.

\subsection{REPORTS}

The MS ACCESS report application was used to generate hardcopy printouts of the element averages. A "generic report" was created to be used as a template for reports on elements and /or transects specified by the user. Since the report changes with the element or transect being considered, a general methodology for creating the reports follows.

\subsection{Creating a report from the "generic report"}

\subsubsection{Copy the generic report.}

4.1.2 Select the "Properties" icon; this will cause the properties dialogue box to appear.

4.1.3 Change the "Record Source" in the properties dialogue box to the underlying table. The appropriate underlying table is created by using the action queries described in section 1.2.2. The queries must be performed in a specified order to insure that the data in the report is correct. The following methodology is used to create the underlying table.

4.1.3.1 "Open" the appropriate action query, (i.e., select Transect X - Wghted Conc and StDev for Exp 0 and Porewater, where $X=A, B, E$, etc.) Note that the SAMPLE_ID and ISOTOPE fields must have the "ascending" attribute set in the query.

\subsubsection{2 "Open" the appropriate action query for either Expmt 0 or Porewater, (i.e.,} select either Transect X - Elemental Averages for Exp 0 or Transect X - Elemental Averages for Porewater). Note that the element to be considered must be specified by the user.

4.1.4 Edit the label in the report to reflect the change of the "Record Source" in the properties dialogue box to the underlying table. Print the report. 
Table D-1. Sample identification scheme. Depth units are in feet. Samples marked in the Water column have an associated water sample.

\begin{tabular}{|c|c|c|c|c|c|c|c|c|c|}
\hline \multirow{20}{*}{$\frac{\text { Transect }}{\mathrm{A}}$} & Hole & Depth & ID & Water & Transect & Hole & Depth & ID & Water \\
\hline & \multirow[t]{4}{*}{1} & 63 & A11 & & \multirow[t]{4}{*}{$\mathrm{C}$} & \multirow[t]{4}{*}{1} & 24 & C11 & \\
\hline & & 73 & A12 & $\mathbf{x}$ & & & 57 & $\mathrm{C} 12$ & \\
\hline & & 88 & A13 & $\mathrm{X}$ & & & 67 & $\mathrm{C} 13$ & $\mathrm{x}$ \\
\hline & & 88 & Al3(R) & $\mathbf{x}$ & & & 82 & $\mathrm{Cl} 4$ & $\mathrm{x}$ \\
\hline & \multirow[t]{4}{*}{2} & 66 & A21 & & & \multirow[t]{4}{*}{2} & 55 & $\mathrm{C} 21$ & \\
\hline & & 76 & A22 & $\mathbf{x}$ & & & 55 & C21(R) & \\
\hline & & 91 & A23 & $\mathbf{x}$ & & & 65 & $\mathrm{C} 22$ & $\mathbf{x}$ \\
\hline & & & & & & & 80 & $\mathrm{C} 23$ & $\mathbf{x}$ \\
\hline & \multirow[t]{3}{*}{3} & 56 & A31 & $\mathbf{x}$ & & & & & \\
\hline & & 71 & A32 & $\mathbf{x}$ & & 3 & 46 & C31 & \\
\hline & & & & & & & 56 & C32 & $\mathbf{X}$ \\
\hline & \multirow[t]{3}{*}{4} & 39 & A41 & $\mathrm{x}$ & & & 71 & C33 & $\mathrm{x}$ \\
\hline & & 54 & A42 & $\mathbf{X}$ & & & & & \\
\hline & & & & & & 4 & 38 & $\mathrm{C} 41$ & \\
\hline & \multirow[t]{6}{*}{5} & 18 & As1 & & & & 48 & $\mathrm{C}_{42}$ & $\mathbf{x}$ \\
\hline & & 28 & A52 & $\mathrm{x}$ & & & 63 & $\mathrm{C} 43$ & $\mathrm{x}$ \\
\hline & & 38 & A53 & $\mathbf{x}$ & & & & & \\
\hline & & 38 & A53(R) & $\mathrm{x}$ & D & 1 & 32 & D11 & \\
\hline & & 110 & A55V & no soil & & & 63 & D12 & $\mathrm{x}$ \\
\hline & & & & & & & 102 & D13 & $\mathrm{x}$ \\
\hline \multirow[t]{19}{*}{ B } & \multirow[t]{4}{*}{1} & 48 & B11 & & & & 100 & $\mathrm{D} 13(\mathrm{R})(\mathrm{A})$ & d) $x$ \\
\hline & & 58 & B12 & & & & 106 & D13(R)(B) & \\
\hline & & 68 & B13 & $\mathrm{x}$ & & & & & \\
\hline & & 78 & B14 & $\mathrm{x}$ & & 2 & 63 & D21 & \\
\hline & \multirow[t]{5}{*}{2} & 47 & B21 & & & 4 & 67 & D41 & \\
\hline & & 57 & B22 & & & & & & \\
\hline & & 67 & B23 & $\mathrm{X}$ & & 5 & 64 & D51 & \\
\hline & & 67 & B23(R) & $\mathrm{x}$ & & & & & \\
\hline & & 77 & B24 & $\mathbf{x}$ & & & & & \\
\hline & \multirow[t]{3}{*}{3} & 51 & B31 & & & & & & \\
\hline & & 61 & B32 & $\mathrm{X}$ & & & & & \\
\hline & & 71 & B33 & $\mathrm{X}$ & & & & & \\
\hline & \multirow[t]{4}{*}{4} & 43 & B41 & & & & & & \\
\hline & & 53 & B42 & $\mathrm{X}$ & & & & & \\
\hline & & 73 & B43 & $\mathrm{X}$ & & & & & \\
\hline & & 145 & $\mathrm{~B} 45 \mathrm{~V}$ & no soil & & & & & \\
\hline & \multirow[t]{3}{*}{5} & 40 & B51 & $\mathrm{x}$ & & & & & \\
\hline & & 55 & B52 & $\mathrm{x}$ & & & & & \\
\hline & & 55 & $\mathrm{~B} 52(\mathrm{R})$ & $\mathrm{x}$ & & & & & \\
\hline
\end{tabular}


Table D-1 (con't). Sample identification scheme. Depth units are in feet. Samples marked in the Water column have an associated water sample.

\begin{tabular}{|c|c|c|c|c|}
\hline Transect & Hole & Dept & ID & Water \\
\hline $\mathrm{E}$ & 1 & 29 & E11 & \\
\hline & & 34 & E12 & \\
\hline & & 34 & E12(R) & \\
\hline & & 44 & E13 & $\mathrm{x}$ \\
\hline & & 49 & E14 & $\mathrm{x}$ \\
\hline & 2 & 27 & E21 & \\
\hline & & 32 & E22 & \\
\hline & & 42 & E23 & $\mathrm{X}$ \\
\hline & & 42 & E23(R) & $\mathrm{X}$ \\
\hline & & 47 & E24 & $\mathrm{X}$ \\
\hline & 3 & 13 & E31 & \\
\hline & & 18 & E32 & \\
\hline & & 28 & E33 & $\mathrm{X}$ \\
\hline & & 33 & E34 & $\mathrm{X}$ \\
\hline & 4 & 9 & E41 & $\mathrm{X}$ \\
\hline & & 14 & E42 & $\mathrm{X}$ \\
\hline $\mathbf{F}$ & 1 & 23 & F11 & \\
\hline & & 30 & F12 & $\mathrm{X}$ \\
\hline & & 35 & F13 & $\mathrm{X}$ \\
\hline & 2 & 22 & F21 & \\
\hline & & 22 & $F 21(R)$ & \\
\hline & & 29 & F22 & $\mathrm{X}$ \\
\hline & & 34 & F23 & $\mathbf{X}$ \\
\hline & 3 & 20 & F31 & \\
\hline & & 27 & F32 & $\mathrm{X}$ \\
\hline & & 32 & F33 & $\mathrm{X}$ \\
\hline & 4 & 17 & F41 & \\
\hline & & 27 & F42 & $\mathrm{X}$ \\
\hline & & 32 & F43 & $\mathrm{X}$ \\
\hline & 5 & 13 & F51 & \\
\hline & & 21 & F52 & $\mathrm{X}$ \\
\hline & & 26 & F53 & $\mathrm{X}$ \\
\hline
\end{tabular}


Appendix E

\section{MICROSOFT ACCESS BASIC MODULES}




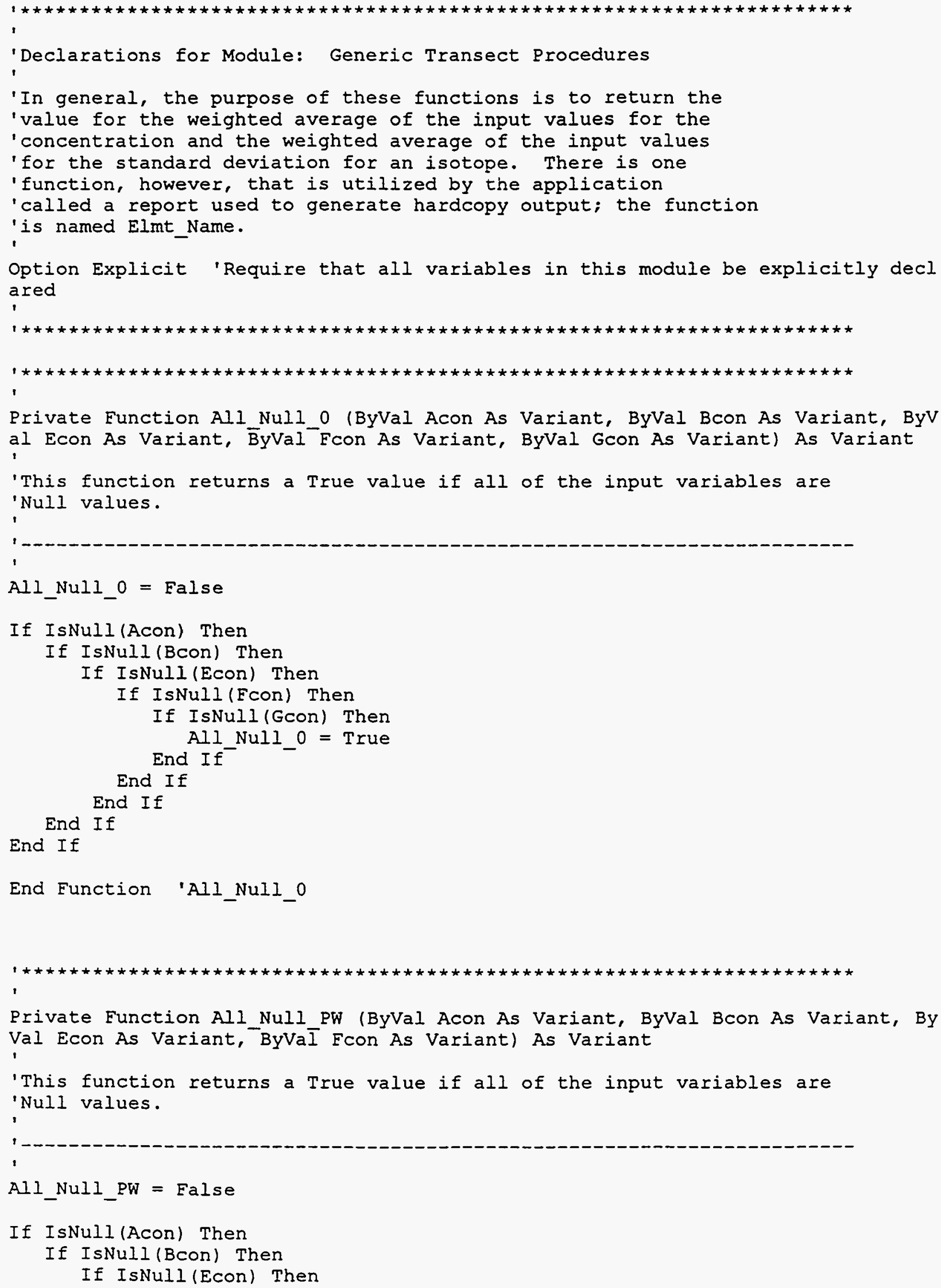




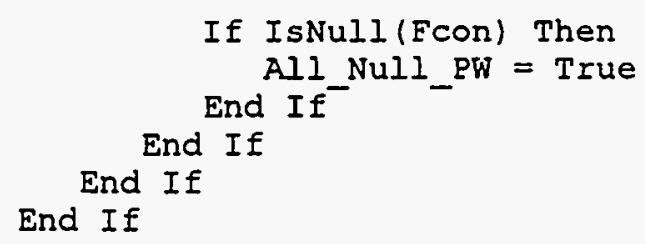




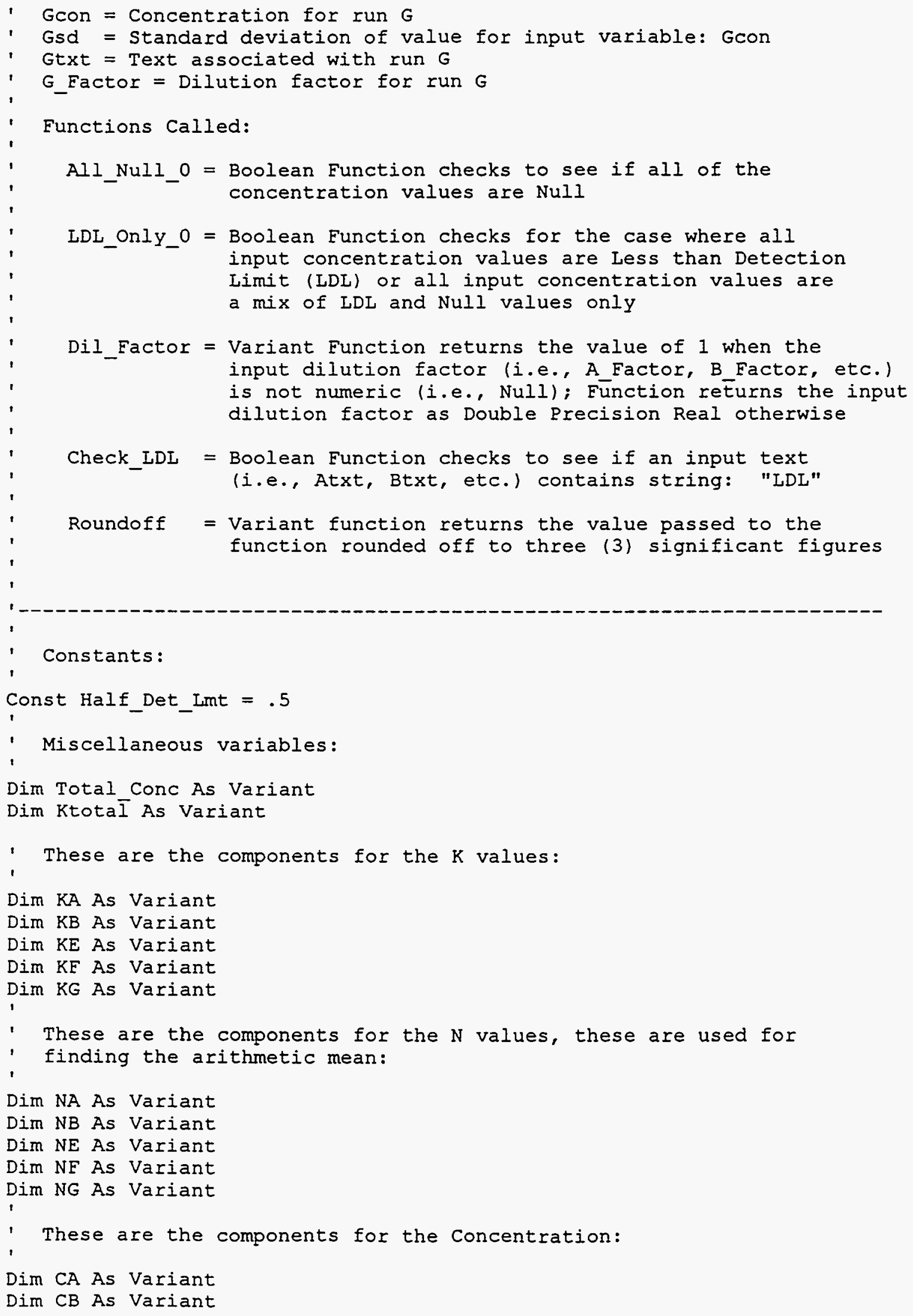




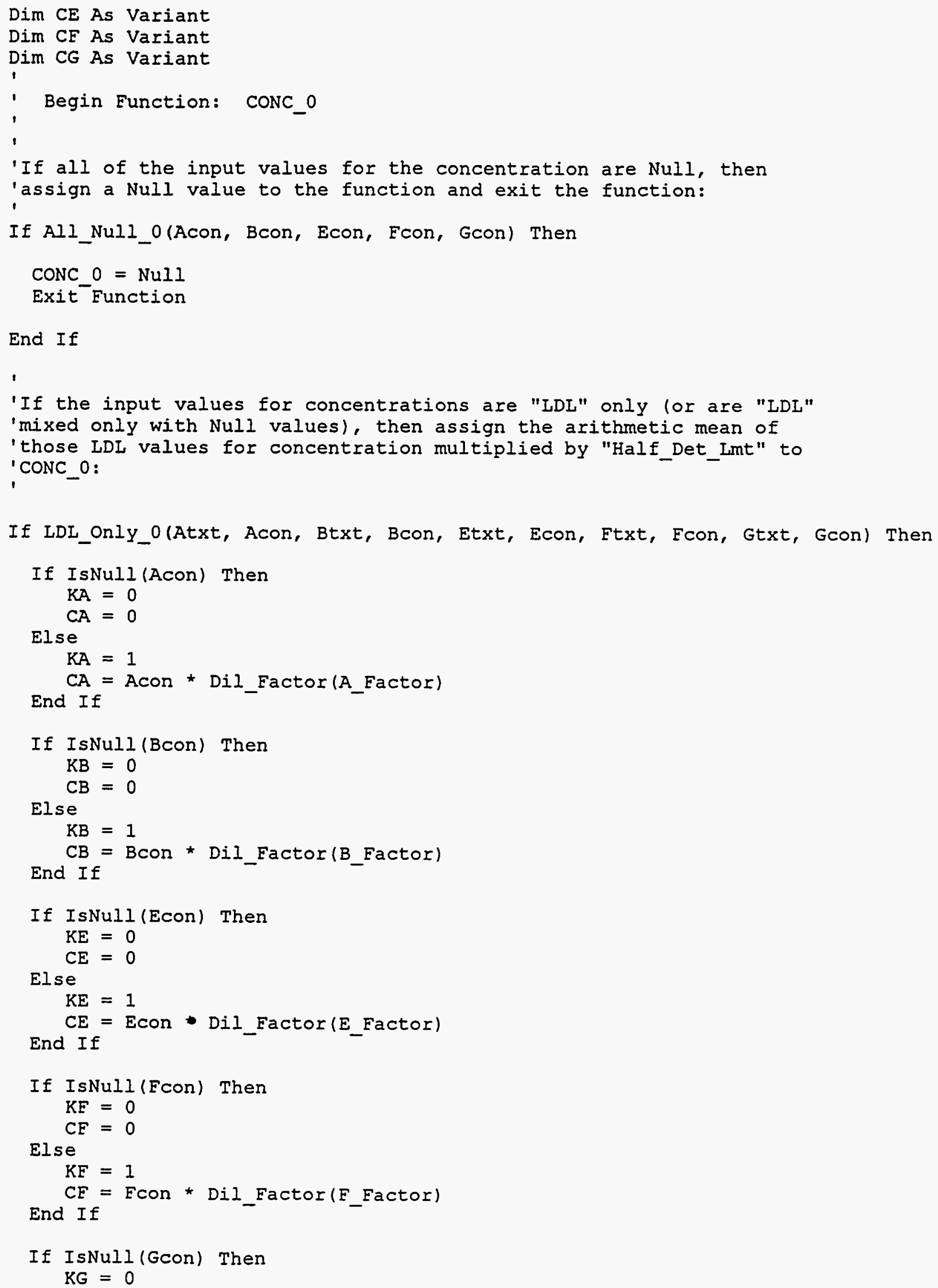




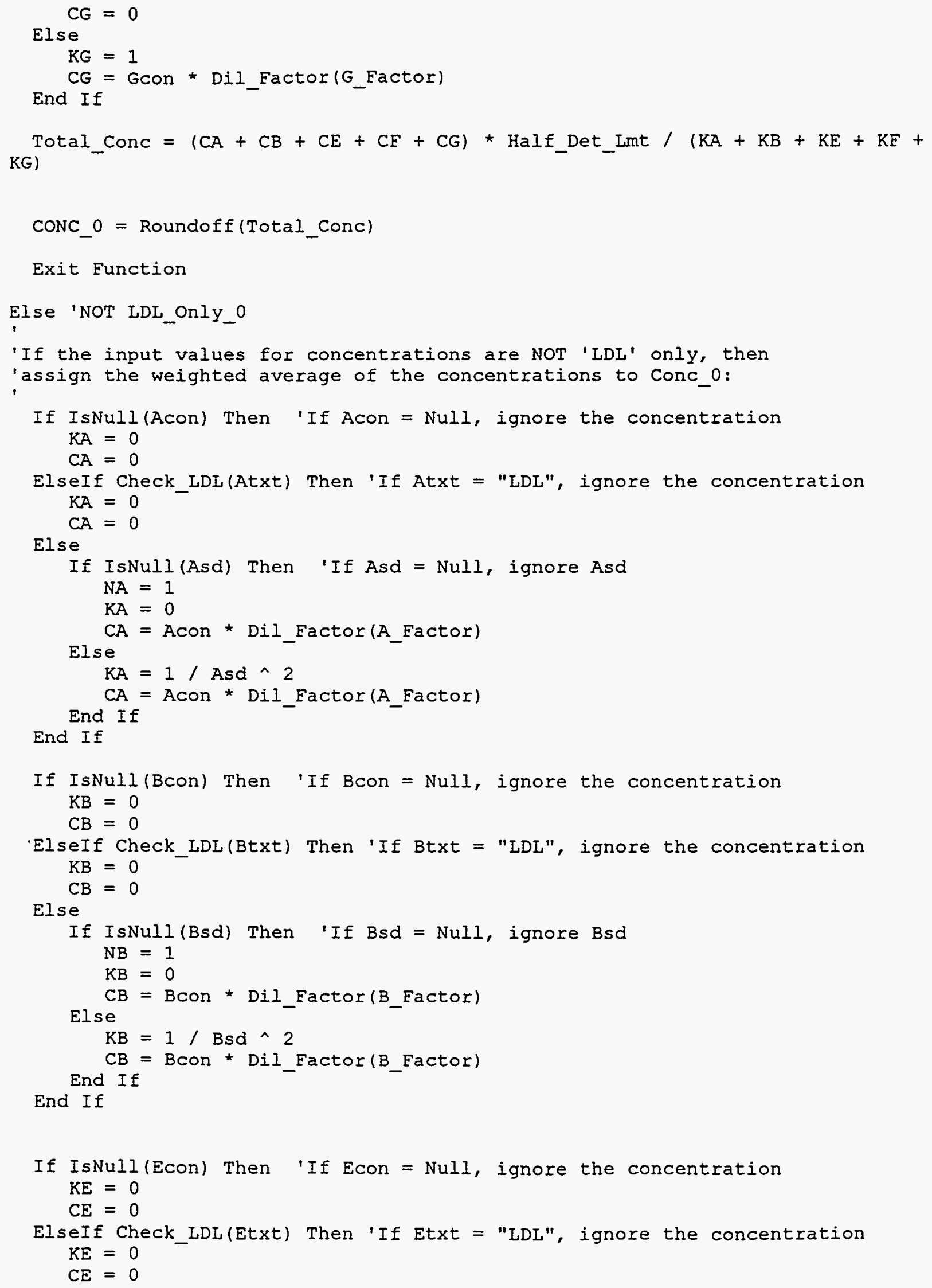


Else

If IsNull(Esd) Then 'If Esd = Null, ignore Esd

$\mathrm{NE}=1$

$\mathrm{KE}=0$

Else

$\mathrm{CE}=$ Econ * DiI_Factor (E_Factor $)$

$\mathrm{KE}=1 / \mathrm{Esd} \wedge 2$

End If

$\mathrm{CE}=\mathrm{Econ} \cdot$ Dil_Factor(E_Factor)

End If

If IsNull(Fcon) Then 'If Fcon = Null, ignore the concentration $\mathrm{KF}=0$

$\mathrm{CE}=0$

ElseIf Check_LDL(Ftxt) Then 'If Ftxt = "LDL", ignore the concentration $\mathrm{KE}=0$

Else $C F=0$

If IsNull(Fsd) Then 'If Fsd = Null, ignore Fsd $\mathrm{NF}=1$

$\mathrm{KE}=0$

Else

$C F=F C o n *$ Dil_Factor $\left(F_{\text {_Eactor }}\right)$

$\mathrm{KF}=1 /$ Fsd $\wedge 2$

End If

$C F=$ FCon *Dil_Factor $\left(F_{-}\right.$Factor $)$

End If

If IsNull(Gcon) Then 'If Gcon = Null, ignore the concentration $\mathrm{KG}=0$

$C G=0$

ElseIf Check_LDL(Gtxt) Then 'If Gtxt = "LDL", ignore the concentration $K G=0$ $C G=0$

Else

If IsNull(Gsd) Then 'If Gsd = Null, ignore Gsd

$$
\begin{aligned}
& \text { NG }=1 \\
& K G=0
\end{aligned}
$$

End If

Ktotal $=K A+K B+K E+K F+K G$

If (Ktotal $=0$ ) Then 'There were no values for std. dev, so

'find the arithmetic mean of the concentration

Total_ConC $=(\mathrm{CA}+\mathrm{CB}+\mathrm{CE}+\mathrm{CF}+\mathrm{CG}) /(\mathrm{NA}+\mathrm{NB}+\mathrm{NE}+\mathrm{NF}+\mathrm{NG})$

Else 'Find the weighted concentration Ktotal

Total_Conc $=((C A * K A)+(C B * K B)+(C E * K E)+(C F * K F)+(C G * K G))$

End If

CONC_0 $=$ Roundoff (Total_Conc) 
End If 'NOT LDL_Only_O

End Function 'CONC 0

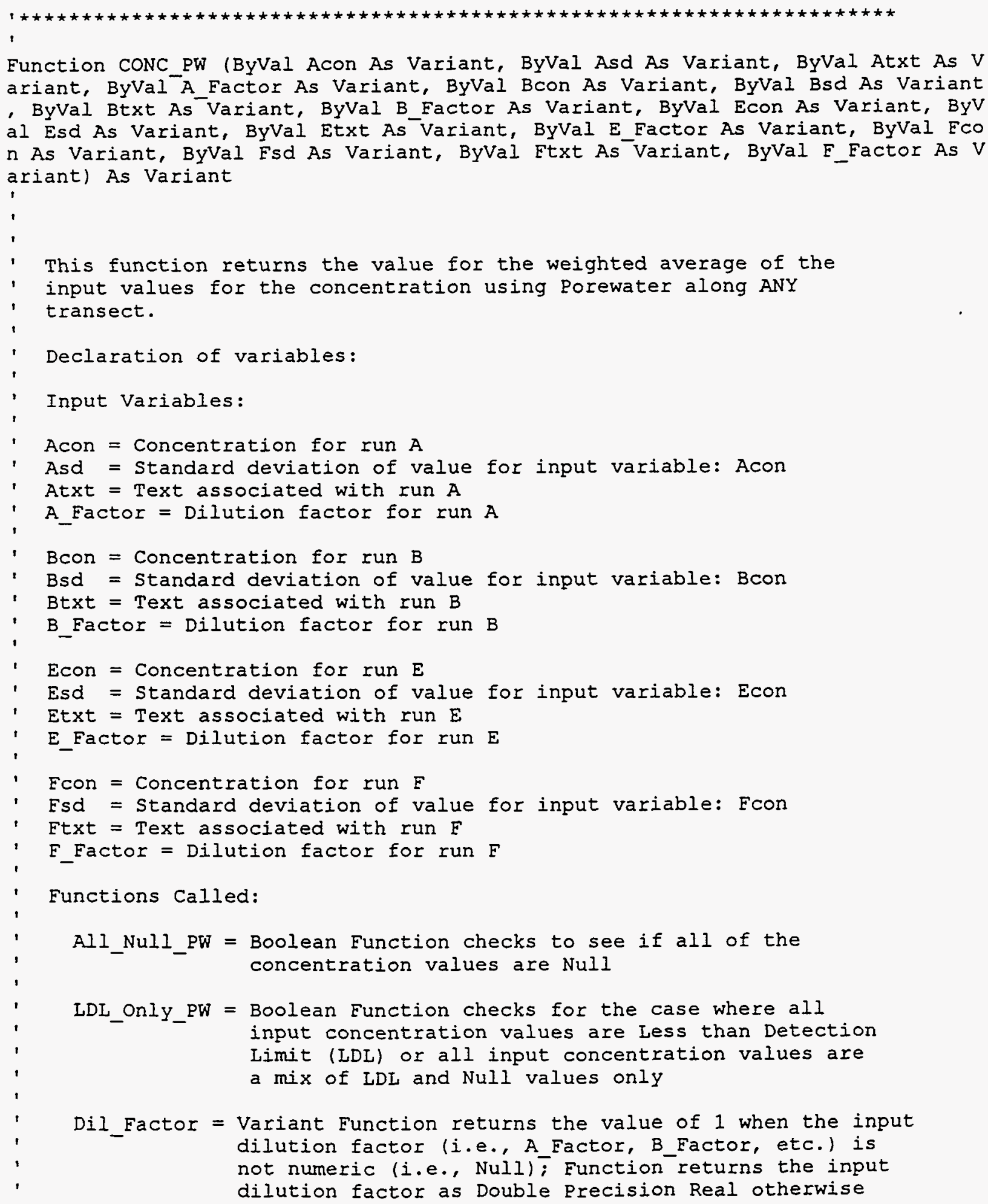

Function CONC PW (ByVal Acon As Variant, ByVal Asd As Variant, ByVal Atxt As V ariant, ByVal A Factor As Variant, ByVal Bcon As Variant, ByVal Bsd As Variant - ByVal Btxt As Variant, ByVal B Factor As Variant, ByVal Econ As Variant, ByV al Esd As Variant, ByVal Etxt As Variant, ByVal E_Factor As Variant, ByVal Eco $n$ As Variant, ByVal Fsd As Variant, ByVal Etxt As Variant, ByVal E_Factor As V ariant) As Variant 


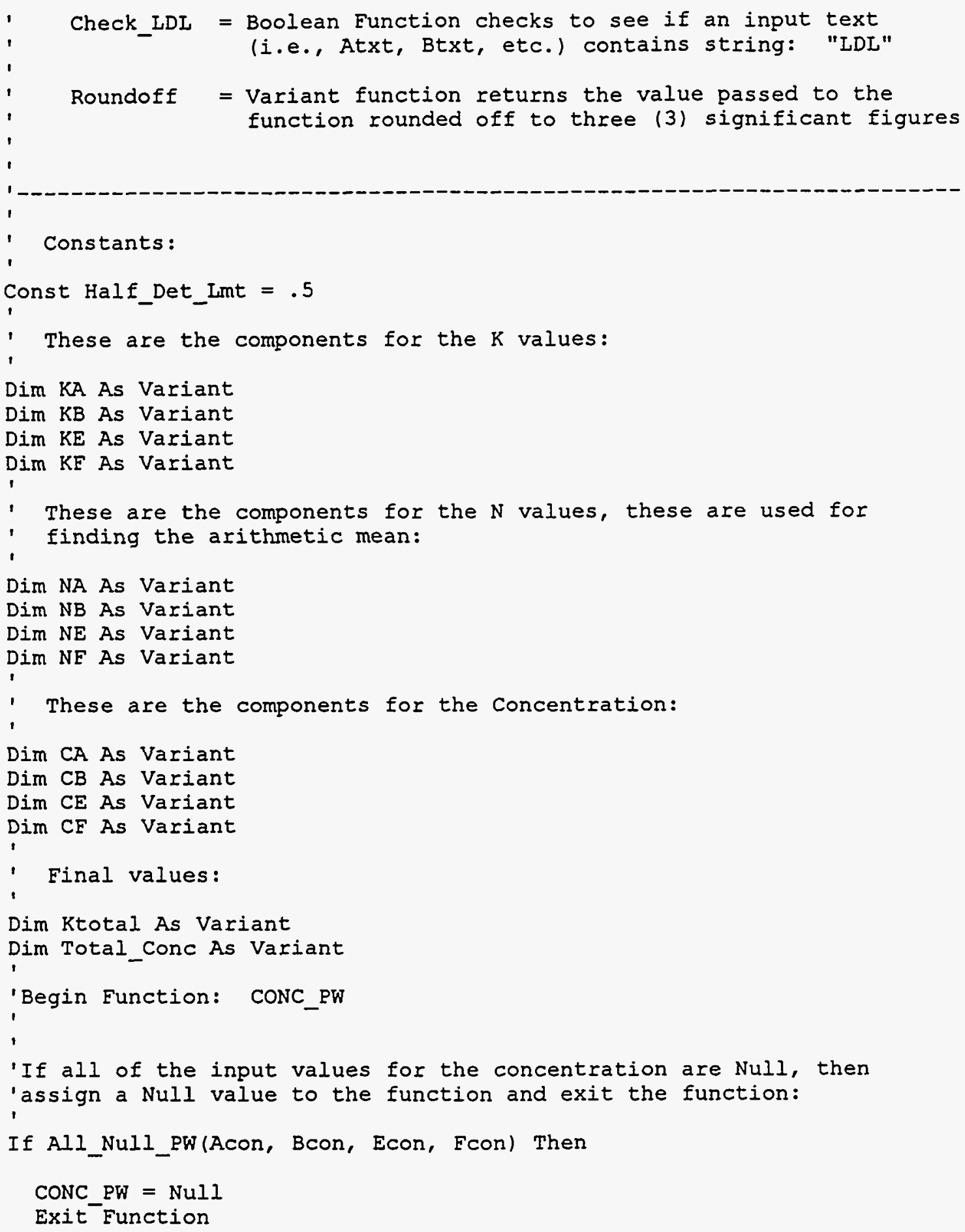




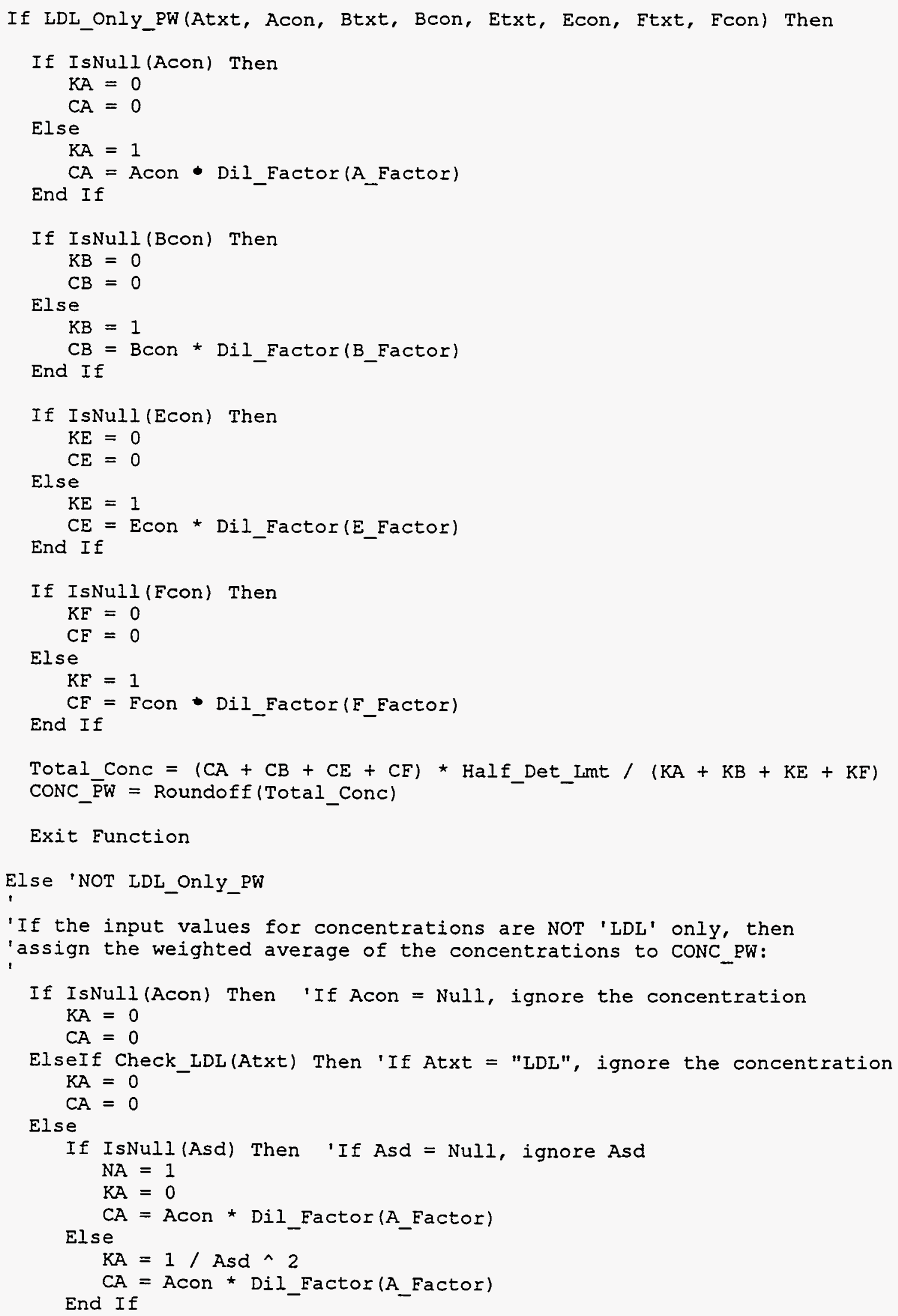


End If

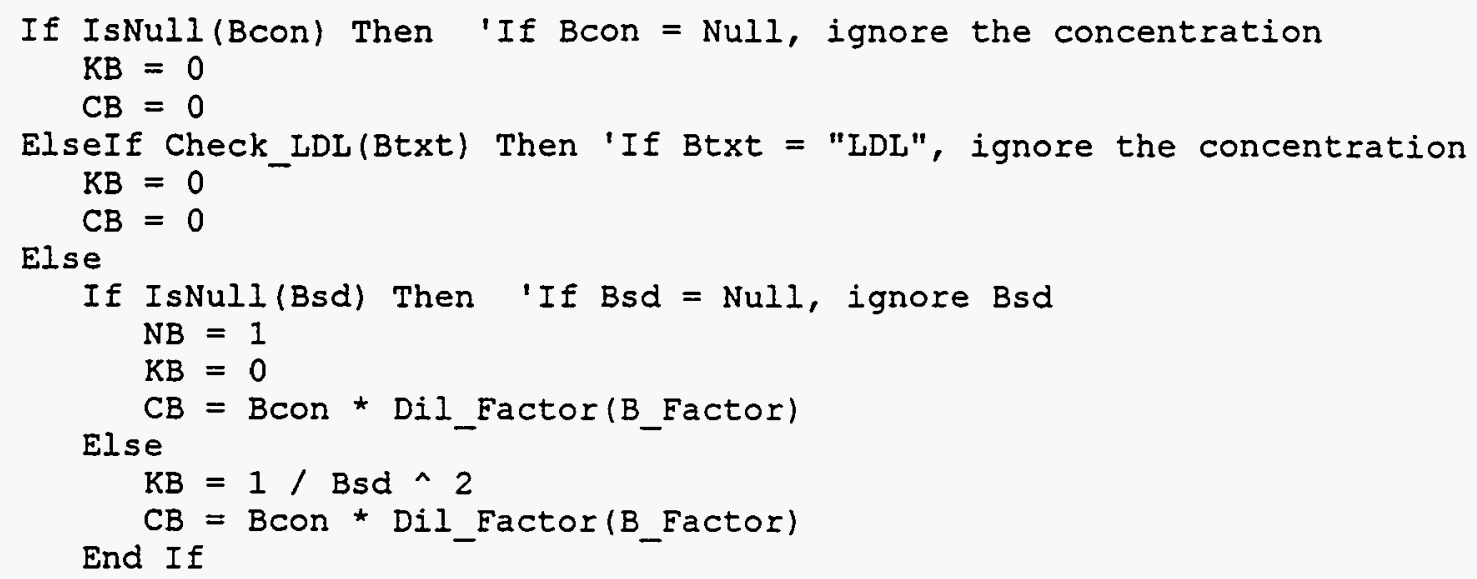




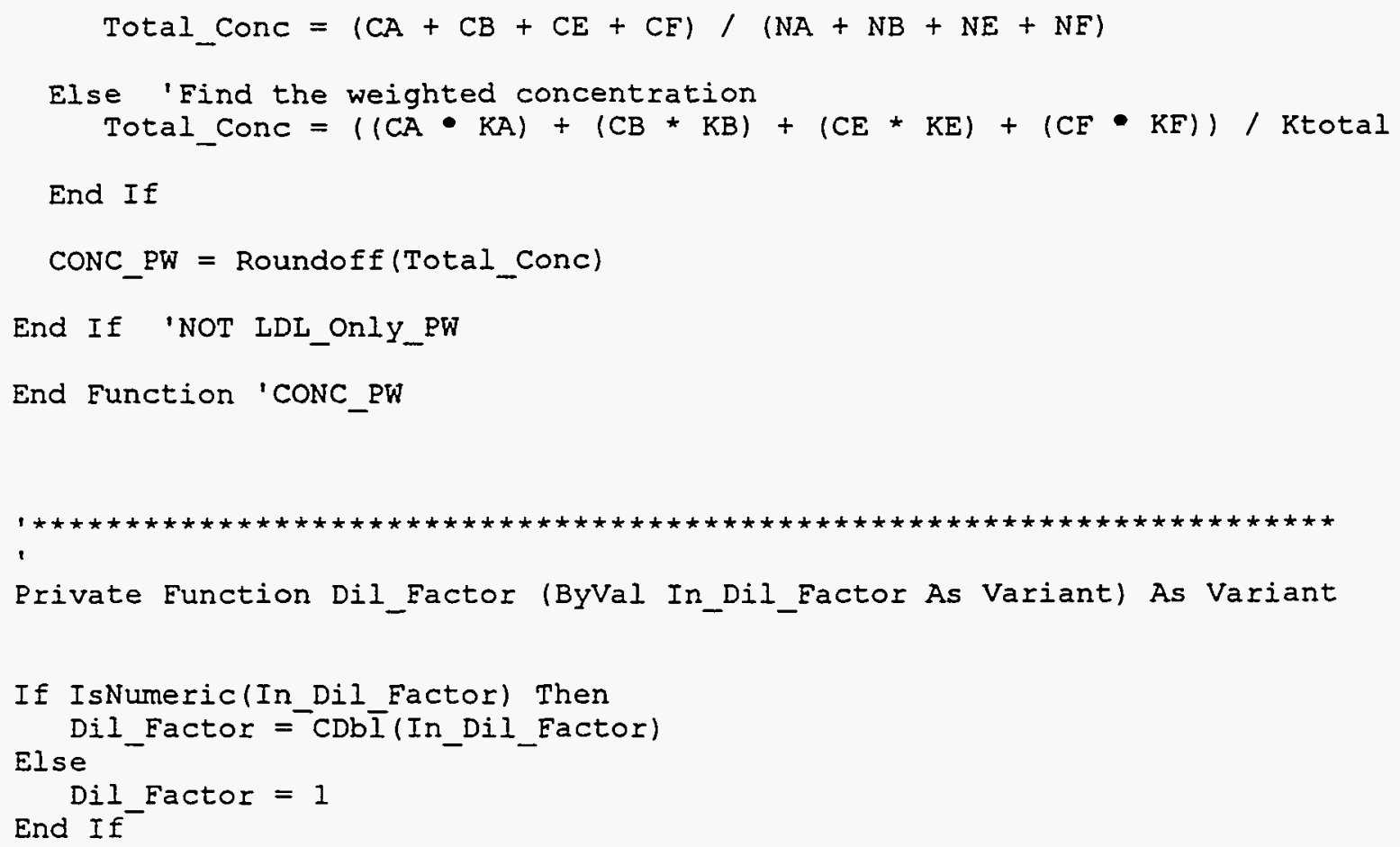


ElseIf In isotope $=$ "Ca46" Then Elmt Name = "Calcium"

ElseIf In isotope = "Ca48" Then Elmt Nāme = "Calcium"

ElseIf In_isotope = "Cd111" Then Elmt Name = "Cadmium"

ElseIf $\overline{I n}$ isotope = "Cd114" Then Elmt Name = "Cadmium"

ElseIf $\bar{I}$ _isotope $=$ "Cl" Then Elmt Name = "Chlorine"

ElseIf $\overline{I n}$ isotope $=$ "Cm243" Then Elmt Nāme = "Curium"

ElseIf In isotope $=$ "Cm245" Then Elmt Name = "Curium"

ElseIf $\overline{I n}$ isotope = "Co" Then Elmt Name = "Cobalt"

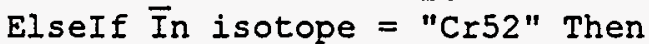
Elmt Name = "Chromium"

ElseIf In isotope = "Cr53" Then Elmt Name = "Chromium"

ElseIf $\overline{I n}$ isotope = "Cs" Then Elmt Nāme = "Cesium"

ElseIf In_isotope = "Cs137" Then Elmt Name = "Cesium"

ElseIf In isotope = "Cu63" Then Elmt Näme = "Copper"

ElseIf $\overline{I n}_{n}$ isotope $=$ "Cu65" Then Elmt Name = "Copper"

ElseIf $\bar{I}$ isotope = "F" Then Elmt Name = "Eluorine"

ElseIf $\overline{I n}$ isotope $=$ "Fe" Then Elmt Name = "Iron"

ElseIf $\overline{I n}$ isotope = "gross alpha" Then Elmt Name = "Gross Alpha"

ElseIf In isotope = "gross beta" Then Elmt Näme = "Gross Beta"

ElseIf In isotope $=$ "H3" Then Elmt Name = "Tritium"

ElseIf $\bar{I}_{n}$ isotope = "Hg199" Then " Elmt Name = "Mercury"

ElseIf In_isotope $=$ "Hg200" Then Elmt Name = "Mercury"

ElseIf $\overline{I n}$ isotope = "Hg201" Then Elmt Name = "Mercury"

ElseIf In_isotope = "Hg202" Then Elmt Nāme = "Mercury"

ElseIf $\overline{I n}$ isotope = " $\mathrm{K}$ " Then Elmt Name = "Potasium"

ElseIf In_isotope = "Mg24" Then Elmt Name = "Magnesium"

ElseIf $\overline{I n}$ isotope $=$ "Mg25" Then Elmt Name = "Magnesium"

ElseIf $\bar{I}_{\text {n }}$ isotope $=$ "Mg26" Then Elmt Näme = "Magnesium"

ElseIf $\overline{I n}$ isotope $=$ "Mn" Then Elmt Näme = "Manganese"

ElseIf $\overline{I n}$ isotope = "Na" Then Elmt Name = "Sodium"

ElseIf $\overline{\text { In isotope }=~ " N i 60 " ~ T h e n ~}$ Elmt_Näme = "Nickel" 
ElseIf In isotope $=$ "Ni61" Then Elmt Näme = "Nickel"

ElseIf In isotope = "Ni62" Then Elmt Näme = "Nickel"

ElseIf $\overline{I n}$ isotope $=$ "NO2" Then Elmt Name = "Nitrogen Dioxide"

ElseIf In_isotope = "NO3" Then Elmt Name = "Nitrate"

ElseIf In isotope = "Np237" Then Elmt Name = "Neptunium"

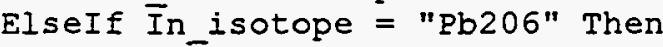
Elmt Name = "Lead"

ElseIf In isotope $=$ "Pb207" Then Elmt Name = "Lead"

Elself $\bar{I}_{n}$ isotope $=$ "Pb208" Then Elmt Name = "Lead"

ElseIf In_isotope = "PO4" Then Elmt Name = "Phosphate"

ElseIf $\overline{\mathrm{I}}$ isotope = "Pu239" Then Elmt Name = "Plutomium"

ElseIf In isotope = "Pu240" Then Elmt Näme = "Plutonium"

ElseIf In isotope = "Pu242" Then Elmt Name = "Plutonium"

ElseIf $\overline{\text { In }}$ isotope = "Ra226" Then Elmt Name = "Radium"

ElseIf $\bar{I}_{n}$ isotope $=$ "Se77" Then Elmt Name = "Selenium"

ElseIf $\bar{I}_{n \text { isotope }}=$ "Se82" Then Elmt Name = "Selenium"

ElseIf $\overline{I n}$ isotope $=$ "Sn118" Then Elmt Näme = "Tin"

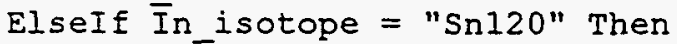
Elmt Name = "Tin"

ElseIf $\bar{I}_{n}$ isotope = "SO4" Then Elmt Name = "Sulfate"

ElseIf In isotope = "Sr" Then Elmt Nāme = "Strontium"

ElseIf In isotope = "Sr90" Then Elmt Näme = "Strontium"

ElseIf $\overline{I n}$ isotope $=$ "Th232" Then Elmt Name = "Thorium"

ElseIf $\bar{I}$ isotope = "Ti" Then Elmt Name = "Tianium"

ElseIf $\bar{I}$ isotope $=$ "U234" Then Elmt Näme = "Uranium"

ElseIf $\bar{I}$ isotope = "U235" Then Elmt Name = "Uranium-235"

ElseIf $\overline{I n}$ isotope $=$ "U238" Then

Elmt Name = "Uranium-238"

ElseIf $\bar{I}$ isotope = "V" Then Elmt Name = "Vanadium"

ElseIf $\bar{I}$ isotope = "Y" Then Elmt Näme = "Yttrium"

ElseIf $\overline{I n}$ isotope $=$ "Zn64" Then Elmt Name = "Zinc"

ElseIf In isotope $=$ "Zn66" Then Elmt Name = "Zinc"

ElseIf $\overline{I n}$ isotope $=$ "Zn67" Then Elmt_Näme = "Zinc" 
ElseIf In isotope $=$ "Zr90" Then

Elmt Name = "Zirconium"

ElseIf $\overline{\text { In }}$ isotope $=$ "Zr9l" Then

Elmt_Name $=$ "zirconium"

End If

End Function

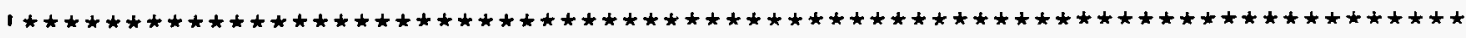

Private Function LDL Only_o (ByVal Atxt As Variant, ByVal Acon As Variant, ByV al Btxt As Variant, $\bar{B} y$ Val Bcon As Variant, ByVal Etxt As Variant, ByVal Econ A s Variant, ByVal Ftxt As Variant, ByVal Econ As Variant, ByVal Gtxt As Variant : ByVal Gcon As Variant) As Variant

'This function returns a True value when either both the input values

' for concentration and the text are equal to Null, or when only the

'input text is equal to "LDL"

'

1

'Flags are True when either both Acon and Atxt are NuIl, or when

'Atxt is "LDL" .....(this is true for all of the variables B, E, etc.)

Dim $A$ is OK As Variant

Dim $B$ is OK As Variant

Dim $\mathrm{E}^{-}$is $\mathrm{OK}$ As Variant

Dim $\mathrm{F}^{-}$is $\mathrm{OK}$ As Variant

Dim G_is_oK As Variant

$$
\begin{aligned}
& \text { A_is_OK }=\text { False } \\
& \text { B-is_OK }=\text { False } \\
& \text { E-is_OK }=\text { False } \\
& \text { F_is_OK }=\text { False } \\
& \text { G_is_OK }=\text { False } \\
& \text { LDI_Only_O = False 'The function is False until all five }
\end{aligned}
$$$$
\text { 'variables, } A, B, E, F \text {, and } G \text { are determined }
$$$$
\text { to be "OK" }
$$

If IsNull (Acon) And IsNull (Atxt) Then

$$
A \text { is } O K=\text { True }
$$

Else If Ātxt $=$ "LDL" Then

End $\bar{I} f$

A_is_OK = True

If IsNull(Bcon) And IsNull(Btxt) Then $B$ is $O K=$ True

Else If $\bar{B}$ txt $=$ "LDL" Then $B$ is $O K=$ True

End $\overline{\mathrm{I}} \mathrm{f}$

If IsNull(Econ) And IsNull (Etxt) Then $E$ is $O K=$ True

Else $\bar{I} f \bar{E} t x t=$ "LDL" Then

End $\overline{I f}$

$E$ is_OK $=$ True

If IsNull(Fcon) And IsNuIl(Ftxt) Then 


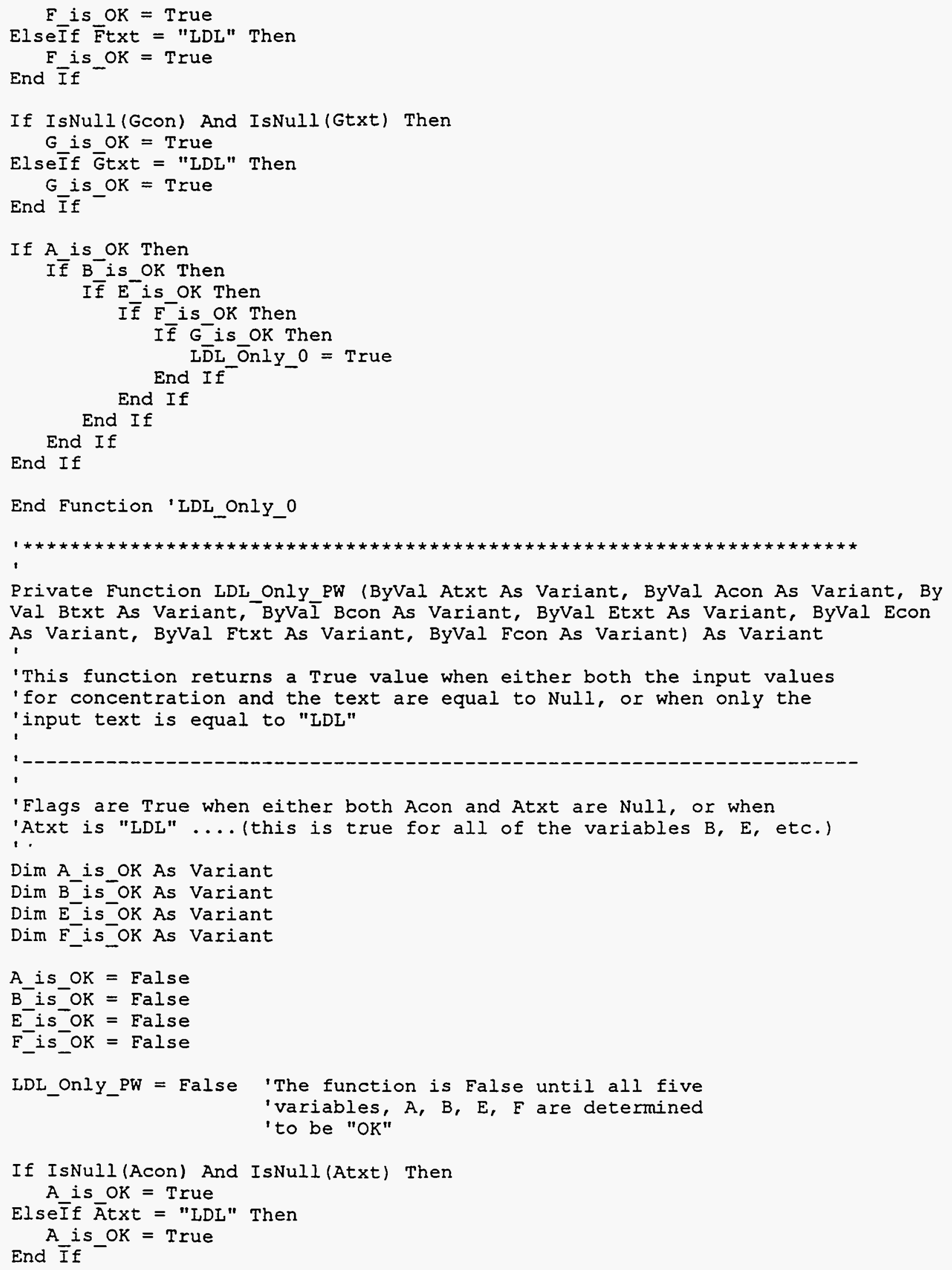


If IsNull(Bcon) And IsNull (Btxt) Then $B$ is $O K=$ True

Else $\bar{I} f$ Btxt $=$ "LDL" Then

End $\bar{I} f$

B_is_OK = True

If IsNull(Econ) And IsNull(Etxt) Then

$E$ is $O K=T r u e$

Else If Etxt $=$ "LDL" Then

End $\bar{I} f$

E_is_OK $=$ True

If IsNull(Fcon) And IsNuII (Ftxt) Then

$\mathrm{F}$ is_OK = True

Else $\bar{I} \bar{F}$ txt $=$ "LDL" Then

$E$ is $\mathrm{OK}=$ True

End $\overline{\mathrm{I}} \mathrm{f}$

If $A$ is $O K$ Then

I $\bar{f}$ B is OK Then

I $\bar{f} E^{-}$is OK Then

$I \bar{f} F^{-}$is OK Then IDI ONIY $P W=$ True

End If

End If

End If

End Function 'IDL_Only_PW

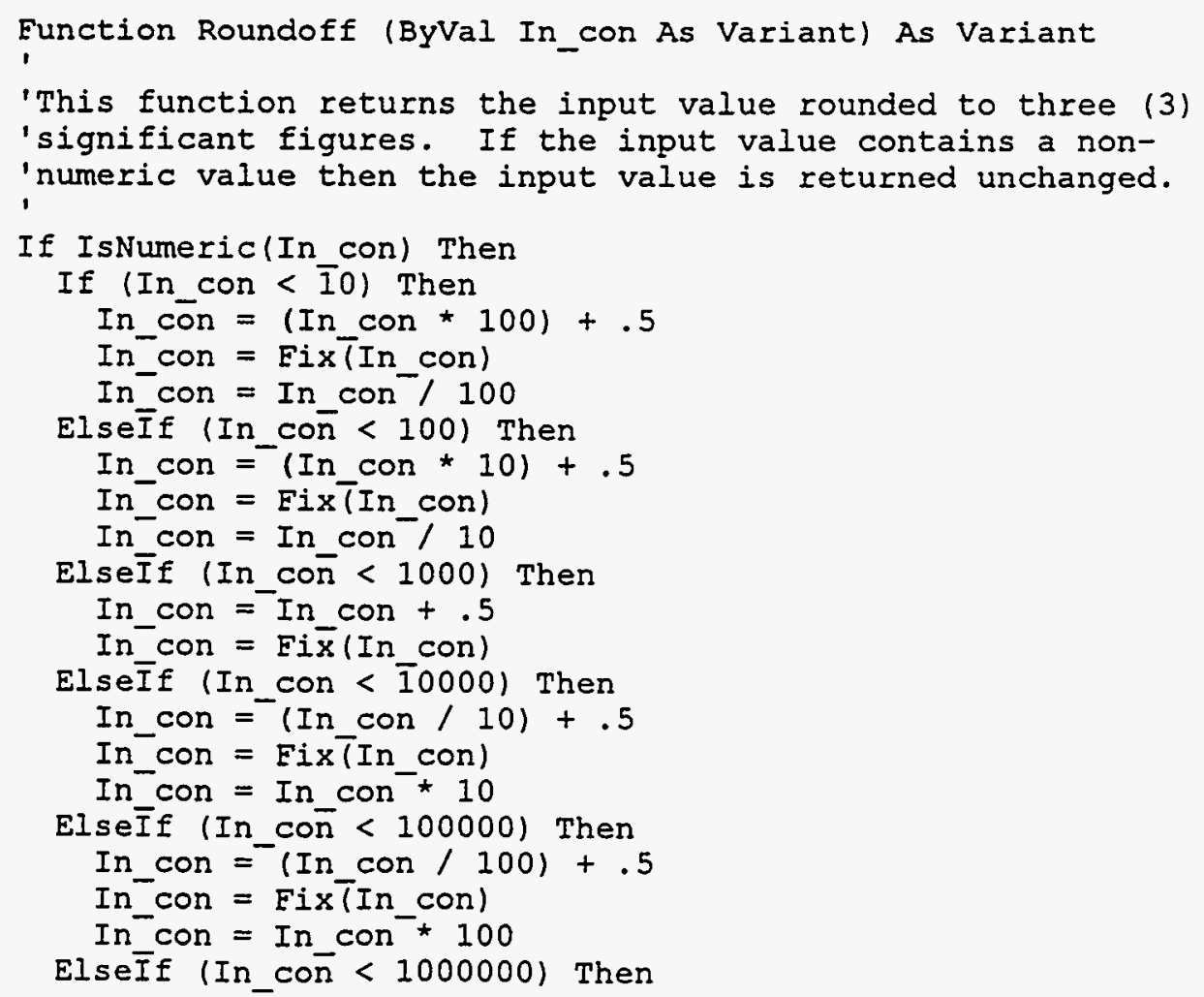




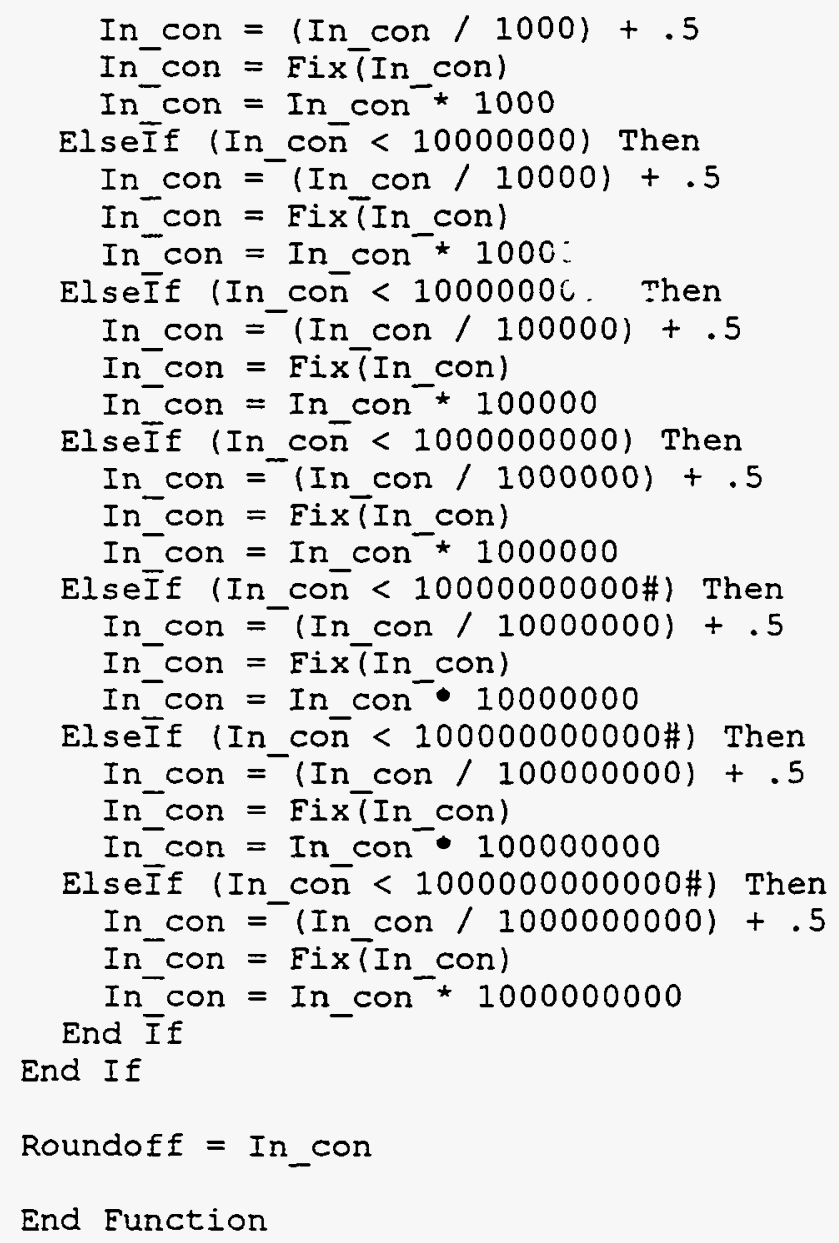




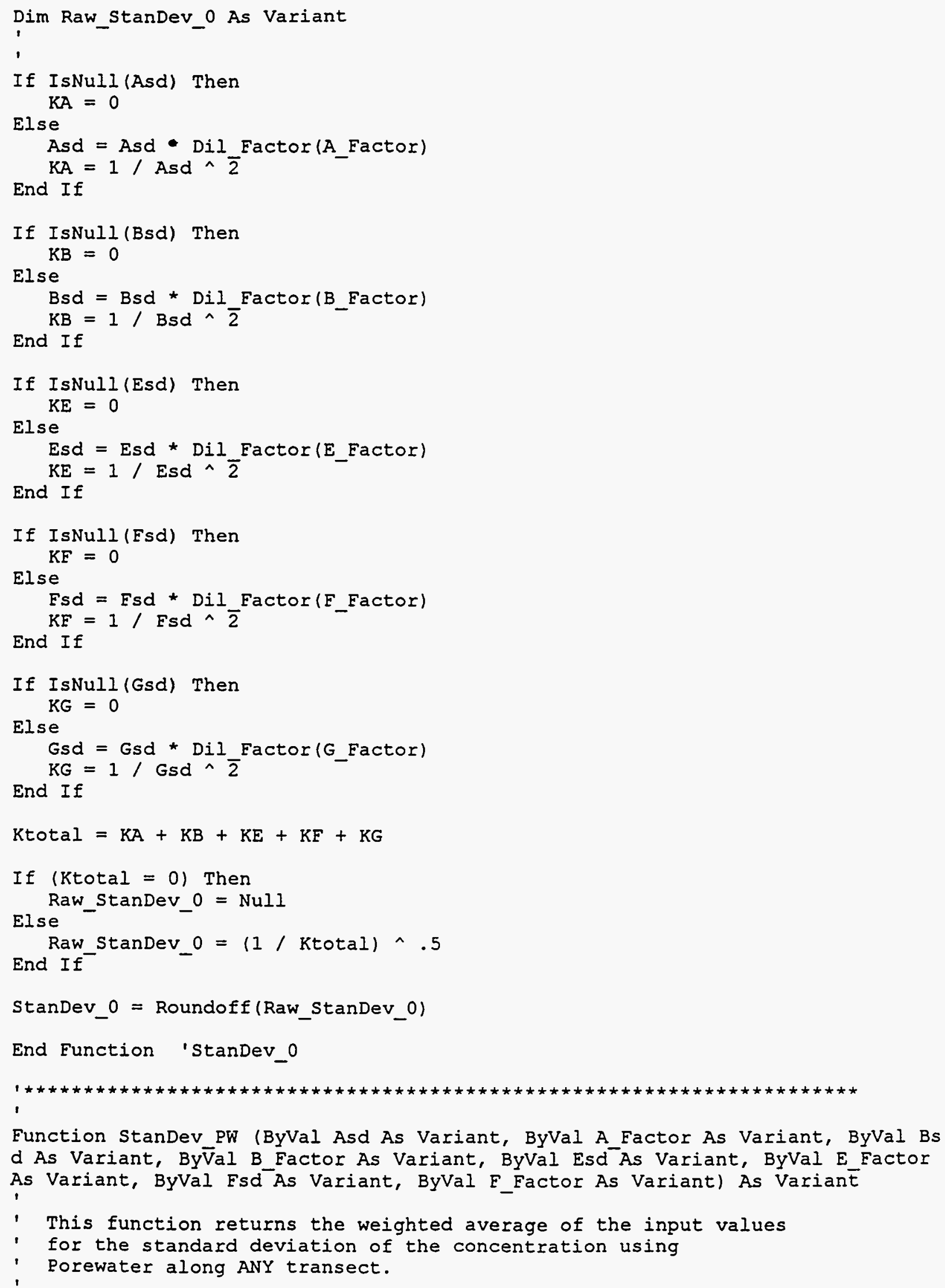




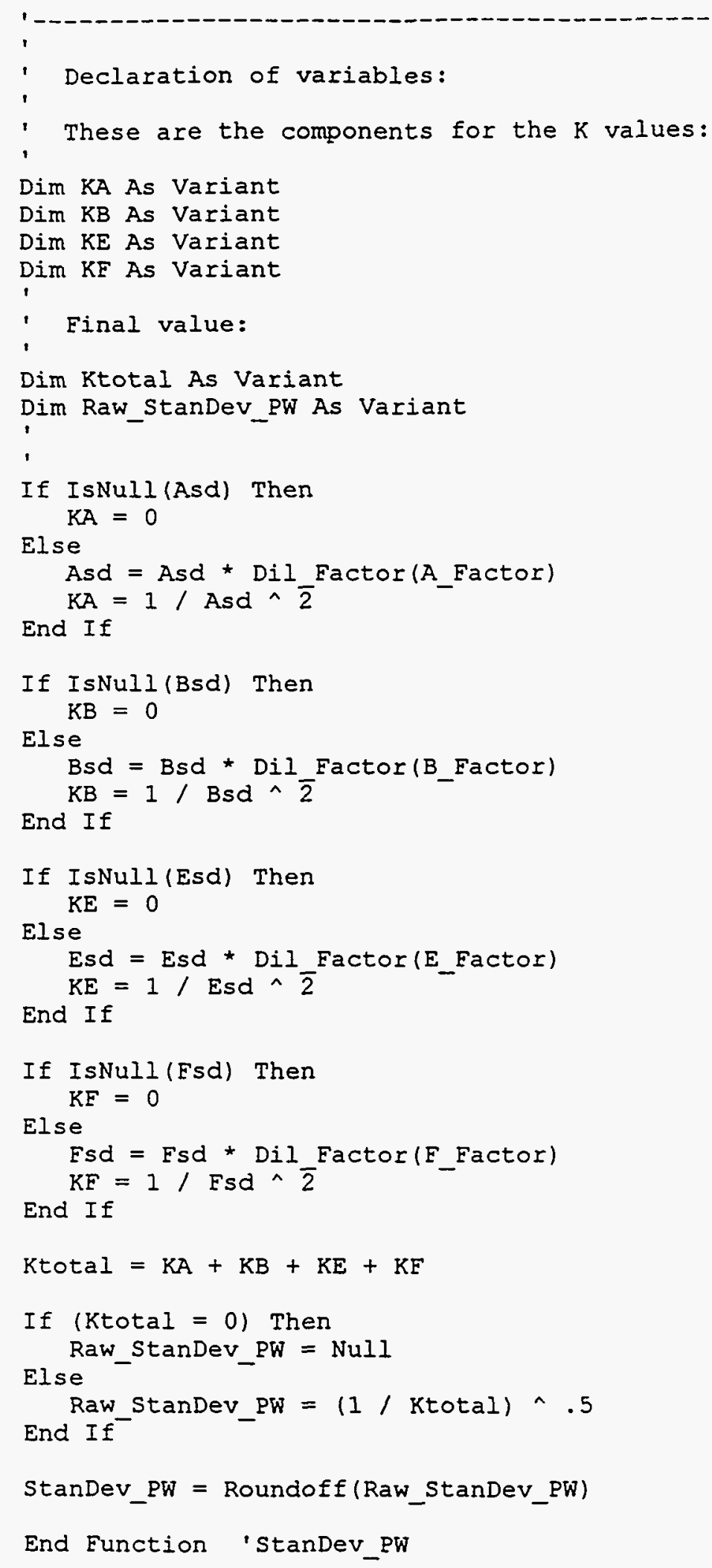




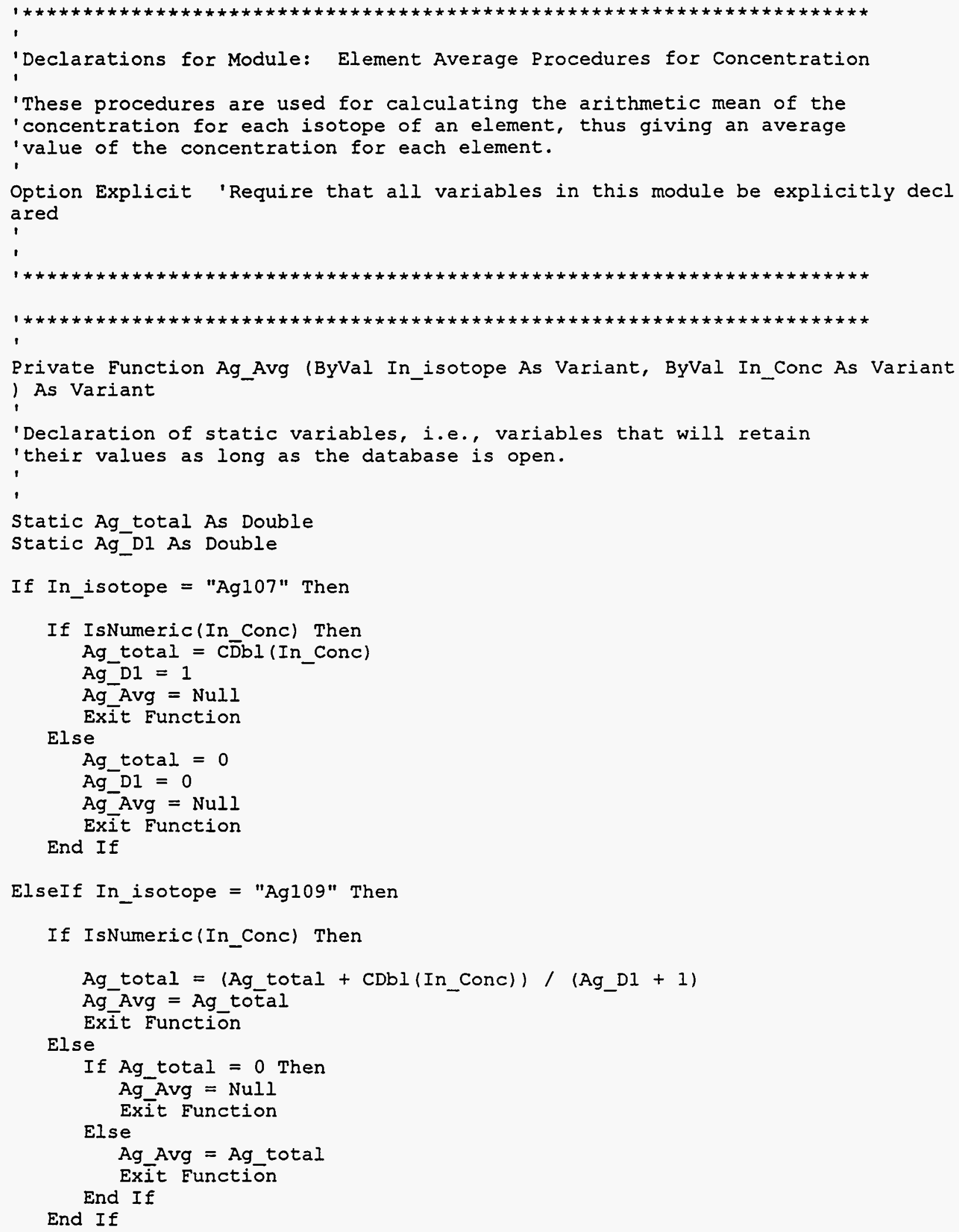

End If

End Function 


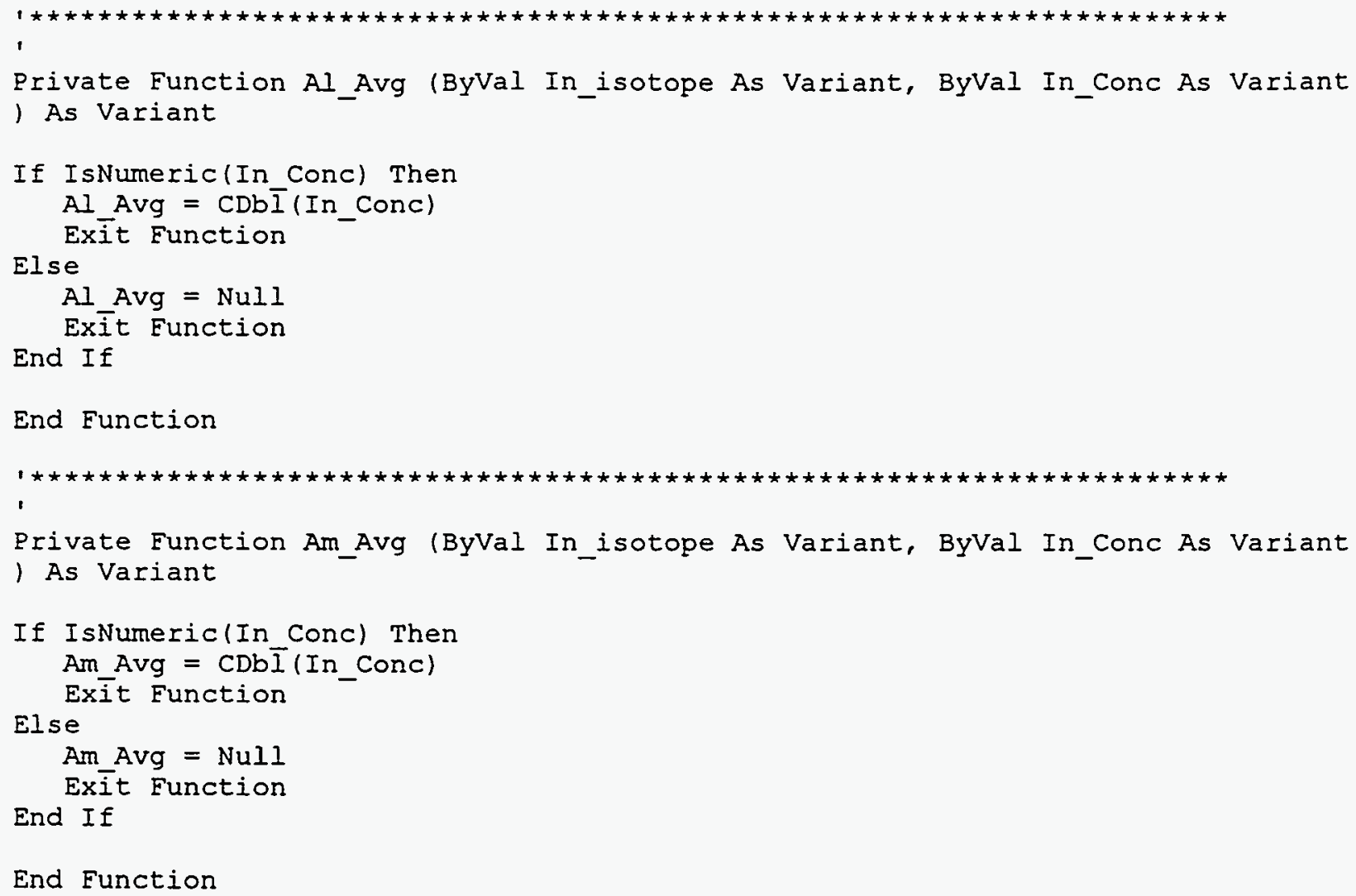

End Function

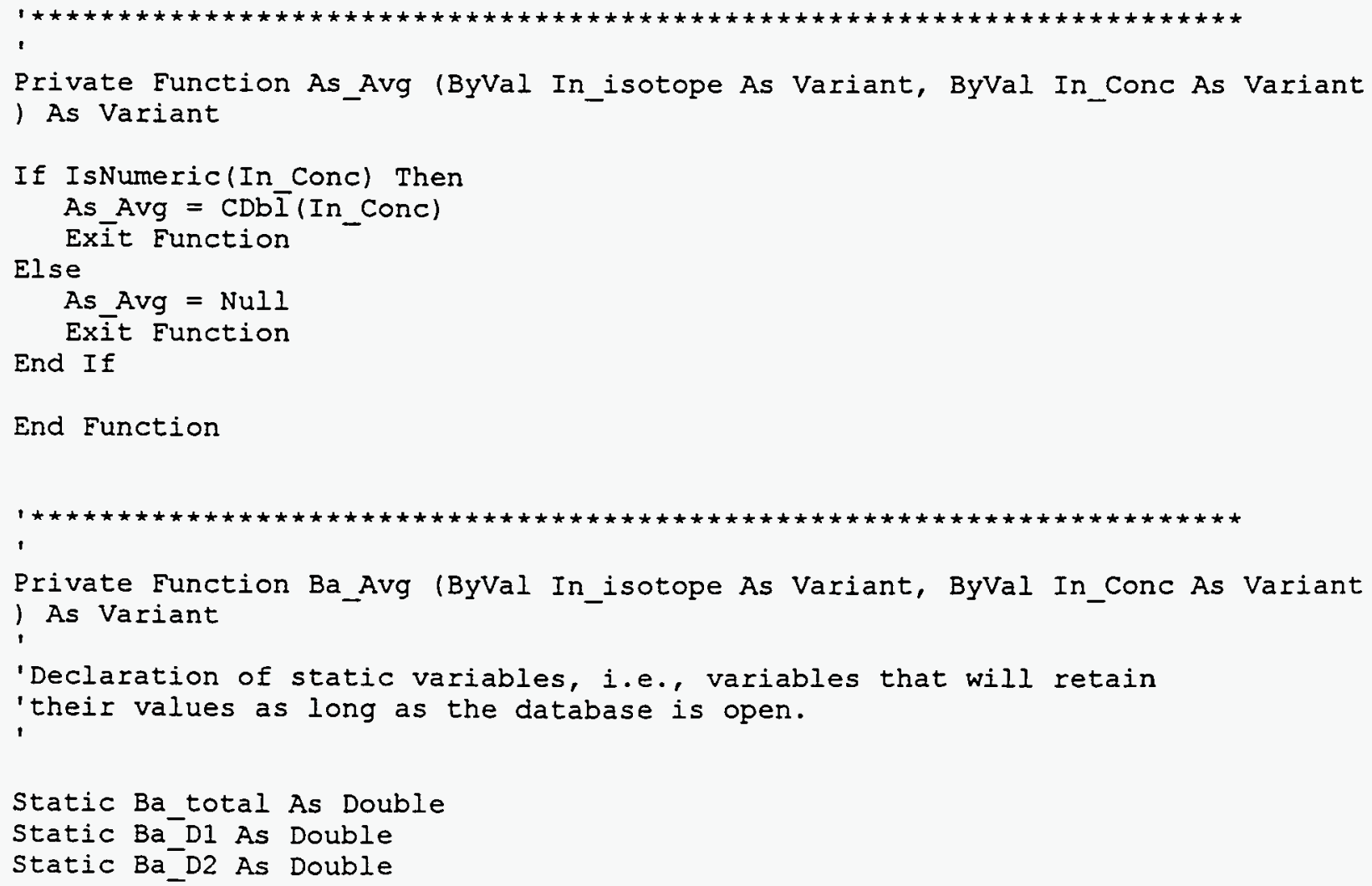




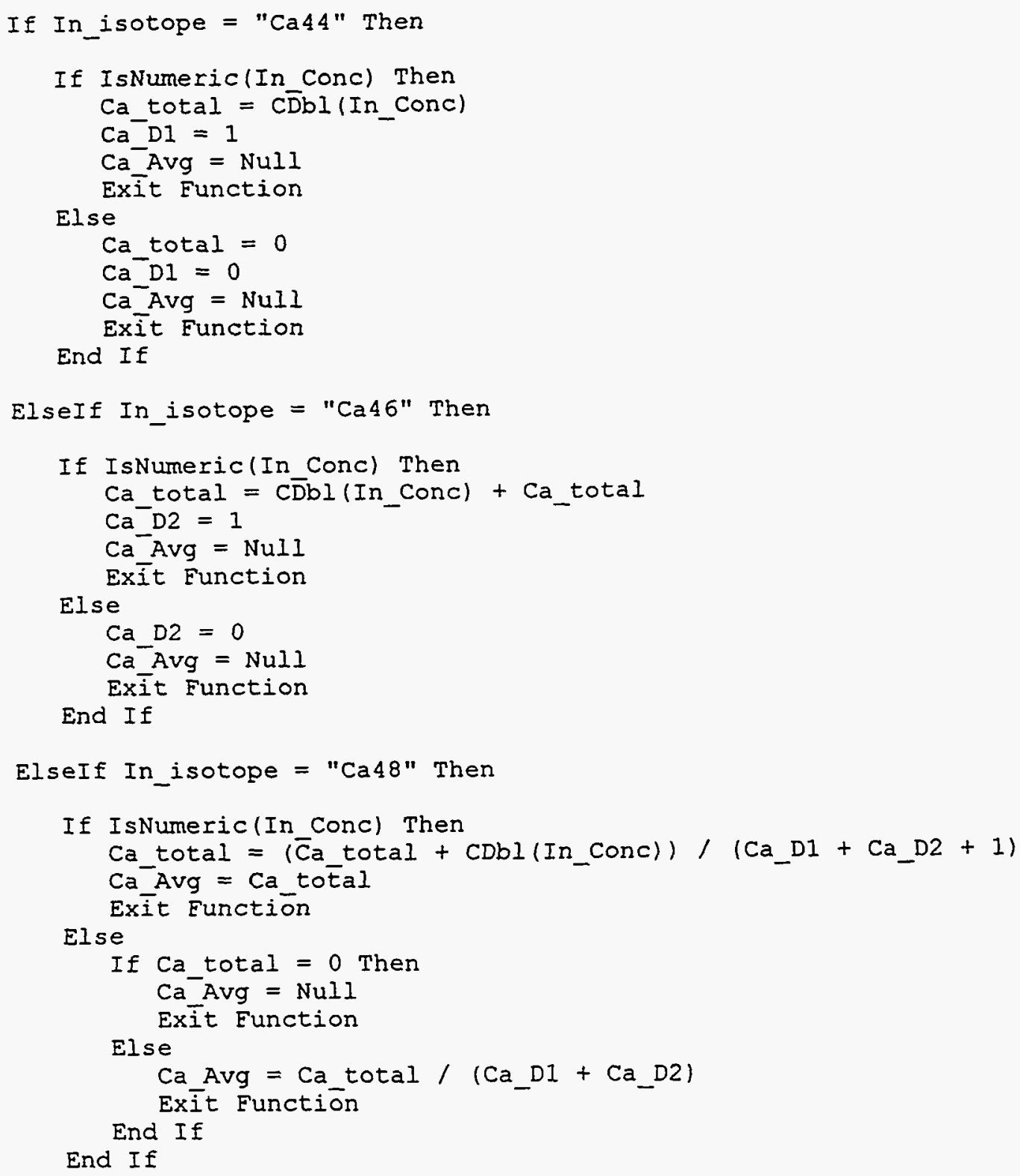

End If

End Function

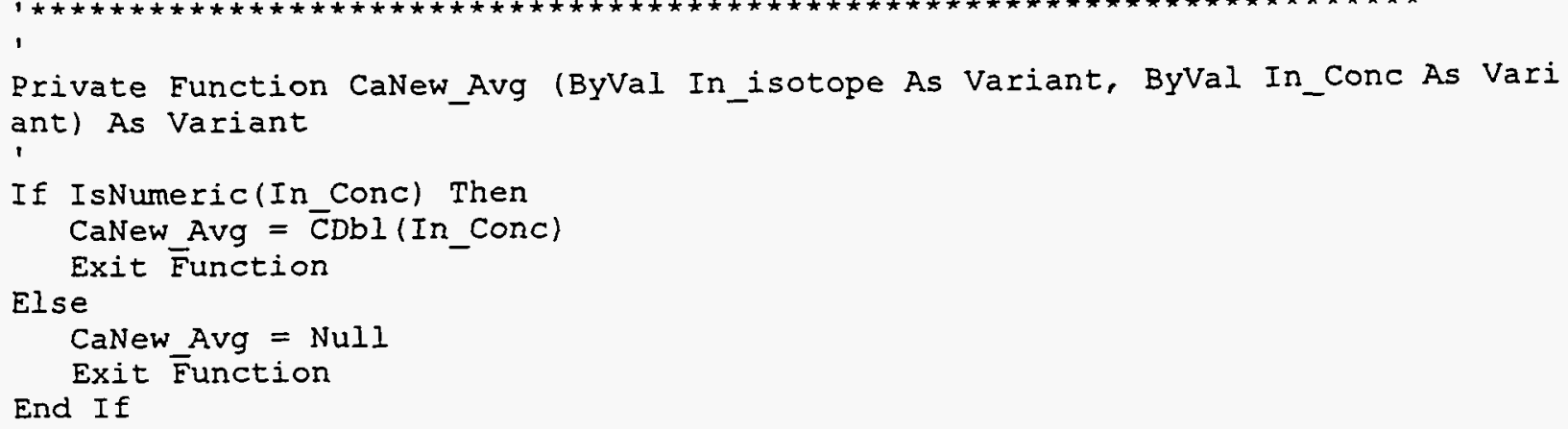


End Function

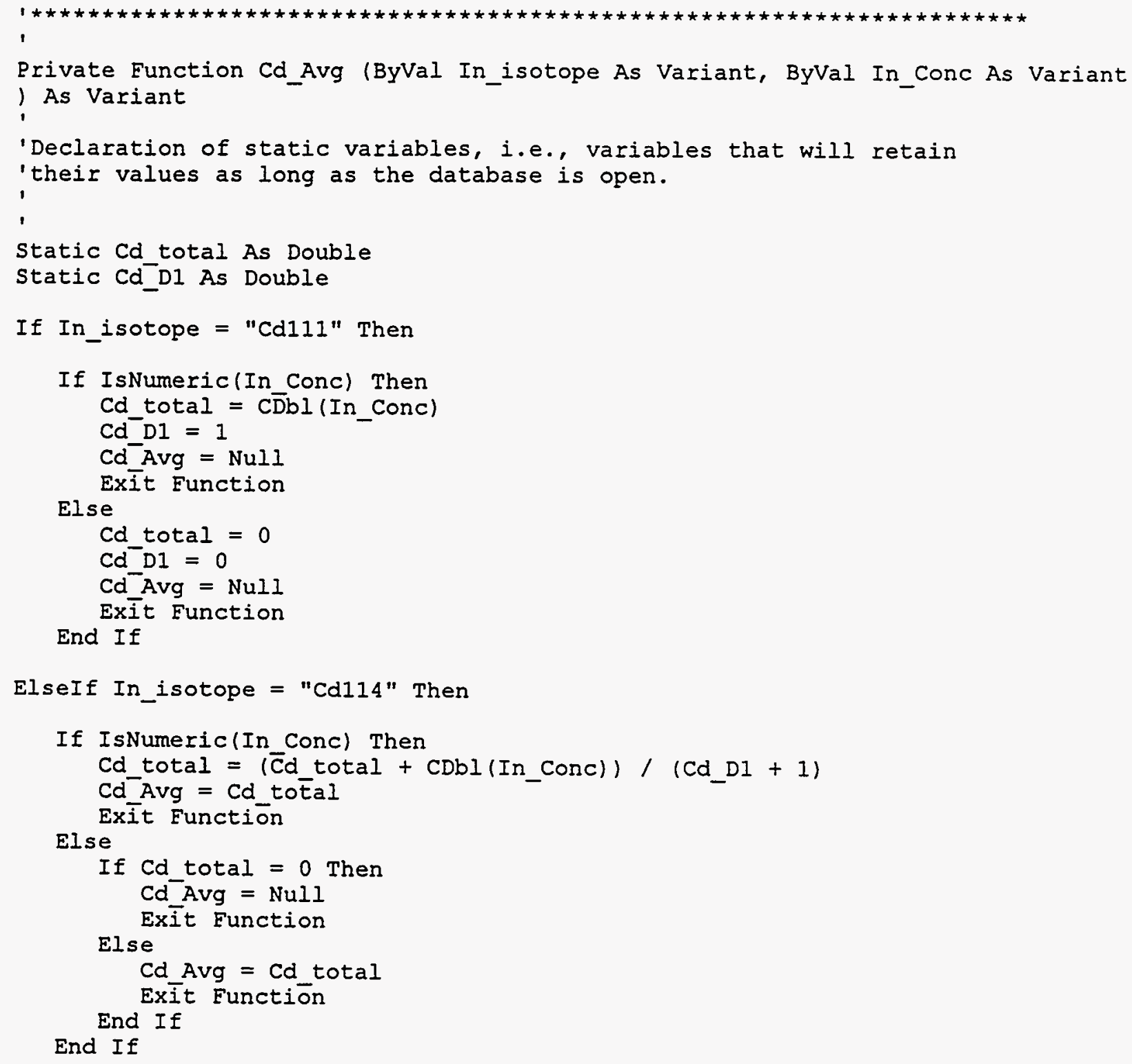

End If

End Function

Private Function Cl_Avg (ByVal In_isotope As Variant, ByVal In_Conc As Variant ) As Variant

If IsNumeric(In Conc) Then $\mathrm{Cl}$ Avg $=\mathrm{CDbI}$ (In_Conc)

Else Exit Function

Cl_Avg $=$ Null 


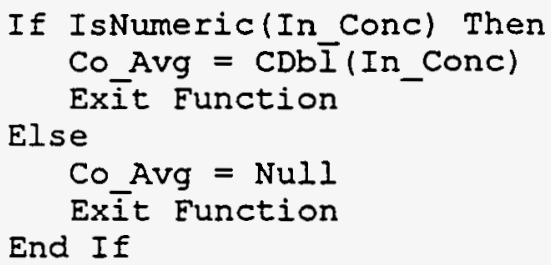

End Function

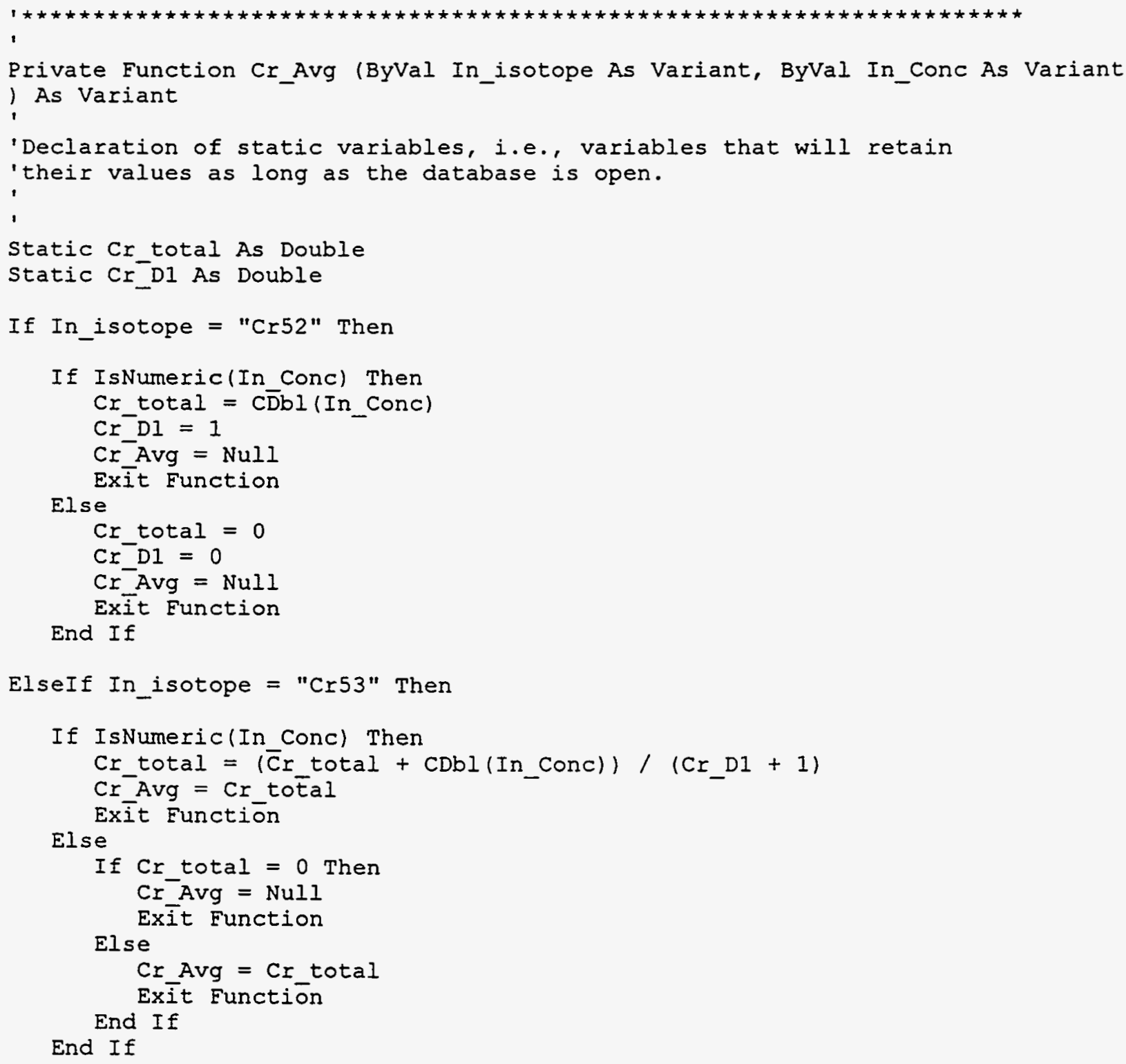

If IsNumeric(In_Conc) Then

Cr_total $=\left(\bar{C} r\right.$ total $+\operatorname{CDbl}\left(I_{n}\right.$ Conc $\left.)\right) /\left(C r \_D 1+1\right)$

$\mathrm{Cr}^{-}$Avg $=\mathrm{Cr}$ total

End If

End Function 


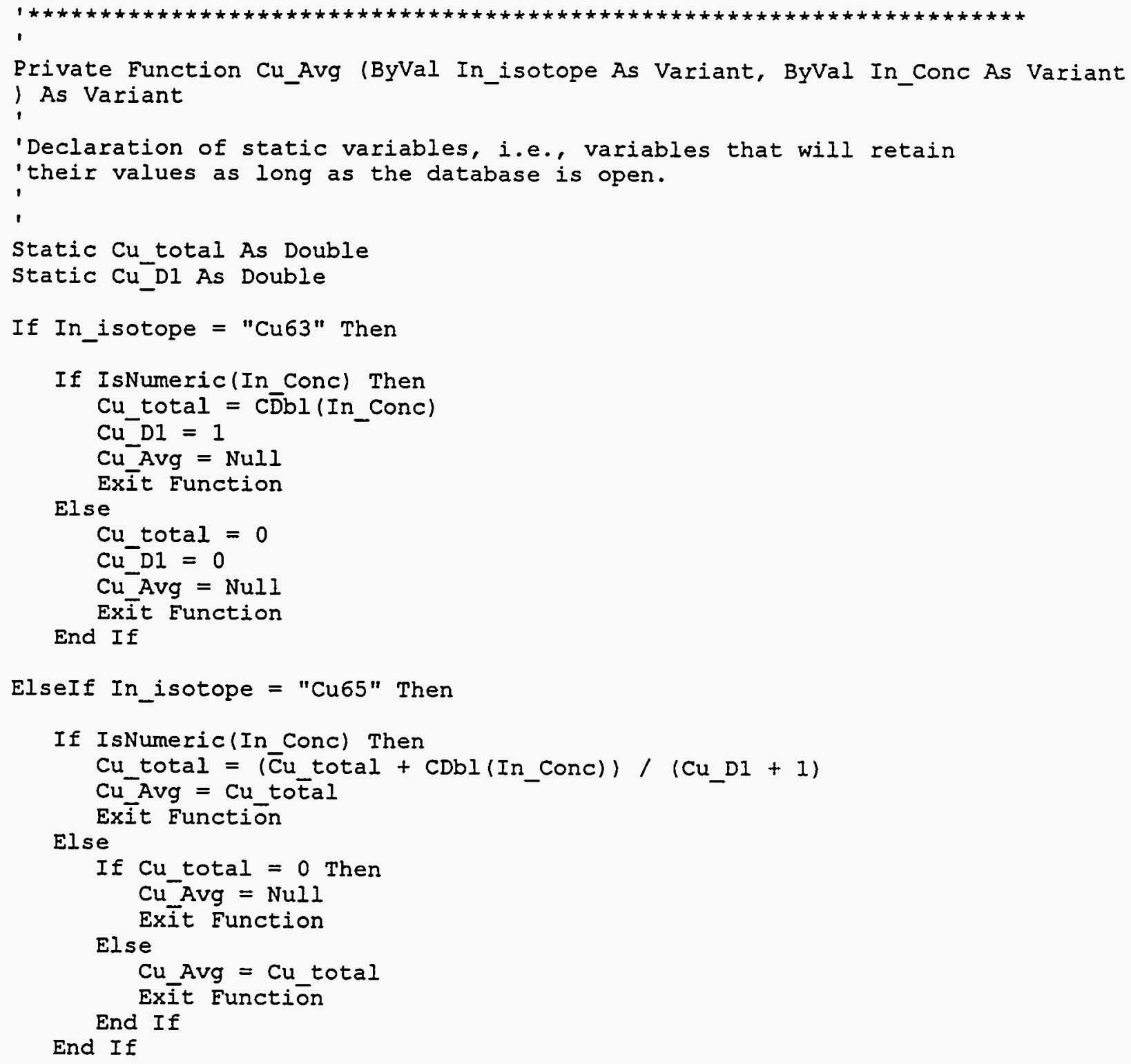

End If

End Function

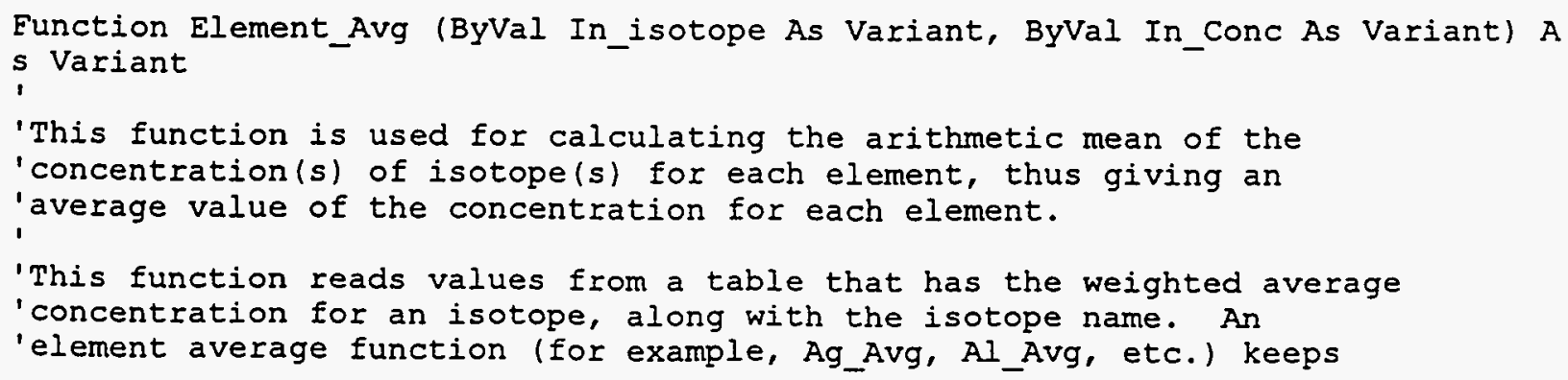




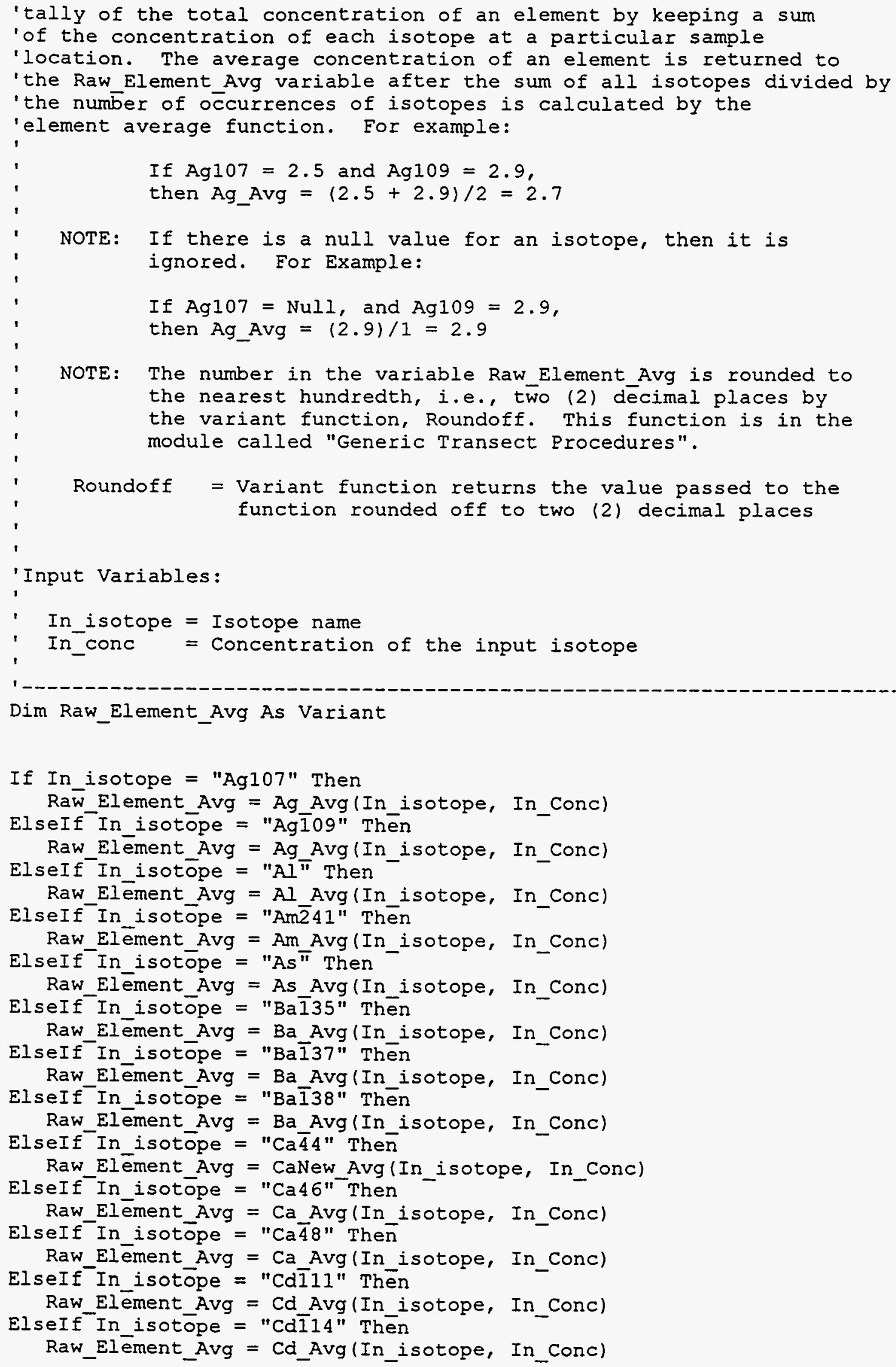




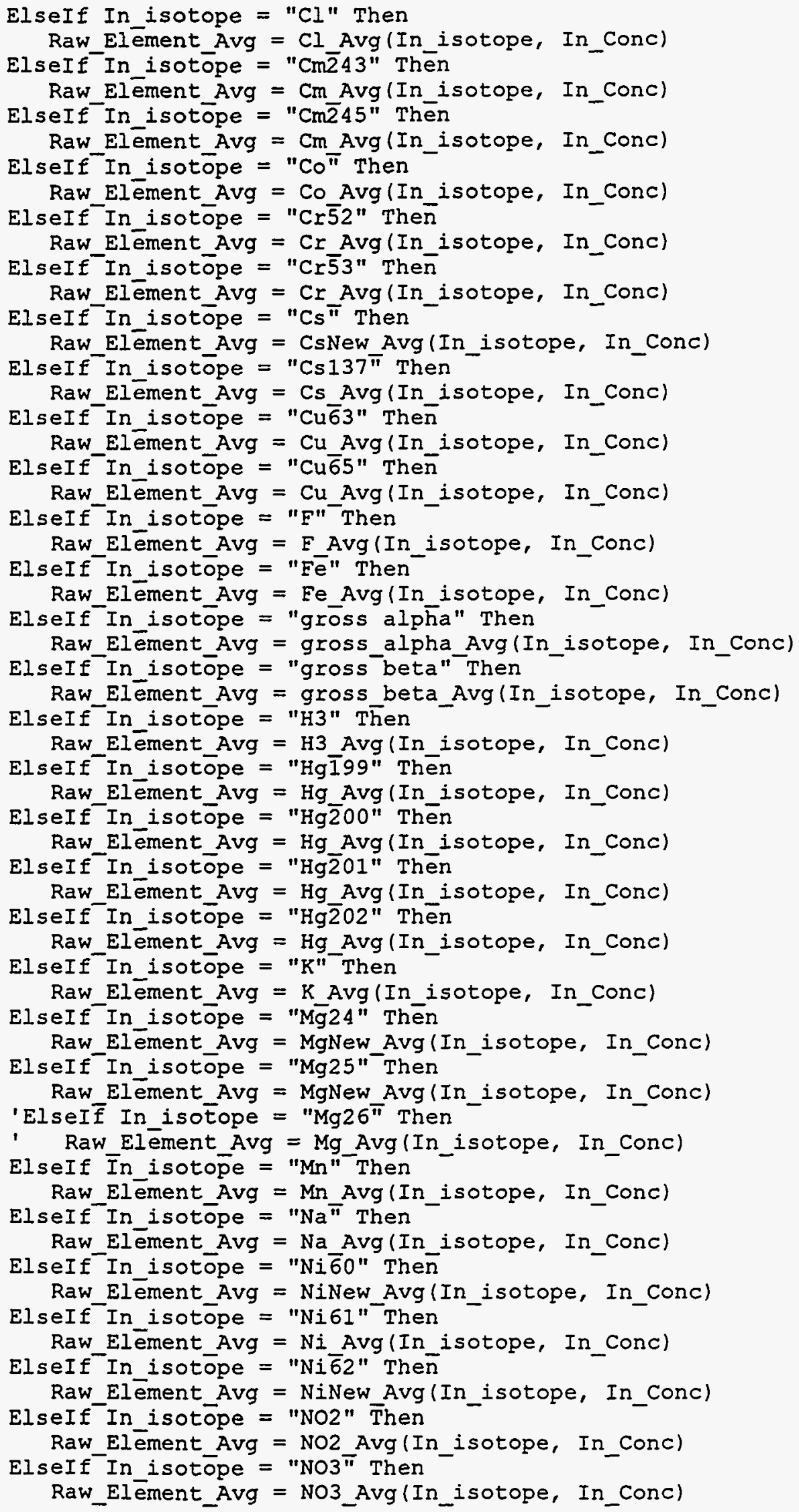


ElseIf In_isotope $=$ "Np237" Then

Raw_Elēment_Avg $=\mathrm{Np} A v g$ (In_isotope, In_Conc)

ElseIf In isotope = "Pb206" Then

Raw_Element_Avg $=\mathrm{Pb}$ Avg (In_isotope, In_Conc)

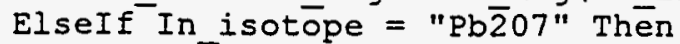

Raw_Element_Avg $=\mathrm{Pb}$ Avg (In isotope, In Conc)

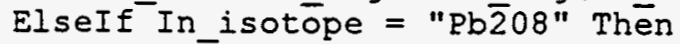

Raw_Element_Avg $=\mathrm{Pb}$ _Avg (In_isotope, In_Conc)

ElseIf In_isotope $=$ "POA" $"$ Then

Raw_Elēement_Avg $=$ PO4_Avg (In_isotope, In_Conc)

ElseIf In_isotope = "Pu2 $\overline{3} 9 "$ Then

Raw_Elēment_Avg $=\mathrm{Pu}$ Avg ( In_isotope, In_Conc)

ElseIf In isotope = "Pu $\overline{2} 40 "$ Then

Raw_Elēment_Avg = Pu_Avg (In_isotope, In Conc)

ElseIf In_isotope $=$ "Pū 42 " Then

Raw_Element_Avg $=\mathrm{Pu}$ Avg (In_isotope, In Conc)

ElseIf In_isotōpe $=$ "Rā̄26" Then

Raw_Element_Avg = Ra_Avg (In_isotope, In Conc)

ElseIf' In isotope = "Se $\overline{7} 7$ " Then

Raw_Elēment_Avg $=\mathrm{Se} A \mathrm{Avg}$ (In_isotope, In Conc)

ElseIf In_isotope = "Se $\overline{8} 2$ " Then

Raw_Element_Avg $=\mathrm{Se}$ Avg (In_isotope, In_Conc)

ElseIf In isotope = "Sn'118" Then

Raw_Element_Avg $=\mathrm{Sn} A v g$ (In_isotope, In_Conc)

ElseIf In isotope = "Sn'̄20" Then

Raw_Element_Avg $=\mathrm{Sn}$ Avg (In_isotope, In Conc)

ElseIf In_isotope = "SO4" Then

Raw_Element_Avg $=S O 4$ Avg (In_isotope, In Conc)

ElseIf In_isotope = "SI" Then

Raw_Element_Avg = SrNew_Avg (In_isotope, In_Conc)

ElseIf In_isotope = "Sr90" Then

Raw_Elèment_Avg = Sr_Avg (In_isotope, In_Conc)

ElseIf In isotope $=$ "Th $\overline{2} 32 "$ Then

Raw_Element_Avg = Th_Avg(In_isotope, In_Conc)

ElseIf In isotope = "Ti" Then

Raw_Element_Avg $=\mathrm{Ti}$ Avg (In isotope, In Conc)

ElseIf In_isotope $=$ "U2 $\overline{3} 4$ " The

Raw_Elēement_Avg = U Avg (In_isotope, In_Conc)

ElseIf In isotope = "U2̌35" Then

Raw_Element_Avg $=U 235$ Avg (In_isotope, In Conc)

ElseIf In_isotope = "U238" Then

Raw_Element_Avg $=$ U238_Avg (In_isotope, In Conc)

ElseIf In isotope = "V" Then Raw_Element_Avg $=\mathrm{V}$ Avg (In_isotope, In Conc)

ElseIf In isotope $=$ "Y" Then Raw_Element_Avg $=Y$ Avg (In_isotope, In Conc)

ElseIf In isotope $=" z \bar{n} 64$ " Then Raw_Element_Avg $=\mathrm{Zn}$ Avg (In_isotope, In Conc)

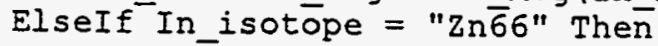
Raw_Element_Avg = ZnNew Avg (In isotope, In Conc)

ElseIf In isotope $=$ "Zn67" Then Raw_Element_Avg $=\mathrm{Zn}$ Avg (In isotope, In Conc)

ElseIf In isotope $=" Z r \overline{9} 0 "$ Then Raw_Elèment_Avg $=\mathrm{Zr}$ Avg (In_isotope, In_Conc)

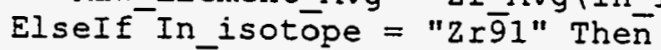
End If Raw_Elèment_Avg $=\mathrm{Zr}$ _Avg(In_isotope, In_Conc)

Element_Avg $=$ Roundoff (Raw_Element_Avg) 
End Function

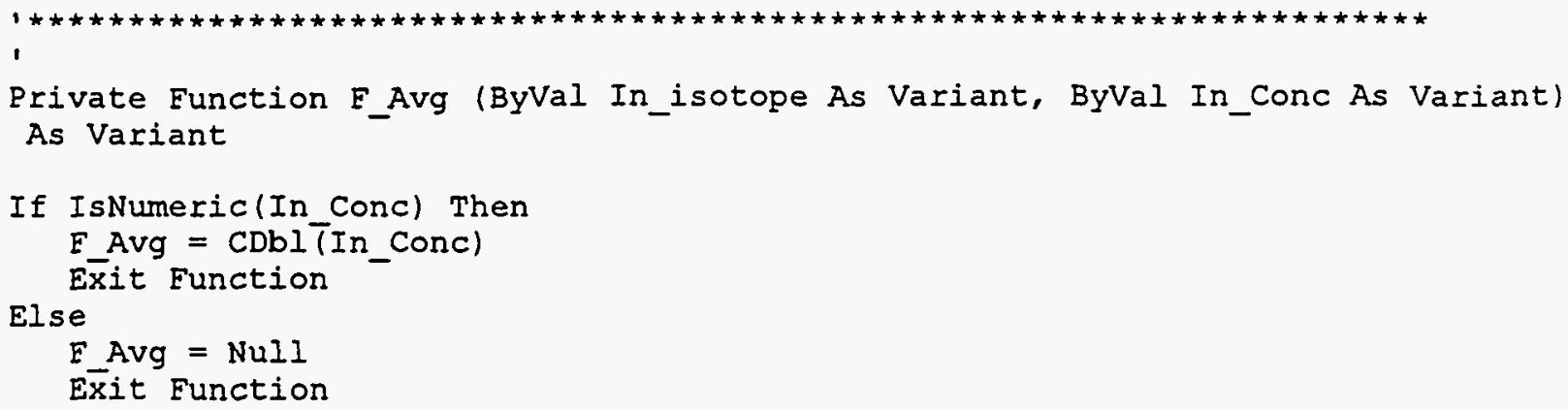

End Function

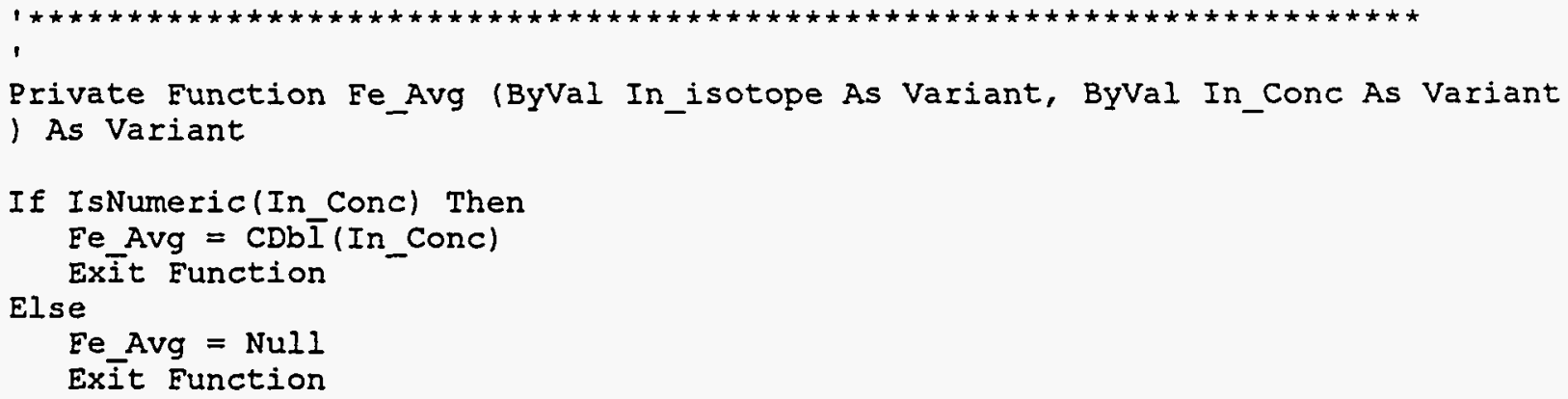

End Function

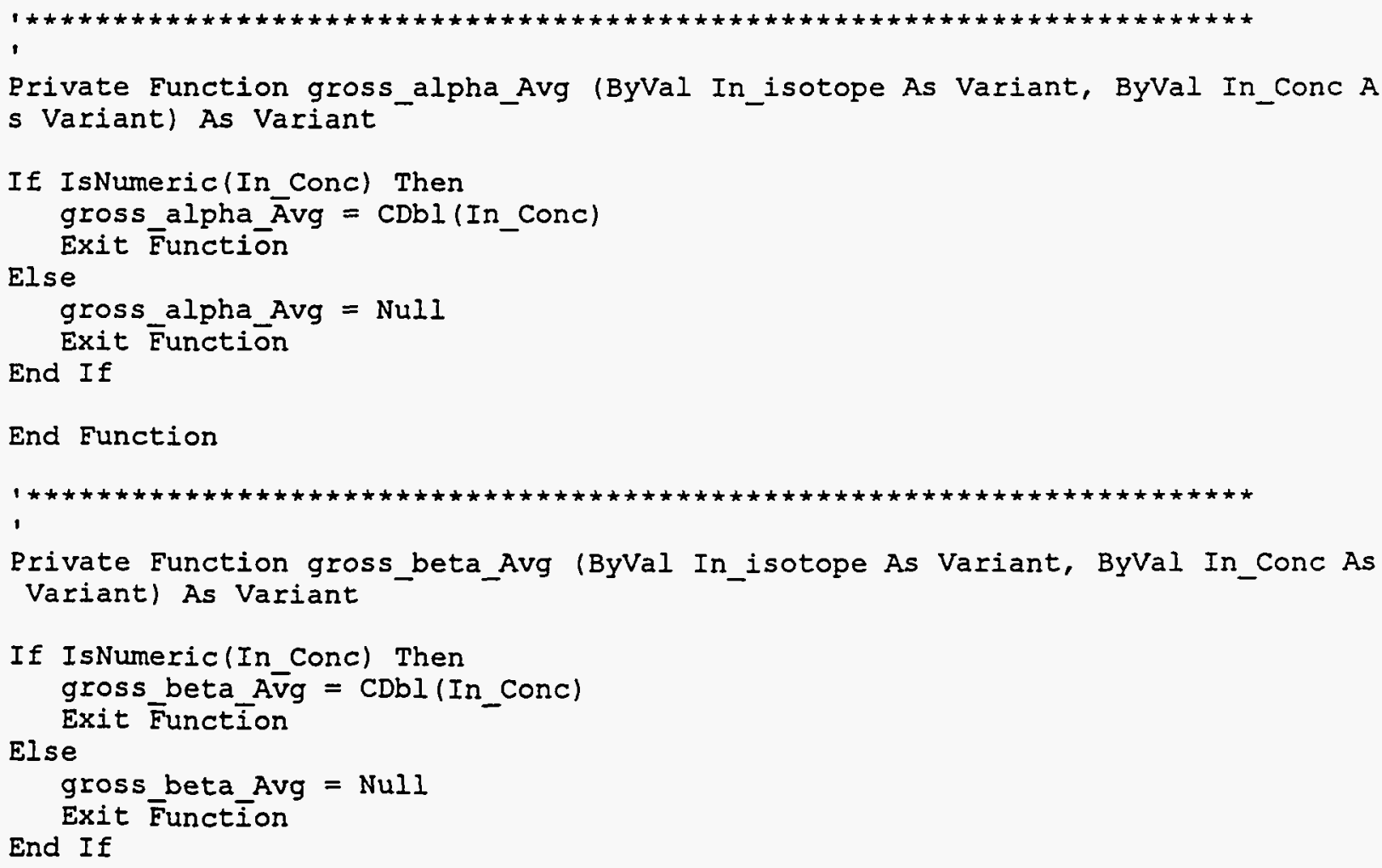

End Function 
End Function

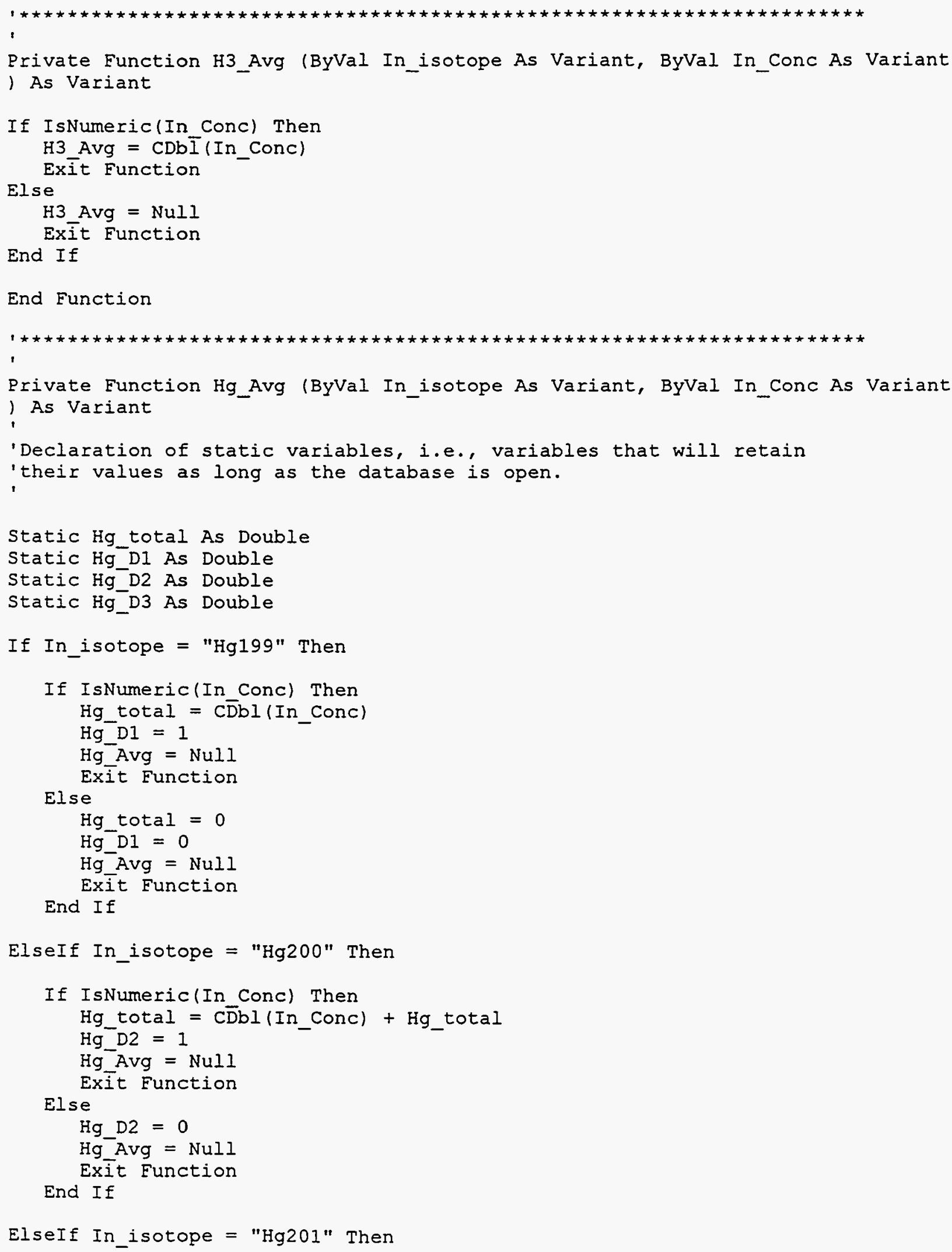


If IsNumeric(In Conc) Then

Hg_total $=\mathrm{CD} b I$ (In_Conc) + Hg_total

Hg_D3 $=1$

Hg Avg $=$ NuIl

Else

Exit Function

$\mathrm{Hg}$ D3 $=0$

Hg Avg $=\mathrm{Null}$

Exit Function

End If

ElseIf In_isotope $=$ "Hg202" Then

If IsNumeric(In Conc) Then

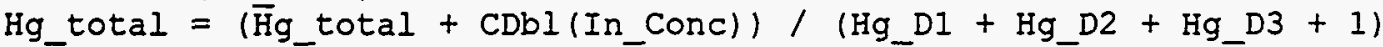

Hg_Avg = Hg_total

Exit Function

Else

If $\mathrm{Hg}$ total $=0$ Then

Hg_Avg $=$ Null

Exit Function

Else

Hg_Avg $=$ Hg_total / (Hg_D1 + Hg_D2 + Hg_D3 $)$

Exit Function

End If

End If

End If

End Function

,

Private Function $K_{-}$Avg (ByVal In_isotope As Variant, ByVal In_Conc As Variant) As Variant

If IsNumeric(In Conc) Then

$K_{-}$Avg $=\mathrm{CDbl}\left(\mathrm{In} \_\right.$Conc $)$

Exit Function

Else

$\mathrm{K}$ Avg $=$ Null

Exit Function

End If

End Function

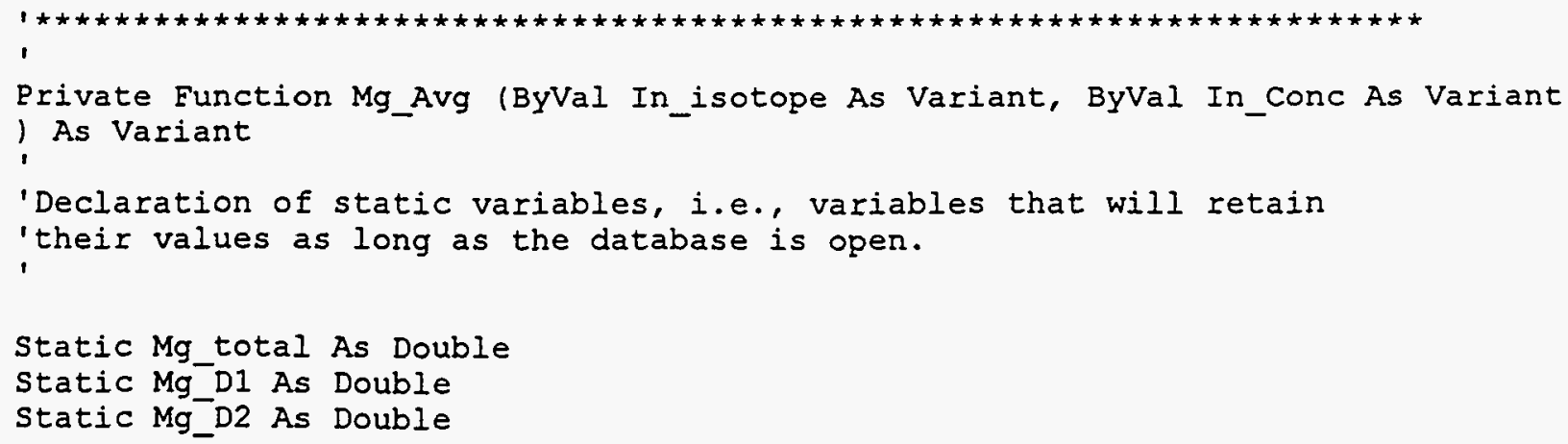




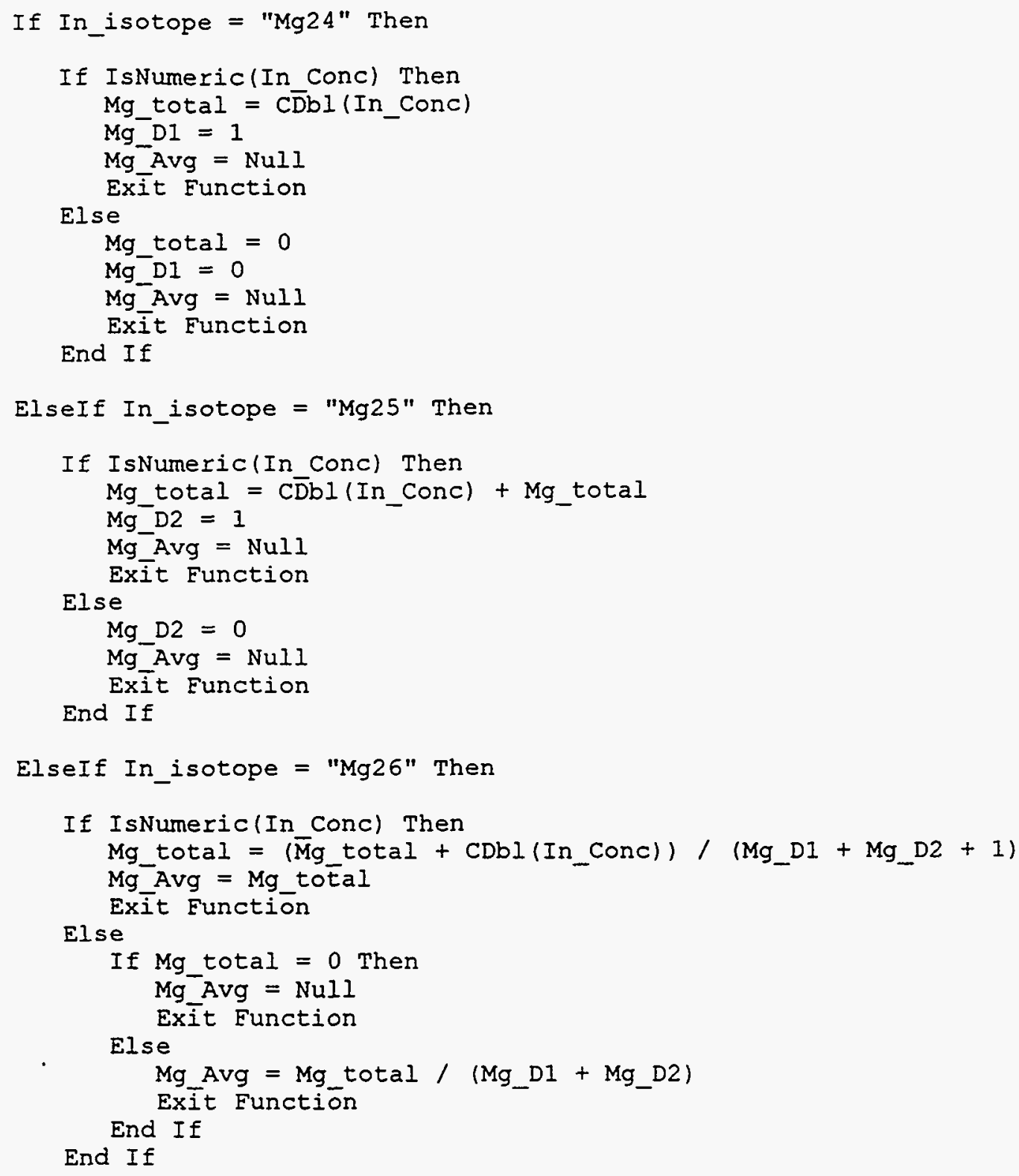

End If

End Function

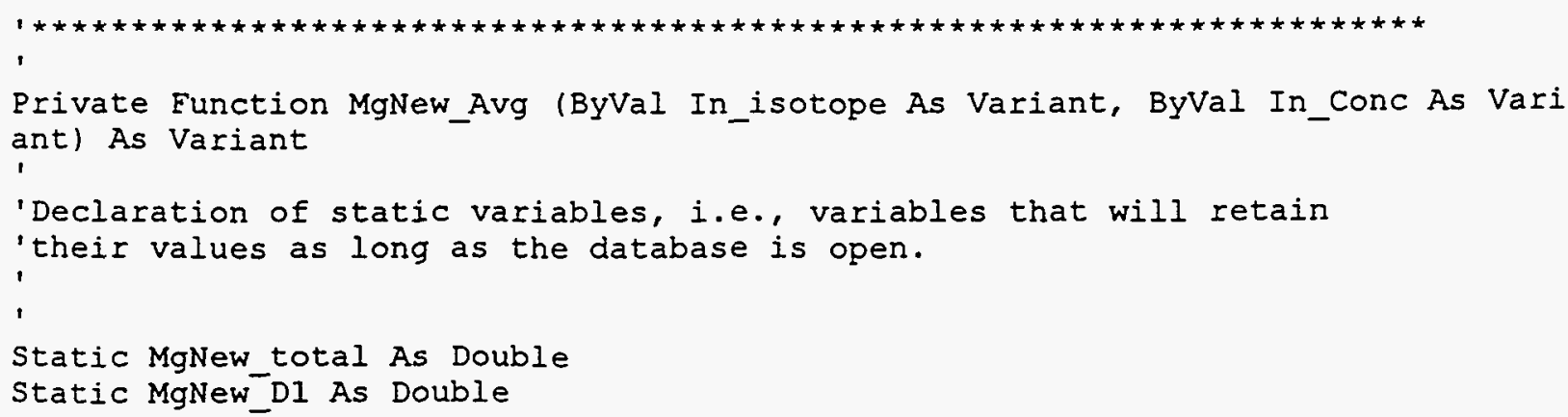




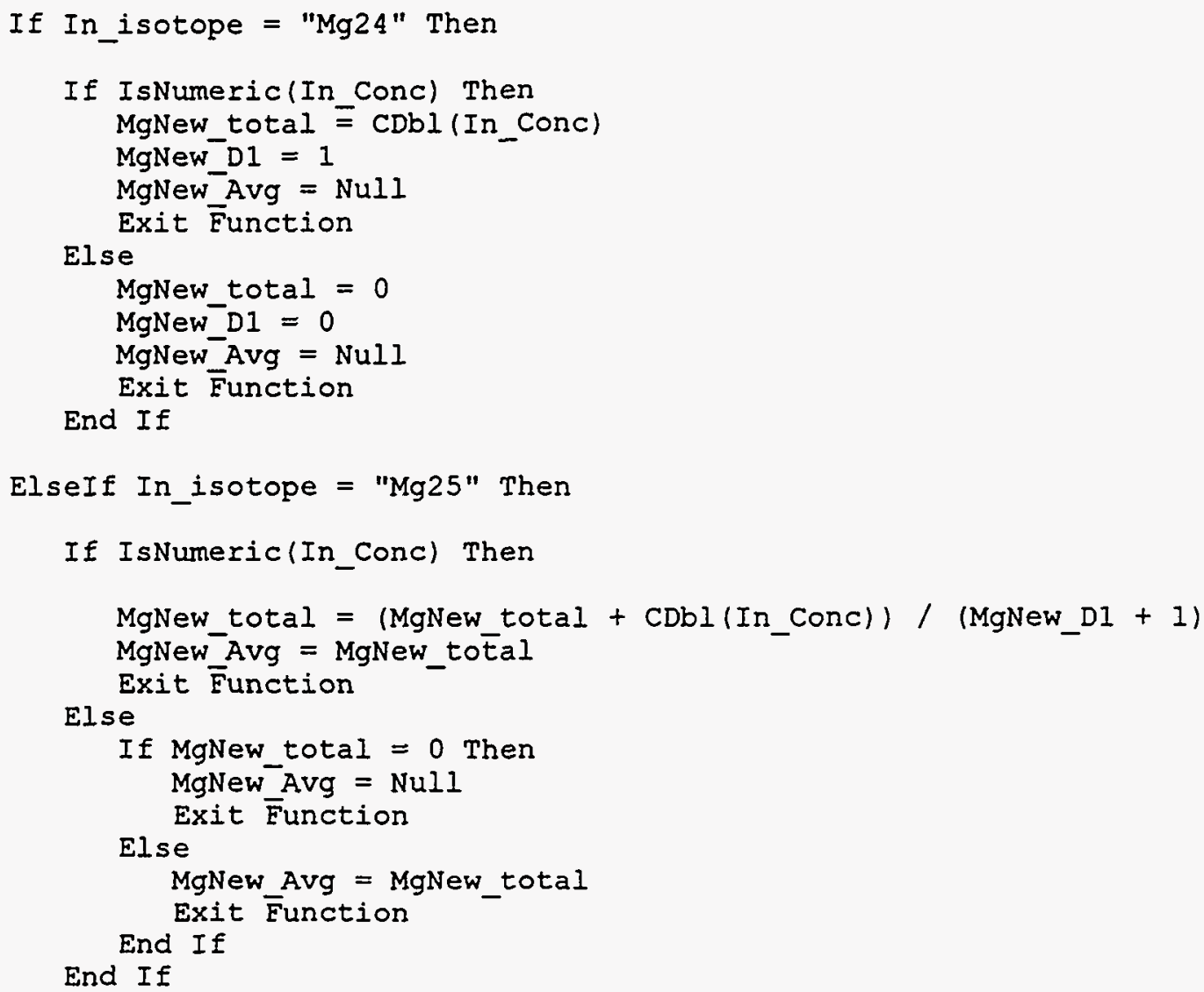

End If

End Eunction

1

Private Function Mn_Avg (ByVal In_isotope As Variant, ByVal In_Conc As Variant ) As Variant

If IsNumeric(In Conc) Then

$\mathrm{Mn}$ Avg $=\mathrm{CDb} \bar{I}(\mathrm{In}$ Conc)

Exit Function

Else

$\mathrm{Mn}$ Avg $=\mathrm{Null}$

Exit Function

End If

End Function

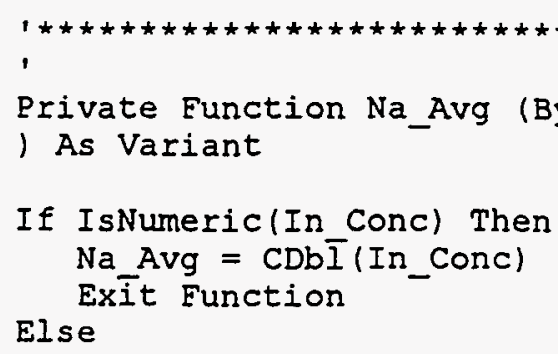




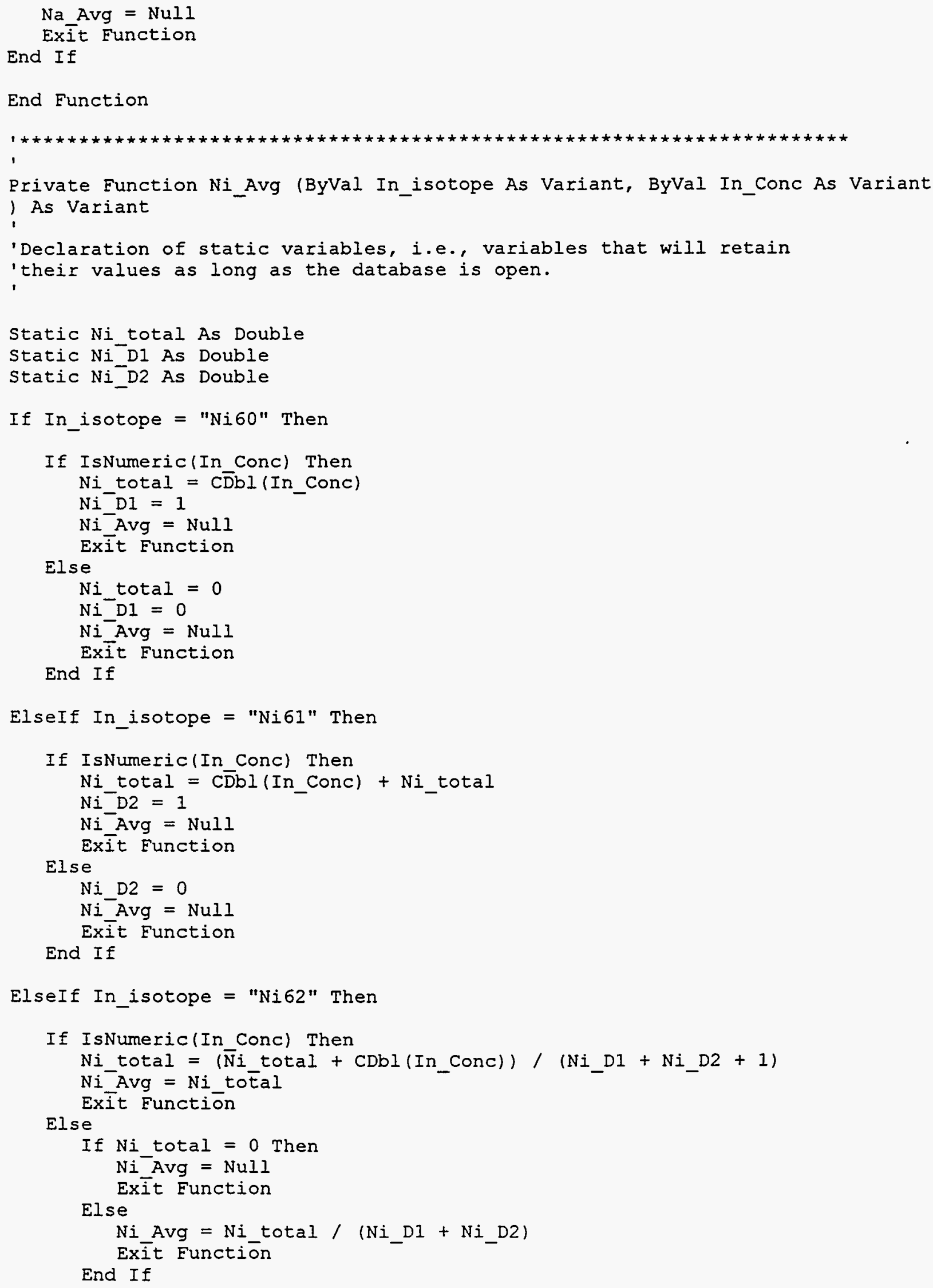


End If

End If

End Function

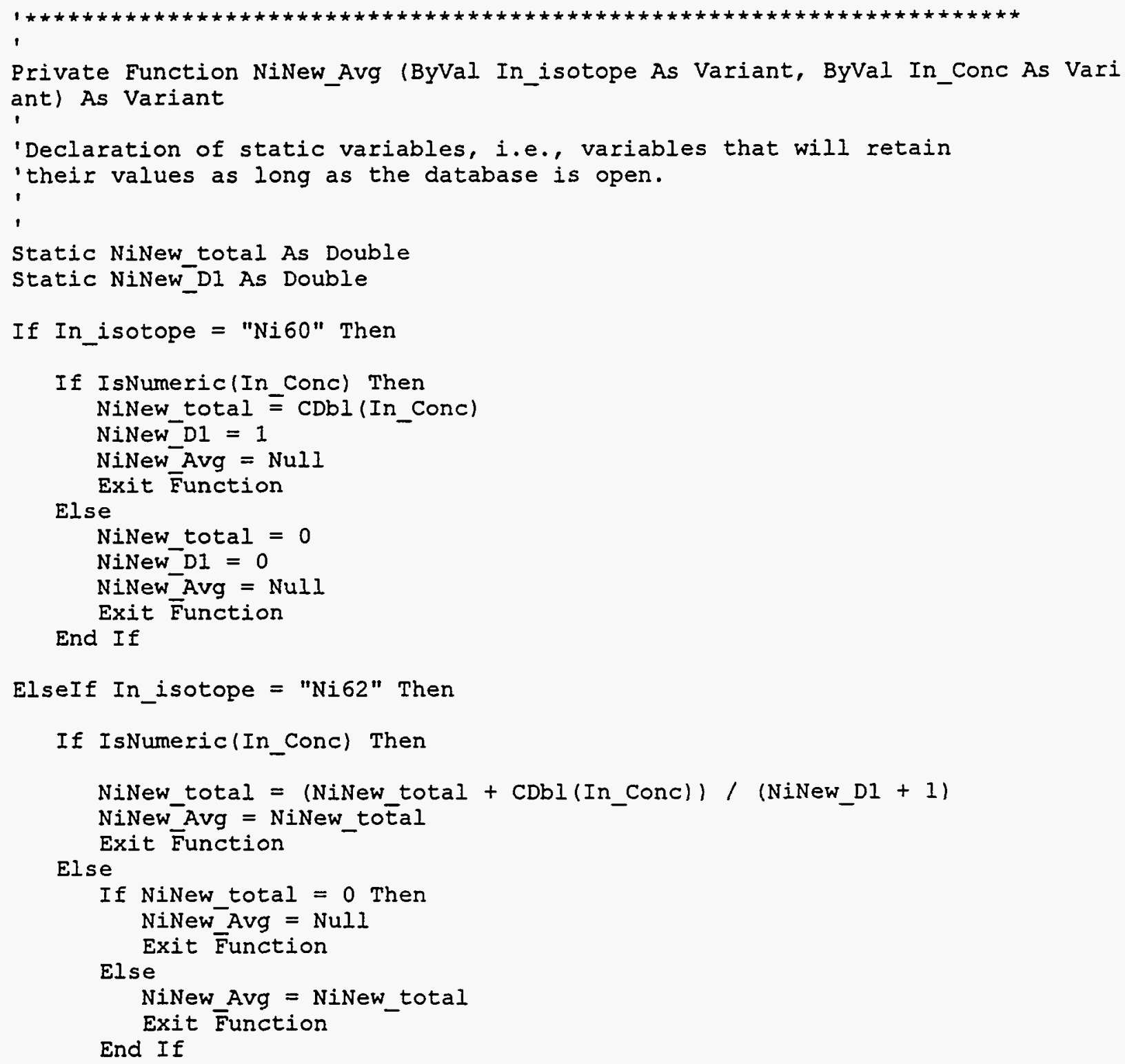

End If

End Function

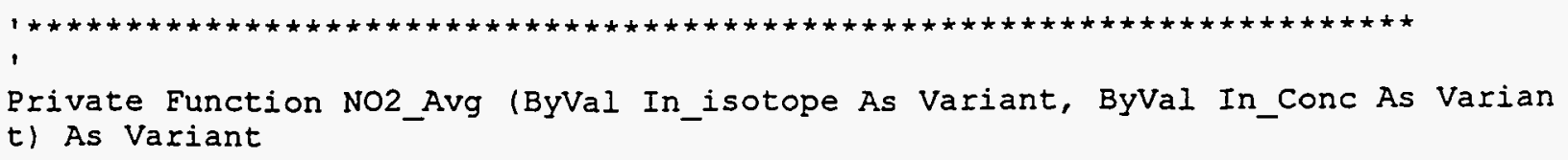




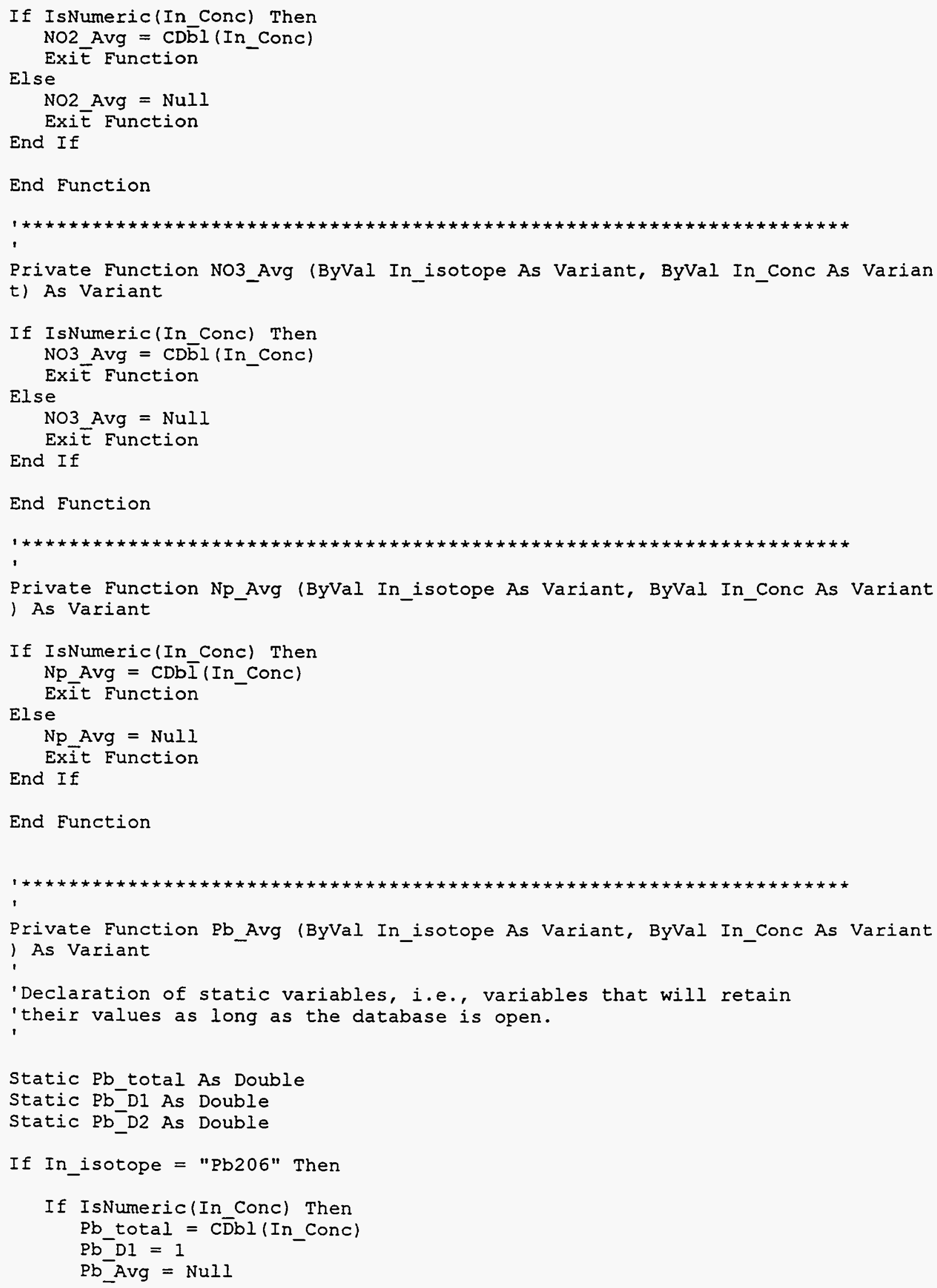




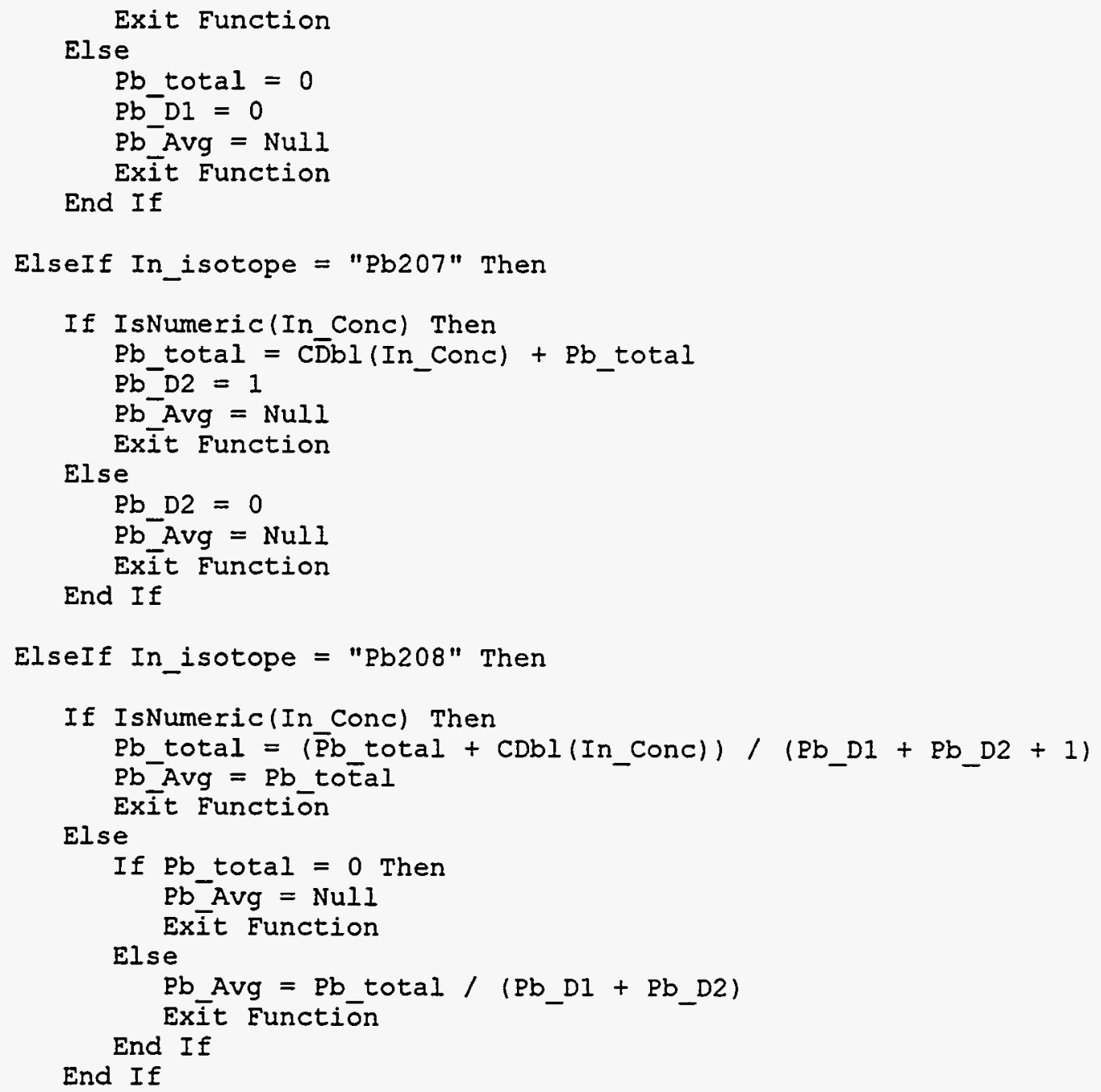

End If

End Function

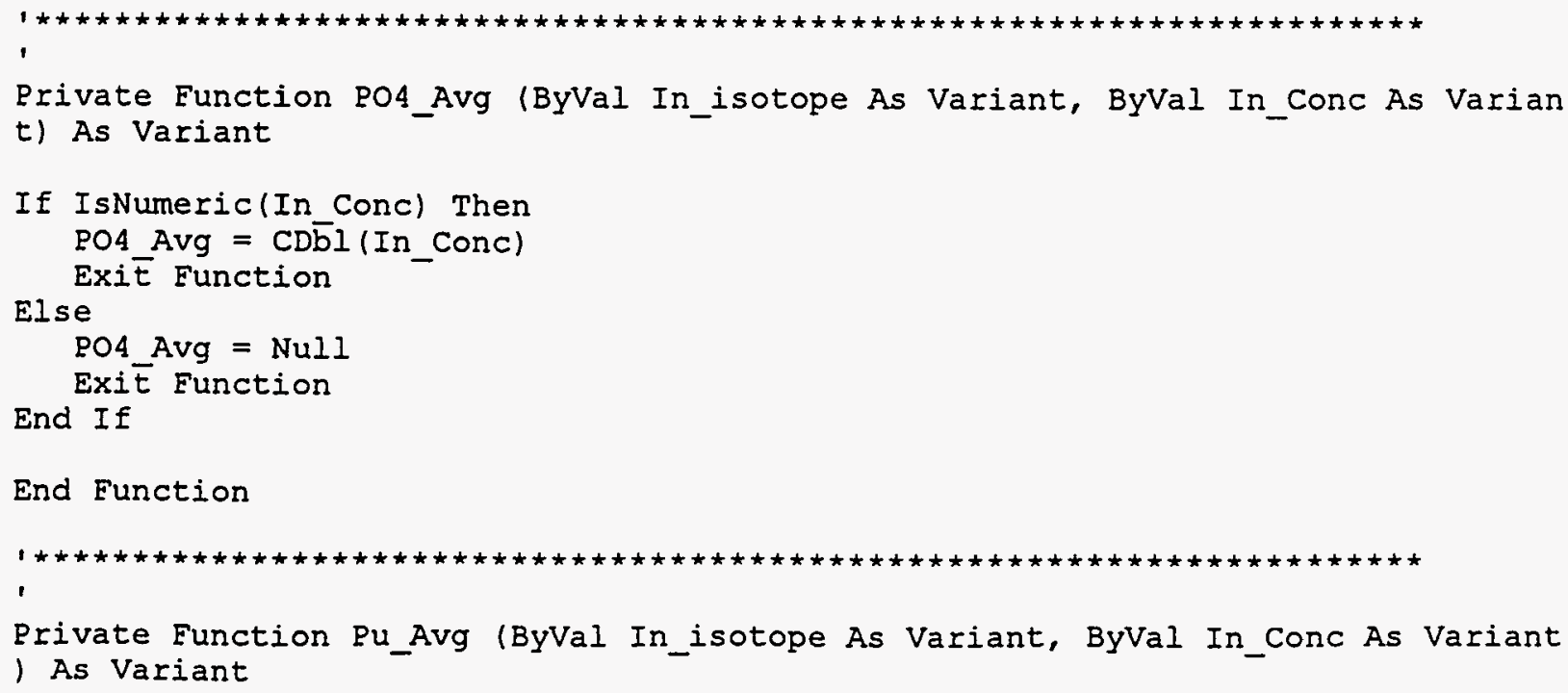


,

'Declaration of static variables, i.e., variables that will retain

'their values as long as the database is open.

Static Pu total As Double

Static Pu-D1 As Double

Static Pu_D2 As Double

If In_isotope $=$ "Pu239" Then

If IsNumeric(In_Conc) Then

Pu_total $=\mathrm{CDbl}$ (In_Conc)

$\mathrm{Pu} D 1=1$

Pu Avg $=$ Null

Else

Exit Function

Pu total $=0$

Pu-D1 $=0$

Pu Avg $=$ NuII

Exit Function

End If

ElseIf In_isotope $=$ "Pu240" Then

If IsNumeric(In Conc) Then

Pu_total $=\mathrm{CD} b l\left(I n \_C o n c\right)+P u \_t o t a l$

$\mathrm{Pu}$ D2 $=1$

$\mathrm{Pu}^{-}$Avg $=\mathrm{Null}$

Exit Eunction

Else

$\mathrm{Pu} D 2=0$

Pu_Avg $=$ Null

Exit Function

End If

Elself In_isotope $=$ "Pu242" Then

If IsNumeric(In Conc) Then

$\mathrm{Pu}$ total $=(\overline{\mathrm{Pu}}$ total $+\mathrm{CDbl}(\mathrm{In}$ Conc $)) /(\mathrm{Pu} D \mathrm{D} 1+\mathrm{Pu} D 2+1)$

$\mathrm{Pu}^{-} \mathrm{Avg}=\mathrm{Pu}$ total

Exit Function

Else

If Pu_total $=0$ Then

Pu Avg $=$ Null

Else

Exit Function

Pu_Avg $=$ Pu_total / (Pu_D1 + Pu_D2)

Exit Function

End If

End If

End If

End Function

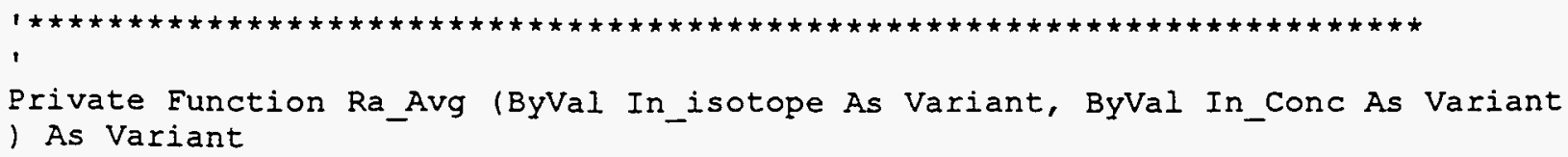


If IsNumeric(In Conc) Then

Ra_Avg $=\mathrm{CDb} \bar{I}$ (In_Conc)

Exit Function

Else

Ra Avg $=$ Null

Exit Function

End If

End Function

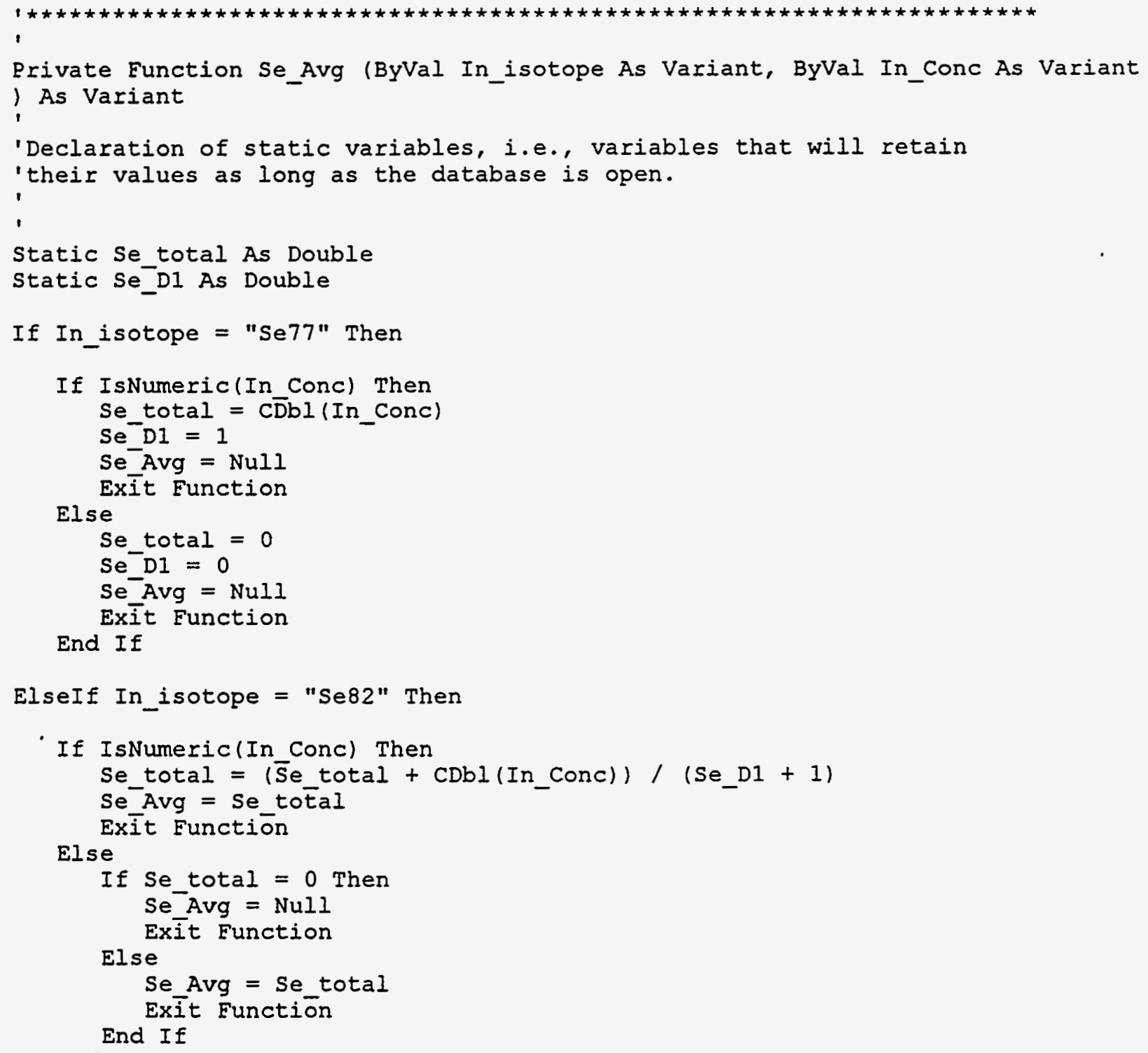

End Function 


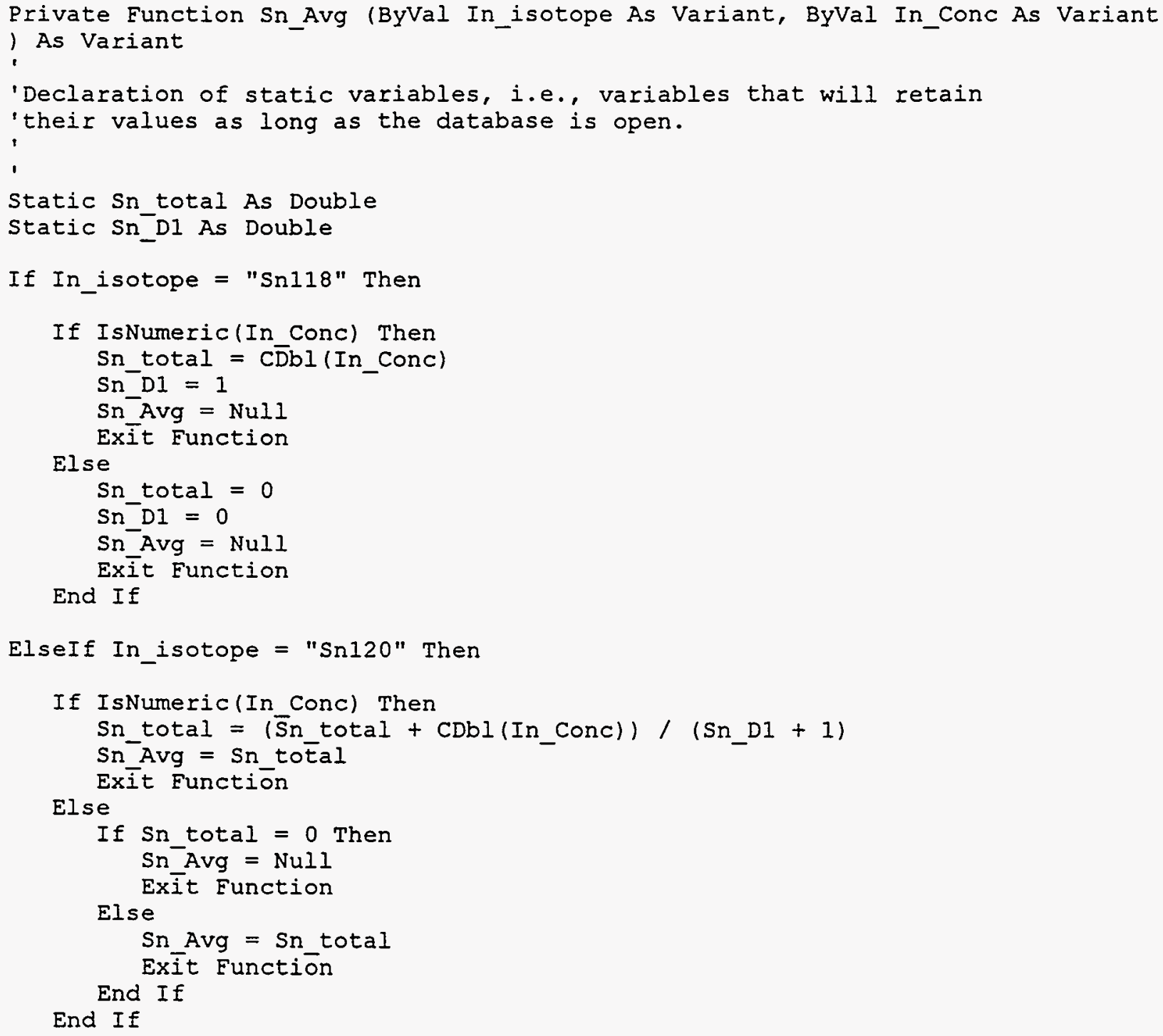

End If

End Function

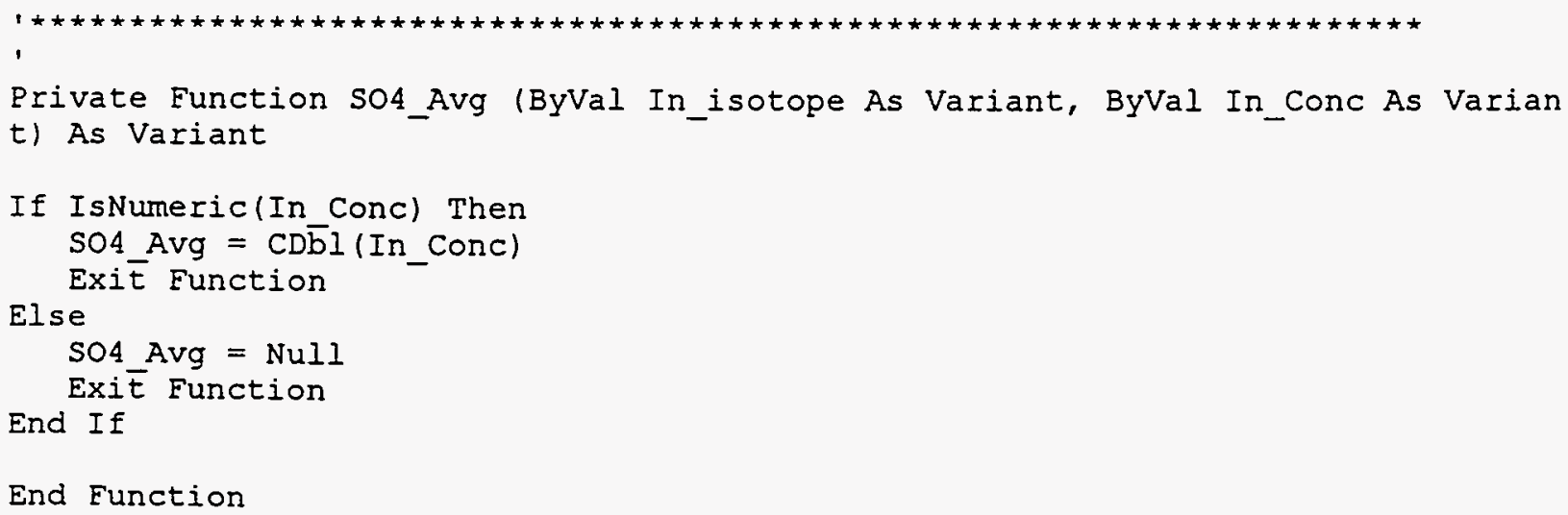


Private Function Sr_Avg (ByVal In_isotope As Variant, ByVal In_Conc As Variant ) As Variant

'Declaration of static variables, i.e., variables that will retain 'their values as long as the database is open.

I

Static Sr total As Double

Static Sr_D1 As Double

If In_isotope $=$ "Sr" Then

If IsNumeric(In Conc) Then

Sr_total $=\mathrm{C} \overline{\mathrm{D}} \mathrm{bl}$ (In_Conc)

Sr_D1 = 1

$\mathrm{Sr}^{-} \mathrm{Avg}=\mathrm{NuII}$

Else

Exit Function

Sr total $=0$

$S{ }^{-} D 1=0$

Sr-Avg $=$ Null

Exit Function

End If

ElseIf In_isotope $=$ "Sr90" Then

If IsNumeric(In Conc) Then

$S r_{\text {ttotal }}=\left(\bar{S} r_{\text {total }}+\mathrm{CDbl}(\mathrm{In}\right.$ Conc $\left.)\right) /(\mathrm{Sr} D 1+1)$

Sr_Avg $=$ Sr_total

Else

Exit Function

If $S r$ total $=0$ Then

Sr-Avg $=$ Null

Exit Function

Else

Sr Avg = Sr total

Exit Function

End If

End If

End If

End Function

$$
\text { i }
$$

Private Function SrNew_Avg (ByVal In_isotope As Variant, ByVal In_Conc As Vari ant) As Variant

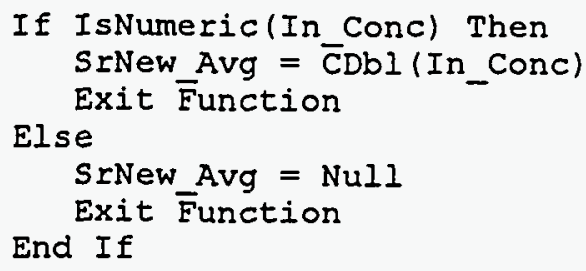

End Function 


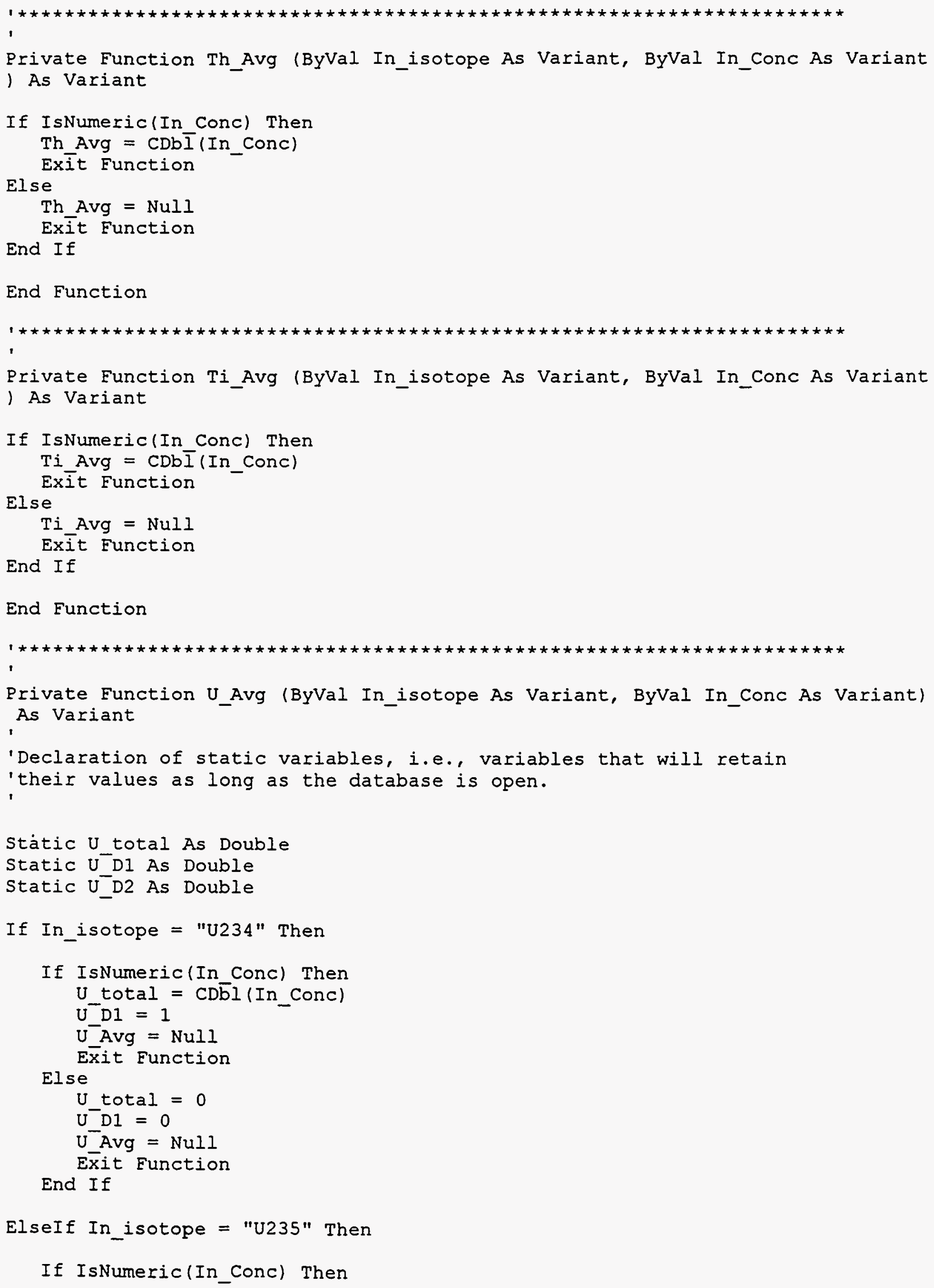




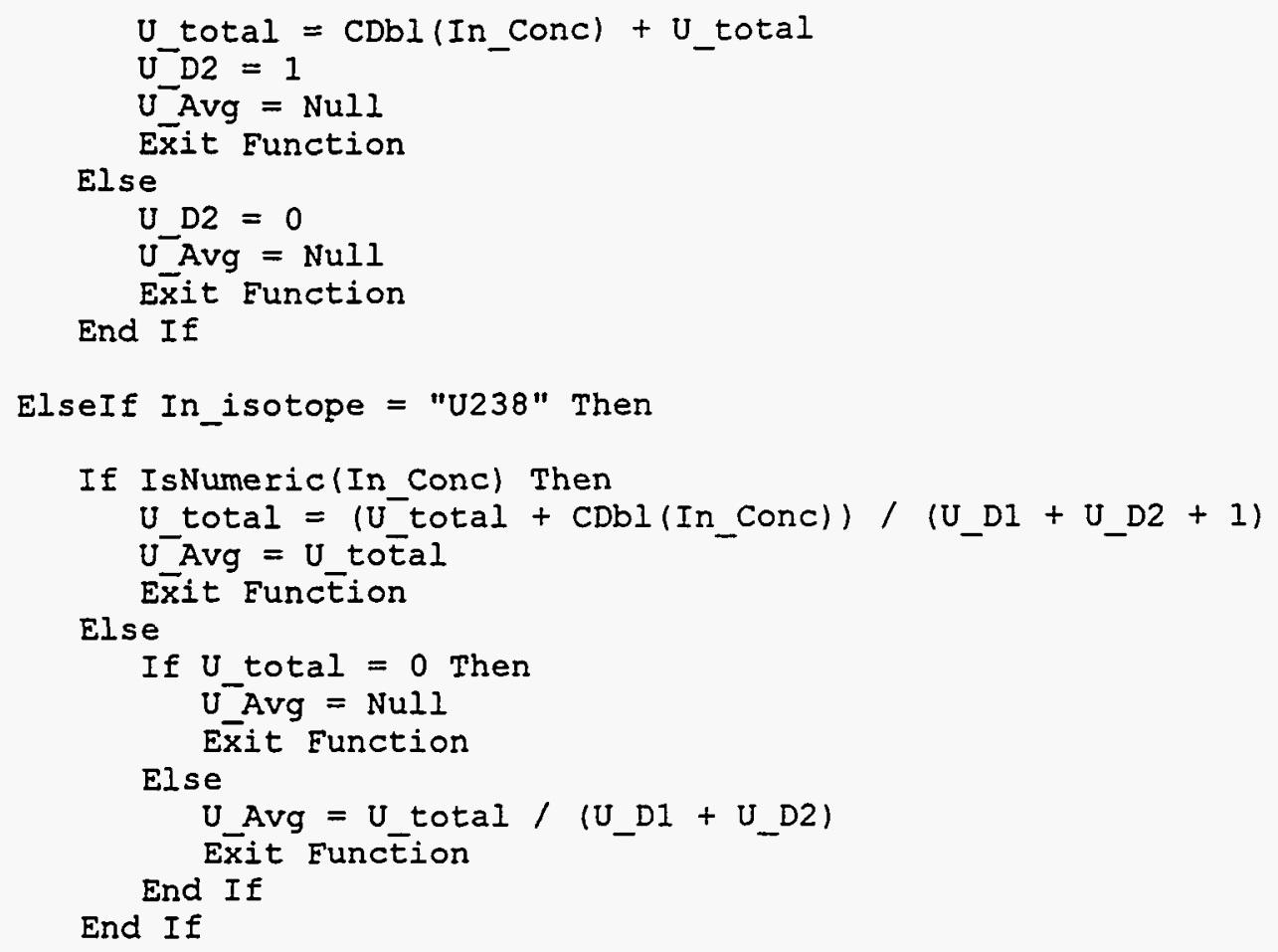

End If

End Function

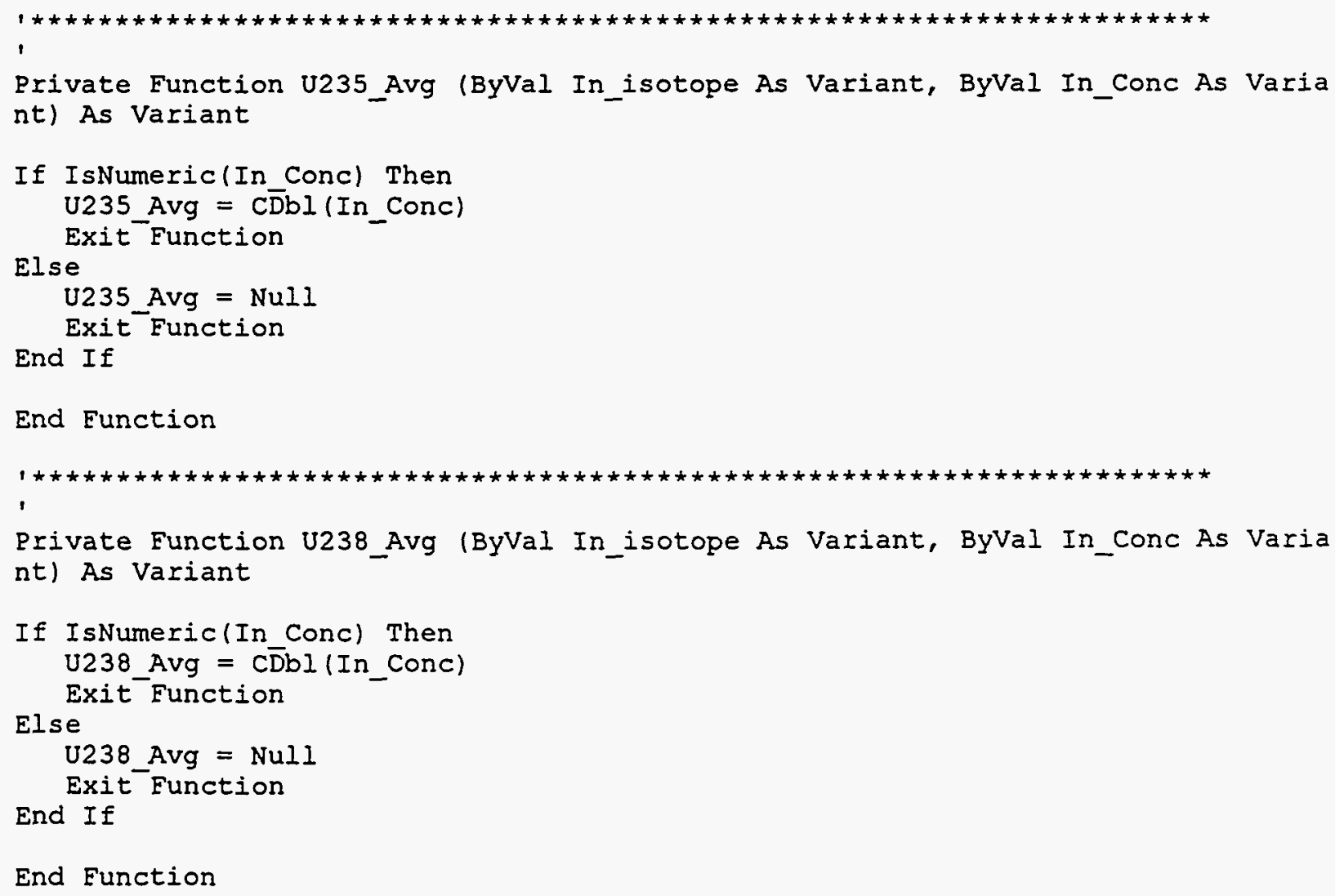




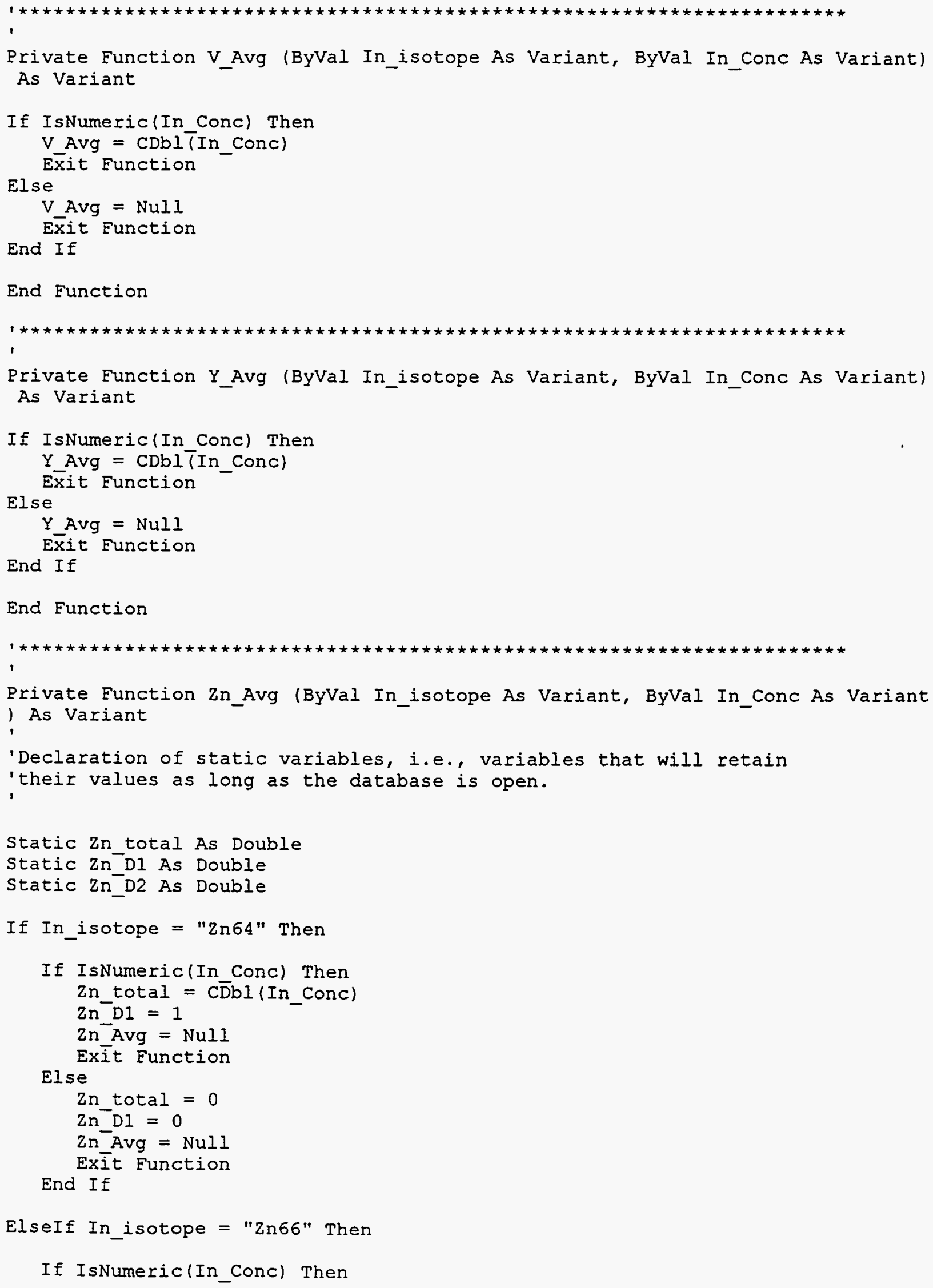




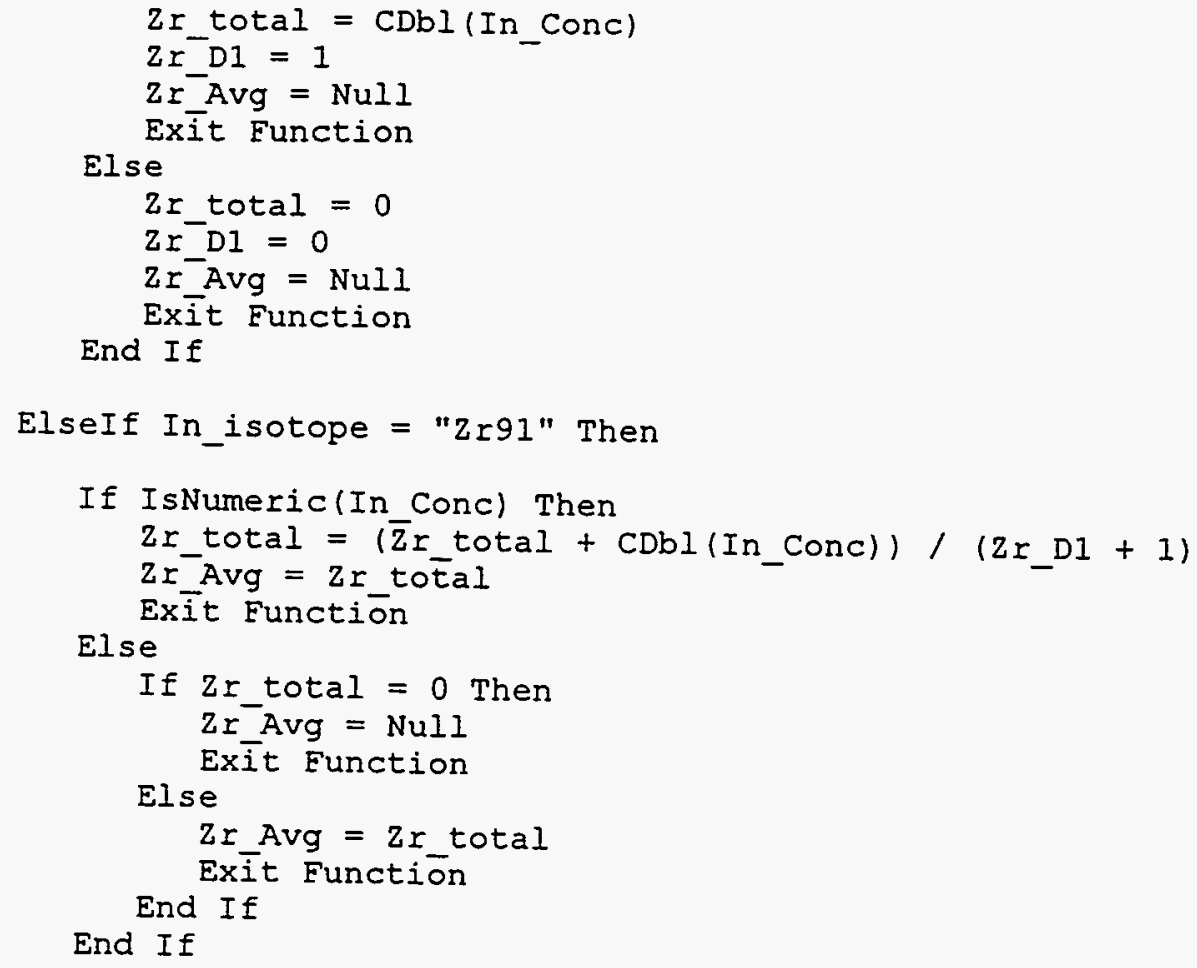

End If

End Function 


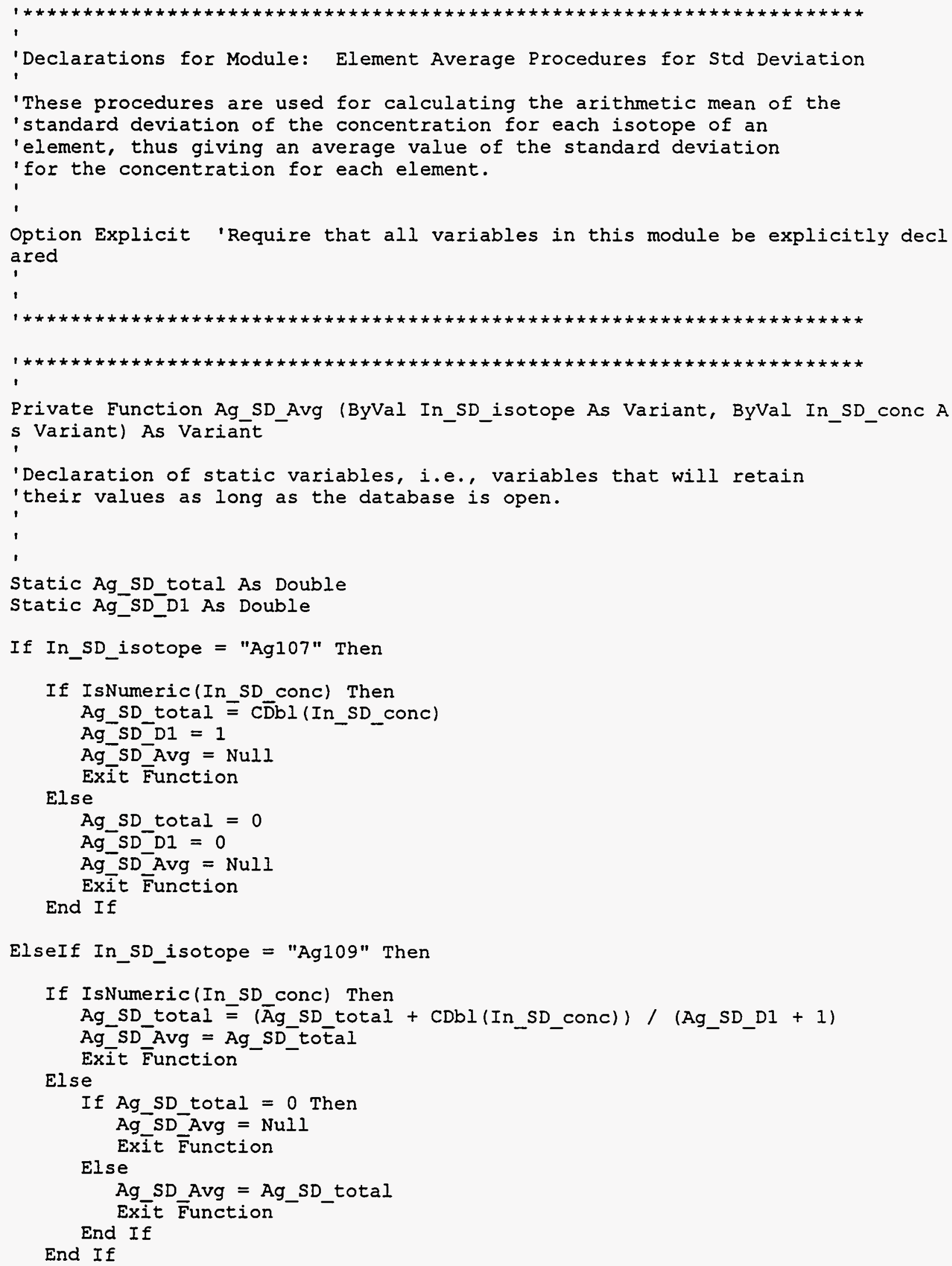


Static Ba_SD_D2 As Double

If In_SD_isotope $=$ "Ba135" Then

If IsNumeric(In_SD_conc) Then

Ba_SD_total $\equiv \mathrm{C} \overline{\mathrm{D}} \mathrm{bl}$ (In_SD_conc)

$\mathrm{Ba}^{-} \mathrm{SD}^{-} \mathrm{D} 1=1$

$\mathrm{Ba}^{-} \mathrm{SD}^{-} \mathrm{Avg}=\mathrm{Null}$

Else

Exīt Function

Ba_SD_total $=0$

$\mathrm{Ba} \mathrm{SD}^{-} \mathrm{Dl}=0$

$\mathrm{Ba}-\mathrm{SD}^{-\mathrm{Avg}}=\mathrm{Null}$

Exit Function

End If

ElseIf In_SD_isotope $=$ "Ba137" Then

If IsNumeric(In_SD_conc) Then

Ba_SD_total $=\bar{C} \overline{D b l}\left(I n \_S D \_c o n c\right)+B a$ SD_total

$\mathrm{Ba} S \mathrm{SD} D 2=1$

$\mathrm{Ba}^{-} \mathrm{SD}^{-} \mathrm{Avg}=\mathrm{Null}$

Else

Exīt Eunction

$\mathrm{Ba} S \mathrm{SD} 2=0$

$\mathrm{Ba} S \mathrm{SD}^{-} \mathrm{Avg}=\mathrm{Null}$

Exitt Function

End If

ElseIf In_SD_isotope $=$ "Ba138" Then

If IsNumeric(In_SD_conc) Then

1)

Ba_SD_total $\equiv\left(\overline{B a}{ }_{-} S D_{-} t o t a l+C D b I\left(I n \_S D \_c o n c\right)\right) /\left(B a \_S D \_D 1+B a \_S D \_D 2+\right.$

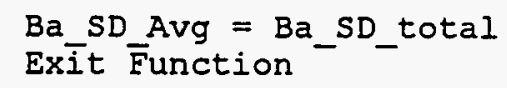

End If

End If

End Function

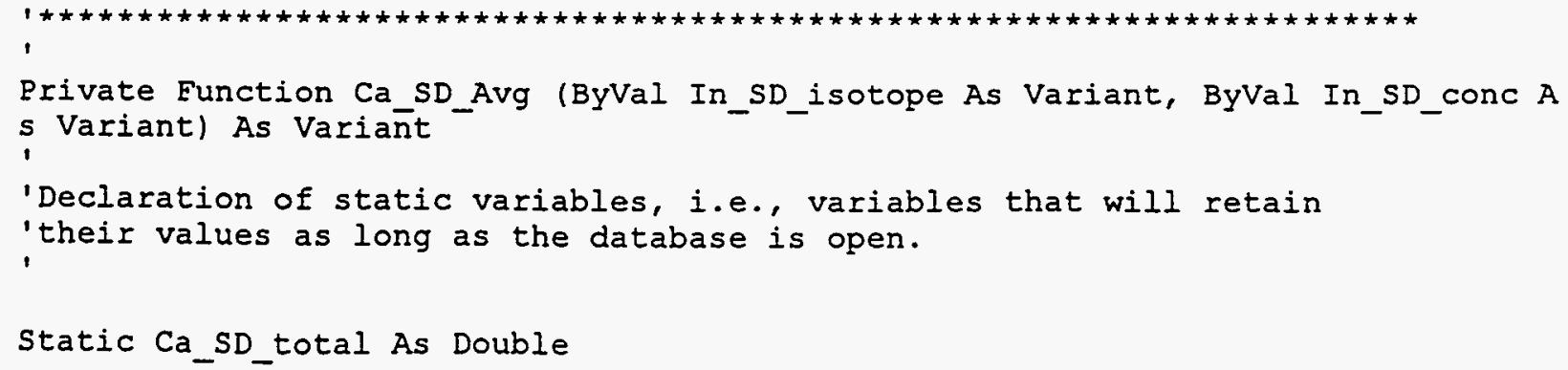




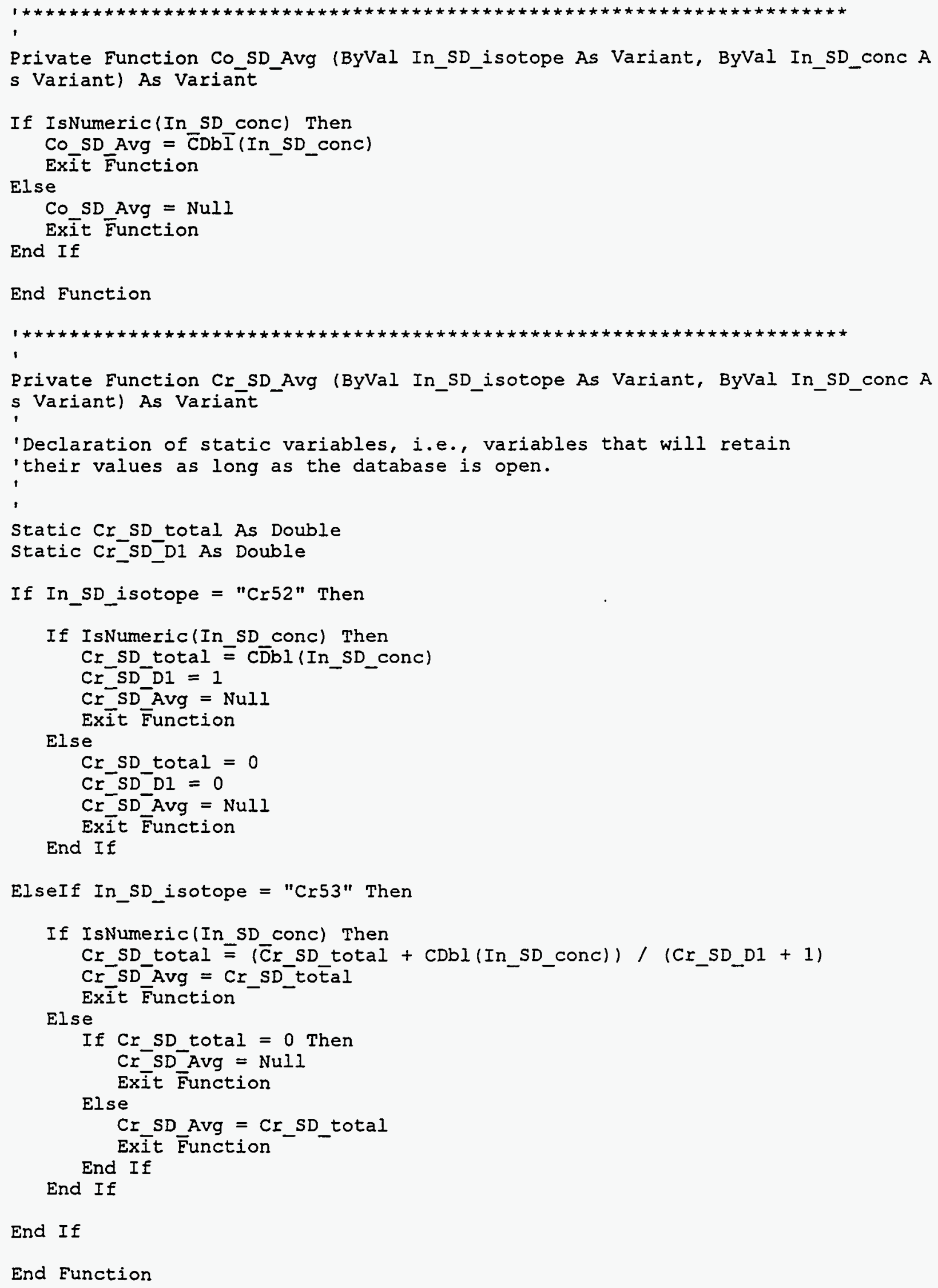

End Function 
Private Function Cs SD Avg (ByVal In_SD_isotope As Variant, ByVal In_SD_conc A s Variant) As Variañt

'Declaration of static variables, i.e., variables that will retain

'their values as long as the database is open.

Static Cs SD total As Double

Static Cs_SD_D1 As Double

If In_SD_isotope $=$ "Cs" Then

If IsNumeric(In SD conc) Then

Cs SD total $\equiv C \overline{D b} l(I n S D$ Conc)

Cs SDDD1 = 1

$\mathrm{Cs}^{-} \mathrm{SD}^{-}$Avg $=\mathrm{Null}$

Exit Function

Else

Cs $S D$ total $=0$

$\mathrm{Cs}^{-} \mathrm{SD}^{-} \mathrm{D1}=0$

$\mathrm{Cs}^{-} \mathrm{SD}^{-}$Avg $=\mathrm{Null}$

Exit $\overline{\text { Function }}$

End If

ElseIf In_SD_isotope $=$ "Cs137" Then

If IsNumeric(In SD conc) Then

Cs_SD_total $\equiv(\bar{C} s$ SD_total $+\operatorname{CDbl}(I n$ SD_conc) $) /(C s$ SD_D1 + 1)

Cs_SD_Avg = Cs_SD_total

Exīt Function

Else

If Cs SD total $=0$ Then

$\mathrm{Cs}^{-} \mathrm{SD}^{-} \mathrm{Avg}=\mathrm{Null}$

Exit Function

Else

Cs_SD_Avg = Cs_SD_total

Exīt Function

End If

End If

End If

End Function

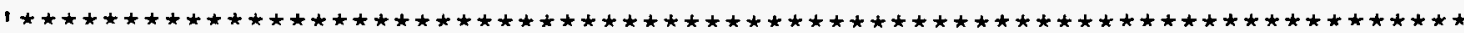

Private Function CsNew_SD_Avg (ByVal In_SD_isotope As Variant, ByVal In_SD_con c As Variant) As Variañt

If IsNumeric(In SD conc) Then

CsNew SD_Avg ${ }^{-}=\bar{C}$ Dbl (In SD conc)

Exit $\bar{F} u n \bar{c} t i o n$

Else

CsNew_SD_Avg $=\mathrm{Null}$ 
End If

Exit Function

End Function

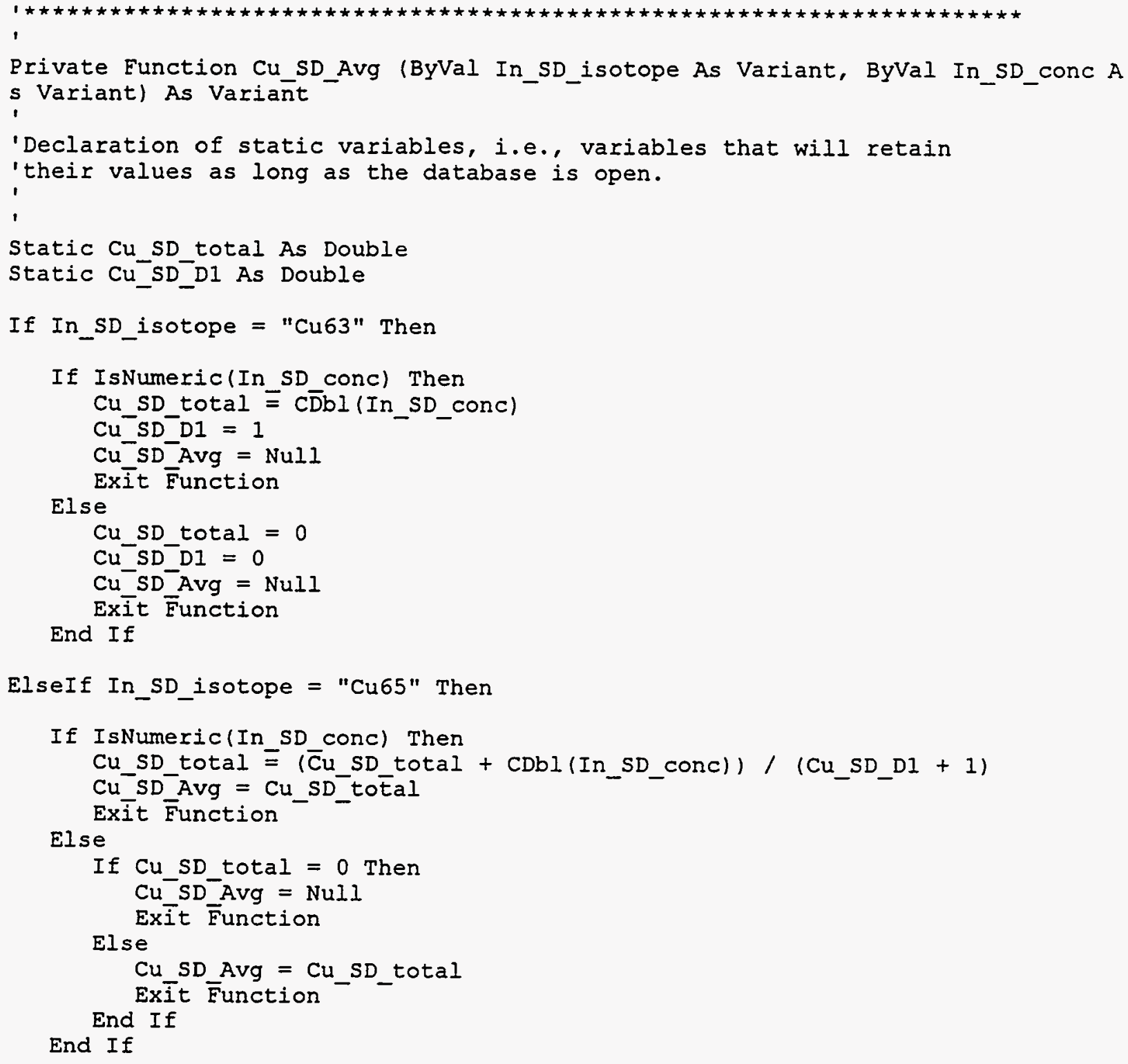

End If

End Function

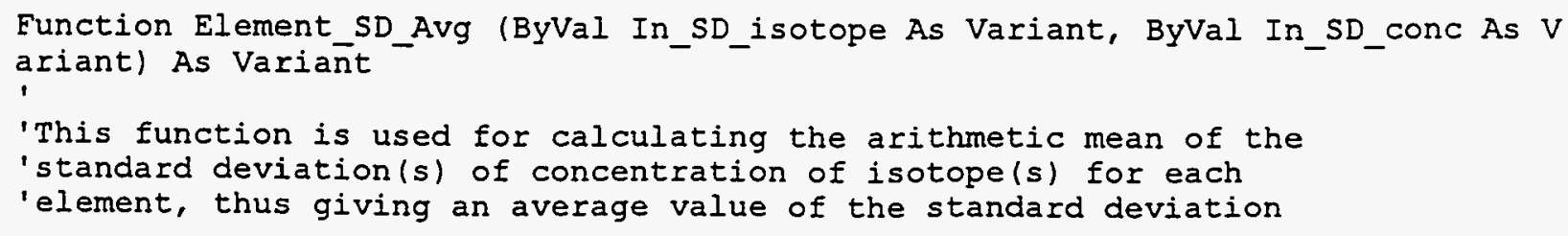




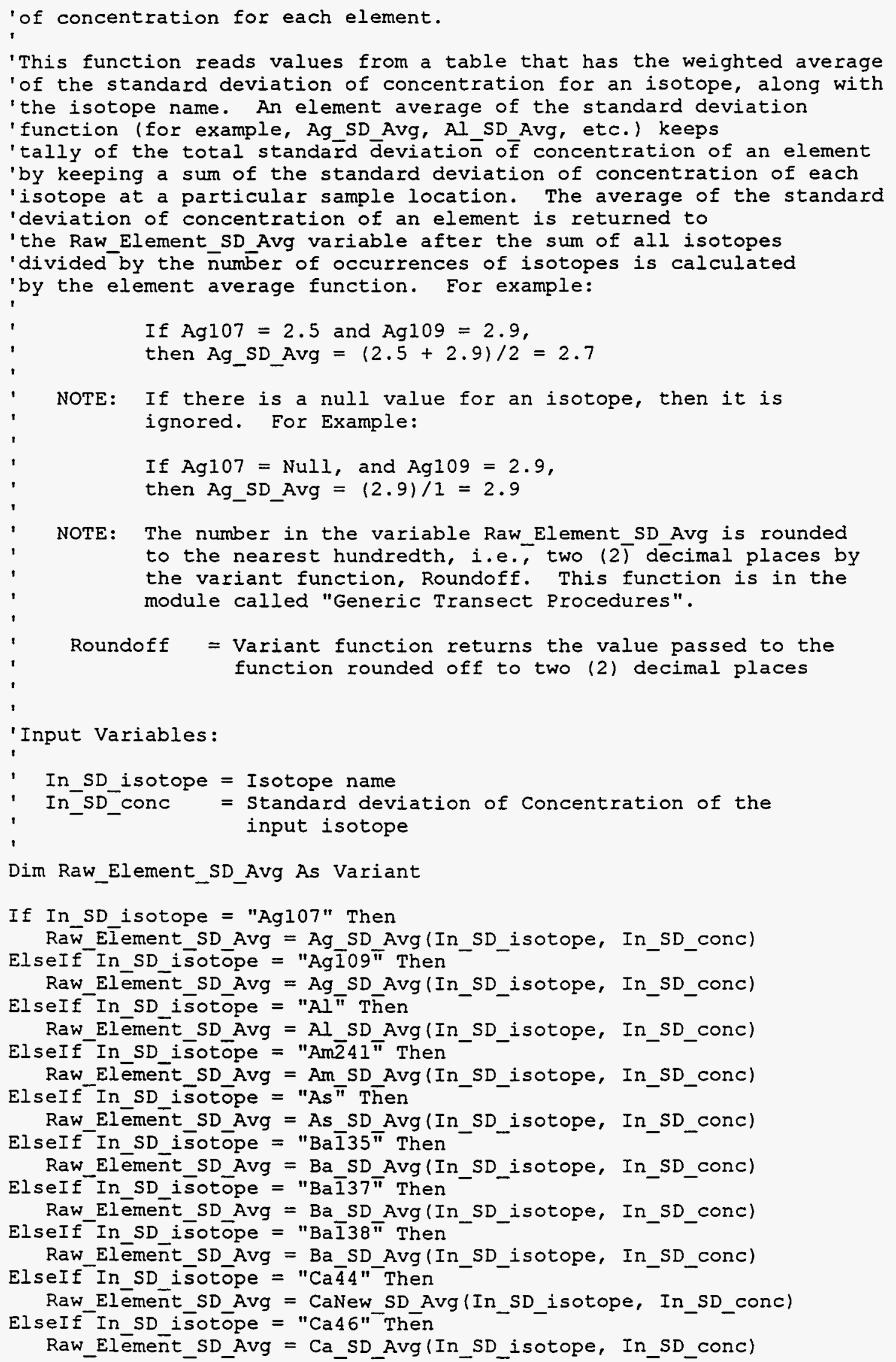


ElseIf In SD isotope $=$ "Ca48" Then

Raw Element SD Avg $=$ Ca SD Avg (In SD_isotope, In SD conc)

ElseIf In SD isotope = "Cdil11" Then

Raw Elemeñt SD_Avg = Cd SD_Avg (In_SD_isotope, In_SD_conc)

ElseIf In SD isotope $=$ "Cdī14" Then

Raw Element SD Avg = Cd SD Avg (In SD isotope, In_SD_conc)

ElseIf In SD is sotope $=$ "Cl" Then

Raw_Element_SD_Avg $=\mathrm{Cl}$ SD_Avg (In_SD_isotope, In_SD_conc)

ElseIf In SD isotope $=" \mathrm{Cm} \overline{2} 43^{\overline{1}}$ Then

Raw_Elemeñt_SD_Avg $=$ Cm_SD_Avg (In_SD_isotope, In_SD_conc)

ElseIf In_SD_isotope $=$ "Cm $\overline{2} 45 "$ Then

Raw_Elemeñt_SD_Avg $=C m$ SD_Avg (In_SD_isotope, In_SD_conc)

ElseIf In SD isotope $=$ "Co" Then

Raw_Elementt_SD_Avg $=$ Co_SD_Avg (In_SD_isotope, In_SD_conc)

ElseIf In_SD_isotope $=$ "Cr52" Then

Raw Elemeñt SD Avg $=C r$ SD_Avg (In_SD_isotope, In_SD_conc)

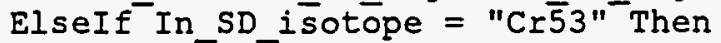

Raw_Element_SD_Avg $=C r$ SD_Avg (In_SD_isotope, In_SD_conc)

ElseIf In_SD_išotope $=$ "Cs" Then

Raw Elementt SD Avg = CsNew SD Avg (In_SD isotope, In_SD_conc)

ElseIf In_SD_isotope $=$ "Cs137" Then

Raw_Element_SD_Avg $=$ Cs_SD_Avg (In_SD_isotope, In_SD_conc)

ElseIf In SD isotope $=$ "Cuб 3 " Then

Raw_Element SD_Avg $=\mathrm{Cu} S \mathrm{SD}_{\text {Avg }}$ (In_SD_isotope, In_SD_conc)

ElseIf In_SD_išotope $=$ "Cu $\overline{6} 5$ " Then

Raw_ElementtSD_Avg $=C u$ SD_Avg (In_SD_isotope, In_SD_conc)

ElseIf In SD isotope $=$ "F" Then

Raw_Element_SD_Avg $=F$ _SD_Avg (In_SD_isotope, In_SD_conc)

ElseIf In_SD_isotope $=" \overline{\mathrm{E}} "$ Then

Raw Elementt SD Avg = Fe SD Avg (In SD isotope, In SD conc)

ElseIf-In_SD_is̄otope = "grōss'alpha" Then

Raw_Elemeñt_SD_Avg = gross_alpha_SD_Avg (In_SD_isotope, In_SD_conc)

ElseIf In SD isotope = "gross beta" Then Raw_ElementtSD_Avg = grossbbeta_SD_Avg(In_SD_isotope, In_SD_conc)

ElseIf-In_SD_is̄otope $=$ "H3" Then

Raw Elementt SD Avg $=$ H3 SD Avg (In SD isotope, In SD conc)

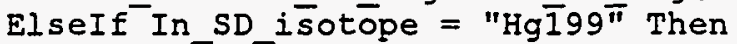
Raw_Element_SD_Avg $=$ Hg_SD_Avg (In_SD_isotope, In_SD_conc)

ElseIf In_SD_i sotope $=$ "Hg$\overline{2} 00 \bar{"}$ Then Raw Elemeñt SD Avg $=\mathrm{Hg} S \mathrm{SD}$ Avg (In_SD_isotope, In_SD_conc)

ElseIf In_SD_is̄otope $=$ "Hg$\overline{2} 01 \bar{"}$ Then Raw_Element_SD_Avg $=$ Hg_SD_Avg (In_SD_isotope, In_SD_conc)

Elself In SD isotope $=$ "Hg$\overline{2} 02 \bar{\pi}$ Then Raw_Elemeñt_SD_Avg $=\mathrm{Hg} S \mathrm{SD} A \mathrm{Avg}$ (In_SD_isotope, In_SD_conc)

ElseIf In_SD_isotope $=$ " $K "$ Then Raw_Element_SD_Avg $=K$ SD_Avg (In_SD_isotope, In_SD_conc)

ElseIf In SD isotope $=$ "Mğ24" Then Raw_Elemeñt_SD_Avg $=$ MgNew_SD_Avg (In_SD_isotope, In_SD_conc)

ElseIf In_SD i sotope = "Mg25" Then Raw Element SD Avg = MgNew SD Avg (In_SD isotope, In_SD_conc)

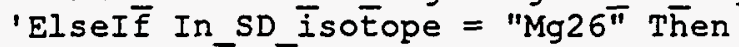

- Raw_ElementtSD_Avg = Mg_SD_Avg (In_SD_isotope, In_SD_conc)

ElseIf In_SD_isotope $=$ "Mn" Then

Raw Elemeñt SD Avg $=M n$ SD Avg (In_SD_isotope, In_SD_conc)

ElseIf In_SD_isotope $=$ "Na" Then

Raw_Elemeñt_SD_Avg $=\mathrm{Na} S \mathrm{SD} A v g$ (In_SD_isotope, In_SD_conc)

ElseIf In SD isotope $=$ "Ni $\overline{6} 0 "$ "Then Raw_Element $S D$ Avg $=$ NiNew_SD_Avg (In_SD_isotope, In_SD_conc)

ElseIf In SD isotope = "Ni61" Then Raw_Elemeñt_SD_Avg $=N i$ SD_Avg (In_SD_isotope, In_SD_conc) 
ElseIf In_SD isotope = "Ni62" Then

Raw_Elemeñt_SD_Avg $=$ NiNew_SD_Avg (In_SD_isotope, In_SD_conc)

ElseIf In SD isotōpe = "NO2" Then

Raw_Element_SD_Avg $=$ NO2 SD_Avg (In_SD_isotope, In_SD_conc)

ElseIf In SD isotope = "NO3" Then

Raw_Element_SD_Avg $=N O 3$ SD_Avg (In_SD_isotope, In_SD_conc)

ElseIf In SD isotope $=$ "Np2 $\overline{3} 7{ }^{-}$Then

Raw_Elemeñt_SD_Avg $=$ Np_SD_Avg (In_SD_isotope, In_SD_conc)

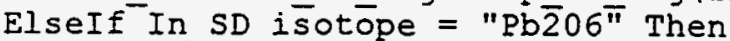

Raw_Element_SD_Avg $=\mathrm{Pb} S \mathrm{PD}_{\text {Avg }}$ (In_SD_isotope, In_SD_conc)

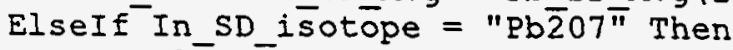

Raw_ElementtSD_Avg $=\mathrm{Pb}_{\mathrm{e}} \mathrm{SD}$ Avg (In_SD_isotope, In_SD_conc)

ElseIf In SD isotope $=" \mathrm{~Pb} \overline{2} 0 \overline{8}^{\bar{s}}$ Then

Raw_Element_SD_Avg $=$ Pb_SD_Avg (In_SD_isotope, In_SD_conc)

ElseIf In SD isotope = "POA " Then

Raw_Elementt_SD_Avg $=$ PO4_SD_Avg(In_SD_isotope, In_SD_conc)

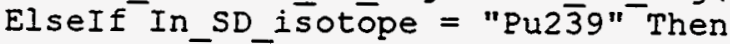

Raw_Elemeñt_SD_Avg $=$ Pu_SD_Avg (In_SD_isotope, In_SD_conc)

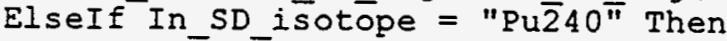

Raw_Elementt_SD_Avg $=$ Pu_SD_Avg (In_SD_isotope, In_SD_conc)

ElseIf In SD isotope = "Pu' $42 \overline{2}$ Then

Raw_Elementt_SD_Avg $=$ Pu_SD_Avg (In_SD_isotope, In SD_conc)

ElseIf In_SD_isotope $=$ "Ra $\overline{2} 26$ " Then

Raw_Elementt_SD_Avg $=$ Ra_SD_Avg (In_SD_isotope, In_SD_conc)

ElseIf In SD isotope $=$ "Se $\overline{7} 7{ }^{-}$Then

Raw_Elemeñt_SD_Avg = Se_SD_Avg (In_SD_isotope, In_SD_conc)

ElseIf In SD isotope = "Se $\overline{8} 2$ " Then

Raw_Element_ SD_Avg = Se_SD_Avg (In_SD_isotope, In SD conc)

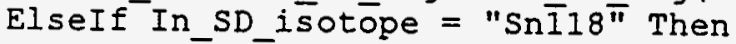

Raw_Elemeñt_SD_Avg $=S n \_S D \_A v g$ (In_SD_isotope, In_SD_conc)

ElseIf In_SD_isotope $=$ "Sn $\overline{1} 20 "$ Then

Raw_Element_SD_Avg $=$ Sn_SD_Avg (In_SD_isotope, In_SD_conc)

ElseIf In SD isotope = "SOT" Then

Raw_Element_SD_Avg $=$ SO4_SD_Avg (In_SD_isotope, In_SD_conc)

ElseIf In SD isotope = "Sr" Then

Raw_Element_SD_Avg $=$ SrNew_SD_Avg (In_SD_isotope, In_SD_conc)

ElseIf In SD isotōpe = "Sr90" Then

Raw_Elemeñt_SD_Avg $=S r$ SD_Avg (In_SD isotope, In SD_conc)

ElseIf In_SD_isotope $=$ "Th $\overline{2} 32 \overline{2}$ Then

Raw_Elementt_SD_Avg $=T h \_S D$ Avg (In_SD_isotope, In_SD_conc)

ElseIf In SD isotope = "Ti" Then

Raw_Element_SD_Avg $=T i \_S D$ Avg (In_SD_isotope, In_SD_conc)

ElseIf In_SD isotope = "U2 $\overline{3} 4$ " Then

Raw_Element_SD_Avg $=U$ SD_Avg (In_SD_isotope, In_SD_conc)

ElseIf In_SD_isotope $=$ "U⿳亠丷厂 $35^{\overline{1}}$ Then

Raw_Element_SD_Avg $=U 235$ SD_Avg (In_SD_isotope, In_SD_conc)

ElseIf In_SD_isotope $=$ "U238" Then

Raw_Element_SD_Avg $=0238$ SD_Avg (In_SD_isotope, In_SD_conc)

ElseIf In_SD_isotope = "V" Then

Raw_Element_SD_Avg $=V$ V SD_Avg (In_SD_isotope, In_SD_conc)

ElseIf In SD isotōpe $=$ "Y" Then

Raw_Element_SD_Avg $=Y$ YSD_Avg (In_SD_isotope, In_SD_conc)

ElseIf In_SD_isotope $=$ "Zñ $64 \bar{"}$ Then

Raw_Element_SD_Avg $=\mathrm{Zn} S \mathrm{SD} A v g$ (In_SD_isotope, In SD conc)

ElseIf In_SD_isotope $=$ " $\ln \bar{\sigma} \sigma "$ Then

Raw_Elementt_SD_Avg $=$ ZnNew_SD_Avg (In_SD_isotope, In_SD_conc)

ElseIf In_SD_isotope $=" \mathrm{Zn} 67{ }^{\prime}$ Then

Raw_Element_SD_Avg $=\mathrm{Zn} S \mathrm{SD}_{\mathrm{Avg}}$ (In_SD_isotope, In SD conc)

ElseIf In SD isotope = "Zrḡo" Then

Raw_Element_SD_Avg $=$ Zr_SD_Avg (In_SD_isotope, In_SD_conc) 
ElseIf In_SD_isotope = "Zr91" Then

Raw_Elemeñt_SD_Avg $=2 r$ SD_Avg (In_SD_isotope, In_SD_conc)

End If

Element_SD_Avg $=$ Roundoff (Raw_Element_SD_Avg)

End Function

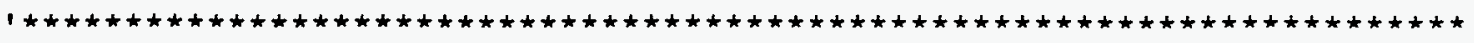

Private Function F_SD_Avg (Byval In_SD_isotope As Variant, ByVal In_SD_Conc As Variant) As Variant

If IsNumeric(In SD conc) Then

E_SD_Avg $=C \bar{D} b l\left(I n \_S D \_c o n c\right)$

Else

Exit Eunction

E_SD_Avg $=$ Null

Exit Function

End If

End Function

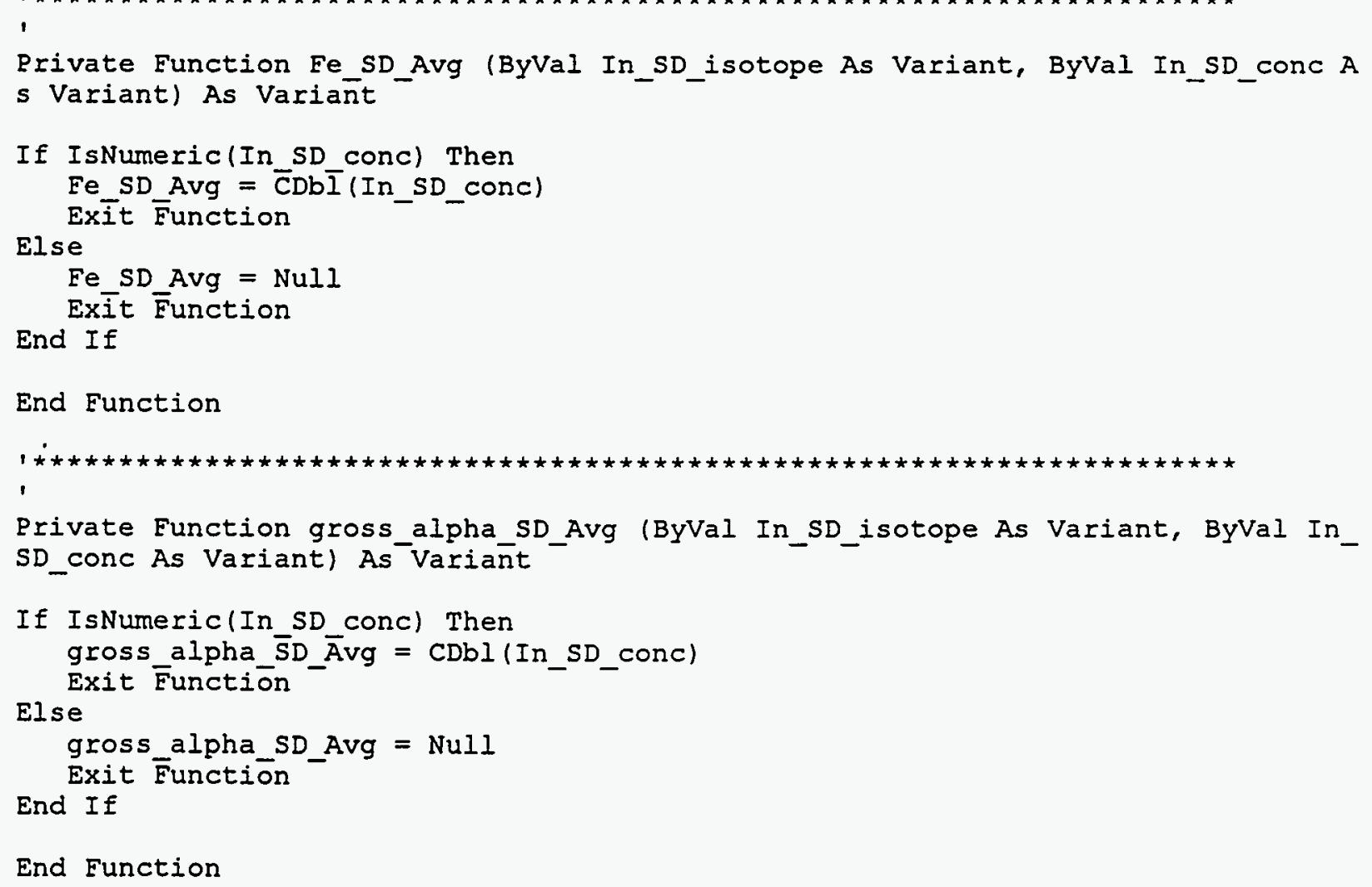

End Function

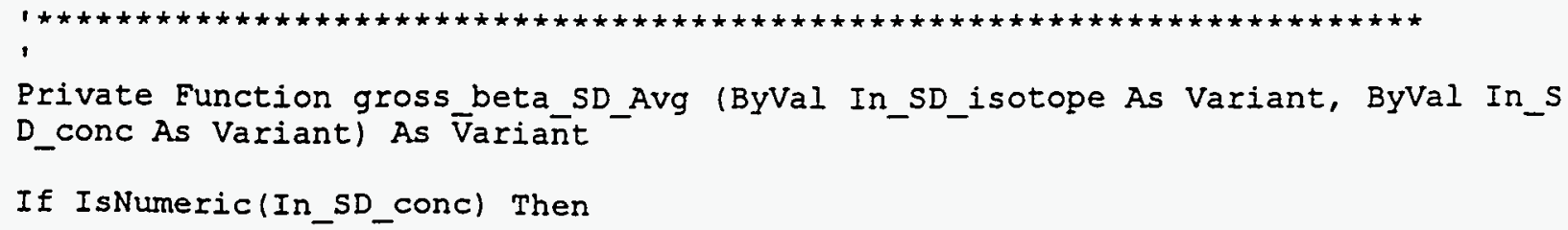




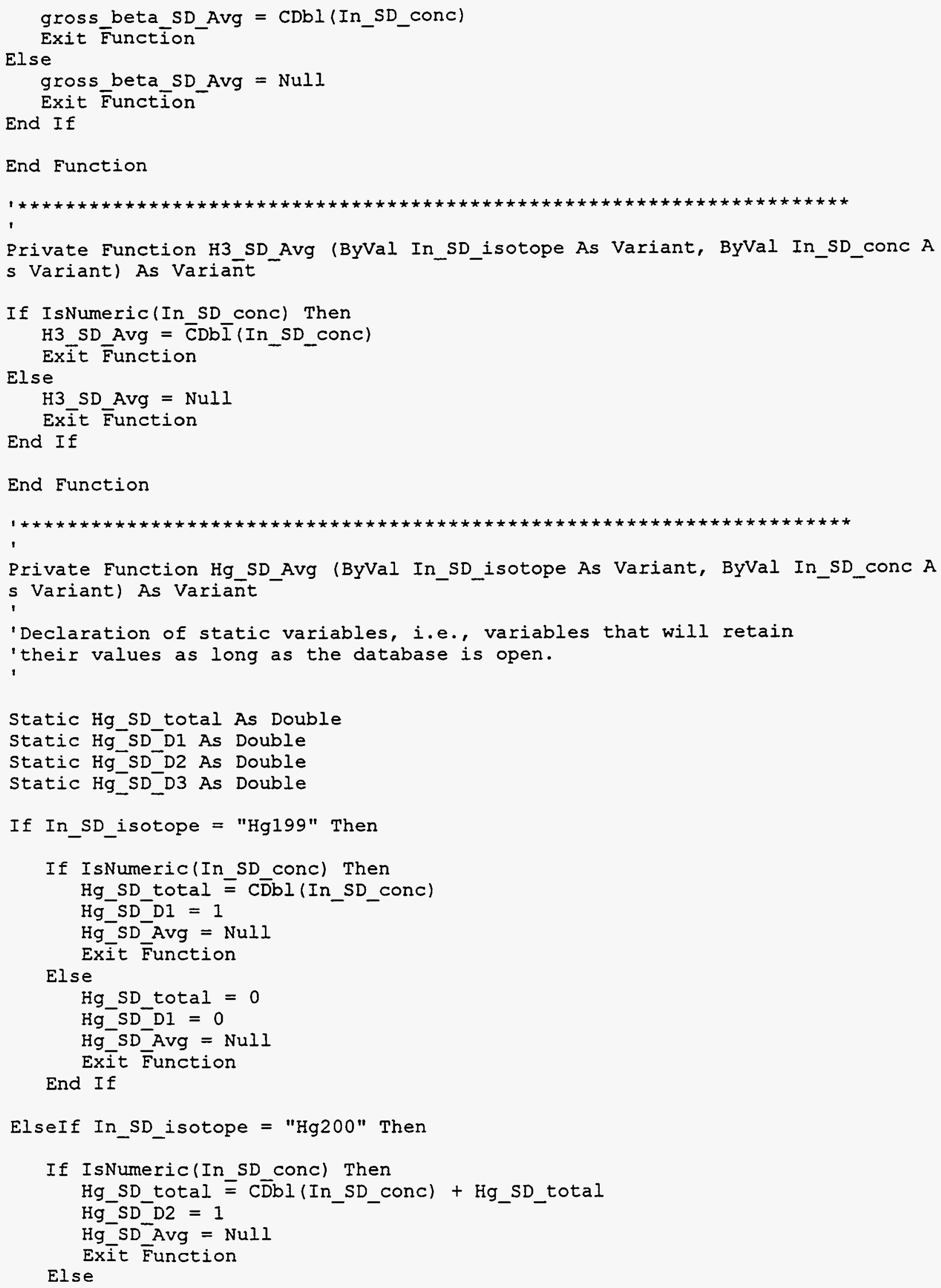




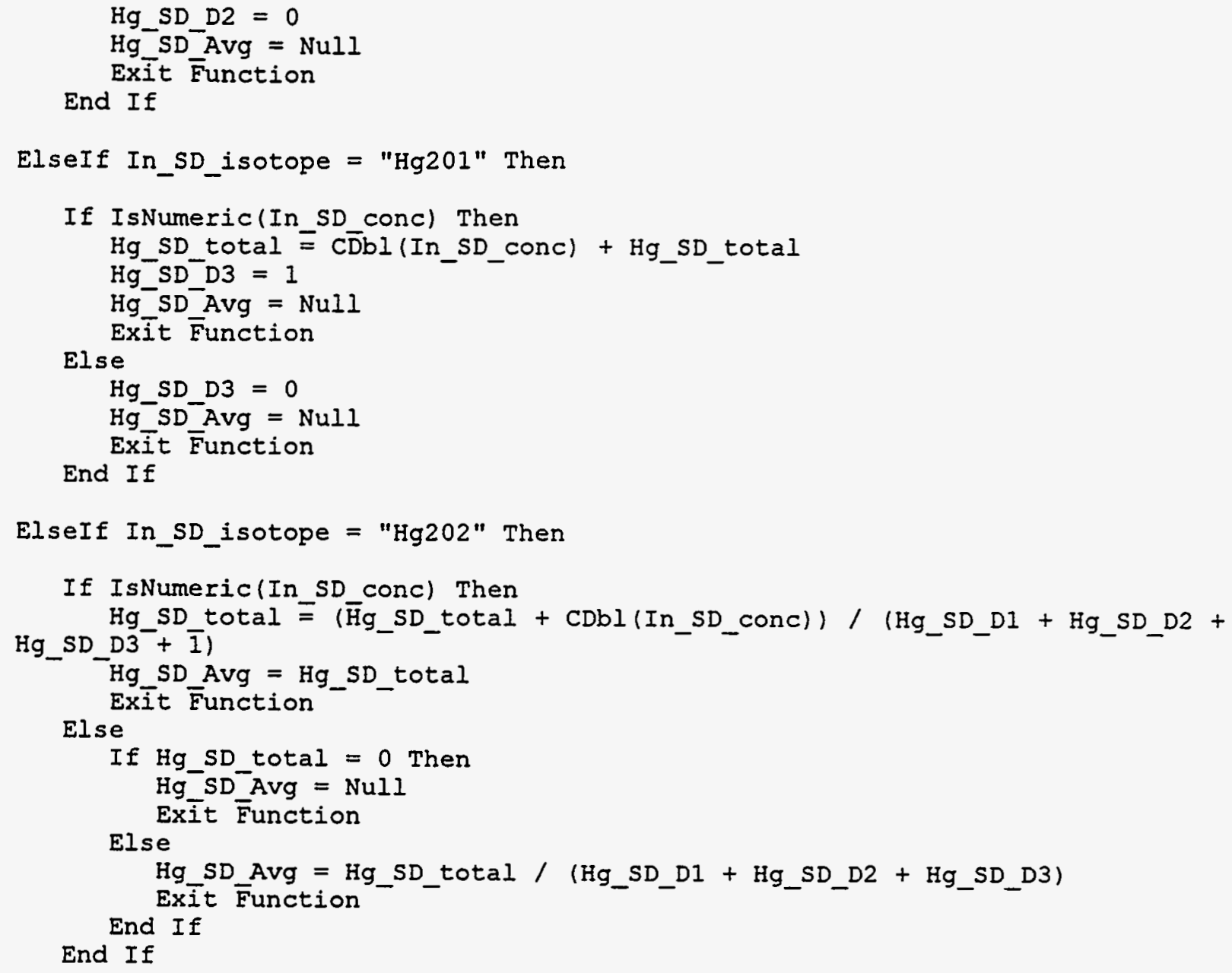

Private Function K_SD_Avg (ByVal In_SD_isotope As Variant, ByVal In_SD_conc As Variant) As Variañt

If IsNumeric(In SD conc) Then

K_SD_Avg $=$ CDDITIn_SD_conc)

Else

Exit Function

K_SD_Avg $=$ Null

Exit Function

End If

End Function

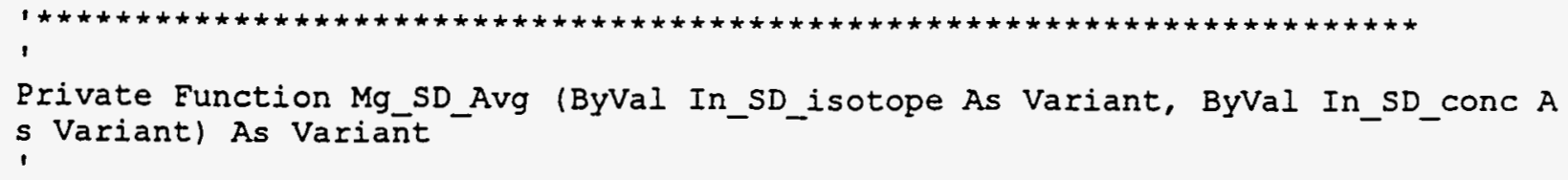


'Declaration of static variables, i.e., variables that will retain

'their values as long as the database is open.

Static Mg_SD_total As Double

Static Mg_SD_D1 As Double

static Mg_SD_D2 As Double

If In_SD_isotope $=$ "Mg24" Then

If IsNumeric(In_SD conc) Then

$M g$ SD_total $\equiv C \bar{D} b l\left(I n \_S D \_c o n c\right)$

Mg_SD_D1 $=1$

$\mathrm{Mg}^{-} \mathrm{SD}^{-}$Avg $=\mathrm{Null}$

Else

Exit Function

Mg_SD_total $=0$

Mg_SD_D1 = 0

$\mathrm{Mg} S D^{-} \mathrm{Avg}=\mathrm{Null}$

Exit Function

End If

ElseIf In_SD_isotope $=$ "Mg25" Then

If IsNumeric(In SD_conc) Then

Mg_SD_total $\equiv C \overline{D b}($ In_SD_conc) + Mg_SD_total

Mg_SD_D2 $=1$

$\mathrm{Mg}^{-} \mathrm{SD}^{-} \mathrm{Avg}=\mathrm{Null}$

Else

Exit $\bar{F}$ unction

$\mathrm{Mg} \mathrm{SD} \mathrm{D2}=0$

$\mathrm{Mg}^{-} \mathrm{SD}^{-} \mathrm{Avg}=\mathrm{NuIl}$

Exit $\overline{\text { Function }}$

End If

ElseIf In_SD_isotope $=" \mathrm{Mg} 26 "$ Then

If IsNumeric(In_SD_conc) Then

1)

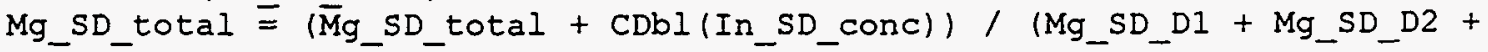

Mg_SD_Avg $=$ Mg_SD_total

Else

Exit Function

If Mg_SD_total $=0$ Then

Mg_SD_Avg $=\mathrm{Null}$

Else

Exit Function

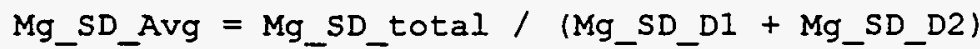

End If

Exit Function

End If

End If

End Function

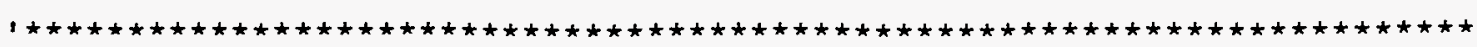

1 


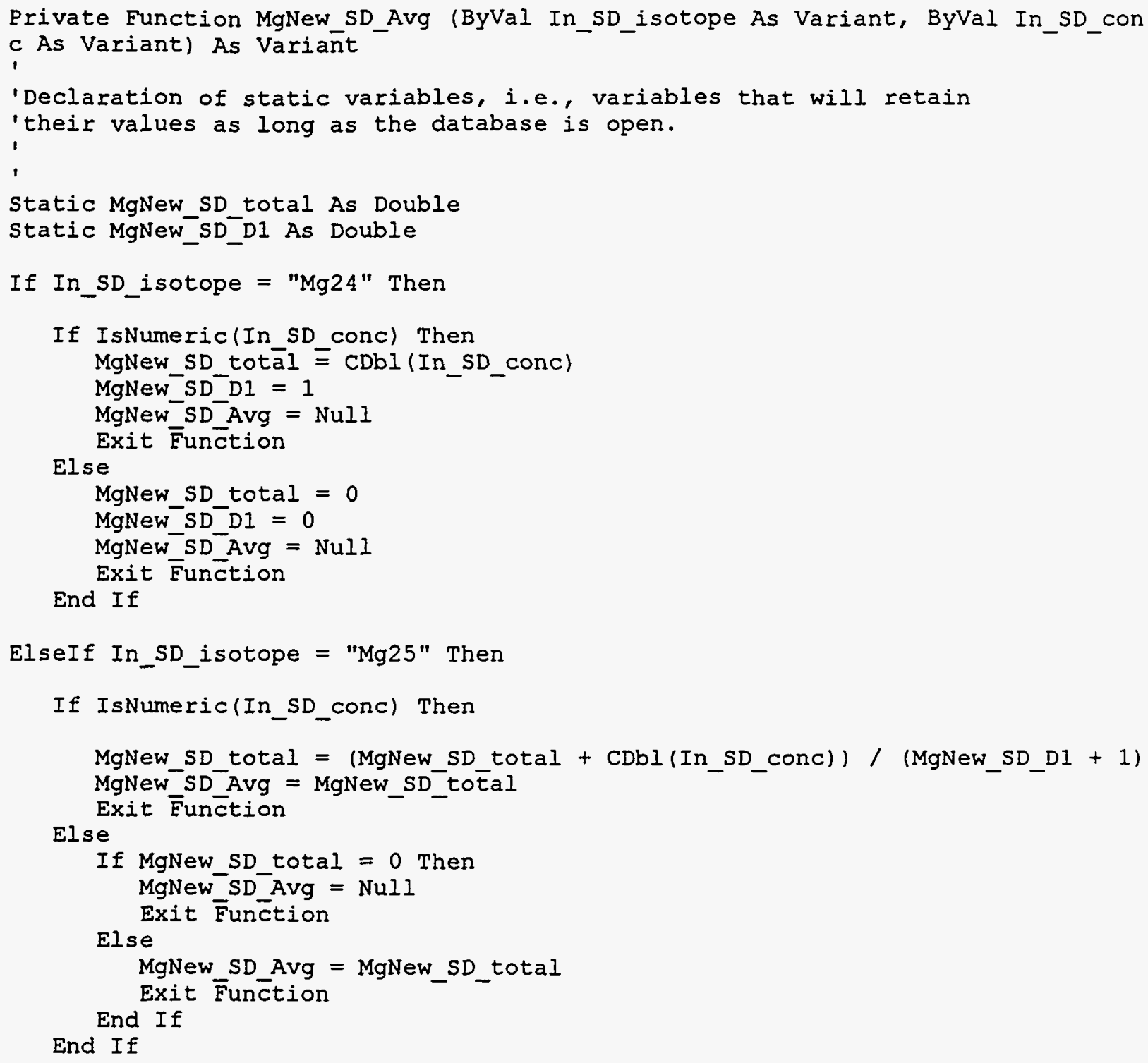

End If

End Function

,

Private Function Mn_SD_Avg (ByVal In_SD_isotope As Variant, ByVal In_SD_conc A $s$ Variant) As Variañt

If IsNumeric(In SD conc) Then Mn_SD_Avg $=\overline{C D b} \bar{l}\left(I n \_S D \_c o n c\right)$

Else

Exit $\overline{\text { Eunction }}$

Mn SD Avg $=\mathrm{Null}$

Exit $\overline{\text { Function }}$

End If

End Function 


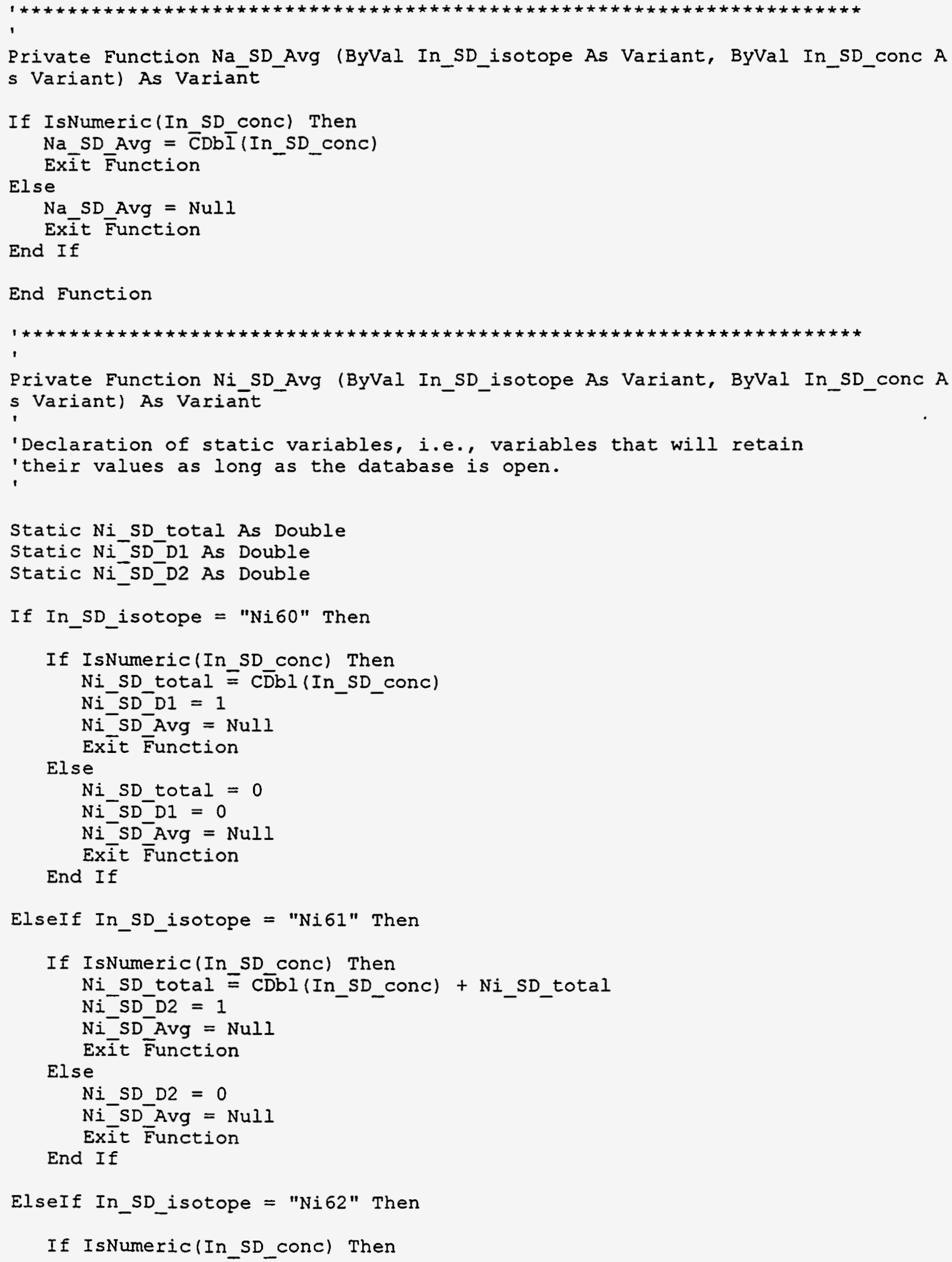


1)

$\mathrm{Ni}$ _SD_total $=\left(\mathrm{Ni} \mathrm{ND}_{-}\right.$total $+\mathrm{CDbl}\left(\mathrm{In} \_\mathrm{SD} \_\right.$conc $\left.)\right) /(\mathrm{Ni}$ _SD_D1 $+\mathrm{Ni}$ _SD_D2 +

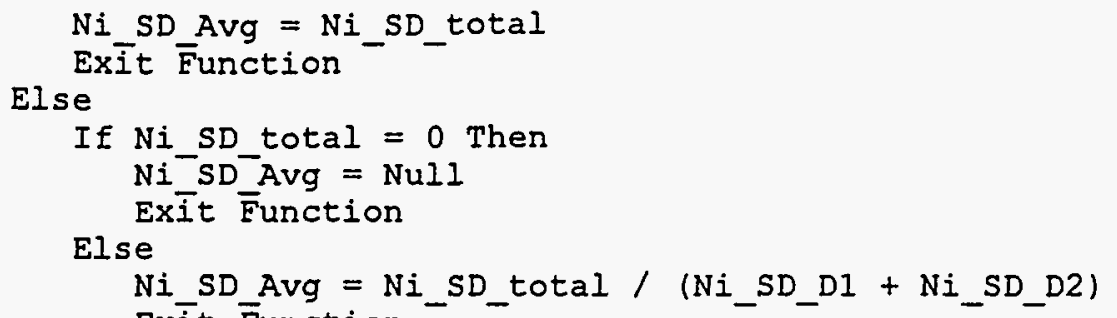

End If

End Function

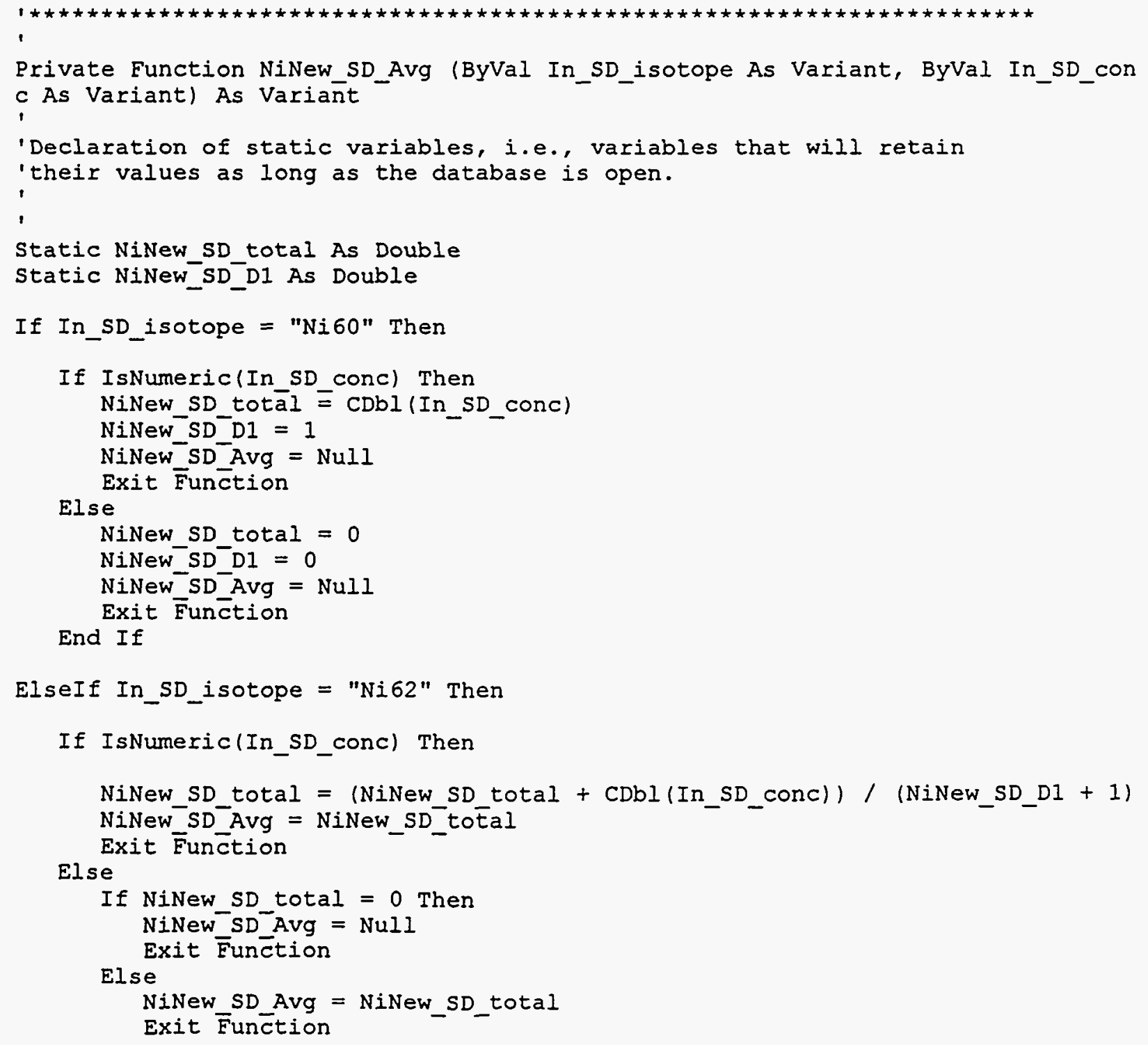


'their values as long as the database is open.

Static Pb_SD_total As Double

Static $\mathrm{Pb} S \mathrm{SD}$ - 1 As Double

static Pb_SD_D2 As Double

If In_SD_isotope $=$ "Pb206" Then

If IsNumeric(In_SD_conc) Then

$\mathrm{Pb} S D$ total $\equiv \mathrm{CD} \overline{\mathrm{Db}}$ (In SD_conc)

$\mathrm{Pb}-\mathrm{SD}^{-} \mathrm{DI}=1$

$\mathrm{Pb}^{-} \mathrm{SD}^{-} \mathrm{Avg}=\mathrm{Null}$

Exitt $\bar{F} u n c t i o n$

Else

Pb_SD_total $=0$

$\mathrm{Pb}-\mathrm{SD}-\mathrm{DI}=0$

$\mathrm{Pb}^{-} \mathrm{SD}^{-} \mathrm{Avg}=\mathrm{Null}$

End If

Exit F̄unction

ElseIf In_SD_isotope $=$ "Pb207" Then

If IsNumeric(In_SD_conc) Then

$\mathrm{Pb}$ SD_total $\equiv \mathrm{CD} \overline{\mathrm{D}} \mathrm{l}\left(\mathrm{In} \_\mathrm{SD}\right.$ _conc) + $\mathrm{Pb}$ SD_total

$\mathrm{Pb}^{-} \mathrm{SD}^{-} \mathrm{D} 2=1$

$\mathrm{Pb}^{-} \mathrm{SD}^{-} \mathrm{Avg}=\mathrm{Null}$

Else

Exit F̄unction

$\mathrm{Pb} S \mathrm{SD} 2=0$

$\mathrm{Pb}-\mathrm{SD}^{-} \mathrm{Avg}=\mathrm{Null}$

End If

Exitt Function

ElseIf In_SD_isotope $=$ "Pb208" Then

If IsNumeric(In_SD conc) Then

1)

Pb_SD_total $\equiv\left(\overline{\mathrm{Pb}} \mathrm{SD}_{\text {_total }}+\mathrm{CDbI}(\mathrm{In}\right.$ SD_conc $\left.)\right) /(\mathrm{Pb}$ SD_D1 + Pb_SD_D2 +

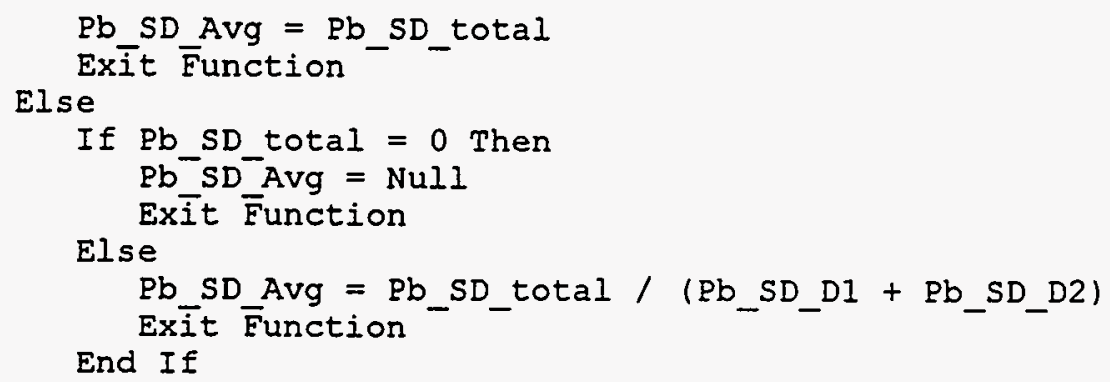

End If

End Function

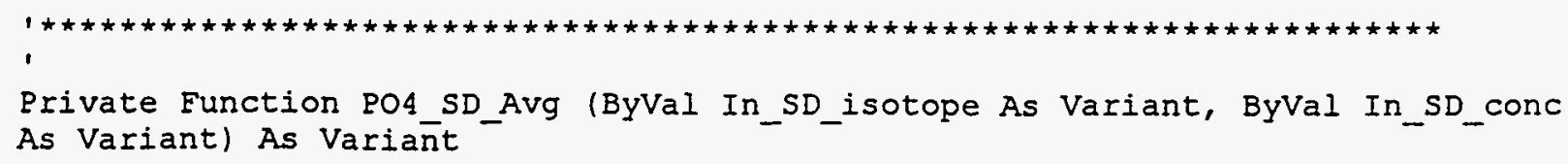


If IsNumeric(In_SD_conc) Then

PO4_SD_Avg $=-\mathrm{CD} \overline{\mathrm{b}}$ l(In_SD_COnC)

Exit Fünction

Else

PO4 SD Avg $=$ Null

Exit Function

End If

End Function

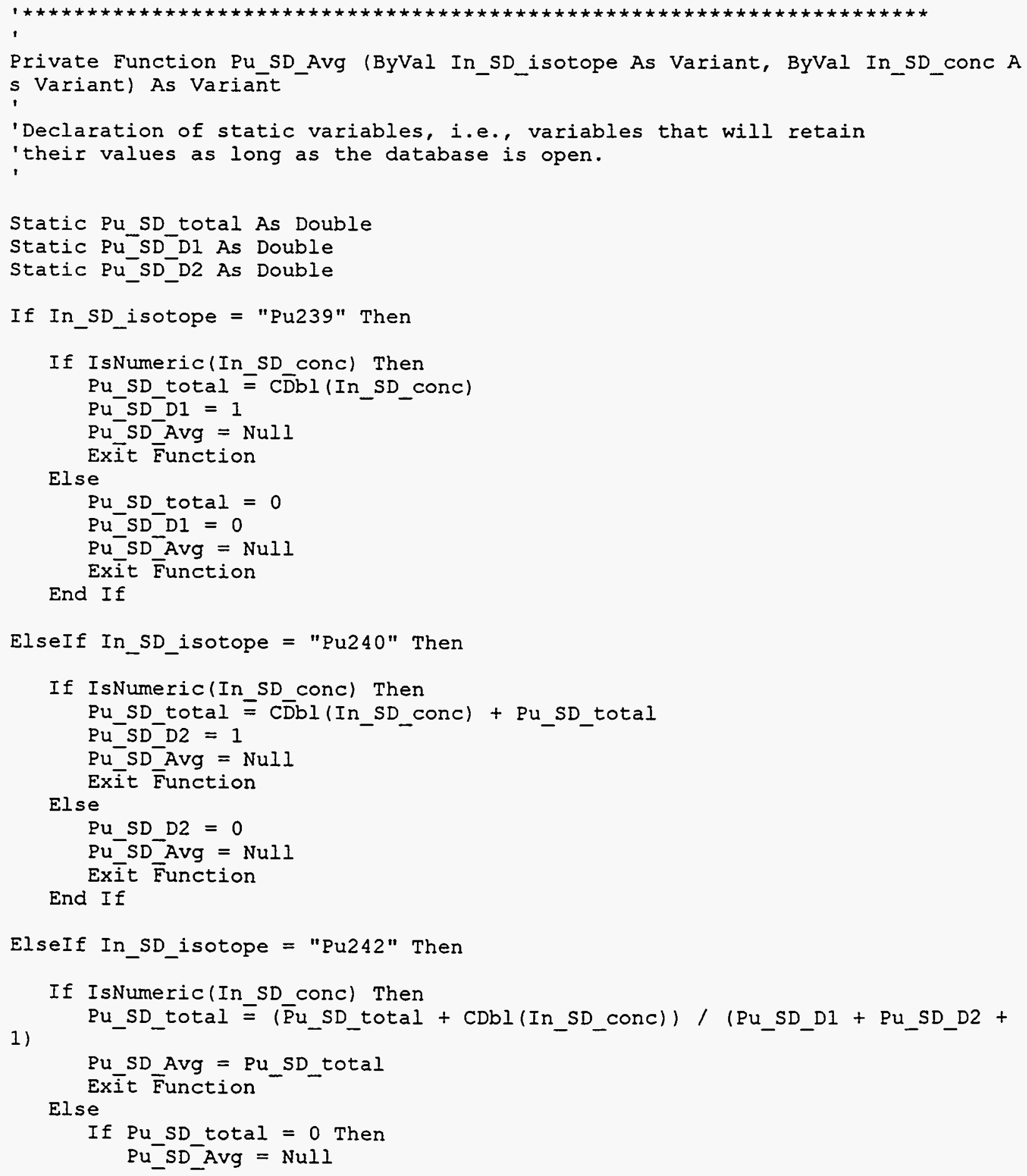




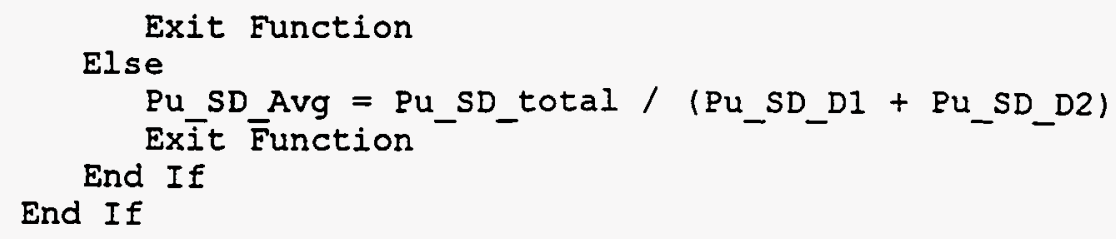

End Function

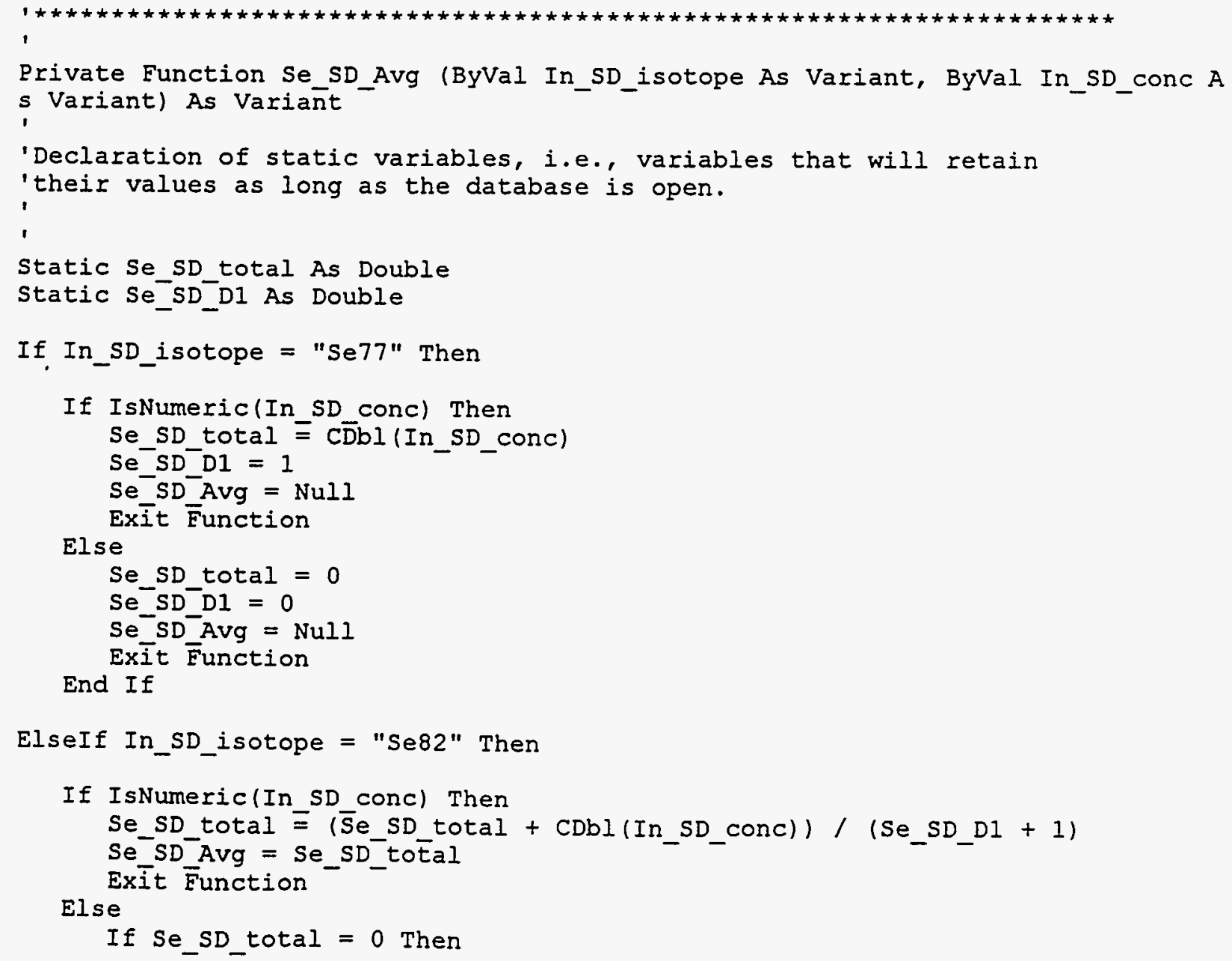




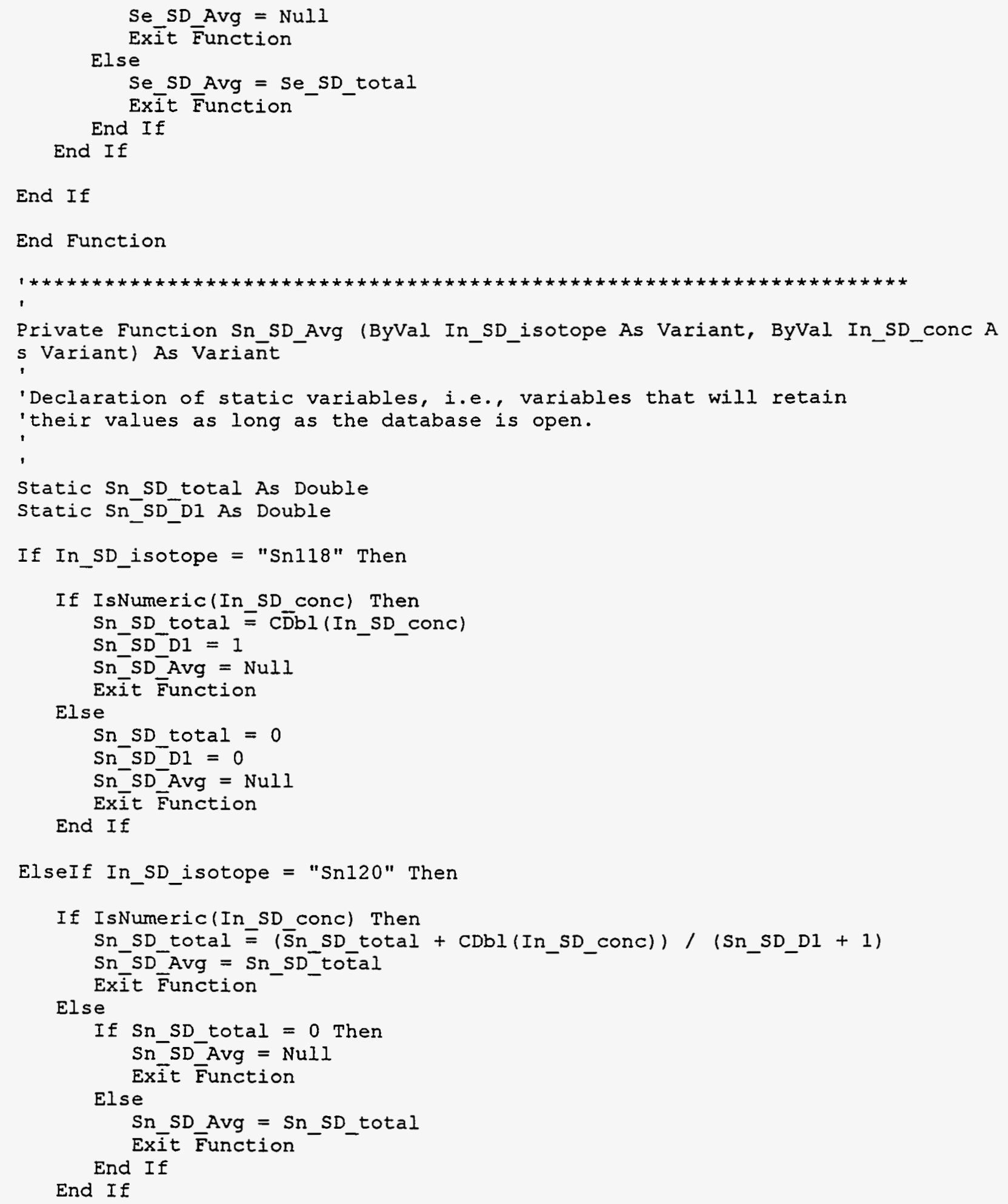

End If

End Function 
Private Function SO4_SD_Avg (ByVal In_SD_isotope As Variant, ByVal In_SD_conc As Variant) As Variañt

If IsNumeric(In SD conc) Then

SO4_SD_Avg $=-\mathrm{CD} \overline{\mathrm{b}} \mathbf{I}$ (In_SD_conc)

ExiE Fünction

Else

SO4_SD_Avg $=$ Null

Exit Function

End If

End Function

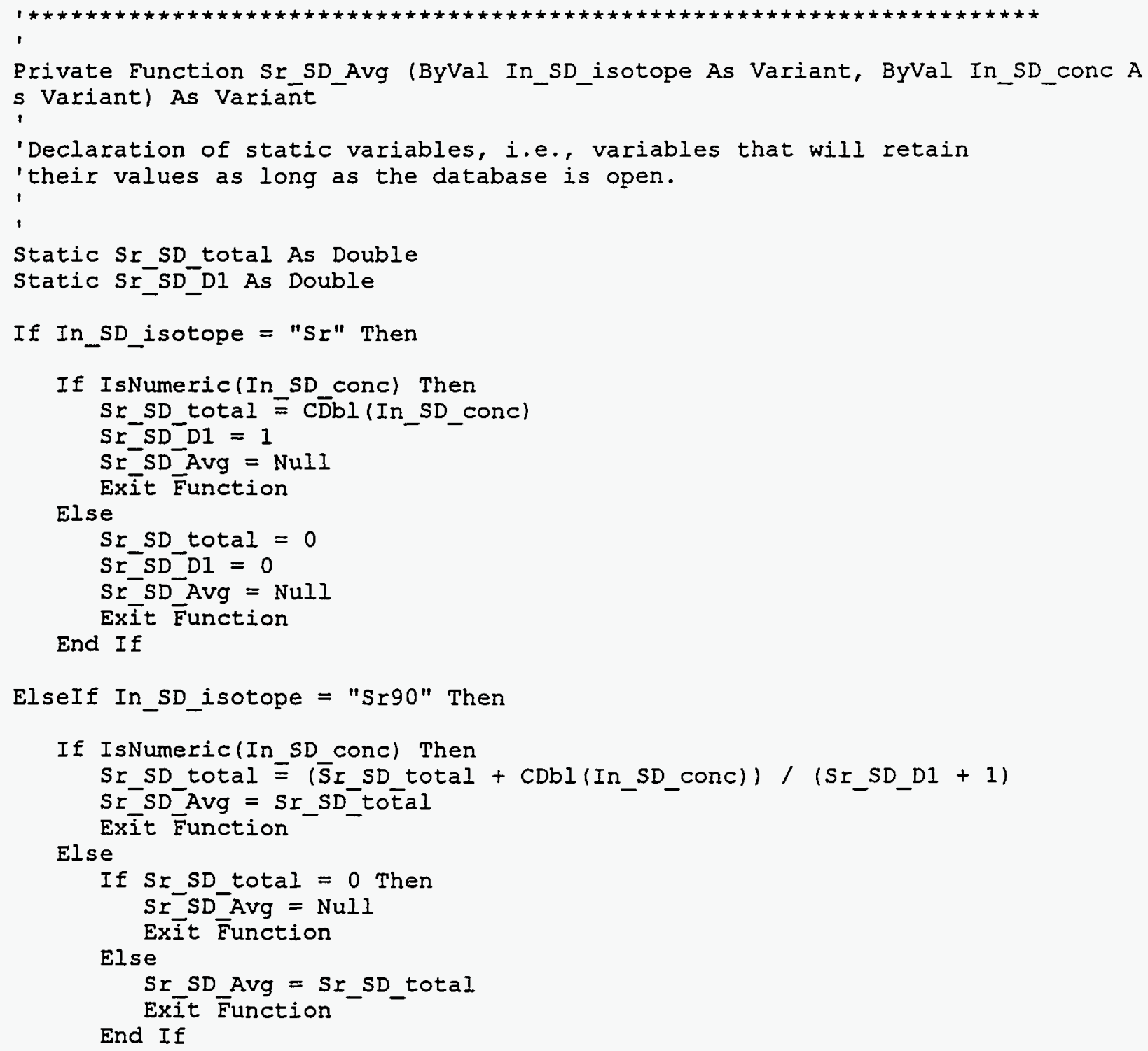

End If

End Function 
s

Private Function SrNew_SD_Avg (ByVal In_SD_isotope As Variant, ByVal In_SD_con c As Variant) As Variañt

If IsNumeric(In $S D$ conc) Then

SrNew_SD_Avg $=\bar{C}$ Dbl (In_SD_Conc)

Exit $\overline{\text { Function }}$

Else

SrNew_SD_Avg $=$ Null

Exit Function

End If

End Function

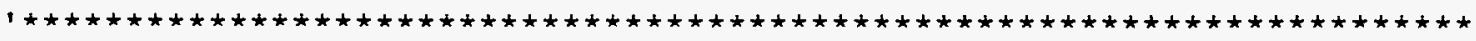

Private Function Th_SD_Avg (ByVal In_SD_isotope As Variant, ByVal In_SD_conc A $s$ Variant) As Variañt

If IsNumeric(In_SD_conc) Then

Th_SD_Avg $=\bar{C} D b \bar{I}$ (In_SD_ConC)

Exit Function

Else

Th_SD_Avg $=$ NulI

Exit Function

End If

End Function

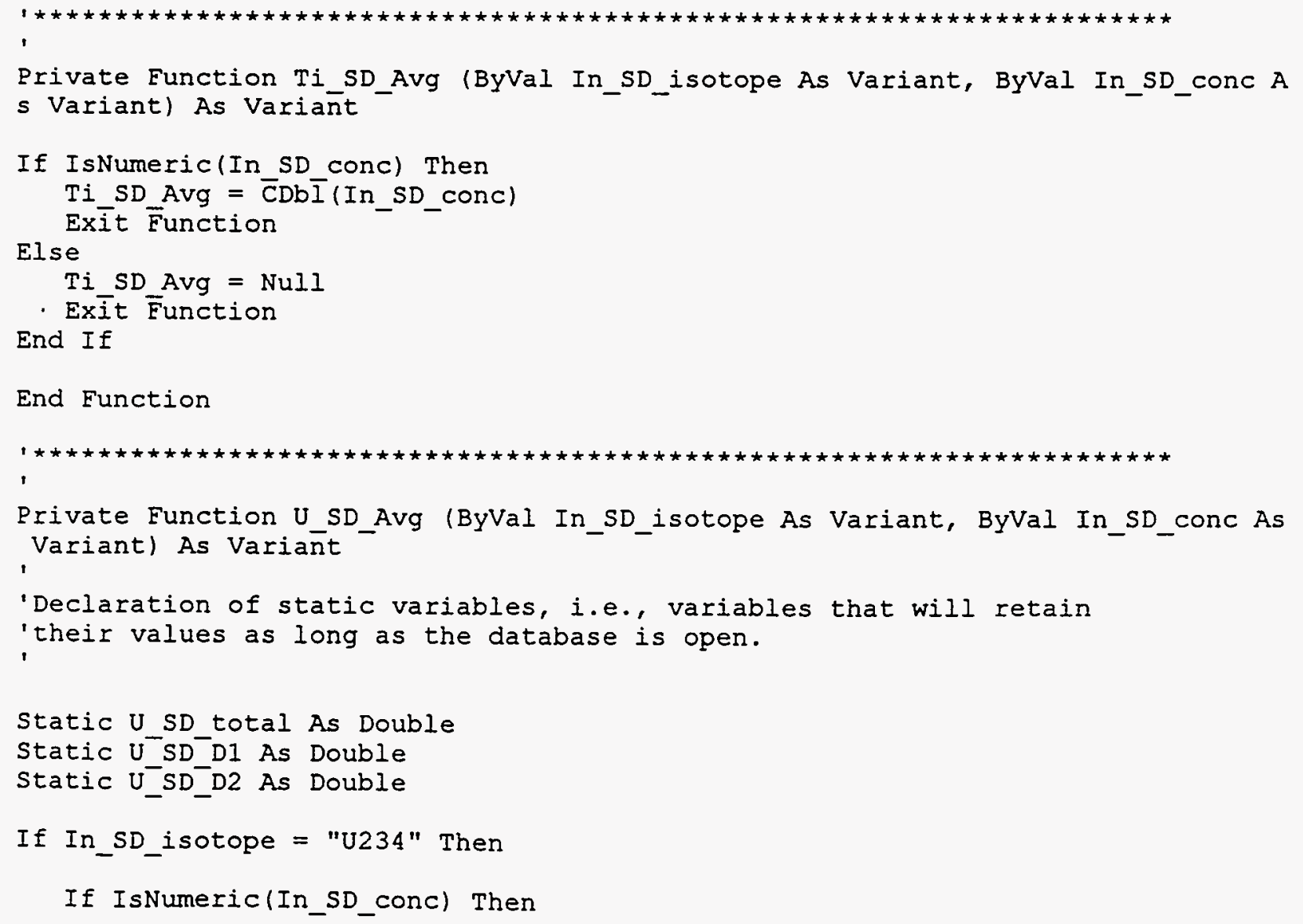




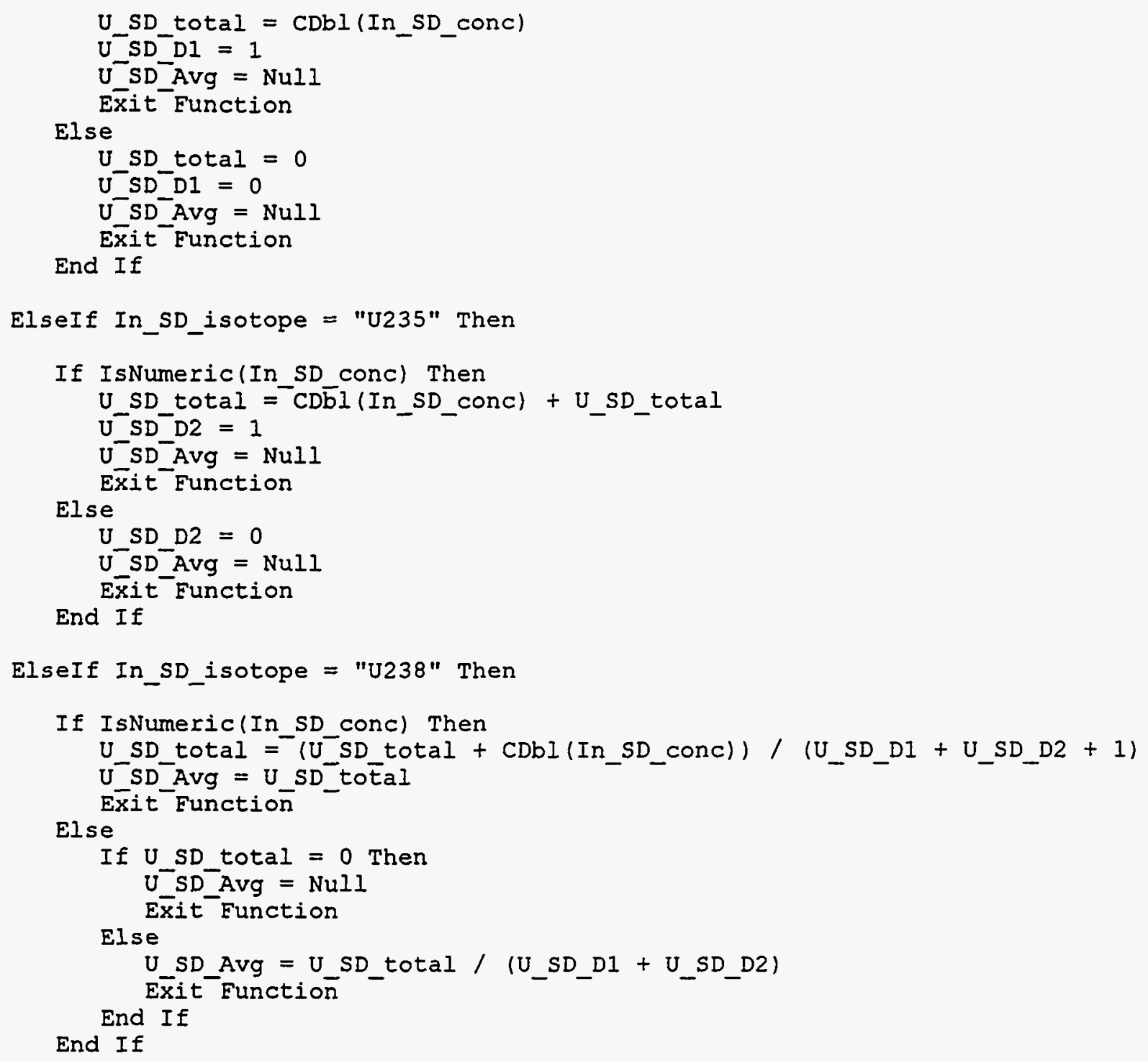

End If

End Function

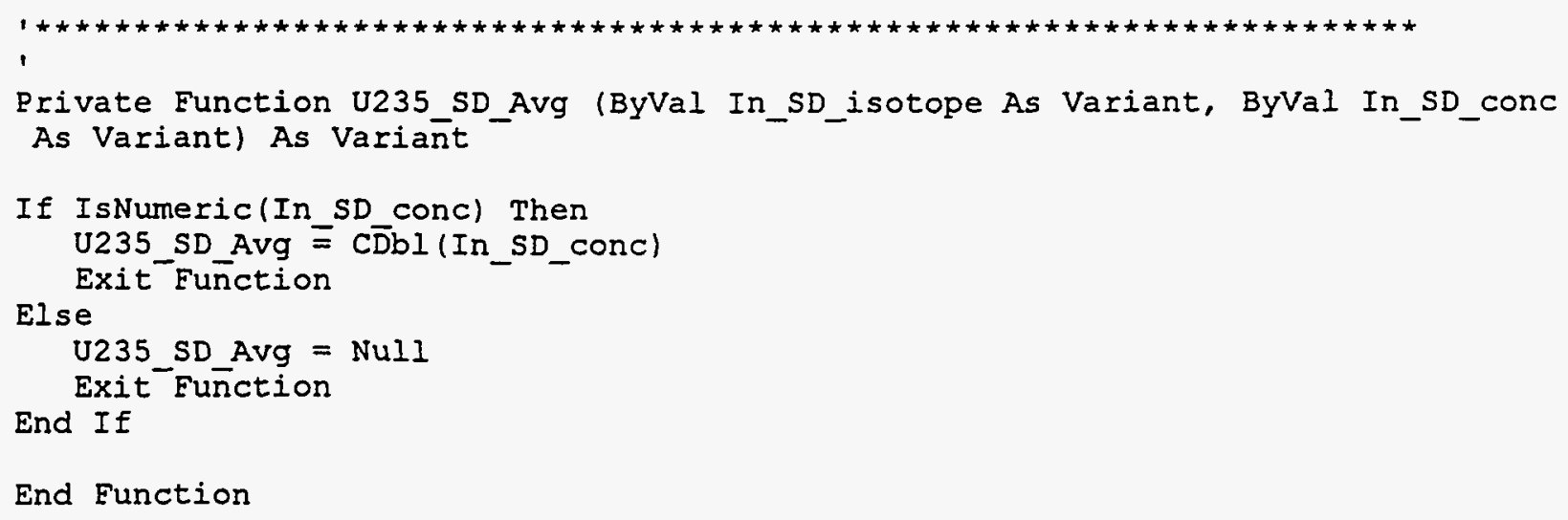


,

Private Function U238_SD_Avg (ByVal In_SD_isotope As Variant, ByVal In_SD_conc As Variant) As Variant

If IsNumeric(In SD conc) Then

U238 SD Avg $\equiv \mathrm{CDBl}$ (In_SD conc)

Exit Fuñction

Else

U238 SD_Avg $=\mathrm{Null}$

Exit Fuñction

End If

End Function

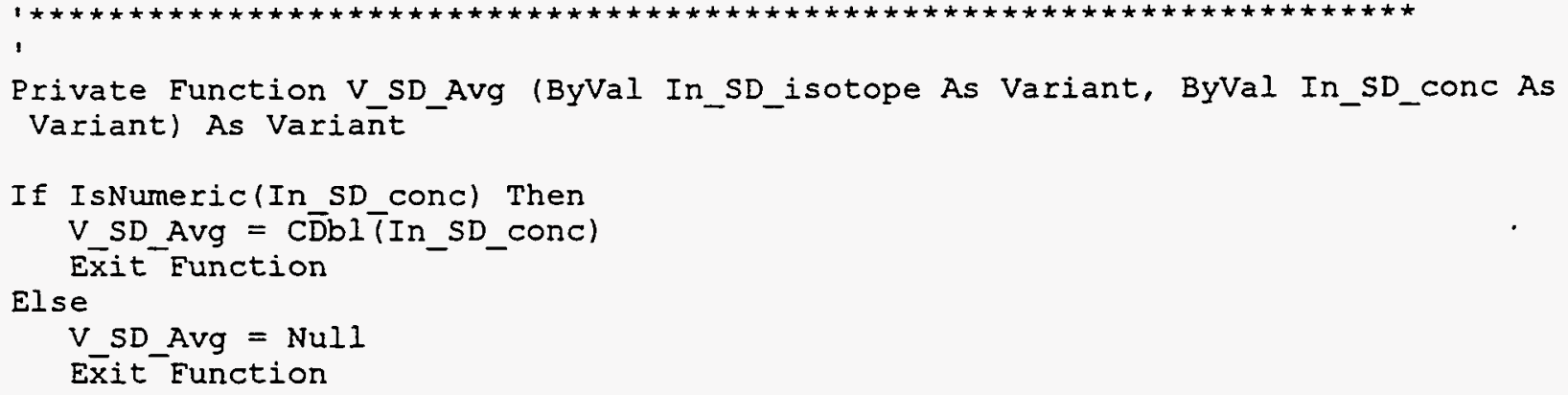

End Function

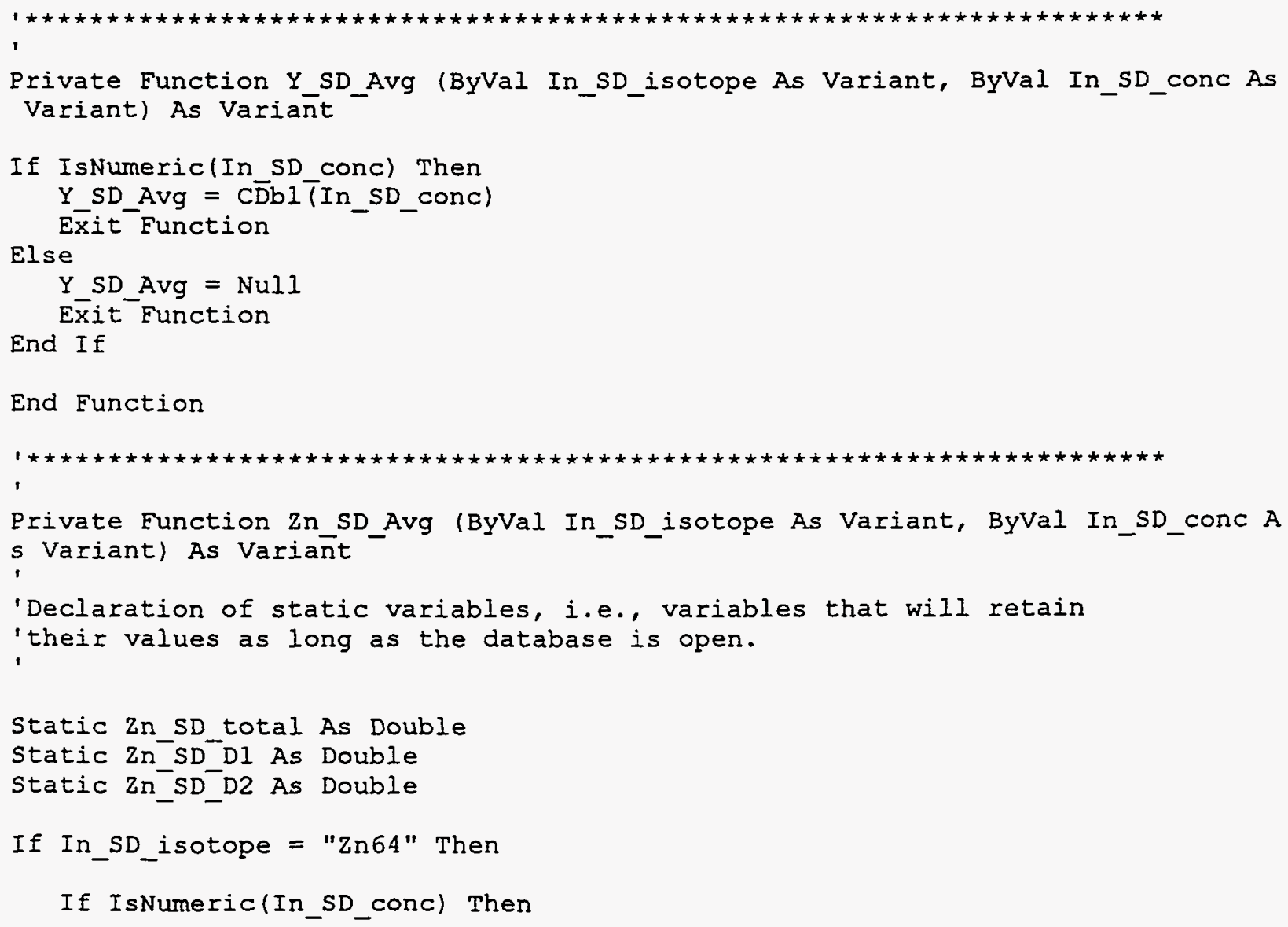

End Function 


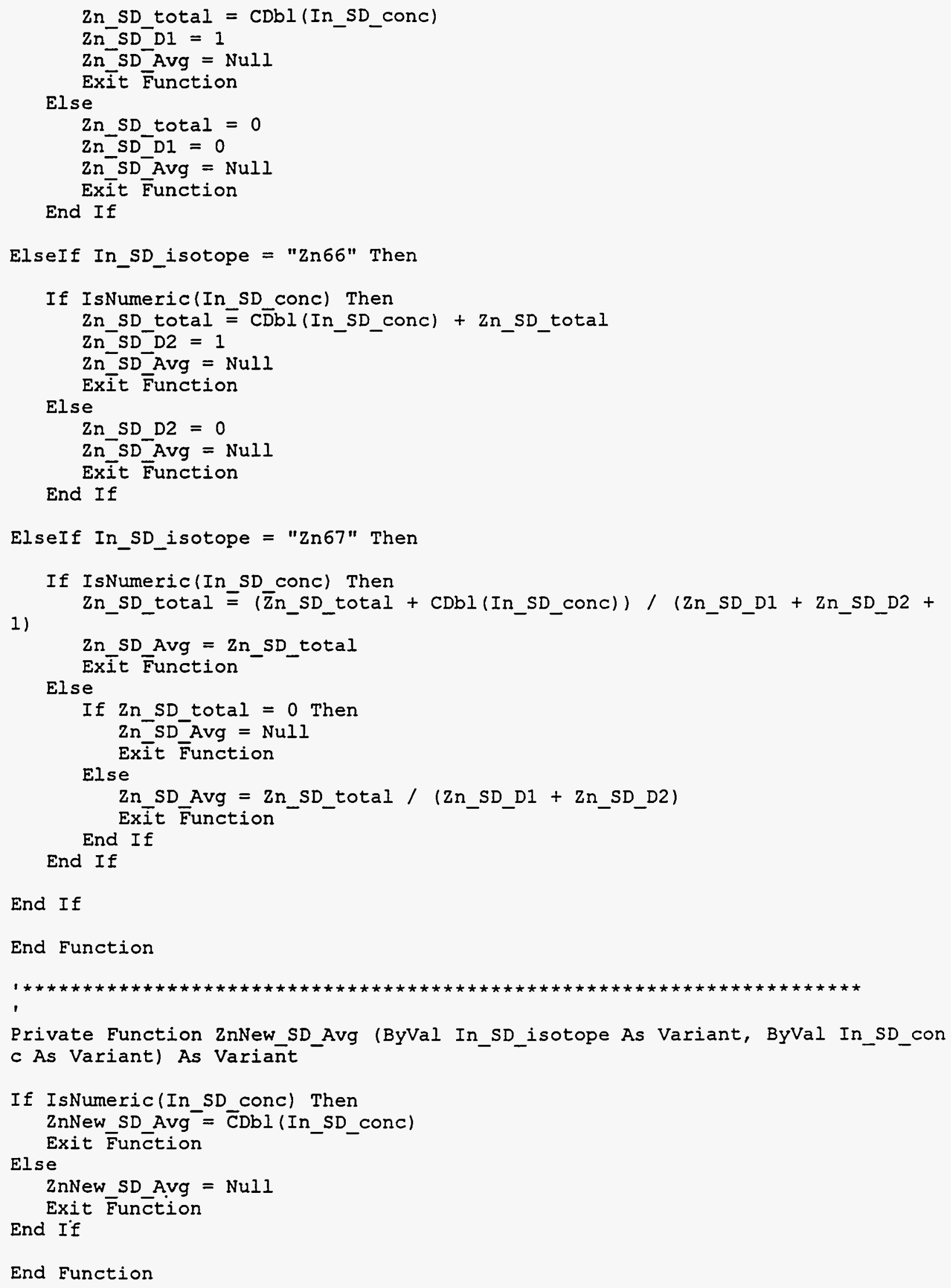




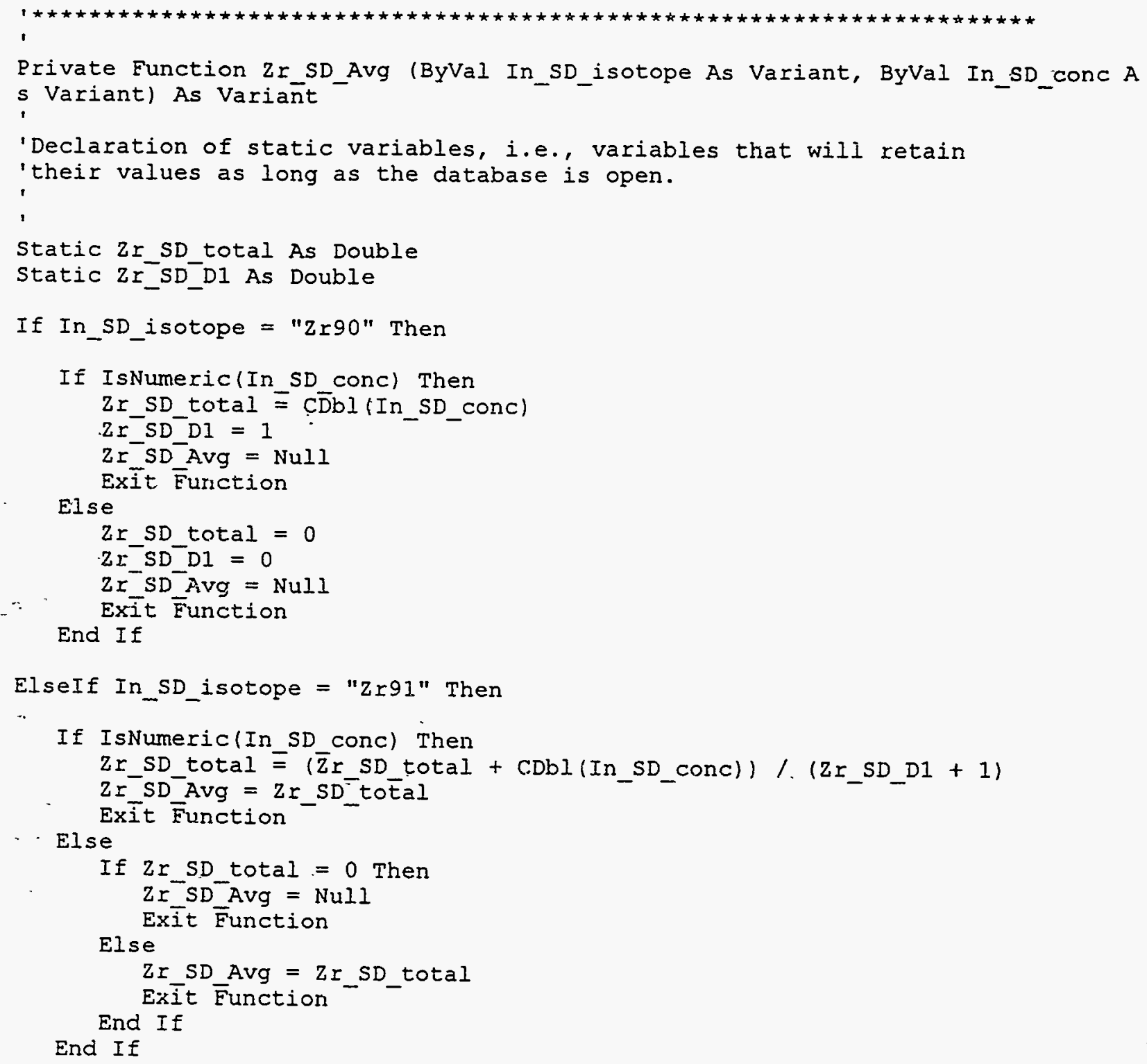

